\title{
RECENT ADVANCES IN
} Pharmaceutical

\section{SCIENCES}

\author{
EDITOR
}

DEEGO MUNOZ-TORRERO
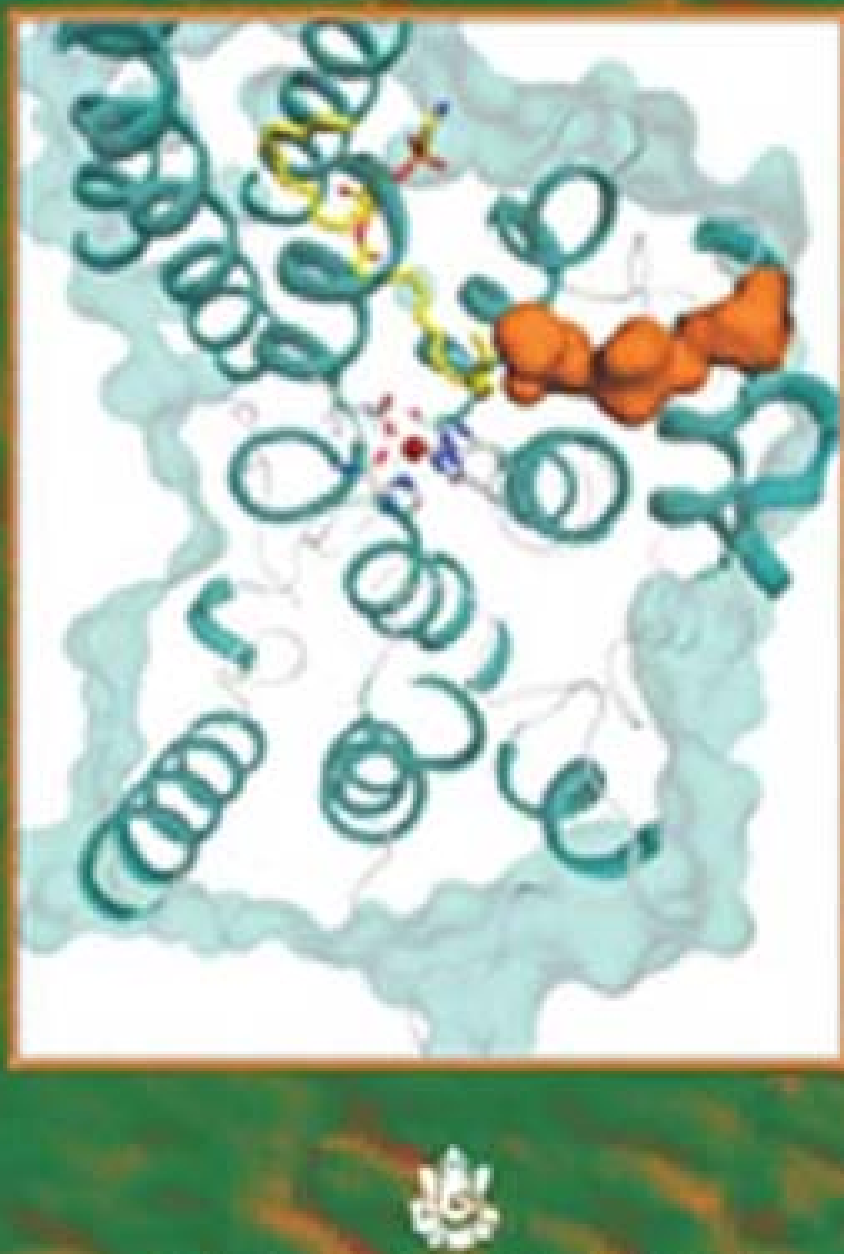

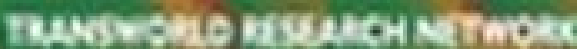




\title{
Recent Advances in Pharmaceutical Sciences
}

\author{
Editor \\ Diego Muñoz-Torrero
}

Voe-Dean of Research, Faculty of Pharmacy, University of Barcolone, Spain

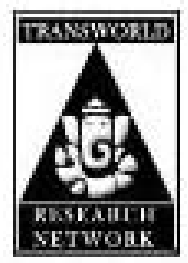

Transworld Research Network, T.C. 37/661 (2), Fort P.O., Trivandrum-695 023 Kerala, India 
Published by Transworld Research Network

2011; Rights Reserved

Transworld Research Network

T.C. 37/661(2), Fort P.O.,

Trivandrum696023, Kerala, India

Editor

Diego Mñaz-Tanero

Managing Editor

S.G Pandalai

Publication Manager

A Gayeathri

Transworld Research Network and the Editar assume no responsibility for the opinions and statements advanoed by contributars

ISEN $978-81-7896-528-5$ 


\section{Preface}

Pharmaceutical sciences constitute a multidisciplinary field which encompasses a number of areas of both basic and applied sciences, with boundaries becoming increasingly less apparent, with interconnections increasingly growing, and sharing common fundamental concepts and aims, particularly the generation of knowledge around all aspects related to drugs (design and discovery, synthesis or extraction from natural sources, formulation, delivery, pharmacokinetics, pharmacodynamics, disposition, safety, analysis, etc.), identification and control of organisms causing human disease, food technology and hygiene, as well as public and environmental health.

This E-book compiles some of the most recent contributions to the general field of pharmaceutical sciences by internationally recognized research groups of the Faculty of Pharmacy of the University of Barcelone, one of the faculties most active in research of the Spain's leading research university. This E-book consists of 12 chapters, which cover all the above-mentioned aims through contributions in areas such as biochemistry and molecular biology, medicinal chemistry, plant physiology, physiology, nutrition and food science, physical chemistry (molecular modeling), pharmacology, pharmacy and pharmaceutical technology (biopharmacy and pharmacokinetics), botanics, edaphology, microbiology, and parasitology. Chapters 1-5 report recent findings on different therapeutic agents, namely molecular biology or medicinal chemistry approaches to get novel active compounds against cancer (Chapter 1) or influenza virus infections (Chapter 2) or the therapeutic potential of naturally occurring compounds such as Amaryllidaceae alkaloids (galantamine and others) (Chapter 3), resveratrol (Chapter 4), or cocoa phytochemicals (flavonoids) (Chapter 5). Chapter 6 deals with molecular modeling studies addressing the structure and function of heme proteins. Chapter 7 focuses on the study of the neurotoxic effects of the abuse drug MDMA (ecstasy) and their pharmacological prevention. In Chapter 8, the biopharmaceutical and pharmacokinetic aspects of novel dosage forms through the skin and mucosal membranes are discussed. Chapter 9 reports on the evolution of plants of the genus Artemisia, widely distributed in the Northem hemisphere, on the basis of karyological, molecular cytogenetic, and phylogenetic data. In Chapter 10, some phytoremediation and biodegradation strategies against chlorinated solvent soil and groundwater pollution are discussed. In Chapter 11, the first molecular structure of a prokaryotic lipooxigenase and a preliminary phylogenic study of this group of enzymes, which could be involved in biodegradation processes, are reported. Finally, Chapter 12 deals with the qualitative and 
quantitative determination of the presence in swimming pools of oocysts of Cryptosporidium parasites, which are responsible of parasitic diseases of the human intestinal tract, constituting one of the most important waterborne pathogen in developed countries.

Some of these chapters are examples of multidisciplinary works involving collaborations among researchers of different areas of pharmaceutical sciences, an approach which needs to be actively pursued in order to enhance our contributions to human health.

Dr. Diego Muñoz-Torrero 


\section{Contents}

\section{Chapter 1}

Generation of biological association networks: A novel strategy to detect new targets in cancer therapy

Elisabet Selga, Cristina de Almagro, Carlota Oleaga, Núria Mencia

Sara Ramírez, F. Xavier Ruiz, Joume Farrés, Xavier Parés

Rémi Thibaut, Cinta Porte, Veromique Noé and Carlos .I. Ciudad

\section{Chapter 2}

Inhibitors of the $\mathrm{M} 2$ channel of influenza A virus

Maria D. Duque, Eva Torres, Elena Valverde, Marta Barniol

Salvador Guardiola, Matias Rey and Santiago Vázquez.

\section{Chapter 3}

Chemical and biological aspects of Amaryllidaceae alkaloids

Jaune Bastida, Strahil Berkov, Laura Torras, Natalia Belén Pigni

Jean Paulo de Andrade, Vanessa Martínez, Carles Codina

and Francesc Viladomat

\section{Chapter 4}

Resveratrol: A polyphenol with multiple effects

Joana M. Planas, Helena Colom and M. Emilia .Juan

\section{Chapter 5}

Effect of cocoa powder in the prevention of cardiovascular disease: Biological, consumption and inflammatory biomarkers. A metabolomic approach

Mireia Urpi-Sarda, Rafael Llorach, Maria Monagas, Nasiruddin Khan

Moria Rotches-Ribalta, Elena Roura, Rosa Lamuela-Raventos

Ramón Estruch and Cristina Andres-Lacueva 


\section{Chapter 6}

Molecular simulations of globins: Exploring the relationship between structure, dynamics and function

Flavio Forti, Leonardo Boechi, Ana Novo de Oliveira, Damian Bikiel

Pau Arroyo, Alejandro Nadra, Luciana Capece, Axel Bidon-Chanal

Marcelo A. Martí, Darío Estrín and F. Javier Luque

\section{Chapter 7}

Involvement of nicotinic receptors in methamphetamine and MDMA

induced neurotoxicity: Pharmacological studies

Elena Escubedo, Sara García-Ratés, Jordi Camarasa and David Pubill

\section{Chapter 8}

Technological, biopharmaceutical and pharmacokinetic advances:

New formulations of application on the skin and oral mucosa

Ana C. Calpena, Beatriz Clares and Francisco Femúndez

\section{Chapter 9}

Artemisia (Asteraceae): Understanding its evolution using cytogenetic and molecular systematic tools, with emphasis on subgenus Dracunculus Jatme Pellicer, Teresa Garnatje and Joan Vallès

\section{Chapter 10}

Biological remediation approach involving soils and groundwaters polluted with chlorinated solvents in a Mediterranean context

Amparo Cortés, Diana Puigserver, José Maria Carmoma

and Manuel Viladevall

\section{Chapter 11}

Crystallization and resolution of the lipoxygenase of Pseudomonas aeruginosa $42 \mathrm{~A} 2$ and phylogenetic study of the subfamilies of the lipoxygenases Albert Garreta, Xavier Carpena, Montse Busquets, M. Carme Fusté Isnacio Fita and Àngels Manresa

\section{Chapter 12}

Cryptosporidium sp. in public swimming pools in Barcelona 


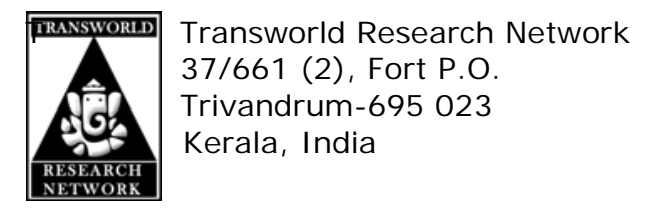

Recent Advances in Pharmaceutical Sciences, 2011: 1-33 ISBN: 978-81-7895-528-5

Editor: Diego Muñoz-Torrero

\title{
1. Generation of biological association networks: A novel strategy to detect new targets in cancer therapy
}

\author{
Elisabet Selga', Cristina de Almagro', Carlota Oleaga', Núria Mencia' \\ Sara Ramírez', F. Xavier Ruiz ${ }^{2}$, Jaume Farrés ${ }^{2}$, Xavier Parés ${ }^{2}$, Rémi Thibaut ${ }^{3}$ \\ Cinta Porte ${ }^{3}$, Veronique Noé' and Carlos J. Ciudad' \\ 'Department of Biochemistry and Molecular Biology, School of Pharmacy, UB, 08028 \\ Barcelona, Spain; ${ }^{2}$ Department of Biochemistry and Molecular Biology, UAB, and \\ ${ }^{3}$ Department of Environmental Chemistry, CSIC, Barcelona, Spain
}

\begin{abstract}
The aim of this work was to design a novel strategy to detect new targets for anticancer treatments. The rationale was to build Biological Association Networks from differentially expressed genes in drug-resistant cells to identify important nodes within the Networks. These nodes may represent putative targets to attack in cancer therapy, as a way to destabilize the gene network developed by the resistant cells to escape from the drug pressure. As a model we used cells resistant to methotrexate (MTX), an inhibitor of DHFR. Selected node-genes were analyzed at the transcriptional level and from a genotypic point of view. In colon cancer cells, DHFR, the AKR1 family, PKC $\alpha$, S100A4, DKK1, and CAV1 were overexpressed while E-cadherin was lost. In breast cancer cells, the UGT1A family was overexpressed, whereas EEF1A1 was
\end{abstract}

Correspondence/Reprint request: Dr. Carlos J. Ciudad, Department of Biochemistry and Molecular Biology School of Pharmacy. University of Barcelona, Spain. E-mail: Cciudad@ub.edu 
overexpressed in pancreatic cells. Interference RNAs directed against these targets sensitized cells towards MTX.

\section{Introduction}

Cells regulate their functions by timely changing the expression of genes in a complex and precise way. This complexity cannot be explained by a collection of separate parts or pathways [1], but through larger networks, which are more informative and real [2]. Biochemical networks can be constructed at several levels and can represent different types of interactions. Literature mining allows the extraction of meaningful biological information from publications to generate networks [3] that, when built from properly curated interactions, can help to uncover the complete biochemical networks of the cells.

In this work, we used biological association networks (BANs) as a tool to define putative targets for gene therapy in combination with methotrexate (MTX). Basically, human cell lines resistant to MTX were developed by stepwise selection with this drug over a long period of time (0.5-1 year). Using total RNA from sensitive and resistant cells, expression microarrays were performed to obtain lists of differentially expressed genes. These lists were used as the input to build networks using the Pathway Architect software. This software confronts the genes in those lists with the information stored in Interaction Databases extracted from the literature using a Natural Language Processing (NLP) tool to construct novel views as to how the entities (the genes) in a list could be interacting with each other, even including entities not present in the original list.

Each generated network shows interplays of relationships among the differentially expressed genes and also includes highly connected nodes. Those gene-nodes support the infrastructure of the network developed in response to the sustained treatment with the drug responsible for the resistance. A novel strategy to select new targets to be used in cancer therapy is to concentrate on a variety of node-genes and test them, alone or in combination, for their effect when inhibited or overexpressed, on the final sensitivity to the main drug (MTX in this case).

The contribution to the resistance to MTX by some of the genes identified by this novel method were new to our knowledge (DKK1, UGT1A, EEF1A1) and some had already been the subject of study of our group since they appeared as differentially expressed in previous works of our team (DHFR, AKR1C1, SP1). 


\section{Identification of gene-nodes}

\subsection{Development of cell lines resistant to methotrexate}

Cell lines representative of 5 types of human cancer were used: HT29 and Caco-2 of colon cancer, MCF-7 and MDA-MB-468 of breast cancer, MIA PaCa-2 of pancreatic cancer, K562 of erythroblastic leukemia, and Saos-2 of osteosarcoma. These cell lines are sensitive to MTX, with a $\mathrm{IC}_{50}$ of 16.7 nM MTX for HT29, $48.7 \mathrm{nM}$ MTX for MDA-MB-468 and 11.6 nM MTX for MIA PaCa-2 cells. IC $_{50}$ were calculated using GraphPad Prism 5 version 5.0a for Macintosh (GraphPad Software, San Diego, California, USA). Resistant cells were obtained in the laboratory upon incubation with stepwise concentrations of MTX (Lederle) as previously described [4]. HT29, Caco-2 and K562 resistant cells were able to grow in $10 \mu \mathrm{M}$ MTX; MIA PaCa-2, Saos-2, MCF-7 and MDA-MB-246 were resistant to $1 \mu \mathrm{M}$ MTX.

\subsection{Microarray data analyses}

Gene expression analyses were performed using three samples of both sensitive and resistant cells for each of the 7 cell lines studied. These analyses were carried out with the GeneSpring GX software v 7.3.1 (Agilent Technologies), using the latest gene annotations available (March 2009). This software package allows multi-filter comparisons using data from different experiments to perform the normalization, generation of restriction (filtered) lists and functional classifications of the differentially expressed genes. The expression of each gene was reported as the ratio of the value obtained for each condition relative to the control condition after normalization of the data. Additionally, a filter was performed by selecting the genes that displayed a p-value, corrected by false discovery rate (Benjamini and Hochberg FDR), of less than 0.05 . The output of this analysis was then filtered by fold expression. Thus, lists of differentially expressed genes by 2 -fold were generated for each of the 7 resistant cell lines.

\subsection{Common genes between cell lines}

The lists of genes differentially expressed by 2 -fold with a $p$-value $<0.05$ including multiple testing correction for each cell line were split in overexpressed and underexpressed genes. Lists of overexpressed genes were compared by using Venn Diagrams in GeneSpring GX. Lists of underexpressed genes were also compared among them using the same 
approach. All lists were compared in pairs and lists of genes in common between each pair were generated.

\subsection{BANs generation}

Biological Association Networks (BANs) were constructed with the aid of Pathway Architect software v3.0 (Stratagene-Agilent). Briefly, this software package generates interaction networks starting with the genes in a given list (entities) taking into account the information present in a database of known molecular interactions. The lists correspond to the collection of differentially expressed genes under specific conditions. The database of molecular interactions is composed by millions of interactions divided into different classes (binding, regulation, promoter binding, transport, metabolism, protein metabolism and expression). The interactions are extracted from literature using a Natural Language Processing tool run on Medline Abstracts (NLP references), plus those obtained from external curated databases like BIND [5] and MINT [6]. Interactions in the interaction database are scored into 5 different categories: maximum, high, medium, low and minimum. Curated interactions (BIND and MINT sources) get the maximum quality scores as do any interactions that have at least 3 NLP references. Pathway Architect gathers all that information to construct novel views as to how the entities in a list could be interacting with each other, including entities not present in the original list (neighbours resulting from the expanded interaction). Customized analyses were performed to select relevance interaction networks with an associated high confidence index since such networks are likely to mirror biological significance. One-step expansion (expand network) of the original set of entities with maximum score interaction was then analyzed by setting an advanced filter that included the categories of binding, expression, metabolism, promoter binding, protein modification and regulation. This procedure gives a final view formed by a collection of nodes, with different degrees of interrelationship. On the other hand, a number of gene products from the original lists were not significantly connected with the other members or neighbours, and therefore were removed from the final view. Finally, overlapping of the expression levels to the members of the network was also performed.

When generating the BAN out of the list of the common genes differentially expressed in HT2929 and $\mathrm{CaCo} 2$ colon cancer cells, different nodes including DKK1, AKR1C and DHFR were detected (Fig. 1). 


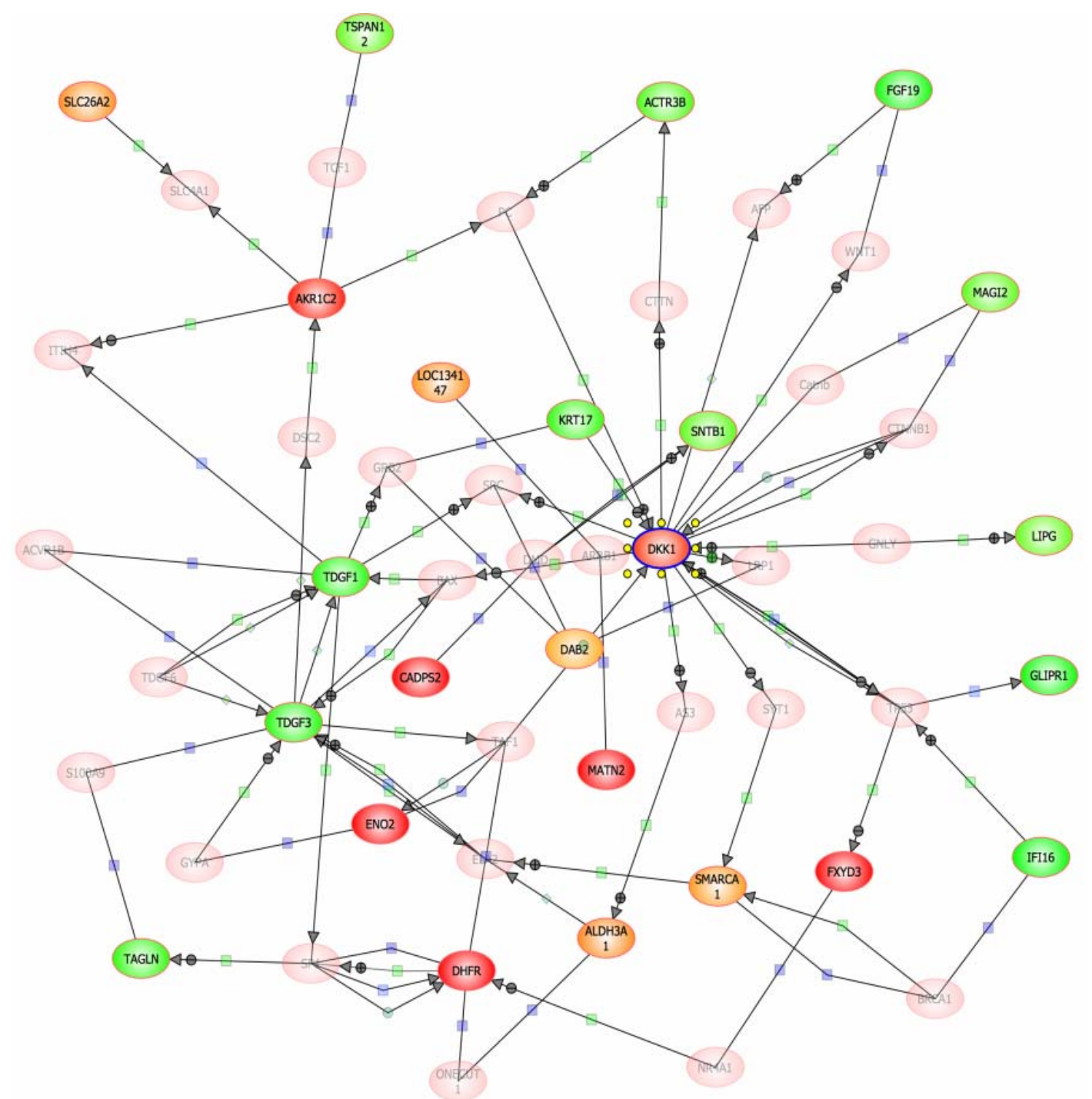

Figure 1. Network constructed with differentially expressed genes in common between HT29 and $\mathrm{CaCo} 2$ cells. Overexpressed genes are displayed in red while underexpressed genes are shown in green. Translucent shading represents genes that were not in the list and were added by the program from the interaction database.

When the BAN was generated from the list of differentially expressed genes in common between MCF7 and MDA-MB468 breast cancer cells, a main node including different members of the UGT1A family was formed (Fig. 2).

On the other hand, when the list came from the intersection of the differentially expressed genes in $\mathrm{K} 562$, MiaPaCa and $\mathrm{SaOs} 2$ cells, a node coresponding to EEF1A1 was obtained (Fig. 3). 


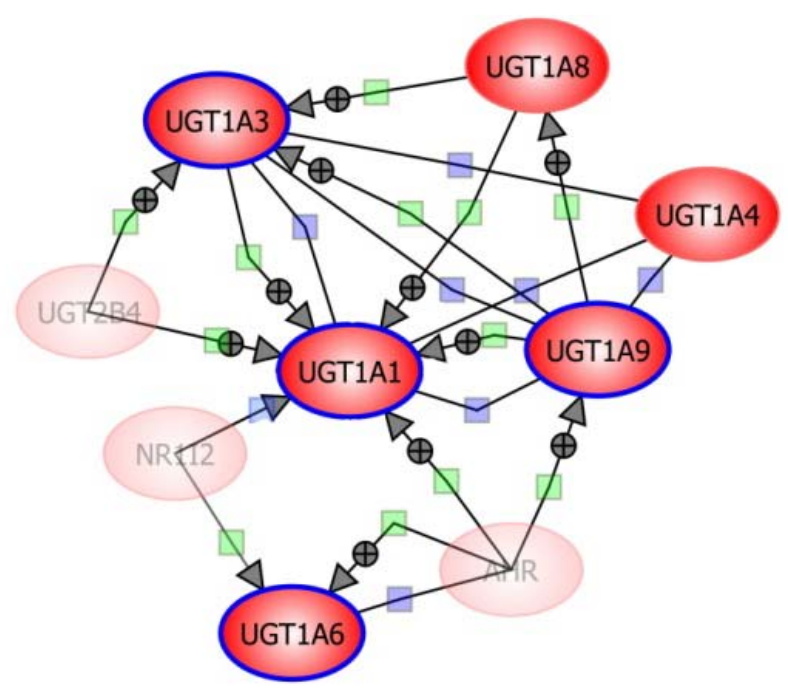

Figure 2. BAN generated using a list of differentially expressed genes in common between MCF7 and MDA-MB-468 breast cancer cells. Overexpressed genes are displayed in red while underexpressed genes are shown in green. Translucent shading represents genes that were not in the list and were added by the program from the interaction database.

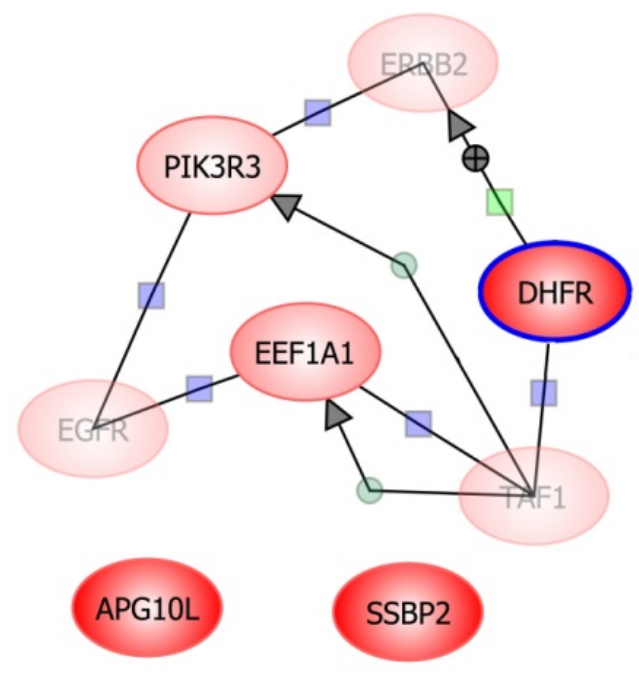

Figure 3. BAN generated using the differentially expressed genes among K562, $\mathrm{MiaPaCa}$ and $\mathrm{SaOs} 2$ cells. Overexpressed genes are displayed in red while underexpressed genes are shown in green. Translucent shading represents genes that were not in the list and were added by the program from the interaction database.

An additional BAN is presented which was generated with a list including all the entities from the three lists of differentially expressed genes (from colon cancer, breast cancer and among K562, MiaPaCa and $\mathrm{SaOs} 2$ ) (Fig. 4). In this case, DKK1, UGT1A1, EEF1A1 and AKR1C can be seen as 


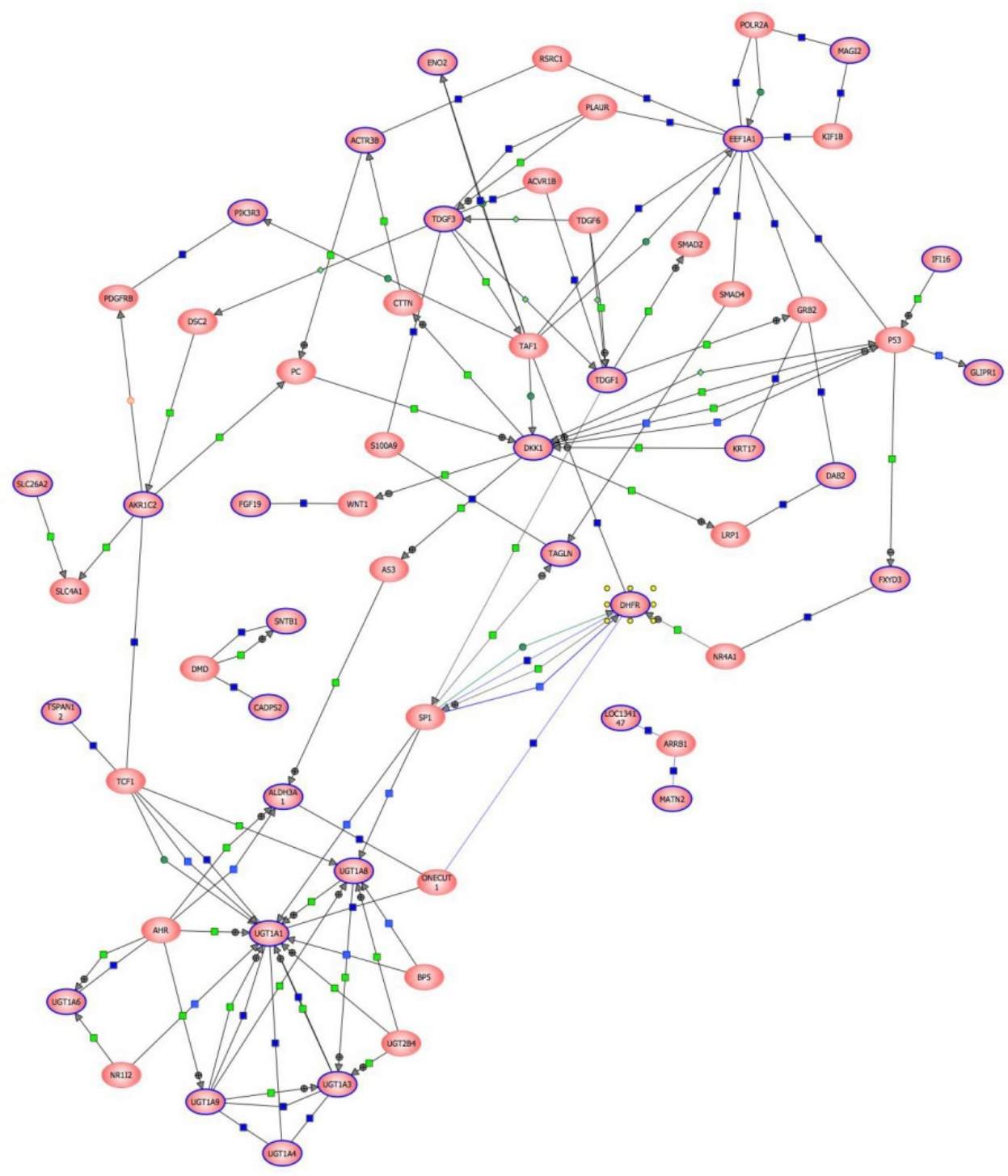

Figure 4. BAN generated using the list of the common differentially expressed genes in cells from colon cancer, breast cancer and among K562, MiaPaCa and $\mathrm{SaOs} 2$.

major nodes of the network, although other minor nodes can also be observed, such as Sp1.

The description of the regulation and characteristics of each target gene is given below considering the cell type and from the point of view of their contribution to resistance to the chemotherapeutic agent methotrexate.

\section{MTX resistance in colon cancer}

Colorectal cancer is the third most common form of cancer and the second leading cause of cancer-related death in the Western world. Colon 
cancer causes 655,000 deaths worldwide per year [7]. Therapy is usually through surgery, followed in many cases by chemotherapy, which is used to slow tumor growth, to shrink tumor size and to reduce the likelihood of metastasis development. Chemotherapy effectiveness in colon cancer cells is usually compromised by the achievement of drug resistance. Therefore, gaining insight into the mechanisms underlying drug resistance is basic to develop more effective therapeutic approaches for colon cancer. With this goal in mind, we performed a genome wide analysis of two colon cancer cell lines, namely HT29 and CaCo-2. The expression profiles derived from these microarrays evidenced changes in a wide proportion of genes. We further studied some of these differentially expressed genes and proposed a role for all of them on MTX resistance in HT29 cells.

\subsection{Role of DHFR on MTX resistance}

MTX resistance can be easily acquired through different ways, although amplification of the target gene (Dihydrofolate reductase, $d h f r$ ) has been shown to be the most important mechanism of resistance in cultured cells [8].

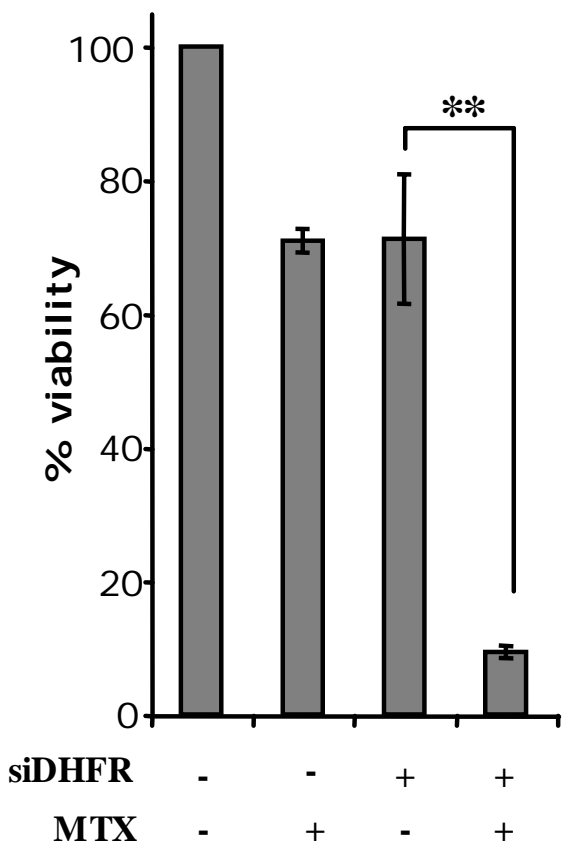

Figure 5. Effects of modulating DHFR mRNA levels on HT29 viability. Cells were treated with a siRNA against DHFR, MTX was added after $48 \mathrm{~h}$, and cell viability was assessed after 3 days from the beginning of the treatment. All results are expressed as percentages referred to a non-related negative control siRNA (NR-siRNA). Values are the mean of three independent experiments \pm SE. ${ }^{* *} \mathrm{p}<0.01$. 
The human colon adenocarcinoma cell line HT29 can be adapted to grow in high concentrations of MTX and concomitantly develop amplification of the $d h f r$ gene. We detected that DHFR was overexpressed by about 10 fold in HT29 resistant cells, and determined that the $d h f r$ gene had undergone an amplification process of about 10 -fold compared to the sensitive cells.

Moreover, use of iRNA technology caused a huge sensitization of HT29 cells toward MTX (Fig. 5), thus confirming a role for DHFR in MTX resistance. CaCo-2 MTX-resistant cells showed a 50-fold overexpression of DHFR, which originated from an increased gene copy number (about 80-fold) in the resistant cells.

\subsection{Role of genes flanking DHFR on MTX resistance}

Amplification of 5q12-14 regions, where $d h f r$ is located, has been described in MTX-resistant HT29 cells [9]. A cluster of genes flanking the dhfr locus in chromosome 5 were overexpressed and amplified in MTX-resistant HT29 cells (Fig. 6). The genes included in this cluster are known to be involved in DNA repair, in cell signalling, in apoptosis prevention or in cell proliferation.

Despite the confirmation of the co-amplification of all these genes with dhfr in HT29 resistant cells, we did not observe a clear sensitization toward MTX when reducing their respective mRNA levels by means of iRNA technology. Our observations indicate that the increase in copy-number and the resulting upregulation of the studied genes in $5 \mathrm{q} 14$ may be a consequence of $d h f r$ amplification more than an adaptation of the cells to MTX resistance. Indeed, many mammalian species (mouse, rat, bull, cock, dog and chimpanzee) show this set of genes in the same order around dhfr as in human chromosome 5 (using the MapViewer at NCBI), indicating a conserved pattern of gene organization. In keeping with this, its overexpression in the resistant cells could have been useful to improve some cellular processes that might facilitate survival. However, the increase in copy number of this set of genes does not directly favour MTX resistance.

\subsection{Role of AKR1C1 on MTX resistance}

Members of the Aldo-Keto Reductase (AKR) superfamily are monomeric cytoplasmic proteins of about 320 amino acid residues, which catalyze the $\mathrm{NAD}(\mathrm{P}) \mathrm{H}$ - dependent oxido-reduction of a wide range of substrates [10]. AKR1C family members' overexpression has been previously related to some types of cancer, and has been correlated with a poor prognostic outcome and 


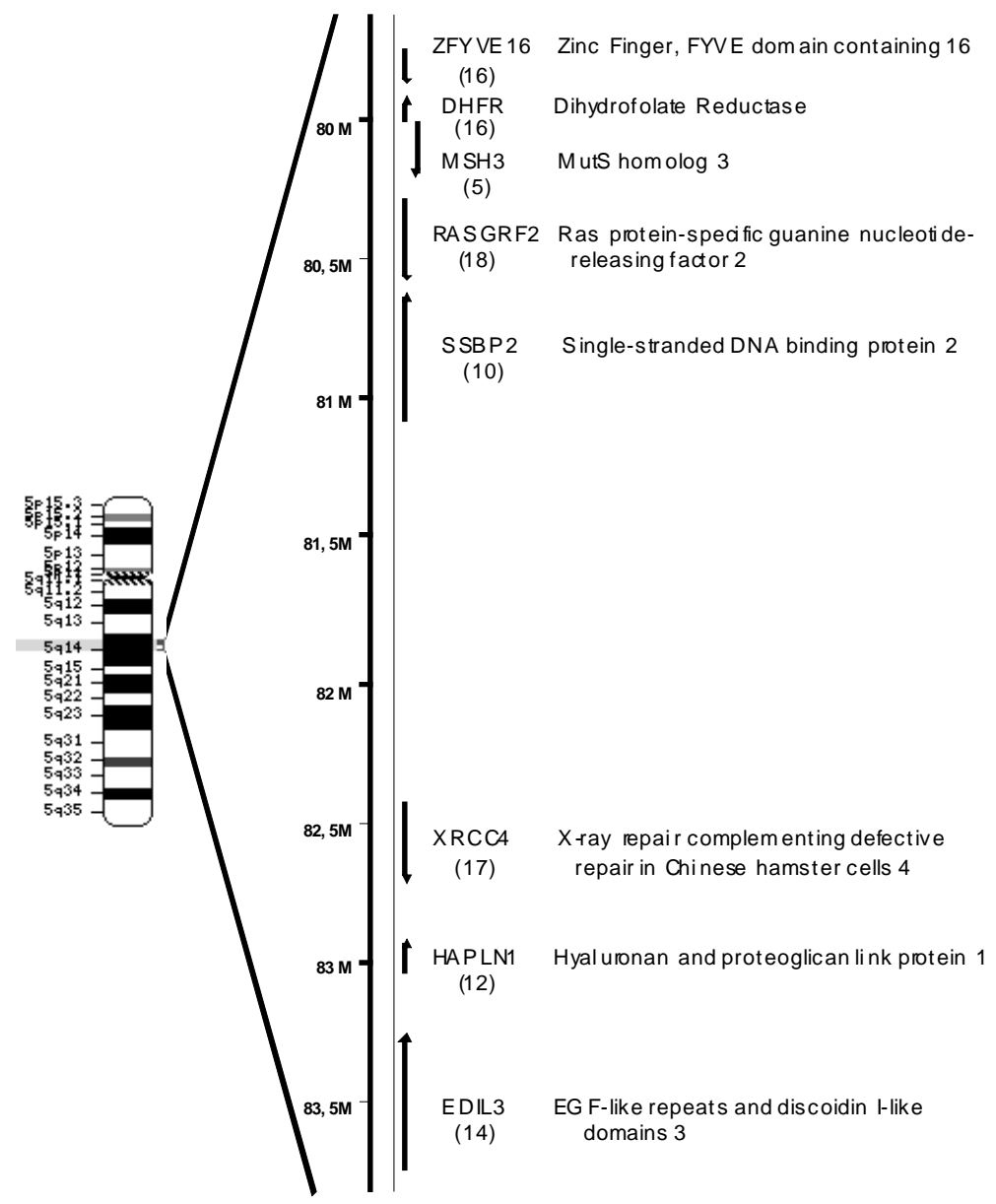

Figure 6. Localization of $d h f r$ and other genes in chromosome 5 that are overexpressed in HT29 MTX-resistant cells. It is presented a magnification of the region in chromosome 5 where $d h f r$ is located (5q14). The left part is an ideogram of chromosome 5; the right part shows the relative position of all genes studied that are located in this chromosome and that were amplified. The arrows indicate their transcription orientation and the values in parentheses under the names correspond to their respective copy-number validated by Real-Time PCR.

with disease progression [11]. We contributed to the field [4] by demonstrating an association between AKR1C1 expression levels and colon cancer. Indeed, this gene showed a 7-fold overexpression in HT29 colon cancer cells resistant to MTX. AKR1C1 upregulation has been previously related to drug (daunorubicin, cisplatin, adriamycin and ethacrynic acid)resistance in a wide variety of cancers [12]. It has been suggested that AKR1C1 could metabolize some anticancer drugs since high similarity exists between drug chemical structures and some compounds that can be processed by this enzyme [11]. Thus, AKR1C1 would have a putative role in detoxification processes. 
Our results showing an increased AKR1C1 activity in HT29 MTXresistant cells (Fig. 7) are in agreement with this possibility. AKR1C1 would play a role on the detoxification of MTX in the resistant cells, a process that would have contributed to the establishment of drug resistance in this cell line.

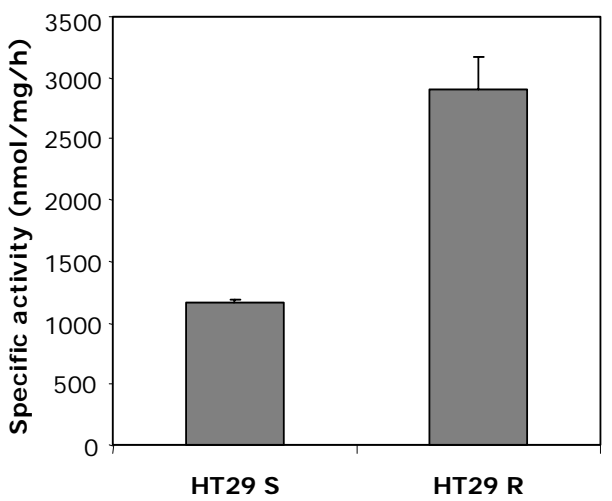

Figure 7. AKR1C specific activity. AKR1C activity was determined in protein extracts from both sensitive and MTX-resistant HT29 cells by measuring the change in absorbance at $340 \mathrm{~nm}$, using 9,10-phenanthrenequinone as a substrate. To distinguish between unspecific and AKR1C specific activity, the AKR1C inhibitor flufenamic acid (FA) was added in the activity assay. The remaining activity upon FA inhibition was considered as non-AKR1C activity and was subtracted from the total activity. Results are shown as means $\pm \mathrm{SE}$ from three independent experiments.

A

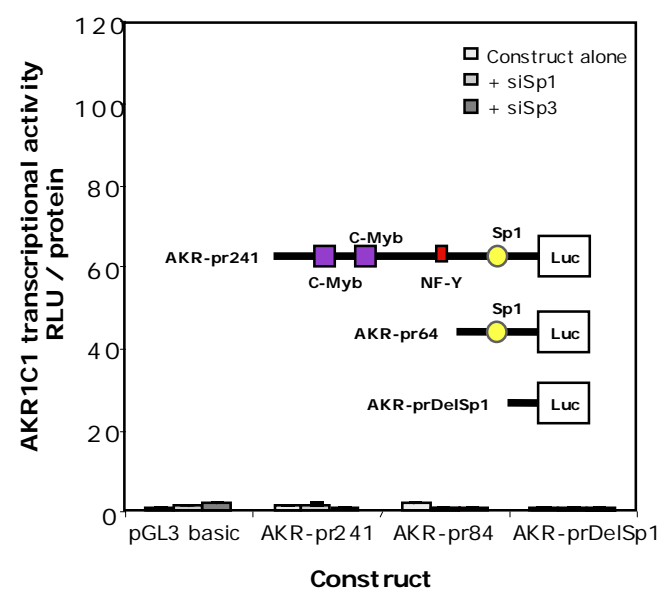

B

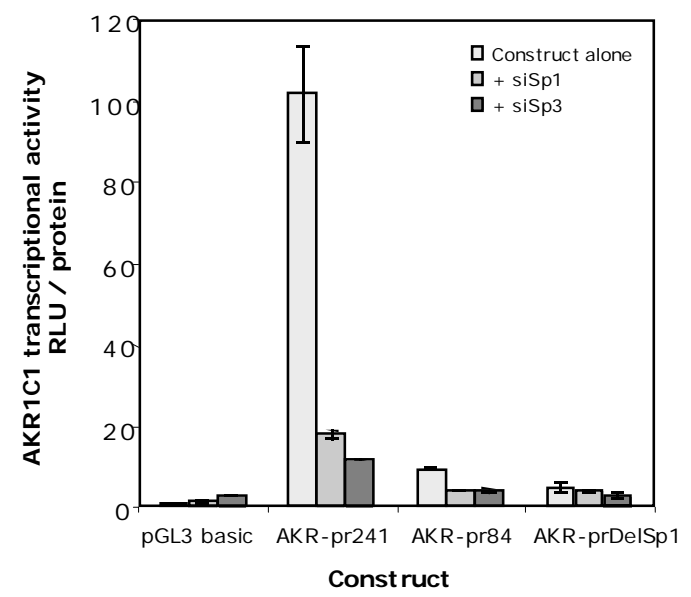

Figure 8. AKR1C1 transcriptional activity. HT29 sensitive (A) and resistant cells (B) were incubated with siRNAs against either $\mathrm{Sp} 1$ or Sp3. Twenty-four hours later, cells were transfected with one of the three different AKR1C1 promoter deletion constructs (see inset), and luciferase (Luc) activity (RLU, relative light units) was assayed after 30h. The protein content was used to normalize the Luc activity for each sample, which is expressed relative to that of pGL3 basic vector (means \pm SE for triplicate wells). 
It is of note that the increase in RNA and $\mathrm{AKR} 1 \mathrm{Cl}$ protein in the resistant cells were at the same level, about 7-fold, which suggests that the overexpression is caused mainly by an increase in mRNA abundance rather than by a translational control. The increase in mRNA was not due to amplification of the akr1c1 locus but to transcriptional regulation. In this regard, we could conclude, from luciferase experiments (Fig. 8), that: i) there is a vast increase in transcriptional activity originating from the AKR1C1 promoter in the resistant cells compared to the sensitive cells; and ii) AKR1C1 transcriptional activation in the resistant but not in the sensitive cells depends at least in part on Sp1 and also Sp3. Gel-shift experiments (Fig. 9) also corroborate the role of $\mathrm{Sp} 1$ and $\mathrm{Sp} 3$ in regulating $\mathrm{AKR} 1 \mathrm{C} 1$ in the resistant cells.

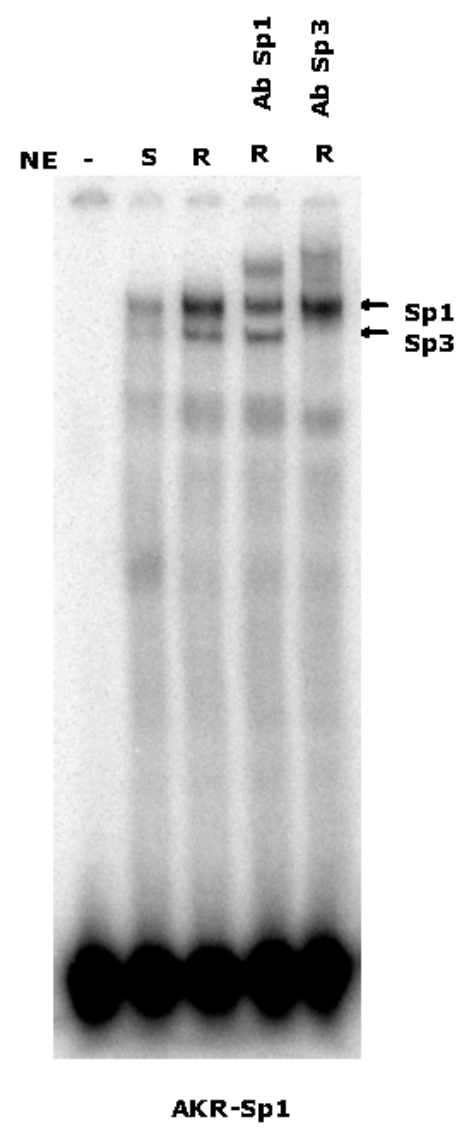

Figure 9. Characterization of $\mathrm{Sp} 1$ and $\mathrm{Sp} 3$ binding to AKR1C1 promoter. Binding reactions were performed with $20,000 \mathrm{cpm}$ of each ds probe, $2 \mu \mathrm{g}$ nuclear extracts (NE) from exponentially growing HT29 sensitive (S) or MTX-resistant (R) cells and $1 \mu \mathrm{g}$ poly $[\mathrm{d}(\mathrm{I}-\mathrm{C})]$ as the nonspecific competitor. Supershift mobility assays were performed in the presence of specific antibodies against either $\mathrm{Sp} 1$ or $\mathrm{Sp} 3$ (Ab Sp1 or $\mathrm{Ab} \mathrm{Sp} 3$, respectively). Shifted and supershifted bands are indicated by arrows. 
As there was no difference in the levels of $\mathrm{Sp} 1$ protein between resistant and sensitive cells, we explored the phosphorylation status of this transcription factor in the two types of cells, taking into account that phosphorylated Sp1 shows increased binding compared to the nonphosphorylated form [13]. Indeed, the increase in Sp1 binding in the resistant condition could be due to a higher phosphorylation state of Sp1 as dephosphorylation of the extracts coursed with a decrease in its binding. In this direction, we searched for the differential expression, in the microarray data, of those kinases and phosphatases known to control the phosphorylation state of Sp1 [14]. Within the kinases, PKC alpha was overexpressed. The role of protein kinase $\mathrm{C}$ alpha $(\mathrm{PKC} \alpha)$ on MTX resistance in HT29 cells is discussed below.

Trying to mimic the overexpression of AKR1C1 present in the resistant cells, we transiently transfected an expression vector for this gene into HT29 sensitive cells, which resulted in a decrease in sensitivity toward methotrexate. We also decreased AKR1C1 mRNA levels by means of iRNA technology, and observed an increase in sensitivity toward MTX. These results supported the idea that the overexpression of AKR1C1 in the resistant cells may contribute, at least partially, to the resistant phenotype.

A relationship between AKR and proliferation has been reported [15]. We demonstrated that AKR1C1 overexpression counteracts the cell cycle S-phase arrest caused by MTX (Fig. 10A), suggesting that AKR1C1 activity may be needed for the cells to progress throughout the cell cycle. This could be a strategy, concomitant with the amplification of the $d h f r$ locus, for the resistant cells to bypass the metabolic pressure exerted by MTX on nucleotide synthesis. Since AKR1C1 is functionally involved in the metabolism of xenobiotics, its activity might be acting either on MTX directly or on one of its metabolites to decrease the concentration of a DHFR inhibitor. In addition, AKR activity inversely correlates with apoptosis [16] and has been proposed to lead to apoptosis-related development of drug resistance. Our results are in keeping with this possibility since overexpression of AKR1C1 counteracts the apoptosis induced by MTX (Fig. 10B).

In summary, AKR1C1 overexpression may represent a mechanism, parallel to DHFR amplification, which can contribute to the establishment of MTX resistance. Its overexpression partially reverses both the MTXinduced S-phase arrest of the cell cycle and the apoptosis caused by this chemoterapeutic agent. The increase in AKR1C1 mRNA is transcriptionally regulated, at least in part, by Sp1. Silencing of AKR1C1 by iRNA technology improves the sensitivity toward MTX. 


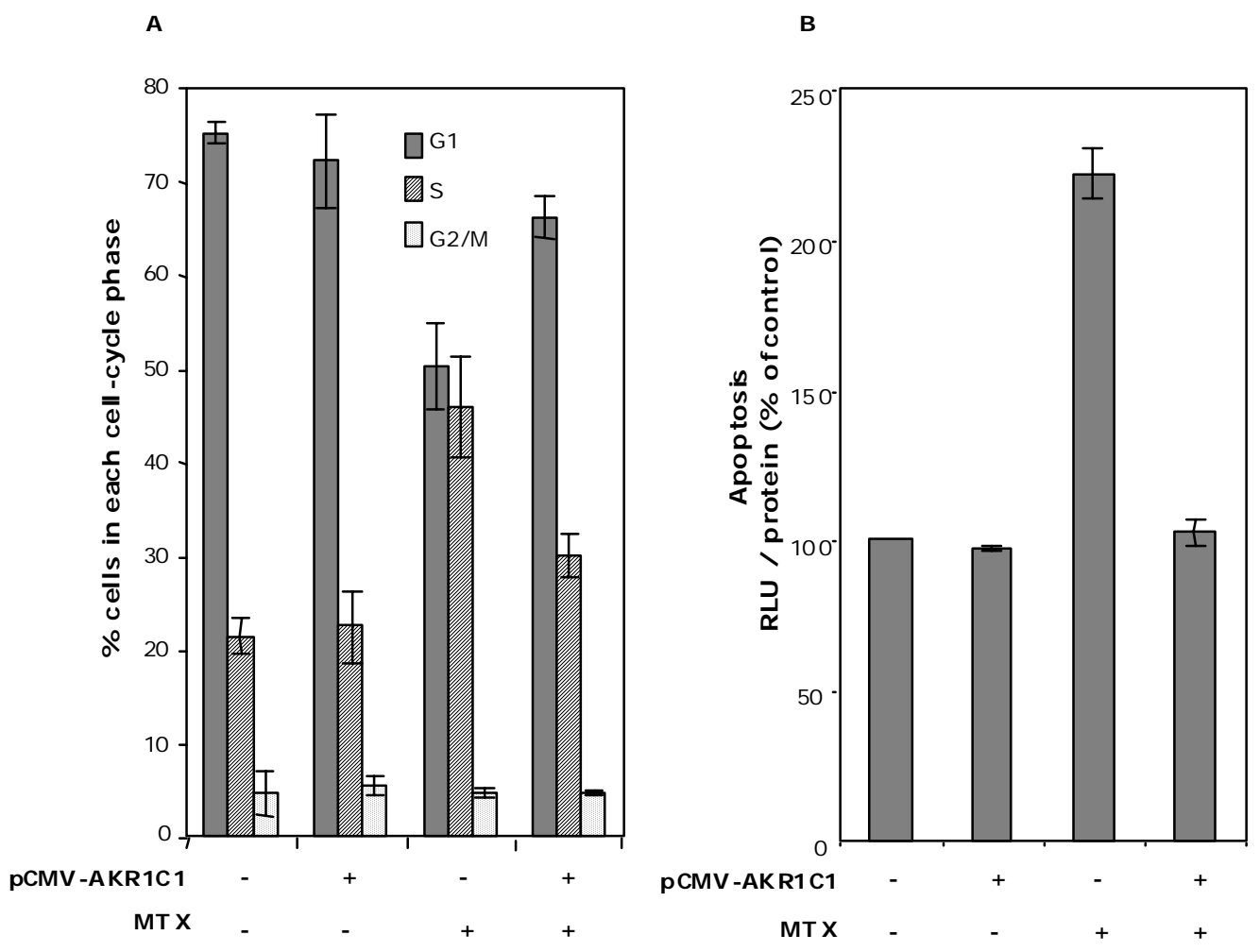

Figure 10. Changes in the cell cycle and in the apoptosis caused by AKR1C1 overexpression. A) Changes in cell cycle distribution. AKR1C1 was transiently overexpressed by means of an expression vector (pCMV6-XL5-AKR1C1). MTX was added 1 hour after plasmid treatment. After forty-eight hours of drug incubation, cells were collected, stained with propidium iodide and analyzed for DNA content using flow cytometry. Results are shown as means \pm SE from at least three independent experiments. B) Changes in apoptosis. Overexpression of AKR1C1 was performed by transfecting its expression plasmid, either alone or in combination with MTX, for 24 hours in HT29 sensitive cells. MTX was added during the last six hours of treatment. Caspase 3/7 activation was finally measured and expressed as percentage of relative light units (RLU) referred to the control and normalized by protein content (means \pm SE from at least three independent experiments).

\subsection{Role of PKC $\alpha$ on MTX resistance}

PKC $\alpha$-mediated protein phosphorylation triggers a wide variety of cellular responses including proliferation, differentiation, membrane transport, gene expression and tumor promotion [17]. Chemical inhibitors of PKC activity have been proposed as resistance modulators in MTX chemotherapy [18]. Furthermore, decreasing PKC $\alpha$ mRNA levels attenuates the MDR phenotype in tumor cells and increases the sensitivity to anticancer drugs, both in vitro and in vivo. These observations are in accordance with our results [19] 
showing that the decrease of PKC $\alpha$ mRNA levels by means of iRNA technology causes a sensitization of the cells toward MTX (Fig. 11A). It is interesting to note that this kinase has already been related to MTX resistance through a mechanism involving transcription factor Sp1 [13]. In HT29 resistant cells, this relation could be explained by the regulation of AKR1C1 transcriptional activity through Sp1 (see above).

\subsection{Role of CAV1 on MTX resistance}

Caveolin 1 (CAV1), the principal component of caveolae, has been associated with progression of colon and breast carcinomas and with enhanced invasiveness in lung adenocarcinoma cells [20]. Although suggested as tumor suppressor gene, and downregulated in some oncogene-transformed and tumor-derived cells [21], overexpression of CAV1 has been found in prostate and esophageal cancer [22]. Moreover, re-expression of CAV1 at latter stages of tumor development has been described in human and mouse prostate adenocarcinomas, a scenario that could resemble chemotherapy resistance. Indeed, Bender et al. [23] found significantly higher levels of CAV1 in MTX resistant HT29 clones. Our experiments using iRNA technology against CAV1 (Fig. 11B) allowed us to confirm the implication of this gene in MTX resistance in our HT29 cell line.

\subsection{Role of E-cadherin on MTX resistance}

Loss of E-cadherin, frequently observed in epithelial tumors, has been associated with tumor progression and is considered a crucial event that favours metastasis and invasiveness [24]. There is a functional correlation between E-cadherin levels and malignancy. Our experiments show a decrease of 3-fold in E-cadherin levels in resistant cells, and also that a mild overexpression of E-cadherin causes a higher sensitivity toward MTX (Fig. 11C).

\subsection{Role of S100A4 on MTX resistance}

S100A4 is a member of the S100 calcium binding protein family. S100A4 is involved in a wide variety of intra- and extracellular processes, such as cell cycle progression, cell motility and as a modulator of intercellular adhesion and of the invasive properties of cells [25]. Overexpression of S100A4 has been associated with tumor malignancy as well as to metastasis, angiogenesis and chemoresistance [26]. A correlation between S100A4 expression levels 

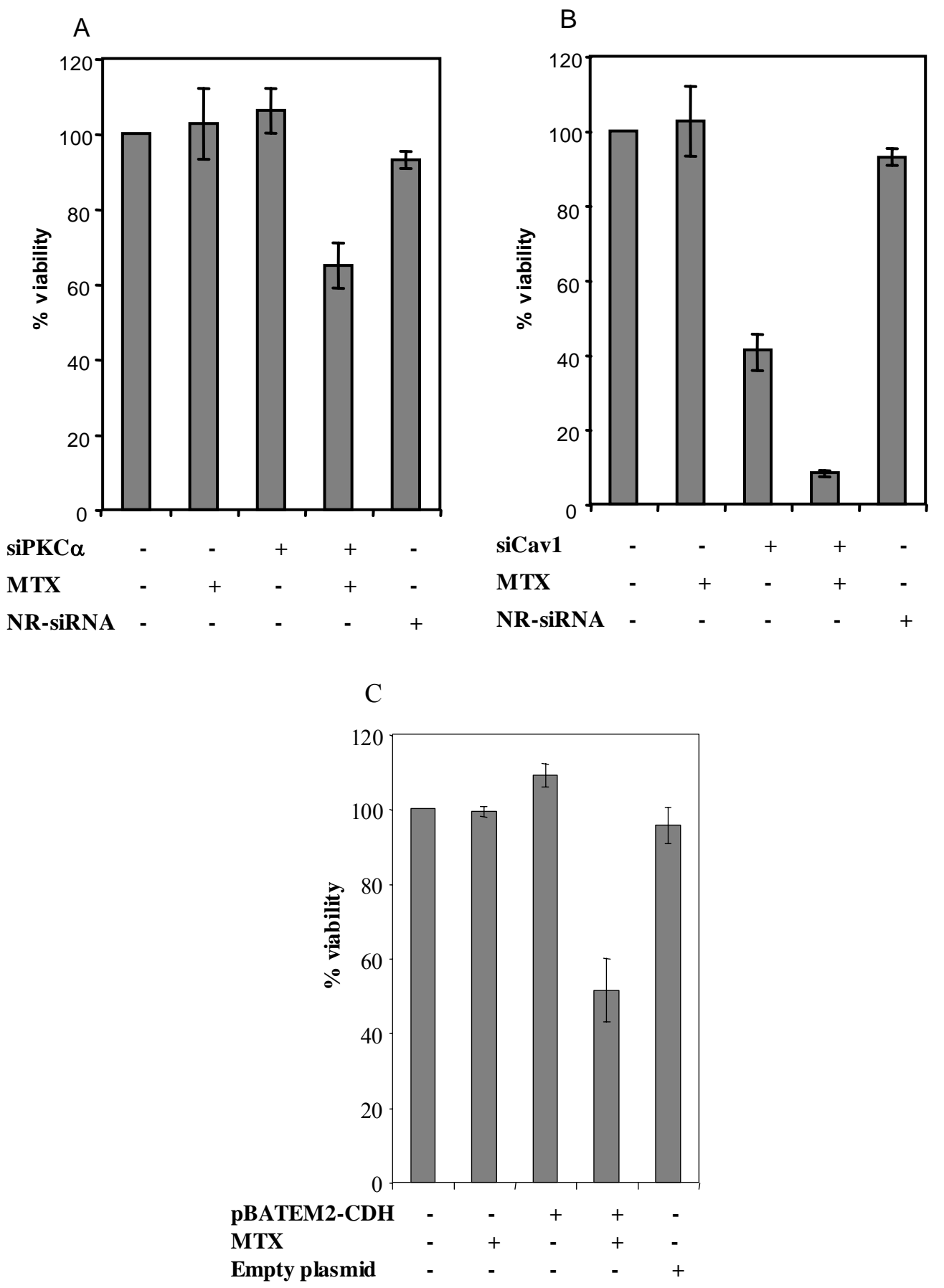

Figure 11. Effects of modulating PKC $\alpha$, Cav1 or E-Cadherin mRNA levels on cell viability. Cells were treated with a siRNA against PKC $\alpha$ (A) or against Cav1 (B), or with an expression plasmid for E-Cadherin (pBATEM2-CDH; C). MTX was added after $48 \mathrm{~h}$, and cell viability was assessed after 5 days from the beginning of the treatment. All results are expressed as percentages referred to untreated cells. A nonrelated (NR) siRNA or an empty plasmid was used as negative control. The mean values $\pm \mathrm{SE}$ of three independent experiments are depicted. 
and the invasive potential of HT29 cells has been suggested. Importantly, we could observe a reversion of the cytotoxicity caused by MTX upon transfection with S100A4 expression vector, and transfection of a siRNA against S100A4 in HT29 sensitive cells caused chemosensitization of these cells toward MTX (Fig. 12) [27].

The present results are in agreement with Mahon et al. [28] that showed that inhibition of S100A4 expression results in an increased sensitivity of pancreatic ductal adenocarcinoma cell lines to gemcitabine treatment and induced apoptosis. Our results provide evidence that S100A4 acts as a pro-survival factor that contributes to chemoresistance in HT29 MTXresistant cells.
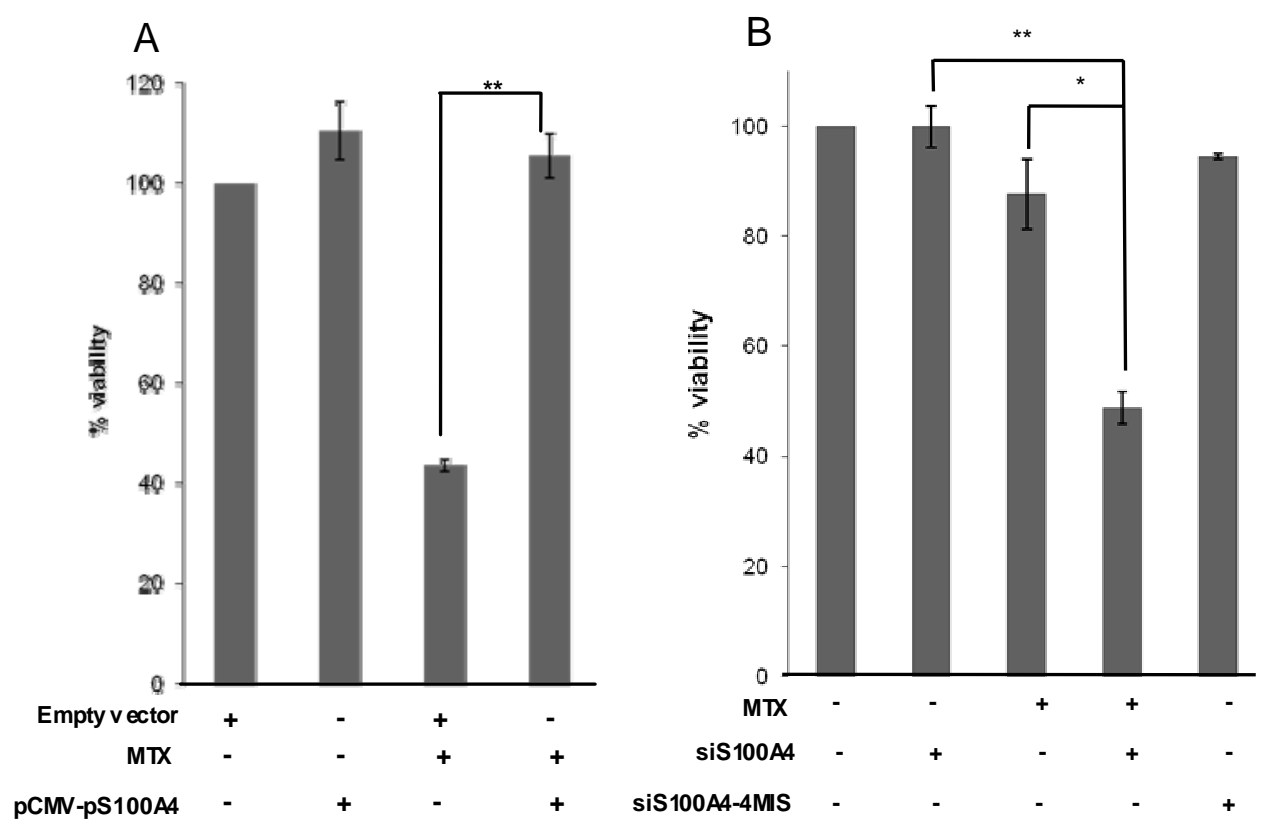

Figure 12. Effects of modulating S100A4 mRNA levels on cell viability. HT29 cells were treated with either the expression plasmid for S100A4 (pCMV-S100A4; A) or with a siRNA against its RNA (siS100A4; B), and MTX was added 48h later. Cell viability was assessed by the MTT assay 3 days after MTX treatment. Results are expressed as percentages referred to the untreated cells. Values are the mean of three independent experiments \pm SE. ${ }^{*} p<0.05,{ }^{* *} p<0.01$.

\subsection{Role of DKK1 on MTX resistance}

Dikkopf homolog 1 (DKK1) is a secreted protein involved in embryonic development and it is classically considered to function as an inhibitor of the canonical Wnt signaling pathway (see [29] for a review). However, DKK1 does not take an active part in Wnt / $\beta$-catenin pathway in colon cancer cells, 
as mutation of adenomatous polyposis coli (APC, one of the components of Wnt pathway) occurs in most human colon cancers, thus disconnecting the effector part of the signaling cascade from the Wnt receptors, where DKK1 plays its inhibitory effect [30]. This situation led us to the hypothesis that DKK1 could have other cellular functions aside from its role in Wnt pathway. Indeed, a role for DKK1 overexpression in cancer and proliferation has been previously stated, although its precise mechanism of action has not yet been elucidated. In the case of HT29 MTX-resistant cells, the role of DKK1 is unclear, although it seems to be related to the resistant phenotype, since treatment with a siRNA against DKK1 mRNA showed a chemosensitization toward MTX (Fig. 13). In keeping with this, Katula and collaborators showed that folate deficiency led to the downregulation of DKK1, and that MTX inhibited DKK1 transcription [31]. Thus, DKK1 overexpression in HT29 MTX-resistant cells could constitute a mechanism to overcome the transcriptional repression exerted by MTX [32].

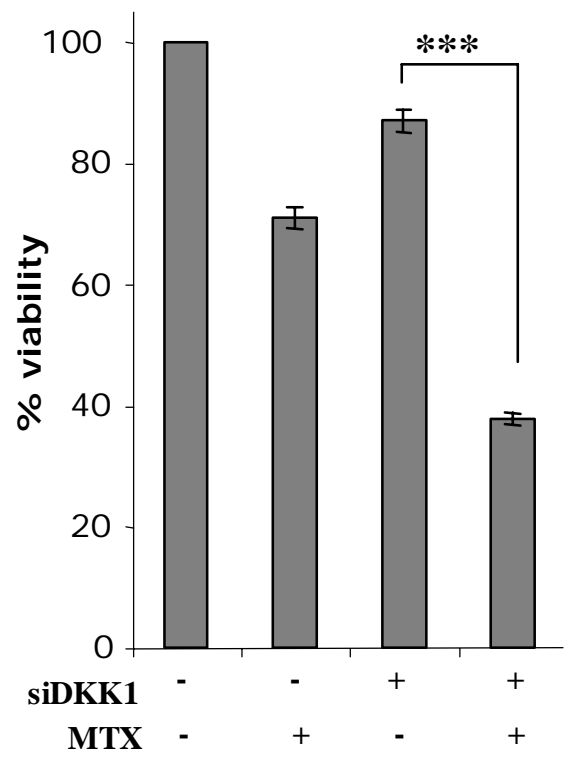

Figure 13. Effects of modulating DKK1 mRNA levels on cell viability. Cells were treated with a siRNA against DKK1, MTX was added after $48 \mathrm{~h}$, and cell viability was assessed after 3 days from the beginning of the treatment. All results are expressed as percentages referred to a non-related negative control siRNA (NR-siRNA). Values are the mean of three independent experiments \pm SE. ${ }^{* * *} \mathrm{p}<0.001$.

\subsection{Integrative view of MTX resistance in $\mathrm{HT29}$ colon cancer cells}

It has been shown that activated PKC $\alpha$ translocates from the nucleus to the membrane, where it associates with caveolae, and regulates the function 
and formation of such biological structures [33]. PKC $\alpha$ has been described to directly interact with Cav1. Further, activation of $\mathrm{PKC} \alpha$ by phorbol esters dislocates the enzyme from caveolae. These observations indicate that PKC $\alpha$ interacts functionally with these membrane structures. Moreover, PKC $\alpha$ has been proposed to be involved in the rearrangement of the cytoskeleton. Masur et al. showed that a high level of PKCa expression plus a low E-cadherin level predicts an elevated migratory activity of colon carcinoma cells, which could be derived more easily to metastasis [34]. Lahn et al. speculated that $\mathrm{PKC} \alpha$ overexpression may represent an important cellular event leading to enhanced tumor progression, as they showed that MCF-7 breast cancer cells transfected with PKC $\alpha$ had reduced expression of E-cadherin and $\beta$-catenin, resulting in a loss of cell-cell adhesion and thus in a more aggressive tumor phenotype [35].

Specific protein-protein interactions between CAV1 and other proteins have been proposed to regulate cell signalling. Indeed, CAV1 is known to control cell proliferation and viability via a transcriptional mechanism involving the $\mathrm{Wnt} / \beta$-catenin-Tcf/Lef-1 pathway [36]. One of the possible locations of $\beta$-catenin is within a complex with E-cadherin in the adherence junctions, specialized cell-cell adhesion sites that link the cadherin molecules to the actin microfilaments. E-cadherin promotes co-localization and co-immunoprecipitation of CAV1 with $\beta$-catenin, as well as inhibition of $\beta$-catenin-Tcf/Lef-1 dependent transcription of a wide variety of genes regulated by this pathway. However, the ability of CAV1 to regulate gene expression and cell proliferation is severely impaired in metastasic cancer cells lacking E-cadherin. If E-cadherin is lost, $\beta$-catenin is not retained in the plasma membrane and can be then translocated into the nucleus [37], thus activating Tcf/Lef-1 transcription factors-mediated expression of genes implicated in cell proliferation and tumor progression [38]. E-cadherin has been shown to be an important permissive element in defining the functions of CAV1, since several characteristics potentially relevant to CAV1 function as a tumor suppressor are compromised in E-cadherin-deficient HT29 cells. In this sense, we demonstrated that combination treatments to simultaneously decrease the expression levels of CAV1 and overexpress E-Cadherin were able to reduce cell viability by about $85 \%$ in the sensitive cells, and importantly, also in HT29 resistant cells (Fig. 14). These results give evidence of CAV1 and E-Cadherin interrelations in HT29 cells.

In order to shed some light on the possible role of Wnt/ $\beta$-catenin-Tcf/Lef-1 pathway in HT29 resistant cells, we performed transient transfection experiments with a luciferase reporter of $\beta$-catenin-mediated transcriptional activation. These experiments showed that Wnt / $\beta$-catenin-Tcf/Lef-1 pathway 

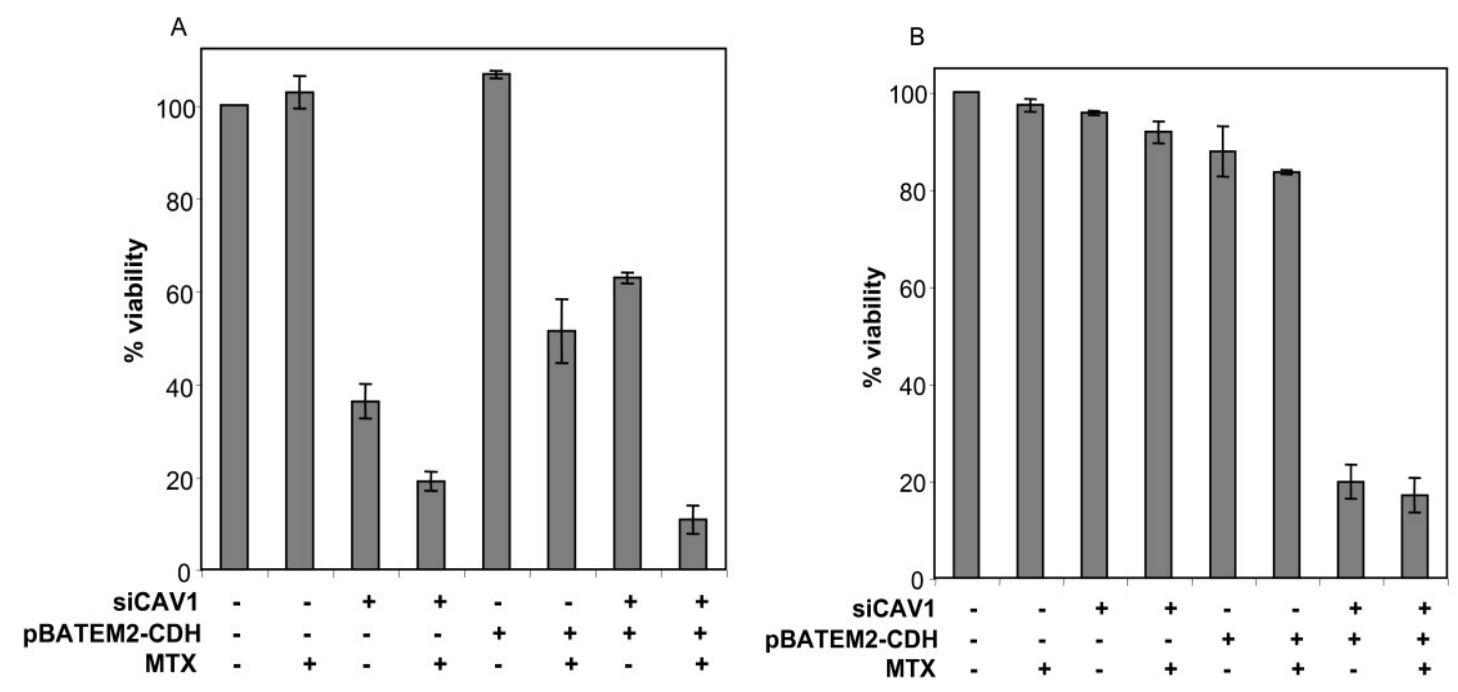

Figure 14. Effect of combining the siRNA against CAV1 and the expression plasmid for E-cadherin. Simultaneous treatments with a siRNA against CAV1 (siCAV1) and an expression plasmid for E-cadherin (pBATEM2-CDH) were performed in both sensitive (A) and resistant (B) HT29 cells. MTX was added 48 hours after transfection and the MTT assay was used to determine cell viability. Results are expressed as percentages referred to untreated cells. Values are the mean of three independent experiments $\pm \mathrm{SE}$.

was more active in the HT29 resistant cells than in HT29 sensitive cells, and that re-expression of E-Cadherin in the resistant cells results in a lower $\beta$-catenin-mediated transcriptional activation probably due to recruitment of $\beta$-Catenin to the adherent junctions (Fig. 15). Interestingly, DKK1 is transcriptionally regulated by Wnt / $\beta$-catenin signaling. Thus, constitutive activation of this signaling pathway through $\beta$-catenin, downstream of APC, could represent a mechanism for DKK1 overexpression in HT29 MTX-resistant cells.

It has been shown that $\mathrm{S} 100 \mathrm{~A} 4$ is also a target of Wnt signaling pathway in colon cancer, and a functional TCF binding site has been identified in its promoter sequence [39]. In this direction, an inverse correlation has been established between the expression levels of S100A4 and E-Cadherin [40] and has been associated with poor differentiation of cancer cells. Moreover, transfection of an E-Cadherin expression vector has been reported to cause a decrease in S100A4 expression levels. We showed a two-fold increase in S100A4 mRNA expression upon $\beta$-catenin overexpression (Fig. 16), thus giving support to a Wnt/ $\beta$-catenin pathway-mediated S100A4 transcription. 


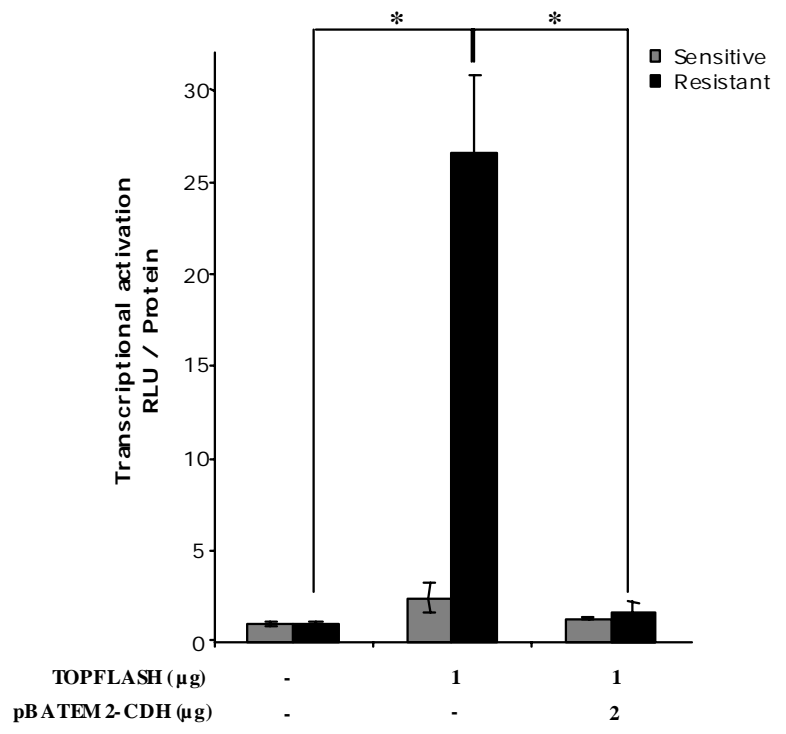

Figure 15. Transcriptional activation of Wnt pathway in MTX-resistant cells. HT29 cells were transiently transfected with a luciferase reporter of $\beta$-catenin-medi-ated transcriptional activation (TOPFLASH) in the presence or in the absence of E-Cadherin expression vector (pBATEM2-CDH). Thirty hours after transfection, luciferase activity (relative light units, RLU) was assayed. The protein content was used to normalize the luciferase acti-vity for each sample and is expressed relative to that of pGL3 basic vector (mean \pm S.E.M. for triplicate wells). ${ }^{*} \mathrm{p}<0.05$.

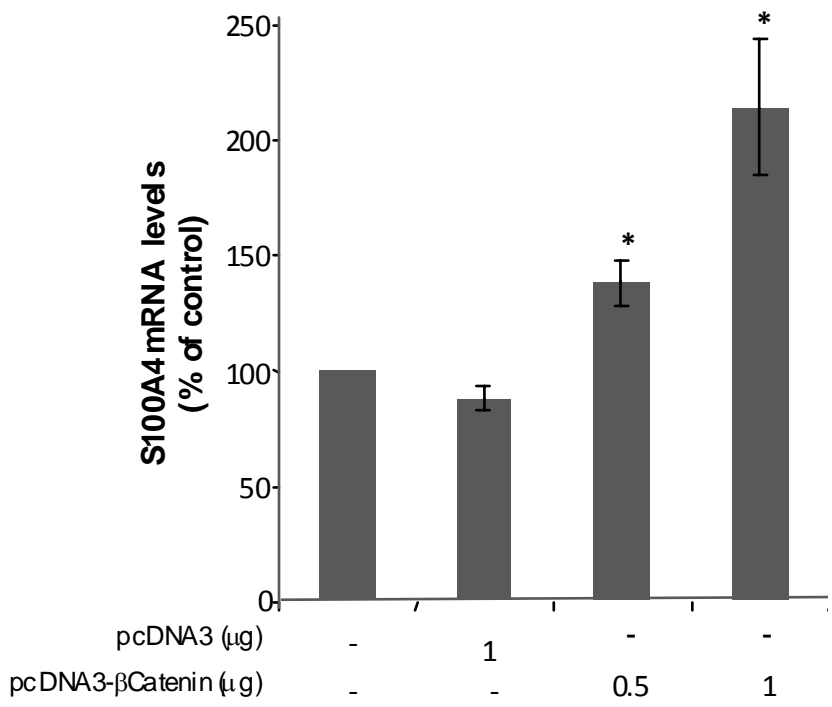

Figure 16. Effects of transfecting an expression vector encoding for $\beta$-Catenin on S100A4 mRNA levels. Transfection with $\beta$-Catenin expres-sion vector (pcDNA3- $\beta$ Catenin) was performed in HT29 cells. S100A4 mRNA levels were determined by RTReal-Time PCR 48h after trans-fection. All results are expressed as percentages referred to untreated cells. Values are the mean of three independent experiments \pm SE. ${ }^{*} \mathrm{p}<0.05$. 


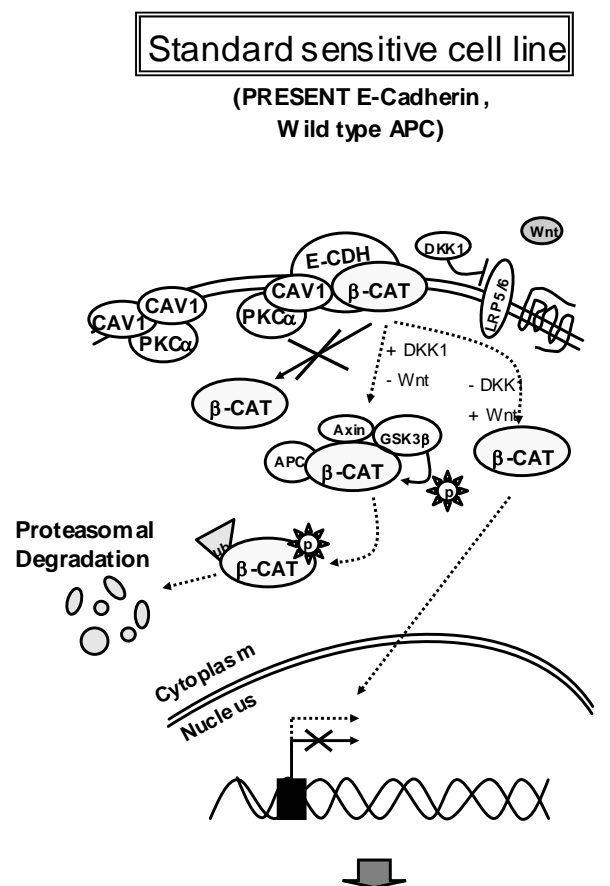

Decreased Growth

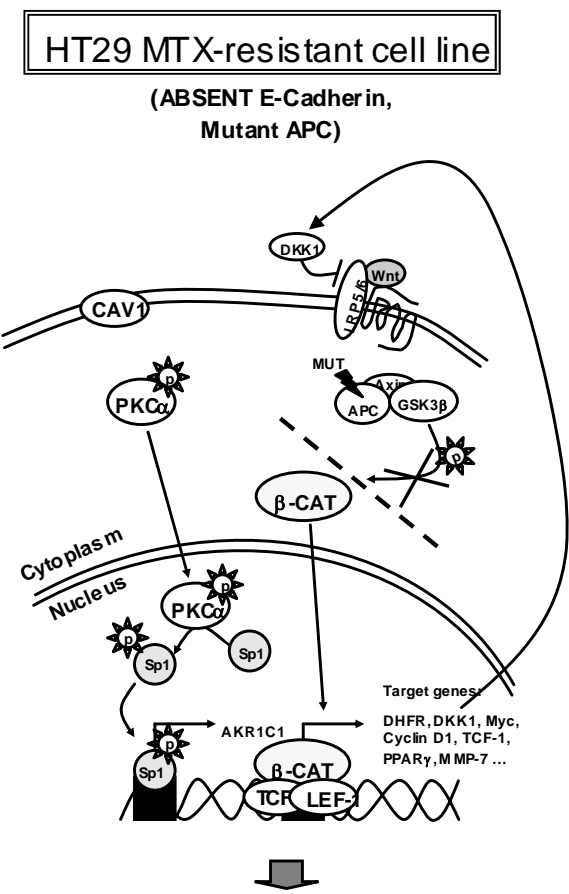

Increased Growth

Scheme 1. Regulation of the $\beta$-catenin-Tcf/Lef-1 pathway in sensitive and MTXresistant cells.

The interrelationship of AKR1C1 with the scenario described until now would be mediated trough PKC $\alpha$. This kinase could be responsible for Sp1 phosphoryaltion in HT29 MTX-resistant cells, thus being important for AKR1C1 transcriptional regulation in this cell line. No reports exist on a possible regulation of $\mathrm{AKR} 1 \mathrm{C} 1$ promoter through the $\beta$-catenin-Tcf/Lef- 1 signaling pathway. However, our results show the interrelationship between AKR1C1, cell proliferation, and apoptosis, two processes that can be regulated trough the $\beta$-catenin-Tcf/Lef- 1 pathway. One could hypothesize that AKR1C1 may favour or help the proteins from genes regulated throug the $\beta$-catenin-Tcf/Lef-1 pathway exhert their functions, maybe metabolizing a compound that could be interrfering in its pro-proliferative or anti-apoptotic functions. Thus, AKR1C1 overexpression in HT29 MTX-resistant cells would represent a parallel mechanism that could positvely contribute to the effects of the $\beta$-catenin-Tcf/Lef-1 signaling pathway.

In summary, HT29 MTX-resistant colon cancer cells bear many expression changes with respect to their sensitive counterparts. This scenario leads to changes in proteins that play a role in many processes, that are somehow interconnected (see scheme 1), and finally lead to increased growth, and thus to drug resistance. 


\section{MTX resistance in breast cancer}

Breast cancer is the most common cancer in women in developed countries, and after lung cancer, the tumor that causes more deaths among females [41]. One of the possible treatments for this type of cancer is MTX, usually given in combination with cyclophosphamide and 5-fluorouracil. With the aim to get further insight into the mechanisms of MTX resistance, functional genomics analysis using microarrays were performed in two breast cancer cell lines sensitive and resistant to MTX. We identified DHFR and UGT1A as differentially expressed genes in common between two breast cancer cell lines, namely MCF-7 and MDA-MB-468, resistant to MTX [42].

\subsection{Role of DHFR on MTX resistance}

DHFR was overexpressed in both breast cancer cell lines studied, by about 30 fold in MCF-7 resistant cells and about 3 fold in MDA-MB-468 resistant cells. Moreover, use of iRNA technology caused a sensitization of

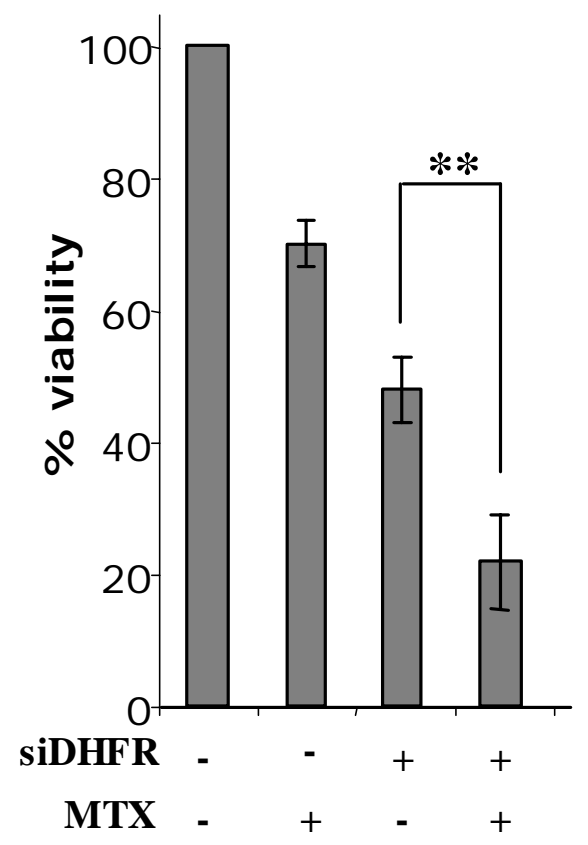

Figure 17. Effects of modulating DHFR mRNA levels on MCF-7 viability. Cells were treated with a siRNA against DHFR, MTX was added after $48 \mathrm{~h}$, and cell viability was assessed after 3 days from the beginning of the treatment. All results are expressed as percentages referred to a non-related negative control siRNA (NR-siRNA). Values are the mean of three independent experiments \pm SE. ${ }^{* *} \mathrm{p}<0.01$. 
MCF-7 cells toward MTX (Fig. 17), thus confirming a role for DHFR in MTX resistance in this cell line.

We also determined DHFR copy number in both cell lines. While 10-fold amplification was observed in MCF-7 resistant cells, no changes in gene copy number were detected in MDA-MB-468 resistant cells. Thus, gene amplification of the $d h f r$ locus could represent a mechanism for MTX resistance in MCF-7 cells, while drug resistance in MDA-MB-468 cells may obey to any of the other known mechanisms for MTX resistance [32]. Additionally, one has to take into account that MTX causes the differential expression of many genes that may be direct or indirect regulators of cell proliferation, survival or apoptosis, and that this expression pattern can contribute to modulate the resistance phenotype. In breast cancer cells, UGTs play a role in MTX resistance (see below).

\subsection{Role of the UGT1A family on MTX resistance}

UDP-glucuronosyltransferases (UGTs) are a family of enzymes involved in phase II metabolism. The addition of a glycosyl group from uridine

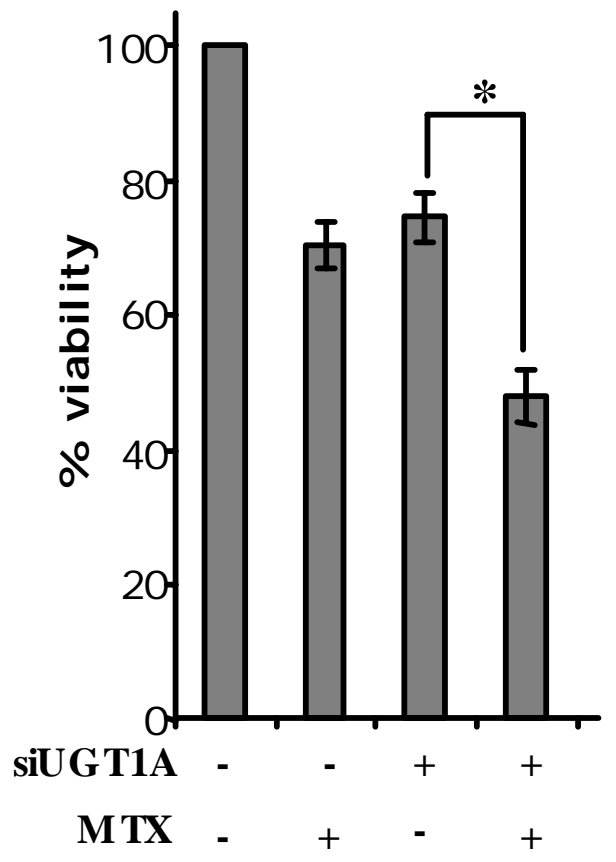

Figure 18. Effects of modulating UGT1A mRNA levels on cell viability. Cells were treated with a siRNA against UGT1A, MTX was added after 48h, and cell viability was assessed after 3 days from the beginning of the treatment. All results are expressed as percentages referred to a non-related negative control siRNA (NR-siRNA). Values are the mean of three independent experiments \pm SE. ${ }^{*} p<0.05$. 
diphosphoglucuronic acid (UDPGA) renders hydrophobic compounds more soluble for their elimination via bile and urine. UGTs catalyze the glucuronidation of many lipophilic endogenous substrates such as bilirubin and estrogens, and xenobiotics. Anticancer agents such as irinotecan, topotecan, doxorubicin and tamoxifen as well as carcinogens, are glucuronidated, contributing significantly to the overall availability and pharmacological effect of these xenobiotics [43]. The UGT1 gene family expresses nine functional UGT1A proteins by alternative splicing of 13 different tandem exons 1 with the common exons 2-5 [44]. Thus, all the UGT1A isoenzymes have a highly conserved "common" region, which is believed to contain the cofactor-binding site, and a variable region containing unique substrate-binding sites. Treatments that combine a siRNA designed to decrease the mRNA levels of some members of the UGT1A family and MTX showed an increased sensitivity toward the drug (Fig. 18), thus demonstrating a role for this gene family in MTX resistance in breast cancer.

\subsection{Role of UGT1A6 on MTX resistance}

Among the different members of UGT1A family, the cluster formed by UGT1A1 through 6, and specifically UGT1A6, showed to be the main responsible for the rise of UGT1A expression in breast cancer resistant cells. UGT1A6 is a major UGT in humans that mediates glucuronidation and is responsible for the metabolism of some drugs, carcinogens, and endogenous substrates [45]. UGT1A6 could be responsible for metabolising MTX in a certain degree, as it shares a phenolic structure common to other UGT1A6 substrates. A detoxification role can be hypothesized for UGT1A6 since its ectopic overexpression in sensitive breast cancer cells counteracts the cytotoxic effect of MTX (Fig. 19).

UGT1A family members' overexpression in both breast cancer cell lines resistant to MTX was not due to gene amplification, but to an increase in UGT1A transcription that led to an increased UGT1A activity. UGT1A family is characterized by its induction by a wide range of compounds. MTX could be an UGT1A6 inducer according to our results of increased mRNA levels and UGT1A enzymatic activity (Fig. 20).

Some transcription factors have been described to be involved in UGT1A induction. AhR has been described to provoke moderate UGT1A6 induction in humans and rodents [46]. This transcription factor is located in the cytoplasm and upon activation by ligand binding, it enters the nucleus and interacts with the AhR nuclear translocator protein (ARNT), forming the AhR/ARNT heterodimer that binds to a specific DNA sequence called 

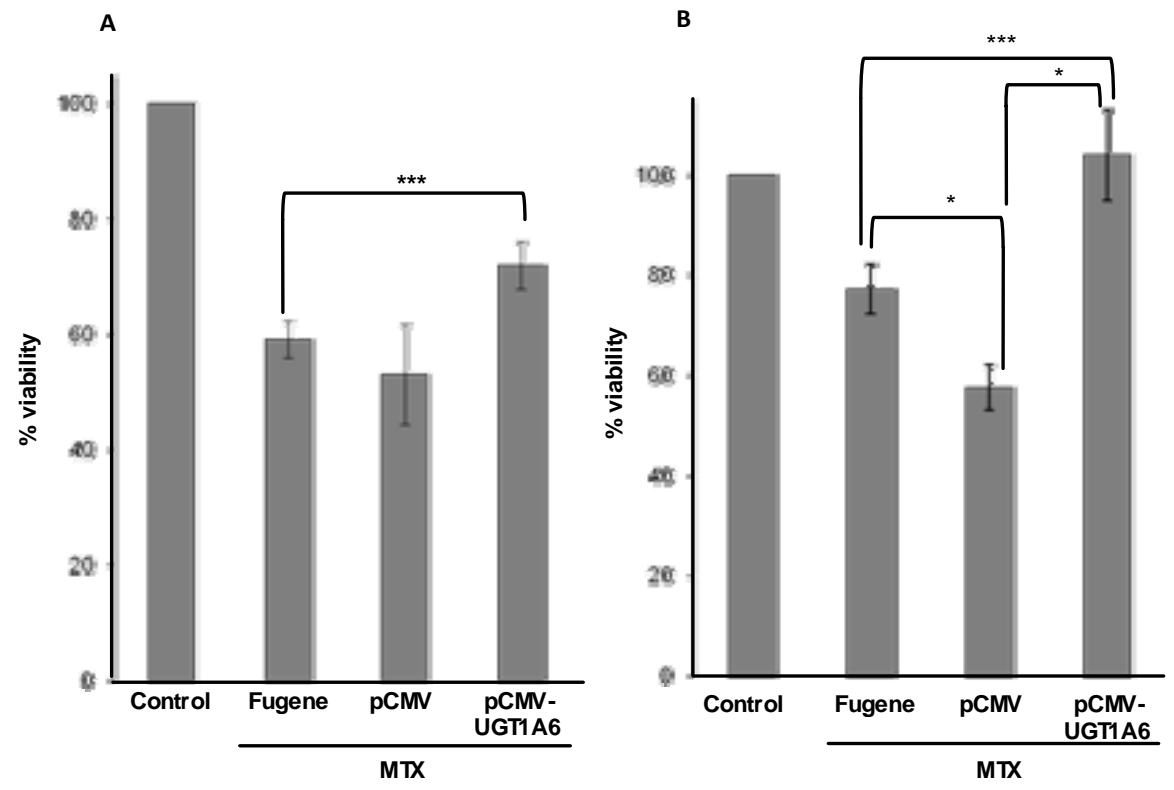

Figure 19. Methotrexate cytotoxicity upon UGT1A6 overexpression. Cells were transfected with either the expression plasmid for UGT1A6 (pCMV-UGT1A6) or with the empty vector $\mathrm{pCMV}$, which was used as a negative control. MTX was added 48 hours after transfection and cell viability was determined a week after transfection. A) MCF-7 cells. B) MDA-MB-468 cells. Results are presented as percentages referred to control cells. Values are the mean $\pm \mathrm{SE}$ of at least five experiments. $* \mathrm{p}<0.05$ $* * * \mathrm{p}<0.005$.
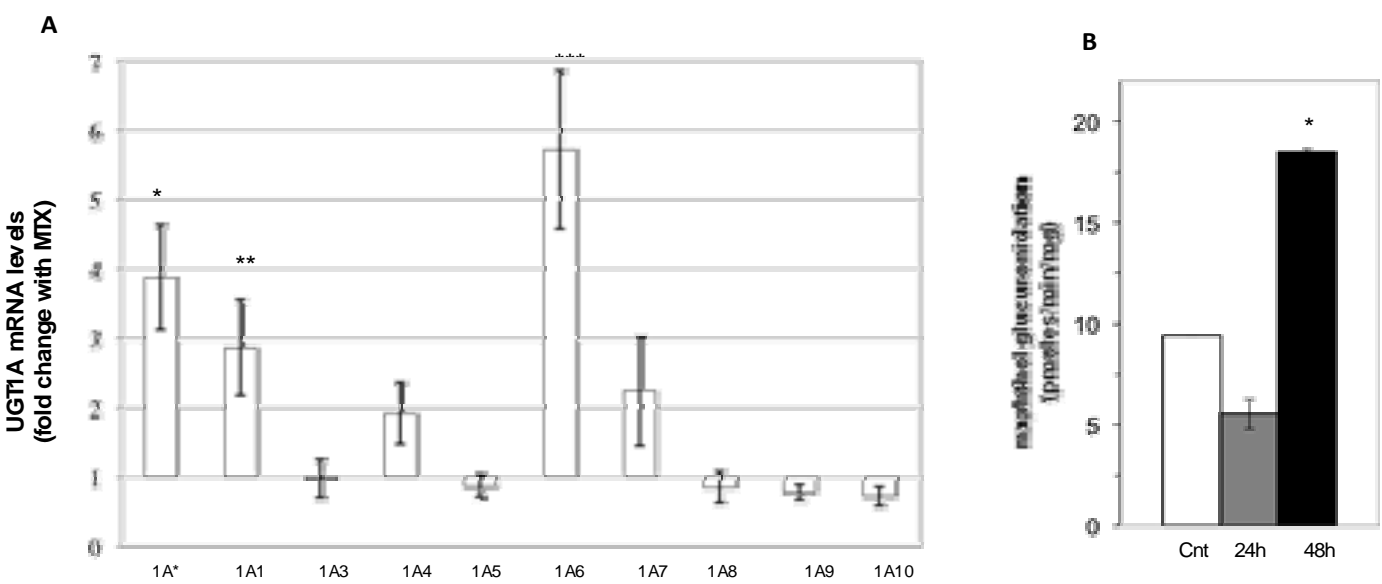

Figure 20. UGT1A mRNA and activity after MTX incubation. A) MDA-MB-468 cells were incubated for 24 hours with MTX. Total mRNA was obtained and subjected to DNAase treatment prior to RT-Real Time PCR. Data represent the fold change referred to control cells. Values are the mean $\pm \mathrm{SE}$ of at least three experiments. B) MDA-MB468 cells were incubated with MTX for 24 and 48 hours. The microsomal fraction was obtained and UGT1A activity was analysed through [1-14C]- $\alpha$-naphthol glucuronidation. Values are the mean \pm SE of two experiments. $* \mathrm{p}<0.05, * * \mathrm{p}<0.01$, or $* * * \mathrm{p}<0.005$. 


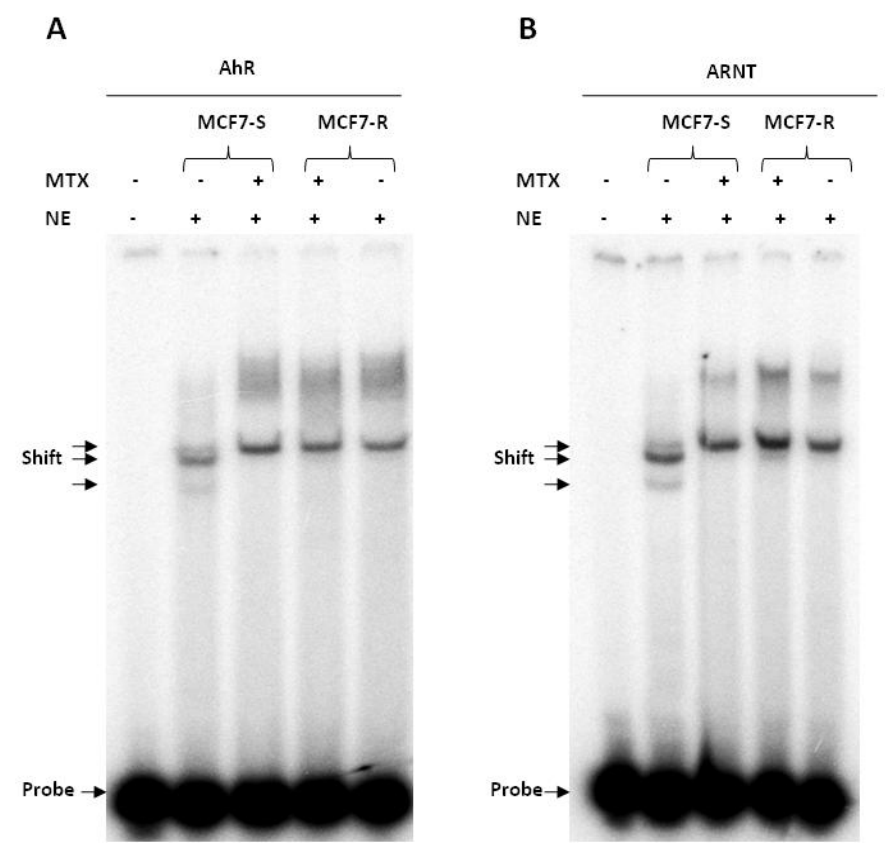

Figure 21. Binding of AhR and ARNT to UGT1A6 promoter. Cells were incubated with or without MTX for 24 hours prior to nuclear extracts harvest. Nuclear extracts were incubated with a radiolabelled probe corresponding to: (A) UGT1A6 AhR/ARNT promoter sequence. (B) UGT1A6 ARNT promoter sequence. Shifted bands are indicated by arrows.

xenobiotic response element (XRE, 5'-GCGTG-3'). AhR/ARNT binding to XRE activates the expression of a battery of genes involved in drug and hormone metabolism, among which UGT1A6 is found [47]. Some AhR agonists cause coordinate induction of both phase-I CYPs and UGTs to attenuate the generation of mutagenic benzo[a]pyrene metabolites, facilitating detoxification of the carcinogen. Our gel-shift results (Fig. 21) support the idea that MTX could be inducing UGT1A6 expression through AhR/ARNT heterodimers and ARNT itself. Another possibility would be that MTX, instead of directly activating AhR or ARNT, could bind to an ARNT inhibitor or activate ARNT through coactivators.

MTX induction of UGT1A6 may have important toxicological, pharmacological and physiological consequences, as it would decrease the bioavailability of many dietary constituents and drugs susceptible to glucuronidation, such as irinotecan and tamoxifen, which would become less active when administered simultaneously with MTX, as indicated by our results. UGTs have been reported to be responsible in part for the resistance to chemotherapeutic drugs such as daunorubicin and mycophenolic acid, and as shown here they constitute an important element in MTX resistance in breast cancer cells. This type of cross-resistance between MTX and other 
chemotherapeutics has significant pharmacological repercussions and could represent a handicap to tumor treatment, since MTX is given in combination with other drugs such as tamoxifen to treat breast cancer. Additionally, any other drug susceptible to glucuronidation, such as paracetamol, given to a patient treated with MTX, would decrease its bioavailability and therapeutic effect.

In summary, we show that UGT1A6 is overexpressed in breast cancer cells resistant to MTX, and that this drug induces UGT1A6 mRNA and enzymatic activity, through a mechanism mediated by ARNT and AhR/ARNT. The pharmacological effect of this induction should be taken into account when combining MTX with other drugs susceptible to glucuronidation.

\section{MTX resistance in other cancer cells}

\subsection{Role of DHFR on MTX resistance in pancreatic cancer, erythroblastic leukemia and osteosarcoma}

We also studied DHFR expression and copy number in three other cell lines representative of pancreatic cancer (MIA $\mathrm{PaCa}-2$ ), erythroblastic leukemia (K562) and osteosarcoma (SaOs-2). DHFR is overexpressed in all three cell lines, but only displays clear gene amplification in MIA PaCa-2 cells (Table 1).

DHFR expression levels are presented both as the values found in the microarrays and as validated by RT-PCR. DHFR copy number was determined by Real-Time PCR. Values are the mean (in fold change relative to the sensitive cells) of three independent experiments \pm SE.

We further studied the role of DHFR on MTX resistance in MIA PaCa-2 cells. Transfection of a siRNA designed against DHFR RNA caused a sensitization toward MTX (Fig. 22).

Table 1. Validation of DHFR overexpression and copy number determination in the different cell lines.

\begin{tabular}{llll}
\hline \multirow{3}{*}{ Cell Line } & \multicolumn{2}{l}{ Expression } & Copy-number \\
\cline { 2 - 4 } & Microarray & $\begin{array}{l}\text { RT-PCR } \\
\text { Validation }\end{array}$ & \\
\hline MIA PaCa-2 & 9.5 & $8.2 \pm 1.1$ & $32.2 \pm 2.2$ \\
\hline K562 & 9.4 & $9.8 \pm 0.2$ & $1.9 \pm 0.1$ \\
\hline Saos-2 & 4.1 & $4.1 \pm 1.1$ & $0.6 \pm 0.1$ \\
\hline
\end{tabular}




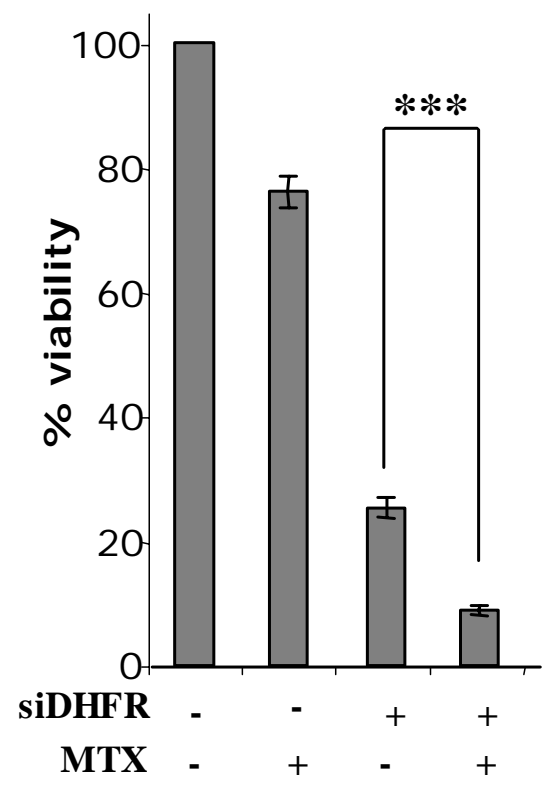

Figure 22. Effects of modulating DHFR mRNA levels on Mia PaCa-2 viability. Cells were treated with a siRNA against DHFR, MTX was added after $48 \mathrm{~h}$, and cell viability was assessed after 3 days from the beginning of the treatment. All results are expressed as percentages referred to a non-related negative control siRNA (NR-siRNA). Values are the mean of three independent experiments \pm SE. $* * * p<0.001$.

\subsection{Role of EEF1A1 on MTX resistance in pancreatic cancer}

Eukaryotic translation elongation factor 1A1 (EEF1A1) was a gene overexpressed in common among MIA PaCa-2, K562 and Saos-2 resistant cell lines. EEF1A1 is a ubiquitously expressed protein elongation factor that recruits amino-acetylated tRNAs to the A site of the ribosome (see [48] for a review). Although it has been traditionally described as a cellular housekeeper enzyme, overexpression of EEF1A1 is found in melanomas and in some tumors [49]. It has been demonstrated that EEF1A expression is related to increased cell proliferation, oncogenic transformation, delayed cell senescense and metastasis. Moreover, increased EEF1A1 expression has been related to cisplatin, doxorubicin and MTX resistance, maybe due to its ability to inhibit apoptosis. It has been proposed that EEF1A overexpression promotes cell growth and replication by contributing to an overall increase in protein translation. Antisense-mediated abrogation of EEF1A1 expression inhibits tumorigenesis and anchorage-independent cell replication in prostate tumor cells [50]. Our functional analyses using siRNA technology against EEF1A1 (Fig. 23) are in keeping with these results, and show chemosensitization of MIA PaCa-2 cells. Thus, we could state a role for EEF1A1 in MTX resistance in this cell line. 


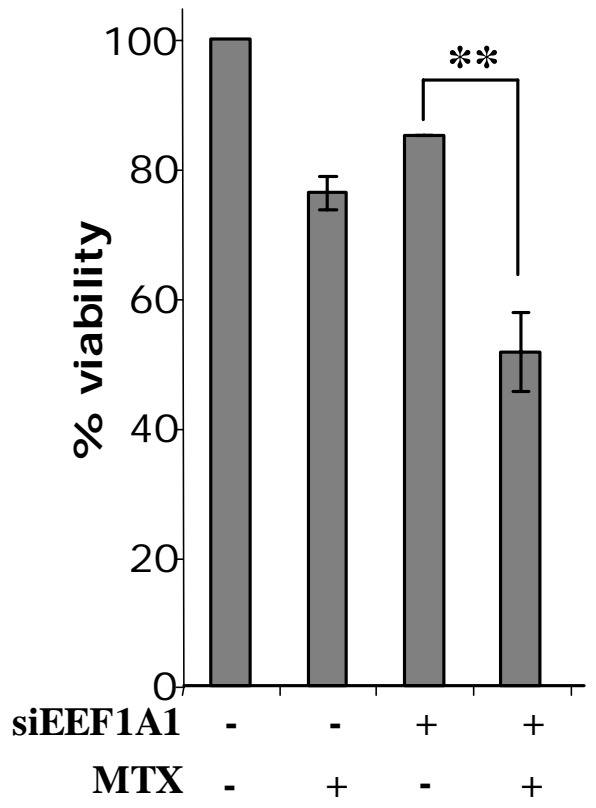

Figure 23. Effects of modulating EEF1A1 mRNA levels on cell viability. Cells were treated with a siRNA against EEF1A1, MTX was added after 48h, and cell viability was assessed after 3 days from the beginning of the treatment. All results are expressed as percentages referred to a non-related negative control siRNA (NR-siRNA). Values are the mean of three independent experiments \pm SE. $* *$ p $<0.01$.

\section{Conclusions}

- Gene Networks can be constructed, using specific software, from lists of differentially expressed genes by correlating them with the information already available in interaction databases. Then, significant node-genes within the networks can be selected and explored as putative targets for different types of therapy (e.g. cancer).

- We have applied this methodology to detect genes that could be used as novel targets in cancer chemotherapy. We used as a model cells resistant to methotrexate representative of 5 types of human cancer: colon, breast, pancreas, osteosarcoma and leukemia.

- In human colon cancer cells, DKK1, AKR1C and DHFR were detected as node-genes. The appearance of DHFR in the network was obvious and served as positive control; AKR1C had already been studied by our group since it appeared as a clear overexpressed gene in the lists of differentially expressed genes; but DKK1 had been missed in our previous studies. The methodology described in this chapter was able to detect it and subsequently its relationship with E-Cadherin, ß-Catenin, Caveolin 1 and PKC $\alpha$. 
- When analyzing breast cancer cells, network generation enabled the detection of the UGT1A family; and EEF1A1 as well as DHFR were common overexpressed nodes in the BAN constructed for pancreatic cancer, erythroblastic leukemia and osteosarcoma.

- In all cases, functional validation of the detected node-genes in the corresponding cell lines were performed using siRNA technology, obtaining an increased sensitivity toward methotrexate when combining this drug with the antisense molecule.

\section{Acknowledgements}

The authors are grateful to Professor M. Cascante for helpful suggestions on the manuscript. This work was supported by Grants for the Scientific Research from the Ministry of Education, Science and Technology of Spain: SAF94-0177, SAF99-120, SAF05-247 and SAF08-043, and BFU2008-02945.

\section{References}

1. Dohr, S., Klingenhoff, A., Maier, H., Hrabe de Angelis, M., Werner, T., Schneider, R. 2005, Nucleic Acids Res., 33, 864.

2. Brazhnik P, de la Fuente A, Mendes P. 2002, Trends Biotechnol., 20, 467.

3. Natarajan J, Berrar D, Dubitzky W, Hack C, Zhang Y, DeSesa C, Van Brocklyn JR, Bremer EG. 2006, BMC Bioinformatics, 7, 373.

4. Selga E, Noe V, Ciudad CJ. 2008, Biochem. Pharmacol., 75, 414.

5. Bader GD, Donaldson I, Wolting C, Ouellette BF, Pawson T, Hogue CW. 2001, Nucleic Acids Res., 29, 242.

6. Zanzoni A, Montecchi-Palazzi L, Quondam M, Ausiello G, Helmer-Citterich M, Cesareni G. 2002, FEBS Lett., 513, 135.

7. World Health Organization. http://www.who.int.

8. Singer MJ, Mesner LD, Friedman CL, Trask BJ, Hamlin JL. 2000, Proc. Natl. Acad. Sci. U. S.A., 97, 7921.

9. Morales C, Ribas M, Aiza G, Peinado MA. 2005, Oncogene, 24, 6842.

10. Jez JM, Flynn TG, Penning TM. 1997, Biochem. Pharmacol., 54, 639.

11. Hsu NY, Ho HC, Chow KC, Lin TY, Shih CS, Wang LS, Tsai CM. 2001, Cancer Res., 61, 2727.

12. Ax W, Soldan M, Koch L, Maser E. 2000, Biochem. Pharmacol., 59, 293.

13. Noe V, Alemany C, Nicolas M, Ciudad CJ. 2001, Eur. J. Biochem., 268, 3163.

14. Chu S, Ferro TJ. 2005, Gene, 348, 1.

15. Tanaka NM, Shiota K, Noda K, Kadosawa T, Mochizuki M, Nishimura R, Takahashi M, Sasaki N. 1996, J. Vet. Med. Sci., 58, 623.

16. Li D, Hinshelwood A, Gardner R, McGarvie G, Ellis EM. 2006, Toxicology, 226, 172. 
17. Martelli AM, Sang N, Borgatti P, Capitani S, Neri LM. 1999, J. Cell Biochem., $74,499$.

18. Noe V, Ciudad CJ. 1995, Biochem. Pharmacol., 50, 337.

19. Selga E, Morales C, Noe V, Peinado MA, Ciudad CJ. 2008, BMC Med. Genomics, $1: 35$.

20. Ho CC, Huang PH, Huang HY, Chen YH, Yang PC, Hsu SM. 2002, Am. J. Pathol., 161, 1647.

21. Lavie Y, Fiucci G, Liscovitch M. 1998, J. Biol. Chem., 273, 32380.

22. Kato K, Hida Y, Miyamoto M, Hashida H, Shinohara T, Itoh T, Okushiba S, Kondo S, Katoh H. 2002, Cancer, 94, 929.

23. Bender FC, Reymond MA, Bron C, Quest AF. 2000, Cancer Res., 60, 5870.

24. Frixen UH, Behrens J, Sachs M, Eberle G, Voss B, Warda A, Lochner D, Birchmeier W. 1991, J. Cell Biol., 113, 173.

25. Donato R. 2001, Int. J. Biochem. Cell Biol., 33, 637.

26. Bertram J, Palfner K, Hiddemann W, Kneba M. 1998, Anticancer Drugs, 9, 311.

27. Mencia N, Selga E, Rico I, de Almagro MC, Villalobos X, Ramirez S, Adan J, Hernandez JL, Noe V, Ciudad CJ. 2010, BMC Cancer, 10, 250.

28. Mahon PC, Baril P, Bhakta V, Chelala C, Caulee K, Harada T, Lemoine NR. 2007, Cancer Res., 67, 6786.

29. Nelson WJ, Nusse R. 2004, Science, 303, 1483.

30. Polakis P. 2000, Genes Dev., 14, 1837.

31. Katula KS, Heinloth AN, Paules RS. 2007, J. Nutr. Biochem., 18, 541.

32. Selga E, Oleaga C, Ramirez S, de Almagro MC, Noe V, Ciudad CJ. 2009, Genome Med., 1, 83.

33. Lisanti MP, Scherer PE, Vidugiriene J, Tang Z, Hermanowski-Vosatka A, Tu YH, Cook RF, Sargiacomo M. 1994, J. Cell Bio., 126, 111.

34. Masur K, Lang K, Niggemann B, Zanker KS, Entschladen F. 2001, Mol. Biol. Cel., 12, 1973.

35. Lahn M, Kohler G, Sundell K, Su C, Li S, Paterson BM, Bumol TF. 2004, Oncology, 67, 1.

36. Torres VA, Tapia JC, Rodriguez DA, Lladser A, Arredondo C, Leyton L, Quest AF. 2007, Mol. Cell Biol., 27, 7703.

37. Brabletz T, Jung A, Reu S, Porzner M, Hlubek F, Kunz-Schughart LA, Knuechel R, Kirchner T. 2001, Proc. Natl. Acad. Sci. U.S.A., 98, 10356.

38. Cavallaro U, Christofori G. 2004, Nat. Rev. Cancer, 4, 118.

39. Stein U, Arlt F, Walther W, Smith J, Waldman T, Harris ED, Mertins SD, Heizmann CW, Allard D, Birchmeier W, Shhlag PM, Shoemaker RH. 2006, Gastroenterology, 131, 1486.

40. Li Y, Zhang KL, Sun Y, Yang Y, Chen XY, Kong QY, Wu ML, Liu J, Li H. 2008, Transl. Oncol., 1, 165.

41. Mayo Clinic http://www.mayoclinic.com/.

42. de Almagro MC, Selga E, Thibaut R, Porte C, Noe V, Ciudad CJ. 2011, Biochem. Pharmacol., 81, 60.

43. Nagar S, Remmel RP. 2006, Oncogene, 25, 1659. 
44. Gong QH, Cho JW, Huang T, Potter C, Gholami N, Basu NK, Kubota S, Carvalho S, Pennington MW, Owens IS, Popescu NC. 2001, Pharmacogenetics, 11, 357.

45. Krishnaswamy S, Hao Q, Al-Rohaimi A, Hesse LM, von Moltke LL, Greenblatt DJ, Court MH. 2005, J. Pharmacol. Exp. Ther., 313, 1331.

46. Auyeung DJ, Kessler FK, Ritter JK. 2003, Mol. Pharmacol., 63, 119.

47. Klinge CM, Jernigan SC, Risinger KE, Lee JE, Tyulmenkov VV, Falkner KC, Prough RA. 2001, Arch. Biochem. Biophys., 390, 64.

48. Thornton S, Anand N, Purcell D, Lee J. 2003, J. Mol. Med. 81, 536.

49. Alon U, Barkai N, Notterman DA, Gish K, Ybarra S, Mack D, Levine AJ. 1999, Proc. Natl. Acad. Sci. U.S.A., 96, 6745.

50. $\mathrm{Su}$ Z, Goldstein NI, Fisher PB. 1998, Proc. Natl. Acad. Sci. U.S.A., 95, 1764. 


\title{
2. Inhibitors of the M2 channel of influenza A virus
}

\author{
María D. Duque, Eva Torres, Elena Valverde, Marta Barniol \\ Salvador Guardiola, Matias Rey and Santiago Vázquez \\ Laboratori de Química Farmacèutica, Facultat de Farmàcia and Institut de Biomedicina (IBUB) \\ Universitat de Barcelona, Av. Diagonal, 643, Barcelona, E-08028, Spain
}

\begin{abstract}
Influenza is a highly contagious, major respiratory tract disease affecting millions of people each year. At present, two classes of antivirals are available: the neuraminidase inhibitors and the M2 proton channel blockers amantadine and rimantadine. However, rapid emergence of M2 blockers resistance makes imperative the development of new anti-influenza drugs. In the last few years several groups have synthesized and evaluated several analogs of amantadine. While several of them are active against wild-type M2 channel only a few are able to inhibit the mutant ion channels that lead to amantadine-resistance.
\end{abstract}

\section{Introduction}

Influenza is a worldwide epidemic that causes substantial morbidity and mortality. Of the three types of influenza viruses, A, B and C, influenza A and B 
cause seasonal epidemics. Moreover, influenza A viruses are responsible for sporadic pandemics that usually cause higher mortality rates than seasonal influenza epidemics. The most severe pandemic, the "Spanish flu", occurred in 1918, is thought to have killed more individuals than any disease outbreak in history, resulting in approximately 40 million deaths worldwide [1]. More recent pandemics in 1957 (“Asian flu”, H2N2 strain) and 1968 ("Hong Kong flu”, H3N2 strain) were not as deadly, yet influenza remains a grave health hazard [2]. For example, in the United States, according to the Center for Disease Control and Prevention (CDC), influenza and its complications are currently the leading cause of death due to any infectious disease. In fact, in 2009, a new influenza virus ("swine flu”, H1N1 strain) originated a new pandemic that caused much concern, although, thankfully, was not as deadly as initially thought [3]. In addition, H5N1 viruses ("bird flu”), which are also currently worldwide circulating, are extremely virulent in humans but have not acquired the ability for efficient human-to-human transmission yet [4].

Influenza A viruses infect a wide range of avian and mammalian hosts, unlike influenza B viruses, which infect only humans. Influenza A and B viruses are enveloped negative-strand segmented RNA viruses. The envelope of influenza A viruses contains two different surface glycoproteins, hemagglutinin (HA) and neuraminidase (NA) [5]. Influenza A viruses are categorized into antigenic HA and NA subtypes: 16 HA (H1-H16) and 9 NA (N1-N9) antigenic subtypes have been identified so far. For example, the 2009 "swine flu" is an H1N1 virus because it contains a H1 subtype HA and a N1 subtype NA.

The major influenza A subtypes that have infected humans during seasonal epidemics are H1N1, H2N2 and H3N2. Within a subtype, different strains arise as a result of point mutations in a process known as 'genetic drift'. These new strains cause seasonal epidemics. A new pandemic can emergence by two different mechanisms: by direct transmission from animals, usually birds, to humans, as happened in 1918 with the "Spanish flu"; or through reassortment of an avian influenza virus with a human influenza virus, as occurred in 1957 with the "Asian flu" (H2N2) and, again, in 1968 with the "Hong Kong flu" (H3N2) [6]. The H1N1 virus of the 2009 "swine flu" is an apparent reassortment of four endemic strains of influenza: one from humans, one from birds, and two from pigs, a fact that further exemplifies the versatility of the influenza A virus [3]. When a new pandemic starts, the HA of the new strain differs substantially from recent HAs of seasonal influenza A viruses and, consequently, most of the human population lacks immunological protection against this virus, resulting in a pandemic.

There are two different strategies for combating influenza: vaccination and chemotherapy. The primary defense against influenza $A$ has been 
vaccination with inactivated or live-attenuated virus. However, vaccination effectiveness is limited due to the antigenic drifts and shifts that the influenza virus undergoes from year to year, and new influenza vaccines must be designed every year by predicting the genetic drift of seasonal influenza A. Antivirals have also been used for both prophylactic and therapeutic treatments during seasonal epidemics [5b]. Additionally, antivirals are particularly important at the beginning of a fast-spreading pandemic because the timely production of sufficient amounts of an effective vaccine is difficult. Current antivirals are directed against the M2 ion-channel protein of the influenza A virus (amantadine and rimantadine, Fig. 1) and the NA of the influenza $A$ and $B$ virus (zanamivir and oseltamivir) [8]. However, many influenza virus strains have developed resistance to adamantanes and/or oseltamivir (the only orally bioavailable NA inhibitor), highlighting a major health risk [9]. For example, after four decades of effective use of amantadine, resistance by influenza viruses of the A/H3N2 subtype currently exceeds $90 \%$ in the United States, and virus mutants are as fit as the wild-type (wt) virus. The situation is even worst with the new 2009 pandemic H1N1 influenza. In both strains, the basis for resistance is a single Ser to Asn amino acid replacement (S31N) in the matrix M2 ion channel, which interferes with the drug's ability to block M2 ion channel activity and viral replication [10].

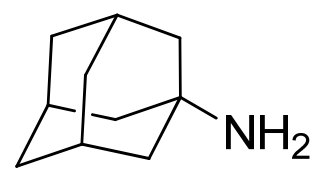

amantadine

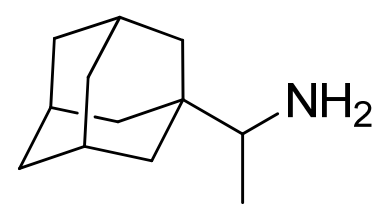

rimantadine

Figure 1. The structures of the anti-influenza A drugs amantadine and rimantadine.

Another important problem encountered in the administration of amantadine and rimantadine is related to the central nervous system side effects of both drugs. In fact, amantadine has been used in the treatment of Parkinson's disease, although its antiparkinsonian effect is poorly understood [11]. The side effects of rimantadine are analogous, but somewhat less pronunced than those of amantadine [12].

The appearance of pandemic H1N1 and highly pathogenic avian influenza viruses of the H5N1 subtype being able to infect humans, the emergence of resistances, and the side effects of amantadine and rimantadine reveal the urgent need for the development of new antiviral drugs [13]. 
In this review, we will focus on the design of new amantadine analogs targeting the matrix M2 ion channel of influenza A virus.

\section{The M2 protein}

The influenza A virus M2 protein is a homotetrameric protein containing four parts: a short unstructured N-terminal extracellular domain, important for incorporation into the virion; a transmembrane helix that is necessary for tetramerization, proton conductance and drug-binding; a cytoplasmic amphiphilic helix, involved in cholesterol-binding, membrane localization, budding and scission; and a disordered tail that interacts with the matrix protein M1 [14].

The influenza virus enters its target cells by receptor-mediated endocytosis, which is followed by acid-induced fusion of the viral and endosomal membranes. This fusion event is mediated by a conformational change of the influenza HA proteins, triggered by the low $\mathrm{pH}$ in the endosome lumen [15]. The transmembrane region of the M2 protein forms a $\mathrm{pH}$ activated channel that selectively conducts protons along a chain of water molecules and ionizable sidechains, including His37, playing an essential role for viral replication equilibrating the $\mathrm{pH}$ of the virus interior with that of the acidic endosome. When the endosome is acidified, His37 residues in the transmembrane region of M2 become protonated, leading to the opening of the M2 channel and to a proton influx from the endosome into the virus interior [16]. The acidification of the virus interior enables the release of the viral RNA into the host's cytoplasm after membrane fusion has taken place. In addition, it has been shown that, for some strains of influenza A virus, the M2 proton channel function is required for preventing a premature HA conformation transition when newly synthesized viral proteins are trafficked through the trans-Golgi network [17].

The replication of the influenza A virus can be stopped by inhibiting the activity of the M2 channel, using amantadine and rimantadine.

Although the role of the M2 protein as the target for amantadine and rimantadine has been known for more than twenty years [18], only very recent functional, structural [19] and computational [20] studies have revealed that the drugs inhibit proton conduction by binding to an aqueous cavity adjacent to M2's proton-selective filter, thereby blocking access of proton to the filter [14]. These recent works enable novel insights into the adamantanesresistance and provide a solid basis for structure-based drug design.

The number of drug-resistant variants of influenza A M2 channel is limited by the very conserved nature of the binding site within the channel. Thus, only a few amantadine-resistant mutations, namely V27A, L26F and 
S31N, have been widely observed in transmissible strains of the virus in the past eight decades for which a genetic record is available [21], although other mutations can easily be observed in vitro [22]. The mutations that cause the greatest decrease in inhibition, S31N and V27A, increase the polarity of porelining residues.

\section{1-Substituted-adamantanes}

Amantadine and rimantadine exhibit their inhibitory activity at micromolar concentrations. Rimantadine has a superior intrinsic antiviral activity compared to amantadine, but peak plasma levels of rimantadine are 2-3 fold lower than those achieved with amantadine when given at the same dose [23]. Amantadine was initially licensed in USA in 1966. Interestingly, for many years, amantadine was mainly used in western countries, while rimantadine was used in the former USSR and eastern European countries [24].

Both drugs are rather old, therefore it is not a great surprise that hundreds of derivatives have been synthesized and pharmacologically tested. In fact, soon after the publication of the antiviral activivity of amantadine by du Pont de Nemours' researchers [25], several amantadine derivatives were synthesized and evaluated as anti-influenza agents [26]. Most of these analogs were alkylaminoalkyl derivatives of adamantane (Fig. 2), although some derivatives featuring aditional polar groups, such as alcohols, amines, ethers, or derivatives lacking an amino group were also synthesized and tested (Fig. 3).
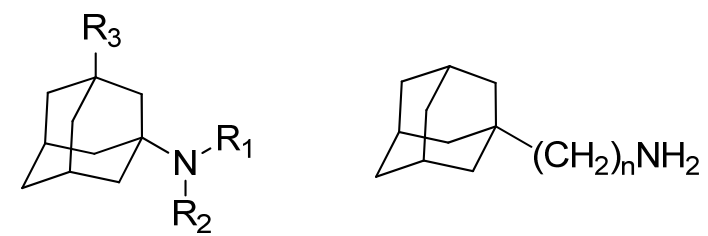
$\mathrm{R}_{2}$
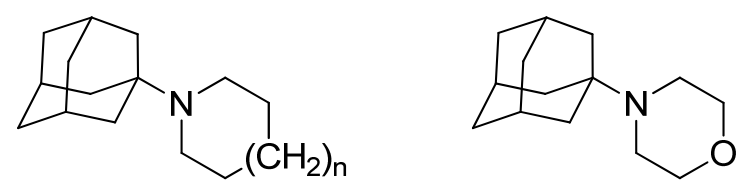

$R_{1}, R_{2}=H$, methyl, ethyl, allyl, propargyl, propyl, butyl, etc.; $R_{3}=H$, methyl, phenyl, etc.; $n=0,1,2$

Figure 2. Alkylamantadines and related compounds.<smiles>CN(C)CCCNC12CC3CC(CC(C3)C1)C2</smiles><smiles>OCCCNC12CC3CC(CC(C3)C1)C2</smiles><smiles>N=C(N)CC12CC3CC(CC(C3)C1)C2</smiles>

Figure 3. Amantadine analogs featuring polar groups. 
Although several of these compounds displayed anti-viral activities similar to that of amantadine, they showed cross-resistance with amantadine and rimantadine, so their therapeutical interest is rather low.

Worthy of note, while amantadine is, for the most part, excreted without metabolism [27], rimantadine is extensively metabolized by hydroxylation before excretion in the urine [23c,28]. Manchand and coworkers reported the synthesis of three hydroxylated metabolites of rimantadine and showed that 2-hydroxyrimantadine was as active against several influenza A virus as amantadine, while the 3 - and the 4-hydroxy derivatives showed only very modest inhibitory activity (Fig. 4). Unfortunately, rimantadineresistant strains exhibited cross-resistance to the 2-hydroxyamantadine [29].

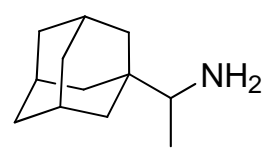

rimantadine

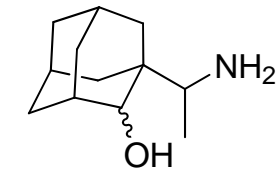

2-hydroxyrimantadine

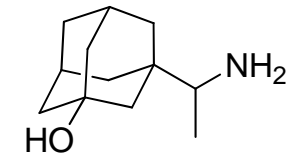

3-hydroxyrimantadine

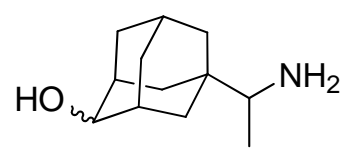

4-hydroxyrimantadine

Figure 4. Rimantadine and its hydroxylated metabolites.

The antiviral activity of 2-hydroxyrimantadine was the first example of a trend that has also been observed in much more recent work using amantadine and other polycyclic systems, that is, the introduction of polar groups in the polycyclic scaffold is tolerated, but does not enhance the potency of amantadine and related aminopolycyclic derivatives. For example, in 2011, Wang et al. described that the aminoalcohol 3 showed an $\mathrm{IC}_{50}=16 \mu \mathrm{M}$ against the wt M2 channel from influenza A virus, exactly the same value than that reported for amantadine [30]. Surprisingly, they found that 1-adamantanol, $\mathbf{1}$, and 2-methyl-2-adamantanol, 2, showed $\mathrm{IC}_{50}$ values very close to that of amantadine, while the 3-amino-1-adamantanol, 4 , showed to be inactive (Fig. 5).

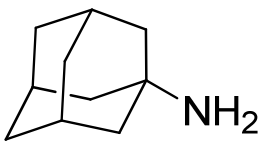

amantadine

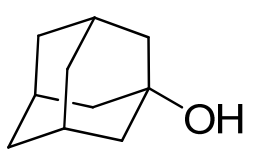

1

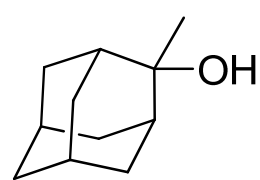

2

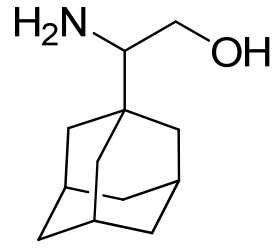

3

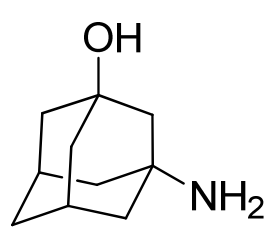

4

Figure 5. Several hydroxylated analogs of amantadine. $\mathrm{IC}_{50}$ values (against wt $\mathrm{M} 2$ channel): amantadine (16 $\mu \mathrm{M}), \mathbf{1}(20 \mu \mathrm{M}), 2$ (14 $\mu \mathrm{M}), 3$ (16 $\mu \mathrm{M}), \mathbf{4}$ (not active). 
Kolocouris' group has reported the synthesis and anti-influenza activity of a series of heterocyclic rimantadine analogs (Fig. 6) [31]. The aziridine and the azepine derivatives were much less active than amantadine, while azetidine $\mathbf{6}$ $\left(\mathrm{R}_{1}=\mathrm{H}\right)$, pirrolidines $7 \mathbf{a}\left(\mathrm{R}_{1}=\mathrm{R}_{2}=\mathrm{H}\right)$ and $\mathbf{7 b}\left(\mathrm{R}_{1}=\mathrm{H}, \mathrm{R}_{2}=\mathrm{CH}_{3}\right)$, and piperidine 8 $\left(\mathrm{R}_{1}=\mathrm{H}\right)$ showed to be more potent than amantadine and rimantadine against the influenza $\mathrm{A}_{2} / \mathrm{Japan} / 305 / 57$ (H2N2) strain. Compounds 6 and $\mathbf{7 b}$ also showed good inhibitory activity against the influenza A/Hong Kong/68 (H3N2) strain. While amantadine displayed $\mathrm{IC}_{50}$ of 42 and $6 \mu \mathrm{M}$ against $\mathrm{A}_{2} / \mathrm{Japan} / 305 / 57$ and A/Hong Kong/68, respectively, the most potent compound within this series, $\mathbf{7 b}$, showed $\mathrm{IC}_{50}$ of 1.6 and $1.8 \mu \mathrm{M}$ against $\mathrm{A}_{2} / \mathrm{Japan} / 305 / 57$ and $\mathrm{A} / \mathrm{Hong} \mathrm{Kong} / 68$, respectively. The introduction of an additional alkyl group in the nitrogen atom caused a dramatic reduction in anti-influenza activity.

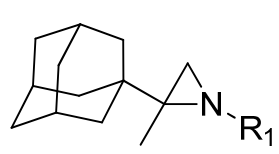

5

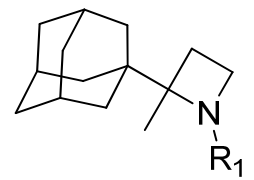

6

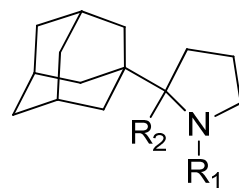

$7 a, b$

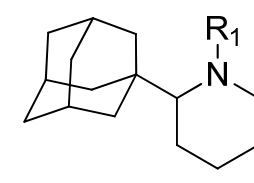

8

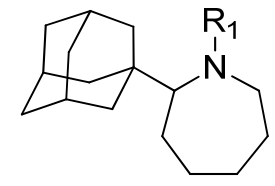

9

Figure 6. Heterocyclic rimantadine analogs.

Later on, the same group reported analogs of rimantadine featuring a second nitrogen atom, the aim of this modification being the incorporation of additional hydrogen bonding interactions with the M2 protein [32]. Although the presence of this second amino group was compatible with anti-influenza activity, the new analogs were not more potent than rimantadine. Thus, compounds $\mathbf{1 0}$ and $\mathbf{1 1}$ (Fig. 7) displayed $\mathrm{EC}_{50}$ of 18.3 and $24.1 \mu \mathrm{M}$, respectively, against A/Hong Kong/68 (H3N2) strain, very similar values to that of rimantadine $\left(\mathrm{EC}_{50}=19.1 \mu \mathrm{M}\right)$.

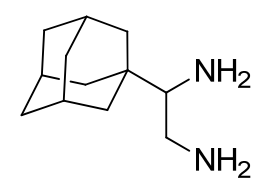

10

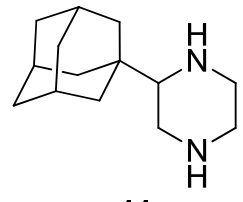

11

Figure 7. Rimantadine analogs featuring a second nitrogen atom.

As previously stated, amantadine interferes with the ion channel function of the M2 protein of influenza A virus at low micromolar concentrations. Interestingly, a second mechanism of action of amantadine, at least in some influenza A strains, is on the hemagglutinin, at concentrations around 100 times 
higher. Theoretically, an amantadine derivative able to simultaneously interact with both targets at the same concentrations should have a reduced probability to develop resistance. In this case, two mutations, one in each target protein would be necessary at once. With this aim, Scholtissek and coworkers reported the synthesis and evaluation of forty adamantane derivatives and tested them against the influenza A/Singapore/1/57 (H2N2) strain [33]. They found several analogs active against this strain and also against A/Swine/1976/31 and $\mathrm{A} / \mathrm{Udorn} / 307 / 72$, although all the products were inactive against A/WSN/33, which is amantadine-resistant. Most of the active compounds at low micromolar concentrations (e. g., 12-14) interacted with the M2 protein; the corresponding escape mutants produced with them had amino acid replacements at positions 27, 30 or 31 of the M2 protein. Interestingly, they found two compounds, 15 and 16 (Fig. 8), able to interact with both the ion channel and the hemagglutinin at about the same concentration. It was expected that in order to become resistant the virus should mutate both proteins. However, the resistant mutants to these compounds showed mutations only in the HA protein [33].

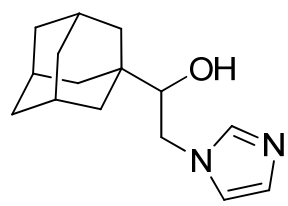

12

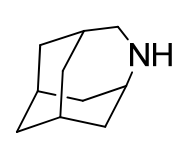

13

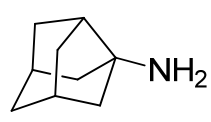

14

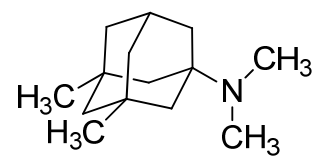

15

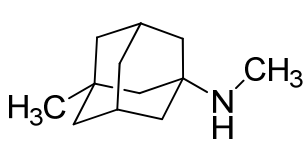

16

Figure 8. Some of the amantadine analogs reported by Scholtissek and coworkers.

Very recently, Zarubaev and coworkers have reported the synthesis and the anti-influenza activity of a series of di-, tri- and tetrazole derivatives of amantadine. Interestingly, several compounds were active against the amantadine-resistant influenza A/Puerto Rico/8/34 strain, which bears the S31N mutation in its M2 channel. Tetrazoles such as 17, 18 and 19 (Fig. 9), showed micromolar values of $\mathrm{EC}_{50}$ and higher selectivity index (SI) than rimantadine [34]. It remains to be clarified if the target of these adamantane derivatives is the M2 channel of the influenza virus.

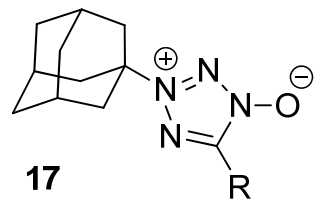

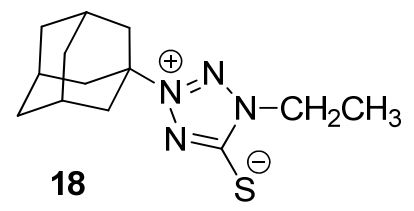

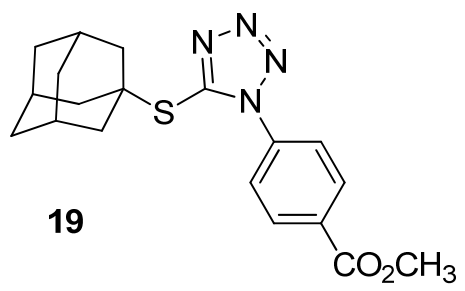

Figure 9. Tetrazolo-adamantanes with anti-influenza A virus activity. 
Finally, Zhang et al., have reported that an $L$-histidine derivative of adamantane, 20 (Fig. 10), was able to inhibit the wt, the S31N, and the double mutant S31N/L26I M2 channels of avian H5N1 influenza expressed in cell lines of transformed HEK 293. The $\mathrm{IC}_{50}$ of $\mathbf{2 0}$ against the wt, the S31N mutant and the double mutant S31N/L26I channels were 5.84, 10.96 and $9.77 \mu \mathrm{M}$, respectively [35]. However, these data were not confirmed with viral inhibition assays.

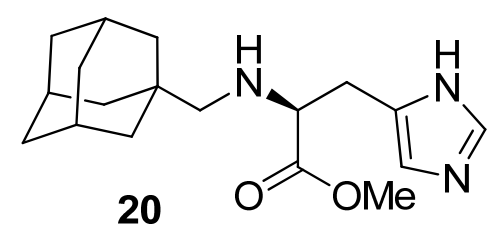

Figure 10. Structure of histidine derivative 20.

\section{2-Substituted-adamantanes}

2-Amantadine is only moderately active against influenza virus. The antiviral activity improved by the incorporation of a 2-ethyl or 2-n-propyl group, although the introduction of a methyl group in C-2 diminished the activity. Interestingly, 2-methyl-2-adamantanol, 2a, showed an $\mathrm{EC}_{50}$ of $3 \mu \mathrm{M}$ against influenza A/Japan/305/57 (H2N2) strain, very similar to the $\mathrm{EC}_{50}$ of amantadine against this strain $(1.1 \mu \mathrm{M})$ [36]. As previously stated, 2a inhibits the wt M2 channel of influenza A with an $\mathrm{IC}_{50}$ of $14 \mu \mathrm{M}$ [30]. In 2010, Kolocouris' group reported that several adamantanaminoalcohols such as $\mathbf{2 3}$ and 24 (Fig. 11) had potent anti-influenza activity. For example, aminoalcohol 23, displayed submicromolar activity against the influenza A /Hong Kong/7/87 (H3N2) strain [37].

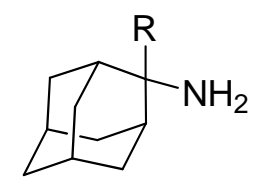

21a-c

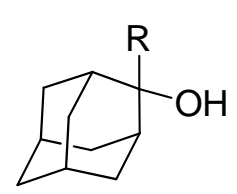

2a-c

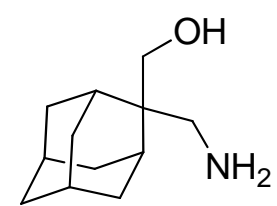

22

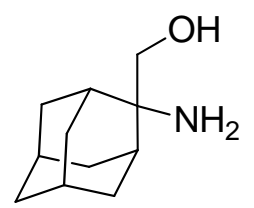

23

Figure 11. 2,2-Disubstituted adamantanes. a, $\mathrm{R}=$ methyl; b, $\mathrm{R}=$ ethyl; $\mathrm{c}, \mathrm{R}=n$-propyl.

Although 2-amantadine is only moderately active against influenza virus, the 2-isomer of rimantadine, 24 (Fig. 12), was found to be 4 times more potent than rimantadine against the influenza $\mathrm{A}_{2} / \mathrm{Japan} / 305 / 57$ (H2N2) strain. This 
finding led to Kolocouris' and De Clercq's groups to investigate the antiviral activity of several 2-alkyl and 2-cycloalkyl analogs of rimantadine [38]. They found that alkylation of the nitrogen atom reduced the anti-viral activity as did the introduction of a methyl group in the C-2 of the adamantane, as in $\mathbf{2 5}$. Unfortunately, 24 was much less potent against X-31, a reassortant influenza A H3N2 strain (A/Hong Kong/1/68 with A/Puerto Rico/8/34) carrying the S31N mutation.

They also investigated the activity of 2-(2-adamantyl)piperidines, 2-(2adamantylmethyl)piperidines and 3-(2-adamantyl)pyrrolidines. In these series they found that while the alkylation of the nitrogen atom reduced the activity, as in going from $\mathbf{2 6} \mathbf{a}$ to $\mathbf{2 6} \mathbf{b}$, the introduction of a further nitrogen atom two carbon away from the heterocyclic ring, as in $\mathbf{2 7 a - c}$ or $\mathbf{2 8}$ led to high anti-viral potency. For example, compounds 27 a-c showed $\mathrm{EC}_{50}$ between 3 and $7 \mu \mathrm{M}$, against the $\mathrm{X}-31$ strain, much lower than amantadine $\left(\mathrm{EC}_{50}=49 \mu \mathrm{M}\right)$ or rimantadine $\left(\mathrm{EC}_{50}=19 \mu \mathrm{M}\right)$. Taking into account the size of the diamines, it seems like the M2 receptor site can accommodate cages much larger than the adamantane. Unfortunately, the selectivity index (SI) of these compounds was much lower than that of amantadine or rimantadine [39].

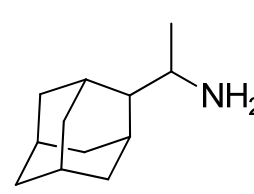

24

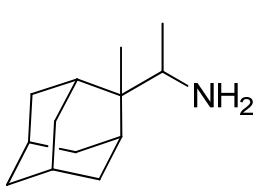

25

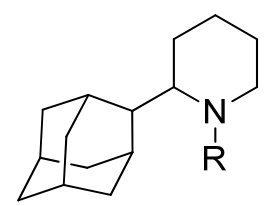

26a, $R=H$

26b, $R=M e$

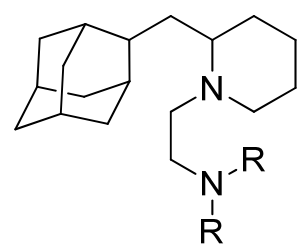

27a, $R=M e$

27b, $R=E t$

27c, $\mathrm{R}=-\left(\mathrm{CH}_{2}\right)_{5}-$

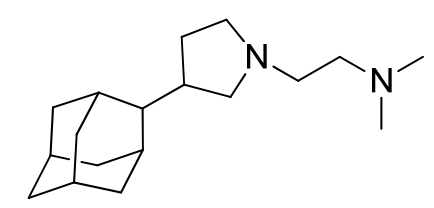

28

Figure 12. 2-Substituted analogs of rimantadine.

\section{Azaspiroadamantanes}

A unique kind of 2-substituted adamantanes is the group of the azaspiroadamantanes, because several of these derivatives have very potent anti-influenza activity.

Forty years ago, researchers at N. V. Philips-Duphar synthesized a series of azaspiroadamantanes (Fig. 13) [40]. Several of these amantadine analogs showed anti-influenza activity and, in fact, one of them, DU 34796, that had an antiviral spectrum in vitro wider than that of amantadine and was more potent than amantadine against mouse influenza, entered clinical trials, although finally the drug was not further developed [41]. The main problem of these compounds was, once again, the cross-resistance with amantadine and rimantadine. 


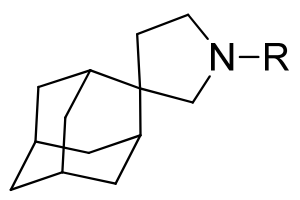

29

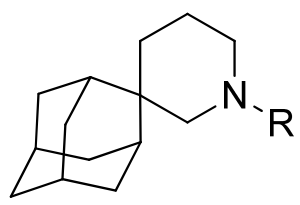

30

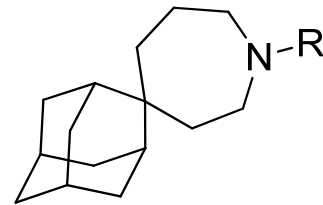

31

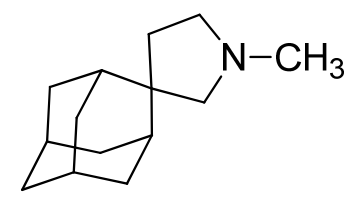

DU 34796

Figure 13. Spiro adamantane derivatives synthesized by N. V. Philips-Duphar. R is a lower alkyl group.

As 2-adamantanamine is only moderately active against influenza virus, the antiviral activity of the aforementioned derivatives points out that a carbon substituent in the vicinity of the 2-adamantyl carbon leads to a remarkable increase in antiviral activity. We will see further examples of this behaviour in the following paragraphs and also in different analogs that will be shown in the next sections.

In the nineties, Kolocouris' group, successfully revisited the topic, synthesizing several azaspiro- and oxazaspiro-adamantanes, such as those shown in Fig. 14. The compounds were examined against several influenza A strains (H1N1, H2N2 and H3N2) by De Clercq's group. Interestingly, against a H2N2 strain, the compound 32b was found to be up to 230 times more active than amantadine. Although 32b showed a SI of 714 in vitro, unfortunately, it proved rather toxic in vivo. Worthy of note, the change of a methylene unit by an oxygen atom was compatible with anti-influenza activity, although these oxa-analogs were less active than amantadine [42].

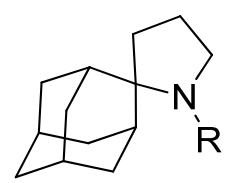

32a-d

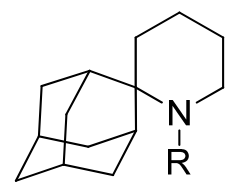

33a-c

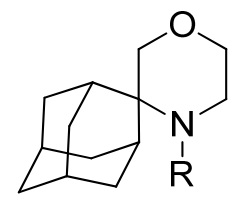

34a-c

Figure 14. Azaspiro- and oxazaspiro-adamantane derivatives. $a, \mathrm{R}=\mathrm{H} ; \mathrm{b}, \mathrm{R}=$ methyl; c, R = ethyl; d, R = cyclopropylmethyl.

Later on, with the aim of improving the antiviral activity, Kolocouris's group explored the introduction of a methyl group in the pyrrolidine ring of 32a and 32b (Fig. 15). While the introduction of a methyl in either C-3 or C-4 of the pyrrolidine ring of 32a and 32b led to slightly less active compounds, introduction of a methyl in C-5 of the pyrrolidine was optimal for biological activity against H2N2 strain. Unfortunately, all pyrrolidines had lower SI than amantadine [43]. 


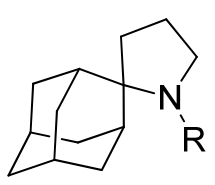

32a-b

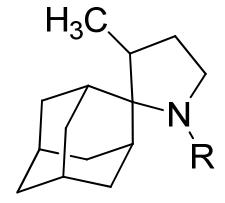

$35 a-b$

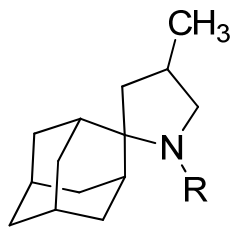

$36 a-b$

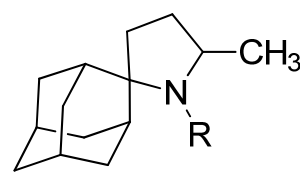

$37 a-b$

Figure 15. $C$-methyl derivatives of azaspiroadamantanes 32a-b. a, $\mathrm{R}=\mathrm{H}$; b, $\mathrm{R}=$ methyl.

More recently, Kolocouris et al. have completed this series with the synthesis of ring-contracted and ring-expanded analogs of 32a (Fig. 16) [44]. Azaspiro derivatives 38-41 were synthesized and tested against an H3N2 strain of influenza A. Whereas aziridine derivatives 38a,b were less potent than amantadine, azetidines 39a,b and $\mathbf{4 0}$, and the piperidine derivatives 41a,b were more potent than amantadine. Piperidine 41a, the most potent of them, showed significant anti-influenza A virus activity, being 12-fold more active than amantadine and about 2-fold more active than rimantadine. Azetidine 36a, while being slightly less potent than 41a showed a better SI (694 vs 106). Methyl substitution at the nitrogen atom of all heterocycles caused reduction in anti-influenza virus A potency.

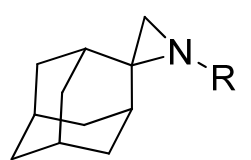

38a-b

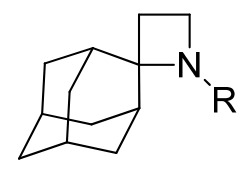

$39 a-b$

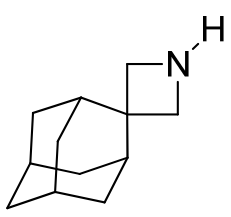

40

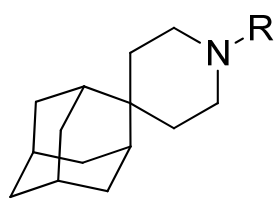

41a-b

Figure 16. Ring-contracted and ring-expanded analogs of 32. a, R = H; b, R = methyl.

Very recently, Kolocouris' group has reported the synthesis of several spiropiperazines of general structure 42 (Figure 17) [45]. These compounds can be regarded as analogs of $\mathbf{3 3}$ and $\mathbf{3 4}$ featuring an additional nitrogen atom. The main aim of this approach was to introduce a further group able to establish additional hydrogen bonds within the channel. However, piperazine derivative 42a was three times less active than spiropiperidine $\mathbf{3 3}$ or amantadine. Moreover, $N$-methylation of 42a to 42b and 42c further reduced the activity, probably by hampering the hydrogen bonding ability of the ligand, 42c being inactive against influenza A/HongKong/68 (H3N2). No significant antiviral effect was observed against the amantadine resistant influenza A/WSN/33 (H1N1) strain. Notwithstanding the introduction of a second nitrogen atom was negative in this spiroadamantanes, other series of adamantane derivatives increased their potency with the introduction of a second amino group, as we have already seen in section 2 . 


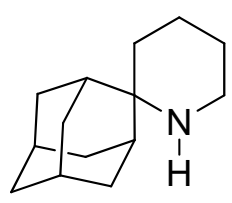

33

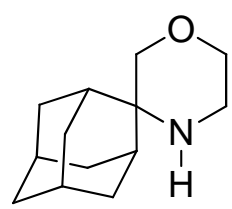

34

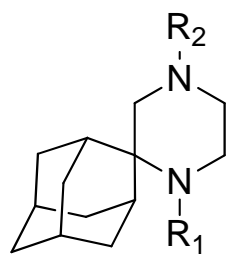

42a-c 42a, $\mathrm{R}_{1}=\mathrm{R}_{2}=\mathrm{H}$

42b, $\mathrm{R}_{1}=\mathrm{CH}_{3}, \mathrm{R}_{2}=\mathrm{H}$

42c, $\mathrm{R}_{1}=\mathrm{R}_{2}=\mathrm{CH}_{3}$

Figure 17. Spiropiperidine 33 and analogs featuring an additional heteroatom.

Finally, it should be noted that, recently, Kolocouris and coworkers have reported the binding constants of some spiroadamantanes against the M2 channel of the influenza A/chicken/Germany/27 (H7N7, Weybridge strain), expressed in E. coli [36]. The binding affinity of spiropiperidine $\mathbf{3 3}$ was in the submicrolar range $\left(K_{d}=0.39 \mu \mathrm{M}\right)$, very similar to that of amantadine $\left(K_{d}=\right.$ $0.32 \mu \mathrm{M})$, although much higher than that of rimantadine $\left(K_{\mathrm{d}}=0.016 \mu \mathrm{M}\right)$. Spiropirrolidines 32a, 32b, and 37b displayed binding affinities in the micromolar range $(1.16,2.93$, and $1.5 \mu \mathrm{M}$, respectively).

Unfortunately, sometimes it is difficult to compare the anti-viral activity of the different adamantane derivatives. This is, at least partly, a reflection of the time-span lasting more than four decades in which these compounds were synthesized and tested. For example, while spiropiperidines 30, published in 1972 [40d], and 33a-c, published in 1996 [42b], were tested against the influenza $\mathrm{A}_{2}$ /Japan, an H2N2 strain, 41a-b, described in 2007 [44], were tested against the influenza A/HongKong/7/87, an H3N2 strain. As the activity of amantadine against these strains is different, it is difficult a quantitative comparison between the activity of all these compounds. Moreover, sometimes the description of the antiviral potency is not very accurate. For example, van Hes and coworkers, in describing the antiviral activity of 30, only reported "activity comparable to that of amantadine or better" without stating a value for the $\mathrm{IC}_{50}$ [40d].

In order to investigate the SAR for their compounds, Kolocouris and coworkers have reported a conformational analysis study by a combination of NMR spectroscopy and theoretical calculations. They found that, in general, for the most active compounds the amine nitrogen atom lies in a distance of 1.5 to $2.5 \AA$ away from the 2-adamantyl carbon [47].

\section{Aminospiroadamantanes}

As part of its monumental work in adamantane chemistry, Kolocouris' group has also reported the synthesis of several aminospiroadamantanes such 
as those shown in Fig. 18. These compounds retain the pharmacophore group of rimantadine in the $\mathrm{C}-2$ position of the adamantane ring. Compounds $\mathbf{4 3 b}$ and 44a showed to be more than 100 times more active than amantadine when tested against the influenza $\mathrm{A}_{2} / \mathrm{Japan} / 305 / 57$ (H2N2) strain [42a] with SI of 83 and 24 , respectively.

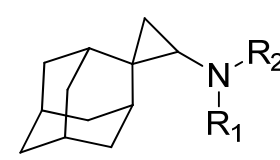

43a-c

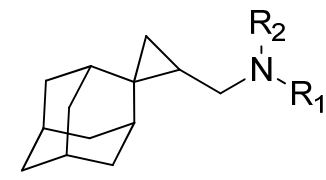

44a-c a, $\mathrm{R}_{1}=\mathrm{R}_{2}=\mathrm{H}$;

b, $\mathrm{R}_{1}=\mathrm{H}, \mathrm{R}_{2}=\mathrm{CH}_{3}$;

c, $\mathrm{R}_{1}=\mathrm{R}_{2}=\mathrm{CH}_{3}$

Figure 18. Aminospiroadamantanes 43 and 44.

Analogs of $\mathbf{4 3}$ featuring a cyclobutyl or cyclopentyl ring have also been synthesized and tested against $\mathrm{A}_{2} / \mathrm{Japan} / 305 / 57$ (H2N2) and X-31 (H3N2, with S31N in the M2 protein) strains [38]. Cyclobutyl derivatives 45 had similar potency against the H3N2 strain than rimantadine. Ring enlargement resulted in spirocyclopentane analogs $\mathbf{4 6}$ which were less potent than their cyclobutane analogs. When tested against the X-31 strain, all the new compounds showed to be less potent than rimantadine (Fig. 19).

Overall, in going from cyclopropyl analogs 43 to cyclopentyl derivatives 46, it appears as if increasing the carbon crowding around the spiro carbon leads to compounds with reduced antiviral potency. However, it must be taken into account that Philips-Duphar researchers reported, in the 1970s, that the cyclopentyl derivative 47 had, against the $A_{2} / J a p a n / 305 / 57$ (H2N2) strain, an antiviral activity of the same order of DU 34796, that, as we have already stated, entered clinical trials [40d]. Moreover, very recently, DeGrado's group disclosed in a patent the structure of the cyclohexyl derivative 48, somehow related to spiropiperidine 41a. Compound 48, when tested against the wt M2 channel of influenza A virus expressed in oocytes of Xenopus laevis, showed an $\mathrm{IC}_{50}$ of $18.7 \mu \mathrm{M}$, very similar to that of amantadine $\left(\mathrm{IC}_{50}=16 \mu \mathrm{M}\right)$ and was slightly less active than rimantadine $\left(\mathrm{IC}_{50}=10.8 \mu \mathrm{M}\right)$. As rimantadine, $\mathbf{4 8}$ was inactive against the mutant S31N. However, compound $\mathbf{4 8}$ revealed to be a submicromolar inhibitor of the clinically important mutant V27A $\left(\mathrm{IC}_{50}=0.31 \mu \mathrm{M}\right)$ and also showed to be active against the mutant $\mathrm{L} 26 \mathrm{~F}\left(\mathrm{IC}_{50}=5.6 \mu \mathrm{M}\right)$. To the best of our knowledge, $\mathbf{4 8}$ is the most potent compound ever reported against the mutant V27A [48]. Unfortunately, there is no data regarding the activity of compounds 43-47 against the V27A mutant M2 channel. Worthy of note, analog $\mathbf{4 9}$ has not been synthesized yet. 


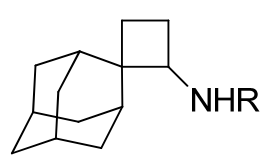

45a-b

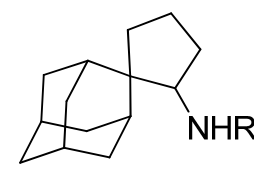

$46 a-b$

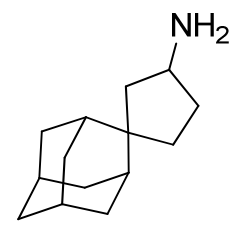

47

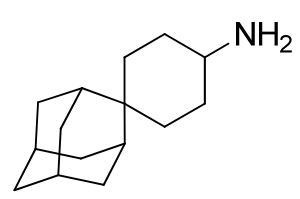

48

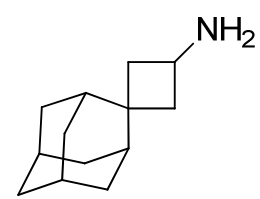

49

Figure 19. Aminospiroadamantanes 45-48 and unkown compound 49. a, $\mathrm{R}=\mathrm{H}$; b, $\mathrm{R}=$ methyl.

Taken together the antiviral acitivity of compounds $\mathbf{4 3}$ to $\mathbf{4 8}$, it seems that the distance and orientation between the nitrogen atom and the adamantyl cage is more important than the steric hindrance around the spiro carbon atom.

\section{1,2-Annulated adamantane derivatives}

In the earlier 1970s, several patents by Squibb claimed anti-influenza activity for a series of 1,2-annulated adamantanopyrrolidines of general structure 50 (Fig. 20), although no much details regarding biological activity were given [49]. Nearly forty years later, Kolocouris's group synthesized several adamantanopyrrolidines 51-52, the related compound 53 and 1,2annulated adamantanopiperidines of general structures 54-56 and tested them against influenza A/Hong Kong/7/87 (H3N2) strain [50].

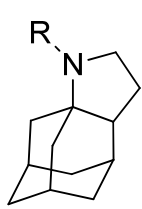

50

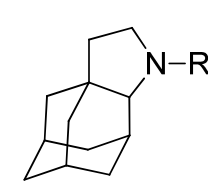

51a-c

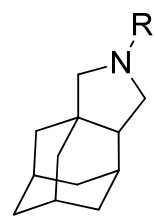

$52 a, c$

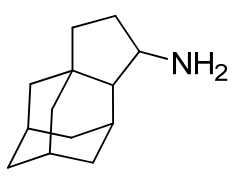

53

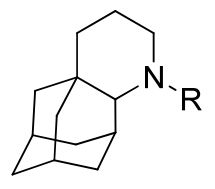

$54 a-b$

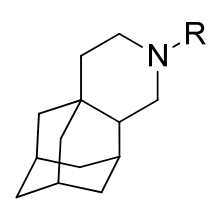

$55 \mathrm{a}-\mathrm{c}$

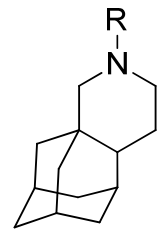

56a-b

Figure 20. 1,2-Annulated adamantane derivatives 50-56. a, $\mathrm{R}=\mathrm{H}$; b, $\mathrm{R}=$ methyl; c, $\mathrm{R}=$ ethyl.

The compounds 52a and 56a elicited submicromolar activities $\left(\mathrm{IC}_{50}\right.$ of 0.5 and $0.6 \mu \mathrm{M}$, respectively) and a SI of 732 and 200, respectively, being equipotent to rimantadine ( $\left.\mathrm{IC}_{50}=0.36 \mu \mathrm{M}\right)$. Compounds 51 and 54, with the nitrogen atom attached directly to the $\mathrm{C}-2$ position of the adamantane ring, showed low micromolar activities (between 2 and $8 \mu \mathrm{M}$ ), similar to that of amantadine $(2.0 \mu \mathrm{M})$.

As previously seen in other series, these results showed that a large lipophilic moiety in the vicinity of adamantane skeleton is compatible with good anti-viral activity, that moving the amine nitrogen atom away from the 
2-adamantyl carbon atom enhaces activity (compare 52a, $\mathrm{IC}_{50}=0.6 \mu \mathrm{M}$, with 51a, $\mathrm{IC}_{50}=2.2 \mu \mathrm{M}$ ), and that $N$-alkylation reduced the potency (compare 52a, $\mathrm{IC}_{50}=0.5 \mu \mathrm{M}$, with 52c, $\mathrm{IC}_{50}=2.4 \mu \mathrm{M}$, or $\mathbf{5 6 a}, \mathrm{IC}_{50}=0.6 \mu \mathrm{M}$, with $\mathbf{5 6 b}$, $\left.\mathrm{IC}_{50}>500 \mu \mathrm{M}\right)$.

In closing sections 2 to 5 , it should be mentioned that in 2009, K.-C. Chou published a fragment-based quantitative structure-activity relationship (FB-QSAR) study with 34 substituted adamantanes. His main conclusion was that position 2 of the adamantane was more sensitive to substitution than position 1 [51].

\section{2-Azaadamantanes and (2-oxaadamant-1-yl)amines}

Geigy has claimed that 2-azaadamantane, 57 (Fig. 21), first described in 1964 by Stetter et al. [52], displayed antiviral activity against three different influenza A H2N2 strains: A/Bethesda/10/63, A/Taiwan/1/62 and A/Singapore/1/57, but not further progress was published later [53].

More recently, we have found that replacement of the methylene unit of C-2 in amantadine by an oxygen atom to obtain (2-oxaadamant-1-yl)amine, 58, reduced the antiviral activity [54].

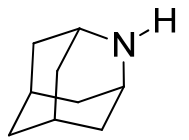

57

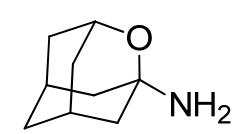

58

Figure 21. 2-azaadamantane and (2-oxaadamant-1-yl)amines.

\section{BL-1743 and related compounds}

In 1995, Bristol-Myers Squibb’s researchers carried out a high-throughput screen based on the ability of inhibitors to reverse the toxicity associated with M2 channels expressed in the yeast Saccharomyces cerevisiae membranes. They found an azaspiro[5.5] undecane derivative, BL-1743, able to efficiently inhibit the activity of wt influenza A M2 channels (Fig. 22) [55]. The mechanism of action of BL-1743 was further characterized by electrophysiological methods. BL-1743 was also able to inhibit the AM2 channel expressed in Xenopus oocytes, as determined using the two-electrode voltage clamp (TEV) technique. It was found that the majority of M2 sequences isolated from influenza viruses resistant to amantadine were also resistant to BL-1743, which suggests that BL-1743 binds competitively with 
amantadine. Interestingly, the kinetics of channel inhibition by BL-1743 were more rapid, showing a fast onset of inhibition as well as a reasonably rapid reversal of inhibition following removal of the compound [56]. This behavior contrasts with that of amantadine, whose second-order rate constant for the onset of inhibition is much slower than the diffusion-controlled rate, and whose off-rate is essentially irreversible on the minute to hour time scale of the experiment. The Hill coefficient for inhibition was 1.0, which is consistent with the binding ratio of one BL-1743 per AM2 tetramer [56].

It should be noted that twenty years before the discovery of BL-1743, A. H. Robins Company Inc., in a US patent [57], claimed anti-influenza activity for a series of aminospiranes that were already known from older literature [58]. Compounds 59-61 (Fig. 22) protected chicken embryos against influenza A/Taiwan/1/64 (H2N2) strain better or similarly than amantadine. No information about the activity of these compounds against amantadineresistant strains was disclosed.

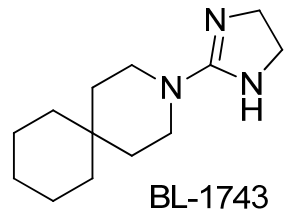

BL-1743

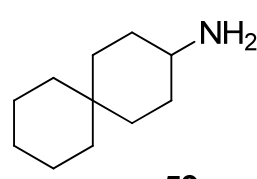

59

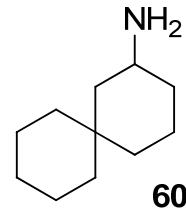

60

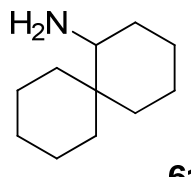

61

Figure 22. BL-1743 and related aminospiro[5.5]undecanes.

Taking into account the recent determination of the 3-D structure of the M2 ion channel of influenza A virus [19], and the structural difference between BL-1743 and the amantadine class of compounds, in 2008 Pinto's and DeGrado's groups started a SAR study of this scaffold with the aim of discovering new inhibitors of amantadine-resistant mutants [59].

Interestingly, spiropiperidine 62a (Fig. 23), an analog of BL-1743 lacking the imidazoline group, had an $\mathrm{IC}_{50}$ of $0.9 \mu \mathrm{M}$ against the influenza A wt M2 channel expressed in the Xenopus oocytes membrane, which is more than one order of magnitude more potent than amantadine $\left(\mathrm{IC}_{50}=16 \mu \mathrm{M}\right)$ and represents a more than 45-fold increase in potency relative to BL-1743 $\left(\mathrm{IC}_{50}=45.3 \mu \mathrm{M}\right)$. Alkylation of 62a with a methyl group to $\mathbf{6 2} \mathbf{b}$ reduced the potency $\left(\mathrm{IC}_{50}=20.6\right.$ $\mu \mathrm{M}$ ), and alkylation with larger groups as in 62c led to inactive compounds. Several $N$-heteroarylmethyl derivatives of $\mathbf{6 2}$ were also inactive. Worthy of note, solid-state NMR data indicated that 62a interacts with influenza A M2 channel differently from amantadine, affecting a longer stretch of the transmembrane helix and immobilizing the G34-I35 region. Ring-contracted analogs 63 and 64 were also active ( $\mathrm{IC}_{50}=8.1$ and $12.0 \mu \mathrm{M}$, respectively) 
although were less potent than 62a. Dithiene 65 was moderately active $\left(\mathrm{IC}_{50}=37.6 \mu \mathrm{M}\right)$, while ketal $\mathbf{6 6}$ was inactive [59a]. Finally, it should be noted that 62a can be seen as a simplified analog of Kolocouris' spiroadamantane 41a (Figure 16), a compound that, when tested against influenza A/Hong Kong/7/87 (H3N2) strain, was found to be 12-fold more active than amantadine [44].<smiles>[R]N1CCC2(CCCCC2)CC1</smiles><smiles>C1CCC2(C1)CCNCC2</smiles><smiles>CN1CCC2(CCCCC2)C1</smiles>

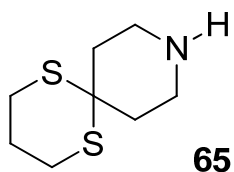<smiles>C1CNC2(CCOCC2)OC1</smiles>

Figure 23. Spiropiperidine 62 and related compounds. a, $\mathrm{R}=\mathrm{H}$; b, R = methyl; c, $\mathrm{R}$ = methylcyclopropyl; d, R = methyl-2-pyridyl; e, $\mathrm{R}$ = methyl-2-imidazolyl.

Moving the nitrogen atom out of the spiro-ring led to the aforementioned amine 59. DeGrado's group found that $\mathbf{5 9}$ had an $\mathrm{IC}_{50}$ of $12.6 \mu \mathrm{M}$, very similar to amantadine. Analogs $\mathbf{6 7}$ and $\mathbf{6 8}$ were also active $\left(\mathrm{IC}_{50}=15.7\right.$ and $14.6 \mu \mathrm{M}$, respectively), while more complex derivatives, such as 69 were inactive (Fig. 24) [59b]. Interestingly, while 59 was less potent than 62a against the wt channel of influenza A, 59 was active against the amantadineresistant L26F and V27A mutants ( $\mathrm{IC}_{50}=30.6$ and $84.9 \mu \mathrm{M}$, respectively) and also inhibited replication of recombinant mutant viruses bearing these mutations in plaque reduction assays. However, $\mathbf{5 9}$ was inactive against the S31N mutant. It is interesting to compare the structure and the activity of $\mathbf{5 9}$ with the spiroadamantane 48 (Fig. 19). While 59 and 48 displayed very similar activities against the wt channel $\left(\mathrm{IC}_{50}=12.6\right.$ and $18.7 \mu \mathrm{M}$, respectively), 48 is much more potent against the amantadine-resistant mutants (V27A, $\mathrm{IC}_{50}=0.3 \mu \mathrm{M}$; L26F, $\left.\mathrm{IC}_{50}=5.6 \mu \mathrm{M}\right)$ [48].

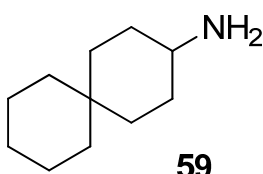

59

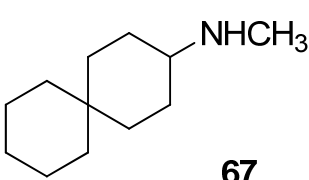

67

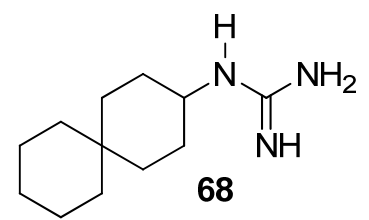<smiles>NCCCNC1CCC2(CCCCC2)CC1</smiles>

Figure 24. Spiro[5.5]undecan-3-amine $\mathbf{5 9}$ and related compounds.

\section{Ring-contracted adamantane analogs}

For the wt M2 protein, the diameter of the hole made from four Ser31 of separate trans-membrane chains is about $8 \AA$. However, after the mutation of residue 31 from Ser to Asn, the diameter of this hole was reduced to $6.32 \AA$ [20i]. As the X-ray structure of the M2-amantadine complex shows that 
amantadine is located in the hole between Ser31 and Ala34, the mutation of Ser to Asn leaves less space for amantadine entering or being stabilized [19d].

Taking into account this reduction in the space available for binding, we synthesized a series of ring-contracted amantadine and rimantadine analogs, featuring noradamantane and bisnoradamantane scaffolds (Fig. 25). Several derivatives showed low micromolar inhibitory activities of the wt M2 channel ranging from $\mathrm{IC}_{50}=2.4 \mu \mathrm{M}$ for guanidine $\mathbf{7 2}$ to $\mathrm{IC}_{50}=17 \mu \mathrm{M}$ for $\mathbf{7 1}$ and $\mathbf{7 5}$. The activity was confirmed by plaque reduction assays with influenza A/Udorn/72 (H3N2) strain, carrying wt M2 protein and, for $\mathbf{7 0}$ it was also confirmed in an assay of inhibitory effect on virus replication using influenza A/Hong Kong/7/87 (H3N2) strain [60]. However, only bisnoradamantane derivative $\mathbf{7 4}$ showed to be moderately active against the S31N channel $\left(\mathrm{IC}_{50}=252 \mu \mathrm{M}\right)$, being less potent than amantadine $\left(\mathrm{IC}_{50}=200 \mu \mathrm{M}\right)[60 \mathrm{~b}]$. Several bisnoradamantanes carrying additional rings were also studied and some of them showed to be slightly less potent than amantadine. For example, pyrrolidine derivative 76, had $\mathrm{IC}_{50}=24 \mu \mathrm{M}$ against the wt channel of influenza A.

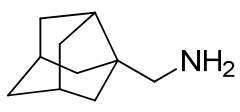

70

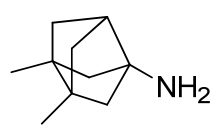

74

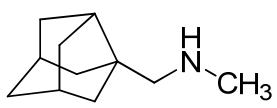

71

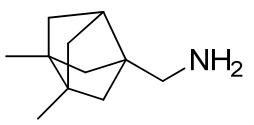

75

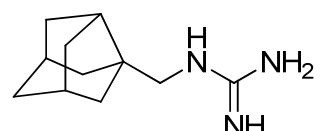

72

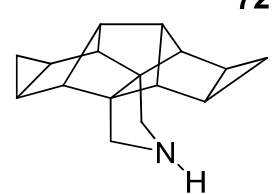

76

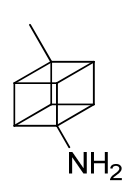

77

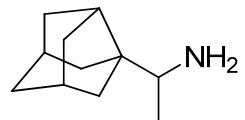

73

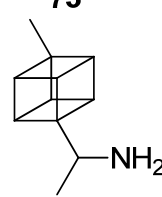

78

Figure 25. Ring-contracted analogs of amantadine and rimantadine.

Cubylamines also can be regarded as ring-contracted analogs of amantadine and rimantadine. In 1971, Du Pont de Nemours, claimed in a patent antiinfluenza activity for several cubane derivatives, such as 4-methylcubane-1amine, 77, and $\alpha$,4-dimethylcubane-1-methylamine, 78 (Fig. 25). When mice infected with the influenza A/Ann Arbor/2/60 (H2N2) strain were treated with the rimantadine analog $\mathbf{7 8}$, there was a $70 \%$ survival rate as compared with $20 \%$ survivors in the infected, non-treated control animals [61].

\section{Aminobicyclo[2.2.1]heptanes, aminobicyclo[2.2.2]octanes and related compounds}

As early as in 1969, Smith Kline \& French disclosed that bicyclo[2.2.1]heptanes 79-81 (Fig. 26) had anti-influenza activity. They 
reported that compound $\mathbf{8 0}$, at oral and subcutaneous doses of $25-100 \mathrm{mg} / \mathrm{kg}$, caused a $35-80 \%$ and a $30-75 \%$ increase in survival of mice infected with the influenza A/Ann Arbor/2/60 (H2N2) strain, and with a swine strain of influenza A, respectively [62]. Although no further details have been published in the western literature related to the antiviral activity of $\mathbf{7 9}$ or $\mathbf{8 0}$, later, Russian researchers found that an isomeric mixture of $\mathbf{8 1}$ and $\mathbf{8 2}$ effectively inhibited replication of influenza viruses and this mixture, as its hydrochloride, known as deitiforin, has been in used as antiviral in the former USSR for several years [63]. As an anti-influenza drug, deitiforin is equal to rimantadine from the standpoint of the protective effect in the treatment of influenza infection, and it can not only efficiently supress virus-specific growth, but can also selectively act on virus-infected cells. It has been found that influenza A/Victoria/35/72 (H3N2) strain resistant to deitiforin mutated the M2 protein in 3 amino acids: Met14Leu, Ala30Val and Met59Leu [64]. Interestingly, compound ICI 130685, which can be regarded as a derivative of $\mathbf{8 1}$ with further rings, advanced into clinical trials, but was not approved for clinical use [65].

García Martínez and coworkers have reported that several 1-norbornylamines were also endowed with potent anti-influenza activity. Secondary amines $\mathbf{8 3}$ and $\mathbf{8 4}$ were more potent than amantadine and showed very high SI [66].

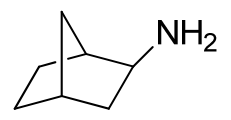

79

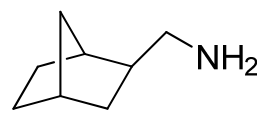

80

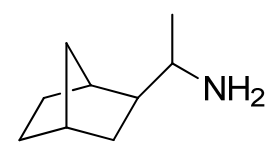

81

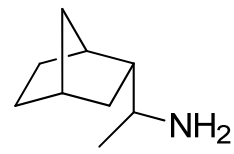

82

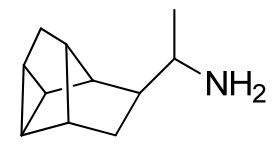

ICI 130685

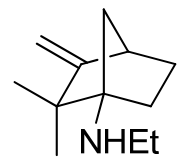

83

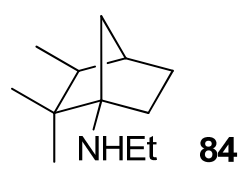

Figure 26. Bicyclo[2.2.1]heptanes with anti-influenza activity.

In 1969, DuPont de Nemours \& Co, also claimed anti-influenza activity for a series of bicyclo[2.2.2]octan-1-amines, 85, bicyclo[2.2.2]oct-2-en-1amines, 86, bicyclo[2.2.2] octane-1-methylamines, 87, and bicyclo[2.2.2]oct2-ene-1-methylamines, 88 [67]. They tested the compounds in mice using the influenza $\mathrm{A} / \mathrm{swine} / \mathrm{S}-15$ strain and found that the unsaturated cage amines were similar in antiviral activity to their saturated counterparts. As seen in other families of polycyclic amines, substitution on the amino group with alkyl groups decreased the anti-viral activity. The addition of a methyl group in C-4 of the bicyclo[2.2.2]octane was optimal but inclusion of a larger group 
reduced the activity. Finally, the presence of $\alpha$-alkyl groups in the bicyclo[2.2.2] octane-1-methylamine series enhances antiviral activity. Overall, rimantadine analog $\mathbf{8 9}$ was the most active compound.

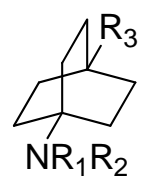

85

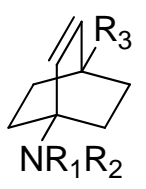

86

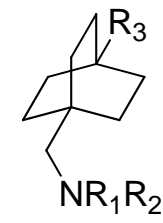

87

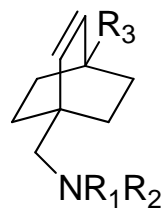

88

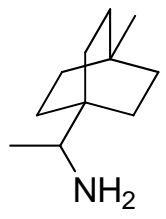

89

Figure 27. Bicyclo[2.2.2] octan-1-amine, 85, and related compounds.

Interestingly, Inamoto and co-workers reported the synthesis of several tricyclo[5.3.1.0 $\left.0^{3,8}\right]$ undecane (4-homoisotwistane) derivatives, such as amines 90 and 91. 4-Homoisotwistanes can be seen as bicyclo[2.2.2]octane derivatives carrying an additional ring. Amines $\mathbf{9 0}$ and $\mathbf{9 1}$ were quite active against the Newcastle disease virus, which is sensitive to amantadine and is the causal agent of a bird disease that, when infecting humans, causes influenza-like symptoms. However, they did not test these compounds against influenza virus [68].

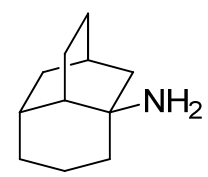

90

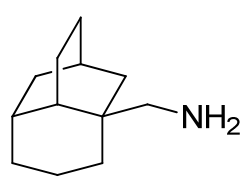

91

Figure 28. Tricyclo[5.3.1.0 $\left.0^{3,8}\right]$ undecane derivatives.

\section{Other polycycloalkanes with anti-influenza activity}

Finally, in this section we will discuss several unrelated polycyclic structures that have shown anti-influenza activity. For example, there are several amines derived from the pentacyclo[5.4.0.0 $\left.0^{2,6} \cdot 0^{3,10} \cdot 0^{5,9}\right]$ undecane that have been biologically tested. In the 1970s, Smith, Kline \& French, reported that amine 92 (Fig. 29) showed marginal activity against influenza A/Ann Arbor, while its isomer 93 was inactive [69]. Very recently, DeGrado and coworkers have found that amine $\mathbf{9 4}$ inhibited the activity of the wt M2 channel of influenza A expressed in oocytes of Xenopus laevis, with an $\mathrm{IC}_{50}=8 \mu \mathrm{M}$, lower than that of amantadine $\left(\mathrm{IC}_{50}=16 \mu \mathrm{M}\right)$ and rimantadine $\left(\mathrm{IC}_{50}=10.8 \mu \mathrm{M}\right)$. As seen in other polycyclic derivatives, the addition of a 
hydroxyl group, as in 95, was compatible with inhibitory activity $\left(\mathrm{IC}_{50}=24 \mu \mathrm{M}\right)$, but not increased the potency [30].

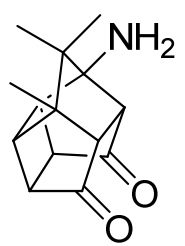

92

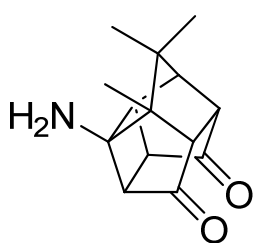

93

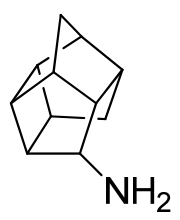

94

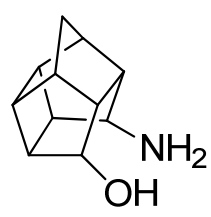

95

Figure 29. Derivatives of pentacyclo[5.4.0.0 $\left.0^{2,6} \cdot 0^{3,10} \cdot 0^{5,9}\right]$ undecane.

In 2010, Hu et al. carried out the screening of a small primary amine library as M2 protein inhibitors. They reported that linear alkyl amines, aromatic amines and unsubstituted monocyclic amines were inactive. However, they found five compounds, 14, previously studied by Scholtissek [33], and 96-99 (Fig. 30), with similar activities to that of amantadine. Isopinocamphenylamine 99, the most potent inhibitor, was three times more active than amantadine $\left(\mathrm{IC}_{50}=1.4 \mu \mathrm{M}\right.$ vs $\mathrm{IC}_{50}=6.0 \mu \mathrm{M}$ ) for viral inhibition of the influenza A/Hong Kong/8/68 (H3N2) strain [70]. Encouraged by these results, the same group has very recently published a small library of derivatives of 99 obtained by keeping the scaffold constant and modifying the amino functionality. The compounds were evaluated for viral inhibiton against influenza A/WS/33 (H1N1), amantadine resistant, and influenza A/Hong Kong/8/68 (H3N2), amantadine sensitive. Although there was no inhibition of the amantadine resistant strain, most of the compounds exhibited antiviral inhibition as good as amantadine against the amantadine sensitive strain. Compound $100\left(\mathrm{IC}_{50}=0.09 \mu \mathrm{M}\right.$ ) was nearly 240-fold more potent than amantadine against wt influenza A virus [71].

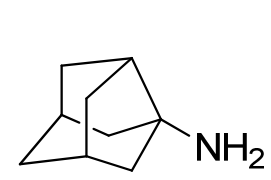

14<smiles>CC(C)(C)C1CCC(N)CC1</smiles>

96<smiles>C[C@H]1C(N)CC2CC1C2(C)C</smiles>

97<smiles>CC1CC2(C)CCC1C2N=Cc1ccc(O)cc1</smiles>

98<smiles>CC1CC2(C)CCCC1C2N</smiles>

100

Figure 30. Primary amine inhibitors of M2 channel and derivative $\mathbf{1 0 0 .}$ 
Finally, DeGrado and coworkers tested the inhibitory activity against wt M2 channel of a series of branched and polycyclic amines (Fig. 21) [30]. Surprisingly, branched alkyl amine $\mathbf{1 0 1}$ was nearly as active as amantadine $\left(\mathrm{IC}_{50}=21 \mu \mathrm{M}\right.$ vs $\left.\mathrm{IC}_{50}=16 \mu \mathrm{M}\right)$, tricyclic amine 102, showed higher activity than amantadine $\left(\mathrm{IC}_{50}=9 \mu \mathrm{M}\right)$ and four homoadamantane derivatives, 103-105 and 13 showed similar activity as amantadine, suggesting that the M2 channel can accommodate a wide range of structural diversity and that is insensitive to minor scaffold modifications, so long as the shape of the molecule conforms to the M2 cavity. All these compounds were found to be less potent or inactive against V27A and/or S31N mutant channels, probably as a consequence of the higher polarity of the mutant channels [30].

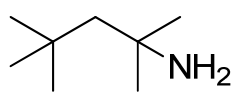

101

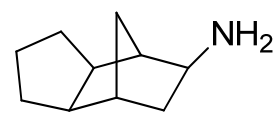

102

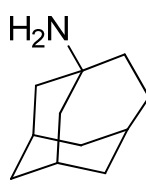

103

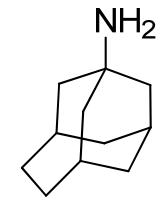

104

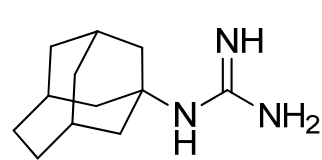

105

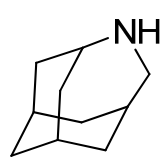

13

Figure 31. Several inhibitors of M2 channel.

\section{Conclusion}

Although amantadine and rimantadine have been in clinical use for many years and hundreds of analogs have been tested as anti-influenza agents, the results obtained so far are a bit disappointing. While several active compounds have been found, occasionally having more potency than amantadine and rimantadine, cross-resistance with both drugs is still an unresolved issue. The above notwithstanding, the recent structural, functional, and computational studies carried out with M2 protein have opened the door to the rational design of new inhibitors [72], and, very recently, some derivatives have shown promising activity against the V27A amantadine-resistant mutant [30,48,59]. The S31N mutant is still even a major challenge.

\section{Acknowledgements}

This work was supported by Grants from Spanish Ministerio de Ciencia e Innovación (FPU fellowship to MDD and to ET; grant CTQ2008-03768). 


\section{References}

1. a) Reid, A. H., Taubenberger, J.K. 2001, Microbes Infect., 3, 81., b) Johnson, N. P. A. S., Mueller, J. 2002, Bull. Hist. Med., 76, 105.

2. Kilbourne, E. D., 2006, Emerging Infect. Dis., 12, 9.

3. Chowell, G., Bertozzi, S. M., Colchonero, A., López-Gatell, H., Alpuche-Aranda, C., Hernández, M., Miller, M. A. 2009, N. Engl. J. Med., 361, 674., b) Garten, R. J., 2009, Science, 325, 197-201., c) Giambenedetto, S., Zileri Dal Verme, L., Sali, M., Farina, S., Di Cristo, V., Manzara, S., De Luca, A., Pignataro, G., Prosperi, M., Franco, A., Gentiloni Silveri, N., Delogu, G., Cauda, R., Fabbiani, M., Fadda, G., 2010, Eur. J. Clin. Microbiol. Infect. Dis. 541., d) Tang, J. W., Shetty, N., Lam, T. T.-Y., 2010, Curr. Opin. Pulmon. Med., 16, 235., e) Schuchat, A., Bell, B. P., Redd, S. C. 2011, Clin. Infect. Dis., 52, S8., f) Ilyushina, N. A., Ducatez, M. F., Rehg, J. E., Marathe, B. M., Marjuki, H., Bovin, N. V., Webster, R. G., Webby, R. J. 2010, mBio, 1, 1.

4. a) Liu, J., Xiao, H., Lei, F., Zhu, Q., Qin, K., Zhang, X. W., Zhang, X. I., Zhao, D., Wang, G., Feng, Y., Ma, J., Liu, W., Wang, J., Gao, G. F. 2005, Science, 309, 1206., b) Normile, D. 2008, Science, 319, 1178., c) The Writing Committee of the Second World Health Organization Consultation on Clinical Aspects of Human Infection with Avian Influenza A (H5N1) Virus. 2008, N. Engl. J. Med., 358, 261. d) Guarner, J., Falcón-Escobedo, R. 2009, Arch. Med. Res., 40, 655.

5. a) Lamb, R. A., and Krug, R. M. 2001, Orthomyxoviridae: the viruses and their replication, in Fields Virology $4^{\text {th }}$ edn., Knipe, D. M., and Howley, P. M. (Eds.), Lippincott, Williams \& Wilkins, Philadelphia (USA), 1487., b) Wright, P. F., Webster, R. G. 2001, Orthomyxoviruses, in Fields Virology $4^{\text {th }}$ edn., Knipe, D. M., and Howley, P. M. (Eds.), Lippincott, Williams \& Wilkins, Philadelphia (USA), 1533.

6. a) Taubenberger, J.K., Morens, D.M. 2006, Emerging Infect. Dis., 12, 15., b) Belshe, R.B. 2005, N. Engl. J. Med., 353, 2209.

7. Kilbourne, E. D. 2006, Emerging Infect. Dis., 12, 9.

8. a) De Clercq, E. 2006, Nat. Rev. Drug Discovery, 5, 1015., b) Jackson, R. J., Cooper, K. L., Tappenden, P., Rees, A., Simpson, E. L., Read, R. C., Nicholson, K. G., 2011, J. Infect., 62, 14.

9. a) Rungrotmongkol, T., Intharathep, P., Malaisree, M., Nunthaboot, N., Kaiyawet, N., Sompornpisut, P., Payungporn, S., Poovorawan, Y., Hannongbua, S. 2009, Biochem. Biophys. Res. Commun., 385, 390., b) Oakley, A. J., Barrett, S., Peat, T. S., Newman, J., Streltsov, V. A., Eaddington, L., Saito, T., Tashiro, M., McKimm-Breschkin, J. L. 2010, J. Med. Chem., 53, 6421., c) Dharan, N. J., Beekmann, S. E., Fiore, A., Finelly, L., Uyeki, T. M., Polgreen, P. M., Fry, A. M. 2010, Antiviral Res., 88, 182., d) Dolin, R. 2011, Clin. Infect. Dis., 52, 432.

10. a) Bright, R. A., Shay, D. K., Shu, B., Cox, N. J., Klimov, A. I. 2006, J. Am. Med. Assoc., 295, 891., b) Nelson, M. I., Simonsen, L., Viboud, C., Miller, M. A., Colmes, E. C. 2009, Virology, 388, 270., c) Gubareva, L. V., Trujillo, A. A., Okomo-Adhiambo, M., Mishin, V. P., Deyde, V. M., Sleeman, K., Nguyen, H. T., 
Sheu, T. G., Garten, R. J., Shaw, M. W., Fry, A. M., Klimov, A. I. 2010, Antivir. Ther., 15, 1151.

11. a) Warnick, J. E., Maleque, M. A., Bakry, N., Eldefrawi, A. T., Albuquerque, E. X. 1982, Mol. Pharmacol., 22, 82., b) Matsubayashi, H., Swanson, K. L., Albuquerque, E. X. 1997, J. Pharmacol. Exp. Ther., 281, 834., c) Blanpied, T. A., Clarke, R. J., Johnson, J. W. 2005, J. Neurosci., 25, 3312., d) Chen, J. J., Swope, D. M. 2007, Pharmacotherapy, 27, 161S., e) Brotchie, J. 2010, Expert Rev. Neurother., 10, 871.

12. a) Dolin, R., Reichman, R. C., Madore, H. P., Maynard, R., Linton, P. N., Webber-Jones, J. 1982, N. Engl. J. Med., 307, 580., b) Van Voris, L. P., Betts, R. F., Hayden, F. G., Christmas, W. A., Douglas, R. G., 1981, J. Am. Med. Assoc., 245, 1128.

13. a) De Clercq, E., Lagoja, I. M. 2008, Med. Res. Rev., 28, 1., b) Gong, J., Fang, H., Li, M., Yang, K., Liu, Y., Xu, W. 2009, Curr. Med. Chem., 16, 3716., c) Krug, R. M., Aramini, J. M. 2009, Trends Pharmacol. Sci., 30, 269., d) Majerova, T., Hoffman, H., Majer, F. 2010, Collect. Czech. Chem. Commun., 75, 81., e) Saladino, R., Barontini, M., Crucianelli, M., Nencioni, L., Sgarbanti, R., Palamara, A. T. 2010, Curr. Med. Chem., 17, 2101., f) Boltz, D. A., Aldridge Jr., J. R., Webster, R. G., Govorkova, E. A. 2010, Drugs, 70, 1349., g) Beigel, J. H. 2010, Drugs Future, 35, 385., h) Ge, H., Wang, Y.-F., Xu, J., Gu, Q., Liu, H.-B., Xiao, P.-G., Zhou, J., Liu, Y., Yang, Z., Su, H. 2010, Nat. Prod. Rep., 27, 1758., i) Guo, X., Wang, J.-F., Zhu, Y., Wei, D.-Q. 2010, Curr. Comput.-Aided Drug Des., 6, 139., j) Das, K., Aramini, J. M., Ma, L.-C., Krug, R. M., Arnold, E. 2010, Nat. Struct. Mol. Biol., 17, 530.

14. a) Pinto, L. H., Lamb, R. A. 2006, J. Biol. Chem., 281, 8997., b) Pinto, L. H., Lamb, R. A. 2007, Mol. BioSyst., 3, 18., c) Betakova, T. 2007, Curr. Pharm. Des., 13, 3231., d) Kozakov, D., Chuang, G.-Y., Beglov, D., Vajda, S. 2010, Trends Biochem. Sci., 35, 471., e) Pielak, R. M., Chou, J. J. 2011, Protein Cell, 1, 246. f) Pielak, R. M., Chou, J. J. 2011, Biochim. Biophys. Acta, Biomembr., 1808, 522., g) Wang, J., Qui, J. X., Soto, C., DeGrado, W. F. 2011, Curr. Opin. Struct. Biol., 21, 68.

15. Skehel, J. J., Wiley, D. C. 2000, Ann. Rev. Biochem., 69, 531.

16. a) Pinto, L. H., Holsinger, L. J., Lamb, R. A. 1992, Cell, 69, 517., b) Wang, C., Lamb, R. A., Pinto, L. H. 1995, Biophys. J., 69, 1363., c) Tang, Y., Venkataraman, P., Knopman, J., Lamb, R. A., Pinto, L. H. 2005, The M2 proteins of influenza A and B viruses are single-pass proton channels, in Viral Membrane Proteins: Strcuture, Function, and Drug Design, Fischer, W. (Ed.), Kluwer Academic / Plenum Publishers, New York, 101.

17. Grambas, S., Hay, A. J. 1992, Virology, 190, 11.

18. a) Hay, A. J., Wolstenholme, A. J., Skehel, J. J., Smith, M. H. 1985, EMBO J., 4, 3021., b) Hay, A. J. 1992, Semin. Virol., 3, 21.

19. a) Hu, J., Fu., R., Nishimura, K., Zhang, L., Zhou, H.-X., Busath, D. D., Vijayvergiya, V., Cross, T. A. 2006, Proc. Natl. Acad. Sci. USA, 103, 6865., b) Hu, J., Asbury, T., Achuthan, S., Li, C., Bertram, R., Quine, J. R., Fu., R., Cross, T. A. 2007, Biophys. J., 92, 4335., c) Schnell, J. R., Chou, J. J. 2008, 
Nature, 451, 591., d) Stouffer, A. L., Acharya, R., Salom, D., Levine, A. S., Di Costanzo, L., Soto, C. S., Tereshko, V., Nanda, V., Stayrook, S., DeGrado, W. F. 2008, Nature, 451, 596., e) Cady, S. D., Hong, M. 2008, Proc. Natl. Acad. Sci. USA, 105, 1483., f) Jing, X., Ma, C., Ohigashi, Y., Oliveira, F. A., Jardetzky, T. S., Pinto, L. H., Lamb, R. A. 2008, Proc. Natl. Acad. Sci. USA, 105, 10967., g) Li, C., Yi, M., Zhou, H.-X. Cross, T. A. 2008, Biophys. J., 94, 1295., h) Luo, W., Cady, S. D., Hong, M. 2009, Biochemistry, 48, 6361., i) Cady, S. D., Luo, W., Hu, F., Hong, M. 2009, Biochemistry, 48, 7356., j) Chuang, G.-Y., Kozakov, D., Brenke, R., Beglov, D., Guarnieri, F., Vajda, S. 2009, Biophys. J., 97, 2846., k) Cady, S. D., Hong, M. 2009, J. Biomol. NMR, 45, 185., l) Cady, S. D., Mishanina, T. V., Hong, M. 2009, J. Mol. Biol., 385, 1127., m) Ma, C., Polishchuk, A. L., Stouffer, A. L., Schön, A., Magavern, E., Jing, X., Lear, J. D., Freire, E., Lamb, R. A., DeGrado, W. F., Pinto, L. H. 2009, Proc. Natl. Acad. Sci. USA, 106, 12283., n) Yi, M., Cross, T. A., Zhou, H.-X. 2009, Proc. Natl. Acad. Sci. USA, 106, 13311., o) Busath, D. D. 2009, Adv. Planar Lipids Bilayers Liposomes, 10, 161., p) Cady, S. D., Schmidt-Rohr, K., Wang, J., Soto, C. S., DeGrado, W. F. 2010, Nature, 463, 689., q) Rosenberg, M. R., Casarotto, M. G. 2010, Proc. Natl. Acad. Sci. USA, 107, 13866., r) Acharya, R., Carnevale, V. Fiorin, G., Levine, B. G., Polishchuk, A. L., Balannik, V., Samish, I., Lamb, R. A., Pinto, L. H. 2010, Proc. Natl. Acad. Sci. USA, 107, 15075., s) Andreas, L. B., Eddy, M. T., Pielak, R. M., Chou, J. J., Griffin, R. G. 2010, J. Am. Chem. Soc., 132, 10958., t) Pielak, R. M., Chou, J. J. 2010, J. Am. Chem. Soc., 132, 17695., u) Pielak, R. M., Chou, J. J. 2010, Biochem. Biophys. Res. Commun., 401, 58., v) Fiorin, G., Carnevale, V., DeGrado, W. F. 2010, Science, 330, 456., w) Hu, F., Luo, W., Hong, M. 2010, Science, 330, 505., x) Sharma, M., Yi, M., Dong, H., Qin, H., Peterson, E., Busath, D. D., Zhou, H.-X., Cross, T. A. 2010, Science, 330, 509., y) Cady, S. D., Wang, J., Wu, Y., DeGrado, W. F., Hong, M. 2011, J. Am. Chem. Soc., 133, 4274., z) Rogers, J. M. G., Polishchuk, A. L., Guo, L., Wang, J., DeGrado, W. F., Gai, F. 2011, Langmuir, 27, 3815., aa) Ghosh, A., Qiu, J., DeGrado, W. F., Hochstrasser, R. M. 2011, Proc. Natl. Acad. Sci. USA, 108, 6115.

20. a) Yi, M., Cross, T. A., Zhou, H.-X. 2008, J. Phys. Chem. B, 112, 7977. , b) Carpenter, T., Bond, P. J., Khalid, S., Sansom, M. S. P. 2008, Biophys. J., 95, 3790., c) Huang, R.-B., Du, Q.-S., Wang, C.-H., Chou, K.C. 2008, Biochem. Biophys. Res. Commun., 377, 1243., d) Intharathep, P., Laohpongspaisan, C., Rungrotmongkol, T., Loisruangsin, A., Malaisree, M., Decha, P., Aruksakunwong, O., Chuenpennit, K., Kaiyawet, N., Sompornpisut, P., Pianwanit, S., Hannongbua, S. 2008. J. Mol. Graphics Modell., 27, 342., e) Khurana, E., Dal Peraro, M., DeVane, R. H., Vemparala, S., DeGrado, W. F., Klein, M. L. 2009, Proc. Natl. Acad. Sci. USA, 106, 1069., f) Leonor, H., Arkin, I. T. 2009, J. Mol. Model., 15, 1317., g) Laohpongspaisan, C., Rungrotmongkol, T., Intharathep, P., Malaisree, M., Decha, P., Aruksakunwong, O., Sompornpisut, P., Hannongbua, S. 2009, J. Chem. Inf. Model., 49, 847., h) Qin, G., Yu, K., Shi, T., Luo, C., Li, G., Zhu, W., Jiang, H. 2010, J. Phys. Chem. B, 114, 8487., i) Carnevale, V., Fiorin, G., Levine, B. G., DeGrado, W. F., Klein, M. L. 2010, J. Phys. Chem. C, 114, 20856., j) Khurana, E., DeVane, R. H., Dal Peraro, M., 
Klein, M. L. 2011, Biochim. Biophys. Acta, 1808, 530., k) Intharathep, P., Rungrotmongkol, T., Decha, P., Nunthaboot, N., Kaiyawet, N., Kerdcharoen, T., Sompornpisut, P., Hannongbua, S. 2011, J. Enzyme Inhib. Med. Chem., 26, 162.

21. a) Deyde, V. M., Xu, X., Bright, R. A., Shaw, M., Smith, C. B., Zhang, Y., Shu, Y. Gubareva, L. V., Cox, N. J., Klimov, A. I. 2007, J. Infect. Dis., 196, $249 .$, b) Furuse, Y.; Suzuki, A.; Kamigaki, T., Oshitani, H. 2009, Virol. J., 6, 67., c) Astrahan, P., Arkin, I. T. 2011, Biochim. Biophys. Acta, Biomembr., 1808, 547.

22. Balannik, V., Carnevale, V., Fiorin, G., Levine, B. G., Lamb, R. A., Klein, M. L., DeGrado, W. F., Pinto, L. H. 2010, Biochemistry, 49, 696.

23. a) Oxford, J. S., Galbraith, A. 1980, Pharmacol. Ther., 11, 181., b) Tilley, J. W., Kramer, M. J. 1981, Progress Med. Chem., 18, 1., c) Hayden, F. G., Minocha, A., Spyker, D. A., Hoffma, H. E. 1985, Antimicrob. Agents Chemother., 28, 216. , d) Belshe, R. B., Burk, B., Newman, F., Cerruti, R. L., Sim, I. S., 1989, J. Infect. Dis., 159, 430.

24. a) Zlydnikov, D. M., Kubar, O. I., Kovaleva, T. P., Kamforin, L. E. 1981, Rev. Infect. Dis., 3, 408., b) Wintermeyer, S. M., Nahata, M. C. 1995, Ann. Pharmacother., 29, 299.

25. Davies, W. L., Grunert, R. R., Haff, R. F., McGahen, J. W., Neumayer, E. M., Paulshock, M., Watts, J. C., Wood, T. R., Hermann, E. C., Hoffmann, C. E. 1964, Science, 144, 862.

26. a) Paulshock, M., Watts, J. C. (E. I. du Pont de Nemours and Co.), 1967, US 3310469., b) Geluk, H. W., Schut, J., Schlatmann, J. L. M. A. 1969, J. Med. Chem., 12, 712., c) Aldrich, P. E., Hermann, E. C., Meier, W. E., Paulshock, M., Prichard, W. W., Snyder, J. A., Watts, J. C. 1971, J. Med. Chem., 14, 535., d) Inamoto, Y., Nakayama, 1971, J. Chem. Eng. Data, 16, 483., e) Aigami, K., Inamoto, Y., Takaishi, N., Hattori, K., Takatsuki, A., Tamura, G. 1975, J. Med. Chem., 18, 713., f) Hess, U., Huhn, D., Neubert-Hils, J., May, A. (HumboldtUniversität zu Berlin), 1989, EP 358152.

27. Koppel, C., Tenczer, J. 1985, Biomed. Mass Spectrom. 12, 499.

28. a) Rubio, F. R., Fukuda, E. K., Garland, W. A. 1988, Drug Metab. Dispos., 16, 773., b) Rubio, F. R., Choma, N., Fukuda, E. K. 1989, J. Chromatogr., Biomed. Appl., 497, 147., c) Loh, A. C., Szuna, A. J., Williams, T. H., Sasso, G. J., Leinweber, F. J. 1991, Drug Metab. Dispos., 19, 381., d) Choma, N., Davis, P. P., Edom, R. W., Fukuda, E. K. 1992, Biomed. Chromatogr., 6, 12.

29. a) Manchand, P. S., Cerruti, R. L., Martin, J. A., Hill, C. H., Merrett, J. H., Keech, E., Belshe, R. B., Connell, E. V., Sim, I. S. 1990, J. Med. Chem., 33, 1992., b) Bailey, P. D., Higgins, S. D., Ridyard, C. H., Roberts, S. M., Rosaire, G. M., Whittaker, R. A., Willets, A. J. 1996, Chem. Commun., 1833.

30. Wang, J., Ma, C., Balannik, V., Pinto, L. H., Lamb, R. A., DeGrado, W. F. 2011, ACS Med. Chem. Lett., 2, 307.

31. a) Fytas, G., Stamatiou, G., Foscolos, G. B., Kolocouris, A., Kolocouris, N., Witvrouw, M., Pannecouque, C., De Clercq, E. 1997, Bioorg. Med. Chem., 7, 1887., b) Stamatiou, G., Foscolos, G. B., Fytas, G., Kolocouris, A., Kolocouris, N., Pannecouque, C., Witvrouw, M., Padalko, E., Neyts, J., De Clercq, E. 2003, Bioorg. Med. Chem., 11, 5485., c) Zoidis, G., Fytas, C., Papanastasiou, I., 
Foscolos, G. B., Fytas, G., Padalko, E., De Clercq, E., Naesens, L., Neyts, J., Kolocouris, N. 2006, Bioorg. Med. Chem., 14, 3341.

32. Tataridis, D., Fytas, G., Kolocouris, A., Fytas, C., Kolocouris, N., Foscolos, G. B., Padalko, E., Neyts, J., De Clercq, E. 2007, Bioorg. Med. Chem., 17, 692.

33. Scholtissek, C., Quack, G., Klenk, H. D., Webster, R. G. 1998, Antiviral Res., 37, 83.

34. Zarubaev, V. V., Golod, E. L., Anfimov, P. M., Shtro, A. A., Saraev, V. V., Gavrilov, A. S., Logvinov, A. V., Kiselev, O. I. 2010, Bioorg. Med. Chem., 18, 839.

35. Zhang, W., Xu, J., Liu, F., Li, C., Jie, Y., Chen, S., Li, Z., Liu, J., Chen, L., Zhou, G. 2010, Chin, J. Chem., 28, 1417.

36. Kolocouris, A., Spearpoint, P., Martin, S. R:, Hay, A. J., López-Querol, M., Sureda, F. X., Padalko, E., Neyts, J., De Clercq, E. 2008, Bioorg. Med. Chem. Lett., 18, 6156.

37. Zoidis, G., Kolocouris, N., Kelly, J. M., Prathalingam, S. R., Naesens, L., De Clercq, E. 2010, Eur. J. Med. Chem., 45, 5022.

38. Zoidis, G., Kolocouris, N., Foscolos, G. B., Kolocouris, A., Fytas, G., Karayannis, P., Padalko, E., Neyts, J., De Clercq, E. 2003, Antiviral Chem. Chemother., 14, 153.

39. a) Kolocouris, A., Tataridis, D., Fytas, G., Mavromoustakos, T., Foscolos, G. B., Kolocouris, N., De Clercq, E. 1999, Bioorg. Med. Chem. Lett., 9, 3465., b) Stamatiou, G., Kolocouris, A., Kolocouris, N., Fytas, G., Foscolos, G. B., Neyts, J., De Clercq, E. 2001, Bioorg. Med. Chem. Lett., 11, 2137., c) Setaki, D., Tataridis, D., Stamatiou, G., Kolocouris, A., Foscolos, G. B., Fytas, G., Kolocouris, N., Padalko, E., Neyts, J., De Clercq, E. 2006, Bioorg. Chem., 34, 248.

40. a) de Bock, C. A., Schlatmann, J. L. M. A. (Koninkl Philips Electronics N. V), 1969, NL 6804904 (see also US 3733412, GB 1220750)., b) N. V. Philips, 1969, NL 6900004 (see also GB 1264500, DE 1965481)., c) Lundahl, K., Schut, J., Schlatmann, J. L. M. A., Paerels, G. B., Peters, A. 1972, J. Med. Chem., 15, 129., d) van Hes, R., Smit, A., Kralt, T., Peers, A. 1972, J. Med. Chem., 15, 132.

41. a) Beare, A. S., Hall, T. S., Tyrrell, D. A. J. 1972, Lancet, 299, 1039., b) Mathur, A., Beare, A. S., Reed, S. E. 1973, Antimicrob. Agents Chemother., 4, 421., c) Togo, Y. 1973, Antimicrob. Agents Chemother., 4, 641.

42. a) Kolocouris, N., Foscolos, G. B., Kolocouris, A., Marakos, P., Pouli, N., Fytas, G., Ikeda, S., De Clercq, E. 1994, J. Med. Chem., 37, 2896., b) Kolocouris, N., Kolocouris, A., Foscolos, G. B., Fytas, G., Neyts, J., Padalko, E., Balzarini, J., Snoeck, R., Andrei, G. De Clercq, E. 1996, J. Med. Chem., 39, 3307.

43. Stylianakis, I., Kolocouris, A., Kolocouris, N., Fytas, G., Foscolos, G. B., Padalko, E., Neyts, J., De Clercq, E. 2003, Bioorg. Med. Chem. Lett., 13, 1699.

44. Kolocouris, N., Zoidis, G., Foscolos, G. B., Fytas, G., Prathalingham, S. R., Kelly, J. M., Naesens, L., De Clercq, E. 2007, Bioorg. Med. Chem. Lett., 17, 4358.

45. Fytas, C., Kolocouris, A., Fytas, G., Zoidis, G., Valmas, C., Basler, C. F. 2010, Bioorg. Chem., 38, 247. 
46. Eleftheratos, S., Spearpoint, P., Ortore, G., Kolocouris, A., Martinelli, A., Martin, S., Hay, A. 2010, Bioorg. Med. Chem. Lett., 20, 4182.

47. Kolocouris, N., Kolocouris, A., Foscolos, G. B., Padalko, E., Neyts, J., De Clercq, E. 2002, In searching for new anti-influenza drugs: heterocyclic and carbocyclic aminoadamantanes bearing a 2-adamantyl group with potent activity against influenza A virus, in Drug Discovery and Design: Medical Aspects, Matsoukas, J., and Mavromoustakos, T. (Eds.), IOS Press, Amsterdam, 103.

48. DeGrado, W. F., Wang, J. (University of Pennsylvania), 2011, WO 2011/022191.

49. a) Narayanan, V. L. (E. R. Squibb \& Sons, Inc.), 1971, US 3624126., b) Narayanan, V. L. (E. R. Squibb \& Sons, Inc.), 1972, US 3705906., c) E. R. Squibb \& Sons, Inc., 1973, GB 1328696.

50. a) Zoidis, G., Tsotinis, A., Kolocouris, N., Kelly, J. M., Prathalingam, S. R., Naesens, L., De Clercq, E. 2008, Org. Biomol. Chem., 6, 3177., b) Zoidis, G., Kolocouris, N., Naesens, L., De Clercq, E. 2009, Bioorg. Med. Chem., 17, 1534. Compounds of general structure $\mathbf{4 8}$ had been patented by Eli Lilly, see US 3668220, 1972.

51. Wei, H., Wang, C.-H., Du, Q.-S., Meng, J., Chou, K.-C. 2009, Med. Chem., 5, 305.

52. Stetter, H., Tacke, P., Gärtner, J. 1964, Chem. Ber., 97, 3480

53. Gagneux, A. R. (Geigy Chemical Corporation), 1971, US 3567829

54. Duque, M. D., Camps, P., Profire, L., Montaner, S., Vázquez, S., Sureda, F. X., Mallol, J., López-Querol, M., Naesens, L., De Clercq, E., Prathalingam, S. R., Kelly, J. M. 2009, Bioorg. Med. Chem., 17, 3198.

55. Kurtz, S., Luo, G. X., Hahnenberger, K. M., Brooks, C., Gecha, O., Ingalls, K., Numata, K. I., Krystal, M. 1995, Antimicrob. Agents Chemother., 39, 2204.

56. a) Tu, Q., Pinto, L. H., Luo, G. X., Shaughnessy, M. A., Mullaney, D., Kurtz, S., Krystal, M., Lamb, R. A. 1996, J. Virol., 70, 4246., b) Gandhi, C. S., Shuck, K., Lear, J. D., Dieckmann, G. R., DeGrado, W. F., Lamb, R. A., Pinto, L. H. 1999, J. Biol. Chem., 274, 5474.

57. Tankersley, Jr., R. W. (A. H. Robins Company, Incorporated), 1977, US 4005224.

58. a) Grogan, C. H (TriKem Corporation), 1965, US 3214470., b) Rice, L. M., Dobbs, E. C., Grogan, C. H 1965, J. Med. Chem., 8, 825., c) Rice, L. M., Sheth, B. S., Zalucky, T. B. 1972, J. Med. Chem., 15, 548.

59. a) Wang, J., Cady, S. D., Balannik, V., Pinto, L. H., DeGrado, W. F., Hong, M. 2009, J. Am. Chem. Soc., 131, 8066., b) Balannik, V., Wang, J., Ohigashi, Y., Jing, X., Magavern, E., Lamb, R. A., DeGrado, W. F., Pinto, L. H. 2009, Biochemistry, 48, 11872.

60. a) Camps, P., Duque, M. D., Vázquez, S., Naesens, L., De Clercq, E., Sureda, F. X., López-Querol, M., Camins, A., Pallàs, M., Prathalingam, S. R., Kelly, J. M., Romero, V., Ivorra, D., Cortés, D. 2008, Bioorg. Med. Chem., 16, 9925., b) Duque, M. D., Ma, C., Torres, E., Wang, J., Naesens, L., Juárez-Jiménez, J., Camps, P., Luque, F. J., DeGrado, W. F., Lamb, R. A., Pinto, L. H., Vázquez, S. 2011, J. Med. Chem., 54, 2646.

61. Gregory, W. A. (E. I. Du Pont de nemours \& Co), 1971, US 3558704.

62. Kormendy, C. G. (Smith Kline \& French Laboratories), 1969, US 3444302. 
63. a) Votiakov, V. I., Boreko, E. I., Rusiaev, V. A., Shashikhina, M. N., Kazak, N. F. 1982, Vopr. Virusol. 27, 172., b) Votiakov, V. I., Gribkova, N. V., Kazak, N. F., Podolskaia, I. A. 1982, Vopr. Virusol., 27, 169., c) Blinov, V. M., Resenchuk, S. M., Karginov, V. A., Mishin, V. P., Kozeletskaya, K. N., Sandakhchiev, L. S., Kiselev, O. I. 1991, Dokl. Akad. Nauk SSSR, 319, 1480., d) Mashkovskii, M. D. Lekarstvennye sredstva (Medicines), Moscow: Meditsina, 1993, vol. 2, p. 396., e) Kiselev, O. I.; Mishin, V. P.; Eroshkin, V. I.; Kozeletskaya, K. N.; Usova, E. V.; Rudenko, V. I.; Chupakhin, O. N. 1994, Mol. Biol. (Moscow), 28, 1009., f) Tandura, S. N., Shumsky, A. N., Litvin, E. F., Kozlova, L. M., Shuvalova, E. V., Sharf, V. Z., Kolesnikov, S. P. 2001, Russ. Chem. Bull., 50, 1014., g) Tandura, S. N., Sheludyakov, V. D., Lebedeva, A. B., Lebedev, A. V. 2007, RU 2307827.

64. Kozeletskaya, K. N., Blinov, V. M., Karginov, V. A., Burmistrova, V. V., Sinkyakv, A. N., Golubev, D. B. 1989, Mol. Genet., Mikrobiol. Virusol., 33.

65. Al-Nakib, W., Higgins, P. G., Willman, J., Tyrrell, D. A. J., Swallow, D. L., Hurst, B. C., Rushton, A. 1986, J. Antimicrob. Chemother., 18, 119.

66. García Martínez, A., Teso Vilar, A., García Fraile, A., de la Moya Cerero, S. Rodríguez Herrero, M. E., Martínez Ruiz, P., Subramanian, L. R., García Cancedo, A. 1995, J. Med. Chem., 38, 4474. Correction in 1996, J. Med. Chem., 39, 2102.

67. a) Kauer, J. C. (E. I. du Pont de Nemours \& Co), 1969, US 3474174., b) Kauer, J. C. (E. I. du Pont de Nemours \& Co), 1970, US 3546290., c) See also (E. I. du Pont de Nemours \& Co), 1967, GB 1054092., d) Whitney, J. G., Gregory, W. A., Kauer, J. C., Roland, J. R., Snyder, J. A., Benson, R. E., Hermann, E. C. 1970, J. Med. Chem., 13, 254.

68. a) Aigami, K., Inamoto, Y., Takaishi, N., Fujikura, Y., Takatsuki, A., Tamura, G. 1976, J. Med. Chem., 19, 536., b) Inamoto and coworkers have also reported the synthesis and antiviral activity of several 1-tricyclo[4.3.1.1 ${ }^{2,5}$ undecylamines, see: Inamoto, Y., Aigami, K., Takaishi, N., Fujikura, Y., Ohsugi, M., Ikeda, H., Takatsuki, A., Tamura, G. 1979, J. Med. Chem., 22, 1206.

69. Chow, A. W., Jakas, D. R., Hoover, J. R. E. 1971, J. Med. Chem., 14, 1242.

70. Hu, W., Zeng, S., Li, C., Jie, Y., Li, Z., Chen, L. 2010, J. Med. Chem., 53, 3831.

71. Zhao, X., Li, C., Zeng, S., Hu, W., 2011, Eur. J. Med. Chem., 46, 52.

72. See, for example, a) Wang, J.-F., Chou, K.-C. 2010, Protein Eng., Des. Select., 23, 663., b) Lin, C.-H., Chang, T.-F., Sun, M.-F., Chen, H.-Y., Tsai, F.-J., Chang, K.-L., Fisher, M., Chen, C. Y.-C. 2011, J. Biomol. Struct. Dyn., 28, 471., c) Lai, C.-Y., Chang, T.-T., Sun, M.-F., Chen, H.-Y., Tsai, F.-J., Lin, J.-G., Chen, C. Y.C. 2011, Mol. Simul., 37, 250., d) Chang, T.-T., Sun, M.-F., Chen, H.-Y., Tsai, F.J., Lin, J.-G., Chen, C. Y.-C. 2011, J. Taiwan Inst. Chem. Eng., 42, (in press, doi:10.1016/j.jtice.2011.01.006). 


\title{
3. Chemical and biological aspects of Amaryllidaceae alkaloids
}

\author{
Jaume Bastida, Strahil Berkov, Laura Torras, Natalia Belén Pigni \\ Jean Paulo de Andrade, Vanessa Martínez, Carles Codina \\ and Francesc Viladomat \\ Department of Natural Products, Plant Biology and Soil Science, Faculty of Pharmacy \\ University of Barcelona, 08028 Barcelona, Spain
}

\begin{abstract}
The Amaryllidaceae alkaloids represent a large (over 300 alkaloids have been isolated) and still expanding group of biogenetically related isoquinoline alkaloids that are found exclusively in plants belonging to this family. In spite of their great variety of pharmacological and/or biological properties, only galanthamine is used therapeutically. First isolated from Galanthus species, this alkaloid is a long-acting, selective, reversible and competitive inhibitor of acetylcholinesterase, and is used for the treatment of Alzheimer's disease. Other Amaryllidaceae alkaloids of pharmacological interest will also be described in this chapter.
\end{abstract}

\section{Introduction}

The Amaryllidaceae are richly represented in the tropics and have pronounced centers of diversity in South-Africa and the Andean region. 
Some genera are also found in the Mediterranean area and temperate regions of Asia.

A particular characteristic of Amaryllidaceae is a consistent presence of an exclusive group of alkaloids, which have been isolated from the plants of all the genera of this family. The Amaryllidaceae alkaloids represent a large and still expanding group of isoquinoline alkaloids, the majority of which are not known to occur in any other family of plants. Since the isolation of the first alkaloid, lycorine, from Narcissus pseudonarcissus in 1877, substantial progress has been made in examining the Amaryllidaceae plants, although they still remain a relatively untapped phytochemical source [1]. At present, over 300 alkaloids have been isolated from plants of this family [2] and, although their structures vary considerably, these alkaloids are considered to be biogenetically related.

The large number of structurally diverse Amaryllidaceae alkaloids are classified mainly into nine skeleton types, for which the representative alkaloids are: norbelladine, lycorine, homolycorine, crinine, haemanthamine, narciclasine, tazettine, montanine and galanthamine (Fig. 1). With the aim of unifying the numbering system of the different skeleton types, Ghosal's model will be used in this review [3].

As the alkaloids of the Amaryllidaceae family species fall mainly into one of these subgroups, they can serve as a classifying tool for including genera and species in this family. Recently, Unver and Jin have proposed subgroups for some skeleton types, according to the structures of new alkaloids isolated from Galanthus species [4,5]. Furthermore, although it is unusual to find other types of alkaloids in this family, if present, they are always accompanied by typical Amaryllidaceae alkaloids. The classical example is the reported presence of the mesembrane (Sceletium) alkaloids, generally found in the Aizoaceae family [6,7], in a few species of Amaryllidaceae such as Hymenocallis arenicola, Crinum oliganthum, Narcissus pallidulus and Narcissus triandrus [8-10]. In turn, the unexpected isolation of (-)-capnoidine and (+)-bulbocapnine from Galanthus nivalis subsp. cilicicus is the first report of the occurrence of classical isoquinoline alkaloids in a typical member of the Amaryllidaceae [11].

Plants of the Amaryllidaceae family have been used for thousands of years as herbal remedies. The alkaloids from their extracts have been the object of active chemical investigation for nearly 200 years. Over the past three decades many have been isolated, screened for different biological activities, and synthesized by a number of research groups.

The structural elucidation of the Amaryllidaceae alkaloids and their biological profiles, as well as their synthesis, have been summarized in the last few years [12-14], which, together with the regular publications of the journal 
<smiles>Oc1ccc(CCNCc2ccc(O)c(O)c2)cc1</smiles><smiles>OC12C=C[C@]3(CN4Cc5cc3c(cc5OCO1)C4)C2</smiles><smiles>CN[C@@]1(O)OCc2cc3c(cc2[C@@]12C=C[C@H](OC)C[C@H]2C)OCO3</smiles>
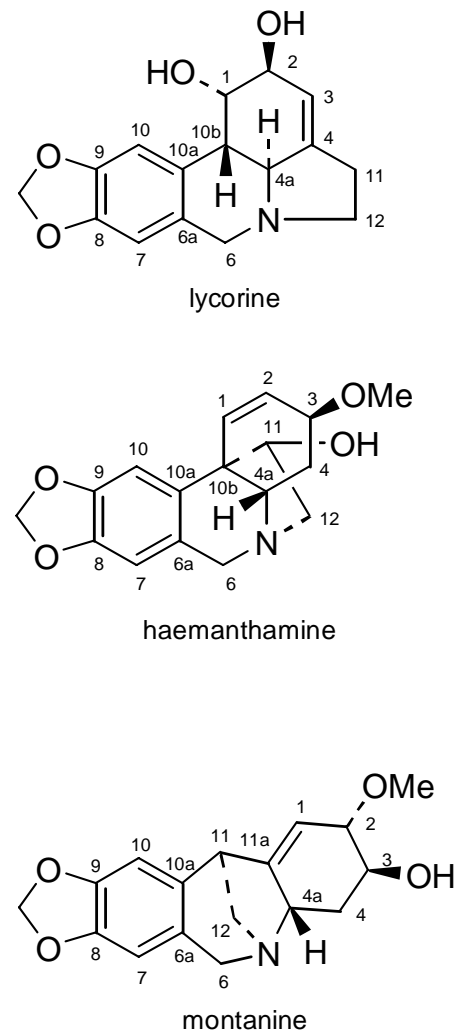

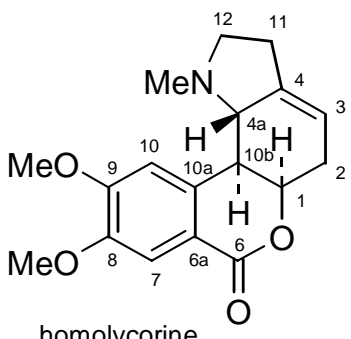<smiles>C[C@@]12NC(=O)c3c(cc4c(c3O)OCO4)C1=CC(O)[C@@H](O)[C@@H]2O</smiles>

narciclasine

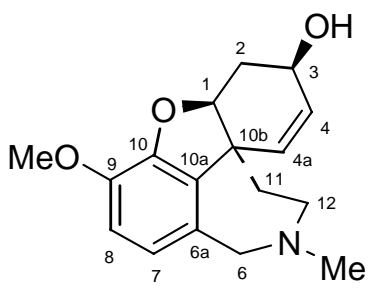

galanthamine

Figure 1. Amaryllidaceae alkaloid types.

Natural Products Reports [5,15-17] over the last decade, represents a valuable source of information.

The present review provides coverage of the biosynthesis, NMR spectroscopy and biological activity of the Amaryllidaceae alkaloids up to the end of 2010.

\section{Biosynthetic pathways}

Most of the biosynthetic research done on Amaryllidaceae alkaloids was carried out in the sixties and early seventies. Since then, the only noteworthy study has been the biosynthesis of galanthamine and related alkaloids [18]. As in most alkaloid biosyntheses, that of the Amaryllidaceae follows a pattern made up of certain steps.

\subsection{Enzymatic preparation of the precursors}

Although L-phenylalanine (L-phe) and L-tyrosine (L-tyr) are closely related in chemical structure, they are not interchangeable in plants. In the Amaryllidaceae alkaloids, L-phe serves as a primary precursor of the $\mathrm{C}_{6}-\mathrm{C}_{1}$ 
fragment, corresponding to ring A and the benzylic position (C-6), and L-tyr is the precursor of ring $\mathrm{C}$, the two-carbon side chain (C-11 and $\mathrm{C}-12$ ) and nitrogen, $\mathrm{C}_{6}-\mathrm{C}_{2}-\mathrm{N}$. The conversion of $\mathrm{L}$-phe to the $\mathrm{C}_{6}-\mathrm{C}_{1}$ unit requires the loss of two carbon atoms from the side chain as well as the introduction of at least two oxygenated substituents into the aromatic ring, which is performed via cinnamic acids. The presence of the enzyme phenylalanine ammonia lyase (PAL) has been demonstrated in Amaryllidaceae plants [19] and the elimination of ammonia mediated by this enzyme is known to occur in an antiperiplanar manner to give trans-cinnamic acid, with loss of the $\beta$-pro-S hydrogen [20]. Thus, it may be expected that L-phe would be incorporated into Amaryllidaceae alkaloids with retention of the $\beta$-pro-R hydrogen. However, feeding experiments in Narcissus 'King Alfred' showed that tritium originally present at C- $\beta$ of L-phe, whatever the configuration, was lost in the formation of several haemanthamine and homolycorine type alkaloids, which led to the conclusion that fragmentation of the cinnamic acids involves oxidation of $C-\beta$ to ketone or acid level, the final product being protocatechuic aldehyde or its derivatives (Fig. 2). On the other hand, L-tyr is degraded no further than tyramine before incorporation into the Amaryllidaceae alkaloids.

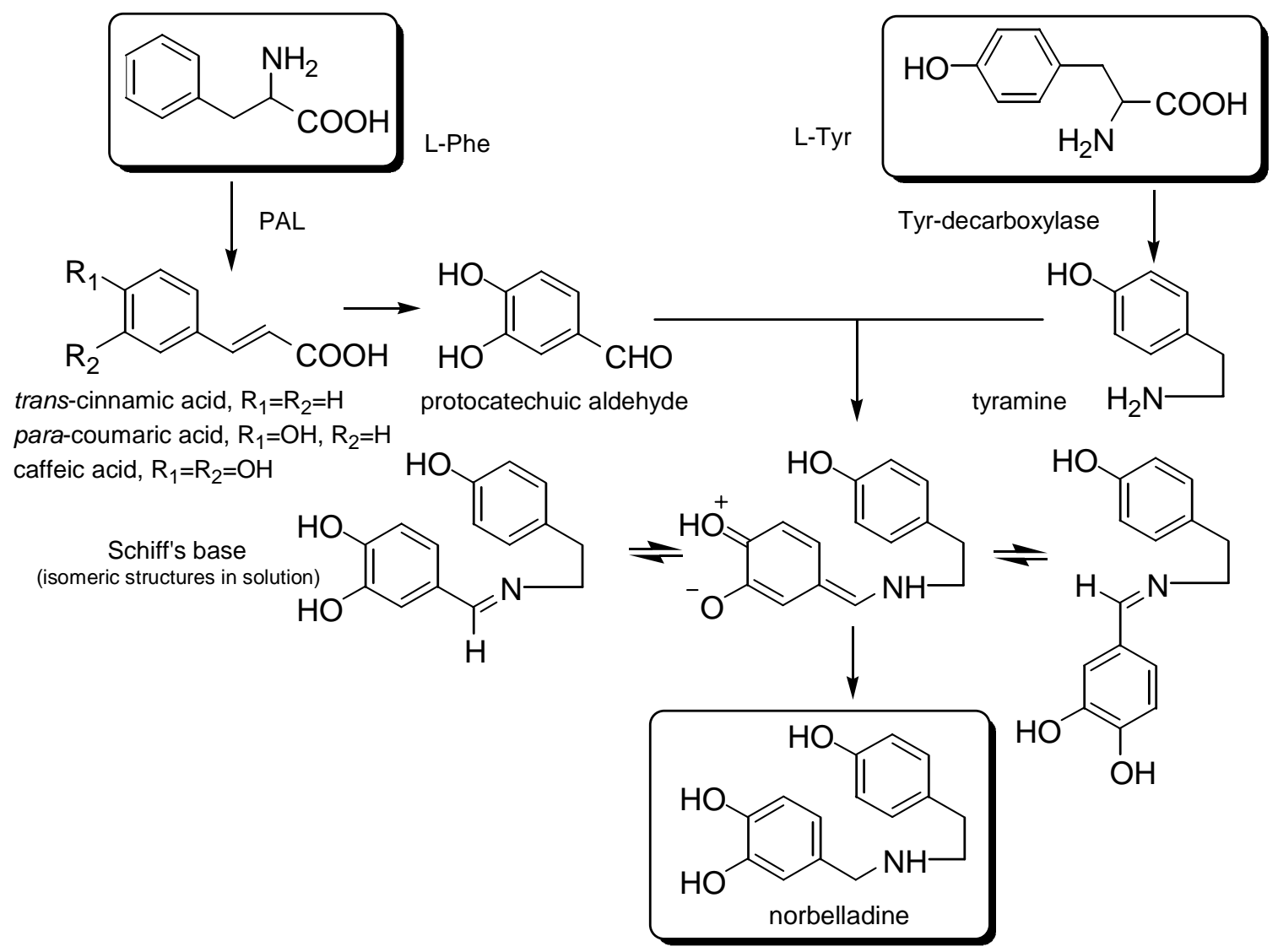

Figure 2. Biosynthetic pathway to norbelladine. 


\subsection{Primary cyclization mechanisms}

Tyramine and protocatechuic aldehyde or its derivatives are logical components for the biosynthesis of the precursor norbelladine. This pivotal reaction represents the entry of primary metabolites into a secondary metabolic pathway. The junction of the amine and the aldehyde results in a Schiff's base, two of which have been isolated up to now from several Crinum species: craugsodine [21] and isocraugsodine [22]. The existence of Schiff's bases in nature as well as their easy conversion into the different ring-systems of the Amaryllidaceae alkaloids suggest that the initial hypothesis about this biosynthetic pathway was correct.

\subsection{Enzymatic preparation of intermediates}

In 1957, Barton and Cohen [23] proposed that norbelladine or related compounds could undergo oxidative coupling in Amaryllidaceae plants, once ring A had been suitably protected by methylation, resulting in the different skeletons of the Amaryllidaceae alkaloids (Fig. 3). The key intermediate in most of cases is $O$-methylnorbelladine.

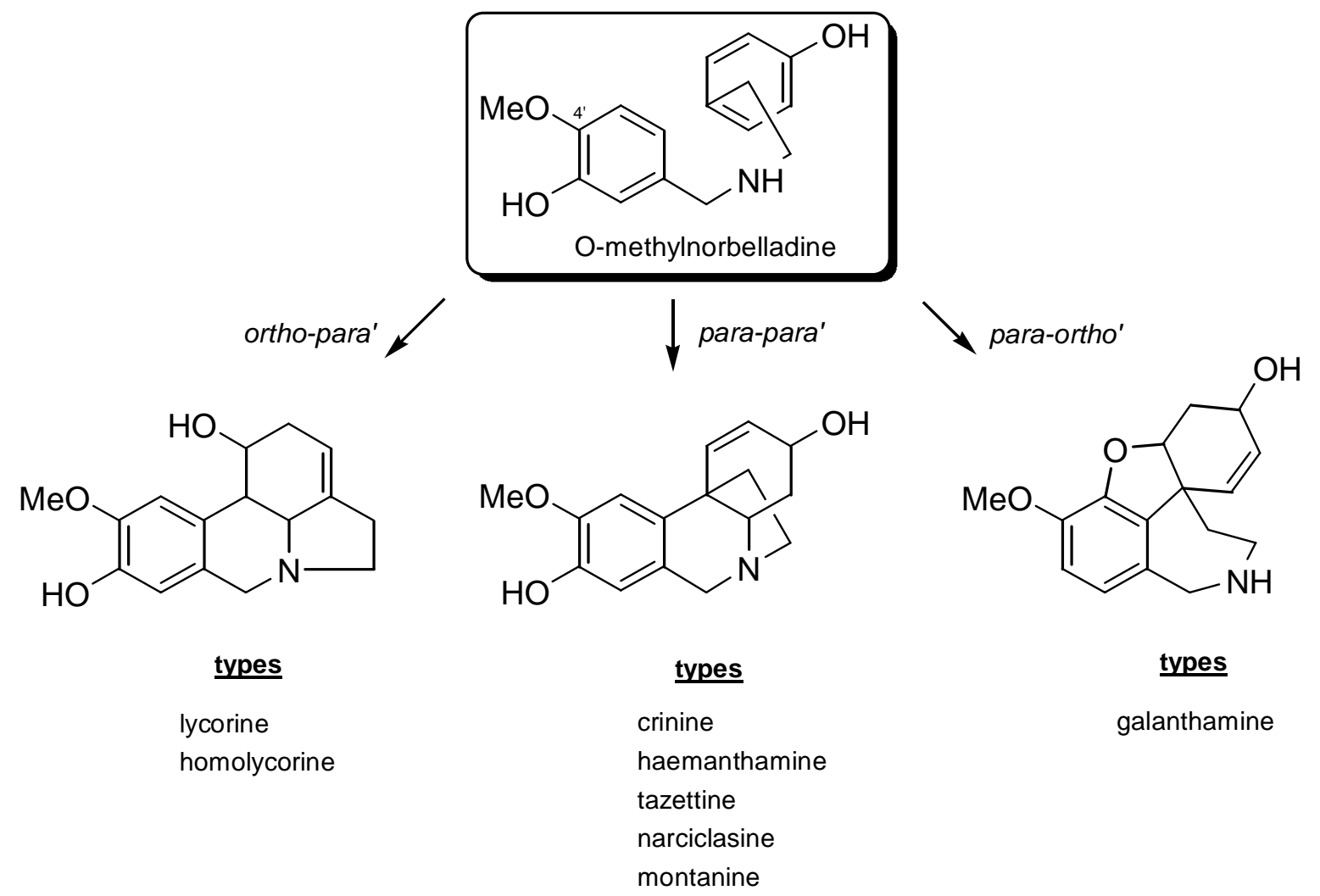

Figure 3. Phenol oxidative coupling in Amaryllidaceae. 


\subsection{Secondary cyclization, diversification and restructuring}

Secondary cyclization is produced by an oxidative coupling of $O$-methylnorbelladine.

\subsubsection{Lycorine and homolycorine types}

The alkaloids of this group are derivatives of the pyrrolo[de]phenanthridine (lycorine type) and the 2-benzopirano-[3,4-g]indole (homolycorine type) skeletons, and both types originate from an ortho-para' phenol oxidative coupling (Fig. 4).

The biological conversion of cinnamic acid via hydroxylated cinnamic acids into the $\mathrm{C}_{6}-\mathrm{C}_{1}$ unit of norpluviine has been used in a study of hydroxylation mechanisms in higher plants [24]. When $\left[3-{ }^{3} \mathrm{H}, \beta-{ }^{14} \mathrm{C}\right]$ cinnamic acid was fed to Narcissus 'Texas' a tritium retention in norpluviine of $28 \%$ was observed, which is very close to the predicted value resulting from parahydroxylation with hydrogen migration and retention.

In the conversion of $O$-methylnorbelladine into lycorine, the labelling position $\left[3-{ }^{3} \mathrm{H}\right]$ on the aromatic ring of L-tyr afterwards appears at C-2 of norpluviine, which is formed as an intermediate, the configuration of the tritium apparently being $\beta$ [25]. This tritium is retained in subsequently formed

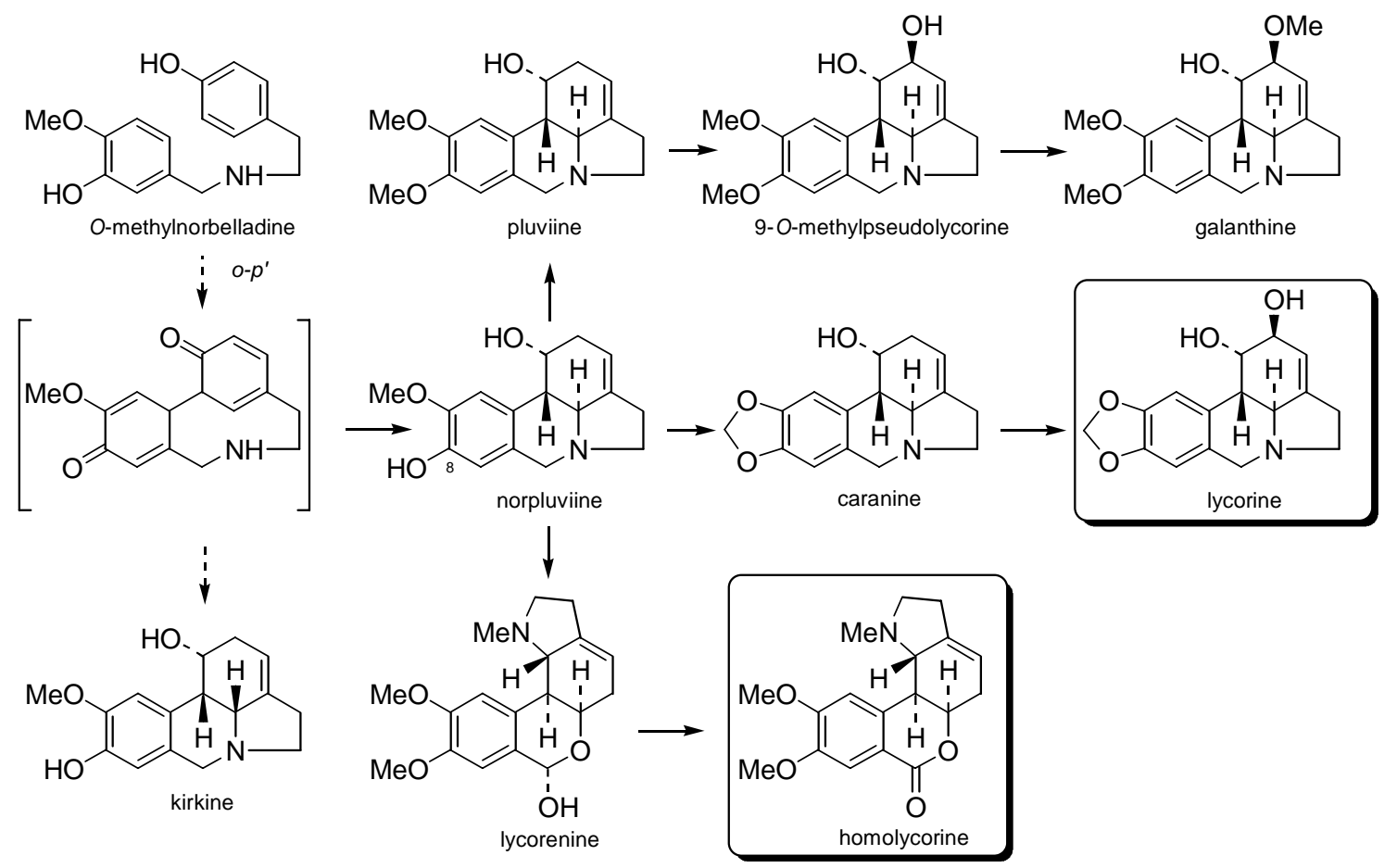

Figure 4. Alkaloids proceeding from an ortho-para' coupling. 
lycorine, which means that hydroxylation at $\mathrm{C}-2$ proceeds with an inversion of configuration [26] by a mechanism involving an epoxide, with ring opening followed by allylic rearrangement of the resulting alcohol (Fig. 5). Supporting evidence comes from the incorporation of $\left[2 \beta-{ }^{3} \mathrm{H}\right]$ caranine into lycorine in Zephyranthes candida [27]. However, an hydroxylation of caranine in Clivia miniata occuring with retention of configuration was also observed [28]. Further, $\left[2 \alpha-{ }^{3} \mathrm{H} ; 11-{ }^{14} \mathrm{C}\right]$ caranine was incorporated into lycorine with high retention of tritium at $\mathrm{C}$-2, indicating that no 2-oxo-compound can be implicated as an intermediate.

The conversion of the $O$-methoxyphenol to the methylenedioxy group may occur late in the biosynthetic pathway. Tritiated norpluviine is converted to tritiated lycorine by Narcissus 'Deanna Durbin', which not only demonstrates the previously mentioned conversion but also indicates that the C-2 hydroxyl group of lycorine is derived by allylic oxidation of either norpluviine or caranine [29].

Regarding the conversion of $\left[2 \beta-{ }^{3} \mathrm{H}, 8-\mathrm{OMe}-{ }^{14} \mathrm{C}\right]$ pluviine into galanthine, in Narcissus 'King Alfred', the retention of $79 \%$ of the tritium label confirms that hydroxylation of C-2 may occur with inversion of configuration [30].

It was considered [31] that another analogous epoxide could give narcissidine in the way shown by loss of the pro-S hydrogen from $\mathrm{C}-11$, galanthine being a suitable substrate for epoxidation. Labelled $\left[\alpha-{ }^{14} \mathrm{C}, \beta-{ }^{3} \mathrm{H}\right]$ $O$-methylnorbelladine, when fed to Narcissus 'Sempre Avanti' afforded galanthine ( $98 \%$ of tritium retention) and narcissidine ( $46 \%$ tritium retention). Loss of hydrogen from C-11 of galanthine was therefore stereospecific. In the nineties, Kihara et al. [32] isolated a new alkaloid, incartine, from flowers of Lycoris incarnata, which could be considered as the biosynthetic intermediate of this pathway (Fig. 6).

The biological conversion of protocatechuic aldehyde into lycorenine, which proceeds via $O$-methylnorbelladine and norpluviine, first involves a reduction of the aldehyde carbonyl, and afterwards, in the generation of lycorenine, oxidation of this same carbon atom. The absolute stereochemistry
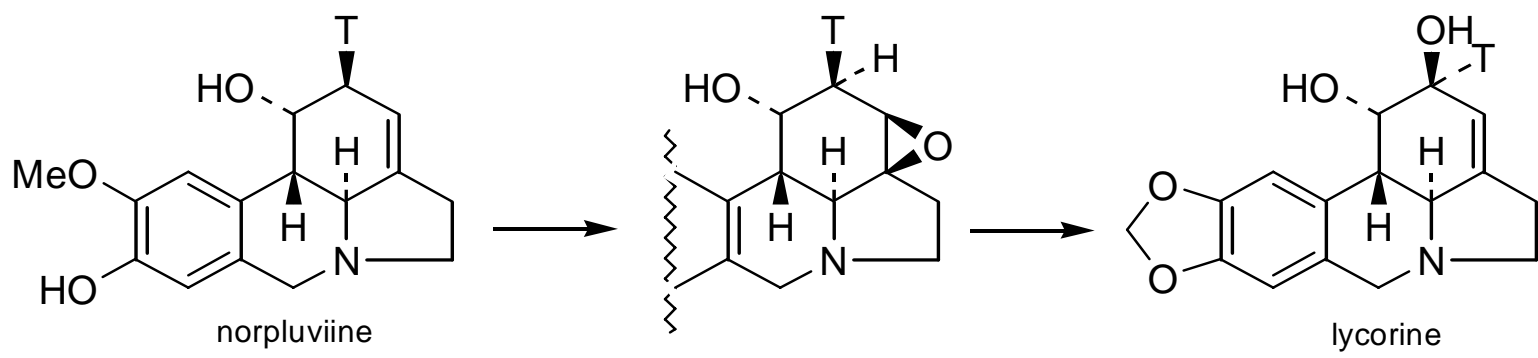

Figure 5. Biosynthesis of lycorine with inversion of the configuration. 


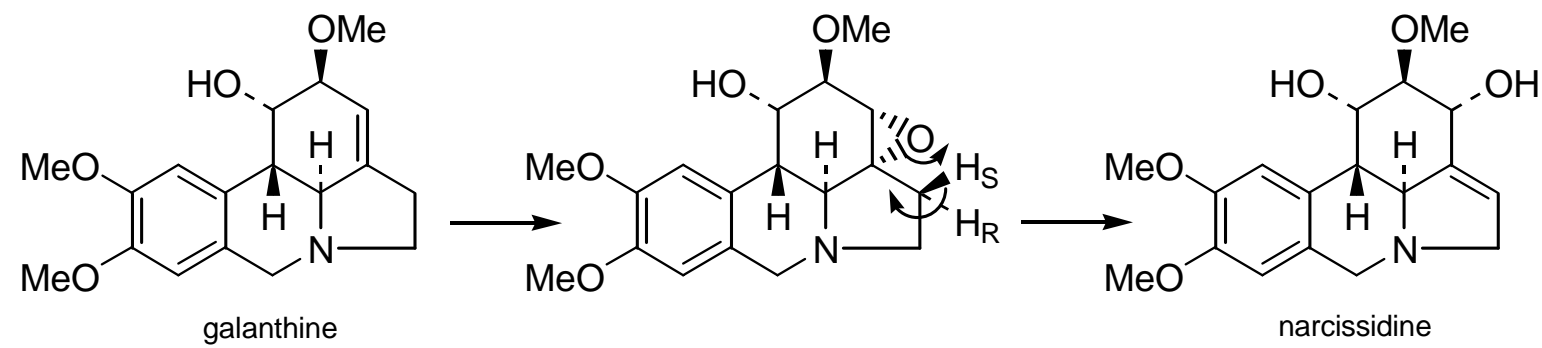

Figure 6. Conversion of galanthine to narcissidine via epoxide.<smiles>COc1cc2c(cc1O)CN1CCC3CC(Cl)CC(C3)C2C1O</smiles><smiles>COc1cc2c(cc1O)[C@@H](O)N1CCC3=CCC(O)[C@H]3[C@H]21</smiles><smiles>COc1cc(C=O)cc(C=O)c1O</smiles><smiles>COc1cc2c(cc1OC)[C@H]1[C@H]3C(CC=C4CCN(C)[C@H]43)C(=O)O[C@@H]1C2</smiles>

[O]

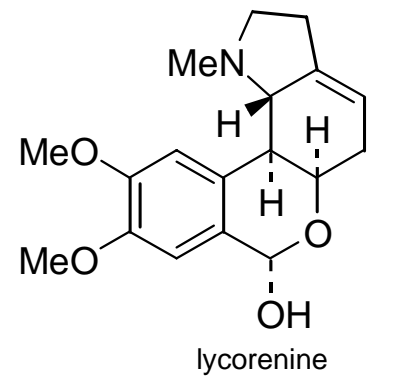

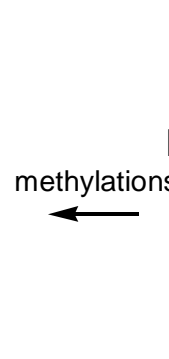
rotation

Figure 7. Conversion of norpluviine to homolycorine type alkaloids.

of these processes has been elucidated in subsequent experiments [33], and the results show that hydrogen addition and removal take place on the re-face of the molecules concerned [34], the initially introduced hydrogen being the one later removed [35]. It is noteworthy that norpluviine, unlike pluviine, is converted in Narcissus 'King Alfred' primarily to alkaloids of the homolycorine type. Benzylic oxidation at position 6 followed by a ring opening forms an amino aldehyde; the formation of hemiacetal and subsequent methylation provides lycorenine [30], which after oxidation gives homolycorine, as shown in Fig. 7.

\subsubsection{Crinine, haemanthamine, tazettine, narciclasine and montanine types}

This group includes the alkaloids derived from 5,10b-ethanophenanthridine (crinine and haemanthamine types), 2-benzopyrano[3,4-c]indole (tazettine type), phenanthridine (narciclasine type) and 5,11- 
methanomorphanthridine (montanine type) skeletons, originating from a parapara' phenol oxidative coupling (Fig. 8).

Results of experiments with labelled crinine, and less conclusively with oxovittatine, indicate that the two naturally occurring enantiomeric series, represented in Fig. 8 by crinine and vittatine, are not interchangeable in Nerine bowdenii [36].

Incorporation of $O$-methylnorbelladine, labelled in the methoxy carbon and also in positions $\left[3,5-{ }^{3} \mathrm{H}\right]$, into the alkaloid haemanthamine was without loss of tritium, half of which was at C-2. Consideration of the possible mechanisms involved in relation to tritium retention led to the suggestion that the tritium which is expected at C-4 of haemanthamine might not be stereospecific [37]. The conversion of $O$-methylnorbelladine into haemanthamine involves loss of the pro- $R$ hydrogen from the C- $\beta$ of the tyramine moiety, as well as a further entry of a hydroxyl group at this site [38]. The subsequent benzylic oxidation results in an epimeric mixture that even HPLC cannot separate. The epimeric forms were proposed to be interchangeable. The biosynthetic conversion of the 5,10b-ethanophenanthridine alkaloids to the 2-benzopyrano[3,4-c]indole was demonstrated by feeding tritium-labelled alkaloids to Sprekelia formosissima. It was shown that this plant converts haemanthamine to haemanthidine/epihaemanthamine and subsequently to pretazettine in an essentially irreversible manner [39]. This transformation was considered to proceed through an intermediate but it has never been detected by spectral methods [40] (Fig. 9).

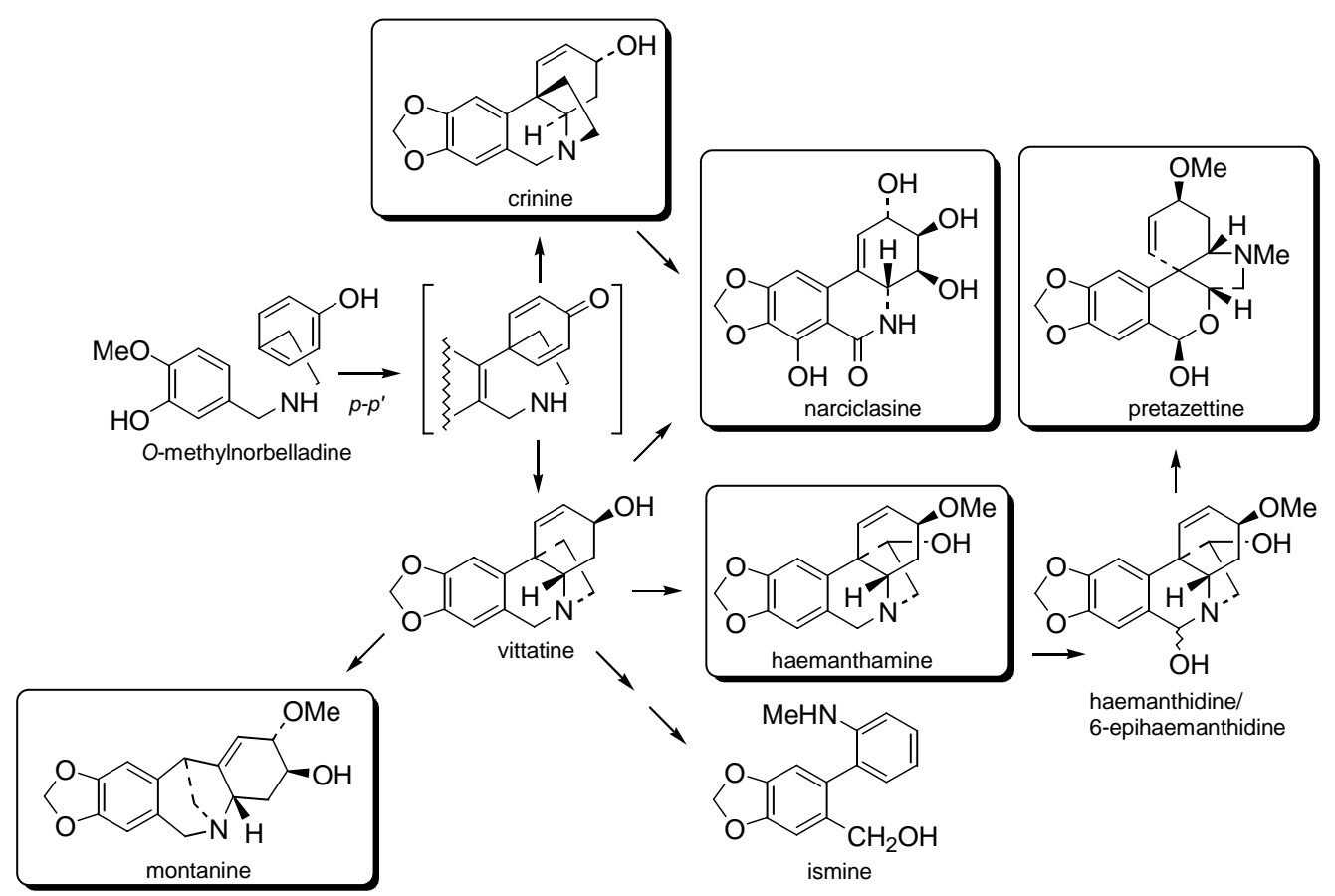

Figure 8. Alkaloids proceeding from a para-para' coupling. 

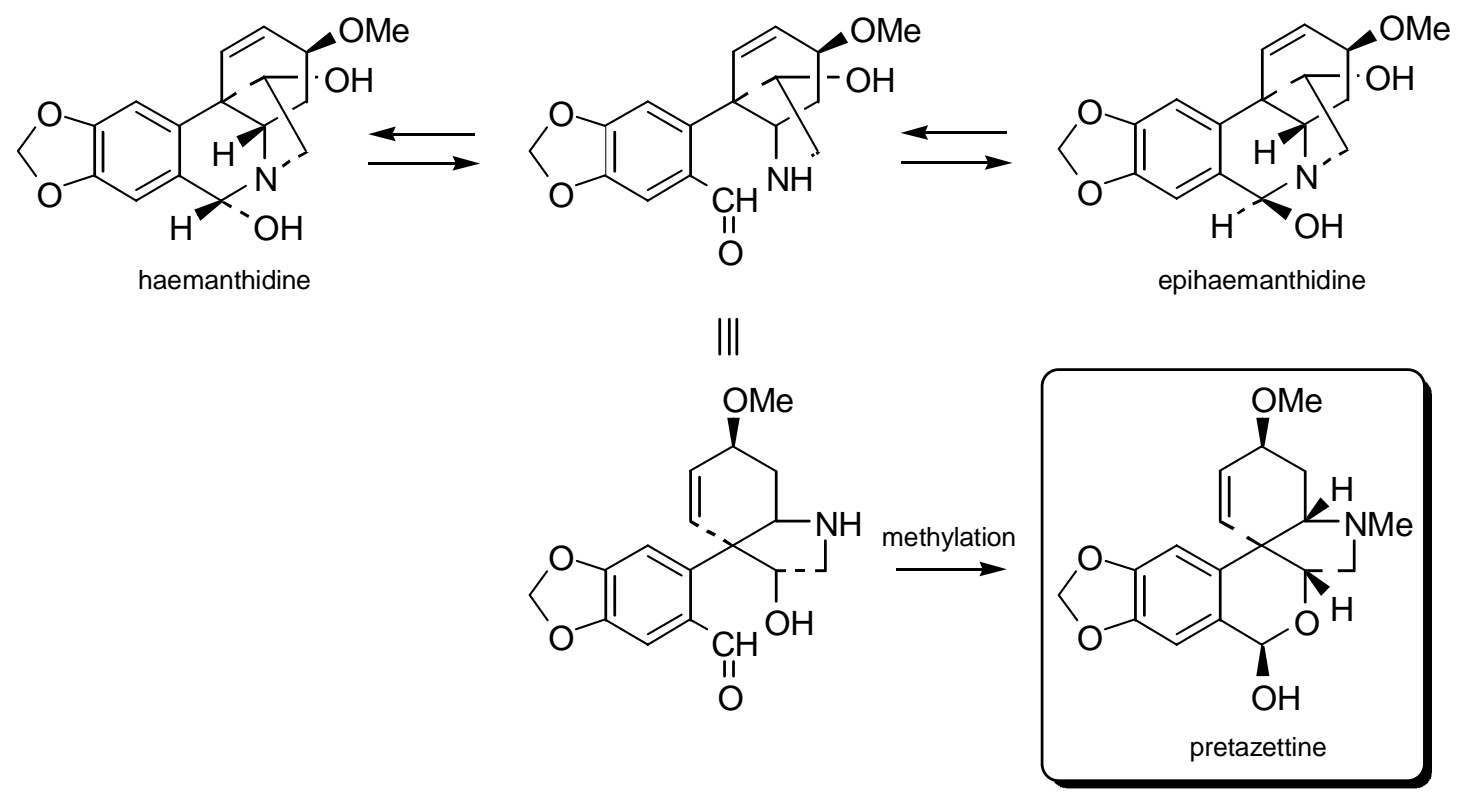

Figure 9. Biosynthesis of pretazettine.

It has also been proved that the alkaloid narciclasine proceeds from the pathway of the biosynthesis of crinine and haemanthamine type alkaloids and not through norpluviine and lycorine derivatives. In fact, in view of its structural affinity to both haemanthamine and lycorine, narciclasine could be derived by either pathway. When $O$-methylnorbelladine labelled in the methoxy carbon and in both protons of position 3 and 5 of the tyramine aromatic ring, was administered to Narcissus plants, all four alkaloids incorporated activity. The isotopic ratio $\left[{ }^{3} \mathrm{H}:{ }^{14} \mathrm{C}\right]$ for norpluviine and lycorine was, as expected, $50 \%$ that of the precursor, because of its ortho-para' coupling. On the contrary, in haemanthamine the ratio was unchanged. These results show clearly that the methoxy group of $O$-methylnorbelladine is completely retained in the alkaloids mentioned, providing a satisfactory internal standard and also, the degree of tritium retention is a reliable guide to the direction of phenol coupling. Narciclasine showed an isotopic ratio (75\%) higher than that of lycorine or norpluviine though lower than that of haemanthamine. However, the fact that more than $50 \%$ of tritium is retained suggests that $O$-methylnorbelladine is incorporated into narciclasine via para-para' phenol oxidative coupling.

$O$-methylnorbelladine and vittatine are implicated as intermediates in the biosynthesis of narciclasine [41-43], and the loss of the ethane bridge from the latter could occur by a retro-Prins reaction on 11-hydroxyvittatine. Strong support for this pathway was obtained by labelling studies. 11-Hydroxyvittatine has also been proposed as an intermediate in the biosynthesis of haemanthamine and montanine (a 5,11-methanomorphanthridine alkaloid) following the observed specific incorporation of vittatine into the two alkaloids in Rhodophiala bifida [36] (Fig. 10). 


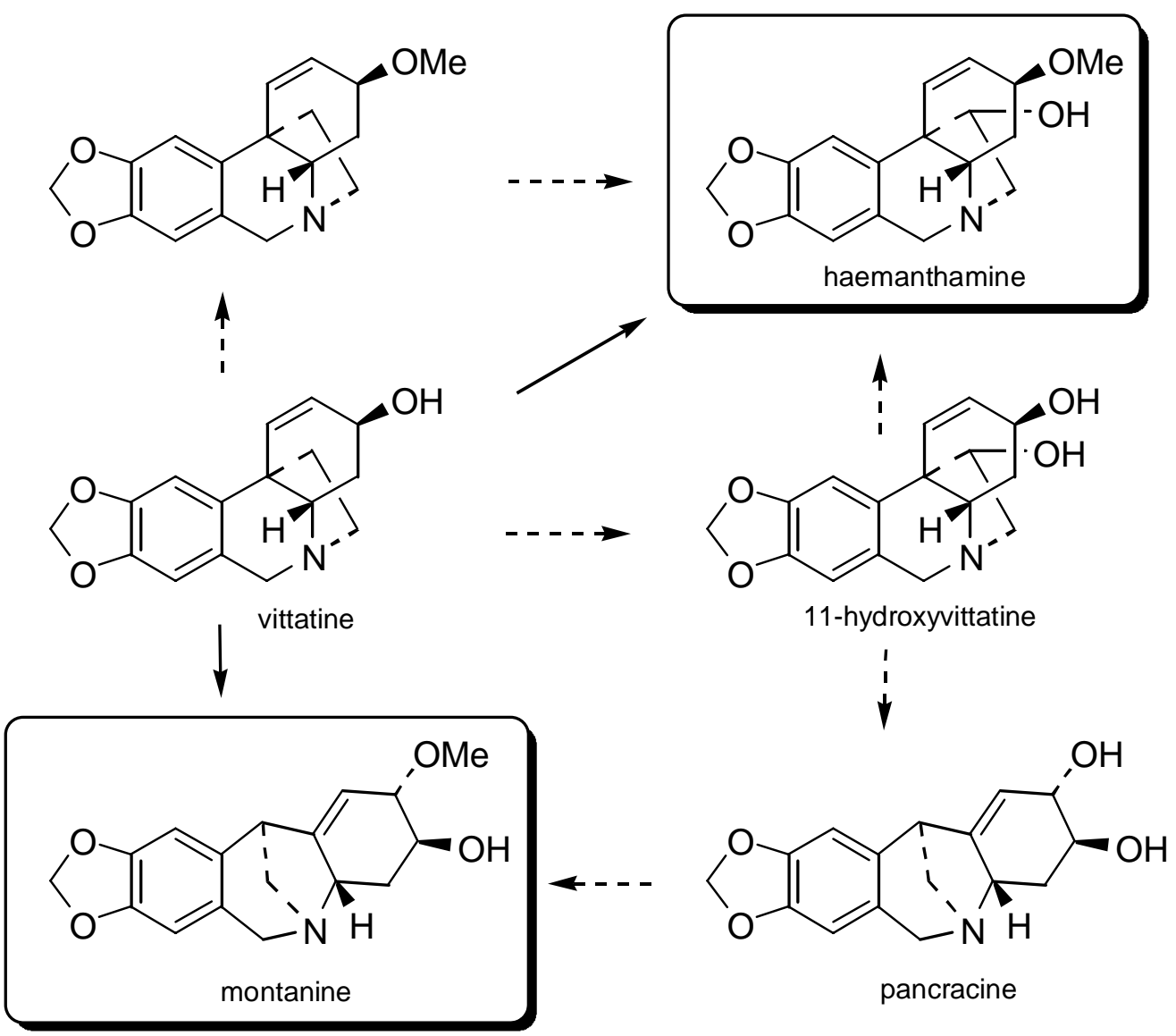

Figure 10. Proposed biosynthetic pathways to haemanthamine and montanine.

Fuganti and Mazza [42,43] concluded that in the late stages of narciclasine biosynthesis, the two-carbon bridge is lost from the oxocrinine skeleton, passing through intermediates bearing a pseudoaxial hydroxy-group at C-3 position and further hydrogen removal from this position does not occur. Noroxomaritidine was also implicated in the biosynthesis of narciclasine and further experiments [44] showed that it is also a precursor for ismine.

The alkaloid ismine has also been shown [45] to be a transformation product of the crinine-haemanthamine series. The precursor, oxocrinine labelled with tritium in the positions 2 and 4, was administered to Sprekelia formosissima plants and the radiactive ismine isolated was shown to be specifically labelled at the expected positions.

\subsubsection{Galanthamine type}

This type of alkaloids have a dibenzofuran nucleus (galanthamine type) and are obtained from a para-ortho' phenol oxidative coupling. 
The initial studies of this pathway suggested that the para-ortho' coupling does not proceed from $O$-methylnorbelladine but from $\mathrm{N}, \mathrm{O}$-dimethylnorbelladine to finally give galanthamine [46]. N,O-dimethylnorbelladine was first isolated from Pancratium maritimum [47] a species that also contains galanthamine.

However, the most recent study seems to contradict the evidence set forth here. Experiments carried out with application of ${ }^{13} \mathrm{C}$-labelled $O$-methylnorbelladine to organs of field grown Leucojum aestivum have shown that the biosynthesis of galanthamine involves the phenol oxidative coupling of $O$-methylnorbelladine to a postulated dienone, which undergoes spontaneous closure of the ether bridge to yield $N$-demethylnarwedine, giving norgalanthamine after stereoselective reduction. Furthermore, it was shown that norgalanthamine is $N$-methylated to galanthamine in the final step of biosynthesis [18] (Fig. 11). In contrast with the literature, N,Odimethylnorbelladine was metabolized to a lesser extent in L. aestivum and incorporated into galanthamine as well as norgalanthamine at about $1 / 3$ of the rate of $O$-methylnorbelladine.

According to Eichhorn et al. [18], narwedine is not the direct precursor of galanthamine, and could possibly exist in equilibrium with galanthamine, a reaction catalyzed by a hypothetically reversible oxido-reductase.

Chlidanthine, by analogy with the known conversion of codeine to morphine, might be expected to arise from galanthamine by $O$-demethylation. This was shown to be true when both galanthamine and narwedine, with tritium labels, were incorporated into chlidanthine [48].

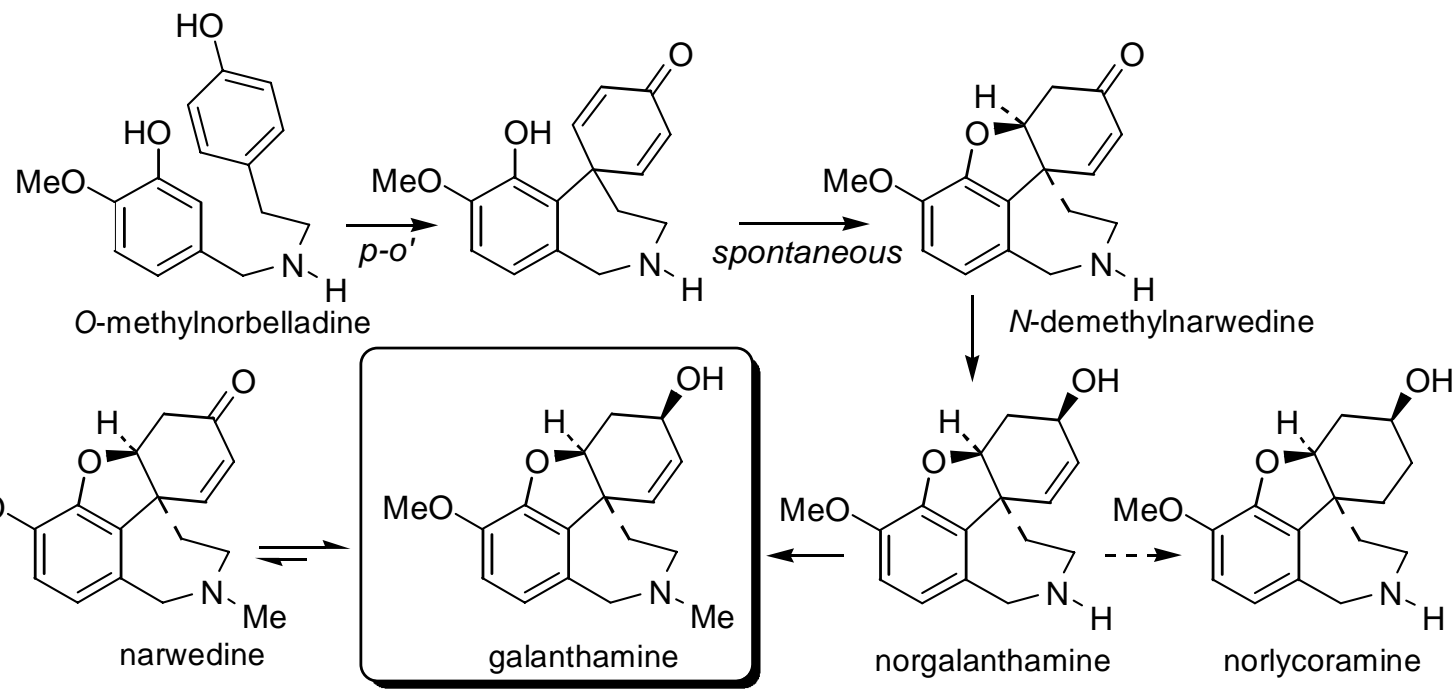

Figure 11. Biosynthesis of galanthamine and derivatives. 


\section{NMR studies}

In a discussion of Proton Nuclear Magnetic Resonance $\left({ }^{1} \mathrm{H}\right.$ NMR) and Carbon Nuclear Magnetic Resonance $\left({ }^{13} \mathrm{C}\right.$ NMR), the most significant characteristics of each of Amaryllidaceae alkaloid-type are outlined, indicating the keys for their identification.

\subsection{Proton nuclear magnetic resonance}

${ }^{1} \mathrm{H}$ NMR spectroscopy gives the most extensive and important information about the different types of Amaryllidaceae alkaloids. In the last 25 years, the routine use of 2D NMR techniques has facilitated the structural assignments and the settling of their stereochemistry.

\subsubsection{Lycorine type}

This group has been subjected to several ${ }^{1} \mathrm{H}$ NMR studies and lycorine, as well as its main derivatives, has been completely assigned. The general characteristics of the ${ }^{1} \mathrm{H}$ NMR spectra are:

a. Two singlets for the para-oriented aromatic protons, together with a unique olefinic proton.

b. Two doublets as an $A B$ system corresponding to the benzylic protons of C- 6 . The deshielding observed in the $\beta$-protons of positions 6 and 12 in relation to their $\alpha$-homologues is due to the effect of the cis-lone pair of the nitrogen atom.

c. Like almost all other lycorine type examples, the alkaloids isolated from the Narcissus genus show a trans B/C ring junction, the coupling constant being $J_{4 \mathrm{a}, 10 \mathrm{~b}} \sim 11 \mathrm{~Hz}$. Only kirkine shows a cis $\mathrm{B} / \mathrm{C}$ ring junction, with a smaller coupling constant $J_{4 \mathrm{a}, 10 \mathrm{~b}} 8 \mathrm{~Hz}$.

In the plant, the alkaloid lycorine is particularly vulnerable to oxidation processes, giving several ring-C aromatized products.

\subsubsection{Homolycorine type}

This group includes lactone, hemiacetal or the more unusual cyclic ether alkaloids. The general traits for this type of compounds could be summarized as follows: 
a. Two singlets for the para-oriented aromatic protons. In lactone alkaloids, the deshielding of $\mathrm{H}-7$ is caused by the peri-carbonyl group.

b. The hemiacetal alkaloids always show the substituent at C-6 in $\alpha$-disposition.

c. The majority of compounds belong to a single enantiomeric series containing a cis $\mathrm{B} / \mathrm{C}$ ring junction, which is congruent with the small size of the coupling constant $J_{1,10 \mathrm{~b}}$. In the Narcissus genus no exception to this rule has been observed.

d. The large coupling constant between $\mathrm{H}-4 \mathrm{a}$ and $\mathrm{H}-10 \mathrm{~b}\left(\mathrm{~J}_{4 \mathrm{a}, 10 \mathrm{~b}} \sim 10 \mathrm{~Hz}\right)$ is only consistent with a trans-diaxial relationship.

e. In general, ring $\mathrm{C}$ presents a vinylic proton. If position 2 is substituted by an $\mathrm{OH}, \mathrm{OMe}$ or OAc group, it always displays an $\alpha$-disposition.

f. The singlet corresponding to the $N$-methyl group is in the range of $\delta 2.0$ $2.2 \mathrm{ppm}$, its absence being very unusual.

g. The $\mathrm{H}-12 \alpha$ is more deshielded than $\mathrm{H}-12 \beta$ as a consequence of the cislone pair of the nitrogen atom.

Homolycorine type alkaloids with a saturated ring $\mathrm{C}$ have been studied by Jeff and co-workers [49]. They describe empirical correlations of $N$-methyl chemical shifts with stereochemical assignments of the $\mathrm{B} / \mathrm{C}$ and $\mathrm{C} / \mathrm{D}$ ring junction.

\subsubsection{Haemanthamine and crinine types}

The absolute configuration of these alkaloids is determined through the circular dichroism spectrum. The alkaloids of the Narcissus genus are exclusively of the haemanthamine type, while in genera such as Brunsvigia, Boophane etc., the crinine type alkaloids are predominant. It is also noteworthy that the alkaloids isolated from the Narcissus genus do not show additional substitutions in the aromatic ring apart from those of C-8 and C-9. On the contrary, in the genera where crinine type alkaloids predominate, the presence of compounds with a methoxy substituent at C-7 is quite common. Thus, haemanthamine type alkaloids show the following characteristics:

a. Two singlets for the para-oriented aromatic protons, although of course only one for crinane type alkaloids substituted at C-7.

b. Using $\mathrm{CDCl}_{3}$ as the solvent, the magnitude of the coupling constants between each olefinic proton ( $\mathrm{H}-1$ and $\mathrm{H}-2)$ and $\mathrm{H}-3$ gives information about the configuration of the $\mathrm{C}-3$ substituent. Thus, in those alkaloids in which the two-carbon bridge $(\mathrm{C}-11$ and $\mathrm{C}-12)$ is cis to the substituent at 
C-3, H-1 shows an allylic coupling with $\mathrm{H}-3\left(J_{1,3} \sim 1-2 \mathrm{~Hz}\right)$ and $\mathrm{H}-2$ shows a smaller coupling with $\mathrm{H}-3\left(J_{2,3} \sim 0-1.5 \mathrm{~Hz}\right)$, as occurs in crinamine. On the contrary, in the corresponding $\mathrm{C}-3$ epimeric series, e.g. haemanthamine, a larger coupling between H-2 and H-3 $\left(J_{2,3} 5 \mathrm{~Hz}\right)$ is shown, the coupling between $\mathrm{H}-1$ and $\mathrm{H}-3$ not being detectable. This rule is also applicable to the crinane type alklaoids.

c. In the haemanthamine series there is frequently an additional $\mathrm{W}$ coupling of $\mathrm{H}-2$ with the equatorial $\mathrm{H}-4 \beta$, while the proton $\mathrm{H}-4 \alpha$ shows a large coupling with $\mathrm{H}-4 \mathrm{a}\left(J_{4 \alpha, 4 \mathrm{a}} \sim 13 \mathrm{~Hz}\right)$ due to their trans-diaxial disposition. The same is applicable in the crinane series.

d. Two doublets for an $\mathrm{AB}$ system corresponding to the benzylic protons of position C-6.

e. The pairs of alkaloids with a hydroxy substituent at C-6, like papyramine/ 6-epipapyramine, haemanthidine/6-epihaemanthidine etc, appear as a mixture of epimers not separable even by HPLC.

f. Also in relation with position C-6, it is interesting to note that ismine, a catabolic product from the haemanthamine series, shows a restricted rotation around the biarylic bond, which makes the methylenic protons at the benzylic position magnetically non-equivalent.

\subsubsection{Tazettine type}

Although tazettine is one of the most widely reported alkaloids in the Amaryllidaceae family, it was found to be an extraction artifact from pretazettine [50].

The presence of an $N$-methyl group (2.4-2.5 ppm) in tazettine type alkaloids immediately distinguishes them from the haemanthamine or crinine types, from which they proceed biosynthetically. Moreover, the ${ }^{1} \mathrm{H}$ NMR spectrum always shows the signal corresponding to the methylenedioxy group.

\subsubsection{Montanine type}

The absolute configuration of Montanine-type alkaloids is determined through the circular dichroism spectrum. Their ${ }^{1} \mathrm{H}$ NMR data are very similar to those of alkaloids with a lycorine skeleton, but Montanine-type alkaloids can be distinguished by the analysis of a COSY spectrum. The signals attributable to the $\mathrm{H}-4$ hydrogens (the most upfield signals) show correlation with those corresponding to $\mathrm{H}-3$ and $\mathrm{H}-4 \mathrm{a}$, while in a lycorine skeleton the most upfield signals correspond to the $\mathrm{H}-11$ hydrogens. 


\subsubsection{Narciclasine type}

The narciclasine-type alkaloids present the highest degree of oxidation. The absolute configuration of the most studied alkaloid of this group pancratistatin, was determined by X-ray diffraction [51]. The main ${ }^{1} \mathrm{H}$ NMR characteristics of the narciclasine-type alkaloids are:

a. The only aromatic hydrogen appears as a singlet with a chemical shift higher than $7 \mathrm{ppm}$.

b. Those alkaloids with a hydrogenated double bond $\mathrm{C}-1 / \mathrm{C}-10 \mathrm{~b}$ possess a trans stereochemistry for the $\mathrm{B}-\mathrm{C}$ ring junction and, consequently, a large coupling constant value for $J_{4 \mathrm{a}-10 \mathrm{~b}}$.

c. The hydrogen attached to the nitrogen atom appears as a broad singlet with a chemical shift around $5 \mathrm{ppm}$, which disappears on the addition of $\mathrm{D}_{2} \mathrm{O}$.

\subsubsection{Galanthamine type}

Among the Amaryllidaceae alkaloids, only the galanthamine type shows an ortho-coupling constant between both aromatic protons of ring A. The general characteristics of their ${ }^{1} \mathrm{H}$ NMR spectra are:

a. Two doublets for the two ortho-oriented aromatic protons with a coupling constant of $J_{7,8} \sim 8 \mathrm{~Hz}$.

b. The assignment of the substituent stereochemistry at C-3 is made in relation with the coupling constants of the olefinic protons $\mathrm{H}-4$ and $\mathrm{H}-4 \mathrm{a}$. When coupling constant $\mathrm{J}_{3,4}$ is about $5 \mathrm{~Hz}$, the substituent is pseudoaxial, while if it is $\sim 0 \mathrm{~Hz}$ this indicates that the substituent at C-3 is pseudoequatorial.

c. Two doublets corresponding to the $\mathrm{AB}$ system of the C-6 benzylic protons.

d. The existence of the furan ring results in a deshielding effect in $\mathrm{H}-1$.

e. This type of alkaloids often shows an $N$-methyl group but occasionally an $N$-formyl group has been reported.

\subsection{Carbon $^{13}$ nuclear magnetic resonance}

${ }^{13} \mathrm{C}$ NMR spectroscopy has been extensively used for determining the carbon framework of Amaryllidaceae alkaloids, and there are several major contributions [52-54]. The assignments are made on the basis of chemical shifts and multiplicities of the signals (by DEPT experiment). The use of 2D 
NMR techniques such as HMQC and HMBC allow the assignments to be corroborated.

The ${ }^{13} \mathrm{C}$ NMR spectra of Amaryllidaceae alkaloids can be divided in two regions. The low-field region ( $>90 \mathrm{ppm})$ contains signals of the carbonyl group, the olefinic and aromatic carbons as well as that of the methylenedioxy group. The other signals corresponding to the saturated carbon resonances are found in the high-field region, the $N$-methyl being the only characteristic group, easily recognizable by a quartet signal between 40-46 ppm.

The effect of the substituent $(\mathrm{OH}, \mathrm{OMe}, \mathrm{OAc})$ on the carbon resonances is of considerable importance in localizing the position of the functional groups.

The analysis of the spectra allows conclusions to be drawn about the following aspects:

- The number of methine olefinic carbons.

- The presence and nature of the nitrogen substituent.

- The existence of a lactonic carbonyl group.

- The presence of a quaternary carbon signal assignable to $\mathrm{C}-10 \mathrm{~b}$ in the chemical shift range of $42-50 \mathrm{ppm}$.

\section{Biological and pharmacological activities}

This section covers the pharmacological and/or biological properties of the most representative Amaryllidaceae alkaloids. Until now only galathamine is being marketed, but the significant activities of other alkaloids in the family demonstrated in recent years could favour their therapeutic use in the near future.

\subsection{Lycorine type}

The most characteristic and common Amaryllidaceae alkaloid is lycorine, reported to be a powerful inhibitor of ascorbic acid (L-Asc) biosynthesis $[55,56]$, and thus a useful tool in studying Asc-dependent metabolic reactions in L-Asc-synthesising organisms [57,58]. Specifically, lycorine is a powerful inhibitor of the activity of L-galactono- $\gamma$-lactone dehydrogenase, the terminal enzyme of L-Asc biosynthesis [59-62], which is thought to be localised in the mitochondrial membrane $[63,64]$. Galanthine also has a high capacity to inhibit ascorbic acid biosynthesis [56].

Lycorine is a powerful inhibitor of cell growth, cell division and organogenesis in higher plants, algae, and yeasts, inhibiting the cell cycle during interphase, which seems to be related with the L-Asc levels [57,65-69]. In plants, it also inhibits cyanide-insensitive respiration, peroxidase activity 
and protein synthesis [70-72]. The effects of lycorine on L-Asc biosynthesis have been reported to occur at concentrations below those at which protein synthesis is affected, but it seems difficult to completely rule out non-specific effects of this alkaloid since it has been reported that, at least in yeasts, lycorine is able to interact directly with mitocondrial DNA. Thus, differing sensitivity to the alkaloid among cells devoid of mitochondrial DNA $\left(r h o^{0}\right)$ and cells with mitochondrial DNA either $r \mathrm{ho}^{+}$or $r h o^{-}$has been found in yeasts $[59,67,73,74]$, rho $o^{0}$ cells being resistant to high concentrations of the drug [69,75-77]. Some strains can even adapt to the presence of lycorine, because they are able to degrade the alkaloid and use its biotransformation products as growth stimulating factors [77]. In contrast, lycorine-1-O- $\beta$-D-glucoside promotes cell growth, seed germination, and rate of development of root and root hairs in higher plants. The glucosyloxy derivatives of lycorine and pseudolycorine and their aglicones form stable complexes with phytosterols and also with divalent metal ions and are able to translocate them from the rhizosphere to the aerial part [78]. Palmilycorine and some acylglucosyloxy conjugates of lycorine, in turn, are frequently encountered among the phytosterols exhibiting membrane-stabilizing action. Plants also use lycorine$1-O-\beta-\mathrm{D}$-glucoside and acylglucosyloxy conjugates of lycorine to recognize and reject microorganisms and parasites [79].

The antitumor activity of lycorine in animals [80,81] has been demonstrated by the inhibition of in vivo and in vitro growth of diverse tumor cells, such as BL6 mouse melanoma, Lewis lung carcinoma, murine ascite or HeLa cells $[3,79,82-86]$. It induces flat morphology in K-ras-NRK cells (transformed fibroblasts) [87], and reduces the cellular activity in femoral bone marrow tissue that results in granulocytic leucopenia and a decrease in the number of erythrocytes. This alkaloid's mechanism of action is thought to be through inhibition of protein synthesis at the ribosomal level, even though the cytotoxic effects of calprotectin can also be suppressed using lycorine [80,81,88-90]. Lycorine also inhibits murine macrophage production of Tumor Necrosis Factor alpha (TNF- $\alpha)$ [91], and shows inhibitory effects on nitric oxide production and induction of inducible nitric oxide synthase (NOS) in lipopolysaccharide-activated macrophages [92]. The molecular mechanism of lycorine against leukaemia (human cell line HL-60) shows that it can suppress cell growth and reduce cell survival by arresting the cell cycle at the $\mathrm{G}_{2} / \mathrm{M}$ phase and inducing apoptosis of tumor cells [93]. Recent studies show that the TNF- $\alpha$ signal transduction pathway and p21-mediated cell-cycle inhibition are involved in the apoptosis of HL-60 cells induced by lycorine [94]. The effects of lycorine on the human multiple myeloma cell line KM3, and the possible mechanisms of these effects have also been studied [95]. The growth rates of the KM3 cells exposed to lycorine clearly slowed down. Cell 
fluorescent apoptotic morphological changes, DNA degradation fragments, and a sub- $\mathrm{G}_{1}$ peak were detected, indicating the occurrence of cell apoptosis after lycorine treatment. Furthermore, the release of mitochondrial cytochrome $\mathrm{c}$, the augmentation of Bas with the attenuation of Bcl-2, and the activation of Caspase-9, -8 , and -3 were also observed, suggesting that the mitochondrial pathway and the death acceptor pathway were involved. The results also showed that lycorine was able to block the cell cycle at the $\mathrm{G}_{0} / \mathrm{G}_{1}$ phase through the downregulation of both cyclin D1 and CDK4. In short, lycorine can suppress the proliferation of KM3 cells and cell survival by arresting cell cycle progression as well as inducing cell apoptosis [96]. A recent paper describes the preparation of a mini-library comprised of synthetic and natural lycorane alkaloids and the investigation of apoptosis-inducing activity in human leukemia (Jurkat) cells. Further insights into the nature of this apoptosis-inducing pharmacophore are described, including the requirement of both free hydroxyl groups in ring-C [97]. Another recent study describes the induction of apoptosis in human leukemia cells by lycorine via an intrinsic mitochondria pathway, causing a rapid turnover of protein level of Mcl-1 before Caspases activation. Pronounced apoptosis accompanied by the down-regulation of Mcl-1 was also observed in blasts from patients with acute myeloid leukemia. Lycorine also displays pronounced cell growth inhibitory activities against both parental and multidrug resistant L5178 mouse lymphoma cell lines, but is almost inactive in inhibiting the glycoprotein responsible for the efflux-pump activity of tumor cells. Assays for interactions with tRNA revealed that the antiproliferative effects of lycorine result from their complex formation with tRNA [98]. Interaction of lycorine, pseudolycorine and 2-O-acetylpseudo-lycorine with DNA has been observed $[99,100]$. Most of the alkaloids that showed promising antiproliferative activities have also proved to be efficient apoptosis inducers [14].

Some other alkaloids of this series, such as caranine, galanthine, pseudolycorine and 2-O-acetylpseudolycorine, are also active against a variety of tumor cells $[84,101,102]$. Pseudolycorine inhibits the protein synthesis in tumor cells at the step of peptide bond formation, but it has a different binding site than lycorine $[89,103]$. Ungeremine, a natural metabolite of lycorine, is responsible, at least partially, for the growth-inhibitory and cytotoxic effects of lycorine, being active against leukemia [104,105]. Lycorine-1-O- $\beta$-Dglucoside, in turn, has the reverse effect of lycorine, and may produce mitogenic activity in animal cells [106].

A mini-panel of semi-synthetic analogs of lycorine was screened for cytochrome P450 3A4 (CYP3A4) inhibitory activity, the most potent of which (1-O-acetyl-2-O-tert-butyldimethylsilyllycorine) exhibited inhibition at a concentration as low as $0.21 \mu \mathrm{M}$. Elements of this unraveled novel 
pharmacophore include bulky lipophilic substitution at C-2 in conjunction with a small hydrogen donor/acceptor bond at C-1, or bulky electron-rich substitution at $\mathrm{C}-1$ in conjunction with a vicinal hydrogen donor/acceptor bond [107]. Two semisynthetic silylated lycorane analogs, accesed via a chemoselective silylation strategy from lycorine exhibited low micromolar activities [108].

Lycorine and pseudolycorine exert antiviral effects on several RNA and DNA-containing viruses [109]. Antiviral activity has been observed in tests with flaviviruses, and to a slightly lesser degree, bunyaviruses. Lycorine and pseudolycorine also show inhibitory activity against the Punta Toro and Rift Valley fever viruses, but with low selectivity [110,111]. Lycorine, in turn, acts as an anti-SARS-CoV (Severe Acute Respiratory Syndrome-associated Coronavirus) and shows pronounced activity against poliomyelitis, coxsackie and herpes type $1[3,112]$. It possesses high antiretroviral activity accompanied by low therapeutic indices [113]. The relationship between its structure and the mechanism of activity has been studied in the Herpes simplex virus, suggesting that alkaloids that may eventually prove to be antiviral agents have a hexahydroindole ring with two functional hydroxyl groups [114]. The activity was found to be due to the inhibition of multiplication, and not to the direct inactivation of extracellular viruses, and the mechanism of the antiviral effect was partially explained as a blocking of viral DNA polymerase activity [109,115-117].

Lycorine has appreciable inhibitory activity against acetylcholinesterase [118]. Cholinesterase activity appears to be associated with the two free hydroxyl groups present in some of the alkaloids of this structural type [119]. The higher acetylcholinesterase inhibitory activity of assoanine and oxoassoanine with respect to the other lycorine-type alkaloids could be explained by an aromatic ring $\mathrm{C}$, which gives a certain planarity to those molecules [120]. Another alkaloid, galanthine, exhibits powerful cholinergic activity and has therefore attracted much interest in the treatment of myasthenia gravis, myopathy and diseases of the central nervous system [121]. Caranine, pseudolycorine, ungiminorine, and in particular, ungeremine, also show an inhibitory effect on acetylcholinesterase $[120,122,123]$. Recently, the synthesis of differentially functionalized analogs of lycorine, accessed via a concise chemoselective silylation strategy, has allowed two of the most potent inhibitors of acetylcholinesterase to be described. Important elements of this novel pharmacophore were elucidated through SAR studies [94].

Lycorine is analgesic, more so than aspirin, and hypotensive [124,125], as are caranine and galanthine. The analgesic activity exhibited by the Amaryllidaceae alkaloids is attributed to their similarity with the morphine 
and codeine skeletons. Lycorine also has antiarrhythmic action, and lycorine hydrochloride is a strong broncholytic [126]. In fact, lycorine shows a relaxant effect on an isolated epinephrine-precontracted pulmonary artery and increases contractility and the rate of an isolated perfused heart. These effects are mediated by stimulation of $\beta$-adrenergic receptors [127].

Lycorine also has a strong inhibitory effect on parasite (Encephalitozoon intestinalis) development [128] and antifungal activity against Candida albicans [129]. Recently, several lycorine derivatives were examined for their activity against Trypanosoma brucei and Plasmodium falciparum. Among them, 2-O-acetyllycorine showed the most potent activity against parasitic T. brucei, while 1-O-(3R)hydroxybutanoyllycorine, 1,2-di-O-butanoyllycorine, and 1-O-propanoyllycorine showed significant activity against $P$. falciparum in an in vitro experiment [130], although the antimalarial activity of lycorine was already known [131-133]. Galanthine, in turn, shows mild in vitro activity against Tripanosoma brucei rhodesiense and Plasmodium falciparum [134]. Additionally, lycorine has antifeedant [135], emetic [136], anti-inflammatory [137], antiplatelet [138] as well as antifertility [125] activities.

\subsection{Homolycorine type}

It is reported that some alkaloids of this series, such as homolycorine, 8-O-demethylhomolycorine, dubiusine, 9-O-demethyl-2 $\alpha$-hydroxyhomoly-corine, hippeastrine, lycorenine or $O$-methyllycorenine present cytotoxic effects against non-tumoral fibroblastic LMTK cells [84], also being moderately active in inhibiting the in vivo and in vitro growth of a variety of tumor cells, such as Molt 4 lymphoma, HepG2 human hepatoma, LNCaP human prostate cancer or HT [84,125,139]. Dubiusine, lycorenine, 8-O-demethylhomolycorine and 9-Odemethyl-2 $\alpha$-hydroxyhomolycorine also show DNA binding activity comparable to that of vinblastine [99]. Homolycorine possesses high antiretroviral activity, accompanied by low therapeutic indices [113]. Hippeastrine, in turn, displays antiviral activity against Herpes simplex type 1 [114].

Dubiusine, homolycorine, 8-O-demethylhomolycorine and lycorenine have a hypotensive effect on the arterial pressure of normatensive rats [140]. Lycorenine also shows a vasodepressor action ascribed to the maintenance of its $\alpha$-adrenergic blocking action, and produces bradycardia by modifying vagal activity [141]. Another feature of lycorenine is its analgesic activity [3].

Homolycorine and masonine are other inductors of delayed hypersensitivity in animals [142]. Hippeastrine, in turn, shows antifungal activity against Candida albicans and it also possesses a weak insect antifeedant activity [129]. 


\subsection{Haemanthamine and crinine types}

Haemanthamine, haemanthidine, crinamine, maritidine and papyramine display pronounced cell growth inhibitory activities against a variety of tumor cells, such as Rauscher viral leukaemia, Molt 4 lymphoma, BL6 mouse melanoma, HepG2 human hepatoma, $\mathrm{HeLa}, \mathrm{LNCaP}$ human prostate cancer or HT [82-84,88,139,143,144]. Some of these alkaloids, namely crinamine, haemanthamine and papyramine, also present a cytotoxic effect against nontumoral fibroblastic LMTK cells [84]. The mechanism of action of haemanthamine is thought to be through inhibition of protein synthesis, blocking the peptide bond formation step on the peptidyl transferase centre of the 60S ribosomal subunit $[89,103]$. Haemanthamine and haemanthidine also display the same pronounced cell growth inhibitory activities against both parental and multidrug resistant L5178 mouse lymphoma cell lines as described above for lycorine [98]. Crinamine, in turn, shows inhibitory effects on nitric oxide (NO) production and induction of inducible nitric oxide synthase (NOS) in lipopolysaccharide-activated macrophages [92]. Crinamine and haemanthamine are potent inducers of apoptosis in tumor cells at micromolecular concentrations [145]. The pharmacophoric elements are the alpha-C-2 bridge as well as a small substituent $(\mathrm{H}$, or $\mathrm{OH})$ at $\mathrm{C}-11$. Studies have also shown that $\alpha$ - or $\beta$-methoxy or the hydroxyl H-bond acceptor are all tolerated at $\mathrm{C}-3$, and that a $\mathrm{C}-1 / \mathrm{C}-2$ double bond modulates, but is not a requirement, for apoptosis-inducing activity [146].

The antimalarial activity against strains of chloroquine-sensitive Plasmodium falciparum observed in haemanthamine and haemanthidine can be attributed to the methylenedioxybenzene part of the molecule and the terciary nitrogen without methyl [131]. Crinamine also exhibits moderate antimalarial activity $[132,147]$. Haemanthidine also works in vitro against Trypanosoma brucei rhodesiense and to a lesser extend against Trypanosoma cruzi [134]. Vittatine has antibacterial activity against the Gram-positive Staphylococcus aureus and the Gram-negative Escherichia coli [129], and the alkaloid crinamine shows strong activity against Bacillus subtilis and Staphylococcus aureus [148].

Like lycorine, haemanthidine has stronger analgesic and anti-inflammatory activity than aspirin [118,137], and vittatine has been found to potentiate the analgesic effect of morphine [149]. Moreover, some alkaloids of this series, such as haemanthamine or papyramine have a hypotensive effect [140,150], and haemanthamine strong antiretroviral activity [113]. 


\subsection{Tazettine type}

Tazettine is mildly active against certain tumor cell lines $[88,139,151]$, with a slight cytotoxicity when tested on fibroblastic LMTK cell lines [84]. Tazettine also displays weak hypotensive and antimalarial activities and interacts with DNA $[99,138,140]$. Its chemically labile precursor, pretazettine, is far more interesting owing to its antiviral and anticancer activities. In fact, when pretazettine is stereochemically rearranged to tazettine, the biological activity of the precursor is to a large extent inactivated $[152,153]$.

Pretazettine shows cytotoxicity against fibroblastic LMTK cell lines and inhibits HeLa cell growth, being therapeutically effective against advanced Rauscher leukaemia, Ehrlich ascites carcinoma, spontaneous AKR lymphocytic leukaemia and Lewis lung carcinoma [151,154-159]. It is one of the most active of the Amaryllidaceae alkaloids against Molt4 lymphoid cells [84], and is used in combination with DNA-binding and alkylating agents in treating the Rauscher leukaemia virus $[151,154]$. In fact, pretazettine strongly inhibits the activity of reverse transcriptase from various oncogenic viruses by binding to the enzyme [3]. It inhibits both the growth of the Rauscher virus and cellular protein synthesis in eukaryotic cells by a mechanism that does not affect DNA and RNA synthesis, even though it has a pronounced DNA binding activity $[88,89,99,101,111,156,160]$. Pretazettine on human MDR1gene-transfected L5158 mouse lymphoma significantly increased the intracellular concentration of $\mathrm{Rh}-123$ and enhanced the antiproliferative activity of doxorubicin in the L5178 MDR cell line [161]. This alkaloid has also been shown to be active against selected RNA-containing flavoviruses (Japanese encephalitis, yellow fewer and dengue) and bunyaviruses (Punta Toro and Rift Valley fever) in organ culture [111]. It also possesses pronounced activity against Herpes simplex type 1 virus [114]. This activity may reflect a general ability to inhibit protein synthesis during viral replication [162].

\subsection{Narciclasine type}

Narciclasine, an antimitotic and antitumoral alkaloid [163], affects cell division at the metaphase stage and inhibits protein synthesis in eukaryotic ribosomes by directly interacting with the 60 s subunit and inhibiting peptide bond formation by preventing binding of the 3' terminal end of the donor substrate to the peptidyl transferase center [89,103,164-166]. It also retards DNA synthesis [167] and inhibits calprotectin-induced cytotoxicity at a more than 10-fold lower concentration than lycorine [90]. The peculiar effects of 
narciclasine seem to arise from the functional groups and conformational freedom of its C-ring [168], with the 7-hydroxyl group believed to be important in its biological activity [169]. This alkaloid, related to pancratistatin [167], is one of the most important antineoplastic Amaryllidaceae alkaloids [80] and shows some promise as an anticancer agent. It inhibits HeLa cell growth, has antileukaemic properties and is active against a variety of tumor cells, such as human and murine lymphocytic leukaemia, larynx and cervix carcinomas and Ehrlich tumor cells [115,167,170-172]. One hemisynthetic derivative of narciclasine demonstrated higher in vivo antitumor activity in human orthotopic glioma models in mice than narciclasine in nontoxic doses [173], by both the i.v and oral routes. No effect has been observed on solid tumors. Narciclasine-4-O- $\beta$ D-glucopiranoside shows a very similar cytotoxic and antitumoral activity to narciclasine [174]. The anticancer activity and preclinical studies of narciclasine and its congeners has been gathered by Kornienko and Evidente in a recent review [175]. Melanomas display poor response rates to adjuvant therapies because of their intrinsic resistance to proapoptotic stimuli. Such resistance can be overcome, at least partly, through the targeting of the eEF1A elongation factor with narciclasine [176]. This alkaloid directly binds to human recombinant and yeast-purified eEF1A in a nanomolar range, but not to actin or elongation factor 2 . Thus, eEF1A is a potential target to combat melanomas regardless of their apoptosis-sensitivity, which has renewed interest in the pleiotropic cytostatic activity of narciclasine. Apoptosis in Jurkat cells was triggered by narciclasine, narciclasine tetraacetate, $C-10 \mathrm{~b}-\mathrm{R}$ hydroxypancratistatin, cis-dihydronarciclasine and trans-dihydronarciclasine [177].

The effect of pancratistatin treatment on cancerous and normal cells has also been reported [178]. The results indicated that pancratistatin selectively induced apoptosis in cancer cells, and the mitochondria may be the site of action. To further explore the structure-activity relationship of pancratistatinrelated compounds, the anticancer efficacy and specificity of two related natural alkaloids were investigated. Both of these compounds lack the polyhydroxylated lycorane element of pancratistatin, instead having a methoxy-substituted crinane skeleton. These results indicated that the phenanthridone skeleton in natural Amaryllidaceae alkaloids may be a significant common element for selectivity against cancer cells. The synergy of pancratistatin and tamoxifen on breast cancer cells in inducing apoptosis by targeting mitochondria has been also reported [179]. The 3,4-O-cyclic phosphate salt of pancratistatin is a novel, water soluble synthetic derivative of pancratistatin that in vivo caused statistically significant tumor growth delays at its maximum-tolerated dose. Significant vascular shutdown and tumor necrosis were also observed [180], 
offering a way forward for improved clinical treatment by greatly enhancing solubility without loss of antitumor activity.

Narciclasine has a prophylactic effect on the adjuvant arthritis model in rats, significantly suppressing the degree of swelling of adjuvant-treated as well as untreated feet [90]. This alkaloid is also active against Corynebacterium fascians, inhibits the pathogenic yeast Cryptococcus neoformans, and modifications like 2,3,4,7-tetra-O-acetylnarciclasine inhibit the growth of the pathogenic bacterium Neisseria gonorrhoeae [181]. Antiviral activity has been observed against RNA-containing flaviviruses and bunyaviruses [111].

At the plant level, narciclasine is a potent inhibitor, showing a broad range of effects, including the ability to inhibit seed germination and seedling growth of some plants in a dose-dependent manner, interacting with hormones in some physiological responses [182]. Thus, indole-3-acetic acid cannot overcome the inhibition of elongation of wheat coleoptile sections caused by narciclasine. Additionally, narciclasine suppresses the gibberellin-induced $\alpha$-amylase production in barley seeds and cytokinin-induced expansion and greening of excised radish cotyledons [183]. Like lycorine, narciclasine also inhibits ascorbic acid biosynthesis [184]. Narciclasine, present in daffodil mucilage, can delay tepal senescence in cut Iris flowers by attenuation of protease activity, which, in turn, is apparently related with the inhibition of the protein synthesis involved in senescence [185]. At the organelle level, narciclasine inhibits both isocitrate lyase (ICL) activity in glyoxysomes and hydroxypyruvate reductase (HPR) activity in peroxisomes. It also blocks the formation of chloroplasts, markedly reducing the chlorophyll content of lightgrown wheat seedlings, probably due to the inhibition of the formation of 5-aminolevulinic acid, an essential chlorophyll precursor [186]. The formation of light harvesting chlorophyll $\mathrm{a} / \mathrm{b}$ binding protein (LHCP) is also inhibited by this alkaloid [187].

Some alkaloids of this series, such as trisphaeridine, possess high antiretroviral activities, accompanied by low therapeutic indices [113]. Ismine, in turn, shows a significant hypotensive effect on the arterial pressure of normotensive rats [140] and is cytotoxic against Molt 4 lymphoid and LMTK fibroblastic cell lines [84].

\subsection{Montanine type}

There is little information about the montanine type alkaloids, only some data about pancracine, which shows antibacterial activity against Staphylococcus aureus and Pseudomonas aeruginosa [129], as well as weak activity against Trypanosoma brucei rhodesiense, T. cruzi and Plasmodium falciparum [188]. Montanine inhibited, in a dose-dependent manner, more 
than $50 \%$ of the enzyme acetylcholinesterase at $1 \mathrm{mM}$ concentration. With the concentrations $500 \mu \mathrm{M}$ and $100 \mu \mathrm{M}, 30-45 \%$ of inhibition was detected [189].

\subsection{Galanthamine type}

Galanthamine, originally isolated from Galanthus nivalis L. in the 1940s, is a long-acting, selective, reversible and competitive inhibitor of acetylcholinesterase. This enzyme is responsible for the degradation of acetylcholine at the neuromuscular junction, in peripheral and central cholinergic synapses and in parasympathetic target organs [190-192]. Galanthamine has the ability to cross the blood-brain barrier and act within the central nervous system [193,194]. It binds at the base of the active site gorge of acetylcholinesterase, interacting with both the choline-binding site and the acyl-binding pocket, having a number of moderate-to-weak interactions with the protein [195-197]. In addition, galanthamine stimulates pre- and postsynaptic nicotinic receptors which can, in turn, increase the release of neurotransmitters, thus directly stimulating neuronal function [192,198]. It is also suggested that the stimulation of nicotinic receptors protects against apoptosis induced by $\beta$-amyloid toxicity $[192,199,200]$. Its dual mode of action [195], coupled with the evidence that galanthamine has reduced side effects, make it a promising candidate for the treatment of nervous diseases, paralysis syndrome, schizophrenia and other forms of dementia, as well as Alzheimer's disease [192,195,196].

Other significant pharmacological actions of Galanthamine include an ability to amplify the nerve-muscle transfer [3], affecting membrane ionic processes [201]. It is also known to cause bradycardia or atrioventricular conduction disturbances [150], has long been used as a reversal agent in anaesthetic practice [18], inhibits traumatic shock and has been patented for use in the treatment of nicotine dependence. Besides this, galanthamine acts as a mild analeptic, shows an analgesic power as strong as morphine, compensates for the effects of opiates on respiration, relieves jet lag, fatigue syndrome, male impotence and alcohol dependence, and when applied in eye drops, reduces the intraocular pressure [3,202-204]. It also acts as a hypotensive and has a weak antimalarial activity $[138,140]$.

At present, Alzheimer's disease cannot be prevented or cured, so the symptomatic relief offered by AChEI therapy is the only approved therapeutic option. Due to the relative lack of alternative treatment, galanthamine is a reasonable approximation of the ideal concept of symptomatic Alzheimer's disease therapy [191,205]. Galanthamine hydrobromide (a third-generation cholinesterase inhibitor used against Alzheimer's disease) offers superior pharmacological profiles and increased tolerance compared to the original 
acetylcholinesterase inhibitors, physostigmine or tacrine [193,206-209]. Galanthamine is effective and well tolerated, resulting in short-term improvements in cognition, function and daily life activities in patients with mild to moderate symptoms [198,210,211]. However, there is doubt about its long-term benefits [212] since persistent elevation of acetylcholine beyond 6 months may lead to over-stimulation of both nicotinic and muscarinic acetylcholine receptors, the former causing receptor desensitisation and the latter potentially causing an increased frequency of cholinergic side effects $[192,198,213]$. The safety profile of galanthamine as well as its clinical effectiveness will only be demonstrated after large-scale clinical trials [213-215].

The development of galanthamine into a widely used Alzheimer's drug can be divided into three main periods: 1- the early development in Eastern Europe for its use in the treatment of poliomyelitis; 2- the pre-clinical development in the 1980s; 3- the clinical development in the 1990s [213]. Galanthamine hydrobromide was first used by Bulgarian and Russian researchers in the 1950s and exploited for a variety of clinical purposes. It has been used clinically for postsurgery reversal of tubocurarine-induced muscle relaxation and for treating post-polio paralysis, myasthenia gravis and other neuromuscular diseases, as well as traumatic brain injuries [216,217]. As early as 1972, Soviet researchers demonstrated that galanthamine could reverse scopolamine-induced amnesia in mice, a finding that was demonstrated in man 4 years later. However, this compound was not applied to Alzheimer's disease until 1986, long after the widely accepted cholinergic hypothesis had been first postulated, when researchers in Western Europe switched their attention to galanthamine due to its ability to penetrate the blood-brain barrier and specifically to augment the central cholinergic function [213,218]. This led to clinical trials of galanthamine in the treatment of Alzheimer's disease. In 1996, Sanochemia Pharmazeutika in Austria first launched galanthamine as 'Nivalin ${ }^{\circledR}$, , but its strictly limited availability meant the international pharmaceutical community adopted a cautions approach [18,194], until Sanochemia Pharmazeutika developed a method to synthetically produce the compound in 1997 [219]. Later, galanthamine was co-developed by Shire Pharmaceuticals (Great Britain) and the Janssen Research Foundation (Belgium), who have launched galanthamine as 'Reminyl ${ }^{\mathbb{R}}$ ' in many countries $[192,213]$. This renewed interest is reflected in the increasing number of scientific reviews dealing exclusively with galanthamine and its derivatives [220-223].

Sanguinine has a more potent acetylcholinesterase inhibitory activity than galanthamine due to an extra hydroxyl group available for potential interaction with acetylcholinesterase [120]. Sanguinine, in turn, is 10-fold 
more selective than galanthamine for acetylcholinesterase (AChE) vs. butyrylcholinesterase (BuChE) [224]. The lack of AChE inhibitory activity of lycoramine and epinorlycoramine could be due to the occurrence of a double bond in ring $\mathrm{C}$, which does not allow these compounds to have the same spatial configuration as the active alkaloids of this series [120].

Narwedine, the biogenic precursor of galanthamine, has been studied as a respiratory stimulator. It increases the amplitude and decreases the frequency of cardiac contractions and would therefore be of value in reducing blood loss during surgery [150]. It also inhibits the action of narcotics and hypnotics, and increases the analgesic effect of morphine [149], as well as the pharmacological effects of caffeine, carbazole, arecoline and nicotine [126].

\subsection{Other alkaloids}

Cherylline is a 4-arylisoquinoline derivative, a group with several potential medicinal properties [188], including a weak acetylcholinesterase inhibitory activity [118]. Mesembrenone, in turn, is mildly active against Molt 4 lymphoid and non-tumoral fibroblastic LMTK cells [84], has a moderate hypotensive effect on arterial pressure and interacts slightly with DNA $[99,140]$.

\section{Acknowledgements}

The authors are grateful to Ms Lucy Brzoska for language corrections. The authors also thank Generalitat de Catalunya (2009-SGR1060) for financial support.

\section{References}

1. Meerow, A. W., Snijman, D. A. 1998, in "The Families and Genera of Vascular Plants" (K. Kubitzki ed.), Vol. III, p. 83. Springer-Verlag, Berlin.

2. Dictionary of Natural Products (Net Database). 2005, Chapman and Hall / CRC Press, London.

3. Ghosal, S., Saini, K. S., Razdan, S. 1985, Phytochemistry 24, 2141.

4. Unver, N. 2007, Phytochem. Rev. 6, 125.

5. Jin, Z. 2009, Nat. Prod. Rep. 26, 363.

6. Jeffs, P. W. 1981, in "The Alkaloids" (R. H. F. Manske and R. G. A. Rodrigo, eds.), Vol. 19, p. 1. Academic Press, New York.

7. Smith, M. T., Crouch, N. R., Gericke, N., Hirst, M. 1996, J. Ethnopharmacol. $50,119$.

8. Döpke, W., Sewerin, E., Trimiño, Z. 1980, Z. Chem. 20, 298. 
9. Bastida, J., Viladomat, F., Llabrés, J. M., Ramírez, G., Codina, C., Rubiralta, M. 1989, J. Nat. Prod. 52, 478.

10. Seijas, J. A., Vázquez-Tato, M. P., Linares, M. T., Ramil-Rego, P., Buján, M. I. 2004, $8^{\text {th }}$ International Electronic Conference on Synthetic Organic Chemistry (ECSOC-8) p. 625.

11. Kaya, G. I., Unver, N., Gözler, B., Bastida, J. 2004, Biochem. Syst. Ecol. 32, 1059.

12. Hudlicky, T., Rinner, U., Gonzalez, D., Akgun, H., Schilling, S., Siengalewicz, P., Martinot, T. A., Pettit, G. R. 2002, J. Org. Chem. 67, 8726.

13. Bastida J., Lavilla, R., Viladomat, F. 2006, in "The Alkaloids: Chemistry and Biology" (G. A. Cordell, ed.), Vol 63, p. 87. Elsevier Inc. Amsterdam.

14. Cedrón, J. C., Del Arco-Aguilar, M., Estévez-Braun, A., Ravelo, Á. G. 2010, in "The Alkaloids: Chemistry and Biology" (G. A. Cordell, ed.), Vol 68, p. 1. Elsevier Inc. Amsterdam.

15. Jin, Z. 2003, Nat. Prod. Rep. 20, 606.

16. Jin, Z. 2005, Nat. Prod. Rep. 22, 111.

17. Jin, Z. 2007, Nat. Prod. Rep. 24, 886.

18. Eichhorn, J., Takada, T., Kita, Y., Zenk, M. H. 1998, Phytochemistry 49, 1037.

19. Suhadolnik, R. J., Fischer, A. G., Zulalian, J. 1963, Biochem. Biophys. Res. Commun. 208.

20. Wightman, R. H., Staunton, J., Battersby, A. R., Hanson, K.R. 1972, J. Chem. Soc.- Perkin Trans. I 2355.

21. Ghosal, S., Kumar, Y., Singh, S. K., Shanthy, A. 1986, J. Chem. Res.-S 28.

22. Ghosal, S., Shanthy, A. Singh, S.K. 1988, Phytochemistry 27, 1849.

23. Barton, D. H. R., Cohen, T. 1957, Festschrift Arthur Stoll, p 117, Birkhäuser Verlag, Basel.

24. Bowman, W. R., Bruce, I. T., Kirby, G. W. 1969, J. Chem. Soc.- Chem. Commun. 1075.

25. Kirby, G. W., Tiwari, H. P. 1966, J. Chem. Soc.-C 676.

26. Bruce, I. T., Kirby, G. W. 1968, J. Chem. Soc.- Chem. Commun. 207.

27. Wildman, W. C., Heimer, N. E. 1967, J. Am. Chem. Soc. 89, 5265.

28. Fuganti, C., Mazza, M. 1972, J. Chem. Soc.- Chem. Commun. 936.

29. Battersby, A. R., Binks, R., Breuer, S. W., Fales, H. M., Wildman, W. C., Highet, R. J. 1964, J. Chem. Soc. 1595.

30. Harken, R. D., Christensen, C. P., Wildman, W. C. 1976, J. Org. Chem. 41, 2450.

31. Fuganti,C, Ghiringhelli, D., Grasselli, P. 1974, J. Chem. Soc.- Chem. Commun. 350.

32. Kihara, M., Xu, L., Konishi, K., Nagao, Y., Kobayashi, S., Shingu, T. 1992, Heterocycles 34, 1299.

33. Fuganti, C., Mazza, M. 1973, J. Chem. Soc.- Perkin Trans. I 954.

34. K. R. Hanson, K.R. 1966, J. Am. Chem. Soc. 88, 2731.

35. Fuganti, C., Mazza, M. 1971, J. Chem. Soc.- Chem. Commun. 1196.

36. Feinstein, A. I., Wildman, W.C. 1976, J. Org. Chem. 41, 2447.

37. Fuganti, C. 1969, Chim. Ind. (Milan) 51, 1254.

38. Battersby, A. R., Kelsey, J. E., Staunton, J. 1971, J. Chem. Soc.- Chem. Commun. 183.

39. Fales, H. M., Wildman, W. C. 1964, J. Am. Chem. Soc. 86, 294. 
40. Wildman, W. C., Bailey, D. T. 1969, J. Am. Chem. Soc. 91, 150.

41. Fuganti, C., Staunton, J., Battersby, A. R. 1971, J. Chem. Soc.- Chem. Commun. 1154.

42. Fuganti, C., Mazza, M. 1971, J. Chem. Soc.- Chem. Commun. 1388.

43. Fuganti, C., Mazza, M. 1972, J. Chem. Soc.- Chem. Commun. 239.

44. Fuganti, C. 1973, Tetrahedron Lett. 1785.

45. Fuganti, C., Mazza, M. 1970, J. Chem. Soc.- Chem. Commun. 1466.

46. Barton, D. H. R., Kirby, G. W., Taylor, J. B., Thomas, G. M. 1963, J. Chem. Soc. 4545.

47. Vázquez-Tato, M. P., Castedo, L., Riguera, R. 1988, Heterocycles 27, 2833.

48. Bhandarkar, J. G., Kirby, G. W. 1970, J. Chem. Soc.-C 1224.

49. Jeffs, P. W., Mueller, L., Abou-Donia, A. H., Seif el-Din, A. A., Campau, D. 1988, J. Nat. Prod. 51, 549.

50. Wildman, W. C., Bailey, D. T. 1967, J. Am. Chem. Soc. 89, 5514.

51. Pettit, G. R., Gaddamidi, V., Venkatswamy, H., Herald, D., Singh, S., Cragg, G., Schmidt, J., Böttner, F., Williams, M., Sagawa, Y. 1986, J. Nat. Prod. 49, 995.

52. Frahm, A. W., Ali, A. A., Ramadan, M. A. 1985, Magn. Reson. Chem. 23, 804.

53. Crain, W. O., Wildman, W. C., Roberts, J. D. 1971, J. Am. Chem. Soc. 93, 990.

54. Zetta, L., Gatti, G., Fuganti, C. 1973, J. Chem. Soc.- Perkin Trans. II 1180.

55. Arrigoni, O., Arrigoni-Liso, R., Calabrese, G. 1975, Nature 256, 513.

56. Evidente, A., Cicala, M. R., Randazzo, G., Riccio, R., Calabrese, G., Liso, R., Arrigoni, O. 1983, Phytochemistry 22, 2193.

57. Arrigoni, O., Calabrese, G., De Gara, L., Bitonti, M. B., Liso, R. 1997, J. Plant Physiol. 150, 302.

58. De Tullio, M. C., De Gara, L., Paciolla, C., Arrigoni, O. 1998, Plant Physiol. Biochem. 36, 433.

59. Davey, M. W., Persiau, G., De Bruyn, A., Van Damme, J., Bauw, G., Van Montagu, M. 1998, Anal. Biochem. 257, 80.

60. De Gara, L., Paciolla, C., Tommasi, F., Liso, R., Arrigoni, O. 1994, J. Plant Physiol. 144, 649.

61. Imai, T., Karita, S., Shiratori, G., Hatturi, M., Nunome, T., Ôba, K. 1998, Plant Cell Physiol. 39, 1350.

62. Kuzniak, E. 2004, Acta Physiol. Plant. 26, 327.

63. Arrigoni, O., Paciolla, C., De Gara, L. 1996, B. Soc. Ital. Biol. Sper. 75, 37.

64. Arrigoni, O., De Gara, L., Paciolla, C., Evidente, A., De Pinto, M. C., Liso, R. 1997, J. Plant Physiol. 150, 362.

65. Arrigoni, O. 1994, J. Bioenerg. Biomembr. 26, 407.

66. Córdoba-Pedregosa, M. C., González-Reyes, J. A., Cañadillas, M. S., Navas, P., Córdoba, F. 1996, Plant Physiol. 112, 1119.

67. Del Giudice, A., Massardo, D. R., Manna, F., Koltovaya, N., Hartings, H., Del Giudice, L., Wolf, K., 1997, Curr. Microbiol. 34, 382.

68. Liso, R., Calabrese, G., Bitonti, M. B., Arrigoni, O. 1984, Exp. Cell Res. 150, 314.

69. Onofri, S., Poerio, E., Serangeli, P., Tosi, S., Garuccio, I., Arrigoni, O. 1997, Antonie Leeuwenhoek 71, 277.

70. Arrigoni, O., Arrigoni-Liso, R., Calabrese, G. 1976, Science 194, 332. 
71. Liso, R., De Gara, L., Tommasi, F., Arrigoni, O. 1985, FEBS Lett. 187, 141.

72. Kukhanova, M., Victorova, L., Krayevsky, A. 1983, FEBS Lett. 160, 129.

73. Del Giudice, A., Massardo, D. R., Manna, F., Evidente, A., Randazzo, G., Wolf, K. 1984, Curr. Genet. 8, 493.

74. Massardo, D. R., Manna, F., Schafer, B., Wolf, K., Del Giudice, L. 1994, Curr. Genet. 25, 80 .

75. Massardo, D. R., Manna, F., Del Giudice, L., Wolf, K. 1990, Curr. Genet. $17,455$.

76. Massardo, D. R., Zweifel, S. G., Gunge, N., Miyakawa, I., Sando, N., Del Giudice, A., Wolf, K., Del Giudice, L. 2000, Can. J. Microbiol. 46, 1058.

77. Onofri, S., Barreca, D., Garuccio, I. 2003, Antonie Leeuwenhoek 83, 57.

78. Ghosal, S., Kumar, Y., Sinhg, S. 1984, Phytochemistry 23, 1167.

79. Ghosal, S., Shanthy, A., Kumar, A., Kumar, Y. 1985, Phytochemistry 24, 2703.

80. Hua, D. H., Saha, S., Takemoto, D. J. 1997, Anticancer Res. 17, 2435.

81. Yui, S., Mikami, M., Kitahara, M., Yamazaki, M. 1998, Immunopharmacology $40,151$.

82. Likhitwitayawuid, K., Angerhofer, C. K., Chai, H., Pezzuto, J. M., Cordell, G. A. 1993, J. Nat. Prod. 56, 1331.

83. Lin, L. Z., Hu, S. F., Chai, H. B., Pengsuparp, T., Pezzuto, J. M., Codell, G. A., Ruangrungsi, N. 1995, Phytochemistry 40, 1295.

84. Weniger, B., Italiano, L., Beck, J. P., Bastida, J., Bergoñon, S., Codina, C., Lobstein, A., Anton, R. 1995, Planta Med. 61, 77.

85. Nair, J. J., Campbell, W. E., Gammon, D. W., Albrecht, C. F., Viladomat, F., Codina, C., Bastida, J. 1998, Phytochemistry 49, 2539.

86. Min, B. S., Gao, J. J., Nakamura, N., Kim, Y. H., Hattori, M. 2001, Chem. Pharm. Bull. 49, 1217.

87. Kushida, N., Atsumi, S., Koyano, T., Umezawa, K. 1997, Drug Exp. Clin. Res. 23, 151.

88. Furusawa, E., Irie, H., Combs, D., Wildman, W. C. 1980, Chemotherapy 26, 36.

89. Jimenez, A., Santos, A., Alonso, G., Vázquez, D. 1976, Biochim. Biophys. Acta, 425,342 .

90. Mikami, M., Kitahara, M., Kitano, M., Ariki, Y., Mimaki, Y., Sashida, Y., Yamazaki, M., Yui, S. 1999, Biol. Pharm. Bull. 22, 674.

91. Yui, S., Mikami, M., Mikami, Y., Sashida, Y., Yamazaki, M. 2001, Yakugaku Zasshi, 121, 167.

92. Abdel-Halim, O. B., Morikawa, T., Ando, S., Matsuda, H., Yoshikawa, M. 2004, J. Nat. Prod. 67, 1119.

93. Liu, J., Hu, W-X., He, L-F., Ye, M., Li, Y. 2004, FEBS Lett. 578, 245.

94. McNulty, J., Nair, J. J., Little, J. R. L., Brennan, J. D., Bastida, J. 2010, Bioorg. Med. Chem. Lett. 20, 5290.

95. Li, Y., Liu, J., Tang, L. J. Shi, Y. W., Ren, W., Hu, W. X. 2007, Oncol. Rep. 17, 377.

96. Griffin, C., Sharda, N., Sood, D., Nair, J. J., McNulty, J., Pandey, S. 2007, Cancer Cell Int. 7, 10.

97. McNulty, J., Nair, J. J., Bastida, J., Pandey, S., Griffin, C. 2009, Phytochemistry $70,913$. 
98. Hohmann, J., Forgo, P., Molnár, J., Wolfard, K., Molnár, A., Thalhammer, T., Máthé, I., Sharples, D. 2002, Planta Med. 68, 454.

99. Schmeda-Hirschmann, G., Astudillo, L., Bastida, J., Viladomat, F., Codina, C. 2000, Bol. Soc. Chil. Quím. 45, 515.

100. Karadeniz, H., Gulmez, B., Sahinci, F., Erdem, A., Kaya, G. I., Unver, N., Kivcak, B., Ozsoz, M. 2003, J. Pharm. Biomed. Anal. 32, 295.

101. Suzuki, N., Tani, S., Furusawa, S., Furusawa, E. 1974, Proc. Soc. Exp. Biol. Med. $145,771$.

102. Pettit, G. R., Gaddamidi, V., Goswami, A., Cragg, G. M. 1984, J. Nat. Prod. 47, 796.

103. Jimenez, A., Sánchez, L., Vázquez, D. 1975, FEBS Lett. 60, 66.

104. Ghosal, S., Singh, S. K., Kumar, Y., Unnikrishnan, S., Chattopadhyay, S. 1988, Planta Med. 54, 114.

105.Zee-Cheng, R. K. Y., Yan, S. J., Cheng,C. C. 1978, J. Med. Chem. 21, 199.

106. Chattopadhyay, U., Chaudhuri, L., Das, S., Kumar, Y., Ghosal, S., 1984, Pharmazie 39, 855.

107. McNulty, J., Nair, J. J., Singh, M., Crankshaw, D. J., Holloway, A. C., Bastida, J. 2010, Nat. Prod. Commun. 5, 1195.

108. McNulty, J., Nair, J. J., Singh, M., Crankshaw, D. J., Holloway, A. C., Bastida, J. 2009, Bioorg. Med. Chem. Lett. 19, 3233.

109. Ieven, M., Van den Bergue, D. A., Vlietinck, A. 1983, Planta Med. 49, 109.

110. Ieven, M., Vlietinck, A., Van den Bergue, D. A., Totte, J., R. Dommisse, R., Esmans, E., Alderweireldt, F., 1982, J. Nat. Prod. 45, 564.

111. Gabrielsen, B., Monath, T. P., Huggins, J. W., Kefauver, D. F., Pettit, G. R., Groszek, G., Hollingshead, M., Kirsi, J. J., Shannon, W. M., Schubert, E. M., Dare, J., Ugarkar, B., Ussery, M. A., Phelan, M. J. 1992, J. Nat. Prod. 55, 1569.

112. Li, S. Y., Chen, C., Zhang, H. Q., Guo, H. Y., Wang, H., Wang, L., Zhang, X., Hua, S. N., Yu, J., Xiao, P. G., Li, R. S., Tan, X. 2005, Antiviral Res. 67, 18.

113. Szlávik, L., Gyuris, A., Minárovits, J., Forgo, P., Molnár, J., Hohmann, J. 2004, Planta Med. 70, 871.

114. Renard-Nozaki, J., Kim, T., Imakura, Y., Kihara, M., Kobayashi, S. 1989, Res. Virol. 140, 115.

115. Hoshino, O. 1998, in "The Alkaloids: Chemistry and Biology" (G. A. Cordell, ed.), Vol 51, p. 323. Academic Press. San Diego. CA.

116. Papas, T. S., Sandhaus, L., Chirigos, M. A., Furusawa, E. 1973, Biochem. Biophys. Res. Commun. 52, 88.

117. Vrijsen, R., Van der Berghe, D. A., Vlietinck, A. J., Boeyé, A. 1986, J. Biol. Chem. 261, 505.

118. Elgorashi, E. E., Stafford, G. I., van Staden, J. 2004, Planta Med. 70, 260.

119. Houghton, P. J., Agbedahunsi, J. M., Adegbulugbe, A. 2004, Phytochemistry $65,2893$.

120. López, S., Bastida, J., Viladomat, F., Codina, C. 2002, Life Sci. 71, 2521.

121. Cordell, G. A. 1981, Introduction to Alkaloids: A biogenetic approach. Wiley, New York. 
122. Ingkaninan, K., Hazekamp, A., de Best, C. M., Irth, H., Tjaden, U. R., van der Heijden, R., van der Greef, J., Verpoorte, R. 2000, J. Nat. Prod. 63, 803.

123. Rhee, I. K., Appels, N., Hofte, B., Karabatak, B., Erkelens, C., Stark, L. M., Flippin, L. A., Verpoorte, R. 2004, Biol. Pharm. Bull. 27, 1804.

124. Tanker, M., Çitoglu, G., Gumusel, B., Sener, B. 1996, Int. J. Pharmacogn. 34, 194.

125. Fennell C. W., van Staden, J. 2001, J. Ethnopharmacol. 78, 15.

126. Cherkasov, O. A., Tolkachev, O. N. 2002, in "Narcissus and daffodil: the genus Narcissus" (G. R. Hanks ed.), Vol. 21 in the series: "Medicinal and aromatic plants - industrial profiles", p. 242. Taylor and Francis, London.

127. Abdalla, S., Abu Zarga, M., Sabri, S. 1993, Fitoterapia, 64, 518.

128. Amara, M. Q., Franetich, J. F., Bouladoux, N., Mazier, D., Eisenbrand, G., Marko, D., Meijer, L., Doerig, C., Desportes-Livage, I. 2001, J. Eukaryot. Microbiol. (Suppl.) 99S (2001).

129. Evidente, A., Andolfi, A., Abou-Donia, A. H., Touema, S. M., Hammoda, H. M., Shawky, E., Motta, A. 2004, Phytochemistry 65, 2113.

130. Toriizuka, Y., Kinoshita, E., Kogure, N., Kitajima, M., Ishiyama, A., Otoguro, K., Yamada, H., Omura, S., Takayama, H. 2008, Bioorg. Med. Chem. 16, 10182.

131. Sener, B., Orham, I., Satayavivad, J. 2003, Phytother. Res. 17, 1220.

132. Campbell, W. E., Nair, J. J., Gammon, D. W., Codina, C., Bastida, J., Viladomat, F., Smith, P. J., Albrecht, C. F. 2000, Phytochemistry 53, 587.

133. Osorio, E. J., Robledo, S. M., Bastida, J. 2008, in “The Alkaloids: Chemistry and Biology" (G. A. Cordell, ed.), Vol 66, p. 113. Elsevier Inc. Amsterdam.

134. Herrera, M. R., Machocho, A. K., Brun, R., Viladomat, F., Codina, C., Bastida, J. 2001, Planta Med. 67, 191.

135. Singh, R. P., Pant, N. C. 1980, Experientia 36, 552.

136. Schultz, A. G., Holoboski, M. A., Smyth, M. S. 1996, J. Am. Chem. Soc. 118,6210 .

137. Çitoglu, G., Tanker, M., Gümüsel, B. 1998, Phytother. Res. 12, 205.

138. Sener, B., Koyuncu, M., Bingöl, F., Muhtar, F. 1997, IUPAC International Conference on Biodiversity and Bioresources: Conservation and Utilization. Phuket, Thailand.

139. Antoun, M. D., Mendoza, N. T., Rios, Y. R. 1993, J. Nat. Prod. 56, 1423.

140. Schmeda-Hirschmann, G., Rodriguez, J. A., Loyola, J. I., Astudillo, L., Bastida, J., Viladomat, F., Codina, C. 2000, Pharm. Pharmacol. Commun. 6, 309.

141. Miyasaka, K., Hiramatsu, Y. 1980, Jpn. J. Pharmacol. 30, 655.

142. Gude, M., Hausen, B. M., Heitsch, H., König, W. A. 1988, Contact Dermatitis 19, 1. 143. Youssef, D. T. A., Frahm, A. W. 1998, Planta Med. 64, 669.

144. Alarcón, M., Cea, G., Weigert, G. 1986, Bull. Environ. Contam. Toxicol. 37, 508.

145. McNulty, J., Nair, J. J., Codina, C., Bastida, J., Pandey, S., Gerasimoff, J., Griffin, C. 2007, Phytochemistry 68, 1068.

146. McNulty, J., Nair, J. J., Bastida, J., Pandey, S., Griffin, C. 2009, Nat. Prod. Commun. 4, 483.

147. Viladomat, F., Bastida, J., Codina, C., Campbell, W. E., Mathee, S. 1995, Phytochemistry 40, 307. 
148. Adesanya, S. A., Olugbade, T. A., Odebiyi, O. O., Aladesanmi, J. A. 1992, Int. J. Pharmacogn. 30, 303.

149. Lewis, J. R. 1998, Nat. Prod. Rep. 15, 107.

150. Martin, S. F. 1987, in "The Alkaloids" (A. Brossi, ed.), Vol. 30, p. 251. Academic Press, New York.

151. Furusawa, E., Furusawa, S., Lee, J. Y. B., Patanavanich, S. 1976, Proc. Soc. Exp. Biol. Med. 152, 186.

152. Kobayashi, S., Kihara, M., Shingu, T., Shingu, K. 1980, Chem. Pharm. Bull. 28, 2924.

153. Rigby, J. H., Cavezza, A., Heeg, M. J. 1998, J. Am. Chem. Soc. 120, 3664.

154. Furusawa, E., Suzuki, N., Furusawa, S., Lee, J. Y. B. 1975, Proc. Soc. Exp. Biol. Med. 149, 771.

155. Furusawa, E., Furusawa, S. 1986, Chemotherapy 32, 521.

156. Furusawa, E., Furusawa, S. 1988, Oncology 45, 180.

157. Furusawa, E., Lockwood, R. H., Furusawa, S., Lum, M. K. M., Lee, J. Y. B. 1979, Chemotherapy 25, 308.

158. Furusawa, E, Lum, M. K. M., Furusawa, S. 1981, Chemotherapy 27, 277.

159. Furusawa, E., Furusawa, S., Sokugawa, L. 1983, Chemotherapy 29, 294.

160. Furusawa, E., Furusawa, S., Lee, J. Y. B., Patanavanich, S. 1978, Chemotherapy 24, 259.

161.Zupkó, I., Réthy, B., Hohmann, J., Molnár, J., Ocsovszki, I., Falkay, G. 2009, In vivo 23, 41.

162. Brown, D. 2002, in "Narcissus and daffodil: the genus Narcissus" (G. R. Hanks ed.), Vol. 21 in the series: "Medicinal and aromatic plants - industrial profiles", p. 332. Taylor and Francis, London.

163. Ceriotti, G. 1967, Nature 213, 595.

164. Baez, A., Vazquez, D. 1978, Biochim. Biophys. Acta 518, 95.

165. Jimenez, A., Sánchez, L., Vázquez, D. 1975, FEBS Lett. 55, 53.

166. Rodríguez-Fonseca, C., Amils, R., Garret, R. A. 1995, J. Mol. Biol. 247, 224.

167. Pettit, G. R., Pettit III, G. R., Backhaus, R. A., Boettner, F. E. 1995, J. Nat. Prod. $58,37$.

168. Evidente, A. 1991, Planta Med. 57, 293.

169. Gonzalez, D., Martinot, T., Hudlicky, T. 1999, Tetrahedron Lett. 40, 3077.

170. Pettit, G. R., Melody, N., Herald, D. L. 2001, J. Org. Chem. 66, 2583.

171. Pettit, G. R., Pettit III, G. R., Groszek, G., Backhaus, R. A., Doubek, D. L., Barr, R. J., Meerow, A. W. 1995, J. Nat. Prod. 58, 756.

172. Pettit, G. R. Pettit III, G. R., Backhaus, R. A., Boyd, M. R., Meerow, A. W. 1993, J. Nat. Prod. 56, 1682.

173. Ingrassia, L., Lefranc, F., Dewelle, J., Pottier, L., Mathieu, V., Spieglkreinecker, S., Sauvage, S., El Yazidi, M., Dehoux, M. Berger, W., Van Quaquebeke, E., Kiss, R. 2009, J. Med. Chem. 52, 1100.

174. Abou-Donia, A. A., De Giulio, A., Evidente, A., Gaber, M., Habib, A. A., Lanzetta, R., Seif El Din, A. A. 1991, Phytochemistry 30, 3445.

175. Kornienko, A., Evidente, A. 2008, Chem. Rev. 108, 1982. 
176. Van Goietsenoven, G., Hutton, J., Becker, J.-P., Lallemand, B., Robert, F., Lefranc, F., Pirker, C., Vandenbussche, G., Van Antwerpen, P., Evidente, A., Berger, W., Prevost, M., Pelletier, J., Kiss, R., Kinzy, T. G., Kornienko, A., Mathieu, V. 2010, FASEB J., 22, 4575.

177. Evidente, A., Kireev, A. S., Jenkins, A. R., Romero, A. E., Steelant, W. F. A., Van Slambrouck, S., Kornienko, A. 2009, Planta Med. 75, 501.

178. McLachlan, A., Kekre, N., McNulty, J., Pandey, S. 2005, Apoptosis 10, 619.

179. Siedlakowski, P., McLachlan-Burgess, A., Griffin, C., Tirumalai, S., McNulty, J., Pandey, S. 2008, Cancer Biol. Ther. 7, 376

180. Shnyder, S. D., Cooper, P. A., Millington, N. J., Gill, J. H., Bibby, M. C. 2008, J. Nat. Prod. 71, 321.

181. Pettit, G. R., Melody, N., Herald, D. L., Schmidt, J. M., Pettit, R. K., Chapuis, J. C. 2002, Heterocycles 56, 139.

182. Ghosal, S., Singh, S., Kumar, Y., Srivastava, S. 1989, Phytochemistry 28, 611.

183. Bi, Y. R., Yung, K. H., Wong, Y. S. 1998, Plant Sci. 135, 103.

184. Evidente, A., Arrigoni, O., Liso, R., Calabrese, G., Randazzo, G. 1986, Phytochemistry 25, 2739.

185. Van Doorn, W. G., Sinz, A., Tomassen, M. M. 2004, Phytochemistry 65, 571.

186. Bi, Y., Guo, J., Zhang, L., Wong, Y. 2003, J. Plant Physiol. 160, 1041.

187. Bi, Y., Zhang, L., Guo, J. K., Yung, K., Wong, Y. 2003, New Zeal. J. Crop Hort. Sci. 31, 335.

188. Labraña, J., Machocho, A. K., Kricsfalusy, V., Brun, R., Codina, C., Viladomat, F., Bastida, J. 2002, Phytochemistry 60, 847.

189. Pagliosa, L. B., Monteiro, S. C., Silva, K. B., De Andrade, J. P., Dutilh, J., Bastida, J., Cammarota, M., Zuanazzi, J. A. S. 2010, Phytomedicine 17, 698.

190. Fulton, B., Benfield, P. 1996, Drug Aging 9, 60.

191. Wilcock, G., Wilkinson, D. 1997, in "Alzheimer's Disease: Biology, Diagnosis and Therapeutics" (K. Iqbal, B. Winblad, T. Nishimura, M. Takeda, and H. M. Wisniewski, eds.), p. 661. John Wiley \& Sons, West Sussex.

192. Sramek, J. J., Frackiewicz, E. J., Cutler, N. R. 2000, Expert Opin. Inv. Drug 9, 2393.

193. Mucke, H. A. M. 1997, Drugs Today 33, 251.

194. Mucke, H. A. M. 1997, Drugs Today 33, 259.

195. Greenblatt, H. M., Kriger, G., Lewis, T., Silman, I., Sussman, J. L. 1999, FEBS Lett. 463, 321.

196. Greenblatt, H. M., Guillou, C., Guenard, D., Argaman, A., Botti, S., Badet, B., Thal, C., Silman, I., Sussman, J. L. 2004, J. Am. Chem. Soc. 126, 15405.

197. Guillou, C., Mary, A., Renko, D. Z., Gras, E., Thal, C. 2000, Bioorg. Med. Chem. Lett. 10, 637.

198. Scott, L. J., Goa, K. L. 2000, Drugs 60, 1095.

199. Arias, E., Ales, E., Gabilan, N. H., Cano-Abad, M. F., Villarroya, M., Garcia, A. G., López, M. G. 2004, Neuropharmacology 46, 103.

200. Kihara, T., Sawada, H., Nakamizo, T., Kanki, R., Yamashita, H., Maelicke, A., Shimohama, S. 2004, Biochem. Biophys. Res. Commun. 325, 976.

201. Radicheva, N., Mileva, K., Stoyanova, N., Georgieva, B. 1999, Method. Find. Exp. Clin. Pharmacol. 21, 5. 
202. Bastida, J., Llabrés, J.M., Viladomat, F., Codina, C., Rubiralta, M., Feliz, M. 1987, J. Nat. Prod. 50, 199.

203. Moraes-Cerdeira, R. M. 2002, in "Narcissus and daffodil: the genus Narcissus" (G.R. Hanks ed.), Vol. 21 in the series: "Medicinal and aromatic plants industrial profiles", p. 273. Taylor and Francis, London.

204. Blacker, C. V. R., Greenwood, D. T., Wesnes, K. A., Wilson, R., Woosward, C., Howe, I., Ali, T. 2004, J. Am. Med. Assoc. 292, 1195.

205. Rainer, M. 1997, Drugs Today 33, 273.

206. Ezio, G. 1998, Neurochem. Int. 32, 413.

207. Nordberg, A., Svensson, A. L. 1998, Drug Saf. 19, 465.

208. Kewitz, H. 1997, Drugs Today 33, 265.

209. Rainer, M. 1997, CNS Drugs 7, 89.

210. Raskind, M. A., Peskind, E. R., Wessel, T., Yuan, W., and the Galantamine USA-1 Study Group, 2000, Neurology 54, 2261.

211.Zhao, Q., Brett, M., Van Osselaer, N., Huang, F., Raoult, A., Van Peer, A., Verhaeghe, T., Hust, R. 2002, J. Clin. Pharmacol. 42, 1002.

212. Patterson, C. E., Passmore, A. P., Crawford, V. L. S. 2004, Int. J. Clin. Pract. $58,144$.

213. Heinrich, M., Teoh, H. L. 2004, J. Ethnopharmacol. 92, 147.

214. Jones, R. W., Soininen, H., Hager, K., Aarsland, D., Passmore, P., Murthy, A., Zhang, R., Bahra, R. 2004, Int. J. Geriatr. Psych. 19, 58.

215. Mannens, G. S. J., Snel, C. A W., Hendrickx, J., Verhaeghe, T., Le Jeune, L., Bode, W., Van Beijsterveldt, L., Lavrijsen, K., Leempoels, J., Van Osselaer, N., Van Peer, A., Meuldermans, W. 2002, Drug Metab. Dispos. 30, 553.

216. Bores, G. M., Kosley, R. W. 1996, Drug Future 21, 621.

217. Radicheva, N., Vydevska, M., Mileva, K. 1996, Method. Find. Exp. Clin. Pharmacol. 18: 301.

218. Allain, H., Bentué-Ferrer, D., Belliard, S., Derouesné, C. 1997, in "Progress in Medicinal Chemistry" (G. P. Ellis and D. K. Luscombe, eds.), Vol. 34, p. 1. Elsevier, Amsterdam.

219. Czollner, L., Frantsits, W., Kuenburg, B., Hedenig, U., Frohlich, J., Jordis, U. 1998, Tetrahedron Lett. 39, 2087.

220. Bullock, R. 2004, Expert Rev. Neurother. 4, 153.

221. Dengiz, A. N., Kershaw, P. 2004, CNS Spectr. 9, 377.

222. Marco-Contelles, J., Perez-Mayoral, E., Van Nhien, A. N., Postel, D. 2007, Targets Heterocycl. Syst. 11, 365.

223. Heinrich, M. 2010, in “The Alkaloids: Chemistry and Biology” (G. A. Cordell, ed.), Vol 68, p. 157. Elsevier Inc. Amsterdam.

224. Bachus, R., Bickel, U., Thomsen, T., Roots, I., Kewitz, H. 1999, Pharmacogenetics 9, 661. 


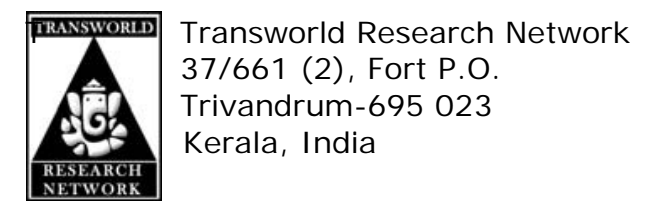

Recent Advances in Pharmaceutical Sciences, 2011: 101-120 ISBN: 978-81-7895-528-5 Editor: Diego Muñoz-Torrero

\title{
4. Resveratrol: A polyphenol with multiple effects
}

\author{
Joana M. Planas', Helena Colom² and M. Emilia Juan' \\ 'Departament de Fisiologia and Institut de Recerca en Nutrició i Seguretat Alimentària (INSA-UB) \\ Facultat de Farmàcia, Universitat de Barcelona (UB), Barcelona, Spain; ${ }^{2}$ Departament de \\ Farmàcia i Tecnologia Farmacèutica, Facultat de Farmàcia, Universitat de Barcelona (UB) \\ Barcelona, Spain
}

\begin{abstract}
Resveratrol (trans-3,5,4'-trihydroxystilbene) is a natural polyphenol that occurs in grapes, berries, peanuts, and several traditional medicines. A number of studies have demonstrated that this polyphenol holds promise against numerous age-associated diseases including cancer, diabetes, Alzheimer, cardiovascular and pulmonary diseases. In view of these studies, resveratrol's prospects for use in the clinics are rapidly accelerating. This review summarizes our work on the mechanisms involved in the intestinal absorption and its population pharmacokinetics. Finally, various targets of resveratrol and its therapeutic potential are described.
\end{abstract}

\section{Introduction}

trans-Resveratrol (3,5,4'-trihydroxystilbene) is a polyphenolic compound naturally occurring in plants and found in dietary products. Along the past

Correspondence/Reprint request: Prof. Joana M. Planas, Departament de Fisiologia, Facultat de Farmàcia Universitat de Barcelona, Av. Joan XXIII s/num, 08028 Barcelona, Spain. E-mail: jmplanas@ub.edu 
decade our research was devoted to study in depth the mechanism involved in its intestinal absorption as the first step to explain the bioavailability of this compound. The present review also discusses the processes implicated on its distribution and elimination as well as its beneficial effects in colon cancer and spermatogenesis.

\section{Chemistry of trans-resveratrol}

trans-Resveratrol (3,5,4'-trihydroxistilbene, Fig. 1) is a polyphenol that belongs to the stilbene family which is characterized by an essential structural skeleton of two aromatic rings joined by an ethylene bridge.

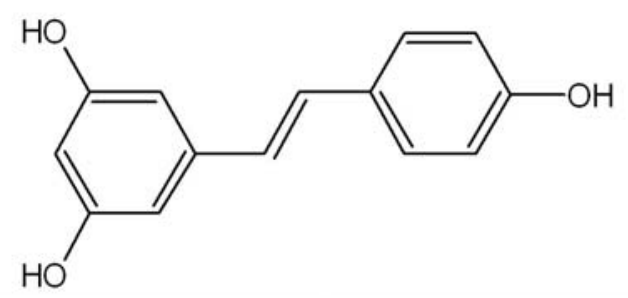

Figure 1. Chemical structure of trans-resveratrol.

trans-Resveratrol is formed via a condensation reaction between three molecules of malonyl-CoA and one molecule of 4-coumaroyl CoA catalyzed by trans-resveratrol synthase. The synthesis of trans-resveratrol in plants is triggered in response to exogenous stress factors, such as injury, ultraviolet radiation and fungal infection. For this reason, this secondary metabolite of plants is defined as a phytoalexin [1]. The concentration of trans-resveratrol peaks approximately 42-72 $\mathrm{h}$ after stress exposure, and declines after $42-72 \mathrm{~h}$ as a result of activation of stilbene oxidase [1].

This polyphenol exists in both cis- and trans-isomeric forms, the transisomer being the most commonly found and extensively studied. In plants, trans-resveratrol is also found as 3-O- $\beta$-D-glucosides, a compound also known as piceid or polydatin, which is more stable and less susceptible to oxidative degradation than the parent compound. Other analogs of trans-resveratrol such as pterostilbene (3,5-trimethoxy-4'-hydroxystibene) or piceatannol $\left(3,4,3\right.$ ', $5^{\prime}$ trans-tetrahydroxystilbene) have also been reported in plants [1].

\section{Sources of trans-resveratrol}

trans-Resveratrol was first isolated in 1940 as a component of the roots of the white hellebore (Veratrum grandiflorum). The term resveratrol comes 
from this plant, being "res" a latin prefix that means "which comes from" and "ol" a suffix indicating the presence of an alcohol moiety in its structure. In 1963, it was identified as an active constituent of the dried roots of Polygonum cuspidatum $(0.524 \mathrm{mg} / \mathrm{g})$, also called kojo-kon and used in traditional Asian medicine against suppurative dermatitis, gonorrhea and hyperlipemia [2]. In Vitis vinifera it was detected in 1976 where this polyphenol is exclusively synthesized in leaf epidermis and in grape skins, but not in the flesh [3], and in wine in 1992 [4]. Afterward this polyphenol has been described in more than 70 plant species [5].

Table 1. Content of trans-resveratrol in dietary sources.

\begin{tabular}{lc}
\hline Source & $\begin{array}{l}\text { trans-Resveratrol } \\
\text { concentration }\end{array}$ \\
\hline Red wine & $0.1-14.3 \mathrm{mg} / \mathrm{L}$ \\
White wines & $0.1-2.1 \mathrm{mg} / \mathrm{L}$ \\
Grapes & $50-100 \mu \mathrm{g} / \mathrm{g}$ \\
Peanuts & $0.02-1.92 \mu \mathrm{g} / \mathrm{g}$ \\
Pistachios & $0.09-1.67 \mu \mathrm{g} / \mathrm{g}$ \\
Blueberries & $\sim 32 \mathrm{ng} / \mathrm{g}$ \\
Bilberries & $\sim 16 \mathrm{ng} / \mathrm{g}$ \\
\hline
\end{tabular}

In grapes the concentrations of trans-resveratrol ranged from 50 to $100 \mu \mathrm{g} / \mathrm{g}$ fresh weight. During mashing, in the winemaking process, part of transresveratrol present in the skin is dissolved in the must. Thus, the concentrations of this polyphenol are higher in red wines since the contact between berry skin and must is more prolonged, than in white wines, where the must is immediately separated from the grape residues. Content of trans-resveratrol in red wine ranges from non-detectable concentrations to $14.3 \mathrm{mg} / \mathrm{L}(62.7 \mu \mathrm{M})$. The average concentrations of trans-piceid in a red wine could be as much as $29.2 \mathrm{mg} / \mathrm{L}$ $(128.1 \mu \mathrm{M})$, i.e., three times that of trans-resveratrol [6].

In addition to grapes and wine (Table 1), trans-resveratrol is found in several edible natural products such as peanuts (Arachis hypogaea), berries (blueberries, cranberries, blueberries, bilberries, from the Vaccinium family), rhubarb (Rheum rhabarbarum), pistachio (Pistacia vera) and hop (Humulus lupulus) $[1,5]$.

The occurrence of trans-resveratrol has also been documented in a number of trees, such as eucalyptus and spruce, as well as the tropical deciduous tree Bauhinia racemosa. It has also been found in a few flowering plants [1]. 


\section{Intestinal absorption of trans-resveratrol}

The processes that take place in the intestine during the absorption of trans-resveratrol have been studied in vivo in rats using a perfusion method [7]. A nutritionally relevant concentration of $25 \mu \mathrm{mol} / \mathrm{L}$ of trans-resveratrol was used to evaluate the absorption across the jejunum as a function of time. Nearly $72 \%$ of the luminally perfused polyphenol disappeared from the buffer after $30 \mathrm{~min}$, indicating that there was an efficient uptake of this polyphenol. At the same time, the result showed that trans-resveratrol was efficiently conjugated inside the enterocyte with glucuronic acid by UDPglucuronosyltransferase or with sulfate by sulfotransferase, and $42 \%$ and $12 \%$, respectively, were subsequently pumped back to the luminal side.

The kinetic study of trans-resveratrol was performed in jejunal and ileal loops perfused with increasing concentrations of this compound. The transport rates of the unconjugated polyphenol were directly proportional to the initially applied trans-resveratrol, indicating that the uptake occurs by simple diffusion, without the participation of a mediated transport, as indicated with perfusion using 2,4-dinitrophenol. The apparent diffusion constant (Kd) normalized to segment dry weight was $8.1 \pm 0.3 \mu \mathrm{L} / 5 \mathrm{~min} \cdot \mathrm{mg}$ dry weight in the jejunum and $10.7 \pm 0.2 \mu \mathrm{L} / 5 \mathrm{~min} \cdot \mathrm{mg}$ dry weight in the ileum [7]. These results are in accordance with findings for Caco-2 cells, where this compound crosses the cells by passive diffusion [8].

The efflux of the intracellulary formed trans-resveratrol glucuronide and sulfate towards the intestinal lumen was higher in the jejunum than in the ileum, indicating a region-dependent metabolism of trans-resveratrol in the small intestine according to the regional distribution of UDPglucuronosyltransferase and sulfotransferase in the intestine [9]. In both segments, the kinetic analysis of trans-resveratrol transformation indicated that in the enterocyte, glucuronidation was favored over sulfation, with similar rates of reaction. Glucuronidation seems to be the predominant conjugation pathway in the rat, and trans-resveratrol glucuronide was excreted form the enterocyte to the intestinal lumen with an average concentration 4.5 times higher than that of the sulfate [7].

The secretion of the conjugates out of the enterocyte was thought to be mediated through the ATP-binding cassette $(\mathrm{ABC})$ transporters which are efflux proteins that act as "gatekeepers" in the intestine thus controlling the oral availability of many substances [10]. This ABC superfamily contains membrane proteins that translocate a wide variety of substrates across extraand intracellular membranes, including metabolic products, lipids, sterols, and drugs. Of the numerous members of the $\mathrm{ABC}$ transporter family, three have been described in the apical membrane of the enterocyte: the P-glycoprotein 
(Pgp; $\mathrm{ABCB} 1$ ), the multidrug resistance-associated protein (MRP2; $\mathrm{ABCC} 2$ ), and the breast cancer resistance protein (BCRP; ABCG2) [10,11]. Pgp recognizes a wide range of structurally and pharmacologically unrelated neutral and positively charged hydrophobic compounds [10]. MRP2 has a relatively hydrophobic substrate spectrum, including glucuronide, glutathione and sulfate conjugates of endogenous and exogenous [12]. BCRP is the most recently discovered of these efflux transporters and recognizes mainly hydrophilic anticancer agents as well as negatively charged drug conjugates[13].

The role of intestinal $\mathrm{ABC}$ transporters in the absorption of transresveratrol was investigated with the use of specific inhibitors (Fig. 2). Consequently, P-glycoprotein was evaluated by using verapamil and cyclosporine [10]. These inhibitors ruled out the possibility that either transresveratrol or its conjugates constituted a substrate of this protein. Intestinal perfusions of trans-resveratrol with the MRP2 inhibitors probenecid [14] and MK571 [15] did not exhibit any effect on trans-resveratrol. However, secretion of glucuronide and sulfate conjugates decreased, thus implicating MRP2 in their efflux. These results are in agreement with findings for isolated perfused livers, which have indicated that MRP2 exclusively mediates the biliary excretion of resveratrol glucuronides and only partly mediates that of sulfates [16]. The role of BCRP was analyzed in the presence of Ko143, a high affinity and specific BCRP inhibitor [17]. Perfusions with Ko143 significantly decreased the secretion of trans-resveratrol glucuronide and sulfate conjugates without affecting the absorption of the parent compound. The role of BCRP was confirmed with the use of $\mathrm{BCRP} 1^{-/}$mice that were orally administered with $60 \mathrm{mg} / \mathrm{kg}$ of trans-resveratrol [18]. trans-Resveratrol and its metabolites were measured in intestinal content at $30 \mathrm{~min}$ after administration and showed a decrease of $71 \%$ and $97 \%$ of resveratrol glucuronide and sulfate respectively, compared to the wild-type mice, thus indicating a lower efflux from the enterocytes [18].

trans-Resveratrol conversion to a mono-glucuronide and a mono-sulfate was the only observed transformation in rat intestine under our experimental conditions with no cytochrome P450-mediated phase I metabolic transformation or methylation [7]. The latter possibility did not take place due to the lack of catechol function in the trans-resveratrol molecule, which is a requirement for catechol-O-methyltransferase (COMT). In addition to the conjugates, another metabolite of trans-resveratrol has been described in the intestine [19]. This polyphenol is quantitatively transformed into dihydroresveratrol, which is a metabolite that may be produced by the intestinal microflora since its formation involves the hydrogenation of the aliphatic double bond of the parent compound. Dihydroresveratrol was found 


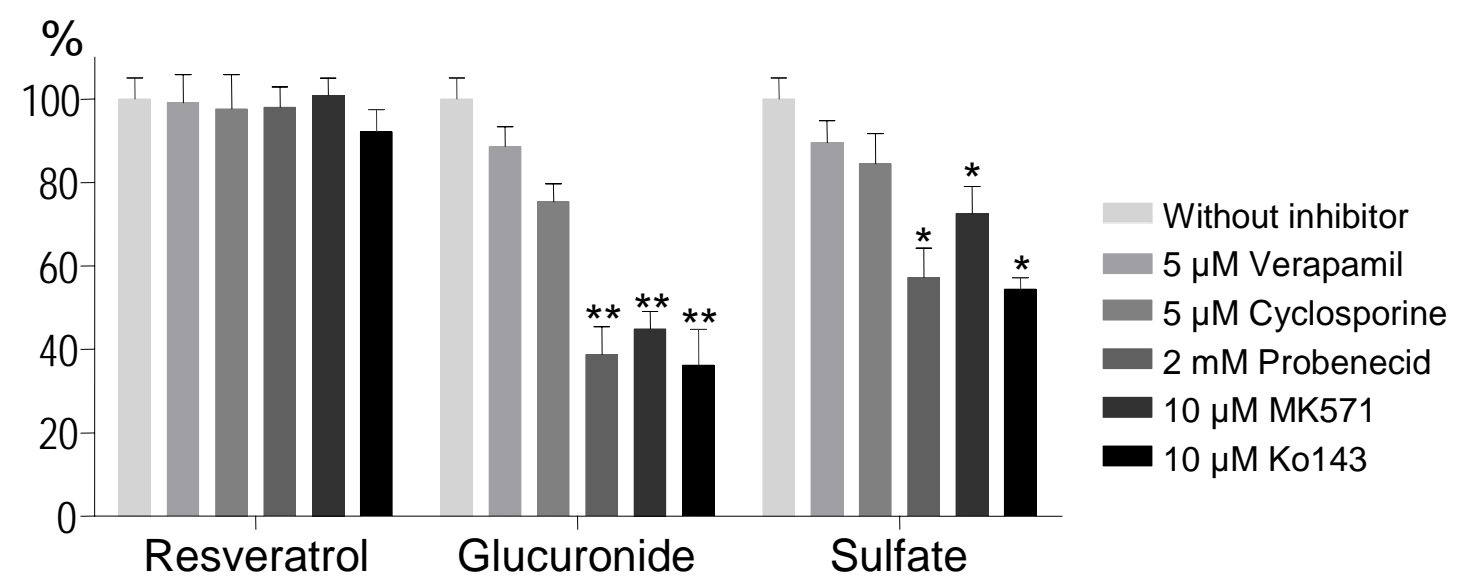

Figure 2. Effects of inhibitors of Pgp (verapamil, cyclosporine), MRP2 (probenecid, MK571) and BCRP (Ko143) on the absorption of trans-resveratrol and the secretion of intracellulary formed glucuronide and sulfate conjugates. Results are expressed as percentage of trans-resveratrol absorbed or glucuronide and sulfate secreted in the absence $(100 \%)$ or presence of the inhibitor under study. Values are expressed as means + SE $(n=4-6) . * P<0.05$, significantly different from perfusion with transresveratrol alone; ${ }^{* *} P<0.001$.

to be the most abundant metabolite in the colon after the oral administration of $60 \mathrm{mg} / \mathrm{kg}$ of trans-resveratrol for 49 days, and 24 hours after the last administration, with concentrations of $303.0 \pm 34.7 \mathrm{nmol} / \mathrm{g}$, 446-fold that of the parent compound [19].

\section{Population pharmacokinetics of trans-resveratrol}

The bioavailability and metabolism of trans-resveratrol have been widely studied in rats and humans given that its efficacy depends on its absorption and metabolism [20]. However, it is difficult to provide an adequate pharmacokinetic description of the intricate processes that determine the bioavailability of this polyphenol. A preliminary evaluation of the plasmatic pharmacokinetics of trans-resveratrol was carried out after the oral administration of $2 \mathrm{mg} / \mathrm{kg}$ to overnight fasted rats [21]. Blood samples were extracted at different time points over an hour, and showed low plasmatic concentrations of unchanged trans-resveratrol with peak concentration of 550 $\mathrm{ng} / \mathrm{mL}$ at 10 minutes [21]. The low bioavailability for trans-resveratrol indicates that the small intestine comes out as the first bottleneck to the entry of this compound to the organism [18,21]. In addition, the metabolism in the liver cannot be underestimated $[22,23]$ before the distribution to tissues where $\mathrm{ABC}$ proteins are also present, given that all these processes influence the 
distribution and subsequent elimination from the organism [18]. As a result of this complex interplay between enzyme activities and efflux transporters, the concentrations of trans-resveratrol in plasma have been reported to be low $[20,21]$.

Given the important metabolism observed for trans-resveratrol, an integrated pharmacokinetic model that could describe the parameters of both the parent compound and its conjugated metabolites was investigated after i.v. and p.o. administration of 2, 10 and $20 \mathrm{mg} / \mathrm{kg}$ of trans-resveratrol in Sprague-Dawley rats [24]. Analyses of the plasmatic data through the population pharmacokinetic approach provided important advantages such as overcoming the limitations of blood sampling in studies using experimental animals, and at the same time preserving animal individuality. In addition, the population approach allows the estimation of the typical pharmacokinetic parameters as well as the inter-animal variability. To this end, all the data (from parent compound and conjugates) obtained after the i.v. administrations of three doses of trans-resveratrol were simultaneously analyzed. Once the best intravenous model was found, the disposition parameters were fixed and the oral data of the three compounds were added to estimate the absorption parameters (absorption rate constants and bioavailability).

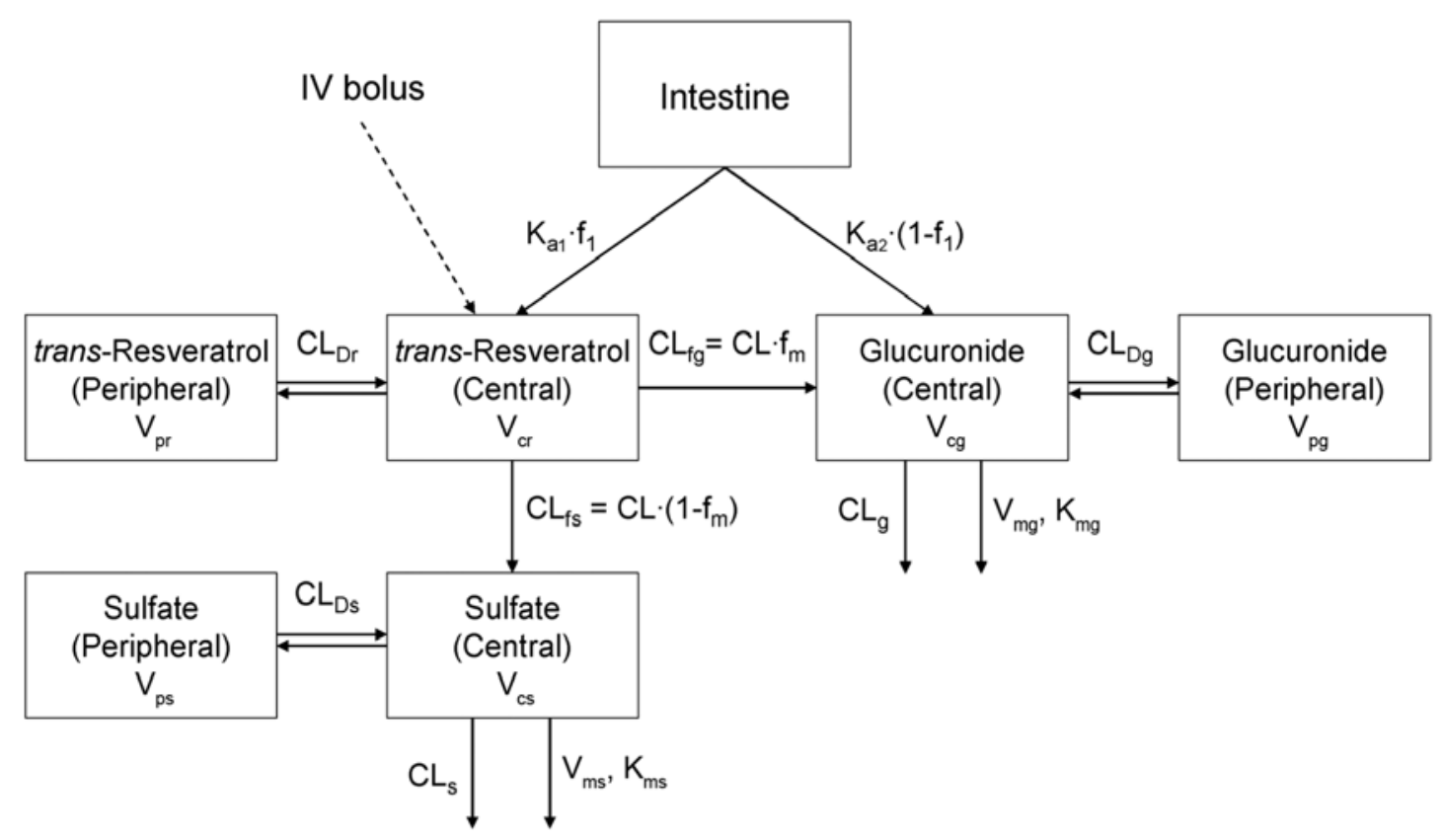

Figure 3. Schematic representation of the pharmacokinetic model to simultaneously describe the data of trans-resveratrol and its glucuronide and sulfate conjugates after i.v. and p.o. administration. 
The pharmacokinetics of trans-resveratrol and its conjugates that best described our results was a three-linked two compartment model (Fig. 3). Elimination of trans-resveratrol by conversion to its glucuronide and sulfate took place by a first-order kinetic process. It is noteworthy that the transformation of the parent compound to its conjugates was not saturable even at the plasmatic concentrations achieved after the highest dose assayed (20 $\left.\mathrm{mg} \cdot \mathrm{kg}^{-1}\right)$. Meanwhile, clearance of trans-resveratrol glucuronide and sulfate was best described by parallel first-order and Michaelis-Menten kinetics. These results are in agreement with existing knowledge about elimination mechanisms of glucuronide and sulfate in tubular cells and hepatocytes $[18,25]$. The total clearance value of trans-resveratrol was slightly higher than $3 / 4$ times the hepatic blood flow in the rat $(0.90 \mathrm{~L} / \mathrm{h}$ for a body weight of $0.25 \mathrm{~kg}$ ). According to the $\mathrm{f}_{\mathrm{m}}$ value obtained (0.540) approximately the same percentage of both metabolites was formed. Clearances of formation of the glucuronide and sulfate were 0.67 and $0.57 \mathrm{~L} \cdot \mathrm{h}^{-1}$, respectively. Distribution volume of trans-resveratrol (total distribution volume $=3.05 \mathrm{~L}$ ) exceeded the total body water in the rat $\left(0.15 \mathrm{~L}^{\mathrm{kg}} \mathrm{kg}^{-1}\right.$ for a body weight of $\left.0.25 \mathrm{~kg}\right)$ suggesting extensive distribution into tissues. A short half-life value was estimated for trans-resveratrol $(0.55 \mathrm{~h})$ that was in agreement with its clearance and distribution volumes values. By contrast smaller distribution volumes were found for the metabolites (total distribution volumes $=0.38$ and $0.14 \mathrm{~L}$ for the glucuronide and sulfate, respectively).

In order to model the oral data, a depot compartment (intestinal compartment) was added to the intravenous model where trans-resveratrol was administered. When going into the gastrointestinal tract, trans-resveratrol was supposed to be subject to first-pass metabolism and to reach the systemic circulation intact or as its glucuronide according to a simple diffusion process. The rate limiting step is most likely the absorption process of trans-resveratrol and its glucuronide rather than the metabolism. The first-order absorption rate constants $\left(\mathrm{K}_{\mathrm{a} 1}=0.442 \mathrm{~h}^{-1}\right.$, absorption half life $=1.57 \mathrm{~h}, \mathrm{~K}_{\mathrm{a} 2}=0.256 \mathrm{~h}^{-1}$, absorption/metabolism half life $=2.71 \mathrm{~h}$ ) confirmed rapid absorption/metabolism kinetics for both compounds. The data did not support the inclusion of first-pass metabolism of trans-resveratrol to sulfate, so the model was simplified to absorption/presystemic metabolism of transresveratrol to its glucuronide and absorption of the formed glucuronide. The inclusion of a fraction of the parent compound never absorbed, due to metabolism to the conjugates and efflux of them back into intestine, did not improve the fit. One of the reasons why trans-resveratrol sulfate could not be included in the presystemic metabolism of trans-resveratrol might be the low concentrations of this metabolite that reached the bloodstream. These low concentrations could be attributed to a lower sulfation compared to the 
glucuronidation of trans-resveratrol in the rat intestine [26,27]. Moreover, the higher affinity and capacity of BCRP for trans-resveratrol sulfate compared to the one observed for the glucuronide could account for a higher efficiency in the secretion of the sulfate [25]. Andlauer et al. [26] appointed that only $0.3 \%$ of the absorbed resveratrol reaches the blood as sulfate. Altogether, these processes could explain why the model did not support the inclusion of presystemic metabolism of trans-resveratrol to its sulfate conjugate.

The proportions of absorbed intact trans-resveratrol and its glucuronide were estimated as $f_{1}$ and $\left(1-f_{1}\right)$, respectively. The results showed that when the dose administered increased a lower fraction of trans-resveratrol remained unchanged $\left(f_{1}=0.420,0.207\right.$ and 0.060 for the doses of 2,10 and $20 \mathrm{mg} \cdot \mathrm{kg}^{-1}$, respectively, and a body weight of $0.25 \mathrm{~kg}$ ). By contrast, the relative fraction of glucuronide formed/absorbed from the intestine increased with the dose with values of $0.580,0.793$ and 0.94 for the doses of 2,10 and $20 \mathrm{mg} \cdot \mathrm{kg}^{-1}$ and a body weight of $0.25 \mathrm{~kg}$, respectively. This suggested a non linear pharmacokinetic behaviour of trans-resveratrol, after p.o. administration from 2 to $20 \mathrm{mg} \cdot \mathrm{kg}^{-1}$.

It is known that trans-resveratrol and its conjugates accumulate significantly in the liver $[18,28]$ and are further excreted into the bile, leading to enterohepatic recirculation [16,29]. The contribution of the biliar pathways to the elimination of the conjugated metabolites could not be captured by the present model although a light rebound was observed after $6 \mathrm{~h}$ of the i.v. administration, supporting the occurrence of the enterohepatic cycle. Previously, Marier et al. [29] have reported that the enterohepatic recirculation using a linked-rat model induced significant increase of plasma concentrations of resveratrol and its glucuronide in bile-recipient rats at 4 to $8 \mathrm{~h}$. The population pharmacokinetic model built adequately described the plasmatic concentrations of trans-resveratrol and its major metabolites in the rat after i.v. and p.o. administrations, as it was verified with internal validation procedures [24]. Its predictive capacity was also evaluated using an external data set obtained after the i.v. administration of $15 \mathrm{mg} \cdot \mathrm{kg}^{-1}$. The results showed a close agreement between the observed data and the predicted concentrations for the dose and administration route assayed, supporting the hypothesis of the robustness of the model. In summary, the population PK model built allowed to increase the knowledge to better understand the PK of this polyphenol but also may be applicable to many other compounds which have similar PK properties. In addition the model may also be useful for planning future PK-PD studies to establish the relative contribution of the conjugates to the overall efficacy.

In humans, Walle et al. showed that the majority of a dose of $25 \mathrm{mg}$ administered p.o and i.v. was converted to trans-resveratrol sulfate within $30 \mathrm{~min}$, and the serum peak concentrations of the parent compound were inferior to 
$22 \mathrm{nM} \mathrm{[30].} \mathrm{Five} \mathrm{metabolites} \mathrm{were} \mathrm{identified} \mathrm{in} \mathrm{urine,} \mathrm{trans-resveratrol}$ monosulphate, two isomeric monoglucuronides, dihydroresveratrol monosulphate and dihydroresveratrol monoglucuronide. The total sulfate and glucuronide conjugates accounted for approximately 37 and 19\%, respectively of the metabolites in urine, and there were only trace amounts of free transresveratrol [30]. A study of the pharmacokinetics of oral trans-resveratrol was performed after the administration of a single dose of $0.5,1,2.5$ or $5 \mathrm{~g}$ in healthy volunteers [31]. An extensive metabolism after the oral administration was also reported since six metabolites were recovered from plasma and urine. Peak plasma concentration of trans-resveratrol was $2.4 \mu \mathrm{M}$ at 1.5 hours after the oral administration of $5 \mathrm{~g}$. Peak levels of two monoglucuronides and resveratrol 3-sulphate were 3- to 8-fold higher. The area under the plasma concentration curve values for trans-resveratrol-3-sulphate and trans-resveratrol monoglucuronides were 23 times greater than those of trans-resveratrol. Urinary excretion of trans-resveratrol and its metabolites was rapid, with $77 \%$ of all urinary agent derived species excreted within $4 \mathrm{~h}$ after the lower dose $(0.5 \mathrm{~g})$.

\section{Toxicology}

Several studies have evaluated the toxicity in mice and rats in short-term and subchronic administration of trans-resveratrol [32-34]. The repeated dosage of $20 \mathrm{mg} / \mathrm{kg}$ of trans-resveratrol during 28 days, which corresponds to 1000 times the amount that may be consumed by a person drinking one glass of red wine a day, did not induce any adverse effect [32]. Body weight, food and water consumption were not altered in rats exposed to trans-resveratrol, when compared to the control group. Hematologic and biochemical variables were not affected by the treatment. An increased relative brain weight and testicular weight normalized to body weight was observed in the group administered with trans-resveratrol whereas no histopatological changes in these organs were detected. In addition, transresveratrol did not alter any vital organ weight or induce histopathologic changes [32].

The effect of higher doses was evaluated by the Crowell et al. [33]. Male and female Sprague-Dawley rats were administered by gavage 300, 1000 and $3000 \mathrm{mg} / \mathrm{kg}$ of trans-resveratrol for 4 weeks. The results showed that no adverse effects were observed in male and female rats exposed to $300 \mathrm{mg} / \mathrm{kg}$. Minor effects were reported for the dose of $1000 \mathrm{mg} / \mathrm{kg}$, such as reduced body weight in females and elevated leukocyte counts in males. The most remarkable adverse events took place at the highest dose of $3000 \mathrm{mg} / \mathrm{kg}$, when a reduced body weight and food consumption, elevated kidney weight, 
and increased incidence of kidney lesions were observed in male and female rats.

Those results were supported by the studies in which trans-resveratrol was incorporated in the diet at 50, 150 and $500 \mathrm{mg} / \mathrm{kg}$ during 28 days [34]. The dietary exposure did not induce any treatment-related effects on body weight clinical signs, hematological, clinical chemistry, histopatology or urinary parameters at any dose.

The reproductive toxicity of trans-resveratrol has been comprehensively evaluated. The administration of trans-resveratrol in the diet at $750 \mathrm{mg} / \mathrm{kg}$ during 90 days did not negatively affect sperm parameters or estrous cycle in the rat or any histopathological effect on reproductive organs [34]. transResveratrol has been administered at $0,120,300$ and $750 \mathrm{mg} / \mathrm{kg}$ to pregnant rats, showing no adverse effects on the number of implantations, resorptions, live young, or pre- and post-implantation losses. In addition no negative findings were observed in the placental, litter and fetal weight; or incidence of major or minor fetal abnormalities at any of the doses tested [34].

In addition to the studies performed in rats, the toxicity has also been evaluated in p53 knockout mice, which is a model accepted for oncogenicity bioassays [35]. The animals were administered trans-resveratrol by gavage at 1000,2000 or $4000 \mathrm{mg} / \mathrm{kg}$ during six months. No effects were observed on body weight, food consumption, or clinical signs in any dose group. However, a dose-related increase in liver weight and serum cholesterol concentrations were reported in both sexes. Histopathology identified the kidney (hydronephrosis) and urinary bladder (epithelial hyperplasia) as target tissues for resveratrol toxicity. The incidences of both benign and malignant tumors in mice exposed to trans-resveratrol were comparable to those in vehicle controls. When administered to p53 knockout mice at its maximum tolerated dose, trans-resveratrol demonstrates no evidence of oncogenicity [35]. This study performed in p53 knockout mice address a key regulatory requirement for the entry of resveratrol into clinical trials for cancer prevention.

A phase I study of the oral administration of single doses of transresveratrol of $0.5,1,2.5$ or $5 \mathrm{~g}$ conducted in 10 healthy volunteers per dose levels did not cause serious adverse events [31]. A safety profile of transresveratrol was carried out in healthy volunteers that received 25, 50, 100 or $150 \mathrm{mg}$, six times/day, for two days. This polyphenol was well tolerated and only mild adverse events were reported [36].

Taken as a whole, the absence of symptoms and the normal appearance of the vital organs in rats and mice suggested that trans-resveratrol is barely toxic even under the conditions that have been described. 


\section{Health benefits of trans-resveratrol}

\subsection{Colon cancer: In vivo and in vitro}

Colorectal cancer is one of the leading causes of death in both men and women in Western countries, being usually lethal when diagnosed at later stages of progression [37]. Genetic predisposition as well as environmental aspects are thought to be involved in colon carcinogenesis, among which, dietary habits play a pivotal role. Mediterranean countries have lower rates of colorectal cancer compared with other Western countries [38]. It has been suggested that the environmental factors are mainly dietary and up to $80 \%$ of sporadic colorectal cancers are therefore potentially preventable [39].

trans-Resveratrol is gaining acceptance as a potential antitumor agent because of its multiple effects described in different experimental models of carcinogenesis $[40,41]$. This bioactive compound was shown to inhibit the growth of tumor cell lines derived from various human cancers, an effect that was associated with its ability to arrest cell cycle progression and to induce programmed cell death [40,41]. These antitumoral activities accompanied with the lack of harmful effects [32,34] makes transresveratrol an attractive chemotherapy and chemopreventive drug for cancer treatment [40]. The low oral bioavailability established for trans-resveratrol both in experimental animals and humans, where the intestine has been pointed out as a bottleneck to its absorption, have promoted the large intestine as a potential target site thus favoring its potential chemopreventive activity in colon cancer.

The chemopreventive activity of trans-resveratrol against colon cancer was evaluated in vitro on the human colorectal carcinoma HT-29 by assessing its anti-proliferative and pro-apoptotic activities. Defects in the regulation of cell cycle progression constitute an important characteristic of transformed cells. Exposure of HT-29 cells to $10-300 \mu \mathrm{M}$ of trans-resveratrol reduced cell growth, with half-maximal effects of around $80 \mu \mathrm{M}$ [42]. Noteworthy, this polyphenol did not induce necrosis even at concentrations that caused full inhibition of cell growth. The antiproliferative activity of trans-resveratrol may be attributed to cell cycle arrest at G2 phase as was previously indicated for HT-29 cells [43] and Caco-2 cells [44,45].

Alterations in programmed cell death mechanisms play important roles in tumor pathogenesis, allowing neoplastic cells to survive beyond their normally intended lifespan, subverting the need for exogenous survival factors, providing protection from hypoxia and oxidative stress as tumor mass expands, and allowing time for accumulative genetic alterations that deregulate cell proliferation, interfere with differentiation, promote 
angiogenesis, and increase cell motility and invasiveness during tumor progression [46]. Consequently, compounds that can eliminate aberrant cell clones by the induction of apoptosis may have a chemopreventive or even a therapeutic potential. The pro-apoptotic activities of trans-resveratrol were evaluated on HT-29 using a series of in vitro assays that allowed the evaluation of different stages of programmed cell death [42]. The activation of the effector caspase- 3 was determined since it represents the converging point of different caspase-dependent apoptosis pathways [47]. trans-Resveratrol increased the activity of caspase- 3 in a time- and a dose-dependent manner. The lowest concentration of trans-resveratrol that induced the activation of this protease was $100 \mu \mathrm{M}$ with an incremented of $200 \%$ that of control cells, and the highest activation was achieved at $250 \mu \mathrm{M}$, with an increase of 7-fold [42]. Execution of apoptosis beyond activation of caspase-3 by transresveratrol led to the characteristic hallmarks of programmed cell death, such as disintegration of the plasma membrane that was characterized by cellstaining with Hoechst 33342 dye. Exposure of HT-29 cells with $150 \mu \mathrm{M}$ of trans-resveratrol accumulated Hoechst dye over time, with an increase of 17.0 $\pm 2.7 \%$ and $33.0 \pm 3.7 \%$ at 20 and $24 \mathrm{~h}$ over the control cells [42]. Full execution of apoptosis with increased fragmentation of DNA and chromatin condensation was evidenced by Hoechst 33258 staining. The presence of apoptotic bodies was evidenced in a $14.9 \pm 1.4 \%$ of cells after $24 \mathrm{~h}$ of exposure to $150 \mu \mathrm{M}$ of trans-resveratrol.

Several studies have indicated that reactive oxygen species (ROS) production in mitochondria play a role in the initiation and execution of apoptosis [48]. HT-29 cells exposed to $150 \mu \mathrm{M}$ of trans-resveratrol showed markedly increased levels of superoxide anion radicals in mitochondria after 4 hours of incubation. Consequently, the apoptotic effect of this polyphenol was mediated, in part, by the intrinsic pathway, through the production of superoxide anions in mitochondria prior to the initiation of the caspase pathway [42]. These results were in agreement with previous studies that pointed out superoxide anions as secondary messengers in apoptosis provoked by anticancer agents, such as paclitaxel and cisplatin $[49,50]$. In addition, trans-resveratrol was also reported to generate reactive oxygen species prior to the release of mitochondrial proteins to the cytosol, activation of effector caspase-3 and caspase-9, and induction of apoptosis in prostate cancer cells [51]. Reactive oxygen species production occurs in an early phase suggesting that the compound triggers a rapid release of cytochrome $\mathrm{c}$ from mitochondria into the cytosol that in turn activates procaspase- 9 and the downstream effectors, including the pro-caspases -3, 6 , and -7 , followed finally by the cleavage of proteins and DNA that characterize the final phase of apoptosis. 
Two principal pathways of apoptosis have been described, such as mitochondria-mediated intrinsic pathway and death receptor-induced extrinsic pathway [47]. However, it has been suggested that lysosomes and the endoplasmic reticulum also play important roles in programmed cell death [52]. In HT-29 cells, trans-resveratrol also triggers apoptosis through lysosome and demonstrates a hierarchy of the proteolytic pathways involved in its cytotoxic mechanism in which lysosomal cathepsin D acts upstream of caspase activation [53]. Furthermore, this polyphenol has been reported to promote apoptosis through the endosplasmic reticulum (ER) [54] and the induction of CHOP/GADD153 gene expression, which has been recognized as a proapoptosis gene [55].

The potential cancer chemopreventive activity of trans-resveratrol in vivo was evaluated in rats that received an intraperitoneal injection of 1,2dimethylhydrazine (DMH) [19]. The development of carcinogenesis in the $\mathrm{DMH}$ rat model takes place through a multistep process as it does in humans, and allowed the measurement of aberrant crypt foci (ACF) and mucindepleted foci (MDF) as valid early preneoplastic markers. ACF have been identified in humans at high risk and are widely used as a surrogate marker of colon cancer [56]. Prior to the performance of the study the experimental conditions were validated. Three subcutaneous injections of DMH $(20 \mathrm{mg} / \mathrm{kg}$, one week apart) followed by an observation period of four weeks proved to be appropriate for the screening of potentially chemopreventive agents [19]. trans-Resveratrol was administered at a dose $60 \mathrm{mg} / \mathrm{kg}$ since it was regarded as a potential nutraceutical and not in any case as an effect encountered after a long period of consumption of this polyphenol in the diet. trans-Resveratrol reduced the number of preneoplastic lesions, since ACF were inhibited by $52 \%$, and the total number of aberrant crypts (AC) by $50 \%$ [19]. In addition, MDF were described in carcinogen-treated rodents [57] and in humans at high risk of colon cancer [56]. MDF are characterized by harboring mutations that show Wnt signaling activation like in colon tumors, suggesting that these lesions are precancerous [56]. trans-Resveratrol oral administration for 49 days reduced the number of MDF by $50 \%$, thus remarking the protecting activity exerted by this compound in the colon mucosa. Given that transresveratrol was administered one week prior to the first exposure to $\mathrm{DMH}$, the results demonstrated that trans-resveratrol acts as an efficient agent inhibiting cancer initiation [19].

The potential cancer chemopreventive activity of trans-resveratrol in vivo was examined previously in long-term studies [45,58-61]. The effect of transresveratrol on azoxymethane-induced colon carcinogenesis was assessed in F344 rats. This phytochemical was administered in drinking water at a dose of $200 \mu \mathrm{g} / \mathrm{kg}$ for $100 \mathrm{day}$, beginning 10 days before administration of the 
carcinogen [59]. trans-Resveratrol reduced the growth of colorectal ACF modulating the expression of bax and $\mathrm{p} 21$, both involved in the regulation of cell proliferation and apoptosis [59]. Sengottuvelan et al. also assessed the anticarcinogenic activity of $8 \mathrm{mg} / \mathrm{kg}$ of trans-resveratrol in a model of colon carcinogenesis induced by $\mathrm{DMH}$, but these were long term experiments that lasted 30 weeks $[58,60]$. Their results showed that in rats, trans-resveratrol markedly reduced the number of 1,2-dimethylhydrazine-induced aberrant crypt foci and incidence and size of tumors, possibly through the modulation of antioxidant defense status and activities of carcinogen-detoxifying enzymes $[58,60]$. In ApcMin mice, which are a model of human familial adenomatous polyposis, trans-resveratrol administered in drinking water at a dose of $15 \mathrm{mg} / \mathrm{kg}$ for 7 weeks prevented the formation of colon tumors and reduced the formation of small intestinal tumor by $70 \%$ by down-regulating genes that are directly involved in cell cycle progression such as cyclin $\mathrm{D}_{1}, \mathrm{D}_{2}$ and DP-1, and in the inhibition of the carcinogenic process and tumor expansion [45]. In the same animal model, trans-resveratrol $(0.2 \%$ in the diet $)$ decreased the number of adenomas, which was associated with inhibition of COX enzymes and interference with prostaglandin $\mathrm{E}_{2}\left(\mathrm{PGE}_{2}\right)$ generation [62]. In contrast, trans-resveratrol administered in the diet at $0,4,20$, or $90 \mathrm{mg} / \mathrm{kg}$ body weight for 7 weeks did not affect intestinal tumorigenesis or COX-2 expression in ApcMin/+ mice [61].

\subsection{Spermatogenesis}

The mammalian testis fulfills two main functions, namely, the synthesis of steroid hormones and the production of spermatozoa, which are controlled by gonadotrophins and testosterone together with locally produced factors [63]. The repeated oral administration of $20 \mathrm{mg} / \mathrm{kg}$ of trans-resveratrol during 90 days enhanced both functions. Sperm counts were significantly greater in the resveratrol treated rats $\left(24.8 \pm 3.30 \cdot 10^{7}\right)$ than in the control group $(14.1 \pm$ $\left.0.80 \cdot 10^{7}\right)$ [64]. The experimental data showed that the repeated oral administration of trans-resveratrol induced a $71 \%$ decrease in the mean diameter of the seminiferous tubules with a $100 \%$ increase in the testicular tubular density. Taken together, these changes result in an overall increase in the size of the spermatogenic tissue. Therefore, this enlargement would be in the basis of the observed increase of sperm production. It is noteworthy that sperm collected from the epididymis showed a correct maturation since the morphological examination evidenced the same percentage of abnormalities between groups. As a conclusion, these results suggest that the transresveratrol-induced increment in total sperm content would be due more to an enlargement in the overall tissue that produces mature sperm cells than to an 
increase in the rhythm of sperm production, which would remain unchanged with respect to the control animals [64].

Spermatogenesis depends on the delicate balance that constitutes the hypothalamic-pituitary-gonadal axis. The endocrine regulation of this axis in the male is intricate with a role for estradiol and testosterone, making obvious the complexity of the steroid-feedback mechanisms [65]. The sites for these feedback effects include cells in the hypothalamus, that are in close proximity to gonadotrophin-releasing hormone $(\mathrm{GnRH})$ neurons, and gonadotrophins in the pituitary, that may respond directly to androgens due to the expression of the androgen receptor (AR), whereas the presence of aromatase and the estrogen receptor (ER) allows the conversion of androgens into estrogens, and the subsequent activation of ER signaling pathways [65]. The oral administration of $20 \mathrm{mg} / \mathrm{kg}$ of trans-resveratrol for 90 days also exerted a stimulatory effect on the secretion of gonadotrophins that are the major endocrine regulators of spermatogenesis [64]. The concentration of FSH, which acts within the tubules to stimulate spermatogenesis, and $\mathrm{LH}$, that signals the production of testosterone in Leydig cells were both elevated in the resveratrol group with respect to the control rats. Testosterone, which is essential for promoting spermatogenesis, was also enhanced. These results seem to indicate that the effect of trans-resveratrol on sperm count was caused by the hypophisary stimulation of the testicular function.

A possible explanation to the described effect could be that transresveratrol binds ER as a mixed weak agonist/antagonist, without estrogenic properties [40]. The fact that trans-resveratrol lacks estrogenic activity could be confirmed by the absence of deleterious effects on testes, in opposition to the toxicity described for diethylstilbestrol (DES) [66]. Rats treated with DES, which is a structural analog of trans-resveratrol, and a potent estrogen agonist, showed reduction in testicular weight, impaired seminiferous tubular morphology and reduced testosterone concentration [66]. The distinct activity shown by two structurally similar compounds can be explained by subtle differences in both molecules. Compared to transresveratrol, DES lacks the $3-\mathrm{OH}$ and $5-\mathrm{OH}$ groups, but on the contrary possesses a 4-OH group and two additional ethyl groups. These features provide differential binding characteristics to ER [67]. DES holds similar affinity as estradiol to ER acting as a full potent agonist, thus accounting for the harmful effects described.

In addition, the daily oral administration of $20 \mathrm{mg} / \mathrm{kg}$ of trans-resveratrol for 90 days did not induce any differences in body weight, food and water consumption in the treated group with respect to the control rats [64]. Given that growth inhibition is a sensitive indicator of estrogenic effects [68], this lack of reduction in body weight in the treated rats substantiates that 
trans-resveratrol does not act as an estrogen agonist, in agreement with other in vivo studies [69,70].

Consequently, trans-resveratrol could interact with ER, thus increasing the secretion of gonadotrophins which leads in turn to an increment in testosterone and sperm output. Furthermore, the effects described above could have been reinforced through androgen antagonism, since trans-resveratrol has also been revealed to antagonize androgen action in prostate cancer cells by inhibiting AR activity as well as by suppressing AR expression [71,72]. Besides the effect of trans-resveratrol on the hypothalamic-pituitary-gonadal axis, its antioxidant and anti-inflammatory activity also warrants attention. Taken as a whole, these actions could also account for the increase on sperm output observed in healthy rats. In Western society, infertility is a growing problem, the causes are diverse and considerable effort is being made to offer effective therapy. In the search for solutions, in the case of male infertility, antioxidants, anti-inflammatories, androgens and antiestrogens are among the treatments used. However, truly effective treatment has still to be found [73]. Therefore, the effect of trans-resveratrol on spermatogenesis merits further research since this compound may be useful in the treatment of male infertility.

\section{Conclusions}

In the present review we summarize the current evidence about the role of $\mathrm{ABC}$ transporters both in the absorption and distribution of trans-resveratrol that contribute to the low bioavailability of this compound. Moreover, we provide evidence that trans-resveratrol, as a pharmacological agent, has an important effect in the prevention of colon cancer as well as in masculine infertility. The wide spectrum of this compound may reflect its simultaneous action on multiple molecular targets.

\section{Acknowledgements}

This work was supported by Ministerio de Ciencia y Tecnología grants PB96-1255, AGL2000-0918, AGL2005-05728 and AGL2009-12866 and the Generalitat de Catalunya grants1999-SGR-00271, 2001-SGR-00142, 2005SGR-00632 and 2009-SGR-00471.

\section{References}

1. Soleas, G.J., Diamandis, E.P., Goldberg, D.M. 1997, Clin Biochem., 30, 91.

2. Nonomura, S., Kanagawa, Makimoto, A. 1963, Yakugaku Zasshi., 83, 988. 
3. Langcake, P., Pryce, R.J. 1976, Physiol. Plant Pathol., 9, 77.

4. Siemann, E.H., Creasy, L.L. 1992, Am. J. Eno. Vitic., 43, 49-52.

5. Baur, J.A., Sinclair, D.A. 2006, Nat. Rev. Drug Discov., 5, 493.

6. Stervo, U., Vang, O., Bonnesen, C. 2007, Food Chemistry, 101, 449.

7. Juan, M.E., González-Pons, E., Planas, J.M. 2010, J. Nutr., 140, 489-495.

8. Maier-Salamon, A., Hagenauer, B., Wirth, M., Gabor, F., Szekeres, T., Jäger, W. 2006, Pharm. Res., 23, 2107.

9. Enokizono, J., Kusuhara, H., Sugiyama, Y. 2007, Mol. Pharmacol., 72, 967.

10. Murakami, T., Takano, M. 2008, Expert Opin. Drug Metab. Toxicol., 4, 923.

11. Oude-Elferink, R.P., de Waart, R. 2007, J. Physiol. Biochem. 63, 75.

12. Nies, A.T., Keppler, D. 2007, Pflugers Arch., 453, 643.

13. van Herwaarden, A.E., Schinkel, A.H. 2006, Trends Pharmacol. Sci., 27, 10.

14. Naruhashi, K., Tamai, I., Inoue, N., Muraoka, H., Sai, Y., Suzuki, N., Tsuji, A. 2002, Antimicrob. Agents Chemother., 46, 344.

15. Gekeler, V., Ise, W., Sanders, K.H., Ulrich, W.R., Beck, J. 1995, Biochem. Biophys. Res. Commun., 208, 345.

16. Maier-Salamon, A., Hagenauer, B., Reznicek, G., Szekeres, T., Thalhammer, T., Jäger, W. 2008, J. Pharm. Sci. 97, 1615.

17. Allen, J.D., van Loevezijn, A., Lakhai, J.M., van der Valk, M., van Tellingen, O., Reid, G., Schellens, J.H., Koomen, G.J., Schinkel, A.H. 2002, Mol. Cancer Ther., 1, 417.

18. Alfaras, I., Pérez, M., Juan, M.E., Merino, G., Prieto, J.G., Planas, J.M., Alvarez, A.I. 2010, J. Agric. Food Chem., 58, 4523.

19. Alfaras, I., Juan, M.E.; Planas, J.M. 2010, J. Agric. Food Chem., 58, 8104.

20. Cottart, C.H., Nivet-Antoine, V., Laguillier-Morizot, C., Beaudeux, J.L. 2010, Mol. Nutr. Food Res., 54, 7.

21. Juan, M.E., Buenafuente, J., Casals, I., Planas, J.M. 2002, Food Res. Inter., 35, 195.

22. Hebbar, V., Shen, G., Hu, R., Kim, B.R., Chen, C., Korytko, P.J., Crowell, J.A., Levine, B.S., Kong, A.N. 2005, Life Sci., 76, 2299.

23. Lançon, A., Hanet, N., Jannin, B., Delmas, D., Heydel, J.M., Lizard, G., Chagnon, M.C., Artur, Y., Latruffe, N. 2007, Drug Metab. Dispos., 35, 699.

24. Colom H., Alfaras, I, Maijó, M., Juan, M.E., Planas, J.M. Pharm. Res., DOI: 10.1007/s11095-011-0395-8

25. van de Wetering, K., Burkon, A., Feddema, W., Bot, A., de Jonge, H., Somoza, V., Borst, P. 2009, Mol. Pharmacol., 75, 876.

26. Andlauer, W., Kolb, J., Siebert, K., Fürst, P. 2000, Drugs Exp. Clin. Res. 26, 47.

27. Kuhnle, G., Spencer, J.P., Chowrimootoo, G., Schroeter, H., Debnam, E.S., Srai, S.K., Rice-Evans, C., Hahn, U. 2000, Biochem. Biophys. Res. Commun., 272, 212.

28. Juan, M.E., Maijó, M., Planas, J.M. 2010, J. Pharm. Biomed. Anal., 51, 391.

29. Marier, J.F., Vachon, P., Gritsas, A., Zhang, J. 2002, J. Pharmacol. Exp. Ther., $302,369$.

30. Walle, T., Hsieh, F., DeLegge, M.H., Oatis, J.E. Jr., Walle, U.K. 2004, Drug Metab. Dispos., 32, 1377.

31. Boocock, D.J., Faust, G.E., Patel, K.R., Schinas, A.M., Brown, V.A., Ducharme, M.P., Booth, T.D., Crowell, J.A., Perloff, M., Gescher, A.J., Steward, W.P., Brenner, D.E. 2007, Cancer Epidemiol. Biomarkers Prev., 16, 1246. 
32. Juan, M.E., Vinardell, M.P., Planas, J.M. 2002, J. Nutr., 132, 257.

33. Crowell, J.A., Korytko, P.J., Morrissey, R.L, Booth, T.D., Levine B.S. 2004, Toxicol. Sci., 82, 614.

34. Williams, L.D., Burdock, G.A., Edwards, J.A., Beck, M., Bausch, J. 2009, Food Chem. Toxicol., 47, 2170.

35. Horn, T.L., Cwik, M.J., Morrissey, R.L., Kapetanovic, I., Crowell, J.A., Booth, T.D., McCormick, D.L. 2007, Food Chem. Toxicol., 45, 55.

36. Almeida, L., Vaz-da-Silva, M., Falcão, A., Soares, E., Costa, R., Loureiro, A.I., Fernandes-Lopes, C., Rocha, J.F., Nunes, T., Wright, L., Soares-da-Silva, P. 2009, Mol. Nutr. Food Res., 53, S7.

37. Ferlay, J., Autier, P., Boniol, M., Heanue, M., Colombet, M., Boyle, P. 2007, Ann. Oncol. 18, 581.

38. Trichopoulou, A., Lagiou, P., Kuper, H., Trichopoulos, D. 2000, Cancer Epidemiol. Biomarkers Prev., 9, 869.

39. Cummings, J.H., Bingham, S.A. 1998, BMJ., 317, 1636.

40. Bishayee, A. 2009, Cancer Prev. Res. (Phila., Pa.), 2, 409.

41. Kraft, T.E., Parisotto, D., Schempp, C., Efferth, T. 2009, Crit. Rev. Food Sci. Nutr., 49, 782.

42. Juan, M.E., Wenzel, U., Daniel, H., Planas, J.M. 2008, J. Agric. Food Chem., $56,4813$.

43. Liang, Y.C., Tsai, S.H., Chen, L., Lin-Shiau, S.Y., Lin, JK. 2003, Biochem. Pharmacol., 65, 1053.

44. Wolter, F., Akoglu, B., Clausnitzer, A., Stein, J. 2001, J. Nutr., 131, 2197.

45. Schneider, Y., Vincent, F., Duranton, B., Badolo, L., Gossé, F., Bergmann, C., Seiler, N., Raul, F. 2000, Cancer Lett., 158, 85.

46. Watson, A.J.M. 2006, Crit. Rev. Oncol., 57, 107.

47. Hengartner, M.O. 2000, Nature, 407, 770.

48. Kroemer, G.; Galluzzi, L.; Brenner, C. 2007, Physiol. Rev., 87, 99.

49. Fawcett, H., Mader, J.S., Robichaud, M., Giacomantonio, C., Hoskin, D.W. 2005, Int. J. Oncol., 27, 1717.

50. Stewart, J.H. $4^{\text {th }}$, Tran, T.L., Levi, N., Tsai, W.S., Schrump, D.S., Nguyen, D.M. 2007, J. Surg. Res. 141, 120.

51. Shankar, S., Siddiqui, I., Srivastava, R.K. 2007, Mol. Cell. Biochem., 304, 273.

52. Bröker, L.E., Kruyt, F.A., Giaccone, G. 2005, Clin. Cancer Res., 11, 3155.

53. Trincheri, N.F., Nicotra, G., Follo, C., Castino, R., Isidoro, C. 2007, Carcinogenesis, 28, 922.

54. Park, J.W., Woo, K.J., Lee, J.T., Lim, J.H., Lee, T.J., Kim, S.H., Choi, Y.H., Kwon, T.K. 2007, Oncol. Rep., 18, 1269.

55. Woo, K.J., Lee, T.J., Lee, S.H., Lee, J.M., Seo, J.H., Jeong, Y.J., Park, J.W., Kwon, T.K. 2007, Biochem. Pharmacol., 73, 68.

56. Femia, A.P., Caderni, G. 2008, Planta Med., 74, 1602.

57. Caderni, G., Femia, A.P., Giannini, A., Favuzza, A., 2003, Cancer Res., 63, 2388.

58. Sengottuvelan, M., Viswanathan, P., Nalini, N. 2006, Carcinogenesis, 27, 1038.

59. Tessitore, L., David, A., Sarotto, I., Caderni, G. 2000, Carcinogenesis 21, 1619.

60. Sengottuvelan, M., Deeptha, K., Nalini, N. 2009, Nutrition, 25, 1169. 
61. Ziegler, C.C., Rainwater, L., Whelan, J., McEntee, M.F. 2004, J. Nutr., 134, 5.

62. Sale, S., Verschoyle, R.D., Boocock, D., Jones, D.J., Wilsher, N., Ruparelia, K.C., Potter, G.A., Farmer, P.B., Steward, W.P., Gescher, A.J. 2004, Br. J. Cancer., 90, 736 .

63. Carreau, S., Bourguiba, S., Lambard, S., Galeraud-Denis, I., Genissel, C., Levallet, J. 2002, Mol. Cell Endocrinol., 193, 137.

64. Juan, M.E., González-Pons, E., Munuera, T., Ballester, J., Rodríguez-Gil, J.E., Planas, J.M. 2005, J Nutr., 135, 757.

65. O’Donnell, L., Roberston, K.M., Jones, M.E., Simpson E.R. 2001, Endocr. Rev., 22, 289.

66. Fritz, W. A., Cotroneo, M. S., Wang, J., Eltoum, I. E., Lamartiniere, C. A. 2003, J. Nutr., 133, 2287.

67. Abou-Zeid, L. A., El-Mowafy, A.M. 2004, Chirality, 16, 190.

68. Hart, J.E. 1990, Pharmacol. Ther., 47, 203.

69. Turner, R.T., Evans, G.L., Zhang, M., Maran, A., Sibonga J.D. 1999, Endocrinology, 140, 50.

70. Kubo, K., Arai, O., Omura, M., Watanabe, R., Ogata, R., Aou, S. 2003, Neurosci. Res., $45,345$.

71. Stewart, J.R., Artime, M.C., O’Brien, C.A. 2003, J. Nutr., 133, 2440 S.

72. Gao, X., Xu, Y.X., Divine, G., Janakiraman, N., Chapman, R.A., Gautam, S.C. 2002, J. Nutr., 132, 2076.

73. Haidl, G. 2002, Drugs, 62, 1741. 


\title{
5. Effect of cocoa powder in the prevention of cardiovascular disease: Biological, consumption and inflammatory biomarkers. A metabolomic approach
}

\author{
Mireia Urpi-Sarda $1,2,5$, Rafael Llorach ${ }^{1,4}$, Maria Monagas ${ }^{2,3}$ \\ Nasiruddin Khan ${ }^{1,2}$, Maria Rotches-Ribalta' ${ }^{1,4}$, Elena Roura' \\ Rosa Lamuela-Raventos ${ }^{1,5}$, Ramón Estruch ${ }^{2,5}$ \\ and Cristina Andres-Lacueva ${ }^{1,4}$ \\ 'Nutrition and Food Science Department, XaRTA. INSA. Pharmacy Faculty, University \\ of Barcelona (UB), Av.Joan XXIII s/n, 08028 Barcelona, Spain; ${ }^{2}$ Department of Internal \\ Medicine, Hospital Clínic. Institut d'Investigació Biomèdica August Pi i Sunyer (IDIBAPS) \\ UB, Barcelona, Spain; ${ }^{3}$ Instituto de Fermentaciones Industriales (CSIC), Madrid, Spain \\ ${ }^{4}$ INGENIO-CONSOLIDER Program, Fun-c-food CSD2007-063 \\ ${ }^{5}$ CIBER de Fisiopatología de la Obesidad y la Nutrición (CIBEROBN) \\ and RETICS RD06/0045, Instituto de Salud Carlos III, Spain
}

\begin{abstract}
Numerous health benefits have been attributed to cocoa and its derived products in the last decade including antioxidant, anti-platelet and positive effects on lipid metabolism and vascular function. Inflammation plays a key role in the initiation and progression of atherosclerosis. However, cocoa feeding trials focused on inflammation are still rare and the results yielded are controversial. Health effects derived from cocoa consumption have
\end{abstract}


been partly attributed to its polyphenol content, in particular of flavanols. Bioavailability is a key issue for cocoa polyphenols in order to be able to exert their biological activities. In the case of flavanols, bioavailability is strongly influenced by several factors, such as their degree of polymerization and the food matrix in which the polyphenols are delivered. Furthermore, gut has become an active site for the metabolism of procyanidins (oligomeric and polymeric flavanols). Estimation of polyphenol consumption or exposure is also a very challenging task in Food and Nutrition Science in order to correlate the intake of phytochemicals with in vivo health effects. In the area of nutrition, modern analytical techniques based on mass spectrometry are leading to considerable advances in targeted metabolite analysis and particularly in Metabolomics or global metabolite analysis.

In this chapter we have summarized the most relevant results of our recent research on the bioavailability of cocoa polyphenols in humans and the effect of the matrix in which cocoa polyphenols are delivered considering both targeted analysis and a metabolomic approach. Furthermore, we have also summarized the effect of long-term consumption of cocoa powder in patients at high risk of cardiovascular disease (CVD) on the inflammatory biomarkers of atherosclerosis.

\section{Introduction}

Cocoa (Theobroma cacao L.) and its related products are rich sources of antioxidant flavonoids, containing higher amounts per serving than other polyphenol rich foods such as red wine or tea [1]. Flavanols are the most abundant flavonoids in cocoa and comprise monomeric flavanols $[(+)$-catechin and (-)-epicatechin], oligomeric (procyanidins B1, B2 and C1) and polymeric forms (procyanidins) [2].

Inflammation is central to cardiovascular disease. The process of atherosclerosis starts with inflammatory changes in the arterial endothelium, which in turn expresses a series of adhesion molecules that attract circulating monocytes, which migrate through the endothelial layer under the influence of various proinflammatory chemoattractants. Once within the arterial intima, the monocytes continue to undergo inflammatory changes, and through these inflammatory processes, the initial lesion of atherosclerosis, the fatty streak, is formed. Also, inflammation is central to the progression from fatty streak to complex plaque that can lead to fatal complications [3].

Evidence based on epidemiological studies supports the belief that regular consumption of cocoa-containing products may confer cardiovascular protection, reducing blood pressure and the risk of CVD mortality $[4,5]$. In addition, a recent meta-analysis on the effectiveness of cocoa human feeding trials has suggested that cocoa and its derived products have a significant effect on endothelial function, increasing flow-mediated dilation and reducing systolic and diastolic blood pressures [6]. Other benefits from cocoa consumption are associated with their capacity to improve lipid levels and 
insulin sensitivity, and to reduce platelet activation and function [7]. In addition, results from in vitro studies have shown that cocoa flavanols and procyanidins also present anti-inflammatory effects, suppressing the production of the proinflammatory molecules such as cytokines and chemokines $[8,9]$, which are produced in the initial phases of atherosclerosis. However, few human feeding trials have focused on the study of the antiinflammatory effects of cocoa, and the results obtained have been contradictory, reporting either neutral effects or changes in a single inflammatory biomarker [10-12].

For these biological activities to be translated into real health effects in vivo, cocoa polyphenols need to be bioavailable. Bioavailability of flavanols is influenced by their degree of polymerization and the food matrix. While monomers are readily absorbed in the small intestine, oligomers and polymers need to be biotransformed by the colonic microbiota before absorption (Fig. 1). Equally important is the development of biomarkers of exposure in

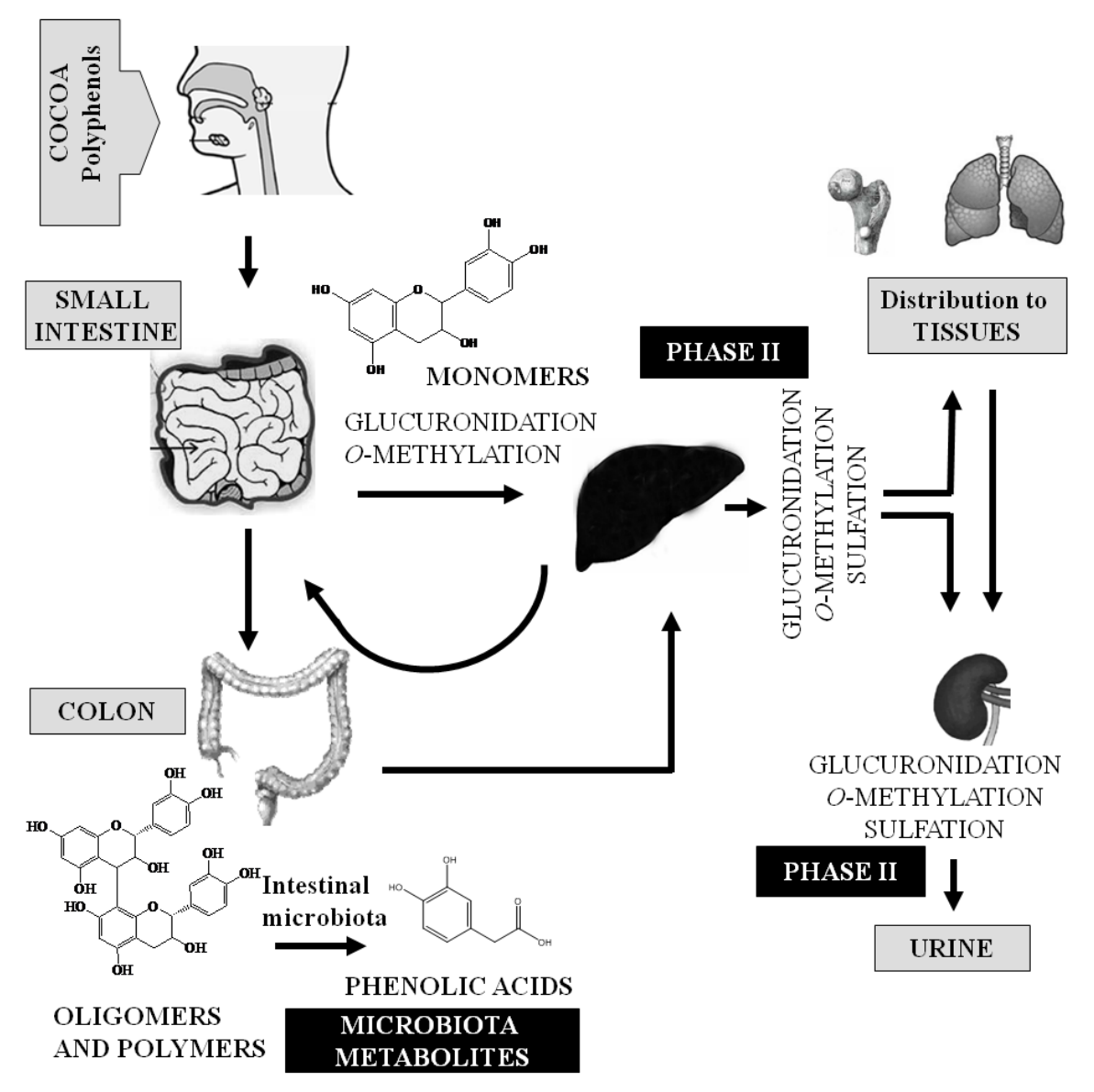

Figure 1. Scheme of bioavailability of cocoa flavanols showing phase II metabolites [conjugation by enzymes catechol-O-methyl transferases (COMT); uridine diphosphate glucuronyltransferases (UDPGT), and sulfotransferases (SULFT)], and microbial metabolites [degradation of oligomers and polymers by intestinal microbiota]. 
order to correlate the intake of flavanols with particular health effects in vivo. In this sense, global analysis of metabolites or metabolomics is becoming an important tool for the determination of new biomarkers of exposure to polyphenols.

The other important aspect that could modulate the bioavailability of polyphenols is the food matrix. Several studies have provided conflicting evidence about the effect of milk on the bioavailability of polyphenols, specifically flavanols, when looking at their phase II conjugate forms from cocoa [13-16]. Recently, it has been suggested that the possible influence of milk on cocoa flavonoid absorption, and consequently on the urinary excretion of phase II metabolites, is more relevant for drinks with lower flavanol content, which is typical of many commercial cocoas, than for drinks with higher content [17]. Therefore, as milk significantly lowered the excretion of urinary phase II metabolites of cocoa flavanols [17], there is a need to study the effect of milk on the colonic microbial metabolism of the non-absorbed cocoa flavanol fraction that reaches the colon [18].

Herein we will summarize the main results of our recent research on the bioavailability of cocoa polyphenols in two interventional studies: 1) A single dose study of cocoa powder whose main aims are: a) Effect of milk on the bioavailability of cocoa polyphenols [18], b) Metabolomic study to assess bioavailability and identify nutritional biomarkers of exposure after cocoa intake in humans [19]; 2) a long-term study of regular consumption of cocoa powder in patients at high risk of CVD whose main aims are: c) Effects of cocoa powder at clinical levels [20], and d) Targeted study of cocoa polyphenol metabolites to assess bioavailability and compliance [21].

\section{Single cocoa powder intake: Short-term study}

\subsection{Effect of milk on the bioavailability of cocoa polyphenols}

The short term study with single intake of cocoa powder was a randomized, crossover and controlled clinical trial. Twenty-one healthy volunteers $(9$ women and 12 men) were included in the study. Participants were instructed to abstain from polyphenol-rich foods for at least $48 \mathrm{~h}$ before the study and during the day of the study. The study was done on three different days with one week between the interventions. After overnight fasting, the volunteers consumed three different meals in random order: a) $40 \mathrm{~g}$ cocoa powder (Nutrexpa, Barcelona, Spain) and $250 \mathrm{~mL}$ whole milk (CC-M); b) $40 \mathrm{~g}$ cocoa powder and $250 \mathrm{~mL}$ water (CC-W); c) $250 \mathrm{~mL}$ whole milk as a control [18].

First, we evaluated the effect of milk on the absorption of (-)-epicatechin from cocoa powder. The only metabolite found in plasma at $2 \mathrm{~h}$ after ingestion 
of cocoa powder was epicatechin-glucuronide. The concentration of epicatechin glucuronide in plasma was $\sim 20 \%$ higher after the intervention of $\mathrm{CC}-\mathrm{W}$ in comparison to the intervention $\mathrm{CC}-\mathrm{M}$ but these differences were almost significant $(\mathrm{P}=0.07)$ [14]. With respect to phase II metabolites of epicatechin in urine (three sulfates and one glucuronide conjugates), milk did not affect the total excretion of metabolites but affected the excretion profile of these conjugates [13].

We have also evaluated the metabolism of cocoa polyphenols at a colonic microbial level (microbiota metabolites) through the measure of the urinary excretion of microbial-derived phenolic acids and evaluated the effect of milk on urinary excretion of these compounds after the intake of a standard portion of cocoa powder with either water or milk in an acute intervention using a targeted quantitative procedure. We have carried out this targeted study through a developed and validated methodology that consist in solid-phase extraction (SPE) of biological samples (urine) followed by LC-MS/MS analysis [22].

We evaluated the excretion of 15 phenolic acids which had been described as microbial degradation products of flavanols [21, 23-26]. Of the 15 phenolic acids studied, the excretion of seven phenolic acids diminished after CC-M when compared with CC-W while two phenolic acids increased. Four phenolic acids of these seven, could come from the direct degradation of procyanidins by the intestinal microbiota following the scheme shown in Fig. 2. Procyanidins could be degraded forming 3,4-dihydroxyphenylacetic acid (Fig. 2) $[18,27]$ and this compound through $\alpha$-oxidation could be degraded to protocatechuic acid [26]. Microbial dehydroxylation of protocatechuic acid could give rise to 4-hydroxybenzoic acid derivative that may undergo glycination in the liver and kidney being converted into 4-hydroxyhippuric acid $[18,24]$.

Two hydroxycinnamic acids, caffeic and ferulic acids, were also significantly reduced ( $50 \%$ and $80 \%$, respectively) by the ingestion of CC-M when compared with $\mathrm{CC}-\mathrm{W}$. In cocoa, these compounds could come from several routes of metabolism, which could include dehydrogenation of 3,4-dihydroxyphenylpropionic acid as well as degradation from other phenolic derivatives present in cocoa powder, such as chlorogenic acid or $N$-phenylpropenoyl-L-amino acids [25, 28]. We observed a high concentration in the last urine fraction suggesting that a longer period of urine collection was required for their complete excretion.

Whereas a higher global urinary excretion of phenolic acids was observed with consumed CC-W, vanillic and phenylacetic acids showed an excretion pattern contrary to those of other metabolites. This result could mean that liposoluble vanillin, when ingested with CC-M, could be absorbed more easily 


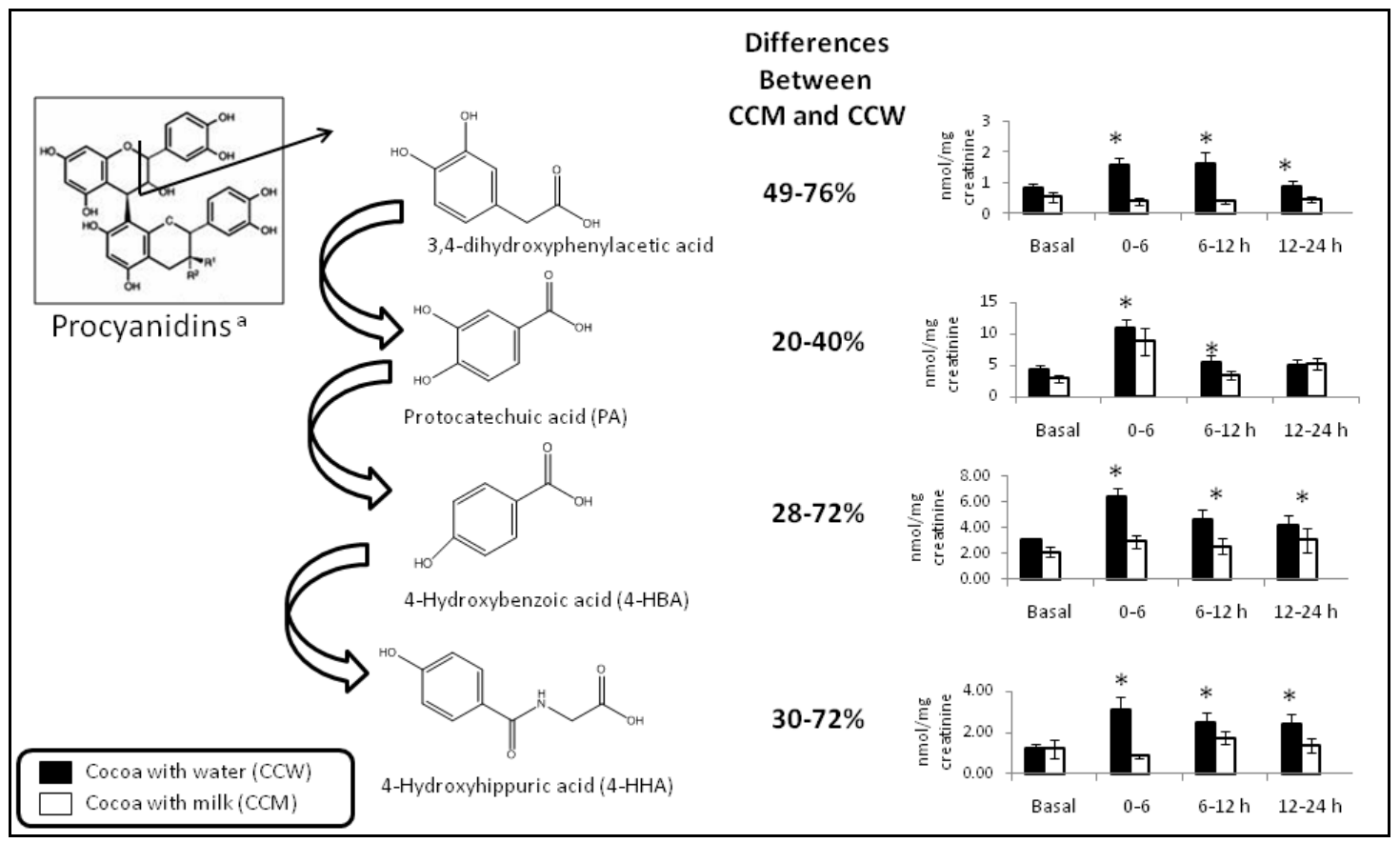

Figure 2. Urinary excretion [mean(SEM)] and percentage difference of four phenolic acids from cocoa procyanidins significantly affected by the consumption of cocoa powder with water or with milk [18]. Bars with an asterisk are significantly different $(\mathrm{P}<0.05$; Wilcoxon's test; $\mathrm{n}=21)$ in the same time period between water and milk. ${ }^{\mathrm{a}}$ Described in [27].

and rapidly than after its intake with $\mathrm{CC}-\mathrm{W}$ and could be oxidized to vanillic acid by aldehyde oxidase of the liver [29]. In the case of phenylacetic acid, its excretion was only significatively higher after $\mathrm{CC}-\mathrm{M}$ than after $\mathrm{CC}-\mathrm{W}$ in the last time period (12-24 h) although the same trend was observed in earlier urine fractions.

\subsection{Metabolomic study to assess bioavailability and identify nutritional biomarkers of exposure after cocoa intake in humans}

Metabolomics aims to assess metabolic changes in a global manner in order to provide the detailed biochemical responses of cellular systems and provide a comprehensive profile of all the metabolites present in a biological sample (metabolome). This approach has been applied to clinical, pharmaceutical and toxicological applications [30] and recently has also emerged as a field of increasing interest to Food and Nutrition Science $[31,32]$. We have applied an HPLC-Q-TOF metabolomics approach followed by multivariate data analysis (PCA, OSC-PLS and OSC-PLSDA) to urine samples collected after a single intake of $40 \mathrm{~g}$ of cocoa powder with milk $(250 \mathrm{~mL})$ or with water $(250 \mathrm{~mL})$ or only milk $(250 \mathrm{~mL})$ as control, by 
healthy subjects ( $\mathrm{n}=10 ; 5$ women and 5 men) in a randomized, crossover and controlled clinical trial [19]. Our aim in this metabolomic approach was to study the influence of cocoa intake on the urinary metabolic profile during the time course of a $24 \mathrm{~h}$-urine excretion period $(0-6,6-12,12-24 \mathrm{~h})$ and to identify the most relevant biomarkers of intake.

The results revealed that modifications in the urine metabolome as a consequence of cocoa intake were explained by the excretion of a complex profile of cocoa-derived phytochemicals. A total of 27 metabolites related to cocoa intake were identified and were grouped as follows: a) purine alkaloid metabolites; b) polyphenol host metabolites; c) polyphenol colonic microbiota metabolites, d) cocoa flavour and taste compounds, and e) vitamins and amino acids.

Among purine alkaloid metabolites, the most important metabolites derived from theobromine metabolism, including 6-amino-5-[ $N$-methylformylamino]-1methyluracil (AMMU), 3- and 7-methyluric acid, 3- and 7-methylxanthine, among others, were identified and were found to be the main contributors to changes in the urine metabolome up to $24 \mathrm{~h}$ after cocoa intake. In the case of polyphenol host metabolites, epicatechin sulfate, $O$-methylepicatechin, vanillic acid, and its derivative vanilloglycine, identified for the first time after cocoa intake, were found to be present $6 \mathrm{~h}$ after cocoa powder consumption (Fig. 1) [25]. On the other hand, microbial-derived phenolic metabolites, such as phenylvaleric acid and phenylvalerolactone derivatives, were found to contribute to the urinary metabolome in the period between 6 and $12 \mathrm{~h}$ after cocoa ingestion (Fig. 1). Cocoa flavoured metabolites identified included 3,5-diethyl-2-methylpyrazine and hydroxyacetophenone, as well as the diketopiperazine derivatives. Finally, trigonellin and hydroxynicotinic acid were identified as metabolites derived from nicotinic acid (niacin) present in cocoa, whereas tyrosine was identified as a metabolite related to cocoa amino acids. Both types of metabolites were found to contribute to changes in urine metabolome in the fraction collected $6 \mathrm{~h}$ after cocoa intake.

These results demonstrate that metabolomics is an important tool for exploring the metabolism of cocoa phytochemicals. Metabolites from different origins and chemical structures could serve as biomarkers of cocoa powder intake and help us to understand if their presence could be related to afford health effects.

\section{Regular cocoa powder intake: Long-term study}

\subsection{Effects of cocoa powder on inflammatory biomarkers of atherosclerosis}

The long-term study with regular consumption of cocoa powder was a randomized, crossover and controlled clinical trial. The subjects included 
were diabetic or had three or more of the following cardiovascular disease risk factors: tobacco smokers, hypertensives, hypercholesterolemics, and/or obese. Forty-two high-risk subjects were included in the study (19 men and 23 women, mean age of 69.7 years). The institutional review board of the hospital approved the study protocol and the trial was registered in the International Standard Randomized Controlled Trial Number, at controlled.trials.com (ISRCTN75176807). Subjects received two 20 g-sachets of soluble cocoa powder per day with $250 \mathrm{~mL}$ of skimmed milk each $(\mathrm{C}+\mathrm{M}$ intervention) or only $500 \mathrm{~mL}$ skimmed milk per day (M intervention) for 4 weeks in a random order [20]. We have analyzed the expression of adhesion molecules [VLA-4 (very late activation antigen-4, CD49-d), LFA-1 (lymphocyte function-associated antigen-1, CD11a), Mac-1 (CD11b/CD18), SLex (Sialil-Lewis X, CD15s), CD36 and CD40] on the surface of peripheral blood mononuclear cells (PBMCs) for the first time after cocoa intake as inflammatory biomarkers of atherosclerosis. Our results showed significant $(p<0.05)$ lower values in the expression of adhesion molecules such as VLA-4 (-7.4\%), CD40 (-6.6\%) and CD36 (-21.2\%) on monocyte surface after consumption of cocoa powder with skimmed milk.

Other circulating inflammatory biomarkers studied included serum levels of P-selectin, E-selectin, VCAM-1, ICAM-1, proinflammatory cytokine IL-6 and high-sensitivity C-reactive protein (hs-CRP). It was found that P-selectin and ICAM-I, which play an important role in leukocyte adhesion cascade, were significantly $(p<0.05)$ decreased by $10.8 \%$ and $9.7 \%$, respectively, after the intake of cocoa powder with skimmed milk. Other biomarkers like MCP-1 and VCAM were also found to be at lower levels (although not significant) after cocoa consumption.

With respect to plasma lipids, the concentration of HDL cholesterol was significantly higher after the intervention of cocoa with skimmed milk in comparison to the skimmed milk intervention ( $4.2 \%$ increase, $\mathrm{P}=0.035)$. No significant changes were observed for total plasma cholesterol, triglycerides and LDL cholesterol. In addition, no significant changes were registered in systolic and diastolic blood pressure, or in heart rate between the two interventions [20].

All the above results strongly support a link between cocoa consumption and the modulation of inflammatory mediators in human subjects at high risk of CVD. These anti-inflammatory effects together with other previously reported effects, including antioxidant, anti-platelet and positive vascular effects may contribute to the overall benefits of cocoa consumption against atherosclerosis and could have significant implications for public health as far as cardiovascular disease is concerned. 


\subsection{Targeted study of cocoa polyphenol metabolites to assess bioavailability and compliance}

In order to exert the beneficial effects, cocoa polyphenols need to be bioavailable. Monomeric flavanols are readily absorbed in the small intestine but polymers need to be degraded by the intestinal microbiota before absorption in the colon. We have carried out a targeted study of main cocoa polyphenol metabolites by HPLC-MS/MS in the plasma and urine samples collected from the clinical study in order to estimate their absorption in vivo and as a measure of protocol compliance [21]. Regular consumption of cocoa powder with skimmed milk resulted in a significant $(p<0.05)$ increase in the urinary excretion of phase II metabolites, including glucuronide and sulfate conjugates of (-)-epicatechin and $O$-methyl-epicatechin, derived from the absorption of epicatechin in the small intestine (Fig. 1). Colonic microbialderived phenolic metabolites, including vanillic, 3,4-dihydroxyphenylacetic and 3-hydroxyphenylacetic acids, and particularly 5-(3,4-dihydroxyphenyl)- $\gamma-$ valerolactone (Fig. 1), were also found in significant higher levels after consumption of cocoa powder with skimmed milk and represented the largest proportion of total phenolic metabolites excreted in urine. These results indicated that cocoa polyphenols are bioavailable and that the colon is an active site for the metabolism of cocoa polyphenols. In vitro studies performed by our group have suggested that some microbial-derived phenolic metabolites possess anti-inflammatory properties, suggesting that they could be partly responsible for the benefits observed in patients at high risk of CVD after cocoa consumption [33].

\section{Conclusion}

The results presented herein provide valuable information that supports the health benefits of cocoa consumption on CVD. New inflammatory biomarkers, such as the expression of adhesion molecules on leukocyte surface, have been used for the first time in a clinical cocoa trial focused on this issue. Although current state of the art does not allow an estimation of CVD risk associated with a given change in these or other inflammatory biomarkers, the positive effects observed after cocoa consumption may contribute together - along with other previously reported effects, in particular on vascular function - to the overall benefits of cocoa consumption against CVD.

Due to their high content in procyanidins, the colonic microbiota plays a key role in the metabolism of cocoa polyphenols, as demonstrated by the increase in certain lactones and phenolic acids after cocoa consumption in our targeted study. 
Given that these metabolites could represent the largest proportion of all phenolic metabolites excreted in urine, more effort needs to be concentrated on the study of the biological properties of these microbial-derived phenolic metabolites, including their effects on the intestinal microbiota, which are still largely unknown.

Finally, our metabolomic approach, combined with visualization strategies, allowed the identification of a series of metabolites derived from cocoa polyphenols, alkaloids, flavour and aroma compounds, vitamins and amino acids that could serve as biomarkers of cocoa powder consumption, thus pushing nutritional information one step closer towards a better understanding of human health.

\section{Acknowledgements}

This research was supported by national grants CICYT (AGL: 200408378-C02-01/02, 2006-14228-C03-02/01 and 2009-13906-C02-01); CIBER 06/03 Fisiopatología de la Obesidad y la Nutrición is an initiative of the Instituto de Salud Carlos III, Spain; Centro Nacional de Investigaciones Cardiovasculares (CNIC-06) and Ingenio-CONSOLIDER program, Fun-cfood (CSD2007-063). M.U.-S. thanks the Sara Borrell postdoctoral program (CD09/00134), M.M. thanks the Ramon y Cajal program, R.Ll. thanks the Fondo de Investigación Sanitaria program (FIS, CD06/00161), and N.K. thanks the FPU fellowship program, all from the Ministry of Science and Innovation. M.R.-R. thanks the FI-DGR2010 fellowship program from the Generalitat de Catalunya. R.E. is recipient of a grant from FIS, Madrid, Spain.

\section{References}

1. Lee, K.W., Kim, Y.J., Lee, H.J. and Lee, C.Y. 2003, J. Agric. Food Chem., 51,7292 .

2. Andres-Lacueva, C., Monagas, M., Khan, N., Izquierdo-Pulido, M., Urpi-Sarda, M., Permanyer, J. and Lamuela-Raventos, R.M. 2008, J. Agric. Food Chem., 56,3111 .

3. Libby, P. 2006, Am. J. Clin. Nutr., 83, 456S.

4. Buijsse, B., Feskens, E.J., Kok, F.J. and Kromhout, D. 2006, Arch. Intern. Med., $166,411$.

5. Kris-Etherton, P.M. and Keen, C.L. 2002, Curr. Opin. Lipidol., 13, 41.

6. Hooper, L., Kroon, P.A., Rimm, E.B., Cohn, J.S., Harvey, I., Le Cornu, K.A., Ryder, J.J., Hall, W.L. and Cassidy, A. 2008, Am. J. Clin. Nutr., 88, 38.

7. Ding, E.L., Hutfless, S.M., Ding, X. and Girotra, S. 2006, Nutr. Metab. (Lond.), $3: 2$.

8. Sanbongi, C., Suzuki, N. and Sakane, T. 1997, Cell. Immunol., 177, 129. 
9. Mao, T.K., Powell, J., Van de Water, J., Keen, C.L., Schmitz, H.H., Hammerstone, J.F. and Gershwin, M.E. 2000, Life Sci., 66, 1377.

10. Farouque, H.M., Leung, M., Hope, S.A., Baldi, M., Schechter, C., Cameron, J.D. and Meredith, I.T. 2006, Clin. Sci. (Lond.), 111, 71.

11. Wang-Polagruto, J.F., Villablanca, A.C., Polagruto, J.A., Lee, L., Holt, R.R., Schrader, H.R., Ensunsa, J.L., Steinberg, F.M., Schmitz, H.H. and Keen, C.L. 2006, J. Cardiovasc. Pharmacol., 47 Suppl 2, S177.

12. Allen, R.R., Carson, L., Kwik-Uribe, C., Evans, E.M. and Erdman, J.W., Jr. 2008, J. Nutr., 138, 725.

13. Roura, E., Andres-Lacueva, C., Estruch, R., Lourdes Mata Bilbao, M., IzquierdoPulido, M. and Lamuela-Raventos, R.M. 2008, Br. J. Nutr., 100, 846.

14. Roura, E., Andres-Lacueva, C., Estruch, R., Mata-Bilbao, M.L., Izquierdo-Pulido, M., Waterhouse, A.L. and Lamuela-Raventos, R.M. 2007, Ann. Nutr. Metab., 51, 493.

15. Schroeter, H., Holt, R.R., Orozco, T.J., Schmitz, H.H. and Keen, C.L. 2003, Nature, 426, 787.

16. Serafini, M., Bugianesi, R., Maiani, G., Valtuena, S., De Santis, S. and Crozier, A. 2003, Nature, 424, 1013.

17. Mullen, W., Borges, G., Donovan, J.L., Edwards, C.A., Serafini, M., Lean, M.E. and Crozier, A. 2009, Am. J. Clin. Nutr., 89, 1784.

18. Urpi-Sarda, M., Llorach, R., Khan, N., Monagas, M., Rotches-Ribalta, M., Lamuela-Raventos, R., Estruch, R., Tinahones, F.J. and Andres-Lacueva, C., 2010, J. Agric. Food Chem., 58, 4706.

19. Llorach, R., Urpi-Sarda, M., Jauregui, O., Monagas, M. and Andres-Lacueva, C. 2009, J. Proteome Res., 8, 5060.

20. Monagas, M., Khan, N., Andres-Lacueva, C., Casas, R., Urpi-Sarda, M., Llorach, R., Lamuela-Raventos, R.M. and Estruch, R. 2009, Am. J. Clin. Nutr., 90, 1144.

21. Urpi-Sarda, M., Monagas, M., Khan, N., Llorach, R., Lamuela-Raventos, R.M., Jauregui, O., Estruch, R., Izquierdo-Pulido, M. and Andres-Lacueva, C. 2009, J. Chromatogr. A, 1216, 7258.

22. Urpi-Sarda, M., Monagas, M., Khan, N., Lamuela-Raventos, R.M., SantosBuelga, C., Sacanella, E., Castell, M., Permanyer, J. and Andres-Lacueva, C. 2009, Anal. Bioanal. Chem., 394, 1545.

23. Gonthier, M.P., Cheynier, V., Donovan, J.L., Manach, C., Morand, C., Mila, I., Lapierre, C., Remesy, C. and Scalbert, A. 2003, J. Nutr., 133, 461.

24. Gonthier, M.P., Donovan, J.L., Texier, O., Felgines, C., Remesy, C. and Scalbert, A. 2003, Free Radic. Biol. Med., 35, 837.

25. Rios, L.Y., Gonthier, M.P., Remesy, C., Mila, I., Lapierre, C., Lazarus, S.A., Williamson, G. and Scalbert, A. 2003, Am. J. Clin. Nutr., 77, 912.

26. Selma, M.V., Espin, J.C. and Tomas-Barberan, F.A. 2009, J. Agric. Food Chem., 57, 6485 .

27. Appeldoorn, M.M., Vincken, J.P., Aura, A.M., Hollman, P.C. and Gruppen, H. 2009, J. Agric. Food Chem., 57, 1084.

28. Tomas-Barberan, F.A., Cienfuegos-Jovellanos, E., Marin, A., Muguerza, B., GilIzquierdo, A., Cerda, B., Zafrilla, P., Morillas, J., Mulero, J., Ibarra, A., Pasamar, M.A., Ramon, D. and Espin, J.C. 2007, J. Agric. Food Chem., 55, 3926. 
29. Panoutsopoulos, G.I. and Beedham, C. 2005, Cell. Physiol. Biochem., 15, 89.

30. Lindon, J.C., Holmes, E. and Nicholson, J.K. 2006, Pharm. Res., 23, 1075.

31. Rezzi, S., Ramadan, Z., Fay, L.B. and Kochhar, S. 2007, J. Proteome Res., 6, 513.

32. Wishart, D. 2008, Trends Food Sci. Technol., 19, 482.

33. Monagas, M., Khan, N., Andres-Lacueva, C., Urpi-Sarda, M., Vazquez-Agell, M., Lamuela-Raventos, R.M. and Estruch, R. 2009, Br. J. Nutr., 102, 201. 


\title{
6. Molecular simulations of globins: Exploring the relationship between structure, dynamics and function
}

\author{
Flavio Forti', Leonardo Boechi' ${ }^{2}$, Ana Novo de Oliveira', Damian Bikiel ${ }^{2}$ \\ Pau Arroyo ${ }^{2}$, Alejandro $\mathrm{Nadra}^{3}$, Luciana Capece ${ }^{2}$, Axel Bidon-Chanal' \\ Marcelo A. Martí ${ }^{2,3}$, Darío Estrín ${ }^{2}$ and F. Javier Luque' \\ 'Departament de Fisicoquímica, Facultat de Farmàcia, and Institut de Biomedicina, Universitat \\ de Barcelona, Avda. Diagonal 643, 08028 Barcelona, Spain; ${ }^{2}$ Departamento de Química \\ Inorgánica, Analítica, y Química Física, INQUIMAE-CONICET, Facultad de Ciencias \\ Exactas y Naturales, Universidad de Buenos Aires, Buenos Aires, Argentina \\ ${ }^{3}$ Departamento de Química Biológica, Facultad de Ciencias Exactas y Naturales \\ Universidad de Buenos Aires, Buenos Aires, Argentina
}

\begin{abstract}
The discovery in the last two decades of novel members of the globin superfamily has challenged the conventional view about the structure and function of globins. Thus, peculiar structural differences are expected to have direct influence on properties related to ligand migration, binding affinity and heme reactivity. Molecular simulations are a valuable tool to gain insigth into the molecular mechanisms that underlie those structural differences, and their relationship with the diversity of functional roles. In this work, the impact of molecular simulations in exploring
\end{abstract}

Correspondence/Reprint request: Dr. F. J. Luque, Departament de Fisicoquímica, Facultat de Farmàcia Universitat de Barcelona, Avda. Diagonal 643, 08028 Barcelona, Spain. E-mail: fjluque@ub.edu Dr. D. Estrín Departamento de Química Inorgánica, Analítica y Química Física, INQUIMAE-CONICET Facultad de Ciencias Exactas y Naturales, Universidad de Buenos Aires, Buenos Aires, Argentina E-mail: dario@qi.fcen.uba.ar 
the linkage between structure, dynamics and function is highlighted for three representative cases: the migration of ligands through the protein matrix of truncated hemoglobins, the modulation of binding affinity by heme distortion in protoglobin, and finally the functional implications due to the equilibrium between penta- and hexacoordination of the heme with distal histidine in neuroglobin.

\section{Introduction}

Globins are a family of heme-containing proteins found in all kingdoms of life. They belong to the hemeprotein superfamily, though they share some distinctive characteristics. In the Protein Data Bank around 2300 hemeproteins can be found, and they can be clustered in 34 different groups with very diverse structural and functional characteristics [1]. As members of this superfamily, globins are supposed to have evolved from a common ancestor and their characteristic tertiary structure is typically known as globin fold. This fold was identified in 1958 in myoglobin (Mb) [2], which was the first protein whose structure became solved by X-ray diffraction. In this sense, the globin fold was the first protein fold to be discovered. Though it originally consisted of a bundle of eight alpha helices, its generalized definition has been challenged in the last decades due to the discovery of new globins, which have a number of peculiar structural features. The globin fold is an all-alpha protein fold, since the only secondary structure found is the alpha-helix. Though primary sequences of globins can have as low as $16 \%$ sequence identity, the globin fold is highly conserved throughout the family.

Globins have evolved to play a variety of biological roles, such as transport and sensing of gases and catalysis of reactions between nitrogen and reactive oxygen species [3-5]. Some of them are present as monomers under physiological conditions, though others form multimeric species, as illustrated by the prototypical cases of mammalian $\mathrm{Mb}$-monomeric- and hemoglobin $(\mathrm{Hb})$-tetrameric- [5-8]. As mentioned before, globins generally adopt a common globin fold characterized by a 3-over-3 helical sandwich $(\mathrm{A} / \mathrm{BC} / \mathrm{E}$ and $\mathrm{F} / \mathrm{G} / \mathrm{H}$ helices), which contains the hydrophobic pocket that accommodates the heme group. The heme iron is coordinated to the only fully conserved residue along this family: the proximal HisF8 [9], leaving the sixth coordination position in the distal side usually free for binding of the exogenous ligand. Typical exogenous ligands are $\mathrm{NO}, \mathrm{CO}$ and $\mathrm{O}_{2}$, being molecular oxygen the most abundant and the one with the lowest affinity for free heme. Therefore, $\mathrm{O}_{2}$ affinity is a key parameter for gaining insight into the function of globins.

The affinity of a protein for a ligand is characterized by the equilibrium constant $\mathrm{K}$, which in turn can be related with the ratio between the apparent kinetic rate constants for the association and dissociation processes, called $k_{o n}$ and $k_{\text {off }}$ respectively (see Fig. 1 for the binding of $\mathrm{O}_{2}$ to the ferrous form of a hemeprotein). 

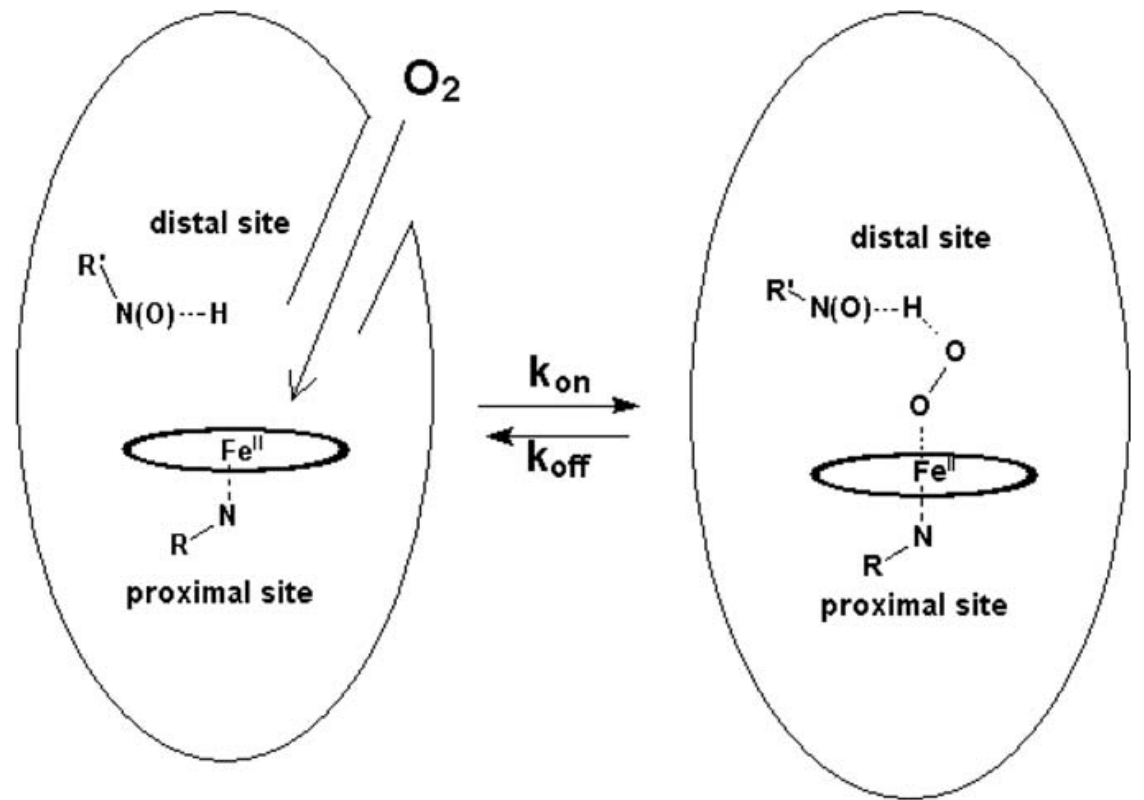

Figure 1. Schematic representation of the association and dissociation processes of small molecules to the heme moiety in globins.

In most globins, the association rate constant depends on two processes: i) ligand migration from the solvent to the heme active site, and ii) ligand coordination to the heme iron [3]. The ligand migration process is regulated by the presence of internal pockets or even tunnels [10-12], and the presence of specific residues that are capable to act as "gates" [7]. In some cases, a distal site residue (mainly His, Tyr) is able to bind to the Fe atom in the so-called internal hexacoordination, which affects the entire process [13-16]. On the other hand, non-coordinated water molecules located in the distal site can also modulate the $k_{o n}$, adding another variable to the complexity of the association process [17-19]. Moreover, the coordination step depends on the spin state of the ligand and the relative in-plane position of the iron. For these reasons, in most cases the value of $k_{o n}$ for $\mathrm{O}_{2}$ is higher than for $\mathrm{CO}$, whereas the $k_{o n}$ value for NO is even higher [7]. The association rates span a wide range of values (spanning up to five orders of magnitude), starting at about $10^{4} \mathrm{M}^{-1} \mathrm{~s}^{-1}$ in those systems with very low accessibility to the iron, and rising to $10^{9} \mathrm{M}^{-1} \mathrm{~s}^{-1}$ when the association rate is mainly controlled by the diffusion from the solvent to reach the protein, as observed for isolated porphyrins $[7,20]$.

The dissociation constant $\left(k_{\text {off }}\right)$ involves two processes: i) thermal breaking of the protein-ligand interactions, and ii) ligand escape from the active site into the solvent. Dissociation rate constants are generally regulated mainly by the protein-ligand thermal breaking step, and span a range of roughly seven orders of magnitude (from $10^{-3} \mathrm{~s}^{-1}$ to $10^{4} \mathrm{~s}^{-1}[3,7,21]$ ). Both 
diffusion and breaking of protein-ligand interaction are processes that vary from ligand to ligand, thus leading to a wide range of ligand affinities. Oxygen binds exclusively to ferrous $\left(\mathrm{Fe}_{\mathrm{II}}\right)$ heme, and its dissociation rate constant is strongly influenced by interactions between the coordinated $\mathrm{O}_{2}$ and the protein matrix [22]. For $\mathrm{CO}$ and $\mathrm{NO}$, the dissociation from ferrous heme is mostly dominated by breaking of the Fe-ligand bond, and similarly low values $\left(\approx 1 \times 10^{-2} \mathrm{~s}^{-1}\right)$ are observed for many different proteins [3]. In the $\mathrm{O}_{2}$ case, the energy required for breaking the protein-ligand bond is regulated by several factors, which include [23,24]:

- distal effect, which accounts for the interaction of the ligand with hydrogen-bond donor residues present in the distal cavity,

- proximal effect, which takes into account the influence of the local structure of the axial histidine, and

- heme distortion, which alters the strength of the Fe-ligand bond.

In the last decades new members of the globin family have been discovered, greatly expanding the globin world. Thus, globins are widely distributed and exhibit an intricate and complex phylogenetic network, which has been proposed to be divided in three main lineages [25]. Novel globins show distinct structural and functional features when compared to the emblematic mammalian $\mathrm{Mb}$ and $\mathrm{Hb}$ (Fig. 2). Apart from the canonical $3 / 3 \mathrm{Mb}$ fold, which encompasses many different globins, another lineage shows a characteristic fold denoted as $2 / 2 \mathrm{Hb}$, which has been found in the three domains of life [25]. The $2 / 2 \mathrm{Hb}$ fold, also referred to as truncated $\mathrm{Hb}$, is around 20-30 residues shorter than $\mathrm{Mb}$ and exhibits a 2-over-2 sandwich fold involving only $\mathrm{BC} / \mathrm{E}$ and $\mathrm{G} / \mathrm{H}$ helices, in contrast to the classical 3 -over-3 fold. The $2 / 2 \mathrm{Hbs}$ have been proposed to act as small gas molecule sensors, oxygen carriers and pseudoenzimes.

Another of these lineages includes protoglobin and globin-coupled sensor (GCS) proteins, whose globin domain is bigger than $\mathrm{Mb}(\sim 190$ amino acids; [25-28]). Protoglobins are the first single-domain GCS-related globins found in Archaea. They can bind $\mathrm{O}_{2}, \mathrm{CO}$ and NO reversibly in vitro, but so far their function is unknown $[25,26,28]$.

In the following sections we firstly describe the main features of $\mathrm{Mb}$, which can be considered a prototypical example found in many textbooks, and three different globins with specific characteristics that make them to be representative examples of the structural and functional diversity of the globin family (Fig. 2):

- Truncated hemoglobins: Members of the 2/2 Hb subgroup, the second lineage of the globin family. In some cases, they have been related to the defense of the bacteria against nitrosative stress. 
- Protoglobin: The 3D structure has been recently solved [29], though its function remains unknown. This protein hosts an unusually distorted heme and exhibits a very low dissociation constant for $\mathrm{O}_{2}$.

- Neuroglobin: Member of the subgroup of globins with relevant internal hexacoordinated phenomena.

\section{Brief survey of mammalian myoglobin (Mb)}

$\mathrm{Mb}$ is not only a member of the first lineage of globins, but one of the most studied proteins. For this reason it is often referred to as the hydrogen atom of biology [30]. Its 3D structure was solved more that 50 years ago by J. Kendrew and coworkers [2], finding that deserved the Nobel Prize in Chemistry in 1962. Despite being one of the most studied proteins, there is still intense research effort due to its complexity, biological relevance and ongoing debate of its physiological function [31].

$\mathrm{Mb}$ is a cytoplasmic globin consisting of 154 amino acids expressed in cardiac mytocytes and oxidative skeletal muscle fibers. Like $\mathrm{Hb}, \mathrm{Mb}$ reversibly binds $\mathrm{O}_{2}$. However, $\mathrm{Mb}$ has a characteristic Michaelis-Menten hyperbolic $\mathrm{O}_{2}$-saturation curve, while a sigmoid-shaped curve is seen in tetrameric $\mathrm{Hb}$, thus reflecting the well known allosterism effect in this latter protein. From a biochemical point of view, $\mathrm{Mb}$ acts as $\mathrm{O}_{2}$ storage protein in muscle, which is especially evident in marine mammals and birds that undergo extended periods of apnea, or in humans and other species living at high altitude. It has also been proposed as a buffer of intracellular $\mathrm{O}_{2}$ pressure in a number of species [31], maintaining $\mathrm{O}_{2}$ concentration relatively constant despite the occurrence of induced changes in $\mathrm{O}_{2}$ level.

There is more controversy about the role of $\mathrm{Mb}$ in assisting $\mathrm{O}_{2}$ diffusion in the cell. Desaturated $\mathrm{Mb}$ close to the cell membrane could bind $\mathrm{O}_{2}$ and diffuse to the mitochondria, thus representing an alternative way of simple $\mathrm{O}_{2}$ diffusion [31]. However, contrary to what it could be expected from its role, knockout experiments in mice with no $\mathrm{Mb}$ in skeletal muscles showed survival of the organism without severe biological consequences. This raises the question of whether other proteins could compensate for $\mathrm{Mb}$ absence. Beyond $\mathrm{O}_{2}$ biochemistry, $\mathrm{Mb}$ has also been related to inactivation of $\mathrm{NO}$ and scavenging reactive $\mathrm{O}_{2}$ species [31].

Besides the described roles and the increasing number of studies done so far, there are still open questions about $\mathrm{Mb}$ that require further research. For instance, what are the factors that regulate its expression in response to hypoxic situations? The discovery of other tissue globins such as neuroglobin (presented in the last section) and cytoglobin raises the question about the complexity of the underneath physiological model governing skeletal muscle biology. 


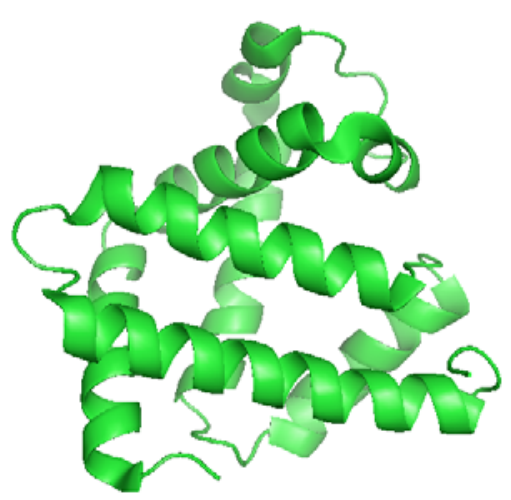

3-on-3 globin fold

Myoglobin
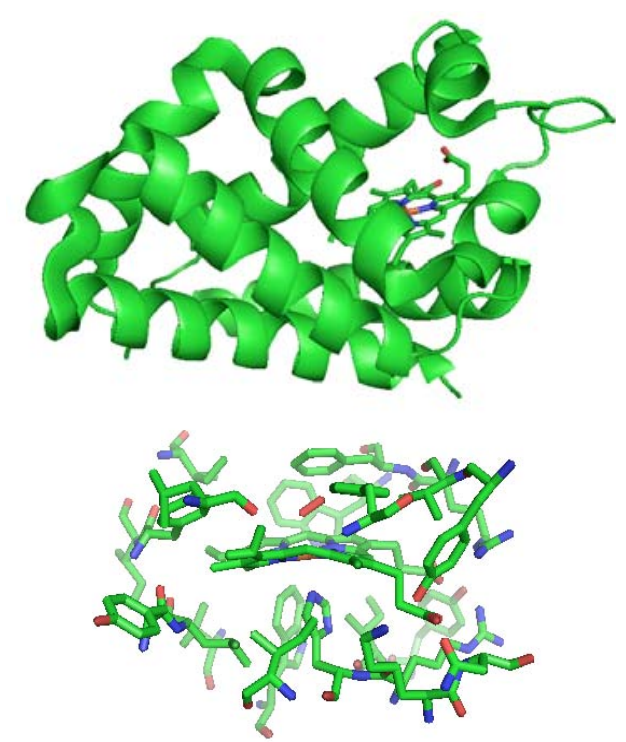

Protoglobin

Highly distorted heme group
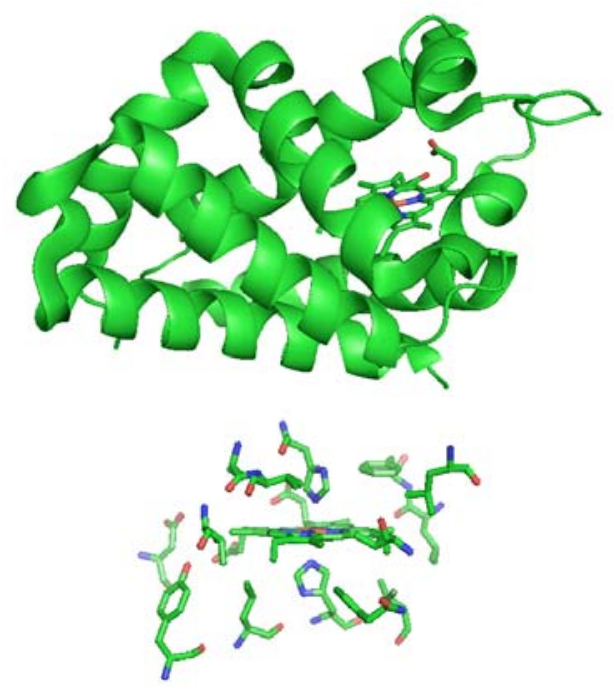

Hexacoordination

Neuroglobin:
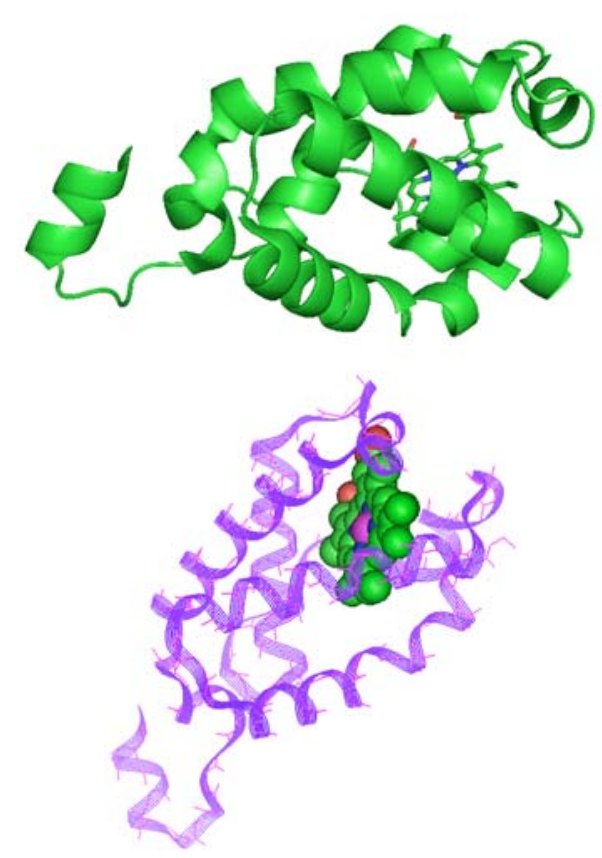

Truncated hemoglobin N:

2-2 Sandwich fold

Figure 2. Globin diversity represented by myoglobin (upper-left), neuroglobin (upperright), protoglobin (lower left) and truncated hemoglobin $N$ (lower right).

\section{2/2 Hbs (truncated) proteins}

The $2 / 2 \mathrm{Hb}$ family can be further divided into 3 groups: I, II, III (also known as $\mathrm{N}, \mathrm{O}, \mathrm{P}$ ). Group I shows a typical tunnel system that connects the solvent with the distal site. Groups II and III share a common TrpG8 and 
generally do not exhibit well delineated tunnels for ligand migration. A network of hydrogen bonds stabilizing the exogenous ligand in the heme distal site is found in all three groups.

Truncated hemoglobin $N$ of Mycobacterium tuberculosis (Mt-trHbN) is the most studied member of the $2 / 2$ globins. For that reason, we will focus on the description of the mechanism of ligand migration from the solvent to the heme pocket in this protein, and later we compare these results with other Hbs belonging to groups $\mathrm{O}$ and $\mathrm{P}$.

\section{The Mt-trHbN case}

Mycobacterium tuberculosis is responsible for tuberculosis in humans [32]. During the first stages of the infection the bacteria is attacked by macrophages that generate large amounts of NO [33]. It has been shown that certain $\mathrm{Hbs}$ that are present in some microorganisms are related to its ability to detoxify NO $[34,35]$. These defense mechanism would be related to $\mathrm{O}_{2}$-bound globins that could convert $\mathrm{NO}$ to nitrate anion following the reaction $\mathrm{Fe}(\mathrm{II})-\mathrm{O}_{2}+\mathrm{NO} \rightarrow \mathrm{Fe}(\mathrm{III})+\mathrm{NO}_{3}{ }^{-}$. In $M$. tuberculosis two $2 / 2 \mathrm{Hbs}$ are capable of performing such detoxifying reaction: truncated $H b N$ and truncated $\mathrm{Hb} \mathrm{O}$. One of the most interesting characteristics of these globins is the presence of an apolar tunel system that connects the solvent with the active site [36], which is postulated to be involved in migration of ligands $\left(\mathrm{O}_{2}, \mathrm{NO}\right)$. In particular, in Mt-trNHb there are two perpendicular tunnels: the so-called Short Tunnel G8 (STG8), which is around $8 \AA$ long and is delineated by residues in helices $\mathrm{G}$ and $\mathrm{B}$, and the Long Tunnel (LT), which is around $20 \AA$ long and is mainly defined by helices B and E (Fig. 3).

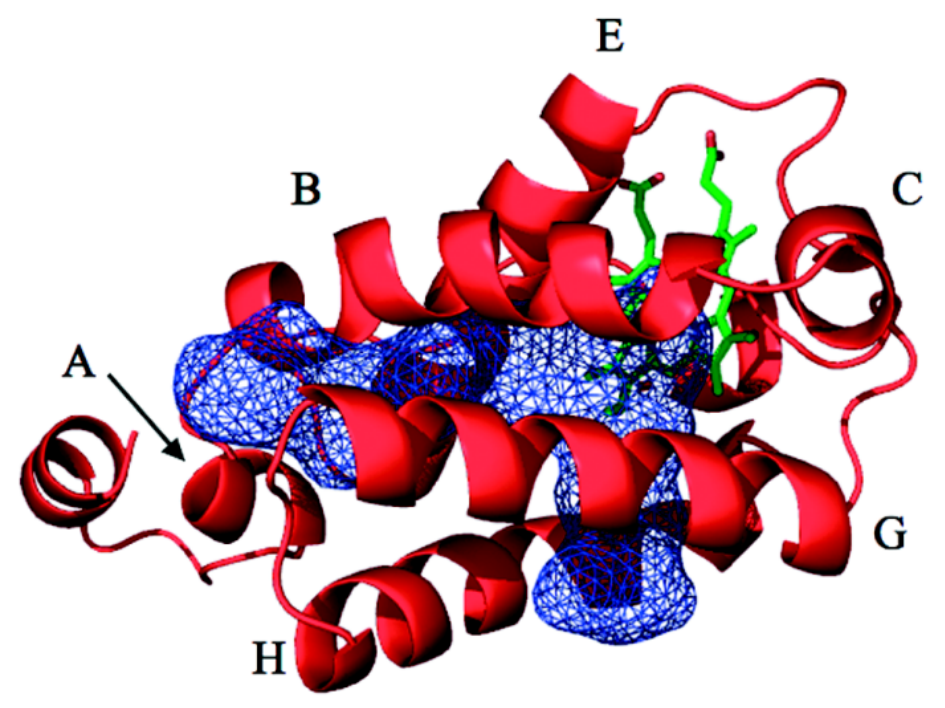

Figure 3. Representation of the two orthogonal branches of the apolar tunnel found in the protein matrix of Mt-trHbN. 
In the crystal structure of the oxygenated globin (PDB code 1IDR [37]) PheE15, which is placed in the middle of the LT, shows two conformations defined by a rotation of $\sim 63^{\circ}$ along the $\mathrm{C}_{\alpha}-\mathrm{C}_{\beta}$ bond. In one conformation the benzene ring is parallel to the tunnel axis, while in the other it is roughly perpendicular. This suggests that the residue could act as a gate for ligand migration being these two conformations, which will be denoted open and closed states, respectively [37].

Molecular dynamics (MD) simulations of the oxygenated form of Mt-trHbN [10] showed several transitions between these two conformational states, in agreement with the conformational flexibility seen in the X-ray structure. In order to explore the functional implications of these findings, Multiple Steered Molecular Dynamics simulations coupled with Jarzinski's equality were used to obtain free energy profiles for NO migration through the tunnel for both open and closed conformations [10]. When PheE15 is in the open conformation, a small barrier (around $2 \mathrm{kcal} / \mathrm{mol}$ ) has to be surpassed in order to access the heme cavity. On the other hand, for the closed state access to the active site is accompanied by a steep increase in the free energy, leading to a barrier of around $5 \mathrm{kcal} / \mathrm{mol}$ (Fig. 4). Similar studies performed for the STG8 show a higher barrier of around $7 \mathrm{kcal} / \mathrm{mol}$. This means that NO entry for the oxygenated Mt-trHbN should mainly occur through the LT.

For the NO detoxifying reaction to take place, the protein must be firstly loaded with $\mathrm{O}_{2}$. MD simulations run for the deoxygenated form of Mt-trHbN showed that PhE15 is only found in the closed state. This is consistent with the free energy barrier for the open/closed torsional transition obtained by

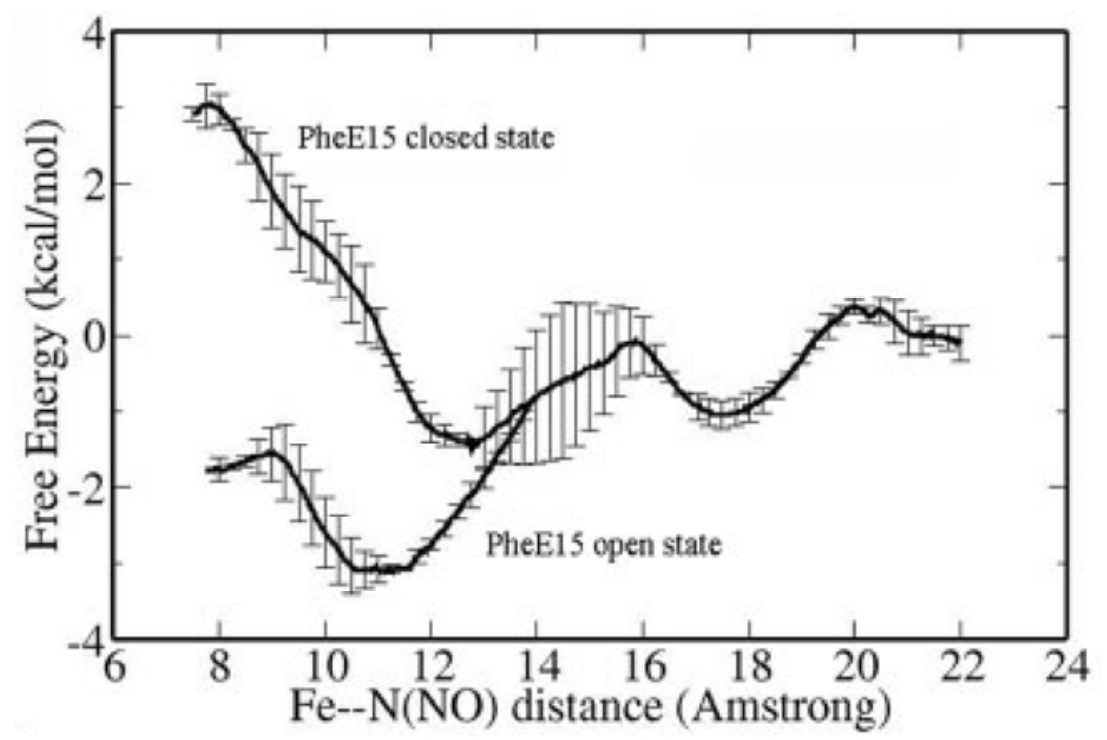

Figure 4. Free energy profile for the migration of NO through the LT in both open and closed states of the PheE15 gate. 
Umbrella Sampling: while the open $\leftrightarrow$ closed transition involves a barrier of $3 \mathrm{kcal} / \mathrm{mol}$ in the oxygenated protein, the barrier increases up to $6 \mathrm{kcal} / \mathrm{mol}$ in the deoxygenated protein. Oxygen entry is then supposed to be achieved through the STG8, as supported by the free energy profiles for ligand migration through this tunnel for the oxygenated and deoxygenated forms. While $7 \mathrm{kcal} / \mathrm{mol}$ are needed for NO to gain access to the active site in the oxygenated form, only $4 \mathrm{kcal} / \mathrm{mol}$ must be surpassed by $\mathrm{O}_{2}$ in the deoxygenated form (Fig. 5).

This behavior suggests that once the Mt-trHbN-O $\mathrm{O}_{2}$ complex is formed, some residues sense the ligand in the distal cavity and favors the conformational change in PheE15, which triggers the aforementioned opening events. This allows NO to enter through the LT and reach the distal cavity for the detoxifying reaction to take place.

A plausible hypothesis to explain the sensing properties of Mt-trHbN relies on residues GlnE11 and TyrB10. In the deoxygenated protein those residues interact by hydrogen bonding between the amide group in GlnE11 and the hydroxyl group in TyrB10. MD simulations show that sometimes GlnE11 is acting as hydrogen-bond donor (and TyrB10 as acceptor), whereas in other snapshots the side chain carbonyl group of GlnE11 is hydrogen-bond acceptor (and TyrB10 is the donor). In all cases GlnE11 is primarily found in an extended all-trans conformation. This fluctuating hydrogen-bond network is drastically altered in the oxygenated protein. Thus, TyrB10 forms a hydrogen-bond with the heme-bound $\mathrm{O}_{2}$ and forces GlnE11 to adopt a folded conformation in order to maintain a hydrogen-bond with TyrB10. In this conformational state, the side chain of GlnE11 is much closer to PheE15, and thermal fluctuations of the side chains would facilitate the opening of the gate.

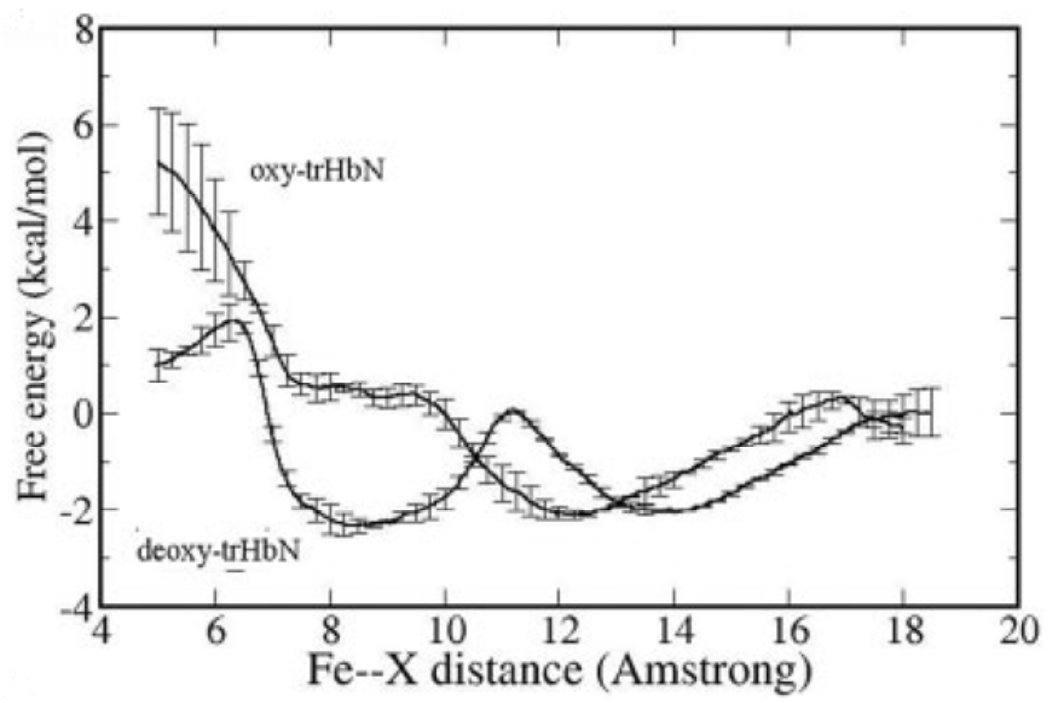

Figure 5. Free energy profile for the migration of NO through the STG8 in both deoxygenated and oxygenated states of the protein. 
Support to this hypothesis also comes from MD simulations run for the TyrB10 $\rightarrow$ Phe mutant [16]. Thus, the analysis of the trajectory reveals an increase in the distance between GlnE11 and PheE15, which implies a reduction in the mechanical pressure exerted by the former residue on the gate. This is due to the fact that in this mutant GlnE11 adopts an extended conformation, which enables the terminal amido group to form a hydrogen-bond directly with the heme-bound $\mathrm{O}_{2}$. For the GlnE11 $\rightarrow$ Ala mutant the protein is predicted to be also inactive, as the lack of the contacts between AlaE11 and PheE15 would make the gate to populate mainly the closed state in the mutant.

These results suggest that multiligand chemistry in Mt-HbN has evolved in such a way that there is a distinct access pathway to the active site for the molecules involved in the reaction: $\mathrm{NO}$ and $\mathrm{O}_{2}$. Oxygen would first enter through the STG8 and bind to the heme. Upon oxygen binding, the hydrogen bond network formed by TyrB10 and GlnE11 is the key feature that regulates the mechanism that triggers the opening of PheE15 gate of the LT for NO entrance.

\section{Other trHbs}

Several efforts have been made in the last years to examine other trHbs by solving X-ray structures, or determining kinetic constants and spectroscopic data, as well as by using MD simulations. At this point, our group has been working on the ligand migration properties in M. tuberculosis trHb (Mt-trHbO) and $B$. subtilis trHb (Bs-trHbO).

For Mt-trHbO, even though a Leu residue occupies the E15 position (thus avoiding the PheE15 gate), the LT is blocked near the heme group. The only accessible tunnel for ligand migration is the Short Tunnel E7 (STE7), which is oriented toward the propionate groups (Fig. 6). This tunnel is topologically

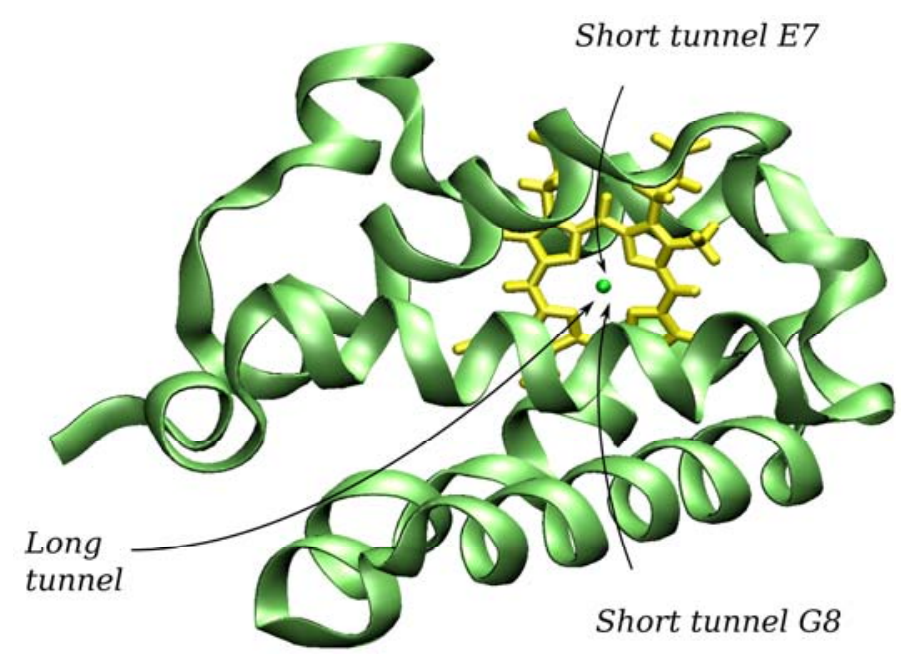

Figure 6. Representation of the backbone in $\mathrm{Mt}-\mathrm{trHbO}$ and schematic view of the three main tunnels found in trHbs. 
related to the E7 "gate" found in Mb. However, although STE7 showed a lower barrier for ligand migration than the LT one [11], it is even higher compared to that observed for Mt-trHbN, a fact that probably explains its low association rate.

Mutation of TrpG8 to smaller residues consistently lowers the $\mathrm{O}_{2}$ entry barrier and increases the association rate, in agreement with the available experimental data [11]. These findings support the important role of TrpG8 in regulating the ligand migration through the LT (Fig. 7). In particular, the mutant TrpG8 $\rightarrow$ Ala opens the STG8 (mentioned above for Mt-trHbN). Therefore, TrpG8 not only blocks LT, but also STG8. Noteworthy, sequence alignment shows that there is a Trp residue at position G8 along the whole O and $\mathrm{P}$ groups of this sub-family.
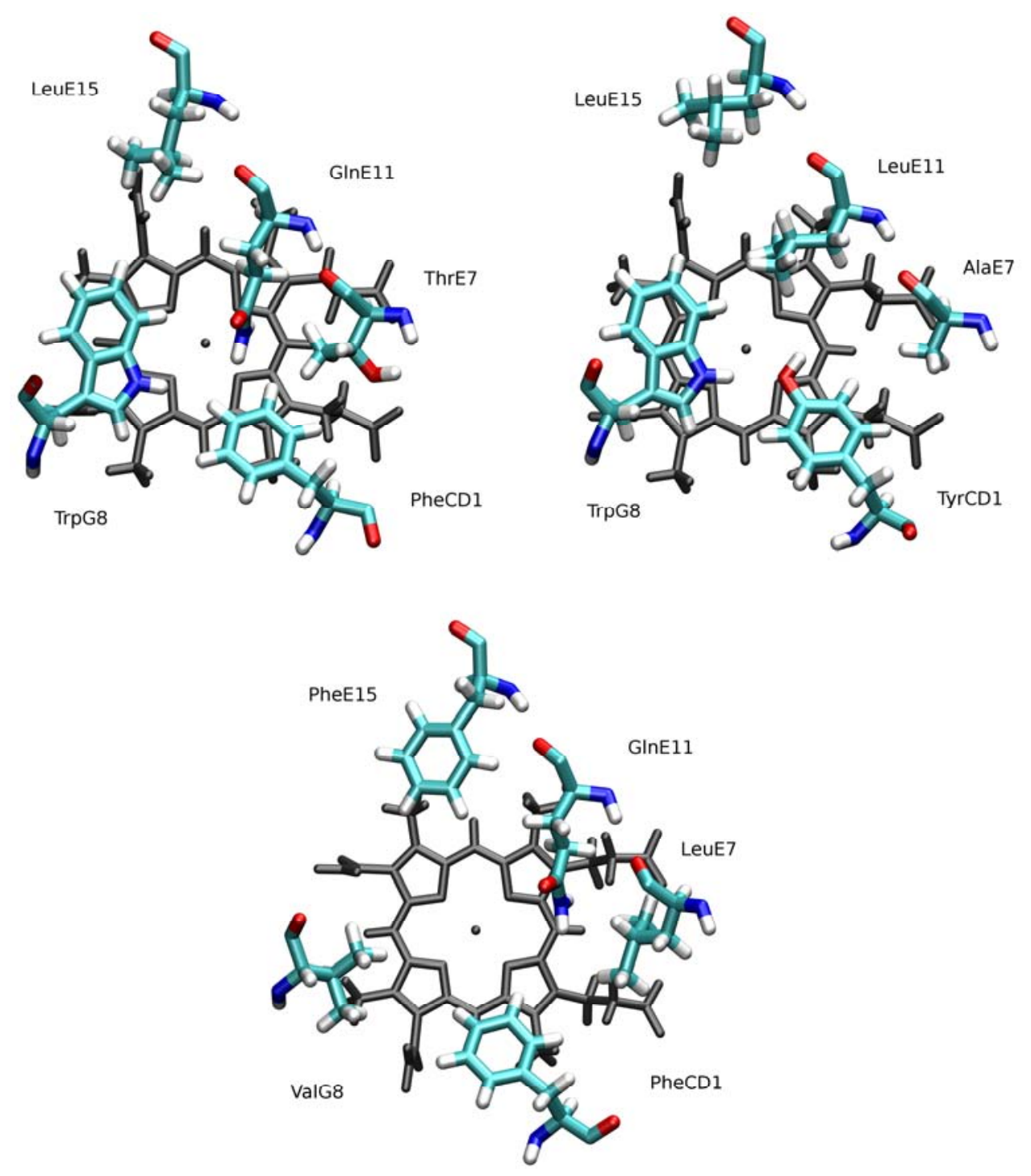

Figure 7. Representation of the main residues at the distal site in (top, left) Bs-trHbO, (top, right) Mt-trHbO, and (bottom) Mt-trHbN. 
The case of Bs-trHbO is even more interesting. Even though inspection of the X-ray structure does not reveal a well-defined tunnel, this protein displays a high association rate [38]. The simulation results showed that GlnE11 adopts a different conformation compared to the position in the crystal structure, opening the LT (Fig. 7). Although Bs-trHbO presents TrpG8 (as in the case of Mt-trHbO), the presence of GlnE11 seems to suffice for opening of the LT. Thus, steered MD simulations run for the Bs-trHbO GlnE11 $\rightarrow$ Leu mutant (designed to mimic $\mathrm{Mt}$-trHbO) showed a large barrier for the migration through the LT, thus indicating that both TrpG8 and LeuE11 are responsible of blocking the LT. Although the presence of TrpG8 was expected to be enough to control ligand entry in these proteins, the presence of GlnE11 -as compared to Leu in Mt-trHbO- clearly contributes to opening of the LT and facilitates ligand entry, in agreement with the experimental values determined for the association rate constants.

Overall, this brief discussion suffices to highlight the close relationship between subtle changes in the nature of certain residues in the interior of the proteins, and the migration properties of gaseous ligands through the protein matrix of structurally related trHbs. Thus, the presence of certain residues at specific positions has a critical role in regulating ligand affinity and reactivity by controlling the barrier for migration of ligands toward the heme active site.

\section{Protoglobin of Methanosarcina acetivorans}

This section is focused in selected structural characteristics of $M$. acetivorans protoglobin (MaPgb), and particularly on the unusually distorted heme found in the X-ray structure of this protein [29], and its possible functional implications.

MaPgb contains around 190 residues and a total of 9 helices, including a pre-A segment named Z. The heme group is highly distorted (Fig. 8) and fully buried in the protein matrix. The propionates are thus inaccessible to the solvent due to the presence of extended CE and FG loops, and to a long (20 residue) N-terminal segment with no secondary structure. In general, globins host an almost planar heme and solvent exposed propionates. Therefore, the large distortion found for MaPgb suggests that heme distortion is due to some sort of tension exerted by the surrounding residues.

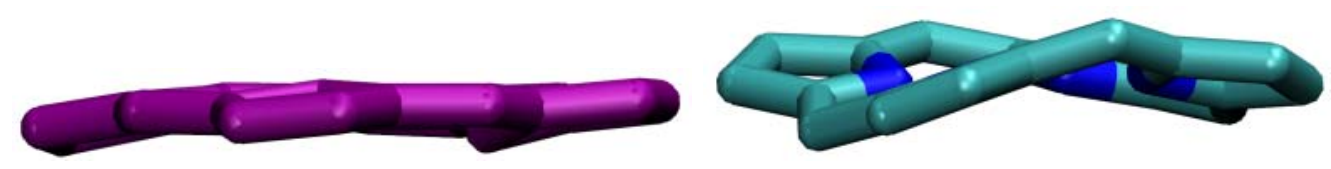

Figure 8. Heme distortion of (right) MaPgb compared to (left) the standard heme of Mb. 
Access of diatomic ligands to the distal site seems feasible through a V-shaped tunnel (Fig. 9), which is topologically different from that found in other globins. Thus, two apolar tunnels connect the solvent with the heme and are delimited by the $\mathrm{B} / \mathrm{G}$ (tunnel 1 ) and $\mathrm{B} / \mathrm{E}$ (tunnel 2) helices.

As mentioned in the Introduction, the $\mathrm{O}_{2}$ dissociation rate can be controlled by distal effects. For instance Mt-trHbN and Ascaris hemoglobin exhibit a very low $k_{\text {off }}$ for $\mathrm{O}_{2}$ due to the presence of multiple hydrogen-bond interactions [23]. In contrast to those proteins, MaPgb has no residues capable of establishing permanent hydrogen bond interactions with the ligand. The only residue that could act as hydrogen-bond donor is TyrB10, but extended MD simulations showed that TyrB10 is mainly involved in hydrogen bonding to LeuE4. Therefore, the low dissociation rate determined experimentally must be related to other mechanisms, like heme distortions.

Bikiel et al. [24] have recently examined the influence played by distinct deformations of the heme on the ligand affinity. A systematic classification of heme distortions, denoted as Normal-Coordinate Structural Decomposition (NSD), has been proposed by Jentzen and coworkers [39]. This technique identifies the most relevant out-of-plane (saddling, ruffling, doming, X-waving, Y-waving and propellering) and in-plane (meso-stretching, N-pyrrole stretching, pyrrole traslation $(\mathrm{X}, \mathrm{Y})$, breathing and pyrrole rotation) normal deformation modes that relate the structure of a distorted heme compared to a reference $\mathrm{D}_{4 \mathrm{~h}}$ structure (Fig. 10).

Table 1 shows the difference in $\mathrm{O}_{2}$ binding affinity for selected deformations of the heme compared to an ideal planar heme determined from

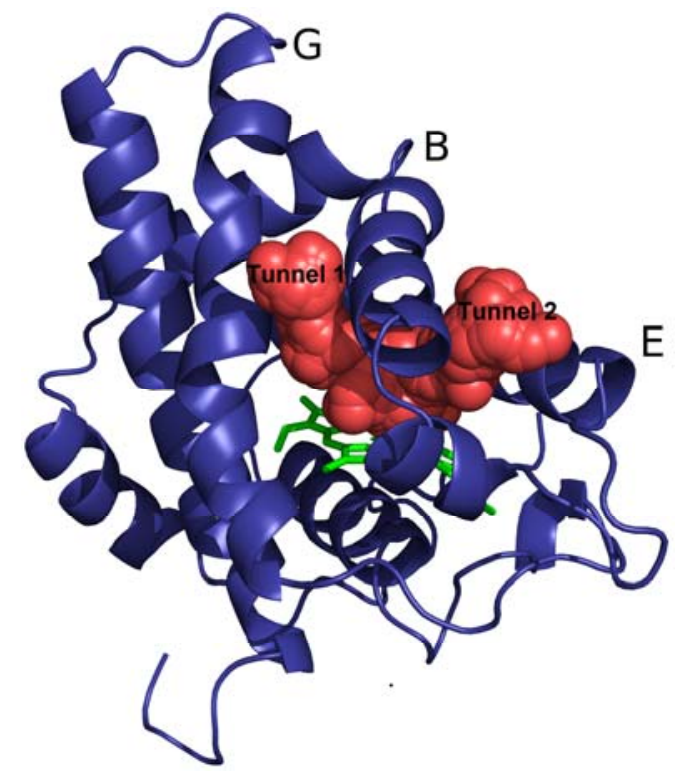

Figure 9. Representation of the V-shaped tunnel found in the X-ray structure of MaPgb. 


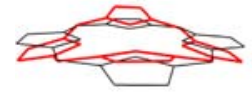

SAD

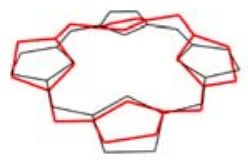

WAX
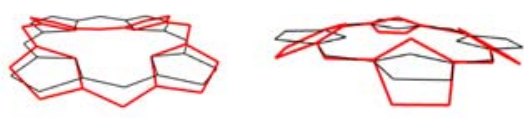

RUF

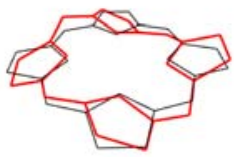

WAY

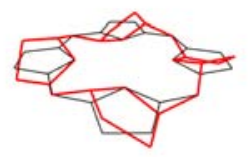

PRO
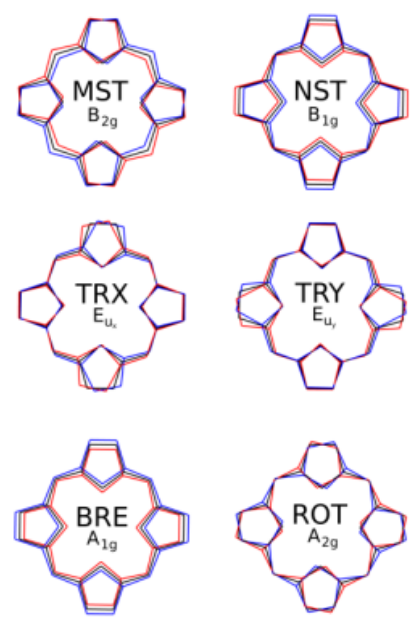

Figure 10. Representation of the normal (left) out-of-plane and (right) in-plane deformation modes of the heme.

Table 1. Difference in binding energy $\left(\Delta\left(\Delta \mathrm{E}_{\mathrm{O} 2}\right) ; \mathrm{kcal} / \mathrm{mol}\right)$ determined for selected distortions $(\AA)$ along out-of-plane and in-plane deformation modes relative to an ideal planar heme. The geometrical deviation from the planarity is measured by the rootmean square deviation (RMSD; $\AA$ ).

\begin{tabular}{|c|c|c|c|c|c|c|c|}
\hline \multicolumn{2}{|l|}{ Out-of-plane } & \multicolumn{6}{|c|}{$\Delta\left(\Delta \mathbf{E}_{\mathrm{O} 2}\right)$} \\
\hline \multicolumn{2}{|l|}{ RMSD } & $-1.2-$ & $-0.6-0$. & 30.0 & 0.3 & 0.61 & 1.2 \\
\hline \multicolumn{2}{|l|}{ Saddling } & 1.9 & $\begin{array}{ll}0.5 & 0.1\end{array}$ & 10.0 & 0.1 & 0.52 & 2.4 \\
\hline \multicolumn{2}{|l|}{ Ruffling } & 2.9 & 0.8 & $4 \quad 0.0$ & -0.1 & $0.1 c$ & 0.6 \\
\hline \multicolumn{2}{|l|}{ Doming } & 7.0 & 2.6 & 00.0 & -0.3 & 0.02 & 2.1 \\
\hline \multicolumn{2}{|l|}{$\mathrm{X}$-waving/Y-waving } & 3.6 & $\begin{array}{ll}0.7 & 0.2\end{array}$ & 20.0 & 0.3 & 1.23 & 3.5 \\
\hline \multicolumn{2}{|c|}{ Propellering } & 2.2 & $\begin{array}{ll}0.5 & 0.1\end{array}$ & 10.0 & 0.1 & 0.52 & 2.2 \\
\hline \multicolumn{2}{|l|}{ In-plane } & \multicolumn{6}{|c|}{$\Delta\left(\Delta \mathrm{E}_{02}\right)$} \\
\hline RMSD & -0.5 & -0.25 & -0.125 & 0.0 & 0.125 & 0.25 & 0.5 \\
\hline Meso-stretching & 2.1 & 0.8 & 0.2 & 0.0 & -0.3 & -0.2 & 0.1 \\
\hline N-pyrrole stretching & 2.0 & 1.1 & 0.3 & 0.0 & 0.3 & 1.1 & 2.0 \\
\hline Pyrrole traslation $\mathrm{X} / \mathrm{Y}$ & 0.3 & 0.1 & 0.0 & 0.0 & 0.0 & 0.0 & 0.2 \\
\hline Breathing & 11.3 & 5.6 & 3.1 & 0.0 & -3.6 & -6.7 & -11.3 \\
\hline Pyrrole rotation & 2.0 & 0.6 & 0.2 & 0.0 & 0.0 & 0.2 & 1.3 \\
\hline
\end{tabular}


B3LYP/6G-31G** calculations [24]. All out-of-plane and in-plane distortions lead to an increase in the energy difference, which tends to reduce the ligand affinity. Fig. 11 shows the energy profiles arising from the ruffling (RUF) deformation as an example of this general trend: both positive and negative values of distortion decrease affinity. Only very small negative values are observed for slight distortions along the MST, DOM and RUF modes, but they do not alter the general trend. The only exception to this behavior is the inplane BRE distortion, since a negative trend in $\Delta(\Delta \mathrm{E})$ is clearly observed for positive values of BRE. This is represented in Fig. 11, where negative values for BRE distortion behave similarly to the rest of the normal modes, while positive values show negative $\Delta(\Delta \mathrm{E})$, that is, compression of the heme (positive BRE) increases the ligand affinity.

The NSD analysis of the porphyrin ring in MaPgb shows that the main out-of-plane contribution to the heme deformation is ruffling, which accounts for a distortion of $1.42 \AA$ (in $\mathrm{Mb}$, this distortion only amounts to $0.02 \AA$ ). Regarding in-plane distortions, MaPgb results in a displacement around 10 -fold larger compared to $\mathrm{Mb}$, which is mainly due to breathing. Whereas the
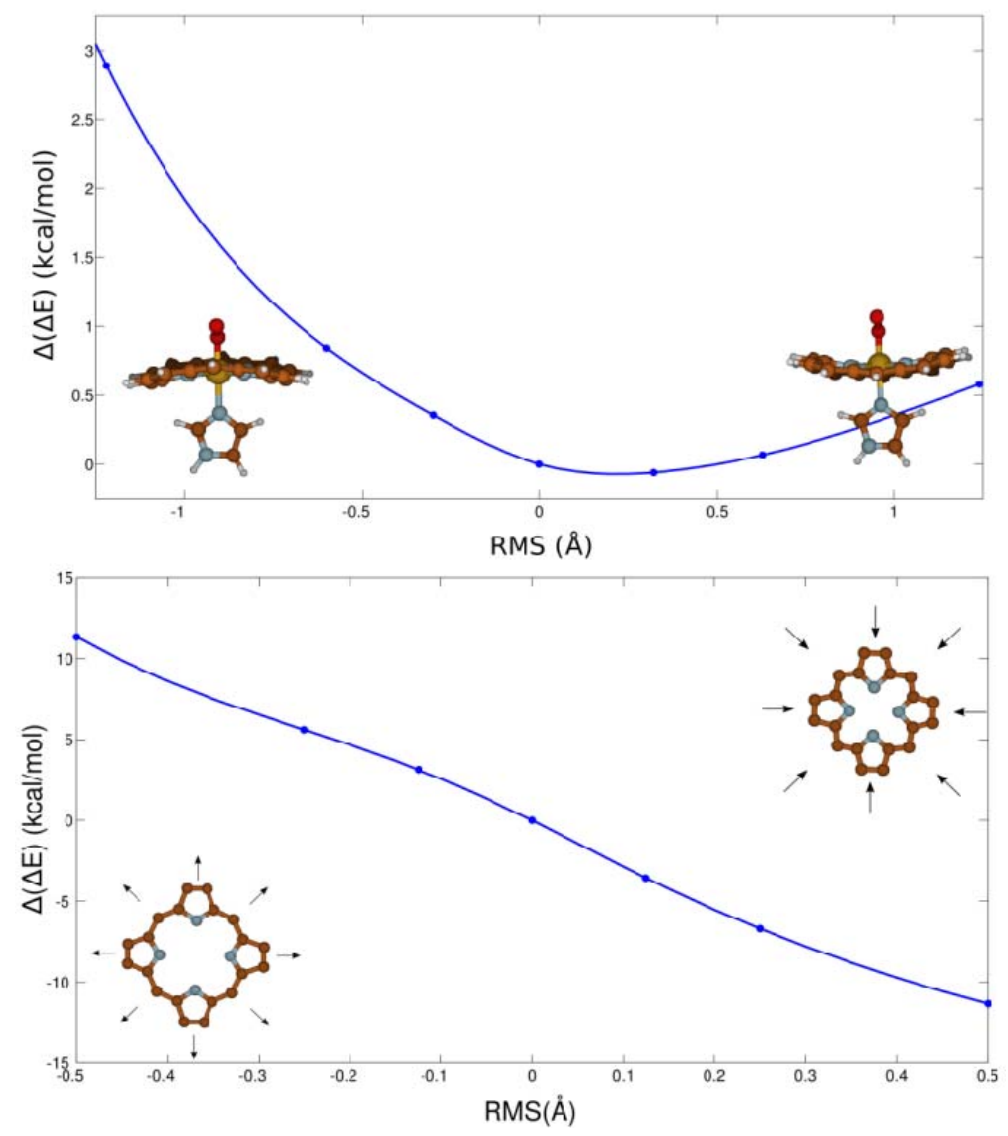

Figure 11. Representation of energy difference profiles due to (top) RUF and (bottom) BRE deformation modes. 
out-of-plane distortions in MaPgb reduce the binding affinity by $1.5 \mathrm{kcal} / \mathrm{mol}$, the positive breathing enhances ligand binding by $4.7 \mathrm{kcal} / \mathrm{mol}$. The net effect is an overall increase in oxygen affinity for MaPgb due to heme distortion.

These findings suggest that evolution has led to the design of a tighter cavity for the heme in MaPgb, which induces an overall heme compression and in turn an increase in oxygen affinity. Although the function of MaPgb is still unkown, these results can shed light on an inherent modulation mechanism for ligand affinity in globins.

\section{Neuroglobin}

Neuroglobin $(\mathrm{Ngb})$ is a member of the group formed by endogenous reversibly hexacoordinated globins. Many globins contain HisE7, normally responsible for $\mathrm{O}_{2}$ stabilization by $\mathrm{H}$ bonding upon heme- $\mathrm{O}_{2}$ complex is formed. However, in some globins, HisE7 is directly bound to the sixth coordination site of $\mathrm{Fe}$ in the heme, forming an hexacoordinated (6c) globin. This is the case of $\mathrm{Ngb}$.

Ngb was firstly identified by Burmester et al. in 2000 [40] in man and mouse, and the X-ray structure of human and murine Ngbs have been solved $[41,42]$. Later Ngb has also been identified in rat, pufferfish and zebrafish [43, 44], which suggests a widespread distribution among vertebrate species. Its function might be related to the protection of cells from stroke damage, amyloid toxicity and injury due to lack of oxygen, and neuroprotection [45]. Although the exact mechanisms by which Ngb protects cells are still unclear, it is suggested that it maintains the function of mitochondria and regulates the concentration of important chemicals in the cell. Increased risk of Alzheimer's disease has been related to low levels of Ngb [46]. Ngb mRNA and protein have been shown to be upregulated by hypoxia and post-anoxia re-oxygenation, which suggest a putative role as a reactive oxygen species scavenger [47].

$\mathrm{Ngb}$ is a monomeric 151-residue globin that shows less than $25 \%$ sequence identity with $\mathrm{Mb}$. Though it retains the canonical 3-over-3 $\alpha$-helical sandwich of mammalian $\mathrm{Mb}$ and $\mathrm{Hb}$, notable structural deviations are found in the CD-D region and in the $\mathrm{N}$-terminal half of the $\mathrm{E}$ helix. As a result of hexacoordination through HisE7, E helix is pulled toward the heme relative to $\mathrm{Mb}$.

Pentacoordinated (5c) Ngb exhibits a very high affinity for $\mathrm{O}_{2}$ but because of the endogenous hexacoordination, it results in a moderate $\mathrm{O}_{2}$ affinity $\left(\mathrm{P}_{\mathrm{O}_{2}}=2\right.$ torr), similar to $\mathrm{Mb}$. The reversible hexacoordination could serve as a way to fine tune the $\mathrm{O}_{2}$ affinity. When $5 \mathrm{c}$ state is favoured affinity should be higher, though it also depends on the residues present in the distal cavity. This hypothesis was confirmed by experiments that drive the $5 \mathrm{c}-6 \mathrm{c}$ equilibrium toward the pentacoordinated state under oxidizing conditions that favor Cys disulfide bridge formation [48, 49]. 
MD simulations (MD), essential dynamics and steered molecular dynamics have been used to gain a molecular-level picture of the $5 \mathrm{c} \leftrightarrow 6 \mathrm{c}$ transition and to estimate its thermodynamic properties [50]. Fig. 12 shows the free energy profile for the $6 \mathrm{c}$ to $5 \mathrm{c}$ transition in Cys reduced and oxidized states, called Cred and Cox, respectively. Reduction of disulfide bridge increases the barrier for the transition from 6.2 to $7.3 \mathrm{kcal} / \mathrm{mol}$, with a $2.6 \mathrm{kcal} / \mathrm{mol}$ increase in the free energy of $5 \mathrm{c}$-Cred state (relative to the 5 c.Cox) state. In terms of rate constant, this implies a decrease by a factor of 6 , and around 78 in the equilibrium constant.

These trends agree with the experimental results reported by Hamdane and coworkers [48, 49]. The overall conclusion is that Cys oxidation stabilizes the 5c state, favoring more avid-for-oxygen species, which supports a mechanism of oxygen release in case of hypoxic conditions, thus suggesting an $\mathrm{O}_{2}$ storage function for $\mathrm{Ngb}$. If oxygen concentration becomes critically low, the disulfide bridge in Ngb would be in the reduced state, which would in turn release oxygen to the cell. If oxygen concentration increases the disulfide bridge will form, triggering an increase in affinity for molecular oxygen.

Capece et al. [51] gained insight into the $5 \mathrm{c} \leftrightarrow 6 \mathrm{c}$ equilibrium molecular determinants by means of MD simulations of $\mathrm{Ngb}$ and $\mathrm{Mb}$ at normal (1 bar) and high ( $3 \mathrm{kbar}$ ) pressure conditions in both coordination states. The overall conclusion of these studies is that the main differences between both proteins are located in the CD loop, whose structure is much more sensitive to both pressure and coordination state in $\mathrm{Ngb}$ than in $\mathrm{Mb}$. This is reflected in the free energy profiles for the $5 \mathrm{c} \leftrightarrow 6 \mathrm{c}$ transition in $\mathrm{Ngb}$ and $\mathrm{Mb}$ (Fig. 13). In Ngb the barrier increases from $7 \mathrm{kcal} / \mathrm{mol}$ at 1 bar to $13 \mathrm{kcal} / \mathrm{mol}$ at $3 \mathrm{kbar}$, with a destabilization of $4 \mathrm{kcal} / \mathrm{mol}$ of the $5 \mathrm{c}$ state. As shown experimentally, the

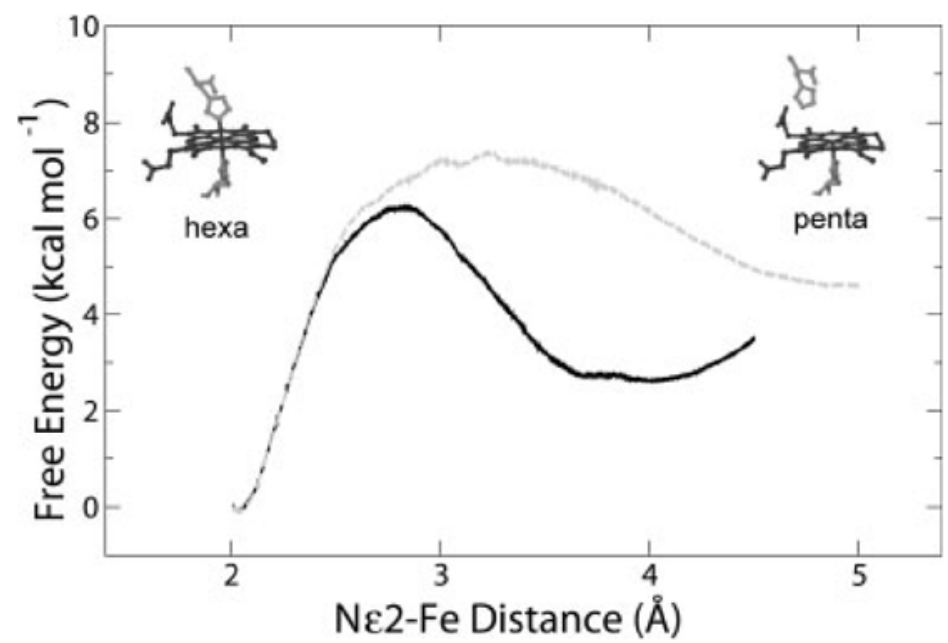

Figure 12. Free energy profile for the $5 \mathrm{c} \leftrightarrow 6 \mathrm{c}$ transition with Cys residues in both reduced (gray) and oxidized (black) states. 
A)

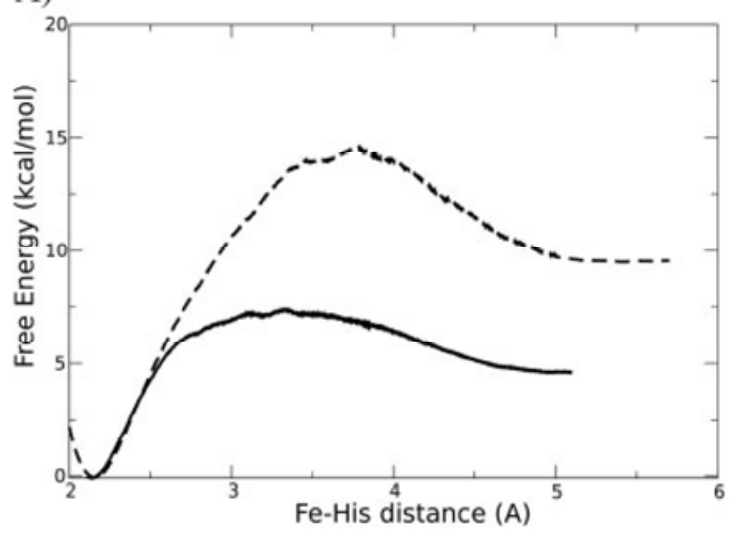

B)

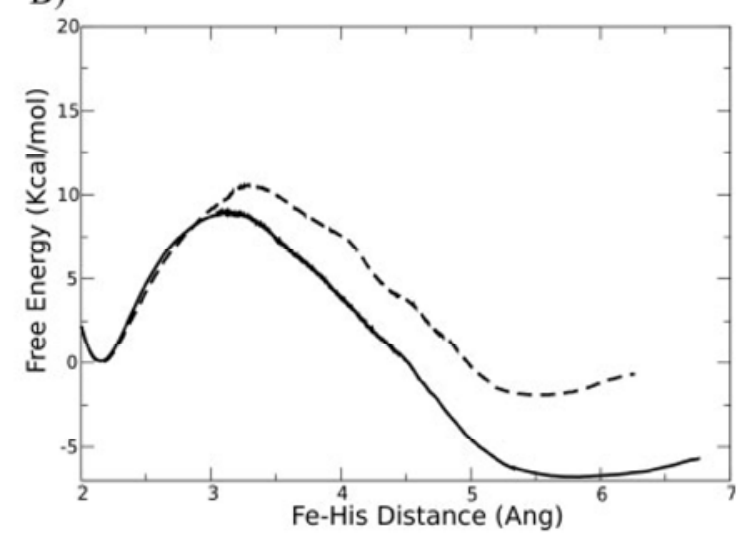

Figure 13. Free energy profile for the $5 \mathrm{c} \leftrightarrow 6 \mathrm{c}$ transition at 1 bar (solid line) and $3 \mathrm{kbar}$ (dashed line) in (A) Ngb and (B) Mb.

hexacoordination state is favored by an increase in pressure [52]. For Mb, the pentacoordinated state in more stable at 1 bar than the hexacoordinated state by $6 \mathrm{kcal} / \mathrm{mol}$, whereas this difference is reduced to $1 \mathrm{kcal} / \mathrm{mol}$ at $3 \mathrm{kbar}$, with a reduction in the barrier for the transition.

Following the previous work by Nadra et al. [50], the disulfide bridge is found to have an important effect on the structure of the CD region in both coordination states, being the effect smaller than the variation with pressure. It seems that the oxidation state of the key Cys has an impact focused on the CD loop, while pressure has a more general influence on the overall structure of the protein.

The preceding results show that pressure alters the dynamics of globins, specifically reducing mobility and shifting the $5 \mathrm{c} \leftrightarrow 6 \mathrm{c}$ equilibrium toward the hexacoordinated state. However the reasons for this shift are different in $\mathrm{Mb}$ and $\mathrm{Ngb}$. In Ngb, pressure mainly affects the mobility in the $\mathrm{CD}$ region and increases the barrier for the $5 \mathrm{c} \leftrightarrow 6 \mathrm{c}$ transition. In contrast, the coordination equilibrium in $\mathrm{Mb}$ involves a more global structural rearrangement and pressure destabilizes the $5 \mathrm{c}$ state. Although the overall trend for the $5 \mathrm{c} \leftrightarrow 6 \mathrm{c}$ transition is the same in both cases -the higher the pressure, the more favored the hexacoordination-, the underlying differences highlight the existence of diverse response mechanisms upon changes in external conditions within the globin world.

\section{Conclusion}

The advances made in the last decade on the structural and functional variation found within the globin world reinforces the idea that the thoroughly studied $\mathrm{Mb}$ and $\mathrm{Hb}$ are just specialized cases in a broad evolutionary 
superfamily that evolved specifically to the demands of circulatory systems and muscles. The three cases presented here ( $\mathrm{Ngb}, \mathrm{MaPgb}$ and $\mathrm{MtHbN}$ ) are representative examples of the growing diversity discovered within globins. Even though they reflect the basic chemical properties of a heme group buried in a conserved globin fold, it is clear that a proper understanding of the structural and dynamical differences between globins is fundamental to gain insight into the functional role of novel globins.

\section{Acknowledgements}

This work was partially supported by the University of Buenos Aires, ANPCyT (PICT-25667), CONICET, the Spanish Ministerio de Innovación y Ciencia (SAF200805595 and PCI2006-A7-0688), the Generalitat de Catalunya (2009-SGR00298), and the EU FP7 program (project NOStress).

\section{References}

1. Smith, L. J., Kahraman, A., Thornton, J. M. Proteins , 78, 2349.

2. Kendrew, J. C., Bodo, G., Dintzis, H. M., Parrish, R. G., Wyckoff, H., Phillips, D. C. 1958, Nature, 181, 662.

3. Ghosh, A. 2007, The smallest biomolecules: diatomics and their interactions with heme proteins, Elsevier.

4. Paoli, M., Marles-Wright, J., Smith, A. 2002, DNA Cell. Biol. 21, 271.

5. Vinogradov, S. N., Moens, L. 2008, J. Biol. Chem., 283, 8773.

6. Frauenfelder, H., McMahon, B. H., Fenimore, P. W. 2003, Proc. Natl. Acad. Sci. USA, 100, 8615.

7. Scott, E. E., Gibson, Q. H., Olson, J. S. 2001, J. Biol. Chem., 276, 5177.

8. Wittenberg, J. B., Wittenberg, B. A. 2003, J. Exp. Biol., 206, 2011.

9. Kapp, O. H., Moens, L., Vanfleteren, J., Trotman, C. N., Suzuki, T., Vinogradov, S. N. 1995, Prot. Sci., 4, 2179.

10. Bidon-Chanal, A., Marti, M. A., Crespo, A., Milani, M., Orozco, M., Bolognesi, M., Luque, F. J., Estrin, D. A. 2006, Proteins, 64, 457.

11. Boechi, L., Marti, M. A., Milani, M., Bolognesi, M., Luque, F. J., Estrin, D. A. 2008, Proteins, 73, 372.

12. Boechi, L., Manez, P. A., Luque, F. J., Marti, M. A., Estrin, D. A. 2009, Proteins, $78,962$.

13. Nadra, A. D., Martí, M. A., Pesce, A., Bolognesi, M., Estrin, D. A. 2007, Proteins, 71, 695.

14. Brunori, M., Vallone, B. 2007, Cell. Mol. Life Sci., 64, 1259.

15. Lama, A., Pawaria, S., Bidon-Chanal, A., Anand, A., Gelpi, J. L., Arya, S., Marti, M., Estrin, D. A., Luque, F. J., Dikshit, K. L. 2009, J. Biol. Chem., 284, 14457.

16. Bidon-Chanal, A., Marti, M. A., Estrin, D. A., Luque, F. J. 2007, J. Am. Chem. Soc., 129, 6782 . 
17. Goldbeck, R. A., Pillsbury, M. L., Jensen, R. A., Mendoza, J. L., Nguyen, R. L., Olson, J. S., Soman, J., Kliger, D. S., Esquerra, R. M. 2009, J. Am. Chem. Soc., $131,12265$.

18. Goldbeck, R. A., Bhaskaran, S., Ortega, C., Mendoza, J. L., Olson, J. S., Soman, J., Kliger, D. S., and Esquerra, R. M. 2006, Proc. Natl. Acad. Sci. USA, 103, 1254

19. Ouellet, Y. H., Daigle, R., Laguee, P., Dantsker, D., Milani, M., Bolognesi, M., Friedman, J. M., Guertin, M. 2008, J. Biol. Chem., 283, 27270.

20. Laverman, L. E., Ford, P. C. 2001, J. Am. Chem. Soc., 123, 11614.

21. Milani, M., Pesce, A., Nardini, M., Ouellet, H., Ouellet, Y., Dewilde, S., Bocedi, A., Ascenzi, P., Guertin, M., Moens, L., Friedman, J. M., Wittenberg, J. B., Bolognesi, M. 2005, J. Inorg. Biochem., 99, 97.

22. Bikiel, D. E., Boechi, L., Capece, L., Crespo, A., De Biase, P. M., Di Lella, S., González Lebrero, M. C., Martí, M. A., Nadra, A. D., Perissinotti, L. L., Scherlis, D. A., Estrin, D. A. 2006, Phys. Chem. Chem. Phys., 8, 5611.

23. Marti, M. A., Crespo, A., Capece, L., Boechi, L., Bikiel, D. E., Scherlis, D. A., Estrin, D. A. 2006, J. Inorg. Biochem., 100, 761.

24. Bikiel, D. E., Forti, F., Boechi, L., Nardini, M., Luque, F. J., Martí, M. A., Estrin, D. A. 2010, J. Phys. Chem. B, 114, 8536.

25. Vinogradov, S. N., Hoogewijs, D., Bailly, X., Mizuguchi, K., Dewilde, S., Moens, L., Vanfleteren, J. R. 2007, Gene, 398, 132.

26. Vinogradov, S., Hoogewijs, D., Bailly, X., Arredondo-Peter, R., Gough, J., Dewilde, S., Moens, L., Vanfleteren, J. 2006, BMC Evol. Biol., 6, 31.

27. Freitas, T. A. K., Hou, S., Dioum, E. M., Saito, J. A., Newhouse, J., Gonzalez, G., Gilles-Gonzalez, M.-A., Alam, M. 2004, Proc. Natl. Acad. Sci. USA, $101,6675$.

28. Hou, S., Freitas, T., Larsen, R. W., Piatibratov, M., Sivozhelezov, V., Yamamoto, A., Meleshkevitch, E. A., Zimmer, M., Ordal, G. W., Alam, M. 2001, Proc. Natl. Acad. Sci. USA, 98, 9353.

29. Nardini, M., Pesce, A., Thijs, L., Saito, J. A., Dewilde, S., Alam, M., Ascenzi, P., Coletta, M., Ciaccio, C., Moens, L., Bolognesi, M. 2008, EMBO Rep., 9, 157.

30. Tomita, A., Sato, T., Ichiyanagi, K., Nozawa, S., Ichikawa, H., Chollet, M., Kawai, F., Park, S.-Y., Tsuduki, T., Yamato, T., Koshihara, S.-Y., Adachi, S.-I. 2009, Proc. Natl. Acad. Sci. USA, 106, 2612.

31. Ordway, G. A., Garry, D. J. 2004, J. Exp. Biol., 207, 3441.

32. Bloom, B. 1994, Tuberculosis: pathogenesis, protection and control, ASM Press, Washington, DC.

33. MacMicking, J. D., North, R. J., LaCourse, R., Mudgett, J. S., Shah, S. K., Nathan, C. F. 1997, Proc. Natl. Acad. Sci. USA, 94, 5243.

34. Ouellet, H., Ouellet, Y., Richard, C., Labarre, M., Wittenberg, B., Wittenberg, J., Guertin, M. 2002, Proc. Natl. Acad. Sci. USA, 99, 5902.

35. Couture, M., Yeh, S.-R., Wittenberg, B. A., Wittenberg, J. B., Ouellet, Y., Rousseau, D. L., Guertin, M. 1999, Proc. Natl. Acad. Sci. USA, 96, 11223.

36. Pesce, A., Nardini, M., Milani, M., Bolognesi, M. 2007, IUBMB Life, 59, 535.

37. Milani, M., Pesce, A., Ouellet, Y., Ascenzi, P., Guertin, M., Bolognesi, M. 2001, EMBO J., 20, 3902. 
38. Giangiacomo, L., Ilari, A., Boffi, A., Morea, V., Chiancone, E. 2005, J. Biol. Chem., 280, 9192.

39. Jentzen, W., Song, X. Z., Shelnutt, J. A. 1997, J. Phys. Chem. B, 101, 1684.

40. Burmester, T., Weich, B., Reinhardt, S., Hankeln, T. 2000, Nature, 407, 520.

41. Pesce, A., Dewilde, S., Nardini, M., Moens, L., Ascenzi, P., Hankeln, T., Burmester, T., Bolognesi, M. 2003, Structure, 11, 1087.

42. Vallone, B., Nienhaus, K., Brunori, M., Nienhaus, G. U. 2004, Proteins, 56, 85.

43. Awenius, C., Hankeln, T., Burmester, T. 2001, Biochem. Biophys. Res. Commun., $287,418$.

44. Zhang, C., Wang, C., Deng, M., Li, L., Wang, H., Fan, M., Xu, W., Meng, F., Qian, L., He, F. 2002, Biochem. Biophys. Res. Commun., 290, 1411.

45. Greenberg, D. A., Jin, K., Khan, A. A. 2008, Curr. Opin. Pharmacol., 8, 20.

46. Raychaudhuri, S., Skommer, J., Henty, K., Birch, N., Brittain, T. 2009, Apoptosis, 15, 401.

47. Nayak, G. H., Milton, S. L., Prentice, H. M. 2007, FASEB J., 21, A924.

48. Hamdane, D., Kiger, L., Dewilde, S., Green, B. N., Pesce, A., Uzan, J., Burmester, T., Hankeln, T., Bolognesi, M., Moens, L., Marden, M. C. 2004, Micron, 35, 59.

49. Hamdane, D., Kiger, L., Dewilde, S., Green, B. N., Pesce, A., Uzan, J., Burmester, T., Hankeln, T., Bolognesi, M., Moens, L., and Marden, M. C. 2003, J Biol. Chem., 278, 51713

50. Nadra, A. D., Martí, M. A., Pesce, A., Bolognesi, M., Estrin, D. A. 2008, Proteins, 71, 695.

51. Capece, L., Marti, M. A., Bidon-Chanal, A., Nadra, A., Luque, F. J., Estrin, D. A. 2008, Proteins, 75, 885.

52. Hamdane, D., Kiger, L., Hoa, G. H. B., Dewilde, S., Uzan, J., Burmester, T., Hankeln, T., Moens, L., Marden, M. C., 2005, J. Biol. Chem., 280, 36809. 


\title{
7. Involvement of nicotinic receptors in methamphetamine and MDMA induced neurotoxicity: Pharmacological studies
}

\author{
Elena Escubedo, Sara García-Ratés, Jordi Camarasa and David Pubill \\ Unitat de Farmacologia i Farmacognòsia. Facultat de Farmàcia \\ Universitat de Barcelona, 08028 Barcelona. Spain
}

\begin{abstract}
During the last years, our emphasis has focused in the study of the neurotoxic effects of 3,4methylenedioxymethamphetamine (MDMA) and methamphetamine (METH) on central nervous system and their pharmacological prevention. In the process of this research, we have used a semipurified synaptosomal preparation from striatum of mice or rats as a reliable in vitro model to study reactive oxygen species (ROS) production by these amphetamine derivatives, which is well correlated with their dopaminergic injury in in vivo models. Using this preparation we have demonstrated that blockade of alpha7 nicotinic receptors with methyllycaconitine (MLA) and memantine (MEM) prevents ROS production induced by MDMA and METH.

Studies at molecular level showed that both, MDMA and METH, displaced competitively the binding of radioligands for homomeric alpha7 and heteromeric nAChRs, indicating that they can directly interact with them. In all the cases MDMA displayed higher affinity than METH and it was higher for heteromeric than
\end{abstract}


for alpha7 subtype. Preincubation of differentiated PC12 cells with MDMA or METH induces nicotinic acetylcholine receptors (nAChR) up-regulation in a concentrationand time-dependent manner, as many nicotinic ligands do, supporting their functional interaction with nAChRs. Such interaction expands the pharmacological profile of amphetamines and can account for some of their effects.

\section{Introduction}

Amphetamine derivatives, such as methamphetamine (METH, speed) and 3,4-methylenedioxymethamphetamine (MDMA, ecstasy) are widely abused drugs, mainly by young people in recreational settings. Besides their stimulatory effects, these drugs have been shown to be neurotoxic in animal models (for reviews see [1, 2]) in which deleterious effects in dopamine (DA) and serotonin (5-HT) nerve terminals have been reported. In addition, signs derived from neurotoxicity have been described in humans who are heavy users of such drugs [3-6]. The patterns of neurotoxicity of MDMA in mice and rats differ in that mice typically exhibit neurotoxicity to both DA- and 5-HT-containing neurons, whereas rats commonly display selective neurotoxicity to 5-HT-containing neurons $[7,8]$.

Two theories have arisen to explain this neurotoxicity. Firstly, the neurotoxicity induced by MDMA may at least partially be a consequence of its metabolism [9]. This hypothesis is based on the fact that a direct intracerebral injection of MDMA failed to reproduce the neurotoxicity profile that appears after its peripheral administration [10]. The other main theory involves reactive oxygen species (ROS), although the two theories cannot be considered mutually exclusive. Oxidative stress appears to be one of the main factors involved in the serotonergic and dopaminergic terminal injury induced by MDMA $[11,12]$.

Although oxidative stress has been proposed as a key neurotoxic mechanism induced by these drugs $[13,14]$, several aspects surrounding the concrete pathways involved in METH- and MDMA-induced ROS generation remain unresolved. Our research group has recently reported not only that METH and MDMA induce ROS production inside rat and mouse striatal synaptosomes, but also that endogenous DA is needed for this reaction to occur [15-17]. We also determined that methyllycaconitine (MLA), an antagonist of $\alpha 7$ nicotinic acetylcholine receptors ( $\alpha 7 \mathrm{nAChR})$, prevented in vitro ROS generation and attenuated in vivo neurotoxicity, thus implicating $\alpha 7 \mathrm{nAChR}$ in the toxicity of amphetamine derivatives.

$\alpha 7 \mathrm{nAChR}$ are homomeric ligand-gated ion channels whose activation induces calcium influx. Calcium entry could favour the activation of $\mathrm{Ca}^{2+}$-dependent enzymes such as protein kinase $\mathrm{C}$ (PKC) and neuronal nitric oxide synthase (nNOS), which have similarly been implicated in the neurotoxicity 
of amphetamines $[18,19]$. In light of these findings, we believed it necessary to assess whether METH and MDMA have a direct interaction with $\alpha 7 \mathrm{nAChR}$.

Certain previous reports have suggested that amphetamines interact with different nicotinic receptors. Liu et al. [20] reported that D-amphetamine acts as an agonist on nicotinic receptors (probably $\alpha 7$ ) in bovine chromaffin cells, inducing catecholamine release. In addition, Skau and Gerald (1978) [21] had reported that $\mathrm{D}$-amphetamine inhibits $\alpha$-bungarotoxin binding at the neuromuscular junction in mice, while Klingler et al. [22] recently identified nAChR as one of the physiological targets of MDMA in the neuromuscular junction. Our previous findings [15-17] relate such an interaction to neurotoxicity. Moreover, as it has been extensively reported that chronic treatment with nicotine and nicotinic ligands induces an up-regulation of nicotinic receptors in central nervous system (CNS) [23, 24], the effect of amphetamines on nicotinic receptor populations warrants further study.

We demonstrated, using radioligand binding assays, the interaction of METH and MDMA with homomeric $\alpha 7 \mathrm{nAChR}$ and heteromeric subtypes of nicotinic receptors, such as $\alpha 4 \beta 2$. We previously demonstrated in vitro that $\mathrm{Ca}^{2+}$ chelation with EGTA prevented ROS production to a similar extent as nAChR blockade [12, 15-17]. This indicates that calcium influx, probably through $\alpha 7 \mathrm{nAChR}$, is a key step in this process. Consequently, one of the objectives of the final work was to use a fluorimetric method to investigate the effect of MDMA on $\mathrm{Ca}^{2+}$ levels in cultured PC12 cells and the involvement of different nAChR subtypes and other cell pathways related to $\mathrm{Ca}^{2+}$ mobilization. In addition, we investigated the effects of pretreatment with METH and MDMA on nAChR densities.

PC12 cells have been utilized by other scientists to study the neurotoxicity of amphetamines [25-27]. In addition, this cell line expresses nAChRs, including the $\alpha 7$ subtype [28-30], and also provides an in vitro model for the up-regulation of $\mathrm{nAChR}$, which occurs following chronic exposure to nicotine [31,32]. Moreover, the pathways involved in cytosolic $\mathrm{Ca}^{2+}$ increase induced by different selective nicotinic agonists have been characterized in this cell line [33]. For this reason, we chose this model and the isolated synaptosomes as the most appropriate for our purposes.

Herein we present the most important points brought out by our research.

\section{DA, PKC and nNOS involvement in METH- and MDMA-induced ROS production}

Our study was undertaken with the goal of developing an alternative in vitro model that might be useful for studying the molecular mechanisms of METH-induced DA neurotoxicity. With this purpose we used the fast and 
simple method for isolating synaptosomes described by Myhre and Fonnum [34]. Using this model the formation of intrasynaptosomal ROS was measured using the conversion of the non fluorescent $2^{\prime}, 7^{\prime}$ - dichlorofluorescein diacetate (DCFH-DA) to the highly fluorescent compound 2',7'dichlorfluorescein (DCF).

METH increases DCF fluorescence when added to our preparation, which indicates that it induces ROS production [15]. Incubation of synaptosomes with METH for a few minutes also causes release of DA from presynaptic nerve terminals and inhibits DA uptake, probably by reversion of dopamine transporter (DAT) functionality. When we used synaptosomes from DA-depleted rats (pretreated with reserpine or reserpine plus alpha-methyl-ptyrosine) the METH-induced ROS production was inhibited, thus corroborating DA as the main source of ROS detected (Fig. 1). Besides, METH, by altering the intracellular $\mathrm{pH}$ gradient, prevents vesicular monoamine transporter (VMAT) function and promotes DA release from vesicles to cytosol [35] where it can be oxidized. By this way, in vitro incubation of synaptosomes with substances that block VMAT (reserpine) prevents METH oxidative effect.

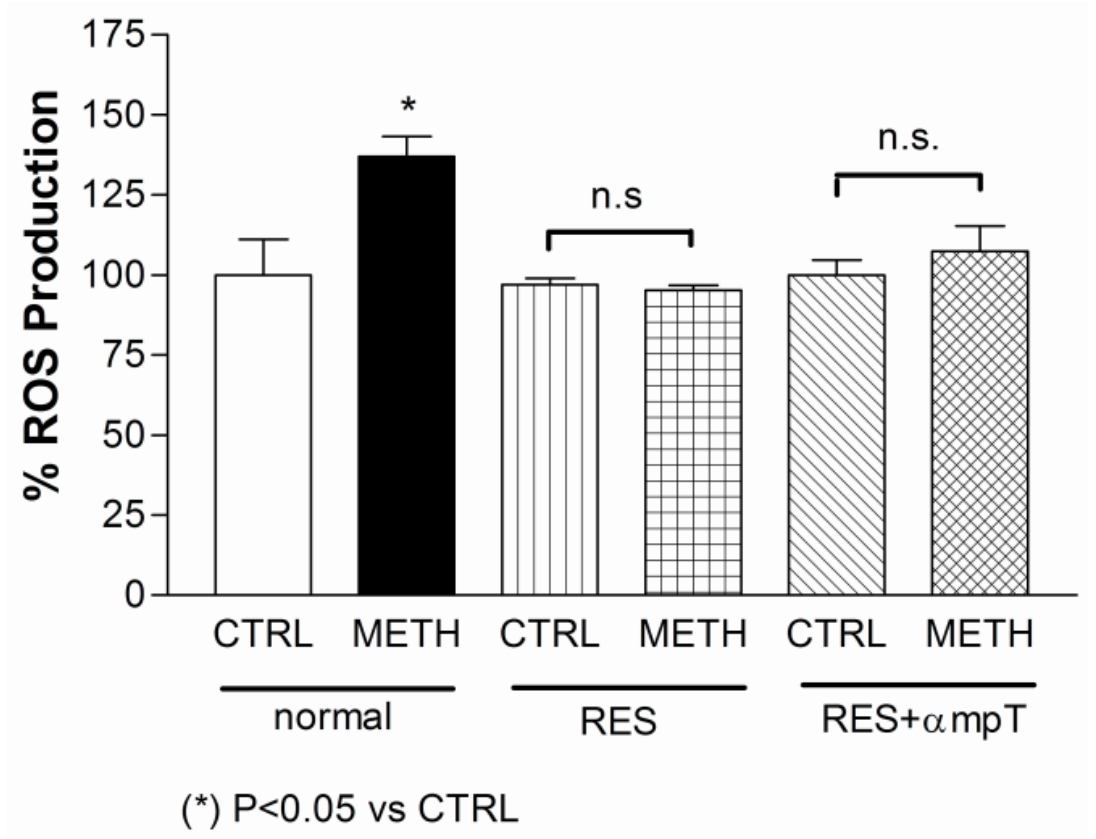

Figure 1. Effect of catecholamine depletion on METH-induced ROS in rat striatal synaptosomes. Rats were pretreated with saline (normal), reserpine (RES), or with reserpine plus alpha-methyl-p-tyrosine (RES + AMPT). Synaptosomes were obtained, and incubated alone (CTRL) or with $2 \mathrm{mM} \mathrm{METH} . * \mathrm{P}<0.05$ versus normal control group. Results are mean \pm S.E.M. of at least three separate experiments run by triplicate. Differences between groups were compared using one-way analysis of variance (ANOVA, two-tailed). Significant $(\mathrm{p}<0.05)$ differences were then analyzed by Tukey's post hoc test for multiple means comparisons where appropriate. 
Activation of nNOS produces NO, which reacts with the peroxide radicals which would originate from DA autooxidation, producing the more toxic radical peroxynitrite $\left(\mathrm{ONOO}^{-}\right)$. In our model, the inhibitor of nNOS, 7-NI, completely abolished METH-induced ROS production, demonstrating a role of the enzyme nNOS in METH oxidative effects.

PKC has been implicated in various aspects of DAT function and its direct phosphorylation [36]. In our model, inhibition of PKC (by NPC 15437, 2,6-diamino- $N$-[[1-(1-oxotridecyl)-2-piperidinyl]methyl]hexanamide dihydrochloride) fully prevented METH-induced ROS, corroborating a key role of $\mathrm{PKC}$ in this process.

Therefore an increase in cytosolic DA and activation of nNOS and PKC (blocking DA transport through DAT) are needed to generate ROS inside the dopaminergic terminal. Both, PKC and nNOS are enzymes that require calcium to be activated. Accordingly, when calcium of the medium was chelated with EGTA, the oxidative effect of METH was prevented.

Also MDMA induces oxidative stress [12]. The MDMA concentrationresponse curve showed an inverted " $U$ " shape and the maximal oxidative effect appeared at 50-100 $\mu \mathrm{M}$ and showed the same dependences.

\section{2. nAChR involvement in METH and MDMA-induced ROS production}

Ionotropic homomeric nicotinic receptors made of five alpha7 subunits are permeant to $\mathrm{Na}^{+}$and $\mathrm{Ca}^{2+}$. Although alpha7 neuronal nicotinic receptors are a minority type in the striatum, they are expressed on DA axon terminals [37]. For this reason we tested the involvement of nicotinic alpha7 receptors in our preparation, using the specific antagonist MLA. MLA completely inhibited METH and MDMA-induced ROS production, as well as alpha-bungarotoxin (another prototypic alpha7 antagonist) while dihydro-beta-erythroidine (DBE), an antagonist that blocks receptors containing beta2 subunits was devoid of effect, thus implicating alpha7 receptors [15]. On the basis of these antecedents, we considered the possibility that MDMA might also exert an oxidative effect dependent on nAChR stimulation. Specific beta2-subunit-containing and alpha7 nAChR antagonists fully inhibited the oxidative stress induced by MDMA.

Additionally, MLA inhibited the remaining effect of MDMA in the presence of catalase plus superoxide dismutase (attributed to NO), pointing a role of alpha7 nAChR in the activation of nNOS induced by MDMA.

\section{2a. In vivo MLA protects from METH- and MDMA-induced dopaminergic neurotoxicity}

Assessment of neurotoxicity markers after an in vivo treatment with a neurotoxic schedule of METH or MDMA was used to determine the 

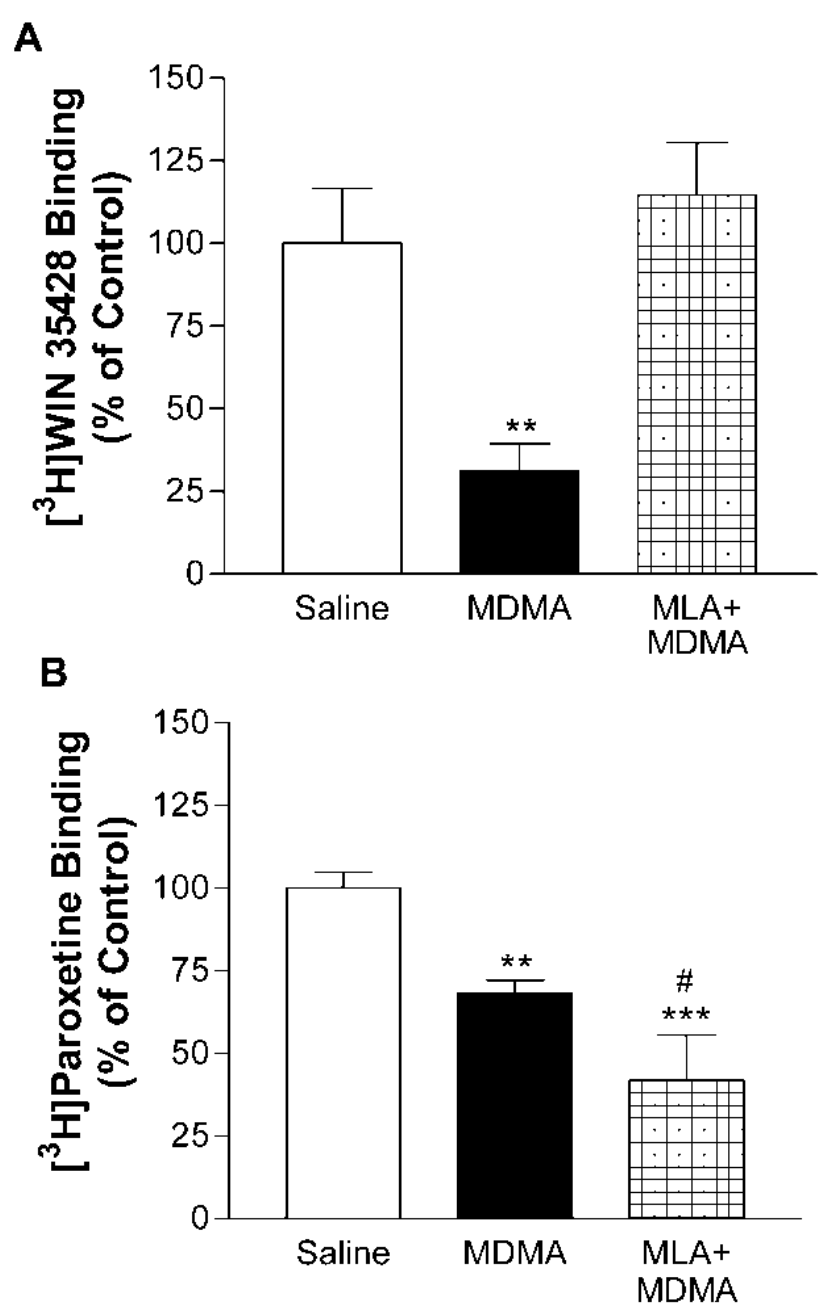

Figure 2. Panel A. Effect of in vivo treatment with MDMA (three injections, $25 \mathrm{mg} / \mathrm{kg}$ s.c., 3-h intervals) or in combination with MLA (three injections, $6 \mathrm{mg} / \mathrm{kg}$ i.p., $20 \mathrm{~min}$ before MDMA) on the density of mouse striatal dopamine reuptake sites at 7 days after treatment, and measured as specific binding of $\left[{ }^{3} \mathrm{H}\right]$ WIN $35428 . * * \mathrm{P}<0.01$ vs salinetreated group. Panel B: Effect of treatment with MDMA alone or in combination with MLA (same dose schedule as above) on the density of mouse striatal serotonin reuptake sites 7 days after treatment, measured as specific binding of $\left[{ }^{3} \mathrm{H}\right]$ paroxetine. $* * \mathrm{P}<0.01, * * * \mathrm{P}<0.001$ vs saline-treated group; \#P<0.05 vs MDMA-treated group. In both panels values are expressed as means \pm S.E.M. of those obtained from $5-6$ animals in each group. One-way ANOVA and Tukey's post hoc test.

neuroprotective effect of MLA (administered 20 min before each dose of METH or MDMA) in mice $[16,17]$.

METH induced, at $72 \mathrm{~h}$ post-treatment, a significant loss of striatal DA reuptake sites of about $73 \%$, measured as specific binding of $\left[{ }^{3} \mathrm{H}\right]$ WIN 35428 $((-)$-2- $\beta$-carbomethoxy-3- $\beta$-(4-fluorophenyl)tropane) in mouse striatum membranes. This dopaminergic injury was attenuated in mice pretreated with MLA (from $73 \%$ to $43 \%$ ). 
The in vivo neurotoxic model of MDMA used was characterized by a significant loss of DA terminals (69\%) and a decrease of tyrosine hydroxylase levels $(65 \%)$ in striatum from mice sacrificed 7 days post-treatment. This terminal loss was prevented by pretreatment with MLA, pointing also to a neuroprotective effect against this amphetamine derivative.

$\left[{ }^{3} \mathrm{H}\right]$ Paroxetine binding, which labels SERT, was measured also in the hippocampus of these animals, as a marker of degeneration of serotonin terminals. Conversely, MLA did not prevent the loss in $\left[{ }^{3} \mathrm{H}\right]$ paroxetine binding sites, indicating that its neuroprotective effect is selective for DA terminals.

\section{2b. Memantine protects from MDMA-induced CNS injury}

Memantine (MEM), a non-competitive antagonist of the NMDA receptor, is a drug used to treat moderate-to-severe Alzheimer's disease. It reduces tonic [38] but not synaptic, NMDA receptor activity.

In 2005, the group of Aracava demonstrated that MEM, at clinically relevant concentrations, can block alpha7 nAChR in a non-competitive manner, and more effectively that it does at NMDA receptors [38].

Our in vitro neurotoxicity studies showed that MEM had not antioxidant effect versus $\mathrm{H}_{2} \mathrm{O}_{2}$ but inhibited the ROS production induced by MDMA at all the concentrations tested. The oxidative effect of amphetamine derivative was reinforced by PNU 282987 an alpha7 specific agonist.

In vivo experiments were carried out with Dark Agouti rats, that are more sensitive to low doses of drugs, and we used it as a model of serotonergic neurotoxicity induced by MDMA. A significant decrease in the SERT density was observed in both, the hippocampus and frontal cortex of MDMA-treated Dark Agouti rats sacrificed 1 or 7 days post-treatment. In both cases, MEM significantly prevented the loss of $\left[{ }^{3} \mathrm{H}\right]$ paroxetine binding sites, suggesting a neuroprotective effect on serotonin terminals [39] (Table 1).

Table 1. Effect of memantine in the abundance of 5-HT (rat hippocampus) transporters (labeled by $\left[{ }^{3} \mathrm{H}\right]$ paroxetine), and also in the glial activation measured by the $\left.{ }^{3} \mathrm{H}\right] \mathrm{PK} 11195$ binding in those tissues in rats killed 7 days post-treatment.

\begin{tabular}{|l|l|l|}
\hline Treatment & $\begin{array}{l}{\left[{ }^{3} \mathrm{H}\right] \text { Paroxetine }} \\
\text { binding }(\%)\end{array}$ & $\begin{array}{l}{\left[{ }^{3} \mathrm{H}\right] \mathrm{PK} \text { 11195 }} \\
\text { binding (\%) }\end{array}$ \\
\hline Saline & $100.00 \pm 9.34$ & $100.00 \pm 6.35$ \\
\hline MDMA & $70.02 \pm 7.85 *$ & $168.22 \pm 20.65 * *$ \\
\hline MEM & $116.40 \pm 3.95$ & $94.90 \pm 7.05$ \\
\hline MDMA + MEM & $94.87 \pm 4.96$ & $104.41 \pm 9.77 \#$ \\
\hline
\end{tabular}

Results are expressed as mean \pm S.E.M. from 6-9 different experiments. ${ }^{*} \mathrm{P}<0.05$ and $* * \mathrm{P}<0.01$ vs Saline; $\# \mathrm{P}<0.05$ and \#\#\# $\mathrm{P}<0.001$ vs. MDMA group. 
Accordingly with our results, MEM could be used not only to treat these addictions or to prevent the effects of these amphetamine derivatives, but it may also have a beneficial effect on the memory impairment that abusers of these drugs usually suffer [40].

\section{Nicotinic receptors and the cognitive impairment induced by MDMA}

In our laboratory we performed some experiments to demonstrate a specific effect of MDMA treatment on the object recognition memory test and the Morris water maze in Long Evans rats [41]. Animals pre-treated with MEM did not exhibit the lack of memory that appeared in the MDMA-treated animals. Therefore, MEM by preventing MDMA-induced neuronal injury contributes to ameliorate cognitive impairment produced by MDMA, this preventive effect on MDMA-induced impairment suggesting a new therapeutic approach to the treatment of long-term adverse effects of amphetamine derivatives.

\section{Amphetamine derivates directly interact with nAChR}

METH and MDMA displaced both $\left[{ }^{3} \mathrm{H}\right]$ epibatidine $\left(\left[{ }^{3} \mathrm{H}\right] \mathrm{EB}\right)$ and $\left[{ }^{3} \mathrm{H}\right] \mathrm{MLA}$ binding in PC12 cells and mouse brain, indicating that they can directly interact with nAChR. MDMA displayed higher affinity than METH for both subtypes of $\mathrm{nAChR}$. The resulting $\mathrm{K}_{\mathrm{i}}$ values fell in the micromolar range, although some of them are in the low micromolar range and other in the high micromolar range (Table 2).

Special attention must be paid in the affinity for heteromeric receptors $\left(\mathrm{K}_{\mathrm{i}}\right.$ about $0.7 \mu \mathrm{M}$ ) which is practically the same that the $\mathrm{K}_{\mathrm{i}}$ displayed by MDMA for the serotonin transporter, its main physiological target $(0.61 \mu \mathrm{M})$ [42]. Therefore an interaction of MDMA on heteromeric $\mathrm{nAChR}$ at recreational doses

Table 2. $\mathrm{K}_{\mathrm{i}}$ values of METH and MDMA against $\left[{ }^{3} \mathrm{H}\right] \mathrm{MLA}$ and $\left[{ }^{3} \mathrm{H}\right] \mathrm{EB}$ binding in mouse brain.

\begin{tabular}{lllll}
\hline & {$\left[{ }^{3} \mathbf{H}\right] \mathbf{E B}$} & \multicolumn{3}{l}{$\mathbf{H}] \mathbf{M L A}$} \\
\hline Drugs & $\mathbf{K}_{\mathbf{i}}(\boldsymbol{\mu} \mathbf{M})$ & $\mathbf{n}_{\mathbf{H}}$ & $\mathbf{K}_{\mathbf{i}}(\boldsymbol{\mu} \mathbf{M})$ & $\mathbf{n}_{\mathbf{H}}$ \\
\hline METH & $23.90 \pm 2.65$ & $1.27 \pm 0.28$ & $369.77 \pm 95.61$ & $0.29 \pm 0.08^{*}$ \\
MDMA & $0.76 \pm 0.11$ & $0.83 \pm 0.12$ & $34.21 \pm 6.71$ & $0.40 \pm 0.27^{*}$ \\
\hline
\end{tabular}

The $\mathrm{K}_{\mathrm{i}}$ values from competition binding curves were calculated using the Cheng-Prusoff equation.* Significantly different from 1 (one sample t-test). Results are expressed as mean \pm S.E.M. from 4-6 different experiments. 
is certainly possible. The fact that the lowest $\mathrm{K}_{\mathrm{i}}$ values were found against $\left[{ }^{3} \mathrm{H}\right]$ epibatidine binding indicates that METH and MDMA displayed higher affinity for heteromeric nAChR which are the most abundant in the CNS.

We also performed binding experiments with MDMA enantiomers in order to determine a difference between $(R)$-MDMA and $(S)$-MDMA in their capability of displacing $\left[{ }^{3} \mathrm{H}\right] \mathrm{EB}$ and $\left[{ }^{3} \mathrm{H}\right]$ MLA from their binding sites. When $\left[{ }^{3} \mathrm{H}\right] \mathrm{EB}$ was used as a radioligand to label $\alpha 4 \beta 2 \mathrm{nAChR},(R)$-MDMA and $(S)$-MDMA induced a concentration-dependent binding displacement, with $\mathrm{IC}_{50}$ values in the micromolar range (see Table 3). The affinity of $(R)$-MDMA for $\left[{ }^{3} \mathrm{H}\right] \mathrm{EB}$ binding sites was higher than (S)-MDMA. The Hill coefficients resulting from the analysis of competition data of MDMA vs $\left[{ }^{3} \mathrm{H}\right] \mathrm{EB}$ were not significantly different from unity, pointing to a competitive displacement.

When $\left[{ }^{3} \mathrm{H}\right]$ MLA was used as a radioligand to label $\alpha 7 \mathrm{nAChR}$, no differences between $(R)$-MDMA and (S)-MDMA were found. The Ki values were similar for both enantiomers.

Table 3. $\mathrm{K}_{\mathrm{i}}$ values of $(R)$ - and $(S)$-MDMA against $\left[{ }^{3} \mathrm{H}\right] \mathrm{MLA}$ and $\left[{ }^{3} \mathrm{H}\right] \mathrm{EB}$ binding to rat membranes.

\begin{tabular}{|l|l|l|l|c|}
\hline Radioligand & Ligand & $\mathrm{IC}_{50} \pm \mathrm{SEM}(\mu \mathrm{M})$ & $\mathrm{Ki} \pm \mathrm{SEM}(\mu \mathrm{M})$ & $\mathrm{n}_{\mathrm{H}}$ \\
\hline$\left[{ }^{3} \mathrm{H}\right] \mathrm{EB}$ & $(R)-\mathrm{MDMA}$ & $0.37 \pm 0.0$ & $0.6 \pm 0.2$ & $1.0 \pm 0.0$ \\
\hline$\left[{ }^{3} \mathrm{H}\right] \mathrm{EB}$ & $(S)-\mathrm{MDMA}$ & $9.7 \pm 1.0$ & $8.2 \pm 2.0$ & $0.8 \pm 0.1$ \\
\hline$\left[{ }^{3} \mathrm{H}\right] \mathrm{MLA}$ & $(R)-\mathrm{MDMA}$ & $149.3 \pm 22$ & $71.8 \pm 10$ & $0.6 \pm 0.1^{*}$ \\
\hline$\left[{ }^{3} \mathrm{H}\right] \mathrm{MLA}$ & $(S)-\mathrm{MDMA}$ & $131 \pm 27$ & $63.1 \pm 17$ & $0.5 \pm 0.3^{*}$ \\
\hline
\end{tabular}

The $\mathrm{K}_{\mathrm{i}}$ values from competition binding curves were calculated using the Cheng-Prusoff equation. .* Significantly different from 1 (one sample t-test). . Results are expressed as mean \pm S.E.M. from 3-4 different experiments.

\section{METH and MDMA increase the density of nicotinic receptors}

A particular feature of $\mathrm{nAChR}$ is that chronic exposure to nicotine induces a higher level of either nicotine or epibatidine binding, termed as upregulation [43]. Accordingly, we tested whether METH and MDMA had any effect on $\alpha 7$ and heteromeric nAChR binding densities and found that both were increased in a time- and concentration- dependent manner (Fig. 3).

The mechanism through which nicotine induces $\mathrm{nAChR}$ up-regulation is complex and not fully clarified to date (reviewed by Gaimarri et al. [44]). There are reports indicating that nicotine-induced increases in nAChR are not accompained by changes in mRNA encoding for the different subunits $[45,46]$. This led to other hypotheses, such as reduced receptor turnover, promotion of the assembly and migration to the plasma membrane of pre- existing 

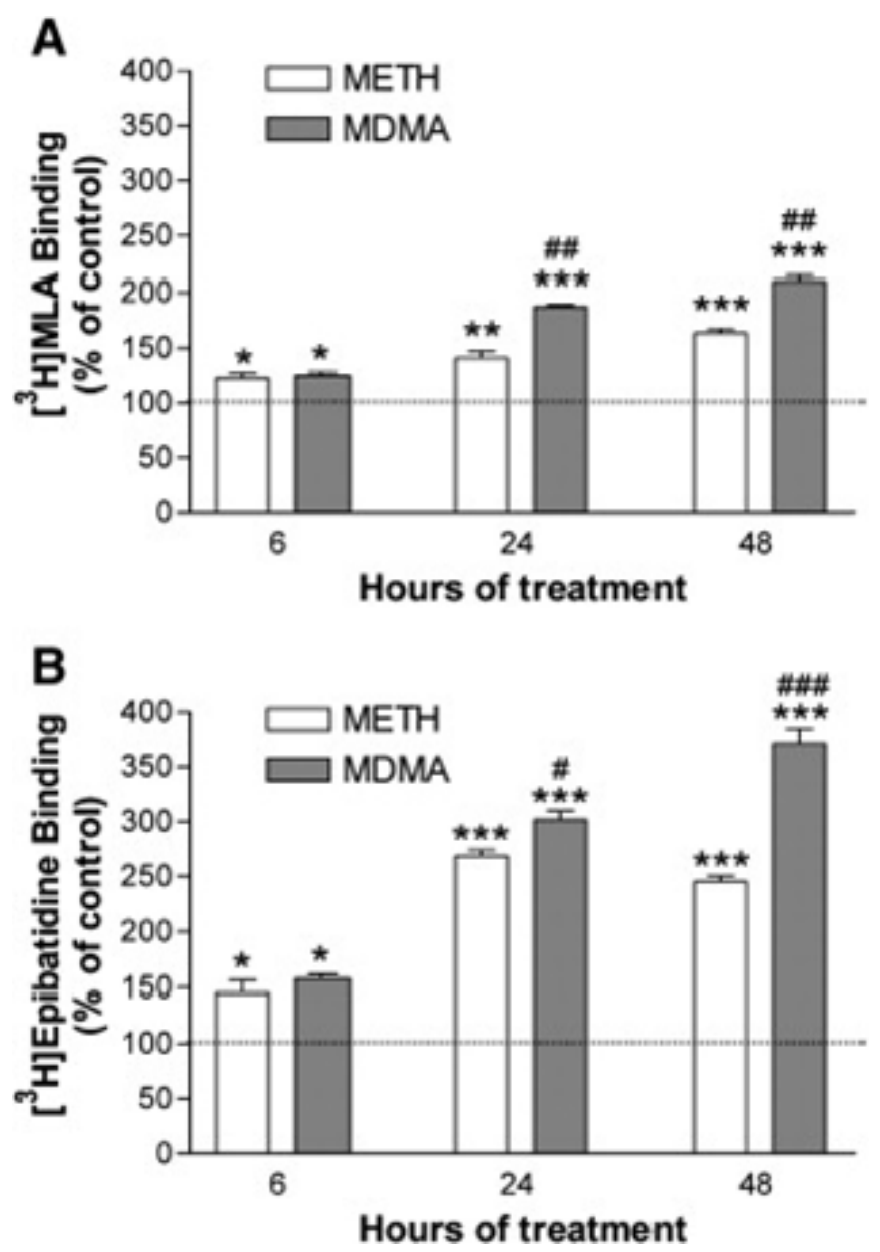

Figure 3. Time-course study of the increases in $\left[{ }^{3} \mathrm{H}\right] \mathrm{MLA}$ (panel A) and $\left[{ }^{3} \mathrm{H}\right]$ epibatidine (panel B) binding sites induced by METH and MDMA $(300 \mu \mathrm{M})$. PC12 cells were incubated with amphetamine derivatives over 6, 24 and $48 \mathrm{~h}$. Thereafter radioligand binding was performed on intact cells in culture. Data represent the means \pm S.E.M. of three separate experiments carried out in triplicates $\left({ }^{*} \mathrm{P}<0.05\right.$, ${ }^{* * *}<0.01,{ }^{* * *} \mathrm{P}<0.001$ vs. untreated cells; ${ }^{\#} \mathrm{P}<0.05,{ }^{\# \#} \mathrm{P}<0.01,{ }^{\# \#} \mathrm{P}<0.001$ vs. METH at the same incubation time).

intracellular subunits [29] or decrease in the rate of receptor turnover [47]. More recently, Sallette et al. [48] demonstrated that nicotine acts as a maturation enhancer of those intracellular nAChR precursors that would otherwise be degraded. However, different authors show controversial results. Vallejo et al. [49] reported that $\alpha 4 \beta 2$ up-regulation by nicotine is due to an increase/stabilisation of the proportion of receptors in a high affinity state and not to an enhancement in receptor maduration.

Regardless the underlying mechanism, according to our competition experiments demonstrating the affinity of METH and MDMA for nAChR, it could be hypothesised that the up-regulation of nAChR induced by these drugs would follow a similar mechanism. 


\section{Role of protein synthesis, cyclophilin A, protein kinase and receptor trafficking}

The protein synthesis inhibitor cycloheximide (CHX) inhibited the increase in both $\left[{ }^{3} \mathrm{H}\right] \mathrm{MLA}$ and $\left[{ }^{3} \mathrm{H}\right]$ epibatidine binding sites, suggesting that such up-regulation require, at least in part, rapid protein synthesis. The chaperone cyclophilin A participates in $\alpha 7-n A C h R$ maturation $[29,50]$. Therefore we tested the cyclophilin A inhibitor cyclosporin A (CsA) on METH/MDMA-induced nAChR up-regulation. CsA inhibited METH/MDMA-induced up-regulation.

To assess whether receptor trafficking to the plasma membrane could be contributing to METH/MDMA-induced nAChR up-regulation we exposed the cells to BFA to inhibit such transport and late steps of maturation. Although BFA induced dramatic decreases in basal binding sites owing to its main mode of action, there was still up-regulation after treatment with METH/MDMA. This indicates that trafficking is not crucial for METH/MDMA-induced nAChR up-regulation and that this takes place previously to surface expression. This result is in agreement with those reported in other studies on the mechanisms involved in nicotine-induced up-regulation of heteromeric receptor [49,51].

PKA- and PKC-dependent pathways have been found to play a role in the up-regulation of $\alpha 4 \beta 2$ receptors induced by cholinergic ligands in several cell lines [52, 53]. For this reason we tested the PKA inhibitor H-89 on METH/MDMA-induced up-regulation. We saw that there was an inhibition by $\mathrm{H}-89$, corroborating that PKA plays a role in heteromeric nAChR regulation, but not in that of $\alpha 7 \mathrm{nAChR}$ [53]. The PKC inhibitor, GF-109203X, did not affect $\alpha 7 \mathrm{nAChR}$ in our experiments with amphetamine derivatives. This result is in agreement with those reported by Nuutinen et al. [53] who found no effect of this compound, at nanomolar concentration, on nicotine-induced $\alpha 7 \mathrm{nAChR}$ up-regulation.

Protein tyrosine kinases have been reported to play a role in $\alpha 7 \mathrm{nAChR}$ regulation $[54,55]$. When genistein, an inhibitor of tyrosine kinases, was assayed for $24 \mathrm{~h}$, it reduced METH/MDMA effects on nAChR densities, indicating that they participate in the up-regulation induced by METH and MDMA.

All these results confirm that METH and MDMA up-regulate $\mathrm{nAChR}$ in a complex process but in a similar manner than nicotine. However, the work done to date indicates that up-regulation can occur if the drug has a particular affinity to one or more nAChR subunits; regardless of the agonist/antagonist properties of the drug (i.e., the antagonist $\mathrm{DH} \beta \mathrm{E}$ is also able to induce it [52]. In addition, up-regulation is enhanced when the drug crosses the cell membrane to interact with immature forms of the receptor [56]. The affinity of MDMA towards both heteromeric and $\alpha 7 \mathrm{nAChRs}$ has been demonstrated 
[30], and this drug can reach the cytosplasm after transport through the dopamine transporter $[57,58]$, which is abundant in PC12 cells. Therefore, the interaction of MDMA with immature receptor subunits is feasible.

\section{Intracellular $\mathrm{Ca}^{2+}$ increase induced by MDMA in PC12 cells}

We used a fluorimetric method [33] to investigate the effect of MDMA on $\mathrm{Ca}^{2+}$ levels in cultured PC12 cells and the involvement of different nAChR subtypes and other cell pathways related to $\mathrm{Ca}^{2+}$ mobilization.

MDMA acutely inhibited the effects of nAChR agonists (ACh, Nic and PNU282987) (Fig. 4a) but, owing to its effects increasing basal calcium levels

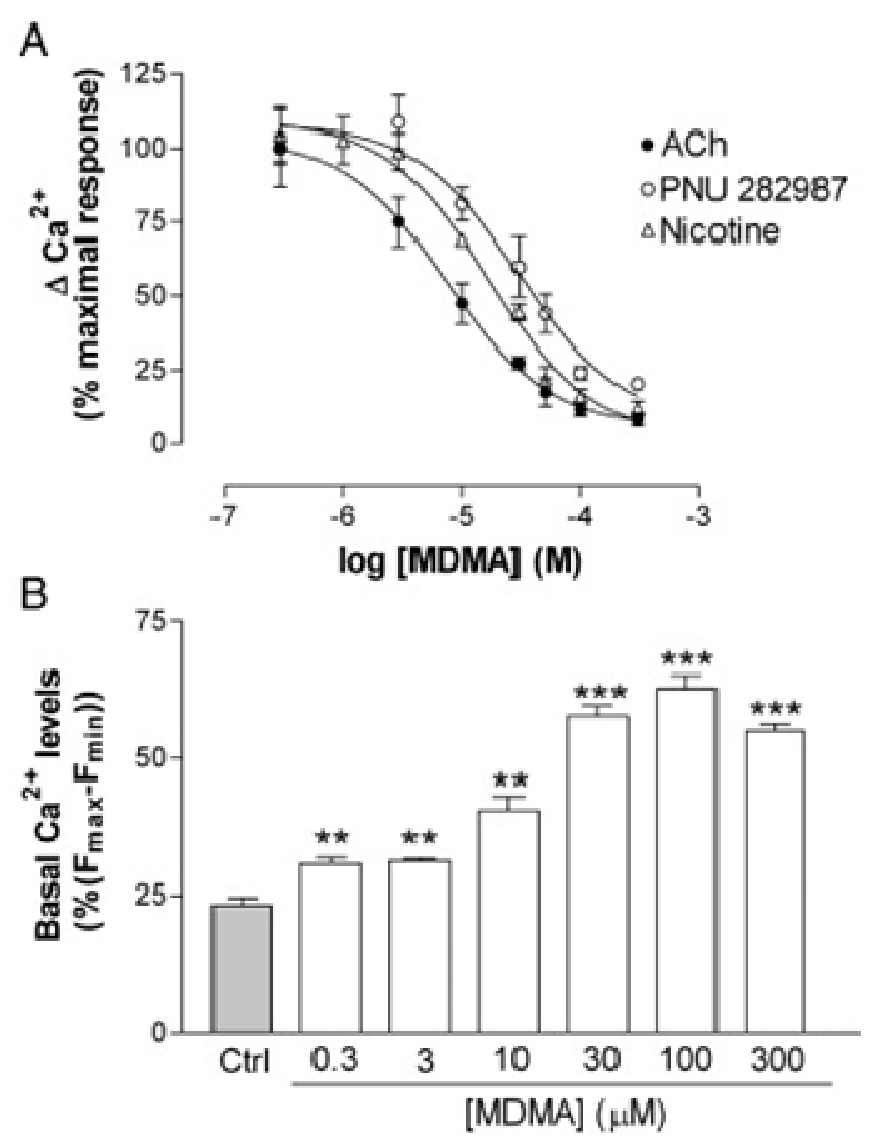

Figure 4. (Panel A) Effect of increasing concentrations of MDMA on the responses to the nicotinic agonists ACh $(100 \mu \mathrm{M})$, nicotine $(100 \mu \mathrm{M})$, and PNU $282987(0.1 \mu \mathrm{M})$ in PC12 cells loaded with Fluo-4. MDMA was added to the cells 5 min before the agonist. Basal fluorescence levels were measured for $5 \mathrm{~s}$ before the agonist and for a further $30 \mathrm{~s}$ after its addition by means of an automated injector. (Panel B) Basal $\mathrm{Ca}^{2+}$ levels $5 \mathrm{~min}$ after the addition of MDMA and before adding the agonist. All the measurements were performed in the presence of the positive allosteric modulator PNU $120596(10 \mu \mathrm{M})$. The results were normalized as percentage $\left(\mathrm{F}_{\max }-\mathrm{F}_{\min }\right)$ and are the means \pm SEM of at least three experiments, carried out in quadruplicates for each condition. $* * \mathrm{P}<0.01, * * * \mathrm{P}<0.001$ vs. control basal levels. 
(Fig. 4b), we explored the possibility of a partial agonist mode of action. We tested the effect of acute application of MDMA and found a concentrationeffect relationship in the micromolar concentration range. The effect of MDMA did not reach the maximum values induced by $\mathrm{ACh}$, which indicates a partial agonist mode of action (Fig. 5). The $\mathrm{EC}_{50}$ value was in the micromolar range, which is in agreement with previous binding results.

The experiments performed with MDMA enantiomers demonstrated that there are no differences in the calcium increase between the enantiomers and the racemic mixture. This results is in agreement with the similar affinity values obtained of both enantiomers in the binding experiments with $\left[{ }^{3} \mathrm{H}\right]$ MLA.
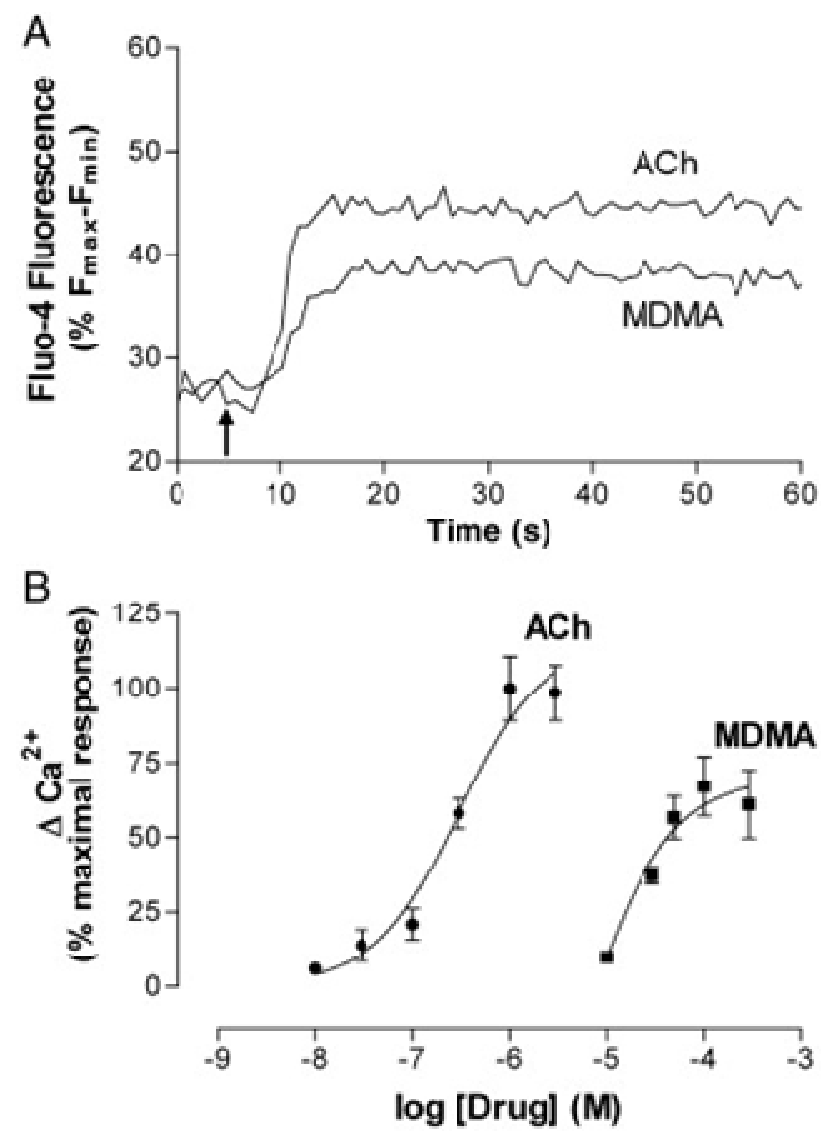

Figure 5. (Panel A) Representative tracings of the increases in Fluo-4 fluorescence in PC12 cells after the addition (arrow) of ACh $(1 \mu \mathrm{M})$ and MDMA $(50 \mu \mathrm{M})$. (Panel B) Representative concentration-response curves showing the increase in cytosolic $\mathrm{Ca}^{2+}$ induced by MDMA and $\mathrm{ACh}$ as a total agonist. Responses were normalized as percentage $\left(\mathrm{F}_{\max }-\mathrm{F}_{\min }\right)$ and represented as a percentage of the maximum response (ACh $100 \mu \mathrm{M}$ ) for both curves. After $5 \mathrm{~s}$ of basal recording, ACh and MDMA were added by means of an automated injector to PC12 cells loaded with Fluo-4 and the fluorescence was measured for a further $30 \mathrm{~s}$. Represented data are the mean $\pm \mathrm{SEM}$ of four replicates for each condition. 


\section{Pathways involved in MDMA-induced $\mathrm{Ca}^{2+}$ increase}

The fact that MDMA induced an increase in cytosolic $\mathrm{Ca}^{2+}$ led us to study the pathways involved using specific blockers (Fig. 6). According to our previous work, the $\alpha 7 \mathrm{nAChR}$ blockers MLA and $\alpha$-bungarotoxine $(\alpha$-BgTX) abolished the effect of MDMA, which showed that such effects take place mainly through activation of these receptors. However, as we had previously described that MDMA also has affinity for heteromeric receptors [12, 30], we tested the effect of DBE, an antagonist of these receptors, and we found that it had no significant effect on MDMA-induced $\mathrm{Ca}^{2+}$ increase, ruling out an antagonistic effect on theses receptors. This finding suggests that MDMA behaves as an antagonist at heteromeric receptors while it is a partial agonist at $\alpha 7$ nAChRs. These receptors are permeant to $\mathrm{Na}^{+}$, which could induce depolarization and open voltage-operated calcium channels (VOCC) [33]. We used nitrendipine, an L-type VOCC inhibitor, and $\mathrm{Cd}^{2+}$, a non selective

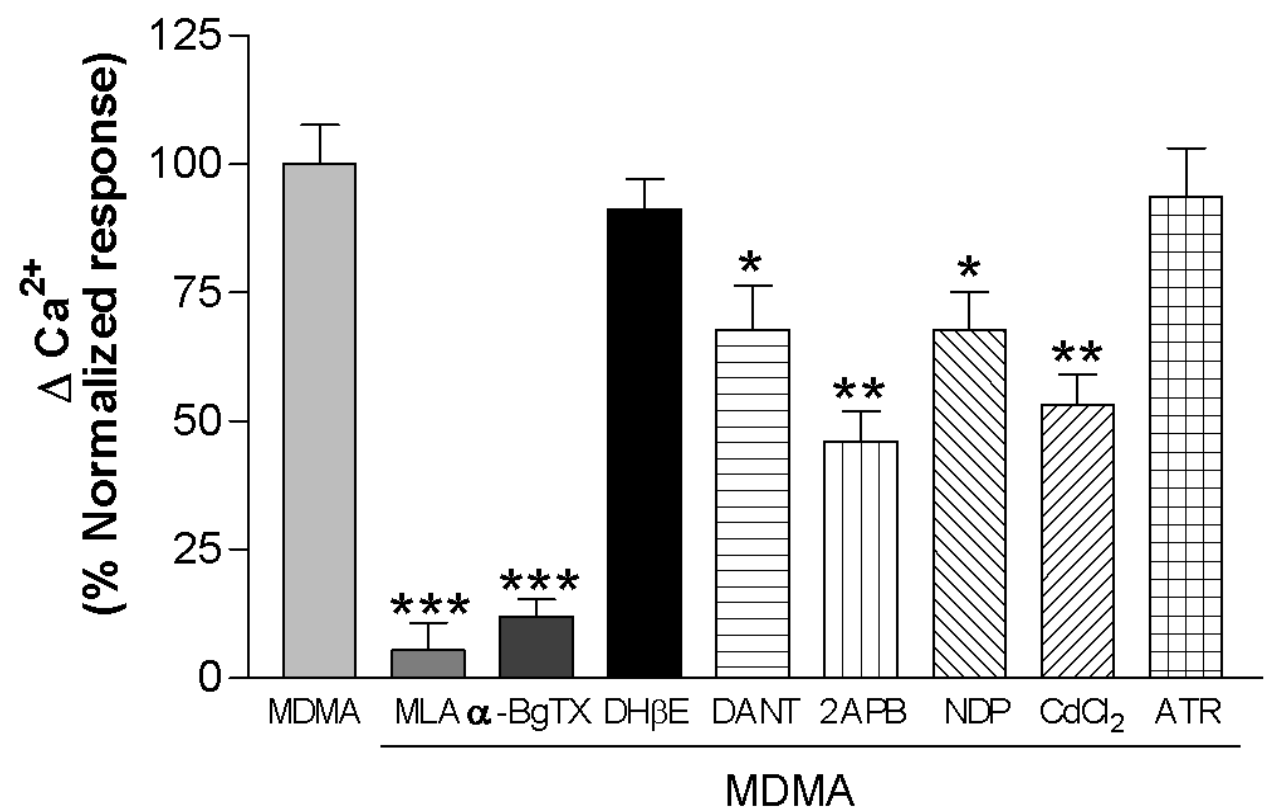

Figure 6. Pharmacological modulation of MDMA-induced increase in cytosolic $\mathrm{Ca}^{2+}$. Drugs were manually added to cultured PC12 cells loaded with Fluo-4, 10 min before the automated addition of MDMA $(50 \mu \mathrm{M})$. The response was measured for $5 \mathrm{~s}$ before and $30 \mathrm{~s}$ after MDMA, then normalized as percentage $\left(\mathrm{F}_{\max }-\mathrm{F}_{\min }\right)$ and expressed as a percentage of the response induced by MDMA alone. All the measurements were carried out in the presence of PNU $120596(10 \mu \mathrm{M})$. Abbreviations and concentrations used: MLA (methyllycaconitine, $1 \mu \mathrm{M}$ ), DH $\beta \mathrm{E}$ (dihydro- $\beta$-erythroidine, $50 \mu \mathrm{M}$ ), DANT (dantrolene, $20 \mu \mathrm{M}$ ), 2-APB (2-aminoethyl diphenyl borate, $150 \mu \mathrm{M}$ ), NDP (nitrendipine, $30 \mu \mathrm{M}$ ), ATR (atropine, $0.1 \mu \mathrm{M}$ ). Results are mean \pm SEM of three experiments carried out in quadruplicates. ${ }^{*} \mathrm{P}<0.05$, ${ }^{*} \mathrm{P}<0.01$, ${ }^{* *} \mathrm{P}<0.001$ vs. MDMA alone. 
blocker of VOCC and found that they inhibited MDMA-induced $\mathrm{Ca}^{2+}$ suggesting that, in our model, the initial depolarization induced after $\alpha 7$ receptor activation by MDMA, partially couples to L-type channel opening.

Calcium-induced calcium release (CICR) from endoplasmic reticulum (ER) stores has been shown to be coupled to a $\alpha 7 \mathrm{nAChR}$ activation $[33,59]$. CICR could occur after activation of two intracellullar calcium release channels localized in the ER: $\mathrm{IP}_{3}$ receptors and ryanodine receptors $[60,61]$. Accordingly we tested two blockers of these receptors (2-APB and dantrolene, respectively) with MDMA. We found that the MDMA effect was significantly inhibited by these blockers, which demonstrates the participation of CICR. In fact, these blockers had also been effective in preventing MDMA-induced ROS generation in striatal synaptosomes [62].

The MDMA response was dependent on extracellular $\mathrm{Ca}^{2+}$, as suppression of this cation totally inhibited its effect. Extracellular $\mathrm{Ca}^{2+}$ could enter through either $\alpha 7$ channels or L-type VOCCs and, as stated above, this $\mathrm{Ca}^{2+}$ increase would also induce subsequent CICR.

Although mechanisms other than nAChR activation cannot be totally ruled out in the MDMA-induced increase in cytosolic $\mathrm{Ca}^{2+}$, the practically complete inhibition by MLA and $\alpha$-BgTX indicates that $\alpha 7 \mathrm{nAChR}$ activation plays a major role in this process.

\section{Effects of MDMA on basal $\mathrm{Ca}^{2+}$ levels}

Preincubation with MDMA for $24 \mathrm{~h}$ induced an increase in basal cytosolic $\mathrm{Ca}^{2+}$ levels, as measured after drug washout. Surprisingly, preincubation with nicotine increased basal levels when it was carried out for $1 \mathrm{~h}$, but not after longer preincubation times. This indicates that cells are able to buffer sustained activation by nicotine, but not that induced by MDMA, which suggests increased vulnerability to this drug.

\section{Calpain/Caspase-3 activation induced by MDMA}

Sustained $\mathrm{Ca}^{2+}$ influx after MDMA treatment could favor cytotoxicity through activation of $\mathrm{Ca}^{2+}$-dependent pathways (i.e., calpain). Calpain is a calcium-dependent protease whose activation is a primary mechanism that contributes to several types of neurodegenerative conditions, including the excitatory amino acid-induced neurotoxicity that is associated with traumatic brain injury, ischemia, and hyperthermia [63,64]. Calpain specifically degrades the cytoskeletal membrane protein, spectrin, into 145 and $150 \mathrm{kDa}$ breakdown products [65]. Caspase 3 is another cysteine protease that is 
involved in apoptotic pathways. It also degrades spectrin but produces a $120 \mathrm{kDa}$ spectrin fragment [66] and also can produce a 150-kDa fragment [67].

Incubation of PC12 cells with MDMA for $24 \mathrm{~h}$ induced a significant increase in $\alpha$-spectrin breakdown products (SBDP) of 145 and $150 \mathrm{kDa}$, which indicates calpain activation, and a rise in the $120 \mathrm{kDa}$ band that, together with the increase in the $150 \mathrm{kDa}$ SBDP, points to caspase 3 activation (Fig. 7). In fact, in a previous work using cerebellar granule cells, we demonstrated the proapoptotic role of caspase 3 in the neurotoxic effects of amphetamines [68]. Moreover, the increases in SBDP induced by MDMA were prevented by MLA, indicating that $\alpha 7 \mathrm{nAChRs}$ play a key role in this process.
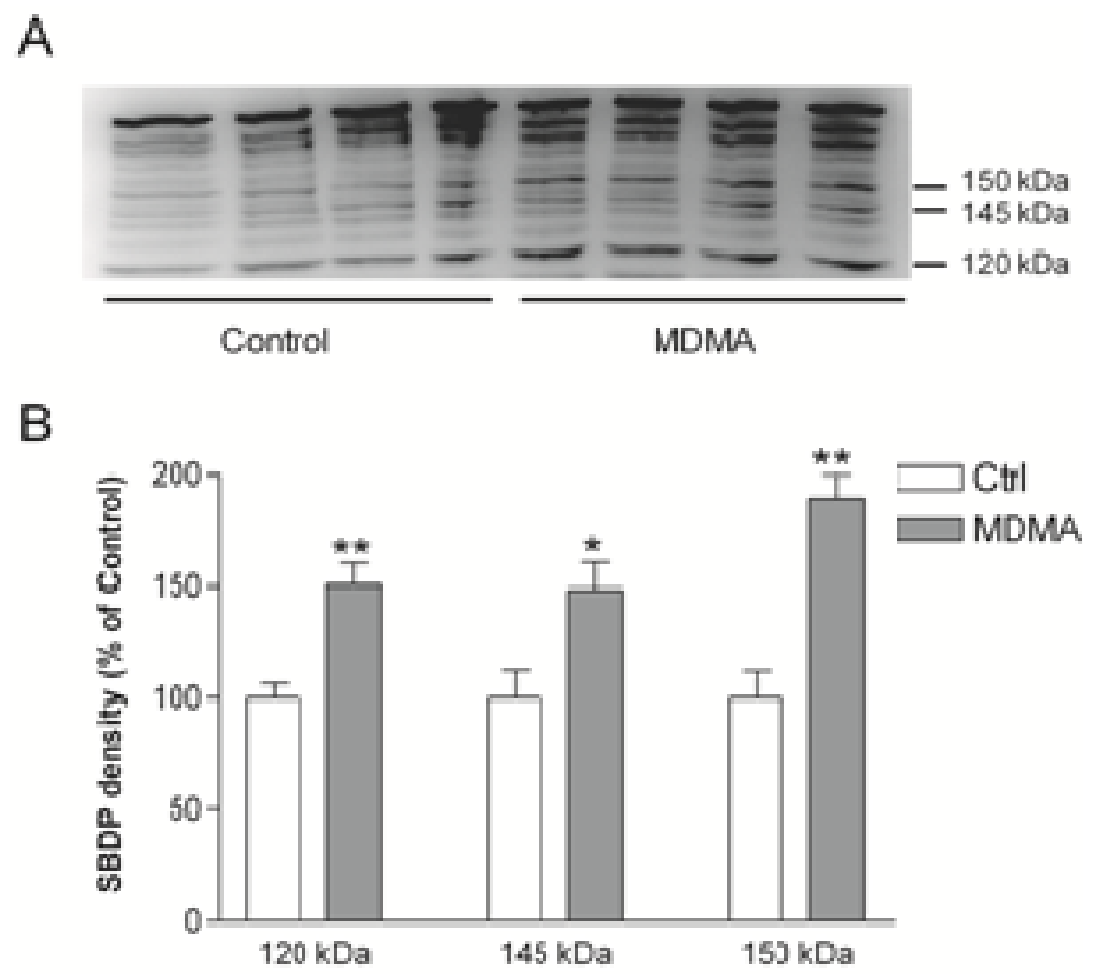

Figure 7. (Panel A) Representative Western blot of $\alpha$-spectrin breakdown products (SBDP) originated by calpain activation (145 and $150 \mathrm{kDa})$ and caspase 3 (120 and $150 \mathrm{kDa}$ ) after 24-h treatment with culture medium (Ctrl), MDMA $(50 \mu \mathrm{M})$, MDMA + MLA (10 nM), and MLA alone. The localization of the molecular weight (MW) markers is shown on the left of the picture. (Panel B) Quantification of dot intensity of the SBDPs. Data are the means \pm SEM of three different cultures, loaded in duplicates. ${ }^{*} \mathrm{P}<0.05$ and ${ }^{* *} \mathrm{P}<0.001$ vs. control.

\section{Functional up-regulation}

After chronic nicotine exposure, some nAChR subtypes undergo radioligand binding up-regulation, changes in stoichiometry, and an increase 
in functional state (functional up-regulation) (reviewed in [44]). Such up-regulation occurs at a post-transcriptional level and has been reported in cell cultures for $\alpha 4 \beta 2 \mathrm{nAChRs}[56,69]$ as well as for $\alpha 7 \mathrm{nAChRs}[70]$. For this reason, we measured cytosolic $\mathrm{Ca}^{2+}$ levels to test whether pretreatment with MDMA induced persistent changes in nAChRs, leading to an increased response to agonists. Our experiments showed that, when incubated for $24 \mathrm{~h}$ with MDMA, PC12 cells exhibited increased responses to PNU282987 $(N$-(3R)-1-azabicyclo[2.2.2]oct-3-yl-4-chlorobenzamide) ( $\alpha 7$-selective) and to 5-I-A-85380 (3-[(2S)-2-azetidinylmethoxy]-5-iodopyridine dihydrochloride) (selective for $\beta 2$ subunit-containing receptors), measured after drug washout. This indicates that MDMA also induces functional nAChR up-regulation.

\section{Final remarks}

Our work demonstrates an additional mode of action for amphetamine derivatives. Their activation of $\alpha 7 \mathrm{nAChR}$ contributes to the toxicity of these drugs, which points to a new target to reduce damage. Moreover, the effects on different $\mathrm{nAChR}$ densities may account for long term effects on neural pathways and addiction processes due the important role of these receptors in CNS functions.

\section{References}

1. Davidson, C., Gow, A.J., Lee, T.H., Ellinwood, E.H. 2001, Brain Res. Rev., 36, 1.

2. Green, A.R., Mechan, A.O., Elliott, J.M., O'Shea, E. and Colado, M.I. 2003, Pharmacol. Rev., 55, 463.

3. Parrott, A.C. 2002, Pharmacol. Biochem. Behav., 71, 837.

4. Quednow, B.B., Jessen, F., Kuhn, K.U., Maier, W., Daum, I., Wagner, M. 2006, J. Psychopharmacol., 20, 373.

5. Reneman, L., Lavalaye, J., Schmand, B., de Wolff, F.A., van den Brink, W., den Heeten, G.J.,Booij, J. 2001, Arch. Gen. Psychiatr., 58, 901.

6. Volkow, N.D., Chang, L., Wang, G.J., Fowler, J.S., Franceschi, D., Sedler, M., Gatley, S.J., Miller, E., Hitzemann, R., Ding, Y.S., Logan, J. 2001, J. Neurosci., 21, 9414.

7. Pubill, D., Canudas, A.M., Pallas, M., Camins, A., Camarasa, J., Escubedo, E. 2003, Naunyn Schmiedeberg's Arch. Pharmacol., 367, 490.

8. Sprague, J.E. and Nichols, D.E. 2005, Trends Pharmacol. Sci., 26, 59.

9. De la Torre, R., Farre, M., Roset, P.N., Lopez, C.H., Mas, M., Ortuño, J., Menoyo, E., Pizarro, N., Segura, J., Cami, J. 2000, Ann. N.Y. Acad. Sci., 914, 225.

10. Esteban, B., O'Shea, E., Camarero, J., Sanchez, V., Green, A.R. and Colado, M.I. 2001, Psychopharmacology, 154, 251. 
11. Jayanthi, S., Ladenheim, B., Andrews, A.M. and Cadet, J.L. 1999, Neuroscience, 91, 1379.

12. Chipana, C., Garcia-Rates, S., Camarasa, J., Pubill, D. and Escubedo, E. 2008, Neurochem. Int., 52, 401.

13. Cadet, J.L., Ladenheim, B., Hirata, H. et al. . 1995, Synapse, 21, 169.

14. Yamamoto, B.K. and Zhu, W. 1998, J. Pharmacol. Exp. Ther., 287, 107.

15. Pubill, D., Chipana, C., Camins, A., Pallas, M., Camarasa, J. and Escubedo, E. 2005, Toxicol. Appl. Pharmacol., 204, 57.

16. Escubedo, E., Chipana, C., Perez-Sanchez, M., Camarasa, J. and Pubill, D. 2005, J. Pharmacol. Exp. Ther., 315, 658.

17. Chipana, C., Camarasa, J., Pubill, D. and Escubedo, E. 2006, Neuropharmacology, 51, 885.

18. Deng, X. and Cadet, J.L. 1999, Brain Res., 851, 254.

19. Kramer, H.K., Poblete, J.C. and Azmitia, E.C. 1998, Neuropsychopharmacology, $19,265$.

20. Liu, P.S., Liaw, C.T., Lin, M.K., Shin, S.H., Kao, L.S. and Lin, L.F. 2003, Eur. J. Pharmacol., 460, 9.

21. Skau, K.A. and Gerald, M.C. 1978, J. Pharmacol. Exp. Ther., 205, 69.

22. Klingler, W., Heffron, J.J., Jurkat-Rott, K., O'sullivan, G., Alt, A., Schlesinger, F., Bufler, J., Lehmann-Horn, F. 2005, J. Pharmacol. Exp. Ther., 314, 1267.

23. Marks, M.J., Burch, J.B. and Collins, A.C. 1983, J. Pharmacol. Exp. Ther., $226,817$.

24. Flores, C.M., Rogers, S.W., Pabreza, L.A., Wolfe, B.B. and Kellar, K.J. 1992, Mol. Pharmacol., 41, 31.

25. Wei, Q., Jurma, O.P. and Andersen, J.K. 1997, J. Neurosci. Res., 50, 618.

26. Imam, S.Z., Newport, G.D., Duhart, H.M., Islam, F., Slikker Jr., W. and Ali, S.F. 2002, Ann. N.Y. Acad. Sci., 965, 204.

27. Fornai, F., Gesi, M., Lenzi, P. et al. 2004, Ann. N.Y. Acad. Sci., 1025, 181.

28. Henderson, L.P., Gdovin, M.J., Liu, C., Gardner, P.D. and Maue, R.A. 1994, J. Neurosci., 14, 1153.

29. Blumenthal, E.M., Conroy, W.G., Romano, S.J., Kassner, P.D. and Berg, D.K. 1997, J. Neurosci., 17, 6094.

30. Garcia-Ratés, S., Camarasa, J., Escubedo, E. and Pubill, D. 2007, Toxicol. Appl. Pharmacol., 223, 195.

31. Takahashi, T., Yamashita, H., Nakamura, S., Ishiguro, H., Nagatsu, T. and Kawakami, H. 1999, Neurosci. Res., 35, 175.

32. Jonnala, R.R. and Buccafusco, J.J. 2001, J. Neurosci. Res., 66, 565.

33. Dickinson, J.A., Hanrott, K.E., Mok, M.H., Kew, J.N. and Wonnacott, S. 2007, J. Neurochem., 100, 1089.

34. Myhre, O. and Fonnum, F. 2001, Biochem. Pharmacol., 62, 119.

35. Sulzer, D. and Rayport, S. 1990, Neuron, 5, 797.

36. Foster, J.D., Pananusorn, B. and Vaughan, R.A. 2002, J. Biol. Chem., 277, 25178.

37. Wonnacott, S. 1997, Trends Neurosci., 20, 92.

38. Aracava, Y., Pereira, E.F., Maelicke, A. and Albuquerque, E.X. 2005, J. Pharmacol. Exp. Ther., 312, 1195. 
39. Chipana, C., Camarasa, J., Pubill, D. and Escubedo, E. 2008, Neurotoxicology, 29, 179.

40. Simon, N.G. and Mattick, R.P. 2002, Addiction, 97, 1523.

41. Camarasa, J., Marimon, J.M., Rodrigo, T., Escubedo, E. and Pubill, D. 2008, Eur. J. Pharmacol., 589, 132.

42. Battaglia, G., Yeh, S.Y. and De Souza, E.B. 1988, Pharmacol. Biochem. Behav., 29, 269.

43. Buisson, B. and Bertrand, D. 2001, J. Neurosci., 21, 1819.

44. Gaimarri, A., Moretti, M., Riganti, L., Zanardi, A., Clementi, F. and Gotti, C. 2007, Brain Res. Rev., 55, 134.

45. Pauly, J.R., Marks, M.J., Robinson, S.F., van de Kamp, J.L. and Collins, A.C. 1996, J. Pharmacol. Exp. Ther., 278, 361.

46. Peng, X., Gerzanich, V., Anand, R., Wang, F. and Lindstrom, J. 1997, Mol. Pharmacol., 51, 776.

47. Peng, X., Gerzanich, V., Anand, R., Whiting, P.J. and Lindstrom, J. 1994, Mol. Pharmacol., 46, 523.

48. Sallette, J., Pons, S., Devillers-Thiery, A., Soudant. M., Prado de Carvalho, L., Changeux, J.P., Corringer, P.J. 2005, Neuron, 46, 595.

49. Vallejo, Y.F., Buisson, B., Bertrand, D. and Green, W.N. 2005, J. Neurosci., $25,5563$.

50. Schroeder, K.M., Wu, J., Zhao, L. and Lukas, R.J. 2003, J. Neurochem., 85, 581.

51. Darsow, T., Booker, T.K., Pina-Crespo, J.C. and Heinemann, S.F. 2005, J. Biol. Chem., 280, 18311.

52. Gopalakrishnan, M., Molinari, E.J. and Sullivan, J.P. 1997, Mol. Pharmacol., 52, 524.

53. Nuutinen, S., Ekokoski, E., Lahdensuo, E. and Tuominen, R.K. 2006, Eur. J. Pharmacol., 544, 21.

54. Cho, C.H., Song, W., Leitzell, K. et al. . 2005, J. Neurosci., 25, 3712.

55. Charpantier, E., Wiesner, A., Huh, K.H., Ogier, R., Hoda, J.C., Allaman, G., Raggenbass, M., Feuerbach, D., Bertrand, D., Fuhrer, C. 2005, J. Neurosci., 25,9836 .

56. Nashmi, R. and Lester, H. 2007, Biochem. Pharmacol., 74, 1145.

57. Metzger, R.R., Hanson, G.R., Gibb, J.W. and Fleckenstein, A.E. 1998, Eur. J. Pharmacol., 349, 205.

58. Hansen, J.P., Riddle, E.L., Sandoval, V., Brown, J.M., Gibb, J.W., Hanson, G.R., Fleckenstein, A.E. 2002, J. Pharmacol. Exp. Ther., 300, 1093.

59. Dajas-Bailador, F. and Wonnacott, S. 2004, Trends Pharmacol. Sci., 25, 317.

60. Ehrlich, B.E., Kaftan, E., Bezprozvannaya, S. and Bezprozvanny, I. 1994, Trends Pharmacol. Sci., 15, 145.

61. Rizzuto, R. 2001, Curr. Opin. Neurobiol., 11, 306.

62. Chipana, C., Torres, I., Camarasa, J., Pubill, D. and Escubedo, E. 2008, Neuropharmacology, 54, 1254.

63. Pike, B.R., Zhao, X., Newcomb, J.K., Posmantur, R.M., Wang, K.K. and Hayes, R.L. 1998, Neuroreport, 9, 2437.

64. Buki, A., Koizumi, H. and Povlishock, J.T. 1999, Exp. Neurol., 159, 319. 
65. Harris, A.S. and Morrow, J.S. 1988, J. Neurosci., 8, 2640.

66. Wang, K.K. 2000, Trends Neurosci., 23, 20.

67. Zhang, Z., Larner, S.F., Liu, M.C., Zheng, W., Hayes, R.L. and Wang, K.K. 2009, Apoptosis, 14, 1289.

68. Jiménez, A., Jorda, E.G., Verdaguer, E., Pubill, D., Sureda, F.X., Canudas , A.M., Escubedo, E., Camarasa, J., Camins, A., Pallàs, M. 2004, Toxicol. Appl. Pharmacol., 196, 223.

69. Gopalakrishnan, M., Molinari, E.J. and Sullivan, J.P. 1997, Mol. Pharmacol., $52,524$.

70. Kawai, H. and Berg, D.K. 2001, J. Neurochem., 78, 1367. 


\title{
8. Technological, biopharmaceutical and pharmacokinetic advances: New formulations of application on the skin and oral mucosa
}

\author{
Ana C. Calpena', Beatriz Clares ${ }^{2}$ and Francisco Fernández' \\ 'Section of Biopharmacy and Pharmacokinetics, Department of Pharmacy and Pharmaceutical \\ Technology, Faculty of Pharmacy, Barcelona University, Avenue Joan XXIII, 08028 Barcelona, Spain \\ ${ }^{2}$ Department of Pharmacy and Pharmaceutical Technology, Faculty of Pharmacy \\ Granada University, Campus de la Cartuja, I807I Granada, Spain
}

\begin{abstract}
Currently a growing interest to improve the pharmacological therapy exists, not only by the production and the appearance of new drugs, but guaranteeing that the uses of those which already exist, become more effective. In fact, the conventional pharmaceutical formulations of different drugs present a few secondary effects due to oral administration. In order to avoid these undesired side effects, the purpose of current therapeutic is the development and research of formulations as an alternative to others routes of administration. Therefore, in spite of the undoubtedly complete parenteral absorption, the transdermal and transbuccal routes appear to be a rather attractive alternative to provide an efficient absorption. In this chapter a new technological, biopharmaceutical and pharmacokinetic approach of strategies for application on skin and buccal mucosa are reported.
\end{abstract}

Correspondence/Reprint request: Dr. Ana C. Calpena Campmany, Section of Biopharmacy and Pharmacokinetic, Department of Pharmacy and Pharmaceutical Technology, Faculty of Pharmacy, Barcelona University, Avenue Joan XXIII, 08028 Barcelona, Spain. E-mail: anacalpena@ub.edu 
In the future new transdermal drug delivery systems will emerge to be more effective, equipped with an improved aesthetic appearance, better adherence and greater diffusion. But to reach these aims, it is necessary previous knowledge of histology and physiology of skin, and factors involved in the penetration of drugs through it.

\section{Introduction}

The pharmaceutical sciences are faced with a need to develop alternative dosage forms for transdermal and transmucosal absorption. In addition to oral and parenteral routes, the suitable sites for administering drugs are the nasal, vaginal, rectal or ocular mucosa. However the oral mucosa represents the most popular route, because of its excellent permeability, good accessibility, high patient acceptance and compliance, the dosage forms can be easily attached to and removed from the mucosa. Moreover oral mucosa is routinely exposed to different compounds and therefore is supposed to be rather robust and less prone to irreversible irritation or damage by the drug, the dosage form, absorption promoters, etc. In fact, the turnover time for the buccal epithelium has been estimated at 5-6 days [1].

Within the oral mucosal cavity, the buccal region offers an attractive route of administration over peroral administration; buccal routes offer the advantages of avoiding hepatic first-pass metabolism, local intestinal enzymes and secretions and are deficient in enzymatic degradation.

In the other hand, the transdermal administration of drugs offers advantages that can enhance the therapeutic benefits of the active substances. This route of administration avoids the gastrointestinal tract and biotransformation due to the first-pass effect and metabolism in the liver. Drug release is targeted at the specific site where it is needed, and the percentage of absorption can be controlled. Delayed release formulations can be used. Systemic secondary effects are reduced, and topical formulations are easy to apply, a factor that improves patient compliance. Substantial concentrations of the drug can be reached in the soft tissues at the site of application. In addition, transdermal formulations can be used in readily accessible sites, and such formulations are nontoxic and easy to use.

These advantages, in both routes, are particularly useful with drugs that can break down in the gastrointestinal tract, or drugs used for long-term treatments, intravenously, or for osteoarticular wounds.

\section{Anatomy, physiology and permeability of the oral mucosa and skin}

Despite the advantages of transdermal and transbuccal pathways, the primary function of skin and oral mucosa is the protection of the underlying tissue. Therefore, to set the stage for subsequent discussion of strategies for 
use of dosage forms in transdermal and transmucosal drug delivery, basic physiological characteristic of the skin and oral mucosa should be mentioned.

\subsection{Oral mucosa}

Drugs can be absorbed from any of the mucosal tissues in the oral cavity: maxillary artery supplies blood to buccal mucosa and blood flow is faster and richer $\left(2.4 \mathrm{~mL} / \mathrm{min} / \mathrm{cm}^{2}\right)$ than that in the sublingual (beneath the tongue), gingival and palatal regions, thus facilitates passive diffusion of drug molecules across the mucosa.

Buccal mucosa is composed of several layers of different cells, but consists principally of two components, an epithelium and an underlying connective tissue (basal lamina, propria lamina and submucosa). Also numerous racemose, mucous, serous glands and major blood vessels and nerves are present in the submucous tissue of the cheeks [2]. The epithelium of the human oral mucosa shows two distinct patterns of maturation, nonkeratinized and keratinized. The most interesting, non-keratinized epithelium forms the surface of the distensible lining of the soft palate, ventral surface of the tongue, floor of the mouth, alveolar mucosa, vestibule, lips, and cheek.

The epithelium and its basal lamina constitute the major resistance barrier [3]. Substances can cross the buccal epithelial membrane by the mechanisms of simple diffusion, carrier-mediated diffusion (intercellular or intracellular), active transport, and endocytosis [4]. Most permeability studies (large molecules) point towards intercellular via [5].

The flux of drug through the membrane under sink condition for paracellular route can be written as Eq. (1)

$$
J_{\mathrm{p}}=\frac{D_{\mathrm{p}} \varepsilon}{h_{\mathrm{p}}} C_{\mathrm{d}}
$$

Where, Dp is the diffusion coefficient of the permeate in the intercellular spaces, $\mathbf{h p}$ is the path length of the paracellular route, $\boldsymbol{\varepsilon}$ is the area fraction of the paracellular route and $\mathbf{C d}$ is the donor drug concentration.

Similarly, flux of drug through the membrane under sink condition for transcellular route can be written as Eq. (2).

$$
J_{\mathrm{c}}=\frac{(1-\varepsilon) D_{\mathrm{c}} K_{\mathrm{c}}}{h_{\mathrm{c}}} C_{\mathrm{d}}
$$

Where, Kc is the partition coefficient between lipophilic cell membrane and the aqueous phase, Dc is the diffusion coefficient of the drug in the transcellular spaces and he is the path length of the transcellular route [6]. 
In general, lipophilic drugs are absorbed through the intracellular route, whereas hydrophilic drugs are absorbed through the intercellular route, but the rate of penetration varies depending on the physicochemical properties of the molecule and the type of tissue being traversed. This has led to the suggestion that materials use one or more of the following routes simultaneously to cross the barrier region in the process of absorption, but one route is predominant over the other depending on the physicochemical properties of the diffusant [7]. So, the absorption potential of the buccal mucosa is influenced by the lipid solubility, molecular weight of the diffusant and carrier $\mathrm{pH}$ [8]. In addition, the microenvironment of the buccal cavity lends itself to modifications, in very few cases also the barriers such as saliva, mucus, membrane coating granules, retard the rate and extent of drug absorption through the buccal mucosa. All affects its bioavailability, hence development of unidirectional release systems with backing layer results high drug bioavailability [9].

\subsection{Skin}

The functions of the skin are thermoregulatory, immunological, mediation of sensation, social and protective. Barrier function plays the most important role in drug development and pharmacokinetic implications for both topically and systemically administered drugs. However the skin is the most accessible organ of the body but it is designed to isolate the organism from the external milieu, and thus poses a challenge to the pharmaceutical development of excipients that yield optimal permeation and cutaneous absorption of the active principles.

The skin is a complex organ consisting of three anatomical layers, the epidermis, the dermis and a subcutaneous fat layer. Moreover, the skin is pierced by the sebaceous and eccrine sweat glands and the hair follicles [10].

The epidermis is in humans $0.02-0.2 \mathrm{~mm}$ thin. The epidermis is made up of two layers: the stratum germinativum and the stratum corneum. The stratum corneum is a dead fully keratinised cells tissue. The functions are: protective, against external environment, occlusive, preventing body water loss (dehydration), and receptor for epidermal metabolic products. In fact, the stratum corneum provides the major barrier to penetration of topically applied drugs due to its lipid-rich nature and its low water content. The water content of the normal stratum corneum is $20-30 \%$. The intracellular lipids consist of ceramides, fatty acids and cholesterol. There are also other intracellular lipids or lipids from the sebaceous glands or from epidermal fat [11].

The region below epidermis is called dermis. It supports and strengthens the epidermis. It ranges 5-20-times thicker than epidermis (approximately 2-3 $\mathrm{mm}$ thick). The dermis contains fibrous protein collagen, elastin, 
histiocytes, mastocytes, water, ions, carbohydrates, blood and lymphatic vessels and nerves. The dermis is therefore a sensitive and highly irrigated tissue. Through the blood vessels in the dermis, the drugs enter the circulation system; firstly they have to cross the stratum corneum.

The subcutaneous fat layer or hipodermis represents the separation zone between the dermis and underlying tissues. It is composed of fat and elastic fibers. The hypodermis is the base of hair follicles and the sweat glands. It is also a well irrigated and innervated layer. Sometimes, the fat deposits may serve as a deep compartment for the drug and this can delay entry into the blood.

Number and type of hair follicles and glands is very relevant for drug penetration. In this zone of appendices, the stratum corneum thickness decreases, and even may disappear. Consequently the appendices are important access roads. The number of appendices varies depending on the species and anatomical region [12].

\section{Cutaneous and transbuccal metabolism}

Drug absorbed through the oral mucosa enters the systemic circulation directly via the jugular vein, avoiding the liver where they might be metabolized. However the drugs which are swallowed in the saliva do not avoid first pass metabolism and will be subjected to degradation by digestive juices. This may be partly overcome by using a drug delivery system which has a unidirectional drug outflow $[13,14]$.

Also, the enzymatic activity on the surface of the buccal mucosa should be evaluated as a barrier to drugs buccal delivery. In this way, the inclusion of enzyme inhibitors in buccal bioadhesive delivery systems could improve buccal bioavailability [15].

The viable part of the epidermis represents an enzymatic barrier for drugs after topical application. The distribution of the enzyme system will depend on the anatomical area and the species [16, 17], causing some differences in absorption and considerable transdermal first pass metabolism [18-20].

In vivo enzymatic activity in the epithelium can activate pro-drugs decreasing the delivery [21].

The stratum corneum can act as a reservoir for drugs, causing the pharmacological response to continue for a short time after the device has been removed. In other cases, depending on the delivery system, the drug will diffuse into underlying layers. The results presented indicate that most of the enzyme activity of the skin may be localized in the epidermal layer.

For this reason, the study of skin metabolism should take into account not only in the field of transdermal drug delivery but also for the safe and efficient local skin treatment with topically applied substances. 
Finally, the oral mucosa contains the greatest variety of micro-organisms, which could alter drugs [22]. The entry into the body of these organisms is limited by the oral epithelium, which is not, as is often suggested, a highly permeable membrane.

Skin surface contains many and some potentially pathogenic microbiota. The drugs topically applied can be metabolised by bacteria on the skin surface [23]. Also, this bacterial population can encourage its growth due to heat or humidity transdermal system. It must be kept in mind when opening pores in skin and oral barrier.

Despite these disadvantages, the enzymes in the skin are essential in order to maintain skin good conditions contribute to the right skin $\mathrm{pH}$ and maintain skin protective capability against pathogens or reactive oxygen species.

Finally, it must be emphasized that the delivery system properties (e.g. permeation enhancers) and regional variations might be considered as a potential reason for inter- and intra-individual variations in metabolism and bioavailability of transdermally administered drugs $[24,25]$.

\section{Pharmaceutical considerations}

To a certain extent, the structure of the oral mucosa resembles that of the skin. For this reason some pharmaceutical considerations are general. Great care needs to be exercised while developing a safe and effective buccal adhesive drug delivery device. Factors influencing drug release and penetration through buccal mucosa, organoleptic factors, and effects of additives used to improve drug release pattern and absorption, the effects of local drug irritation caused at the site of application or texture of buccal mucosa, thickness of the mucus layer, its turn over time and effect of saliva are to be considered while designing a formulation. Also, the buccal epithelium is a lipoidal barrier, hence the majority of absorption of drugs is passive. However, some authors have demonstrated an active transport [26-28].

Another very important factor is the location of drug release system. The dosage form may be used as sublingual, buccal, local or periodontal delivery system. Variations in oral physiology will undoubtedly affect drug absorption.

In the other hand, the permeation of topically applied drugs into the systemic circulation consists of three phases:

- The release from the vehicle: the drug may be dissolved in the excipient and then diffuse to contact the skin surface.

- In a transdermal device, the primary design goal is the maintenance of the desired constant drug concentration at the skin surface for a suitable length of time. 
- Penetration into the epidermis and permeabilization: Once the drug reaches the vehicle/skin surface interphase, it can penetrate through the sweat duct, hair follicles and sebaceous glands, being dissolved in sebum secretion (collectively called the shunt or transappendageal via) or directly across the stratum corneum (transepidermal via).

- The passage through the dermis and the entry into the systemic microcirculation.

There are several routes by which drugs can pass through the skin:

- The intercellular route, the passage of drug through the lipid matrix between corneocytes in the stratum corneum.

- The transcellular route, this occurs when drug passes through both the corneocytes and the lipid matrix within the stratum corneum.

- The appendageal route, the passage of drug through the appendages, such as hair follicles and sweat glands; therefore bypassing absorption through the stratum corneum.

The intercellular and transcellular route are also known as "transepidermal via". It has been suggested that transappendageal via is more rapid that transepidermal via but it is considered unimportant. Transepidermal via is the most important pathway of passage, especially the intercellular route, but all this is depending on the molecules physicochemical properties.

Drug penetration through the skin depends on many factors, dependent on animal, drugs and formulation, which can modify the efficacy of a treatment.

The diffusion of drug across the stratum corneum is driven by a thermodynamic gradient and is not determined solely by a concentration gradient. According to Higuchi [29], drug permeation can be represented in a mathematical model for the purpose of predicting dermal absorption in this equation:

$$
\mathrm{dQ} / \mathrm{dt}=\text { P. D. S. C/h }
$$

Where $\mathbf{S}$ corresponds to the total surface of application, $\mathbf{h}$ is the average thickness of the skin in this surface, $\mathbf{D}$ corresponds to the diffusion coefficient of a given drug across the skin, $\mathbf{C}$ is the applied drug concentration, and $\mathbf{P}$ the partition coefficient between the stratum corneum and the vehicle. From this equation it could be deduced the main factors affecting the rate of penetration:

\subsection{Drug criteria}

- Lipid/water partition coefficient. It is the most important factor of penetration in the stratum corneum, due to the lipid-rich nature of the 
stratum corneum. Absorption of hydrophilic compounds is limited by the lipophilicity of the stratum corneum. However the dermis is much more hydrophilic than the stratum corneum and so act as a barrier to extremely hydrophobic compounds [30], once a hydrophilic compound has penetrated through this, it will partition readily into the epidermis and into systemic circulation [31]. So transdermal administration is ideal for substances with intermediate polarity.

- Molecular weight. This factor also impacts upon drug diffusion through the stratum corneum and dermis. Small molecules are more likely to cross skin. It is defined by its diffusion coefficient $\mathrm{D}$, which is inversely proportional to the cube root of molecular weight. The larger the molecular weight, the lower the diffusion coefficient. However the lipophilic nature of the drug seems to play the most important role in this fact.

- Drug concentration. It is directly proportional to the penetration rate, although drug solubility in the vehicle, should not be exceeded.

- Degree of drug dissociation. It is depending not only on skin $\mathrm{pH}$, but also on formulation $\mathrm{pH}$.

Transdermal administration has several advantages, especially in those drugs with a low water solubility and low bioavailability, and/or those with a great first pass metabolism in comparison with the oral administration. Moreover, pre-systemic metabolism is avoided, permitting lower daily doses. Blood levels of the drug can be kept for extended periods of time prolonging drug action and reducing the dosing frequency. Inter and intra patient variability is reduced, and patient compliance and acceptability improved. Lastly, input of drug can be stopped by removal of the patch [32].

\subsection{Vehicle criteria}

The vehicle in which a drug is applied to the skin must be not only a support, but a delivery system that drives the drug to an appropriate area or biophase with an optimal rate of release. The absorption of a compound into the skin may be impeded if it is more soluble in the vehicle it is applied in than the stratum corneum [33]. Then it must exit an equilibrium between drug-vehicle and drug-skin affinity in order to ensure the maximal thermodynamic activity, and this is possible when the drug contained in the vehicle chamber is saturated. A lipophilic drug dissolved in an aqueous vehicle would be absorbed before than at the same concentration from a vehicle with other lipophilic solvent. 
The vehicle is intended to function as a reservoir to provide a steady supply of drug and prolonging the effect. Therefore, it is important to take into consideration the vehicle effect will have on the dermal absorption drugs.

For a satisfactory release of drug from the vehicle, several conditions must be taken into account:

- Diffusion coefficient of drug in the vehicle must be optimal, for it drug must be solubilized, without possessing a selective affinity toward the vehicle.

- The lower the viscosity the greater the diffusion coefficient.

- Vehicle must improve the permeability by hydration, occlusion or direct penetration.

- Vehicle must not cause irritancy in the skin.

Optimization by a suitable formulation is responsible of residence time, local concentration of the drug in the mucosa and the amount of drug transported across the mucosa into the blood. The products for the buccal cavity must have good patient compliance, an ideal bioadhesive drug delivery system, should be easy to apply to the mucus and withstand salivation, tongue movement, and swallowing for a period of time. Buccal adhesive drug delivery systems with the size $1-3 \mathrm{~cm}^{2}$ and a daily dose of $25 \mathrm{mg}$ or less are preferable. The maximal duration of buccal delivery is approximately 4-6 $\mathrm{h} \mathrm{[34].}$

From a dermal point of view, the aim of skin applied formulations is to reach the skin surface, stratum corneum, viable epidermis, dermis, hair follicles, sweat glands or systemic circulation. Depending on the particular aim, the most appropriate biopharmaceutical and physicochemical aspects must be taken into account.

\subsection{Others factors}

Penetration of the substances into the skin can be modified by some factors as age, gender, location of the skin, skin damage or disease, as well as the well know regional variation in skin permeability to different molecules [35].

Also, tissue vascularity determines the rate of absorption. In man, dermal blood flow is about $2.5 \mathrm{~mL} \mathrm{~min}^{-1} 100 \mathrm{~g}^{-1}$.

Areas of the body where the stratum corneum is thickest, such as the palms of the hands $(400 \mu \mathrm{m})$ and soles of the feet $(600 \mu \mathrm{m})$ will be far less permeable to xenobiotic compounds than an area of the body where the stratum corneum is much thinner, such as the scrotum $(5 \mu \mathrm{m})$ [36].

However several pharmacokinetic studies show bioequivalence of several anatomical sites after patch application: clonidine patch provides similar plasma concentration profile after application on arm and chest [37]. The rate 
and extent of nicotine absorption from Nicoderm ${ }^{\circledR}$ were similar after application on upper outer arm, upper back and upper chest [38]. Plasma concentrations of norelgestromin and ethinyl estradiol from the contraceptive patch remain within the reference ranges throughout the wear period regardless of the application site [39].

Both, temperature and occlusion, increase passive diffusion [40].

Equally, in older people the stratum corneum thickens and becomes less hydrated, decreasing absorption [41].

Recent studies demonstrate that skin properties in the stratum corneum vary considerably among ethnic groups [42].

However, the main factors to consider are two: damage/disease of skin and its hydration. The skin sustains damage [43] or diseases [44, 45] can reduce barrier action and lead to increased permeability of the drugs. These processes are an important obstacle to the selection of the formulation. The degree of hydration of the skin improved contact and hydration of the lipid channels of the stratum corneum. When the skin is hydrated it reduces the evaporation of moisture from underlying tissues, so it is better to use emollients and occlusive substances that moisturizers. As a result, transdermal absorption increases.

\section{Strategies for transdermal and transbuccal drug delivery systems: Current technologies of chemically and physically enhanced diffusive delivery}

The objective of a transdermal and transbuccal delivery system is to provide a sustained concentration of drug for absorption avoiding local irritation. However the slow transport of many drugs across skin limits these administrations.

The administration of active principles on the skin and buccal mucosa with the aim of achieving a systemic and reservoir effect has led to pharmaceutical development of a new form of dosage. There are some methods by which penetration of compounds through the oral mucosa and skin can be improved: by the use of prodrug, co-administration of enzyme inhibitors, delivery systems, enhancers or physical methods. But particular caution should be taken with the creation of pores in the skin, as this has a large number and variety of microbiota, including pathogens.

\subsection{Drug delivery system in buccal mucosa}

Sublingual drug delivery is more commonly used to treat acute disorders, whereas the buccal route is chosen when a prolonged release of drug is needed 
in chronic disorders [46]. However bioavailabilities of some drugs by the buccal route were still low due to its anatomical, physiological and tecnological factors such as texture of buccal mucosa, thickness of the mucus layer, its turnover time, effect of saliva, enzymatic barrier. These factors are to be considered in designing the dosage forms.

For example, one to two litres of fluid are excreted daily into the human mouth and there is a continuous, low basal secretion of $0.5 \mathrm{~mL} \mathrm{~min}{ }^{-1}$ which will rapidly increase to more than $7 \mathrm{~mL} \mathrm{~min}^{-1}$ by the thought, smell or taste of food [47]. Therefore their contact with the oral mucosa is brief. In order to locally treat the mucosa, delivery systems have been designed to prolong residence in this area. These formulations include semisolid, mucoadhesive patches and bioadhesive tablets.

The use of adhesive polymers plays an important role in the development of mucoadhesive dosage forms, prolonging residence on the oral mucosa, improving absorption, targeting of specific tissues and allow for some degree of sustained release of the active principle [48]. Also some bioadhesive polymers, such as poly(acrylic acid), polycarbophil, and carbopol, can also inhibit certain proteolytic enzymes (trypsin, $\alpha$-chymotrypsin, carboxypeptidases A and B, and leucine aminopeptidase) [49].

The polymers must have the following characteristics:

Polymer and its degradation products should be non-toxic, non-irritant, free from leachable impurities and easily available. Also they should have good spreadability, wetting, swelling and solubility, biodegradability, bioadhesive and viscoelastic properties, and should demonstrate local enzyme inhibition and penetration enhancement properties.

In general, buccal dosage forms can be categorized into unidirectional or multidirectional and reservoir or matrix type. They also must possess the requirements described above. If necessary, the drug may be formulated in certain physical states, such as microparticles [50], sponges [51], liposomes $[52,53]$ or nanoparticles $[54,55]$, prior to formulation of dosage form in order to achieve some desirable properties, e.g. enhanced activity and prolonged drug release.

Sprays and fast dissolving tablets are the two most widely used formulations for sublingual delivery. Tablets have been the most commonly investigated dosage form for buccal drug delivery to date, however they have a poor patient compliance.

Others dosage forms, such as buccal films and patches offer advantages due its flexibility and comfort [56]. Different drugs have been successfully studied obtaining optimal permeation values for a systemic effect $[57,58]$.

Certain bioadhesive polymers, e.g. pluronics show a phase change from a liquid to a semisolid with the corporal temperature. They have the advantage 
of easy dispersion throughout the oral mucosa, rich retention at the site of application and adequate drug penetration [59].

In contrast to polymers utilized until now, a new generation of polymers can adhere directly to the cell surface, rather than to mucus [60].

\subsection{Drug delivery system in skin}

Currently the advances on transdermal delivery systems can be divided into three categories; physical methods, chemical methods and other complex systems. These last may include vesicles, eutectic mixtures, pro-drug, micelles, cyclodextrins microemulsions, nanoemulsions, cubic phases, colloids and synergistic mixtures.

It also noted the success in the permeation after the combination of several techniques such as chemical enhancers, physical enhancers and vesicles [61-63].

\subsubsection{Other complex systems}

\subsubsection{Dosage forms (solid, liquid, semisolid)}

Classical ointments, creams, lotions and gels are not suitable for action and/or controlled transdermal delivery.

When an active principle is administered topically, including in a conventional pharmaceutical way (solution, emulsion, gel...) through the skin, the latter has to release the active principle it contains so that it previously dissolves, is absorbed and reaches its area of activity, which in this case must be centred on the coetaneous tissue. Nevertheless, the arrival of the active principle at the area of activity may be insufficient; rather, by means of the circulating fluids, it can be distributed to certain tissues that can determine the emergence of undesirable effects.

On other occasions, the active principle reaches an adequate concentration of its specific receptors, but this concentration is maintained for a short period of time. This forces the administration of the active principle to be repeated at short intervals of time, using conventional dosage methods for immediate release.

From a pharmaceutical point of view, the solution to these problems could lie in the chemical association of the active substance with an appropriate delivery preparation capable of specificity of action [64]. In this way, vectoring is defined as the attainment of maximum efficacy of a drug, by increasing its release in the area where its pharmaceutical receptors are found, thereby minimizing its concentration in other areas of the organism, reducing the adverse effects. So, one of the objectives of pharmaceutical research in recent decades has been the development of systems that release the active 
principle selectively at the level of the damaged organ, without producing changes in healthy tissue.

Several advances to this effect have been made in the last 2-3 decades and novel drug delivery systems have been investigated.

Ability to solubilize and stabilize drugs, their high viscosity hydromiscibility, thermosensitivity and micellar behavior make the Pluronic gels a feasible vehicle for oral and topical control drug delivery.

Gels of pluronic as the vehicles for the percutaneous administration of anti-inflammatory [65], analgesic [66], peptides [67], beta-blockers [68] and other active principles were studied and evaluated. Additionally, in the early 1990s Marty Jones and Lawson Kloesel [69] developed PLO (Pluronic Lecitin Organogel) as a transdermal drug carrier delivery system. Recently, efficacy of transdermal use of different drugs has been demonstrated.

Organogels are semi-solid systems, in which an organic liquid phase is immobilized by a three-dimensional network composed of self-assembled, intertwined gelator fibers $[70,71]$. The application of different organogel systems to transdermal via has been studied [72, 73].

Microemulsions (clear, stable, isotropic mixtures of oil, water and surfactant in combination with a cosurfactant) are systems currently of interest to the pharmaceutical scientist because of their considerable potential to act as drug delivery transdermal vehicles (with diameters in the range of 20-100 nm) $[74,75]$. However, it is necessary large amounts of surfactants to form microemulsions. Therefore, the use of these colloidal carrier systems in the future depends on the choice of well-tolerated surfactants and the restriction of their amounts.

Several excellent reviews discuss the advantages, limitations and opportunities offered by patches reservoir or matrix type in greater details and thus will not be covered in this review.

\subsubsection{Nanosystems or vesicles}

Compared with other external skin preparations, such as creams and liniments, nanosystems provide more adjustable parameters in their preparation, and in treatments offer the advantages of enhancing drug effects, shortening the expected treatment course and lowering side effects.

Among the multiple advantages of these vectors, the following are shown:

- Protects the active substance from deactivation (chemical, enzymatic or immunological), from the area of administration to the biophase.

- Improves transport of the active principles to places difficult to reach.

- Increases the specificity of action and efficacy at cellular and/or molecular level. 
- Increases the average life span of the drug.

- Modifies the soluble properties of the active substance and reduces its immunogenicity and antigenicity.

- Decreases toxicity of certain organs by adjustment of the tissue distribution of the active principle.

- Lacking in toxicity, they are biodegradable and can be prepared industrially on a large scale.

The most important factor for potential nanotoxicity is the lack of biodegradability of many nanomaterials. After generation of these nanomaterials, they will stay forever and pollute the environment and, considering the circle in the environment, finally end up in the human body.

In the other hand, lipid analysis of buccal tissues shows the presence of phospholipid $76.3 \%$, glucosphingolipid $23.0 \%$ and ceramide NS at $0.72 \%$. Other lipids such as acyl glucosylated ceramide, and ceramides [76]. In Stratum corneum, cellular membranes of keratinocytes are composed mainly of phosphatidyl choline and sphingomyelin. Also, the intercorneocyte matrix is rich in phospholipids.

In view of the above, in recent years a great importance has been attached to using lipids as vehicular systems and permeants of active principles through the skin and oral mucosa. The outstanding advantage of lipid vesicles is their easy and complete biodegradation. Lipids are natural materials, are easily degraded by natural processes such as enzymes. Phospholipids as drug carriers have some unique advantages which other conventional external preparations do not have. Phospholipids share a high structural similarity with skin lipids and thus have many advantages. They can moreover affect molecular transport across skin barrier more directly by acting as skin permeation enhancers. This type of application is particularly suitable for certain chronic and relapsing skin diseases, such as chronic eczema, psoriasis, neurodermitis, etc.

Several different kinds of lipid vesicles have been described in the literature.

Liposomes consist of amphiphilic molecules in a bilayer conformation.

In an excess of water these amphiphilic molecules can form one (unilamellar vesicles) or more (multilamellar vesicles) concentric bilayers [77]. Due to its structure, hydrophilic, amphiphilic and lipophilic drugs can be entrapped.

In recent years, most investigators have concentrated on the potential use of liposomes for the transdermal delivery of antibiotics [78], antiviral [79, anesthetics [80] and antiinflamatories [81].

Niosomes are composed of non-ionic amphiphiles (surfactants) and are similar in function to the liposomes [82, 83]. 
Ethosomes are relatively new types of vesicle systems, primarily composed of water, ethanol and phospholipids [79].

Sufficiently deformable and elastic vesicles can enter skin barrier spontaneously, e.g. transfersomes, which cross the skin under the influence of a transepidermal water activity gradient. Transfersomes consist of phospholipids and an edge activator that increases the deformability of the bilayers and is often a single chain surfactant. Recent studies show the effectiveness of elastic liposomes for transdermal delivery of melatonin [84].

Solid Lipid Nanoparticles (SLN) and Nanostructured Lipid Carriers (NLC), SLN are usually aqueous dispersions of solid lipid matrices stabilized by surfactants, or dry powders obtained by lyophilization [85] or spray drying [86], ranging from about 40 to $1000 \mathrm{~nm}$. NCL are produced using blends of solid lipids and liquid lipids (oils) [87]. SLN and NLC exhibit many features for dermal application of cosmetics and pharmaceutics, i.e. controlled release of actives, drug targeting, occlusion and associated with it penetration enhancement and increase of skin hydration. Specifically, since the last decade, SLN have been exploited for delivery of actives via dermal [88-91]. They are an alternative carrier system to emulsions, liposomes and polymeric nanoparticles. Drugs for dermal application using lipid nanoparticles at the present are glucocorticoids, retinoids, non-steroidal anti-inflammatory drugs, COX-2 inhibitors, psoralens and antimycotics. It was shown that it is possible to enhance the percutaneous absorption with lipid nanoparticles. These carriers may even allow drug targeting to the skin or even to its substructures. Thus they might have the potential to improve the benefit/risk ratio of topical drug therapy [92].

In the context it is noteworthy that physico-chemical characteristics of all these vesicles (size, charge, thermodynamic phase, lamellarity and bilayer elasticity) have a significant effect on the behaviour of the vesicles and hence on their effectiveness as a drug delivery system. Taking in account our aim they may serve as:

- A local depot for the sustained release of dermal active compounds.

- Penetration enhancer and facilitate dermal delivery leading to higher localized drug concentrations.

- Rate-limiting membrane barrier for the modulation of systemic absorption or controlled transdermal delivery systems.

\subsubsection{Chemical enhancers}

Generally the usual vehicles are not able to penetrate into stratum corneum by themselves. Penetration enhancement technology is a challenging 
development that would increase the number of drugs available for transdermal administration. Thus for years different compounds capable of penetrating and transporting drugs have been studied, these are chemical substances temporarily diminishing the barrier of the skin and known as penetration promoters [93]. Promoters or accelerant can enhance drug flux, may act by one or more of three main mechanisms [94]:

- Disruption of the highly ordered structure of stratum corneum lipid.

- Interaction with intercellular protein.

- Improved partition of the drug, coenhancer or solvent into the stratum corneum.

The selection of enhancer and its efficacy depends on the physicochemical properties of the drug, site of administration, nature of the vehicle and other excipients. These permeation enhancers should be safe and non-toxic, pharmacologically and chemically inert, non-irritant, and nonallergenic. The different permeation enhancers available are:

- Chelators: EDTA, citric acid, sodium salicylate, methoxy salicylates.

- Surfactants: sodium lauryl sulphate, polyoxyethylene, Polyoxyethylene-9laurylether, Polyoxythylene-20-cetylether, Benzalkonium chloride, 23lauryl ether, cetylpyridinium chloride, cetyltrimethyl ammonium bromide.

- Bile salts: sodium glycocholate, sodium deoxycholate, sodium taurocholate, sodium glycodeoxycholate, sodium taurodeoxycholate.

- Fatty acids: oleic acid, capric acid, lauric acid, lauric acid/propylene glycol, methyloleate, lysophosphatidylcholine, phosphatidylcholine.

- Non-surfactants: unsaturated cyclic ureas.

- Hydrocarbons: alkanes, alkenes, halogenated alkanes, squalane, squalene and mineral oil.

- Alcohols: alkanols, alkenols, glycols, polyglycols and glycerols.

- Amines, amides, esters as isopropyl myristate.

- Others: Terpenes, terpenoids, essential oils and phospholipids.

- Thiolated polymers: chitosan-4-thiobutylamide, chitosan-4thiobutylamide/GSH, chitosan-cysteine, Poly (acrylicacid)-homocysteine, polycarbophil-cysteine, polycarbophil-cysteine/GSH, chitosan-4thioethylamide/GSH, chitosan-4-thioglycholic acid.

In general, it is challenging to strike an optimum balance between the safety and potency of chemical enhancers. In order to develop a transdermal penetration enhancer which a low irritation to the skin, alkyldisiloxanes containing sugar moiety with various alkyl chain length were investigated [95]. 
Nevertheless studies show that treatment with enhancers did not cause major morphological changes in the buccal tissue [96].

Based on such limitations, one can design synthetic enhancers [97] that are significantly more potent in their skin permeabilizing ability as compared to conventional chemicals as Azone (1-dodecylazacycloheptan-2-one).

Also, these limitations can be solved by synergistic effects between enhancers [98] and more polar co-solvents (e.g. ethanol, propylene glycol). Similarly, solvents such as Transcutol ${ }^{\circledR}$ are proposed to act by improving drug solubility within the membrane rather than by increasing drug diffusivity across it [99].

\subsubsection{Physical enhancers: Devices}

The ideal characteristics an active principle should have to penetrate the stratum corneum are: Aqueous solubility $1 \mathrm{mg} \mathrm{mL}^{-1}$, lipophilicity $10 \mathrm{Ko} / \mathrm{w}$ $<1000$, molecular weight $<500 \mathrm{Da}$, melting point $<200{ }^{\circ} \mathrm{C}$, pH of saturated aqueous solution $\mathrm{pH} 5-9$, dose deliverable $10 \mathrm{mg} /$ day.

Unfortunately, drugs such as peptides, proteins and oligonucleotides are usually large, polar and/or charged, characteristics that normally preclude transdermal delivery. However, recent advances in physical enhancement technologies solve these problems.

These methods employed for increasing transport of drug molecules across the skin use some form of mechanical, electrical, magnetic or thermal energy source to promote transport of macromolecules by disrupting the skin membrane. With these new technologies the number of drugs potentially useful for transermal administration has greatly increased, and restrictions related to molecular weight, particle size, dosage, $\mathrm{pH}$, etc. have been reduced.

Examples of physical approaches include the use of iontophoresis, electroporation, sonoporation, high velocity particles, ultrasound, electric current, abrasion, lasers, pressure waves, magnetophoresis and thermophoresis [100].

Other modern methods for skin barrier breaching are micro-scale devices for transdermal macromolecular delivery: liquid jet injectors, powder injectors, microneedles, and thermal microablation [101].

Some of the obstacles in transdermal physical enhancers can be overcome by combining with other physical enhancement techniques for the delivery of macromolecules [102, 103].

Similarly to transdermal, application studies on the mucosa physical enhancers improve the absorption of drugs, e.g. permeation of naltrexone on buccal mucosa was evaluated in presence of chemical enhancers or iontophoresis. No significant differences in penetration rate were observed 
using chemical enhancers; in contrast, Js and $\mathrm{K}_{\mathrm{p}}$ were extensively affected by application of electric fields [104].

A particularly interesting perspective is the application of various physical methods used for gene delivery [105].

Finally, note that some of these methods as iontophoresis, can be used as an in-vivo model for studying physiologic mechanisms and on the analysis and interpretation of dose-response data [106].

\section{Predicting transdermal and transbuccal delivery: In vitro - in vivo correlation}

The main objective in the design and optimization of transdermal and transbuccal dosage forms lies in obtaining a good in vivo performance. Thus, one of the main challenges of biopharmaceutical research is finding a correlation between ex vivo, animal and human studies for prediction of percutaneous and transbuccal absorption in humans.

The permeability of oral mucosa and the efficacy of penetration enhancers have been investigated in numerous in vivo and in vitro models. Various types of diffusion cells, including continuous flow perfusion chambers, Franz cells and Grass-Sweetana cells, have been used to determine the permeability of oral mucosa [107]. Animal models generally are more available than human skin, but only rabbits and pigs have a non-keratinized mucosal lining similar to that in humans.

As for skin studies, several factors should be considered. The ideal model for in vitro permeation studies is the human skin. In this regard, skin from cadavers has approximately the same permeability as living skin, suggesting that the underlying tissues present little resistance to drug adsorption.

A wide range of animal models has been suggested as a suitable replacement for human skin and has been used to evaluate percutaneous permeation of molecules. However the most relevant animal model for human skin is the pig [108] and has less variability than the human skin model [109], also we can see that a synergistic mechanical and chemical on pig skin models exhibit similar permeabilities and pore radii, but the human skin models do not [110]. In fact, drug permeation through human skin at a selected skin site can vary from $46 \%$ to $66 \%$ among individuals [111]. Specifically, pig ear skin, which closely resembles human skin, is a candidate ex vivo alternative model for the investigation of xenobiotics penetration and metabolism. Also, due to its availability, skin of rodents (mice and rats) is commonly used in vitro and in vivo percutaneous permeation studies. 
On the other hand several studies with frozen or fresh skin have been made, highlighting the similarities between the two procedures. However, in general, the results demonstrate that the permeability of the drugs across the skin depends on the storage condition, the length of storage, vehicle and the physicochemical properties of the drug under study [112-114].

To extrapolate the results obtained from experimentation with animal skin in vitro and in vivo to the human situation must be avoided unless scientific validation exists to show that this is appropriate for the given agent.

Cultured epithelial cell lines have also been developed as an in vitro model for studying drug transport and metabolism at biological barriers as well as to elucidate the possible mechanisms of action of penetration enhancers $[115,116]$. However, the overall use of skin cultures is likely to be limited due to questionable performance as a barrier in skin permeation studies, as well as due to their cost and data reproducibility $[117,118]$.

In order to assess the potential of transdermal therapy have been developed some pharmacokinetic models which are based on the physicochemical properties of the penetrant. Most models have focused on the contributions of molecular size and the solubility in stratum corneum lipids. These models provide kinetic equations which allow calculation of the plasma levels achieved after a drug is applied transdermally and transbuccally. A database of in vitro skin permeability coefficient values has been consolidated and over 20 empirical equations have been published estimating permeability coefficients for chemicals penetrating the human skin from aqueous vehicles [119].

Plasma concentration time profiles for most of the transdermal and transbuccal systems are characterized in terms of AUC0-t (area under the time concentration curve from time 0 to time $t$ ), AUC $0-\infty$ (area under the time concentration curve from time 0 to infinity), Cmax (maximal plasma drug concentration) and Tmax (time to maximal plasma drug concentration). Cmax provides a practical estimate of Css (plasma concentration at steady state) [120].

\section{Conclusions}

In many cases buccal and transdermal dose forms can obtain the same bioavailability as intravenous formulations without the disadvantages of this route of administration.

Despite the many advantages of the skin and oral mucosa as sites of drug delivery, only a few of drugs are currently in the market as transdermal delivery system (estradiol, ethinyl estradiol/levonorgestrol, ethinyl estradiol/norelgestromin, ethinyl estradiol/norethindrone, clonidine, 
oxybutinin, fentanyl, methylphenidate, nicotine, nitroglycerin, selegiline, scopolamine, testosterone, rivastigmine and rotigotine).

Because the stratum corneum limits the amount of drug reaching the blood supply and unless the barrier function is reduced with a penetration enhancer it is unlikely that plasma levels above $100 \mathrm{ng} / \mathrm{mL}$ will be attained.

This paper explains why and how skin or oral mucosa preclude essentially any large molecules transport into body, the achievements of transdermal and transmucosal scientists and the new technologies for enhancement the dosage forms across these routes. In this way several physical, chemical and nanosystems methods have been investigated, which can only enhance the drug disposal in the skin and others however, could lead to therapeutic drug concentrations in the systemic circulation.

To face the design of these and others excipients, "in vitro" release studies will constitute an essential tool for its development. It also will let us know the dermal penetration values of drugs and the effectiveness of vehicles.

Therefore, in order to assess the potential of transdermal or transbuccal therapy, it is necessary the development of more experiences and new release vehicles for transdermal and transbuccal drugs.

Lastly it should be noted that other routes, such as nasal, ocular, pulmonary, rectal, and vaginal drug administration, have provided excellent opportunities for the delivery of a variety of compounds.

\section{References}

1. Harris, D., Robinson, J. R. 1992, J. Pharm. Sci., 81, 1.

2. Collins, L. M. C., Dawes, C. 1987, J. Dent. Res., 66, 1300.

3. Squier, C. A., Finkelstein, M. W. 1989, Oral Histology, Development, Structure and Function, A. R. Ten Cate (Ed.), C.V. Mosby, St. Louis, 345.

4. Siegel, I. A. 1984, The Structure and Function of Oral Mucosa, J. Meyer, C. A. Squier, S. J. Gerson (Eds.), Pergamon Press, Oxford, 125.

5. Squier, C. A. 1973, J. Ultrastruct. Res., 43, 160.

6. Shojaei, A. H., Li, X. 1997, Proc. Int. Symp. Control Release Bioact. Mater. $24,427$.

7. Hao, J., Heng, P. W. S. 2003, Drug Dev. Ind. Pharm. 29, 821.

8. Nielsen, H. M., Rassing, M. R. 1999, Int. J. Pharm. 185, 215.

9. Weathercell, J. A., Robinson, C., Rathbone, M. J. 1994, Adv. Drug Del. Rev. $130,24$.

10. Montagna, W. 1961, The Structure and Fuction of Skin, $2^{\text {nd }}$ Ed, Academic Press, New York, 454.

11. Wertz, P. W. 1996, Adv. Drug Deliv. Rev., 18, 283.

12. Monteiro-Riviere N. 1990, Appl. Toxicol., 15, 74. 
13. Darwish, A. M., El-Sayed, A. M., El-Harras, S. A., Khaled, K. A., Ismail, M. A. 2008, Fertil. Steril., 90, 1864.

14. Lei, L., Liu, X., Guo, S., Tang, M., Cheng, L., Tian, L. 2010, J. Control. Release, $146,45$.

15. Walker, G. F., Langoth, N., Bernkop-Schnürch, A. 2002, Int. J Pharm., 233, 141.

16. Martin, R. J., Denyer, S. P., Hadgraft, J. 1987, Int. J. Phann. 39, 23.

17. Moody, R. P., Nadeau, B., Chu, I. 1995, J. Dermatol. Sci., 9, 48.

18. Hsia, S. L., Hao, Y. L. 1966, Biochem. 5, 1469.

19. Goebel, C., Hewitt, N. J., Kunze, G., Wenker, M., Hein, D. W., Beck, H., Skare, J. 2009, Toxicol. Appl. Pharmacol., 235, 114.

20. Mazer, N. A., Heiber, W. E., Moellmer, J. F., Meikle, A. W., Stringham, J. D., Sanders, S. W., Tolman, K. G., Odell, W. D. 1992, J. Control. Release, 19, 347.

21. Adachi, H., Irie, T., Uekama, T., Manako, T., Yano, T., Saita, M. 1993, Eur. J. Pharm. Sci., 1, 117.

22. Yamahara, H., Lee, V. H. L. 1993, Adv. Drug Del. Rev., 12, 25.

23. Denyer, S. P., McNabb, C. 1989, Transdermal Drug Delivery, J. Hadgraft, and R.H. Guy (Eds.), Marcel Dekker, New York, 113.

24. Lefèvre, G., Sedek, G., Huang, H. L., Saltzman, M., Rosenberg, M., Kiese, B., Fordham, P. 2007, J. Clin. Pharmacol. 47, 471.

25. Berner, B., John, V. A., 1994. Clin. Pharmacokinet. 26, 121.

26. Sadoogh-Abrasian F., Evered, D. F. 1979, Br. J. Nutr., 42, 15.

27. Evered, D. F., Sadoogh-Abrasian F., Patel, P. D. 1980, Life Sci., 27, 1649.

28. Evered, D.F., Mallett C. 1983, Life Sci., 32, 1355.

29. Higuchi, T. 1960, J. Soc. Cosm. Chem., 11, 85.

30. Barry, B. W. 2004, Nat. Biotechnol., 22, 165.

31. Bronaugh, R. L., 2004, Dermatotoxicology, 6th edition, H. Zhai, and H.I. Maibach (Eds.), CRC Press New York, 519.

32. Delgado-Charro, M. B., Guy, R. H. 2001, Drug Delivery and Targeting for Pharmacists and Pharmaceutical Scientists, A.M. Hillery, A.W. Lloyd, and J. Swarbrick (Eds.), Harwood Academic Publishers, London, 207.

33. Jacobi, U., Tassopoulos, T., Surber, C., Lademann, J. 2006, Arch. Dermatological Res., 297, 303.

34. Alur, H. H., Johnston, T. P., Mitra, A. K. 2001, Encyclopedia of Pharmaceutical Technology, J. Superbrick, and J. C. Boylan (Eds.), Marcel Dekker Inc., New York, 193.

35. Maibach, H. I., Feldmann, R. J., Hilby, T. H., Servat, W. F. 1971, Arch. Environ. Health, 23, 208.

36. Scheuplein, R. J., Blank, I. H. 1971, Physiol. Rev., 51, 702.

37. MacGregor, T. R., Matzek, K. M., Keirns, J. J., vanWayjen, R. G., van den Ende, A., van Tol, R. G. 1985. Clin. Pharmacol. Ther., 38, 278.

38. Gorsline, J., Okerholm, R. A., Rolf, C. N. 1992. J. Clin. Pharmacol., 32, 576.

39. Abrams, L. S., Skee, D. M., Natarajan, J.,Wong, F. A., Anderson, G. D. 2002, Br. J. Clin. Pharmacol., 53, 141.

40. Feldmann R. J., Maibabach, H. I. 1965, Arch. Dermatol., 91, 661.

41. Barrett, D. A., Rutter, N. 1994, Crit. Rev. Therapeut. Drug Carrier Syst., 11, 1. 
42. Muizzuddin, N., Hellemans, L., Van Overloop, L., Corstjens, H., Declercq, L., Maes, D. 2010, J. Dermatol. Sci., 59, 123.

43. Maibach, H. I. 1976, Dermatol., 152, 11.

44. Turpeinen, M. 1988, Br. J. Dermatol., 118, 517.

45. Kalish, R., Wood, J. A., Wille, J. J., Kydonieus, A. 1996, Contact Dermatitis, 35,76 .

46. Rossi, S., Sandri, G., Caramella, C. M., 2005. Drug Discov. Today: Technol., 2,59 .

47. Shannon, I. L., Prigmore, J. R. 1958, Proc. Soc. Expt. Biol. Med., 97, 825.

48. Leung, S. -H. S., Robinson, J. R., 1991, Water- Soluble Polymers. Synthesis, Solution, Properties and Applications, S. W. Shalaby, C. L. McCormick, and G. B. Butler (Eds.), Washington, DC, 350.

49. Lueßen, H. L. Verhoef, J. C., de Boer, A. G. Junginger, H. E. de Leeuw, B. J. Borchard, G., Lehr, C. M., 1999, Bioadhesive Drug Delivery Systems. Fundamentals, Novel Approaches, and Development, E. Mathiowitz, D. E. Chickering III, and C. M. Lehr (Eds.), Marcel Dekker, New York, 299.

50. Giunchedi, P., Juliano, C., Gavini, E., Cossu, M., Sorrenti, M. 2002, Eur. J. Pharm. Biopharm., 53, 233.

51. Portero, A., Teijeiro-Osorio, D., Alonso, M. J., Remuñán-López, C. 2007, Carbohydr. Polym., 68, 617.

52. El-Samaligy, M. S., Afifi, N. N., Mahmoud, E. A. 2006, Int. J. Pharm., 308, 140.

53. Veuillez, F., Kalia, Y. N., Jacques, Y., Deshusses, J., Buri, P. 2001, Eur. J. Pharm Biopharm., 51, 93.

54. Lopedota, A., Trapani, A., Cutrignelli, A., Chiarantini, L., Pantucci, E., Curci, R., Manuali, E., Trapani, G. 2009, Eur. J. Pharm. Biopharm., 72, 509.

55. He, C., Cui, F., Yin, L., Qian, F., Tang, C., Yin, C. 2009, Eur. Polym. J., 45, 368.

56. Morales, J. O., McConville, J. T. 2011, Eur. J. Pharm Biopharm., in press.

57. Díaz del Consuelo, I., Falson, F., Guy, R. H., Jacques, Y. 2007, J. Contr. Release, $122,135$.

58. Perioli, L., Ambrogi, V., Angelici, F., Ricci, M., Giovagnoli, S., Capuccella, M., Rossi, C. 2004, J. Contr. Release, 99, 73.

59. Morishita, M., Barichello, J. M., Takayama, K., Chiba, Y., Tokiwa, S., Nagai, T. 2001, Int. J. Pharm., 212, 289.

60. Lehr, C. M. 2000, J. Control. Release, 65, 19.

61. Nair, V., Panchagnula, R. 2003, Pharmacol. Res., 43, 555.

62. Qiu, Y., Gao, Y., Hu, K., Li, F. 2008, J. Control. Release, 129, 144.

63. Kajimoto, K., Yamamoto, M., Watanabe, M., Kigasawa, K., Kanamura, K., Harashima, H., Kogure, K. 2011, Int. J Pharm., 403, 57.

64. Ruiz, M. A., Clares, B., Morales, M.E., Gallardo, V. 2008, Drug Dev. Ind. Pharm., 34, 1269.

65. Miyazaki, S., Yokouchi, C., Nakamura, T., Hashiguchi, N., Hou, W. M., Takada, M. 1986, Chem. Pharm. Bull., 34,1801.

66. Lee, B. J., Lee, T. S., Cha, B. J, Kim, S. H., Kim, W. B. 1997, Int. J. Pharm., $159,105$.

67. Pillai, O., Panchagnula, R. 2003. J. Control. Release, 89, 127. 
68. Stamatialis, D. F., Rolevink, H. H. M., Koops, G. H. 2006, J. Contr. Release, 116, e53.

69. Murdan, S. 2005, Hospital Pharmacist, 12, 267.

70. Vintiloiu, A., Leroux, J. C. 2008, J. Control. Release, 125, 179.

71. Ruiz, M. A, Clares, B., Morales, M. E., Gallardo, V. 2007, Pharm. Dev. Technol., $12,637$.

72. Paice, J. A., Von Roenn, J. H., Hudgins, J. C., Luong. L., Krejcie, T. C., Avram, M. J. 2008, J. Pain Symptom Manage., 35, 314.

73. Morales, M. E., Clares, B., López-Viota, V., Ruíz, M. A. 2008, Drug Deliv., $15,373$.

74. Escribano, E., Calpena, A. C., Queralt, J., Obach, R., Doménech, J. 2003, Eur. J. Pharm. Sci. Eur. J. Pharm. Sci., 19, 203.

75. Yuan, J. S., Yip, A., Nguyen, N., Chu, J., Wen, X. S., Acosta, E. J. 2010, Int. J. Pharm., 392, 274.

76. Fox, P. C. Ann. N.Y. Acad. Sci., 842, 132.

77. Bangham, A. D; Standish, M. M; Walkins, J. C. 1965, J. Mol. Biol., 13, 238.

78. Manosroi, A., Kongkaneramit, L., Manosroi J. 2004, Int. J. Pharm., 270, 279.

79. Dubey, V., Mishra, D., Nahar, M., Jain, V., Jain, N. K. 2010, Nanomedicine, 6,590 .

80. Shim, J., Kim, M. J., Kim, H. K., Kim, D. H., Oh, S. G., Ko, S. Y., Jang, H. G., Kim, J. W. 2010, Int. J. Pharm., 388, 251.

81. Clares, B., Medina, M. M., Gallardo, V., Ruíz, M. A. 2009, J. Liposome Res., 19, 197.

82. Choi, M. J., Maibach, H. I. 2005, Skin Pharmacol. Physiol., 18, 209.

83. Uchegbu, I. F., Vyas, S. P. 1998, Int. J. Pharm., 172, 33.

84. Dubey, V., Mishra, D., Asthana, A., Jain, N. K. 2006, Biomaterials, 27, 3491.

85. Lim, S. J., Kim, C. K. 2002, Int. J. Pharm., 243, 135.

86. Freitas, C., Müller, R. H. 1999, Eur. J. Pharm. Biopharm., 47, 125.

87. Müller, R. H., Olbrich, C. 2000, Pharmazeutische Biotechnologie, W. Verlagsgesellschaft (Ed), Wissenschaftliche Verlagsgesellschaft, Stuttgart, 283.

88. Müller, R. H., Petersen, R. D., Hommoss, A., Pardeike, J. 2007. Adv. Drug Del. Rev., 59, 522.

89. Souto, E. B., Almeida, E. J., Müller, R. H. 2007, Adv. Drug Deliv. Rev., 3, 317.

90. Priano, L., Esposti, D., Esposti, R., Castagna, G., De Medeci, C., Fraschini, F., Gasco, M. R. 2007, J. Nanosci. Nanotechnol., 7, 1.

91. Liu, W., Hu, M., Liu, W., Xue, C., Xu, H., Yang, X. L. 2008, Int. J. Pharm., 364, 135.

92. Schäfer-Korting, M., Mehnert, W., Korting, H. C., 2007. Adv. Drug Deliv. Rev., $59,427$.

93. Calpena, A. C., Lauroba, J., Suriol, M., Obach, R., Domenech, J. 1994, Int. J. Pharm., 2, 179.

94. Pathan, I. B, Setty, C. M. 2009, Trop. J. Pharm. Res., 8, 173.

95. Akimoto, T., Nagase, Y. 2003, J. Control. Release, 88, 243.

96. Hu, L., Damaj, B. B., Martin, R., Michniak-Kohn, B. B. 2011, Int. J. Pharm., 404, 66. 
97. Brychtova, K., Jampilek, J., Opatrilova, R., Raich, I., Farsa, O., Csollei, J. 2010, Bioorg. Med. Chem., 18, 73.

98. Lee, P. J., Ahmad, N., Langer, R., Mitragotri, S., Shastri, V. P. 2006, Int. J. Pharm.,308, 33.

99. Harrison, J.E., Watkinson, A.C., Green, D.M., Hadgraft, J., Brain, K. 1996, Pharm. Res., 13, 542.

100. Cevc, G., Vierl, U., 2010, J. Control. Release, 141, 277.

101. Arora, A., Prausnitz, M. R., and Mitragotri, S. 2008, Int. J. Pharm., 364, 227.

102. Chen, H., Zhu, H., Zheng, J., Mou, D., Wan, J., Zhang, J., Shi, T., Zhao, Y., Xu, H., Lefèvre, X., Yang, G. 2009, J. Control. Release, 139, 63.

103. Yan, K., Todo, H., Sugibayashi, K. 2010, Int. J. Pharm., 397, 77.

104. Giannola, L. I., Caro, V. D., Giandalia, G., Siracusa, M. G., Tripodo, C., Florena, A. M., Campisi, G. 2007, Eur. J. Pharm. and Biopharm., 67, 425.

105. Jinturkar, K. A., Rathi, M. N., Misra, A. 2011, Chal. Del. Ther. Genomics Proteomics, 83.

106. Tesselaar, E., Sjöberg, F. 2011, Microvasc. Res., 81, 88.

107. Squier, C. A., Kremer, M.J., and Wertz, P.W. 1997, J. Pharm. Sci., 86, 82.

108. Sekkat, N., Kalia, Y. N., Guy, R. H.. 2004, Pharm. Res., 21, 1390.

109. Barbero, A. M., Frasch, F. 2009, Toxicol. in Vitro, 23, 1.

110. Seto, J. E., Polat, B. E., Lopez, R. F. V., Blankschtein, D., Langer, R. 2010, J. Control. Release, 145, 26.

111. Grond, S., Radbruch, L., Lehmann, K. A., 2000. Clin. Pharmacokinet., 38, 59.

112. Babu, R. J., Kanikkannan, N., Kikwai, L., Ortega, C., Andega, S., Ball, K., Yim, S., Singh, M. 2003, J. Control. Release, 86, 49.

113. Brain, K. R., Walters, K. A., Green, D. M., Brain, S., Loretz, L.J., Sharma, R. K., Dressler, W. D. 2005, Food Chem. Toxicol., 43, 681.

114. Sintov, A. C., Botner, S. 2006, Int. J. Pharm., 311, 55.

115. Audus, K. L. 1996, Oral Mucosal Drug Delivery, M.J. Rathbone (Ed.), Marcel Dekker, New York, 101.

116. Nielsen, H. M., Rassing, M. R. 1999, Int. J. Pharm., 185, 215.

117. Schmook, F. P., Meingassner, J. G., Billich, A. 2001, Int. J. Pharm., 14, 51.

118. Netzlaff, F., Lehr, C. M., Wertz, P. W., Schaefer, U. F. 2005, Eur. J. Pharm. Biopharm., 60, 167.

119. Vecchia, B. E., Bunge, A. L. 2003, Transdermal Drug Delivery, R.H. Guy, and J. Hadgraft,(Eds.), Marcel Dekker, New York, 25.

120. Farahmand, S., Maibach, H. I. 2009, Int. J. Pharm., 367, 1. 


\title{
9. Artemisia (Asteraceae): Understanding its evolution using cytogenetic and molecular systematic tools, with emphasis on subgenus Dracunculus
}

\author{
Jaume Pellicer', Teresa Garnatje ${ }^{2}$ and Joan Vallès ${ }^{3}$ \\ 'Jodrell Laboratory, Royal Botanic Gardens, Kew, Richmond, Surrey, TW9 3AB, United Kingdom \\ ${ }^{2}$ Institut Botànic de Barcelona (IBB-CSIC-ICUB), Passeig del Migdia sn 08038 Barcelona, Catalonia, \\ Spain ${ }^{3}$ Laboratori de Botànica, Facultat de Farmàcia, Universitat de Barcelona, Av. Joan XXIII sn \\ 08028 Barcelona, Catalonia, Spain
}

\begin{abstract}
The genus Artemisia is one of the largest of the Asteraceae family, with more than 500 species. It is widely distributed mainly across the Northern Hemisphere, being profusely represented in the Old World, with a great centre of diversification in Asia, and also reaching the New World. The evolution of this genus has been deeply studied using different approaches, and polyploidy has been found to perform an important role leading to speciation processes. Karyological, molecular cytogenetic and phylogenetic data have been compiled in the present review to provide a genomic characterization throughout some complexes within the genus.
\end{abstract}




\section{Introduction: A general overview on the genus Artemisia}

The genus Artemisia L. is one of the widely distributed genera of the Asteraceae family, and the largest genus of the Anthemideae tribe. The number of representatives considered within the genus is variable depending on the authors consulted, and ranges from about 380 species [1] to more than 500 species [2,3]. Some of the recent revisions of the family [3,4], have considered previously recognized genera as species circumscribed within Artemisia [e.g. Artanacetum (Rzazade) Rzazade, Oligosporus Cass. and Seriphidium (Besser ex Less.) Fourr.].

The genus is distributed worldwide, mainly across the temperate zones of the Northern Hemisphere, some species reaching the Arctic, but a few species can also be found on the Southern Hemisphere [5,6]. The origin of Artemisia, based on fossil data, is in the semi-arid steppes of the temperate Asia at mid Cenozoic, that is, about $20 \mathrm{My}$ ago [7]. In fact, Central Asia is considered its main centre of speciation and diversification, from where is expanding towards the Irano-Turanian, Mediterranean and North American regions.

From the morphological point of view, the leaves of Artemisia are mostly divided (with the exception of a few species, e.g. Artemisia dracunculus L.), alternately or sparsely distributed, and with a wide range of sizes, shapes and textures. The inflorescences, arranged in capitula are small, mainly spheroidal to ovoid, and composed of flosculose florets inserted on a receptacle protected by a bracteal involucre. Corollas are of whitish, yellow and purple color, and not too showy [5]. Fruits are pappus-lacking achenes, of small size and generally obovoid and laterally compressed. Pollen sculpture, although of limited variablility, has been used as a systematic marker [8,9]. The weak (microechinate) ornamentation of Artemisia contrasts with the echinate one present in most of the Anthemideae representatives [10-12].

The genus is mostly composed of perennial plants (Figs. 1b-d, f-i, m), but some of them (ca. 20 species) are known to behave either as annuals (Figs. 1e, j) or biennials (Fig. 1a) [1,2,13,14]. Within the genus there exist a certain variability of biotypes, being predominantly considered as herbs (Artemisia annua L., Artemisia vulgaris L.), subshrubs (Artemisia changaica Krasch., Artemisia crithmifolia L.) and shrubs which may develop highly lignified stems (Artemisia tridentata Nutt.). The ability of this genus to inhabit many different ecosystems and environmental conditions is evident, ranging from deserts and semi-deserts (steppes, tundras and slope hills), forests and deeply anthropized meadows, to humid areas, from sea level to high mountains. 

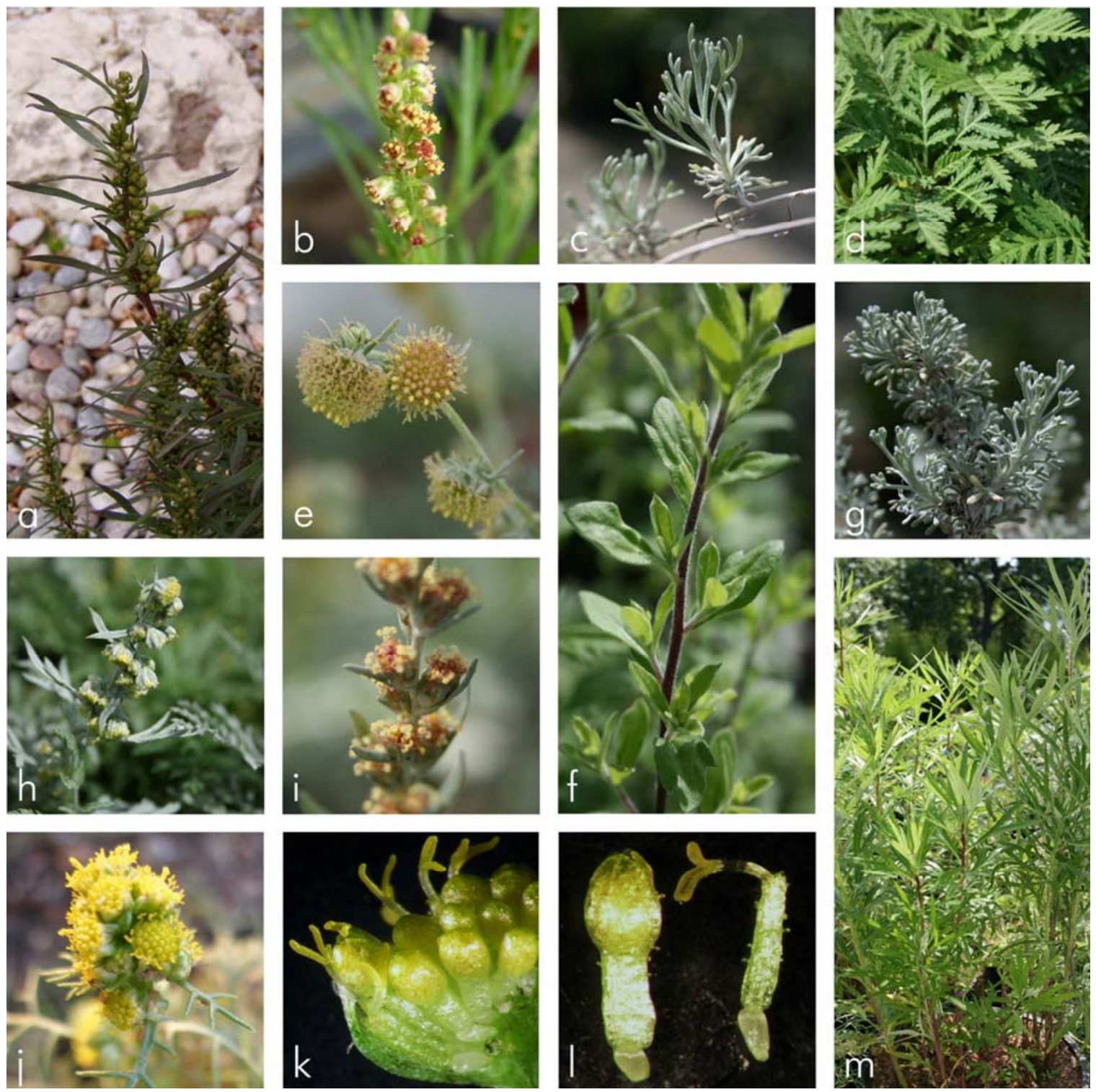

Figure 1. Some representatives of the genus Artemisia: a) Artemisia biennis L.; b) Artemisia desertorum Spreng.; c) Artemisia echegarayi Hieron.; d) Artemisia gmelinii Stechm.; e) Artemisia jacutica Drobow; f) Artemisia keiskeana Miq.; g) Artemisia mendozana DC.; h) Artemisia messerschmidtiana Besser; i) Artemisia nova A. Nelson; $\mathrm{j}, \mathrm{k}, \mathrm{l})$ Artemisia palustris L.; m) Artemisia selengensis Turcz. ex Besser. (Photographs: J. Pellicer).

Many of the species have traditional medicinal (e.g. A. annua, from which artemisinin is extracted to treat malaria), food (e.g. A. dracunculus, a culinary condiment, or Artemisia absinthium L., used to prepare liquors), soil stabilization (Artemisia sphaerocephala Krasch.) and ornamental (Artemisia arborescens L.) uses. A shared characteristic of many of the species is their intense fragrance, a result of the presence of monoterpenic and sesquiterpenic chemicals, localized in trichomes and schizogenic secretory channels [15]. 
Most Artemisia species bloom either at the end of summer or during autumn in contrast to most other Anthemideae genera which typically flower during spring and summer. Another distinctive Artemisia characteristic is that they are almost exclusively wind pollinated (although there are evidences of entomophily in a few species) [16,17]. This wind pollination characteristic is only shared with some closely related genera; the general case in Anthemideae is entomophily with the presence of showy capitula, attractive to insects.

\section{Taxonomical considerations: Conflicts and reorganizations within the genus}

The systematic classification of the genus Artemisia [see 18,19 and references therein], as well as in other Asteraceae genera, has been demonstrated to be complex and is currently under revision because of the lack of general taxonomic agreement. The species currently included in the genus were previously grouped within three independent genera (Abrotanum, Absinthium and Artemisia) by Tournefort [20]. Subsequently, Linné [21] organized them into a single genus (Artemisia), and Cassini [22] followed by Lessing [23], transferred part of the species to a newly created genus Oligosporus, which is currently recognized as the subgenus Dracunculus (Besser) Rydb. Besser [24-27] divided the genus into four groups (with the addition of Seriphidium Besser) which were considered as sections or subgenera (Abrotanum [currently Artemisia], Absinthium, Dracunculus and Seriphidium). This classification was accepted by Candolle [28] and subsequently by Rouy [29], who nevertheless carried out some rearrangements. Rydberg [30] created a new section, Tridentatae, to integrate some of the species previously included in Seriphidium, which was elevated to subgeneric rank by McArthur [31]. More recently, Ling [32,33] proposed that Tridentatae taxa should be subsumed into Seriphidium genus. Bremer and Humphries [2] accepted Ling's proposal in their revision of the Asteracaeae. However, taxonomic consensus remains elusive as witnessed by several additional treatments aimed at clarifying the taxonomy of the genus [i.e. 1,34,35]. Some of the conflicts between traditional and molecular data $[3,4,36,37]$ still remain unsolved.

Nevertheless, subgeneric classification of Artemisia on the basis of morphological characters such as floral structure has been maintained, since it fits at large scales (with some exceptions such as part of the New World species and some of representatives within the subgenera Artemisia and Absinthium) with the classifications based on the analysis of DNA sequences. The floral traits which identify each subgenus are as follows (Fig. 2): 
- Absinthium: Heterogamous capitula with outer florets female and central florets hermaphrodite and fertile. Hairy receptacle.

- Artemisia (= Abrotanum): Heterogamous capitula with outer florets female and central florets hermaphrodite and fertile. Glabrous receptacle.

- Dracunculus: Heterogamous capitula with outer florets female and central florets hermaphrodite but functionally male. Glabrous receptacle.

- Seriphidium and Tridentatae: Homogamous capitula with all florets hermaphrodite and fertile. Glabrous receptacle.
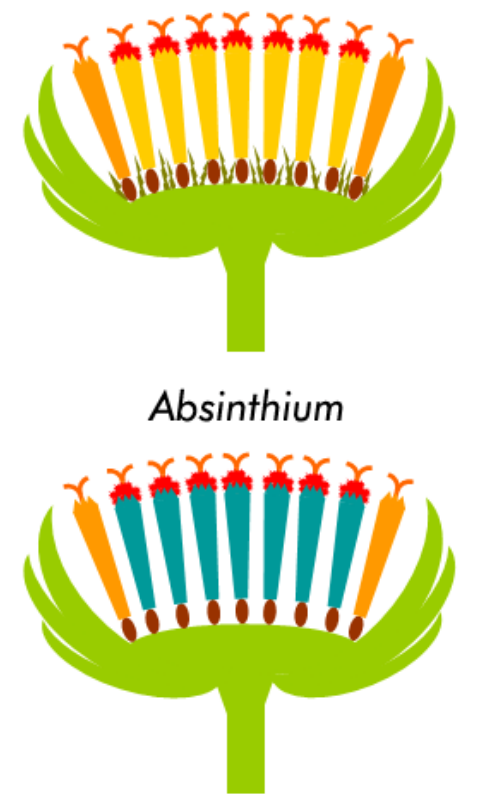

Dracunculus
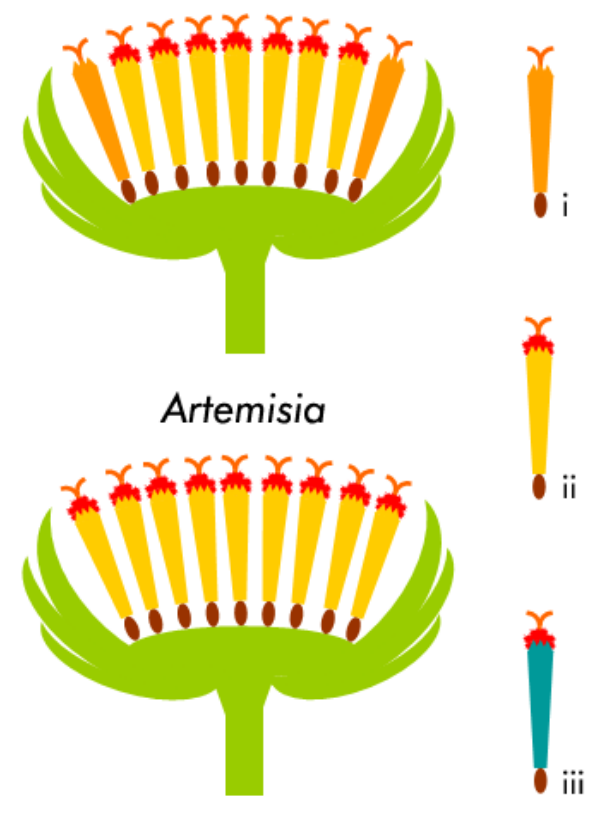

Seriphidium/Tridentatae

Figure 2. Diagrams of the flower head structure of each subgenus in Artemisia. i) Female florets, ii) hermaphrodite fertile florets, iii) hermaphrodite but functionally male florets.

\section{The subgenus Dracunculus (Besser) Rydb.}

As mentioned previously, Cassini [22] described the genus Oligosporus, which included the species that are currently considered as part of the subgenus Dracunculus (Fig. 3), to which we will devote a particular attention in this review. This group, with functionally separate sexes, that is, radial female florets and central male ones as consequence of the abortive ovaries, is composed of about 80 taxa [13,14]. Recently, Ling et al. [1] in their revision of the Anthemideae tribe have proposed a new classification, and as a consequence, the subgenus Dracunculus would be divided into two sections, Dracunculus Besser and Latilobus Y. R. Ling on the basis of their leaf morphology and indumenta. 

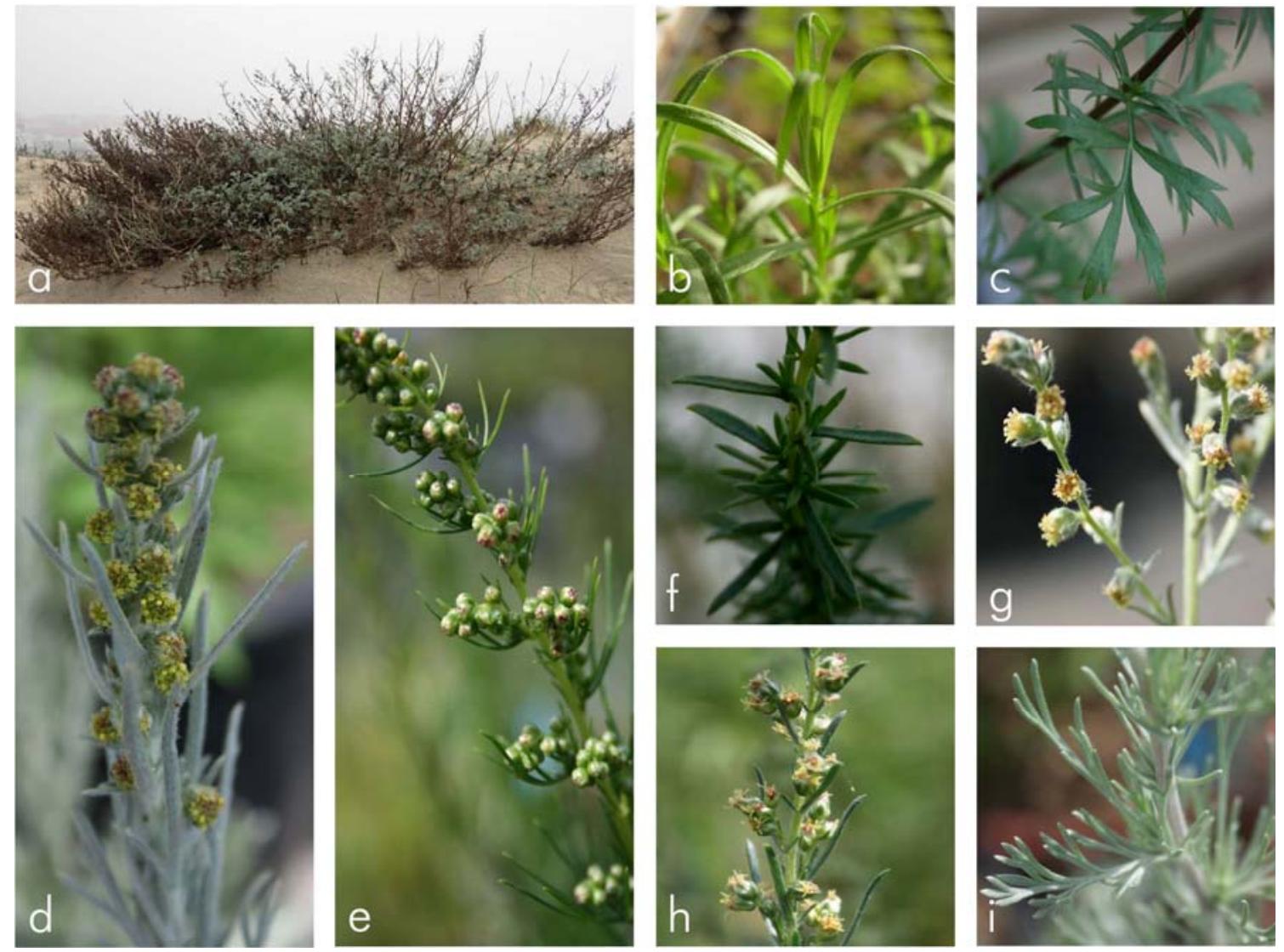

Figure 3. Some representatives of Artemisia subgenus Dracunculus: a) A. crithmifolia; b) A. dracunculus; c) Artemisia eriopoda Bunge; d) Artemisia ledebouriana Besser; e) Artemisia macilenta (Maxim.) Krasch.; f) Artemisia monosperma Delile; g) Artemisia monostachya Maxim. ex Bunge; h) Artemisia pycnorrhiza Ledeb.; i) Artemisia tomentella Trautv. [Photographs: (a) J. Vallès, (b-i) J. Pellicer].

Previous studies based on the analysis of DNA sequences in the genus Artemisia [36,37] have highlighted the relationship of some closely segregated Artemisiinae (Filifolium Kitam., Mausolea Bunge ex Poljakov, Neopallasia Poljakov, Turaniphytum Poljakov) the three last ones having been previously considered within Artemisia as A. eranthema Bunge, A. eriocarpa Bunge and A. pectinata Pall., respectively. In this sense, as we discuss below, different studies have examined in depth the phylogenetic relationships within the subgenus, and to elucidate the systematics between both the New and the Old World representatives as well [36-40].

As for the whole of the genus Artemisia, the representatives of the subgenus Dracunculus are widely spread across the Northern Hemisphere, mainly in the arid zones and semiarid steppes from Europe to Asia, where the subgenus has its main hotspot, but also reaching North America. They are mostly subshrubs and herbs, basically perennial, with few annuals such as 
Artemisia demissa Krasch., Artemisia edgeworthii Balakr., Artemisia pewzowii C. Winkl., and Artemisia scoparia Waldst. et Kit.

Within the representatives of the subgenus, the type species A. dracunculus -tarragon- (Fig. 3b) deserves special attention, since it accounts for a great economic value. This plant is popular worldwide because it is used as culinary condiment in many countries. But some other less known species also have traditional uses either to build windbreaks and soil stabilization (A. sphaerocephala, or Artemisia wudanica Liou et W. Wang) or in medicine (Artemisia capillaris Thunb., with detoxifying and diuretic properties; Artemisia ordosica Krasch., with anti-inflammatory properties).

\section{Chromosome numbers and polyploidy}

The genus Artemisia has been profusely studied from the karyological point of view with many papers reporting chromosome numbers in the genus over several decades covering over $50 \%$, that is, more than 350 species [41]. The genus has two basic chromosome numbers: $x=9$, present in all the subgenera, and the less frequent $x=8$, reported in the subgenera Absinthium, Artemisia and Dracunculus [42-45]. In addition, a $2 n=14$ count has been reported, suggesting a basic number $x=7$ [46]. Recently some authors have suggested the possibility of $x=17$ as a basic chromosome number. Oliva and Vallès [44] reported that in the tetraploid Artemisia umbelliformis Lam. $(2 n=34)$ an event of diploidization might have occurred with subsequent cytotype stabilization. In addition, in a recent paper [47], this new basic chromososme number has been suggested again in a group of restricted Japanese endemic species of subgenus Artemisia (e.g. Artemisia momiyamae Kitam., Artemisia princeps Pamp.). In the case of the $x=8$-based species, the hypothesis of a centric Robertsonian chromosome fusion which may have caused a descending dysploidy from $x=9$ to 8 [48] is widely accepted. Those species with $2 n=16$ frequently present a larger pair of chromosomes (Fig. 4g), sometimes with centromeric fragility, what gives support to the precedent hypothesis. Given that the findings of Oliva and Vallès [44] and Matoba et al. [47] are restricted to few species, and that a significantly larger pair of chromosomes is also present in the metaphase plates, other explanations instead of a $x=17$ basic chromosome number should be taken into account. Thus, these plants might be $x=9$-based tetraploids, but reduced from $2 n=36$ to 34 chromosomes via Robertsonian fusion, and whether this process gives rise to a new base number may be the object of further discussion according to the stability of the reported cases and new ones that might be detected. 


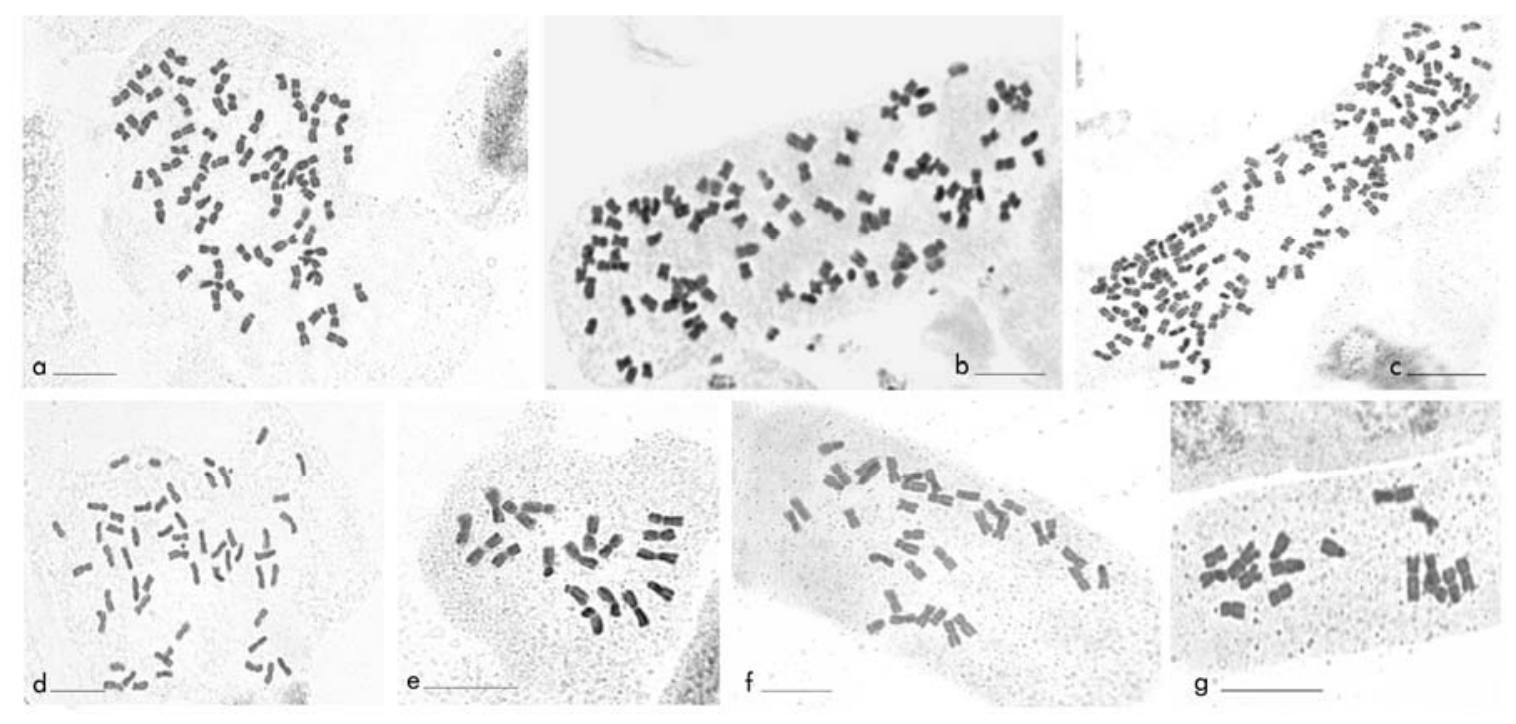

Figure 4. Metaphase plates of some Artemisia species. a) A. echegarayi $(2 n=72)$; b) A. macrantha $(2 n=108)$; c) A. medioxima $(2 n=144)$; d) A. mendozana DC. subsp. paramilloensis F.A. Roig \& J.A. Ambrosseti $(2 n=54)$; e) A. sphaerocephala $(2 n=18) ;$ f) A. subdigitata $(2 n=36) ;$ g) A. sylvatica $(2 n=16)$. Images extracted from Pellicer et al. $[49,50]$ with permission of the authors. (Scale bars $=10 \mu \mathrm{m}$ ).

Both basic chromosome numbers have experienced many episodes of polyploidization. Polyploidy is an important evolutionary trait leading to plant speciation, and has special incidence in the genus Artemisia, being therefore, reported many times in the literature [17,20,31,45,47,49,50-59]. Ploidy levels in the genus are known up to dodecaploid and hexaidecaploid for $x=9$ $(2 n=12 x=108 ; 2 n=16 x=144)$ in Artemisia macrantha Ledeb. and Artemisia medioxima Krasch., respectively [49,60, Figs. 4b-c], and hexaploid for $x=8(2 n=6 x=48)$ in species such as Artemisia verlotiorum Lamotte or Artemisia austriaca Jacq. [17,61].

Some of the subgenera have been revealed to be really active in terms of polyploidy, with species accounting for large polyploid series. This is the case of the subgenus Dracunculus, with its type species (A. dracunculus) displaying ploidy levels of $2 n=2 x, 4 x, 6 x, 8 x, 10 x$ [50,53,62,63]. In fact, most of the representatives of the subgenus are known to present polyploid cytotypes, and many of them, are only known at polyploid level, e.g. A. changaica, A. ledebouriana or A. pycnorrhiza, among others. Other examples of high impact of polyploidy (based on $x=9$ ) can be found in the American endemic representatives. On the one hand, the North American taxa (subgenus Tridentatae) provide cytotypes from diploid to octoploid levels [45], and on the other hand, the South American endemic species might be considered as a polyploid complex with cytotypes ranging from diploid to dodecaploid levels [64]. 


\section{Cytogenetic insights: Ribosomal DNA evolution}

The nuclear genes encoding rRNA have been the subject of many studies in plants and in many other organisms as well. In the case of the genus Artemisia, the distribution and organization of these genes have been deeply studied [64-69]. Whilst in most eukaryotes, both 5S and 45S (18S-5.8S-26S) genes are usually arranged in separated tandem arrays which are transcribed by different RNA polymerases [70, Fig. 5A], there are some exceptions to this organization in some other organisms [71-74] that have evolved towards a linkage of both family genes. This is the case in the genus Artemisia and other related genera, where a co-localized (linked) organization of both 5S and 45S ribosomal sub-units were detected by fluorescent in situ hybridization [66,68,69,75, Fig. 5B]. Given the interest that such structure raised, subsequent studies were carried out in depth. Thus, this linkage was confirmed and unravelled using Southern blot hybridization and polymerase chain reactions (PCR) by Garcia et al. [69]. Besides, and confirming previous evidence, this feature has been recently reported beyond the Artemisiinae (Artemisia and related genera), in at least three of the major lineages within the Asteraceae [76], indicating that it could be more common than previously known in plant species.

Returning to Artemisia, physical mapping of ribosomal DNA using fluorescent in situ hybridization has been conducted with the principal objectives of better understanding the systematic and evolutionary relationships within the genus. Thus, molecular cytogenetic studies focused in different subgenera (Artemisia, Dracunculus, Seriphidium and Tridentatae) have been published [66,68,69,77], albeit some others have been restricted either to specific groups such as the annual one [75] and the South American representatives [64] or to polyploid complexes [78]. It has been reported that not all the subgenera have similar patterns of rDNA loci number and distribution, and furthermore, the changes on the number of loci in polyploid species has been revealed to follow different trends, even within groups of closely related species. As an example, we present some of the results in the subgenus Dracunculus [75].

In the most recent phylogenies of Artemisia [36-38], the subgenus Dracunculus appears segregated in two main clades, one that includes the type species A. dracunculus and its close relatives (A. dracunculus complex) and the main clade, which embraces most of the Eurasian taxa. Within the first group, the number of rDNA loci in diploid cytotypes is 2 (4 rDNA sites), and seems to follow almost a proportional increase of rDNA loci in parallel to ploidy levels. Thus, tetraploids have 4 rDNA loci, hexaploids 6 and the decaploid about 9-10, a trend that does not correlate with the nuclear DNA 

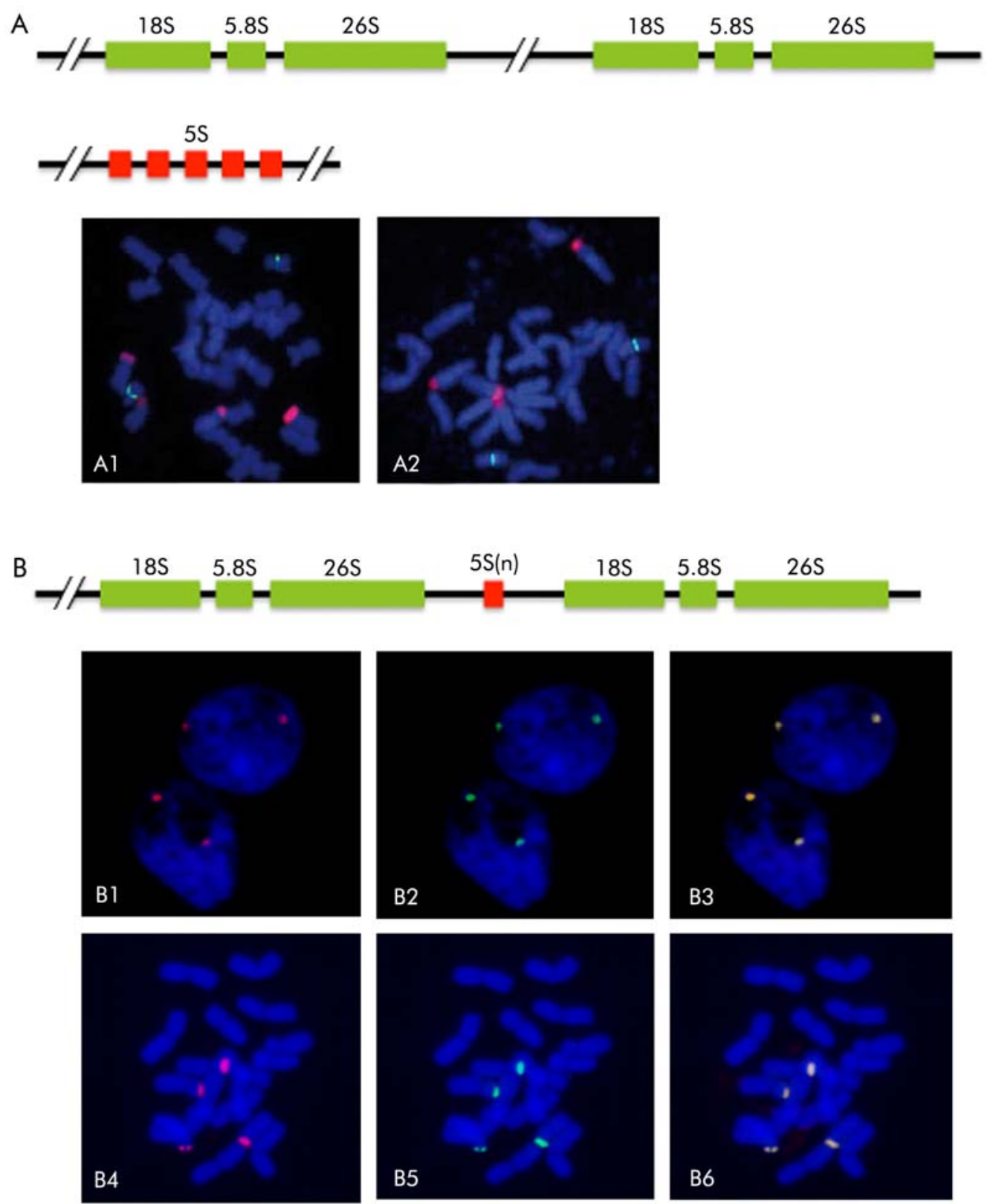

Figure 5. Structural organization of the rRNA genes 5S and 18S-5.8S-26S. A) Most plants and animals (e.g. A1, Myopordon persicum Boiss. and A2, Myopordon aucheri Boiss.; images extracted from Hidalgo et al. [79] with permission of the authors). B) Linked structure of both rRNA genes in Artemisia (B1-3, Artemisia magellanica Sch. Bip.) and relatives (B4-6 Filifolium sibiricum (L.) Kitam.). Note that this linked structure has been also reported in other Asteraceae lineages and other organisms.

content, as the $1 \mathrm{C} x$ values decrease in ascending in ploidy levels, and more remarkably in higher ploidy levels (Fig. 6). Contrary to that, the representatives sampled from the Eurasian clade studied (including previous results from Torrell et al. [66]), showed a different trend. Although the loci number was more variable between diploid cytotypes (from 3-5 rDNA loci), 


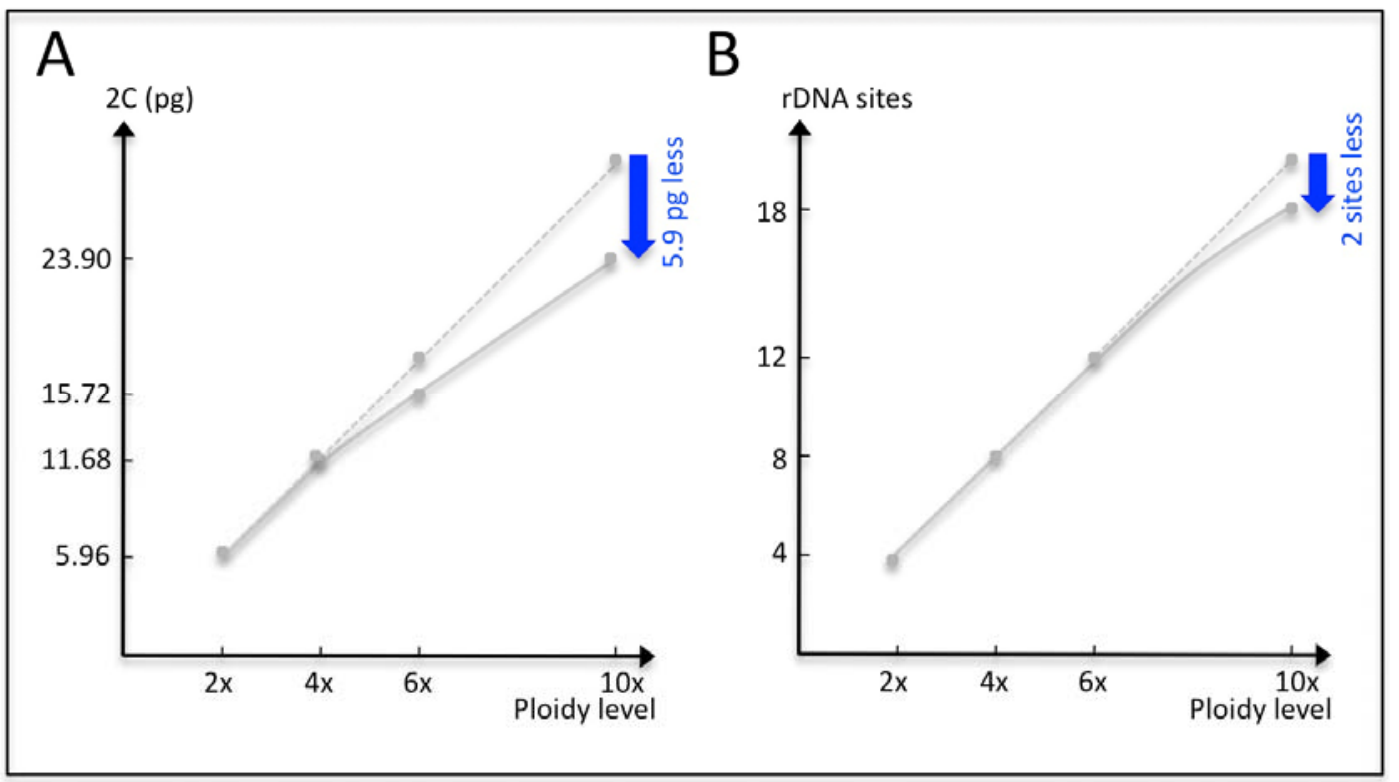

Figure 6. Graphical representation of the variation of genome size (A) and rDNA loci (B) in the polyploid series of the species A. dracunculus. Dotted lines indicate the theoretical patterns expected for a proportional increase of genome size (A) and rDNA loci (B) when ascending in ploidy levels.

this number remained the same on their related tetraploids, pointing towards a loss of rDNA loci during genome polyploidization. In the species where both $2 x$ and $4 x$ populations were studied, such a reduction on rDNA loci was not translated to the $1 \mathrm{C} x$ value, as their values remained about the same, indicating the genome size was doubled but not affecting the number of rDNA loci.

\section{C-values: The genome size dynamics in Artemisia}

Swift [80] coined the term ' $\mathrm{C}$ value' to refer to the DNA amount of the unreplicated haploid complement. Thus, the term monoploid genome size $(1 \mathrm{Cx})$ is related to the nuclear DNA content in a basic chromosome set $(x)$ of a somatic cell [81], and therefore, the $2 \mathrm{C}$ value refers to the whole genome size of a somatic cell. Genome size, estimated by means of the nuclear DNA content, has been revealed variable across plant groups, becoming an important character in biodiversity. As presently understood, differences among angiosperms are about 2400-fold, from the minute genome of Genlisea margaretae Hutch. (Lentibulariaceae) with 2C $=0.128 \mathrm{pg}$ [82] to the recently discovered record holder Paris japonica (Franch. \& Sav.) Franch. (Melanthiaceae), with a 2C $=304.46 \mathrm{pg}$ [83]. The study of genome size has applications in different fields such as ecology, systematics and evolution $[84,85]$. Many correlations 
between this parameter and the biology (e.g. life cycle, guard cell sizes or polyploidy) and the ecology (e.g. plant distribution, environmental stress or insularity) of the species have been reported [e.g. 85-90].

The genus Artemisia, and more in depth some of its subgenera, have been studied from this viewpoint, and many studies concerning genome size values and its evolutionary implications have been published [49,90-93]. To provide a framework about the dynamics of this parameter within the genus we present some of the results from Pellicer et al. [94]. The range of $2 \mathrm{C}$ values within the genus varies about 7.5-fold, a range that increases to ca. 9.2-fold when the annual taxa are taken into account [91,92]. At the present time, the species A. scoparia (2C $=3.54,[92])$ is known to have the smallest genome size within the genus, although such a value could be even slightly smaller in the closely related $A$. capillaris (mean $2 \mathrm{C}=3.37$, [75]). At the upper end of the range, the hexaidecaploid (16x) populations of the species $A$. medioxima reported from Russia, are the largest in the genus with a mean $2 \mathrm{C}=31.01 \mathrm{pg}$. Nonetheless, the latter species, which is the highest polyploid in the genus, becomes the one with the smallest $1 \mathrm{C} x$ value $(1.93 \mathrm{pg})$ together with A. capillaris (1.68 pg), while the annual diploid Artemisia leucodes Schrenk, with $1 \mathrm{C} x=7.7 \mathrm{pg}$ [93], accounts for the largest one.

As a result of the analysis of genome size evolution in Artemisia within a phylogenetic context [94], the authors concluded that at diploid level, although the distribution of nuclear DNA amounts found was fairly consistent with the phylogenetic lineages, the differences were not big enough to discriminate among them. In this sense, those subgenera whose phylogenetic resolution and the traditional classification matched (Dracunculus, Seriphidium and Tridentatae) showed narrower genome size ranges than those with a more conflictive evolutionary history, such as the subgenus Artemisia and Absinthium, both appearing segregated in independent clades (Fig. 7).

At the beginning of this section, we mentioned that genome size can be influenced by several biological factors. One of them is polyploidy, quite frequent in plants [e.g. 94-96], and which is considered one of the major forces inducing genome size changes. Polyploidy is directly linked with different chromosomal and genomic reorganizations which may imply either an elimination of redundant [97,98] and non-random [99-101] sequences, or contrarily, the accumulation of repetitive sequences, often related to retrotransposons [102].

Within the genus, the nuclear DNA content increases with ascending ploidy levels, but not proportionally (Fig. 8), and the differences between the observed and the expected values for a theoretical proportional increase become more evident in high polyploids (with some exceptions, see [94]). This genome downsizing in polyploids can be also detected at the $1 \mathrm{C} x$ values, 
although the changes at this level are less evident. Seeing such a tendency and the quantitative consequences on the genome size in polyploid species, the authors proposed the existence of an upper limit for the nuclear DNA content in the genus. In order to check if this assumption was potentially feasible or not, different mathematical functions were tested to finally reach the conclusion that as the coefficient of correlation for a given Michaelis-Menten model was highly significant $(\mathrm{P}<0.01)$ it could be theoretically assumed that genome size in polyploids increases approaching asymptotically to a maximum.

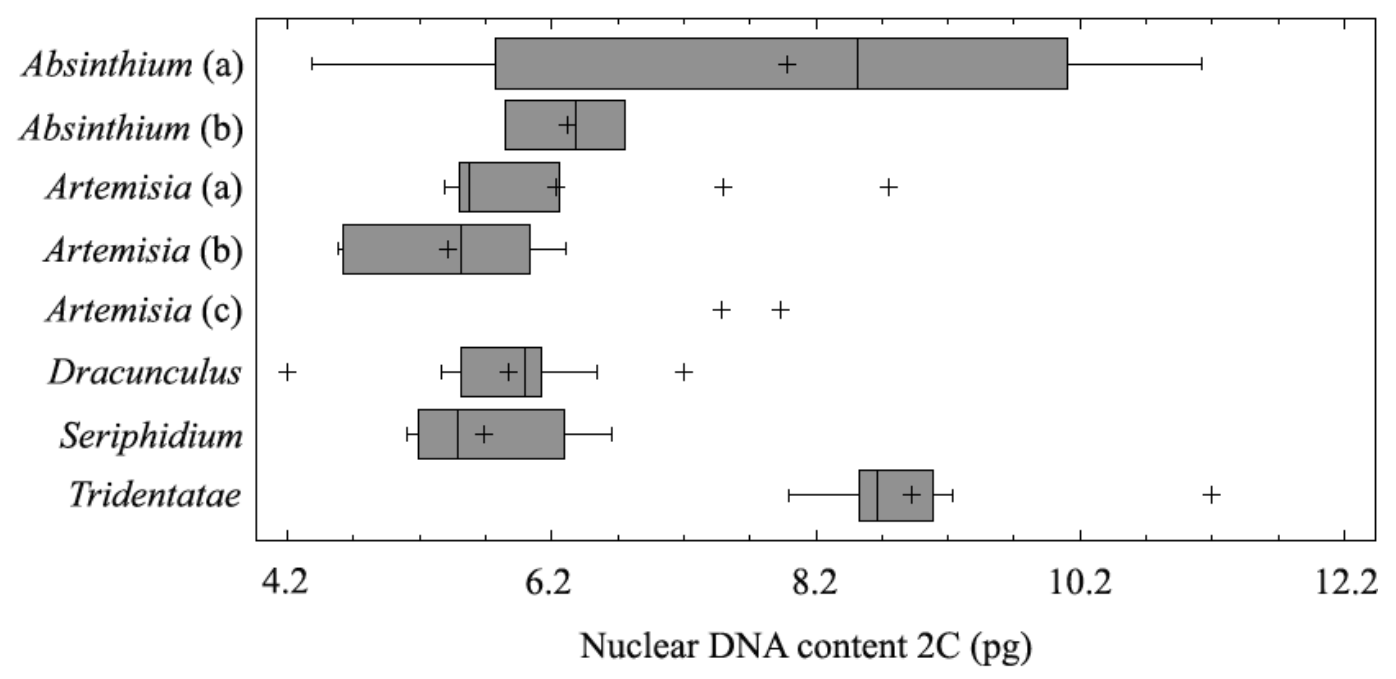

Figure 7. Distribution of genome size (2C values of diploid representatives) in Artemisia. Note that subgenera Absinthium and Artemisia are split in two and three lineages respectively, according to the phylogenetic frame depicted in Pellicer et al. [94]. Image used with permission of the authors.

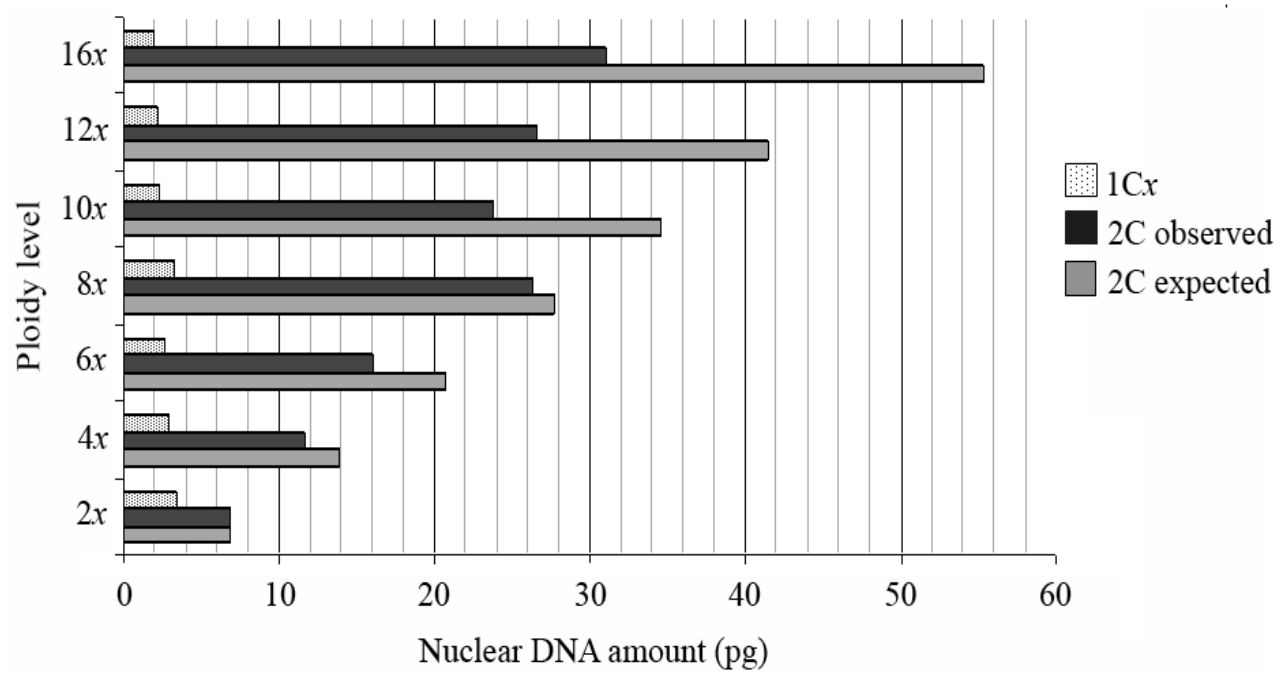

Figure 8. Evolution of DNA content at different ploidy levels in the genus Artemisia (from Pellicer et al. [94] with permission of the authors). 


\section{The phylogenetic relationships in Artemisia and allied genera}

Since late 1990s and up to present, several approaches to unravel the phylogenetic relationships within the genus Artemisia and the subtribe Artemisiinae as well have been published [36-39,103-106], but some groups still lack a complete evolutionary knowledge and are being currently investigated, i.e. subgenera Dracunculus [38] and Tridentatae [39]. The systematics of the genus is challenging to interpret and has engendered controversy among investigators. In some cases processes such as hybridization and polyploidization complicate the relationships between the species at molecular level. In addition, several small segregate genera (i.e. Crossostephium Less., Filifolium, Mausolea or Picrothamnus Nutt. among others), previously recognized under Artemisia, appear scattered along the phylogenies (Fig. 9), increasing the need for an in depth research into the group.

One of the first attempts to investigate from a broad point of view the phylogenetic relationships within the genus was carried out by Torrell et al. [104]. The phylogenetic analyses, based on nuclear DNA sequences (ITS), confirmed the monophyly of the genus including the formerly segregated Seriphidium and Oligosporus, the independence of the subgenus Seriphidium and Tridentatae, and also pointed out the conflictive circumscription of the subgenus Artemisia which appeared scattered along the phylogeny. These previous findings were supported and deeply investigated with the addition of larger datasets by Watson et al. [105] and Vallès et al. [40], who suggested the inclusion within the genus of the segregated genera Artemisiastrum Rydb., Crossostephium, Filifolium, Mausolea, Neopallasia, Picrothamnus, Sphaeromeria Nutt. and Turaniphytum. However, some new conflicts arose; as Watson et al. [105] maintained the generic status of the Seriphidium (even with no phylogenetic support) and expanded it to the subgenus Tridentatae, while Vallès et al. [40] decided to keep both Seriphidium and Tridentatae as independent from each other and at subgeneric level within Artemisia.

The addition of an extra nuclear DNA region (ETS) to the formerly studied ITS phylogenies revealed new linkages which were previously unknown to date [36,37]. Sanz et al. [36] found the Kaschgaria Poljakov group as sister to the remaining Artemisia, a point that has not been further confirmed due to the lack of statistical support in the node splitting the Artemisia and Kaschgaria lineages [37,94, Fig. 9]. Besides, Sanz et al. [36] considered two major lineages within Artemisia, the subgenus Dracunculus clade, including the segregated Filifolium, Mausolea, Neopallasia and Turaniphytum, and a second lineage embracing the remaining representatives of the genus (Absinthium, Artemisia, Seriphidium and Tridentatae) plus the segregated Sphaeromeria and Picrothamnus within the North American endemic clade. 
Similar results were reported by Tkach et al. [37], although the simplicity of the phylogenetic structure proposed by Sanz et al. [36] needed amplification and additional study. In fact, as the data sample was enlarged, new conflicts with the traditional classification rose up, some of them which had been evident since early studies of the genus. Thus, the split of the subgenus Artemisia into independent lineages became more evident along the phylogeny, and also some of the representatives of the subgenus Absinthium (considered by the authors as a section of the former) were placed at undetermined position (Fig. 9). In this sense, it is interesting to remark that the A. vulgaris complex was linked to a group of North American (plus few Arctic) taxa currently recognized to belong to the subgenus Artemisia. This fact would give support to the hypothesis of McArthur and Plummer [107], of a migration via Bering strait of representatives of the subgenus Artemisia from the Old to the New World, but failing to confirm that those would be the ancestors of the subgenus Tridentatae.

As discussed in the previous paragraphs, the North American endemic Tridentatae and the subgenus Dracunculus are being currently investigated, and both have been the subject of research projects $[75,106]$ to clarify, among other issues, their phylogenetic relationships. On the one hand, the Tridentatae has puzzled researchers in many fields for some time because of its geographic circumscription. First approaches to study the molecular systematics of the subgenus were carried out by Kornkven [109]. At that point, conflicts between the nuclear (ITS) and the chloroplast DNA surfaced $[103,110]$, making a proper understanding of the evolution of the group difficult. Many of the species are landscape dominant, so authors pointed out the possibility that interspecific gene flow and subsequent chloroplast capture might have favoured the existence of such conflicts [103]. In addition to the recent taxonomical re-evaluation published by Shultz [35], new phylogenetic frameworks have been constructed and a broader circumscription of the subgenus Tridentatae including other North American endemic previously recognized as non-Tridentatae have been proposed [39].

On the other hand, phylogenetic studies including a representative sample of the subgenus Dracunculus are still very scarce. Previous studies have reported the existence of two major lineages (Fig. 9), one including the A. dracunculus complex (the type species plus some other allied taxa) and the main lineage, which includes the vast majority of the species within the subgenus, mostly distributed across Eurasia. Besides, the inclusion of the segregated Mausolea eriocarpa, Neopallasia pectinata, and Turaniphytum eranthemum has been confirmed by nuclear and chloroplastic DNA sequences $[36,38]$ and therefore, taxonomic rearrangements have been proposed to return this species to Artemisia. In the case of Filifolium sibiricum, while its 


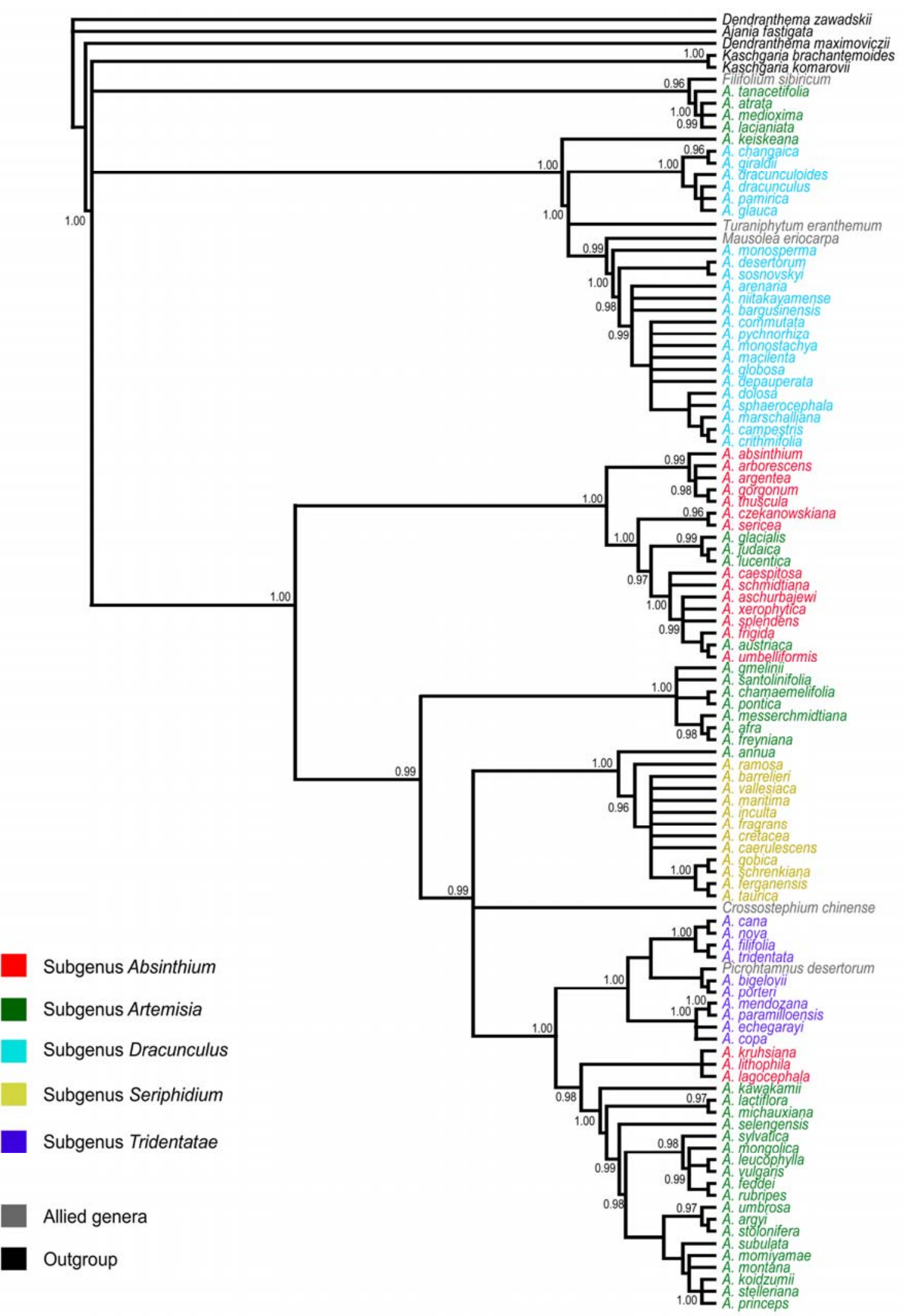

Figure 9. Phylogeny of Artemisia resulting from Bayesian analysis adapted from Pellicer et al. [94] with minor modifications (only PP values $\geq 0.95$ are indicated on branches). 
inclusion within the genus Artemisia is confirmed, its specific placement is not yet resolved. Vallès et al. [40] and Sanz et al. [36] reported this species as sister to the subgenus Dracunculus, but recently Pellicer et al. [94] have found that previous findings could be influenced by sample size, as their study embeds the species in one of the subgenus Artemisia clades next to the Dracunculus lineages but with not enough statistic power to be considered as sister group.

The A. dracunculus complex, including ca. 10 species, is characterized by the synapomorphy of simple linear-lanceolate leaves, with the exception of Artemisia jordanica Danin, which is the unique case of simple leaves beyond the complex [111]. The group is characterized, as previously discussed, by a great incidence of polyploidy. Although most of the representatives have been described at diploid (Artemisia giraldii Pamp., A. glauca Pall., A. pamirica C. Winkl.) or tetraploid levels (e.g. A. changaica, A. glauca, A. subdigitata), a complete polyploid series of $2 n=18,36,56,72,90$ has been reported in the type species (A. dracunculus). The Eurasian clade embeds the majority of the species of the subgenus, which is characterized by a low sequence divergence among species, and therefore, by a lack of enough statistical node support in many subclades, making it sometimes difficult to find convincing explanations for the evolutionary relationships within the group. Among the North American endemic complex, some of the representatives that have been traditionally included in the subgenus Dracunculus (Artemisia filifolia Torr., Artemisia pedatifida Nutt. and Artemisia porteri Cronquist, respectively, Picrothamnus desertorum [= Artemisia spinescens D.C. Eaton], but the molecular evidence has highlighted a strong relationship to the Tridentatae. Thus, the recent taxonomic treatments of the group have proposed the inclusion of these species within the subgenus Tridentatae [35], and the current phylogenetic studies also have pointed out some additional taxonomic rearrangements needed [39].

\section{The pollen grain: A useful systematic marker}

The pollen types in the Asteraceae have been revealed quite variable in terms of shapes and structures [112]. Within the Artemisiinae tribe, two basic pollen types have been described on the basis of the exine ornamentation [113]: the Artemisia-type, with spinules (microechinate), and the Anthemistype, with patent spines (echinate) (Fig. 10). In this sense, the exine ultrastructure has been studied for both pollen types for some time, but the first study describing this spineless form in the genus Artemisia and relatives was Wodehouse [114], being later confirmed by several studies with samples 
across the globe [e.g. 8-11,17,115-118]. In addition, morphometrical comparisons carried out between the Anthemis and Artemisia pollen types have highlighted that differences are not only based on the spines size, but also on the pollen size, shape, and the spine density [12].

The segregation of both pollen types throughout the Artemisiinae has been also the focus of several studies [10-12,36,40,118]. These studies have revealed that each pollen-type defines one of the two main groups within the subtribe: the Dendranthema and relatives clade, characterized by the Anthemis-type pollen, and the Artemisia and allies clade, by the Artemisiatype one. Although some exceptions to the rule are presented in these studies the hypothesis of possible pollen-type reversions along the Artemisiinae via species hybridization may be important and require further study.

Returning back to the genus Artemisia, the number of studies dealing with pollen morphology and its systematic implications has recently notably increased [8-10,119-121]. From the morphologic point of view, these studies consider the pollen of Artemisia as 3-zonocolporate, isopolar, radially symmetrical, prolate to perprolate and, as described above, microechinate.
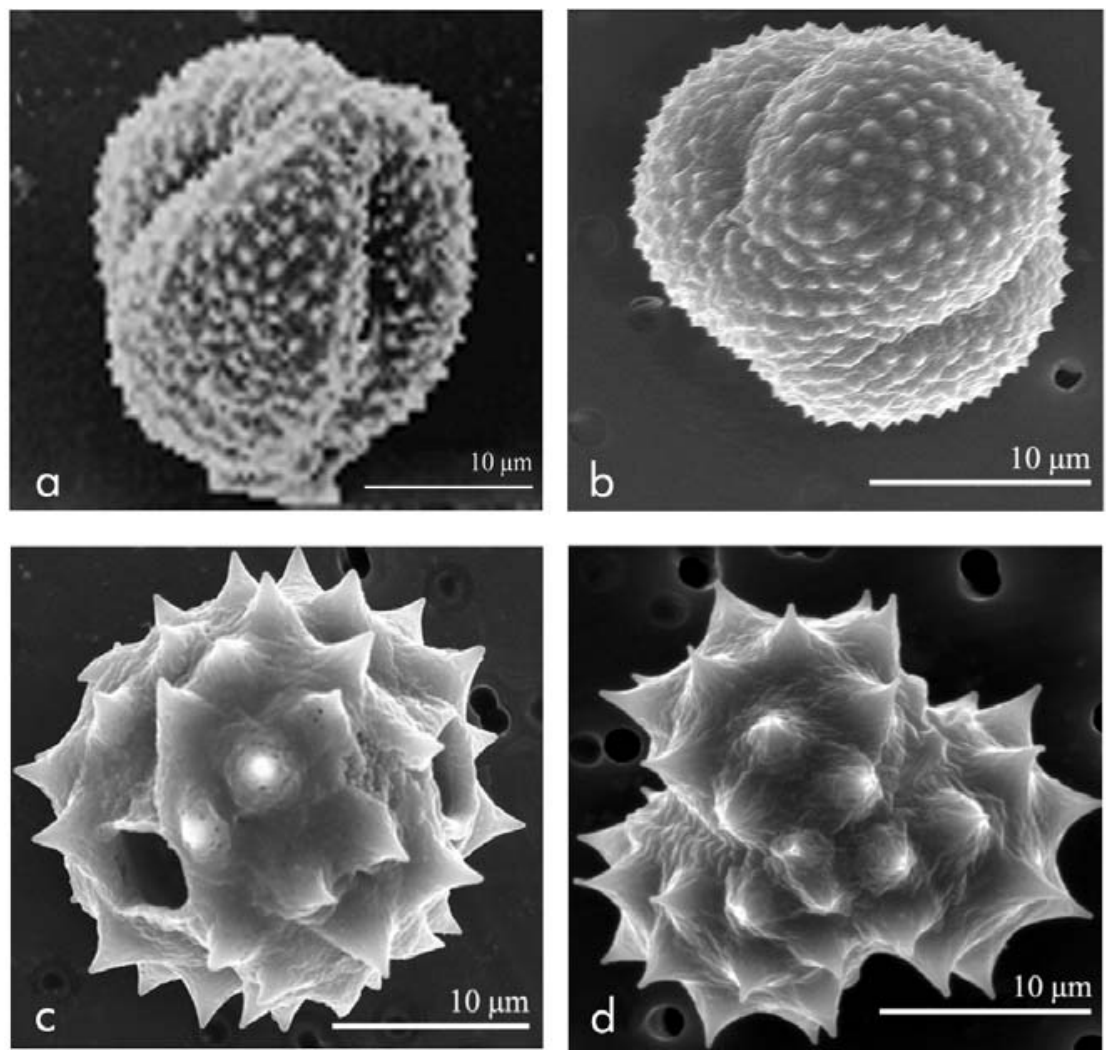

Figure 10. Some examples of pollen types. Artemisia-type: a) Artemisia incana Druce and b) Crossostephium chinense (L.) Makino. Anthemis-type: c) Ajania nubigena (Wall.) C. Shih and d) Cancrinia discoidea (Ledeb.) Poljakov ex Tzvelev. Images extracted from Martín et al. [10] and Pellicer et al. [12] with permission of the authors. 
The study of Jiang et al. [8] further investigated in detail the Artemisia pollen traits and divided it in two main groups, the Myriantha-type and the Mongolica-type, the latter being subdivided in four subtypes (Sacrorum, Oligocarpa, Lavandulaefolia, and Anomala). The authors based this classification on the evolution and distribution of the species, since the Myriantha (primitive) type is widely found in plants inhabiting in high latitudes whereas the subtypes of Mongolica (derived) seem to be related with low latitude distributions. However, while the former (Artemisia and Anthemis) pollen types might be used as a systematic marker at subtribal level, many of the authors have concluded that the differences within Artemisia are few, and have poor diagnostic potential to be useful distinguishing between species $[8,9,120]$.

\section{Conclusions and future prospects}

This review has considered a significant number (around 40, see the literature cited, particularly [75,108] and references therein) of studies and research approaches that have been devoted to elucidate, from different points of view, the evolutionary history of the genus. Artemisia is a giant within the Anthemideae, and that is why such a big volume of work has been and it is currently being carried out on the genus. The combination of results from an important variety of disciplines such as, karyology (chromosome counts, karyotype elaboration and fluorochrome banding), molecular cytogenetics (in situ hybridization of different DNA probes), molecular systematics (DNA sequencing programs and population genetics), taxonomy (including microand macromorphologic approaches) is essential to achieve a level of knowledge which is allowing researchers to propose and test robust hypotheses about the evolution of the genus.

Even so more multidisciplinary approaches covering specific groups and/or complexes within the genus which are currently lacking for a complete evaluation are needed. These will complement the present knowledge of the genus and also may suggest that further taxonomic reconsiderations are needed not only in Artemisia but also within the Artemisiinae.

\section{Acknowledgements}

The authors acknowledge all researchers involved in Artemisia and/or related genera studies for their substantial contributions and improvements to the knowledge of this group of plants from all perspectives, and all our colleagues who have kindly given their permission to use graphics and 
captions already published. Dr. Oriane Hidalgo (Ohio University) is acknowledged for her suggestions and assistance on the draft of this paper. The authors address also their thanks to Prof. E. Durant McArthur (Rocky Mountain Research Station), who reviewed and improved a first version of the manuscript. This work has been supported by different projects from the DGICYT (Spanish government, CGL2007-64839-C02-01 and 02/BOS, CGL2010-22234-C02-01 and 02/BOS) and the Generalitat de Catalunya (Catalan government, 2005SGR00344, 2009SGR439).

\section{References}

1. Ling, Y.R., Humphries, C.J., and Shultz, L. 2006, Flora of China, Vol. 20 (Asteraceae), Editorial Committee (Eds.), Science Press and Missouri Botanical Garden Press. Beiging, - St. Louis. http://flora.huh.harvard.edu/china/ mss/volume20/index.htm

2. Bremer, K., and Humphries C.J. 1993, Bull. Nat. Hist. Mus. London (Bot.), 23, 71.

3. Kubitzki, K. 2007. The families and genera of vascular plants. Vol VIII Flowering plants. Eudicots. Asterales. Kadereit J.W. and Jeffrey C. (Eds.) Springer-Verlag: Berlin Heidelberg, 358.

4. Funk, V.A., Susanna, A., Stuessy, T., and Bayer, R. (Eds.) 2009, Systematics, Evolution and Biogeography of the Compositae. International, Washington D.C., 631

5. Bremer, K. 1994, Asteraceae: cladistics and classification. Timber Press: Portland.

6. Ling, Y.R. 1994, Compositae Newslett., 25, 39.

7. Wang, W.M. 2004, Bot. J. Linn. Soc., 145, 331.

8. Jiang, L., Wang, Q., Ye, L.Z., and Ling, Y.R. 2005, Wuhan Univ. J. Nat. Sci., 10, 448.

9. Hayat, M.Q., Ashraf, M., Khan M.A., Yasmin, G., Shaheen N., and Jabeen, S. 2010, Plant J. Bot., 42, 751.

10. Martín, J., Torrell, M., and Vallès, J. 2001, Plant Biol., 3, 372.

11. Martín, J., Torrell, M., Korobkov, A.A., and Vallès, J., 2003, Plant Biol., 5, 85.

12. Pellicer, J., Hidalgo, O., Garcia, S., Garnatje, T., Korobkov, A.A., Vallès, J., and Martín, J. 2009, Bot. J. Linn. Soc., 161, 171.

13. Poljakov, P.P. 1961, Trudy Instituta Bot. Akad. Nauk Kazaksh. S.S.R., 11, 134.

14. Poljakov P. P. 1961, Rod 1550. Polyn-Artemisia L., Shishkin B.K., and Bobrov E.G. (Eds.) Flora SSSR, Vol. 25. Leningrad.

15. Greger, H. 1977, Anthemideae - Chemical Review, Heywood, V.H., Harborne, J.B., and Turner, B.L. (Eds.), The biology and chemistry of the Compositae, Vol. 2. Academic Press, London, 899.

16. Garnock-Jones, P.J. 1986, Bot. J. Linn. Soc., 92, 285.

17. Vallès, J., Suárez, M., and Seoane, J.A. 1987, Acta Salmanticensia, Ciencias 66, 167.

18. Vallès, J., and McArthur, E.D. 2001, Artemisia systematics and phylogeny: cytogenetic and molecular insights, McArthur, E.D., Fairbanks, D.J. (Eds.) 
Shrubland Ecosystem Genetics and Biodiversity. Department of Agriculture Forest Service, Rocky Mountain Research Station: Utah, 67.

19. Vallès, J., and Garnatje, T. 2005, Artemisia and its allies: genome organization and evolution and their biosystematic, taxonomic and phylogenetic implications in the Artemisiinae and related subtribes (Asteraceae, Anthemideae). In: Sharma AK., Sharma A., (Eds.). Plant genome: biodiversity and evolution, 255. Vol. 1B: Phanerogams (Higher groups). Enfield, M/S Science Publishers.

20. Tournefort, J.P. 1700, Institutiones rei herbariae. Paris.

21. Linné, C. 1735, Systema naturae. Leiden.

22. Cassini, A.H.G. 1817, Bull. Sci. Soc. Philom. Paris, 3, 31.

23. Lessing, C.F. 1832, Synopsis generum Compositarum. Berlin.

24. Besser, W.S.J.G. 1829, Bull. Soc. Imp. Naturalistes Moscou, 1, 219.

25. Besser, W.S.J.G. 1832, Bull. Soc. Imp. Naturalistes Moscou, 3, 5.

26. Besser, W.S.J.G. 1834, Bull. Soc. Imp. Naturalistes Moscou, 7, 5.

27. Besser, W.S.J.G. 1836, Bull. Soc. Imp. Naturalistes Moscou, 8, 3.

28. Candolle, A.P. 1837, Prodromus systematis naturalis regni vegetabilis. Paris.

29. Rouy, G.C.C. 1903, Flore de France 8. Paris.

30. Rydberg, P.A. 1916, North American Flora, 34, 244.

31. McArthur, E.D., Pope, C.L., and Freeman, D.C. 1981, Amer. J. Bot., 68, 589.

32. Ling, Y.R. 1991, Bull. Bot. Res., Harbin, 12, 1.

33. Ling, Y.R. 1995, The New World Seriphidium (Besser) Fourr., Hind, D.J.N., Jeffrey, C., and Pope, G.V. (Eds.). Advances in Compositae systematics. Royal Botanic Gardens, Kew, 283.

34. Shultz, L.M. 2006, "Artemisia”, Flora of North America vol. 21: Asterales. Editorial Committee, (Eds.) Oxford University Press, New York.

35. Shultz, L.M. 2009, Syst. Bot. Monographs, 89, 1.

36. Sanz, M., Vilatersana, R., Hidalgo, O., Garcia-Jacas, N., Susanna, A., Schneeiwess, G.M., and Vallès, J. 2008, Taxon, 57, 66.

37. Tkach, V.N., Hoffmann, M.H., Röser, M., Korobkov, A.A., and Von Hagen, K.B. 2008, Evolution, 62, 184.

38. Pellicer, J.P., Vallès, J., Korobkov, A.A., and Garnatje, T. 2011, Taxon, 60, 691.

39. Garcia, S., McArthur, E.D., Pellicer, J., Sanderson, S.C., Vallès, J., and Garnatje, T. 2010, Amer. J. Bot., 98, 638.

40. Vallès, J., Torrell, M., Garnatje, T., Garcia-Jacas, N., Vilatersana R., and Susanna, A. 2003, Pl. Biol., 5, 274.

41. Watanabe, K. 2004, Compositae Newslett., 41, 64.

42. Solbrig O.T. 1977, Chromosomal cytology and evolution in the family Compositae. In: Heywood, V.H., Harborne, J.B., Turner, B.L. (Eds.). The biology and chemistry of the Compositae, Vol. I. London, New York, San Francisco: Academic Press, 269.

43. Schweizer, D., and Ehrendorfer, F. 1983, Biol. Zentralbl., 102, 637.

44. Oliva, M., and Vallès, J. 1994, Canad. J. Bot., 72, 1126.

45. McArthur, E.D., and Sanderson, S.C., 1999, Amer. J. Bot., 86, 1754.

46. Wiens, D., and Richter, J.A., 1996, Amer. J. Bot., 53, 981.

47. Matoba, H., Nagano, K., and Hoshi, Y. 2007, Cytologia, 72, 181.

48. Vallès, J., and Siljak-Yakovlev, S. 1997, Canad. J. Bot., 75, 595. 
49. Pellicer, J., Garcia, S., Garnatje, T., Hidalgo, O., Korobkov, A.A., Dariimaa, S., and Vallès, J. 2007, Bot. J. Linn. Soc., 153, 301.

50. Pellicer, J., Garcia, S., Garnatje, T., Dariimaa, Sh., Korobkov, A.A., and Vallès, J. 2007, Chromosome Bot., 2, 45.

51. Ehrendorfer, F. 1964, Oesterr. Bot. Z., 111, 84.

52. Estes, J.R. 1969, Brittonia, 21, 29.

53. Kawatani, T., and Ohno, T. 1964, Bull. Natl. Inst. Hyg. Sci. Tokyo, 82, 183.

54. Korobkov, A.A. 1972, Bot. Zhurn., 57, 1316.

55. Persson, K. 1974, Opera Bot. (Lund), 35, 1.

56. McArthur, E.D., and Pope, C.L. 1979, Great Basin Naturalist, 39, 419.

57. Garcia, S., Garnatje, T., Dariimaa, Sh., Tsooj, Sh., and Vallès, J. 2006, Bot. J. Linn. Soc., 150, 203.

58. Sánchez-Jiménez, I., Pellicer, J., Hidalgo, O., Garcia, S., Garnatje. T., and Vallès, J. 2009, Folia Geobot., 44, 307.

59. Zhang, M.J., and He, X.L. 2010, Pl. Syst. Evol., 210, 109.

60. Malakhova, L.A. 1990, Byull. Glavn. Bot. Sada, 155, 60.

61. Torrell, M., Vallès, J., Garcia-Jacas, N., Mozzafarian, V., and Gabrielian, E. 2001, Bot. J. Linn. Soc., 135, 51.

62. Krogulevich, R.E. 1978, Kariologicheskii analiz vidov flory vostochnogo Sayana. In: Malyshev L.I., Peshkova G.A., (Eds.). Flora Pribaikalya. Novosibirsk: Nauka, 19.

63. Kreitschitz, A., and Vallès, J. 2003, Folia Geobot., 38, 333.

64. Pellicer, J., Garnatje, T., Molero, J., Pustahija, F., Siljak-Yakovlev, S., and Vallès, J. 2010, Austr. J. Bot., 58, 605.

65. Torrell, M., Cerbah, M., Siljak-Yakovlev, S., and Vallès, J. 2001, Bocconea, 13, 623.

66. Torrell, M., Cerbah, M., Siljak-Yakovlev, S., and Vallès, J. 2003, Plant Syst. Evol., 239, 141.

67. Hoshi, Y., Matoba, H., and Kondo, K. 2006, Caryologia, 59, 312.

68. Garcia, S., Garnatje, T., Hidalgo, O., McArthur, E.D., Siljak-Yakovlev, S., and Vallès, J. 2007, Pl. Syst. Evol., 267, 79.

69. Garcia, S., Lim, K.Y., Chester, M., Garnatje, T., Pellicer, J., Vallès, J., Leitch, A.R., and Kovarik, A. 2009, Chromosoma, 118, 85.

70. Grummt, I., and Pikaard, C.S. 2003, Nat. Rev. Mol. Cell. Biol., 4, 641.

71. Goyon, C., Rossignol, J.L., and Faugeron, G. 1996, Nucleic Acids Res., 24, 3348.

72. Vahidi, H., Curran, J., Nelson, D.W., Webster J.M., McClure, M.A., and Honda, B.M. 1988, J. Mol. Evol., 27, 222.

73. Belkhiri, A., Buchko, J., and Klassen, G.R. 1992, Mol. Biol. Evol., 110, 86

74. Vitturi, R., Colomba, M.S., Pirrone, A.M., and Mandrioll, M. 2002, J. Hered., 93, 279.

75. Pellicer, J. 2009, Ph.D. dissertation, University of Barcelona, Barcelona, Spain.

76. Garcia, S., Panero, J.L., Siroky, J., and Kovarik, A. 2010, BMC Plant Biol., 10, 176.

77. Pellicer, J., Garcia, S., Garnatje, T., Hidalgo, O., Siljak-Yakovlev, S., and Vallès, J. 2008, Collect. Bot. (Barcelona), 27, 19.

78. Pellicer, J., Garnatje, T., Hidalgo, O., Tagashira, N., Vallès, J. and Kondo, K. 2010, Plant Biosyst., 144, 841. 
79. Hidalgo, O., Garcia-Jacas, N., Garnatje, T., Romashchenko, K., Susanna, A., and Siljak-Yakovlev, S. 2008, Taxon, 57, 769.

80. Swift, H. 1950, Proc. Nat. Acad. Sci. U.S.A., 36, 643.

81. Greilhuber, J., Dolezel, J., Lysak, M.A., and Bennett, M.D. 2005, Ann. Bot., 95, 255.

82. Greilhuber, J., Borsch, T., Müller, K., Worberg, A., Porembski, S., and Barthlott, W. 2006, Pl. Biol., 8, 770.

83. Pellicer, J., Fay, M.F., and Leitch, I.J. 2010, Bot. J. Linn. Soc., 164, 10.

84. Bennett, M.D., and Leitch, I.J. 2005, Ann. Bot., 95, 45.

85. Bennett M.D., and Leitch, I.J. 2005, Ann. Bot., 95, 1.

86. Vinogradov, A.E. 2003, Trends Genet., 19, 609.

87. Albach, D.A., and Greilhuber, J. 2004, Ann. Bot., 94, 897.

88. Garnatje, T., Vallès, J., Garcia, S., Hidalgo, O., Sanz, M., Canela, M.Á., and Siljak-Yakovlev, S. 2004, Biol. Cell, 96, 117.

89. Price, H.J., Dillon, S.L., Hodnett, G., Rooney, W.L., Ross, L., and Johnston, J.S. 2005, Ann. Bot., 95, 219.

90. Beaulieu, J.M., Leitch., I.J., Patel, S., Pendharkar, A., and Knight, C.A. 2008, New Phytol., 179, 975.

91. Garcia, S., Canela, M.Á., Garnatje, T., McArthur, E.D., Pellicer, J., Sanderson, S.C., and Vallès, J. 2008, Biol. J. Linn. Soc., 94, 631.

92. Torrell, M., and Vallès, J. 2001, Genome, 44, 231.

93. Garcia, S., Sanz, M., Garnatje, T., Kreitschitz, A., McArthur, E.D., and Vallès, J. 2004, Genome, 47, 1004.

94. Pellicer, J., Garcia, S., Canela, M.A., Garnatje, T., Korobkov, J.D., and Vallès, J. 2010, Pl. Biol., 12, 820.

95. Soltis, D.E., and Soltis, P.S. 2000, Proc. Nat. Acad. Sci. U.S.A., 97, 7051.

96. Wendel, J.F. 2000, Pl. Mol. Biol., 42: 225.

97. Cui, L., Wall, P.K., Leebens-Mack, J.H., Lindsay, B.G., Soltis, D.E., Doyle, J.J., Soltis, P.S., Carlson, J.E., Arumuganathan, K., Barakat, A., Albert, V.A., Ma, H., and de Pamphilis, C.W. 2006, Genome Res., 16, 738.

98. Petrov, D.A. 2001, Genetica, 114, 81.

99. Bennetzen, J.L., Ma, J., and Devos, K.M. 2005, Ann. Bot., 95, 127.

100. Feldman, M., Liu, B., Segal, G., Abbo, S., Levy, A.A., and Vega, J.M. 1997, Genetics, 147, 1381.

101. Ozkan, H., Levy, A.A., and Feldman, M. 2001, Plant Cell, 13, 1735.

102. Parisod, C., Alix, K., Just, J., Petit, M., Sarilar, V., Mhiri, C., Ainouche, M., Chalboub, B., and Grandbastien M.A. 2010, New Phytol., 186, 37.

103. Kornkven, A.B., Watson, L.E., and Estes, J.R. 1998, Amer. J. Bot., 85, 1787.

104. Torrell, M., Garcia-Jacas, N., Susanna, A., and Vallès, J. 1999, Taxon, 48, 721.

105. Watson, L.E., Bates, P.L., Evans, T.M., Unwin, M.M., and Estes, J.R. 2002, BMC Evol. Biol., 2, 17.

106. D’andrea, S., Caramiello, R., Ghignone, S., and Siniscalco, C. 2003, Plant Biosyst., 137, 121.

107. McArthur, E.D., and Plummer, A.P. 1978, Great Basin Naturalist Mem., 2, 229.

108. Garcia, S. 2007, Ph.D. dissertation, University of Barcelona, Barcelona, Spain. 
109. Kornkven, A.B. 1997, Ph.D. Thesis dissertation, University of Oklahoma, Norman, U.S.A.

110. Kornkven, A.B., Watson, L.E., and Estes, J.R. 1999, Syst. Bot., 24, 69.

111. Danin, A. 1999, Willdenowia, 29, 177.

112. Jeffrey, C. 2007, Introduction with keys to tribes. In: Kadereit, J.W., Jeffrey, C. (Eds.). Flowering plants vol. VIII. Eudicots. Asterales.: Springer-Verlag Press: Berlin Heidelberg, 61.

113. Stix, E. 1960, Grana Palynol., 2, 41.

114. Wodehouse, R.P. 1926, Bull. Torr. Club, 53, 479.

115. Monoszon, M.X. 1948, Morfologiya pyltsy polynei. In: Sukachev, V.N. (Ed.). Trudy konferentsii po sporovo-pyltsemu analizu. Izdatelstvo Moskovskogo Universiteta: Moskva, 37.

116. Straka, H. 1952, Svensk Bot. Tidsskr., 46, 204.

117. Grigoreva, V.V., Korobkov, A.A., and Tokarev, P.I. 2009, Bot. Zhur., 94, 328.

118. Chen, S.G., and Zhang, J.T. 1991, Acta Phytotax. Sin., 29, 246.

119. Hayat, M.Q., Ashraf, M., Khan M.A., Yasmin, G., Shaheen, N., and Jabeen, S. 2009, Afr. J. Biotechnol., 8, 6568.

120. Ghahreman, A., Noorbakhsh, S.N., Mehdigholi, K. and Attar, F. 2007. Iran J. Bot., 13, 21.

121. Sun, H.Z., He, L.X., Chen, T.S., and Zhang, X.J. 2007, Progr. Nat. Sci., 17, 35. 


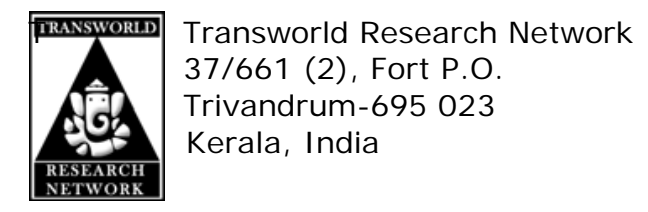

Recent Advances in Pharmaceutical Sciences, 2011: 223-246 ISBN: 978-81-7895-528-5

Editor: Diego Muñoz-Torrero

\title{
10. Biological remediation approach involving soils and groundwaters polluted with chlorinated solvents in a Mediterranean context
}

\author{
Amparo Cortés', Diana Puigserver², José Maria Carmona ${ }^{2}$ \\ and Manuel Viladevall ${ }^{2}$ \\ 'Department of Natural Products, Plant Biology and Soil Science, Faculty of Pharmacy \\ University of Barcelona, 08028 Barcelona, Spain; ${ }^{2}$ Department of Geochemistry, Petrology and \\ Geological Prospecting, Faculty of Geology, University of Barcelona, 08028 Barcelona, Spain
}

\begin{abstract}
Chlorinated solvents such as trichloroethene (TCE) and tetrachloroethene (PCE) form a class of dense non-aqueous phase liquid (DNAPL) compounds that cause a persistent environmental contamination that is difficult to investigate and challenging to remediate. The use of vegetation to enhance degradation by soil microorganisms of chlorinated solvents in soil-groundwater systems (phyto and rhizo-degradation) has been receiving attention since the 90 ' as an attractive low-cost alternative. Remediation strategies must be site-specific, with separate approaches often warranted for the DNAPL source zone and its associated aqueous phase plume. It has been suggested that possible mechanisms for the enhanced bioremediation of TCE in the rhizosphere are excretion of phenolic compounds in root exudates and plant mycorrhization.
\end{abstract}




\section{Introduction}

Tetrachloroethene (PCE) and trichloroethene (TCE) are among the most common groundwater contaminants of industrial origin; their usefulness lead to their wide use in many industries: electronics manufacturing, solvent production, pesticide / herbicide manufacturing, dry cleaning, instrument manufacturing, solvent recycling, engine manufacturing, steel product manufacturing, chemical production, rocket engine / fuel manufacturing, aircraft cleaning / engine degreasing, but also in industrial processes such as: metal cleaning, metal machining, tool and die operations, vapor and liquid degreasing or paint stripping; storage and transfer of solvents are also widespread activities [1]. Although PCE and TCE have been produced and utilized widely since the beginning of the 20th century, their importance as soil and groundwater contaminants was not recognized until the 1980s.

PCE and TCE, as well as carbon tetrachloride (CT) and chloroform (CF) are among the chlorinated solvents that form the primary classes of dense non-aqueous phase liquid (DNAPL) compounds. DNAPLs show common characteristics: a) immiscibility with water, forming separate phases, b) low absolute solubilities: they may persist for decades before dissolving in groundwater, c) relatively high densities, they can therefore sink beneath the water table, polluting the full thickness of the aquifer, and drinking water supplies, d) low viscosities, that allow rapid subsurface migration strongly affected by fluid pressures, which dictate the path the liquid will take, and e) toxicity.

PCE causes irritation of the upper respiratory tract and eyes, kidney dysfunction, and, at lower concentrations, neurological effects, such as reversible mood and behavioral changes, impairment of coordination, dizziness, headache, sleepiness, and unconsciousness. Short-term exposure to TCE causes irritation of the nose and throat and central nervous system (CNS) depression, with symptoms such as drowsiness, dizziness, giddiness, headache, loss of coordination; high concentrations of TCE have caused numbness and facial pain, reduced eyesight, unconsciousness, irregular heartbeat and death. CT is listed as a suspect carcinogen, a laboratory animal carcinogen at relatively high doses; however, human liver cancer has been reported; it can be absorbed through intact skin; it causes CNS depression, damaging kidneys, liver, or lungs, and can cause anemia, rapid and irregular heartbeats; health effects appear to be greatly increased by alcohol consumption. CF is a suspect carcinogen; it causes CNS depression, rapid and irregular heartbeat, and liver and kidney damage. 
The concentrations in groundwater at which chlorinated solvents are harmful to human health are extremely low. The drinking water limit for common chlorinated solvents is $5 \mu \mathrm{g} / \mathrm{L}$, and the groundwater quality standard is $10 \mu \mathrm{g} / \mathrm{L}$.

They cause a persistent environmental contamination, which, in most cases, started decades ago. Some chemical manufacturer material safety data sheets distributed from the 1940s until the early 1970s suggested that 'acceptable practice' for the disposal of waste chlorinated solvents and the residues of distillation was to spread them onto dry ground/soil to allow them to evaporate. These safety data sheets recognized the volatile nature of many chlorinated solvents, but they did not recognize their ability to infiltrate rapidly into the subsurface, causing soil and groundwater pollution. Behind it is the widespread and socially accepted use of shallow soil systems as a location to dispose of unwanted materials.

The DNAPL source zone is defined as the area which has been in contact with DNAPL, and can include: 1) residual DNAPL, 2) pooled (free-phase) DNAPL, 3) DNAPLs sorbed to soil, and 4) dissolved contaminants diffused through fine grained media. Pollution is commonly widespread because plumes of dissolved-phase contamination emanate from the DNAPL source zones, where dissolution of DNAPL continuously supplies solute to the plume, defined as the contaminated groundwater emanating from the source (see Fig. 1).

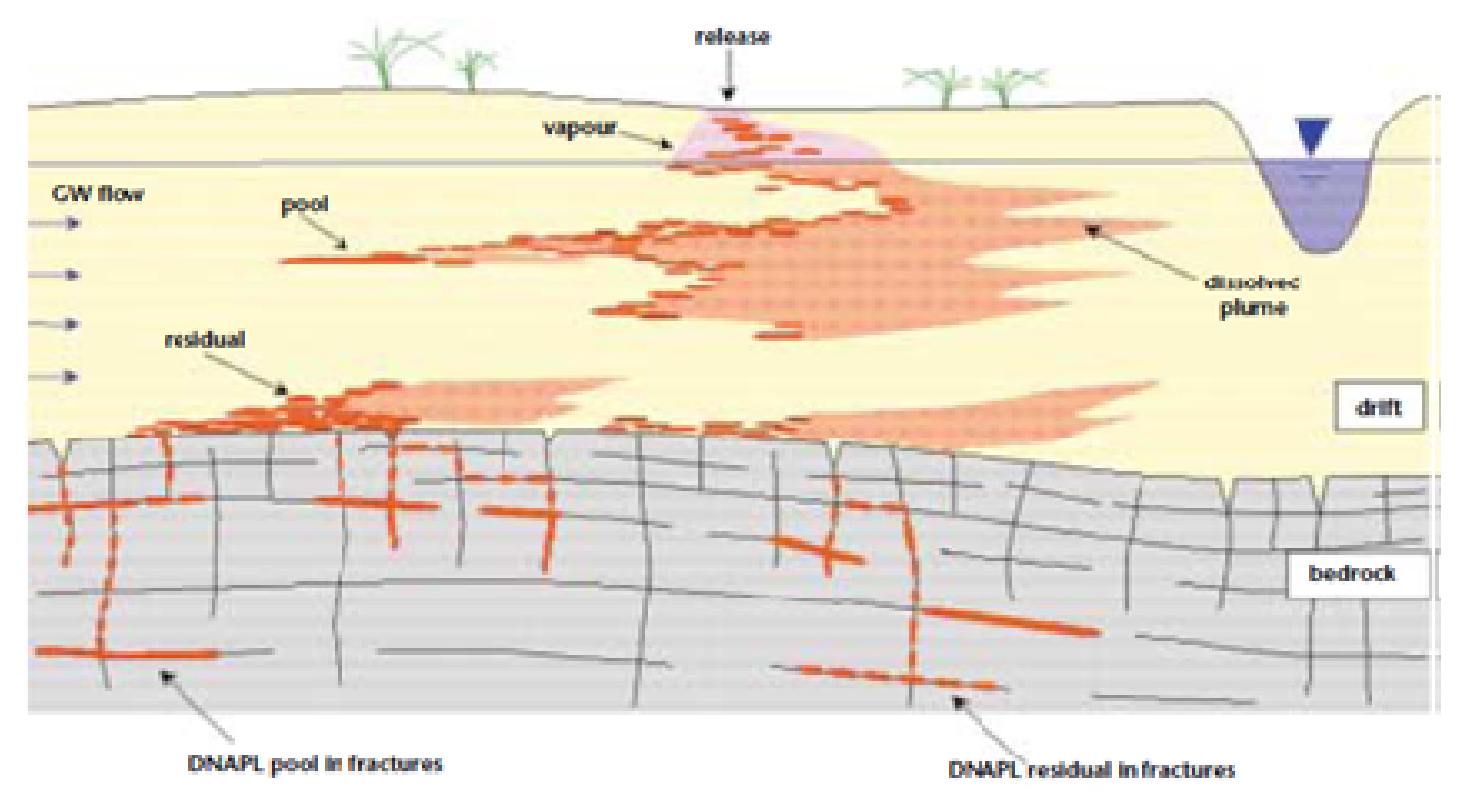

Figure 1. DNAPL source area, plume formation, and DNAPL accumulation in fractured media [2]. 
Natural attenuation that is naturally occurring physical, chemical, and biological processes, such as; biodegradation, dispersion, dilution, adsorption, and volatilization can be significant at some places. Plumes are retarded by sorption, especially in the presence of organic matter, but are also affected by abiotic and biotic degradation processes (see Fig. 2) and to a lesser extent by volatilization [3].

In aerobic environments, TCE and the metabolites of the reductive dehalogenation of PCE and TCE, such as dichloroethylene (DCE) and vinyl chloride (VC), are cometabolically oxidized to $\mathrm{CO}_{2}$ by bacteria that possess oxygenase enzymes. Some of these enzymes are the methane monoxygenase (MMO) of methanotrophs, toluene dioxygenase (TDO) of Pseudomonas putida F1, toluene 2-monooxygenases (TMO) of Burkholderia cepacia G4, propane monoxygenase of Mycobacterium vaccae JOB5, phenol hydroxylase (PH) of Alcaligenes eutrophus JMP134 and Burkholderia cepacia G4, alkene monooxygenase (AMO) of Alcaligenes denitrificans spp., ammonia monooxygenase of Nitrosomonas europaea, and isopropylbenzene dioxygenase (IPB) of Pseudomonas sp. JR1 [4-11].

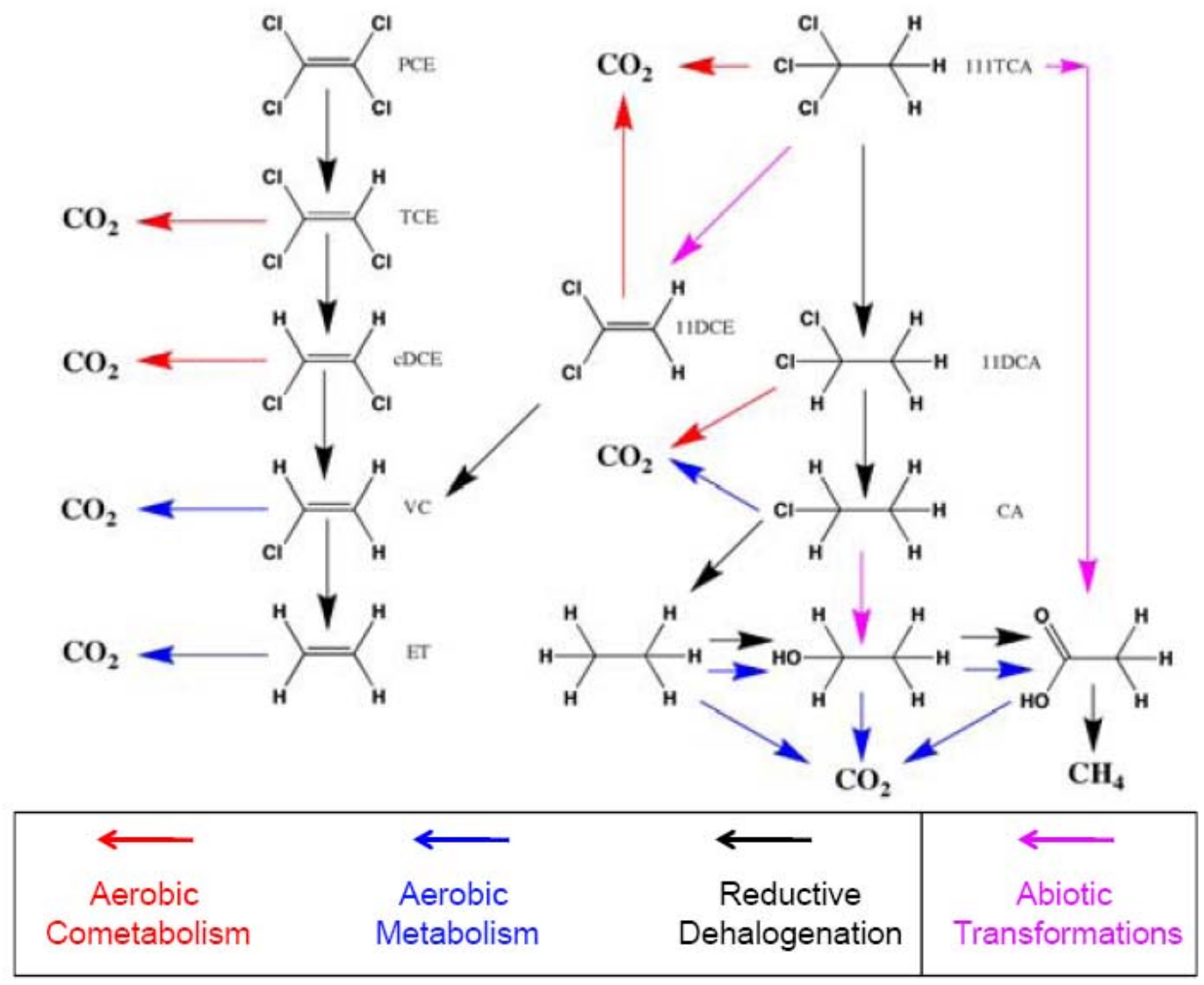

Figure 2. DNAPL main degradation processes. 
TCE can be metabolized to vinyl chloride, a potent carcinogen, if oxygen is not present [12]; this degradation pathway is undesirable where $\mathrm{VC}$ can accumulate with no further breakdown.

Degradation of CT occurs slowly in the environment, indigenous microorganisms may degrade it to CF that may be more persistent than CT. $\mathrm{CF}$ is readily formed under anaerobic conditions [13]. Other degradation products, through reductive dechlorination, are methylene chloride (MC) and chloromethane (CM). For reductive dechlorination to occur, the water has to be anaerobic, i.e., dissolved oxygen must be less than $0.2 \mathrm{mg} / \mathrm{L}$ and redox potential should be less than $-50 \mathrm{mV}$. Therefore, to deplete the oxygen present in the groundwater, organic substrates with sufficient or excess biochemical oxygen demand (BOD) have to be supplied. Further, electrons and hydrogen ions are necessary to replace the chlorine atoms. All these requirements are taken care of by organic compounds such as glucose, acetate, lactate, molasses, or vegetable oils.

Bouwer and McCarty demonstrated CT biodegradation in methanogenic and denitrifying environments with $\mathrm{CF}$ reported as a main metabolite $[14,15]$. Mayotte et al. identified a denitrifying organism, Pseudomonas sp. strain KC, that is capable of transforming CT without accumulation of significant quantities of CF [16].

Biodegradation is conditioned by the existence of a physical-chemical context and by a series of processes occurring in the soil and in the aquifer. For example, the presence of nitrates and/or sulphates could inhibit PCE biodegradation process, as has been noted by $\mathrm{He}$ et al. [17]. Moreover, textural characteristics and subsurface heterogeneities can also exert their influence on the biodegradation rates.

The use of vegetation to enhance degradation of chlorinated solvents in soil-groundwater systems (phyto and rhizo-degradation) has been receiving attention since the 90' as an attractive low-cost alternative to the traditional engineering approaches of soil excavation, incineration and air stripping. Conventional groundwater remediation methods, as "pump \& treat", are not always cost-effective and can even provoque negative impacts such as: a) expansion of the source zone due to mobilization of residual DNAPL, b) undesirable changes in the DNAPL distribution, c) undesirable changes in physical, geochemical, and microbial conditions, d) adverse impact on subsequent remediation technologies, and e) increased life-cycle costs of site cleanup.

When plants are used, a series of mechanisms are involved, including phyto-volatilization, -accumulation, -degradation, -stabilization, and rhizodegradation. The role and contribution of each of these processes to the overall bioremediation system has not been accurately characterized $[18,19]$. 
Soils have many properties that can be dramatically modified at the rhizosphere, giving rise to the so called rhizosphere effect. Rhizosphere exhibits a unique biophysics and biogeochemistry which is different from that of the bulk soil. Rhizosphere responses to seasonal changes, including plant succession, rainfall, and temperature, can significantly influence potential PCE, TCE and CT bioremediation. Chlorinated solvents in soil must be in solution to be absorbed, modified, degraded, or sequestered by either plants or microorganisms.

Water movement and nutrient availability in the rhizosphere is a critical factor as plants take up many times more water than is needed for metabolism and growth. However, all of this water and compounds, dissolved in the soil solution, moves through the rhizosphere, where it is subjected to processing by microorganisms before it enters the roots.

Some rhizosphere microorganisms secrete plant hormones that increase root growth, and thereby the secretion of root exudates that contain metabolites, including proteins and carbohydrates, used by the bacteria. Exudates also contain natural chelating agents such as citric, acetic, and other organic acids that make not only ions of nutrients but contaminants more

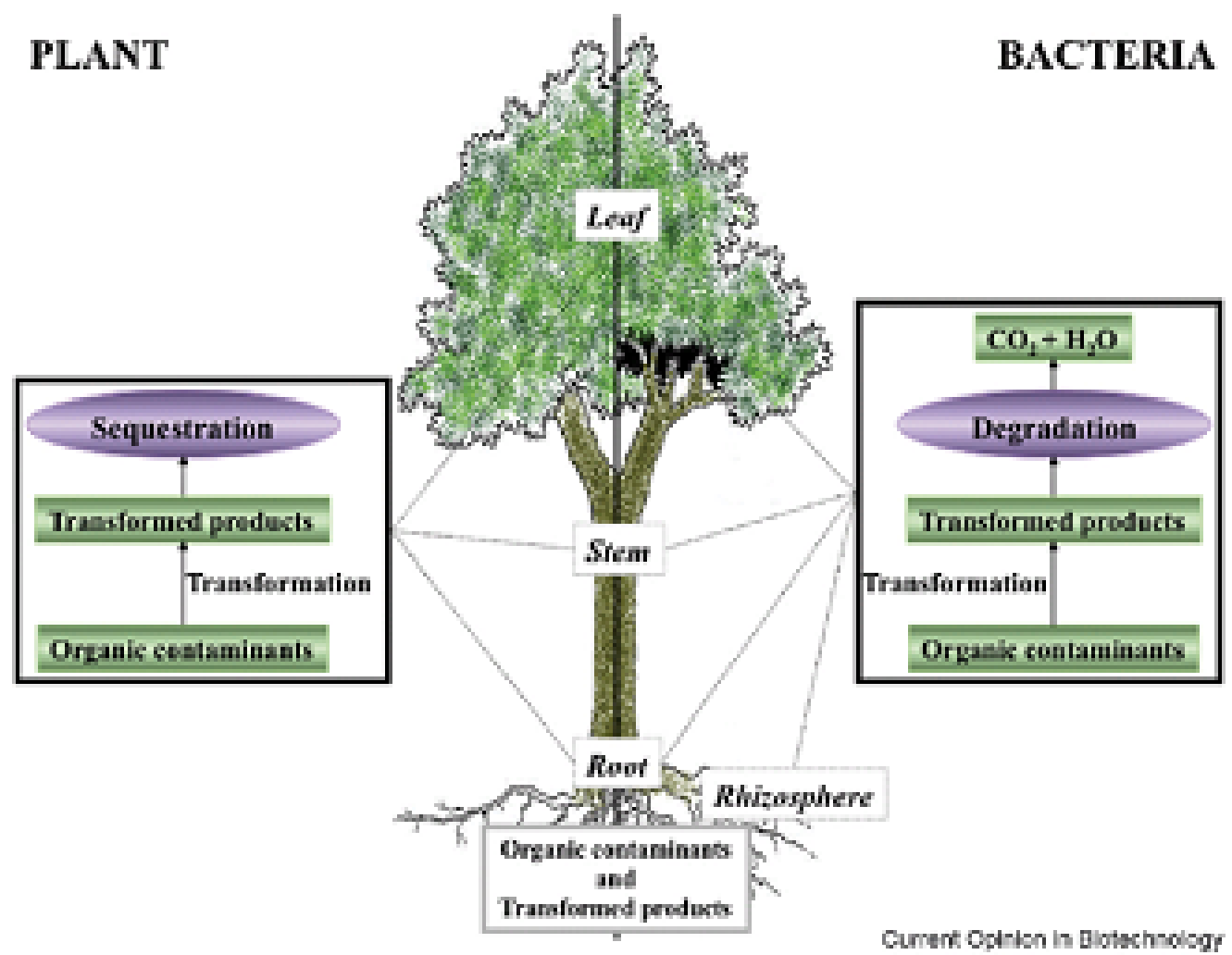

Figure 3. Contribution of plants and their associated bacteria to DNAPLs' phytoremediation. 
mobile in the soil. Exudates may also include enzymes, such as nitroreductases, dehalogenases, and lactases. These enzymes have important natural functions, but may also degrade organic contaminants that contain nitro groups or halogenated compounds. It has been suggested that a possible mechanism for the enhanced microbial mineralization of TCE in the Lespedeza cuneata rhizosphere soil is excretion of phenolic compounds in root exudates. Since phenol is a known inducer of toluene monoxygenase, an enzyme responsible for degradation of TCE, the natural plant exudates could play a role in biodegradation of TCE in the rhizosphere [20]. Exudates have also been found to increase the TCE sorption capacity of soil [21].

The main uncertainty in phytoremediation of chlorinated solvents regards the magnitude of plant uptake, phyto-volatilization, and rhizodegradation. Several studies have reported that TCE disappearance in planted systems is mainly due to plant uptake, followed by phyto-volatilization and diffusion through the stem and/or metabolism by the plant [22]. On the contrary, other studies have observed TCE degradation occurring mainly as a result of rhizosphere microbial metabolism [18]. Uptake and dechlorination of CT by plant tissues is likely the primary mechanism for phytoremediation by poplar [23].

Phytoremediation of chlorinated solvents from soil and groundwater have reported up to $90 \%$ contaminant removal by the use of different plant species [24-26].

Poplar and willow trees are the preferred plant species in temperate climates for PCE, TCE and CT phytoremediation. Valuable poplar and willow common characteristics that make them ideal for this applications are that they are fast-growing, easily propagated, tolerant to relatively high levels of contaminants ( $<550 \mathrm{ppm}$ TCE), resistant to saturated conditions, and they are phreatophytes, that is deep-rooted plants where water uptake is mainly from the groundwater [27]. Willows have been found to consistently utilize groundwater sources even during periods of rainfall [28]. Their transpiration rates can increase from 19 to $200-1000 \mathrm{~L}_{\text {of water day }}{ }^{-1}$ in young to mature trees $[27,29]$. With these high transpiration rates they can extract enough water to depress the water table locally, inducing flow toward the trees and containing the contaminant plume (hydraulic control).

At recent pollution episodes it must be taken into account that TCE is acutely toxic to a variety of crop plants at concentrations of about $2 \mathrm{mM}$ in the gas phase [30].

Box indicated that black willow and eastern poplar are capable of decontaminating solution concentrations of up to $20 \mathrm{mg} / \mathrm{L}$ of PCE and 45 $\mathrm{mg} / \mathrm{L}$ TCE [31]. High concentrations of $45 \mathrm{mg} / \mathrm{L}$ PCE were shown to be toxic to the plants. Poplars are able, according to Gordon et al., to survive when grown in water containing $50 \mathrm{ppm}$ TCE [32]. 
Poplar trees possess endophytic bacteria, including methanotrophs, that live symbiotically within the plants. Some of these bacteria that include members of Pseudomonas sp., Enterobacter-Clostridium species, and methylotroph species such as Methylobacterium populi sp. nov. are known for their bioremediation potential [33-35].

The effectiveness of chlorinated solvent phytoremediation by poplar and willow trees is strongly influenced by the choice of genotypes (clones) that must be compatible with the intended use, site characteristics (soil type, microclimate, pests and diseases), and with the expert opinion concerning use of native versus exotic plants [36].

Other potential tree species that have been studied for chlorinated solvent phytoremediation are conifers [37]. Loblolly pine (Pinus taeda) is capable of up to $90 \%$ TCE removal from soils and groundwater at some areas [25]. This species is characterized by the production of significant quantities of oil extracts, composed mainly of monoterpenes. Therefore, these compounds may influence the microbial processes occurring in the rhizosphere, either as root exudates or leachates from the decaying foliage at the soil surface.

Schnabel et al. investigated in 1997 the uptake and transformation of TCE by edible garden plants: carrots, spinach, and tomatoes [21]. 74\% to $95 \%$ of TCE was volatilized through the leaves and $5 \%$ to $25 \%$ was sorbed to the soil. $1 \%$ to $2 \%$ was found in the plant material and could not be extracted.

Schnoor proposed that TCE metabolism in plants is the result of dehalogenase degrading enzyme that oxidizes alkanes, alkenes, methanes, and their halogenated analogues [38]. An antibody assay has been developed to determine the presence of the dehalogenase enzyme in plants. This technique can be used to aid in the selection of viable plants for a particular site [39]. Cunningham et al. stated that many of the enzyme systems present in mammalian metabolism of TCE are also present in plants (e.g., cytochrome P-450 and glutathione) [40]. Schnabel et al. suggested that transformation of TCE after plant uptake may be similar to the mammalian breakdown of TCE. This theory is based on similar metabolites being observed in both plants and animals [21].

Soil and groundwater remediation are undertaken in European countries to manage unacceptable risks to human health and/or the environment. The application of more sustainable remediation technologies that do not require large amounts of inputs (inorganic fertilizers, surfactants,...) and promote the application of biological systems to solve anthropogenic environmental problems are highly recommended if knowledge about soil-plant system is as complete as possible.

Experience from the past years has demonstrated that DNAPL contaminated sites are difficult to investigate and challenging to remediate. 
DNAPL can penetrate fractured rock and clay forming pools and, for this reason, many decades are required to dissipate DNAPL secondary sources in most hydrogeological environments. It is intended to assist site investigators, site owners and regulators in conducting site investigations and in selecting sustainable remediation approaches.

\section{Mediterranean area and chlorinated solvent pollution}

At least 4 attributes make the Mediterranean area indeed different and largely determine the nature of its soils: I) specific climate, II) topography and mountains, III) dust from the desert, and IV) long term effects of man.

The soils with such singular characteristics extend over 420 million ha approximately; they are old (product of interactions that have taken place for millennia between the natural processes of pedogenesis and the human activities), frequently polygenetic, affected by climatic fluctuations and man's management of landscape. Some soils are polycyclic, namely, composed of the superposition of several young soils, and resulting from the succession of several phases of pedogenesis over very long periods of time; others are, by contrast, truncated, amputated by the erosion of their surface horizons. Dry summer periods frequently coincide with the occurrence of violent rainstorms, causing average yearly soil losses above 15 tons/ha in more than one third of the Mediterranean basin. Such unreasonable soil losses drive a principle mechanism of irreversible soil degradation that disperses away pollutants when present.

Loss of organic matter and fertility, erosion, salinization, chemical pollution and its related problems have affected these soils since land was first settled and cultivated.

The annual rate of loss of organic matter varies greatly. High temperatures during Mediterranean summer lead to a rapid decline in the organic matter content, especially in cultivated soils.

Preliminary estimates of the UE indicate that $74 \%$ of the land in Southern Europe is covered by soils containing less that $2 \%$ organic carbon (less than $3.4 \%$ organic matter) in the topsoil $(0-30 \mathrm{~cm})$ (see Fig. 4). Global climatic change (GCC) may further decrease organic matter content and soil biological activity.

Solvent production and metal degreasing are processes that are done in a great number of small plants. Concentrations of these plants in a certain industrial area may be considered as point sources at the regional level. In Catalonia, Spain, a representative industrial area of the Mediterranean basin, there are 20 main point sources of halogenated solvents. 
A quarter of the inquiries opened in Catalonia because of groundwater pollution are due to chlorinated solvent releases (internal information of the Catalan Water Agency). Information about total volume of DNAPL released into soils and groundwaters during the last 30 years is not available.

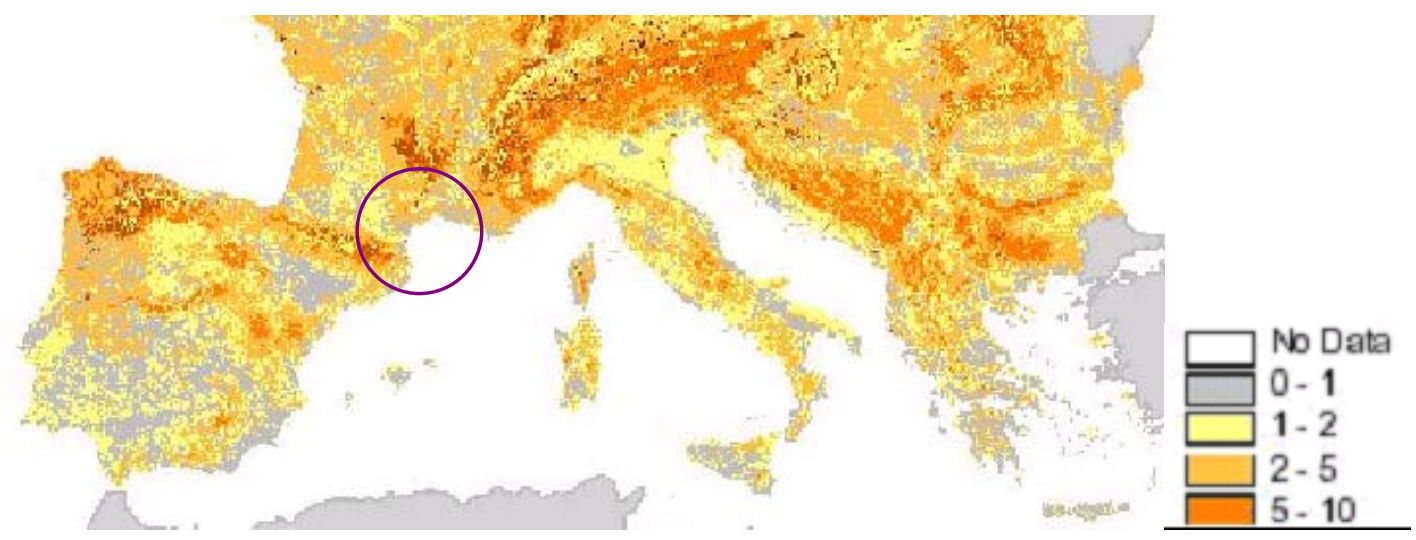

Figure 4. Organic Carbon Content (\%) in topsoils of South Europe (UE, 2003).

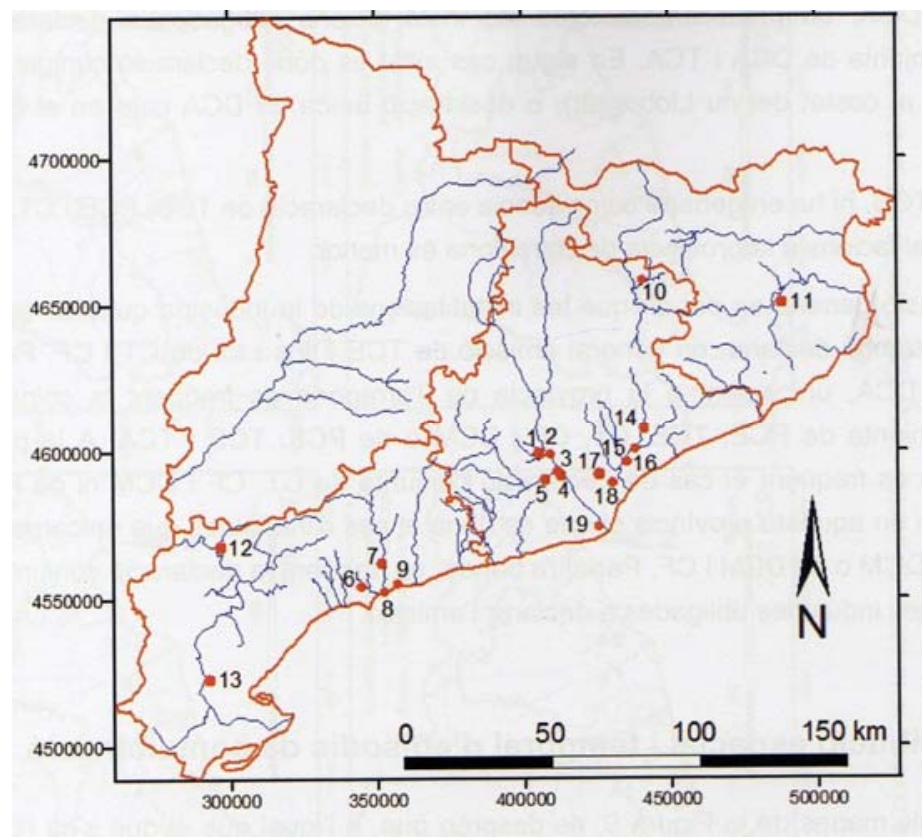

Figure 5. Chlorinated solvents production distribution at Mediterranean river basins in Catalonia [41].

\section{Site and pollution characterization}

Two polluted areas have been chosen to conduct site investigations and to study phytoremediation viability. 


\section{Site A}

The site is located at a Quaternary alluvial irregular overburden varying in thickness between 3 and $12 \mathrm{~m}$. The geological formation exhibits an important textural variability, consisting on fine sands and silts crossed by paleochannels made up of silty sands and gravels and sandy gravels with thin silty layers interbedded. These materials are distributed on terraces that are geomorphologically differentiated but hydraulically connected. These alluvial materials overlie a thick formation of Eocene fractured marls and marlstones containing some amounts of microscopic pyrite.

Natural soils show a stratified profile formed by recent deposits; organic matter decreases irregularly with depth and, on the average, is less than $1 \%$ in average. Soils are calcareous and mildly or moderately alkaline. They have good or moderately good drainage.

The upper layer consists of a horizon about $35 \mathrm{~cm}$ thick subjected to the influence of human activities, more or less nutrient enriched but structurally fairly good. Underneath this horizon, down to a variable depth, is to be found the rest of deposits, unaffected by ploughing, which may be considered as $\mathrm{C}$ horizon, and that can be penetrated by roots. Soil materials are derived from erosion of pre-existing soils, almost all calcareous in character.

Approximately $700 \mathrm{~mm}$ rainfall per year, with a total maximum daily of $125 \mathrm{~mm}$ in autumn, is collected on the area. During the dry period, rainfall does not cover evapotranspiration losses. The depth to the water table ranges from 2 to $8 \mathrm{~m}$. The alluvial formation constitutes an unconfined aquifer connected to the Ges River.

Groundwater contamination was detected in 2000 at a municipal water supply well at levels of $70 \mu \mathrm{g} / \mathrm{L}$ of PCE. The polluted well was closed in January 2001 by the Catalan Hydraulic Administration (ACA), which ordered to do the first works of characterization and rehabilitation.

The groundwater contaminant plume was already displayed in 2001 showing variations from 1,700 to $10,300 \mu \mathrm{g} / \mathrm{L}$ of PCE, and between 38,000 and $338,000 \mu \mathrm{g} / \mathrm{L}$ of PCE, depending on the two source areas. A third area, at the right hand side of the river, was also individualized; this source was caused by a partial lateral migration of PCE free phase through a paleochannel connecting both banks of the river. Currently, the river cuts the Eocene marls but near the last sources it cuts the paleochannel.

The length of the plume was about $1,400 \mathrm{~m}$, with ending concentrations that range between 50 and $200 \mu \mathrm{g} / \mathrm{L}$ of PCE plus TCE; the calculated affected water volume was about $200,000 \mathrm{~m}^{3}$. The applied methods of rehabilitation have been pumping and, air stripping in one case, active charcoal in another.

Since 2005 our team is carrying out studies to characterize adequately the episode rehabilitation fate, in order to improve rehabilitation strategies. 


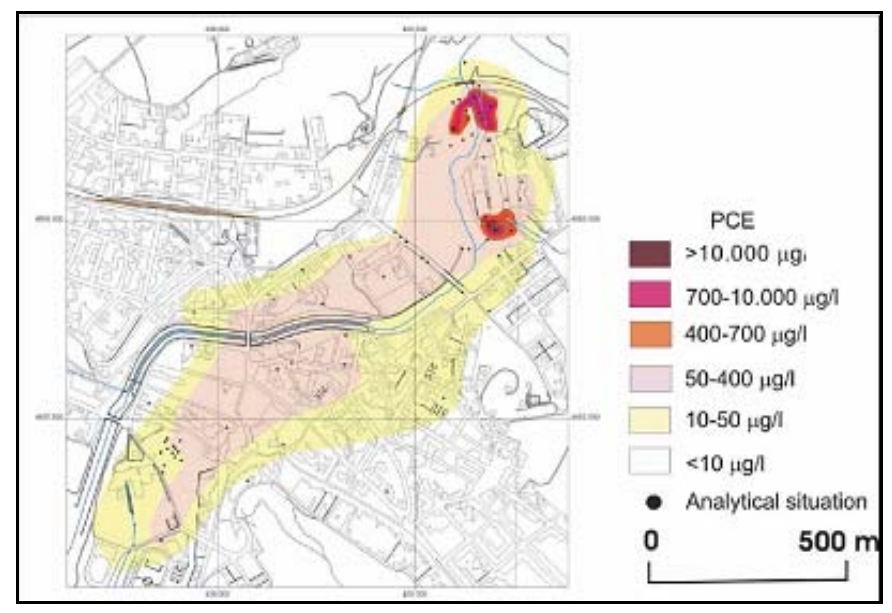

Figure 6. PCE plumes emerging from source areas in July 2001 (ACA).

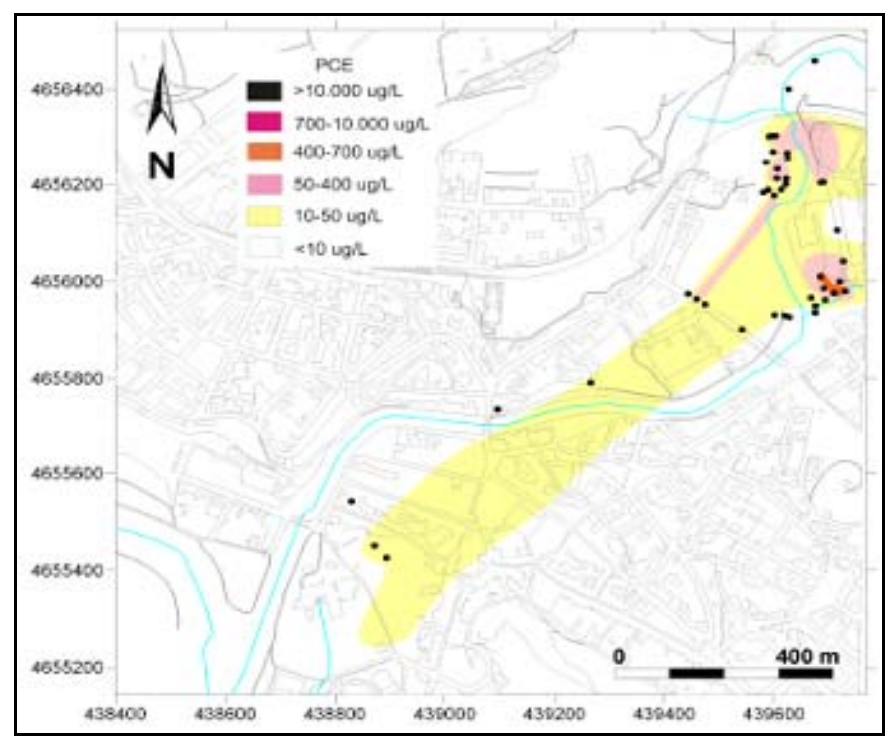

Figure 7. PCE plumes emerging from source areas in July 2006 (ACA).

Table 1. Groundwater physical-chemical parameters determined bimonthly during two years (2005-2007) in sources and downstream, in the proximity of the sources.

\begin{tabular}{|c|c|c|c|c|c|c|c|c|c|c|c|c|}
\hline & \multicolumn{9}{|c|}{ Left bank (River Ges) } & \multicolumn{4}{c|}{ Right bank (River Ges) } \\
\cline { 2 - 15 } & \multicolumn{4}{|c|}{ Source area A } & \multicolumn{4}{|c|}{ Source area B } & \multicolumn{4}{c|}{ Source area C } \\
\cline { 2 - 14 } & min & max & mean & v.c. & min & max & mean & v.c. & min & max & mean & v.c. \\
\hline pH & 6.85 & 8.01 & 7.24 & 0.04 & 6.87 & 8.14 & 7.43 & 0.04 & 6.8 & 9.34 & 7.58 & 0.05 \\
\hline Eh & -108 & 406 & 137.4 & 0.75 & -27 & 197 & 112.6 & 0.70 & -120 & 646 & 87.24 & 1.31 \\
\hline DO & 0.2 & 5.4 & 3.06 & 0.58 & 1.5 & 10.8 & 4.17 & 0.67 & 0.22 & 4 & 1.2 & 0.87 \\
\hline T & 10.2 & 18.5 & 14.51 & 0.1 & 11.7 & 19.8 & 15.42 & 0.13 & 2.5 & 20.9 & 13.5 & 0.34 \\
\hline CE & 493 & 2930 & 964.6 & 0.5 & 436 & 3830 & 1191. & 0.71 & 325 & 2780 & 638.1 & 0.7 \\
\hline
\end{tabular}

Eh: redox potential, DO: dissolved oxygen, T: temperature, $\mathrm{CE}$ : electrical conductivity $\mathrm{CE}(\mu \mathrm{S} / \mathrm{cm})$. 
Table 2. Groundwater chemical parameters determined bimonthly during two years (2005-2007) in sources and downstream, in the proximity of the sources. Concentration is expressed in $\mathrm{mg} / \mathrm{L}$.

\begin{tabular}{|c|c|c|c|c|c|c|c|c|c|c|c|c|}
\hline & \multicolumn{8}{|c|}{ Left bank (River Ges) } & \multirow{2}{*}{\multicolumn{4}{|c|}{$\frac{\text { Right bank (River Ges) }}{\text { Source C }}$}} \\
\hline & \multicolumn{4}{|c|}{ Source A } & \multicolumn{4}{|c|}{ Source B } & & & & \\
\hline & $\min$ & $\max$ & mean & v.c. & $\min$ & $\max$ & mean & v.c. & $\min$ & $\max$ & mean & V.c. \\
\hline $\mathrm{HCO}_{3}{ }^{-}$ & 266 & 734 & 358 & 0.2 & 234 & 755 & 343 & 0.4 & 140 & 1247 & 301 & 0.5 \\
\hline $\mathrm{SO}_{4}{ }^{2-}$ & 65.5 & 1027 & 180 & 1.0 & 37.5 & 661 & 220 & 0.9 & 5.5 & 2352 & 121 & 2.3 \\
\hline $\mathrm{Cl}^{-}$ & 15.1 & 123 & 41.7 & 0.6 & 7.76 & 398 & 69.7 & 1.4 & 6.24 & 73.7 & 18.1 & 0.7 \\
\hline $\mathrm{Na}^{+}$ & 1.3 & 450 & 53.5 & 1.7 & 13.1 & 259 & $\overline{98.4}$ & 0.9 & 7.7 & 1861 & 103 & 2.5 \\
\hline $\mathrm{K}^{+}$ & 4.1 & 13.5 & 7.4 & 0.3 & 3.8 & 96.8 & 19.2 & 1.4 & 0.05 & 49.0 & 6.1 & 1.1 \\
\hline $\mathrm{Ca}^{2+}$ & 84.8 & 277 & 148 & 0.3 & 69.1 & 511 & 153 & 0.7 & 15.9 & 390 & 83.3 & 0.6 \\
\hline $\mathrm{Mg}^{2+}$ & 15.6 & 35.0 & 27.7 & 0.2 & 13.8 & 115 & 32.0 & 0.7 & 5.4 & 71.4 & 17.2 & 0.4 \\
\hline $\mathrm{NO}_{3}{ }^{-}$ & 7.7 & 525 & 93.1 & 1.0 & $\mathrm{ND}$ & 838 & 169 & 1.2 & $\mathrm{ND}$ & 3044 & 109 & 4.0 \\
\hline $\mathrm{NO}_{2}{ }^{\circ}$ & $\overline{N D}$ & 0.26 & 0.01 & 3.9 & $\overline{N D}$ & 0.6 & 0.04 & 3.4 & $\overline{N D}$ & 62.5 & 2.3 & 4.6 \\
\hline $\mathrm{NH}_{4}^{+}$ & ND & 3.45 & 0.18 & 2.9 & ND & 18.3 & 2.02 & 2.4 & ND & 20.5 & 0.41 & $\overline{5.4}$ \\
\hline TOC & 0.89 & 6.10 & 2.31 & 0.5 & 0.71 & 10.7 & 2.20 & 1.2 & 1.20 & 11.9 & 3.13 & 0.7 \\
\hline
\end{tabular}

At the source areas, PCE was present at a range of $99 \%$ of the molar fraction. Chloroethenes concentrations (PCE, TCE) in the plume show considerable spatial and temporal variability. Concentrations increased during the water table recovery periods because of the dissolution of chloroethenes from pools or trails of residual phase in the water saturated zone. Chloroethenes from marlstones, where they remain pooled at the fractures, can contribute to increasing levels in a percentage that ranges from 1 to 10 , depending on the punctual content at the two geological formations. The variability is also due to the influent character of the River Ges, which produces a dilution of contaminants. Concentrations of PCE, TCE and CDCE in the plume vary throughout the year, ranging from $0.3 \mu \mathrm{g} / \mathrm{L}$ of PCE, 0.11 of TCE and 0.1 of $\mathrm{CDCE}$ at periods in which rainfall infiltration and recharge from the river occur, to $950 \mu \mathrm{g} / \mathrm{L}$ of PCE, 410 of TCE and 940 of $\mathrm{cDCE}$ when only recharge from the river takes place.

The applied remediation system (pump and treat) led to an increase in flow velocity, which favored a greater DNAPL dissolution rate and consequently a greater bioavailability.

During our studies, degradation of PCE has been detected in some observation wells (piezometers). Vinyl chloride (VC), which is practically absent in the source areas, has been appearing at the plume in some points.

The particular spatial distribution of anaerobic microorganisms consortia detected during our tests, the prevalence of reducing conditions, the progressive decrease in PCE along the plume together with the increase in CDCE and VC as well as the progressively less negative $\delta^{13} \mathrm{C}$ values [41] are signs of the 
biodegradation of chloroethenes in reducing conditions (reductive dehalogenation).

A competition for electrons between chloroethenes and other electron acceptors with a greater reduction potential such as nitrates or sulphates has been detected.

A high nitrate background characterizes the regional hydrochemical context, because of the practice of widespreading pig manure in the catchment basin. The spreading usually takes place on December-January and October-November, as recommended by the Catalan Department of Agriculture; this fact explains the presence of denitrifying microorganisms. Because of electron competition between dehalogenating microorganisms and those responsible for denitrification, the rate of chloroethenes biodegradation assessed in the field was not high.

A continuous input of sulphates has also been observed. This input comes from oxidation of disseminated pyrites contained in the fractured marlstones.

\section{Site B}

The area is located at a small sedimentary basin, a tectonic depression filled with Plio-Quaternary detritic materials, originating from the dismantling of the adjacent reliefs. The thicknesses of this Quaternary formation do not exceed $14 \mathrm{~m}$ in the study zone, although in some boreholes drilled by the Spanish Geological and Mining Institute (IGME) values as high as $100 \mathrm{~m}$ have been recorded. Beds of heterometric gravels and sands with medium to coarse granulometry predominate; these are paleochannels of alluvial origin with a silt-clay matrix, alternating with silt and clay, together with some levels of caliche crust, which is a hardened calcium carbonate.

Natural soils are related with river sediments. The deposits were formed under flow conditions that were almost torrential; hence the predominance of gravels and rounded stones attaining considerable size sometimes. The soil is about $50 \mathrm{~cm}$ thick, or even less. The soil profile is differentiated from the geological materials by the passage into gravels. The presence of calcium carbonate is the general rule. The organic matter content is around $1 \%$.

The area receives approximately $517 \mathrm{~mm}$ rainfall per year, with a total maximum daily of $113 \mathrm{~mm}$ in spring or autumn. During the dry period, rainfall does not cover evapotranspiration losses. The depth to the water table ranges from 7 to $8 \mathrm{~m}$.

The alluvial fan deposits constitute a surficial aquifer formation, consisting of gravels and sands of different granulometry, structured in paleochannels, showing alternating silts and clays, not more than $14 \mathrm{~m}$ thick. 
It is a free aquifer which is recharged throughout the area where its materials reach the surface; for this reason the aquifer shows a high vulnerability to pollution episodes. It discharges to the sea along the coastline and through pumping wells distributed along its entire surface.

There are many companies devoted to the manufacturing and manipulation of all kinds of oil-derivative products, many of which come into the category of light non-aqueous phase liquids (LNAPLs), e.g. petrol, and DNAPLs (e.g. chlorinated solvents), that began their activity in the 1960's. Various groundwater contaminant episodes with different types of hydrocarbons, such as naphthalene, have been detected since the 1970s.

The pollution episode studied was detected in 1996 at one industrial plant inside a chemical complex, but it is not known when the episode started. Two chlorinated methanes were associated with the episode: carbon tetrachloride (CT) and chloroform (CF) stored independently at the site. Repeated leaks and spills were responsible for it. After obtaining evidence of the existence of $\mathrm{CT}$ and $\mathrm{CF}$ in groundwaters and due to the sale of the plant's facilities to another chemical company, characterization studies, monitoring, and control of the groundwater quality began in 1996. Concentrations recorded at the groundwater, ranged between 15 and $22,600 . \mu \mathrm{g} / \mathrm{L}$ for $\mathrm{CF}$ and between the detection limit and $86 \mu \mathrm{g} / \mathrm{L}$ for CT.

Since 1997, remedial works have been carried out consisting of initial gas extraction and injection of water vapor followed by a pump \& treat (airstripping) system. Despite the substantial reduction observed in groundwater concentrations between 1997 and 2006, pollution continues to be above acceptable values.

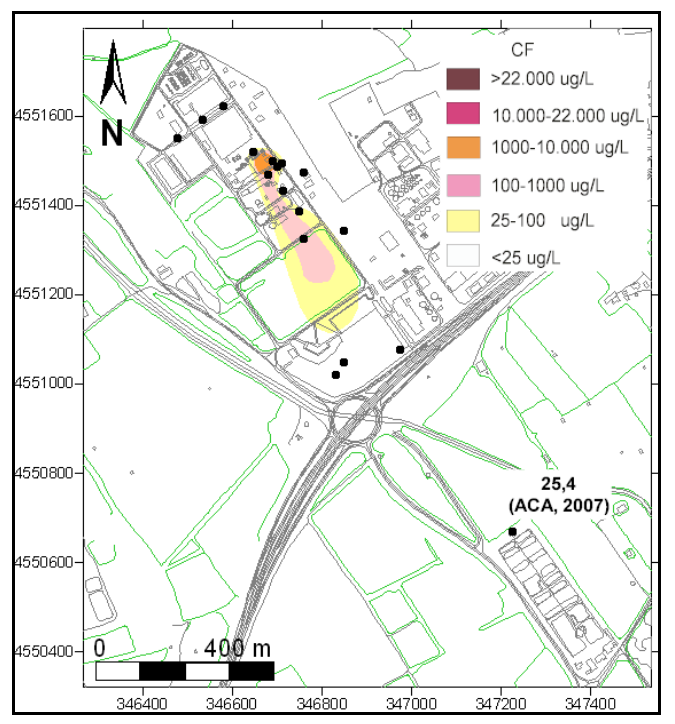

Figure 8. CF plume emerging from the source area in October 2008. 
In April 2008, our team carried out a transect, consisting on two boreholes located $220 \mathrm{~m}$ downstream of the pollution source. The distance from one borehole to the other was $2.5 \mathrm{~m}$ The recovery of the continuous cores made possible to carry out a soil and subsoil sampling at centimetric scale (65 and 71 samples were obtained respectively).

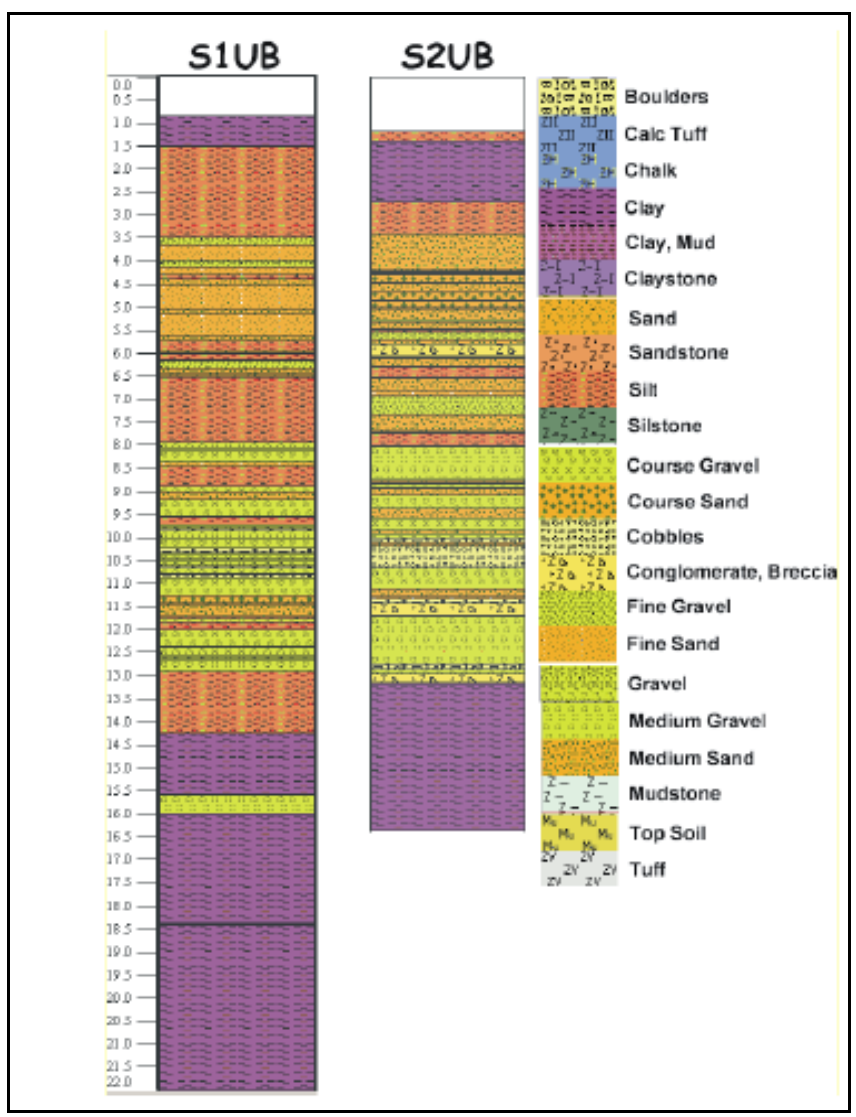

Figure 9. Geological characterization of the cores.

Table 3. Physical-chemical parameters determined at core samples.

\begin{tabular}{|c|c|c|c|c|c|c|c|c|}
\hline S1UB & $\mathrm{pH}$ & $\begin{array}{c}\mathrm{CE} \\
(\mu \mathrm{S} / \mathrm{cm})\end{array}$ & Eh (mV) & $\mathrm{C}_{\mathrm{T}}(\mathrm{ppm})$ & $\mathrm{N}_{\mathrm{T}}(\mathrm{ppm})$ & $\mathrm{C}_{\text {oro }}(\mathrm{ppm})$ & $\mathrm{Cd}(\mathrm{ppm})$ & $\mathrm{Cu}(\mathrm{ppm})$ \\
\hline Max & 8.89 & 523 & 155 & 88.51 & 0.97 & 0.97 & 13.33 & 75 \\
\hline Min & 8.07 & 107.9 & 128 & 0.46 & 0.09 & 0.14 & 3.33 & 5 \\
\hline $\begin{array}{l}\text { Geom } \\
\text { mean }\end{array}$ & 8.65 & 183.51 & 138.76 & 44.8 & 0.22 & 0.32 & 7.18 & 12.22 \\
\hline Std Dev & 0.17 & 76.67 & 8.53 & 21.18 & 0.12 & 0.17 & 2.76 & 11.29 \\
\hline S2UB & $\mathrm{pH}$ & $\begin{array}{c}C E \\
(\mu \mathrm{S} / \mathrm{cm})\end{array}$ & Eh $(\mathrm{mV})$ & CT (ppm) & NT (ppm) & $\begin{array}{l}\text { C org } \\
\text { (ppm) }\end{array}$ & $\mathrm{Cd}(\mathrm{ppm})$ & $\mathrm{Cu}(\mathrm{ppm})$ \\
\hline Max & 9.69 & 320 & & 91.98 & 0.45 & 0.59 & & \\
\hline Min & 8.31 & 15.8 & & 0.8 & 0.05 & 0.11 & & \\
\hline $\begin{array}{l}\text { Geom } \\
\text { mean }\end{array}$ & 9.3 & 142.02 & & 46.29 & 0.19 & 0.28 & & \\
\hline Std Dev & 0.26 & 48.64 & & 21.56 & 0.07 & 0.11 & & \\
\hline
\end{tabular}


Table 4. Chlorinated solvents determined at core samples.

\begin{tabular}{|c|c|c|c|c|c|c|c|c|c|c|c|c|}
\hline \multirow[b]{3}{*}{$\begin{array}{c}\text { \% Samples } \\
>D L\end{array}$} & \multicolumn{12}{|c|}{ S1UB ( $\mu g / L)$} \\
\hline & \multicolumn{3}{|c|}{ Chloromethanes } & \multicolumn{4}{|c|}{ Chloroethanes } & \multicolumn{5}{|c|}{ Chloroethenes } \\
\hline & 33,3 & 81,0 & 41,3 & 25,4 & 15,9 & 17,5 & 33,3 & 42,9 & 17.5 & 6,3 & 22,2 & 20,6 \\
\hline VOCs & CT & CF & DCM & \begin{tabular}{|l|}
$1,1,1,2$ \\
PCA \\
\end{tabular} & \begin{tabular}{|c|}
$1,1,2,2$ \\
PCA \\
\end{tabular} & $\begin{array}{l}1,1,1 \\
\text { TCA }\end{array}$ & $\begin{array}{l}1,1,2 \\
\text { TCA }\end{array}$ & PCE & TCE & $\begin{array}{l}1,1 \\
\text { DCE }\end{array}$ & tDCE & $\mathrm{cDCE}$ \\
\hline Max & 563,56 & 1468,48 & 5730,38 & 131,57 & 2258,95 & 78,83 & 88,47 & 139,37 & 78,63 & 15,28 & 152,48 & 24,92 \\
\hline Min & 1,14 & 3,83 & 1,46 & 1,67 & 1,38 & 1,47 & 1,55 & 1,34 & 1,59 & 1,34 & 1,38 & 2,35 \\
\hline Geom. mean & 11,32 & 87,75 & 31,55 & 8,58 & 5,36 & 5,12 & 7,83 & 11,31 & 5,57 & 4,16 & 5,64 & 4,79 \\
\hline Std. Dev. & 113,27 & 250,64 & 1357,19 & 26,90 & 283,55 & 11,27 & 20,68 & 31,84 & 11,38 & 2,83 & 20,30 & 4,73 \\
\hline \multirow[t]{3}{*}{$\begin{array}{c}\text { Geom. mean + } \\
2 \text { Std. Dev. }\end{array}$} & 237,87 & 589,03 & 2745,92 & 62,39 & 592,46 & 27,65 & 49,30 & 75,19 & 28,34 & 9,82 & 46,24 & 14,24 \\
\hline & \multicolumn{12}{|c|}{ S2UB ( $\mu g / L)$} \\
\hline & \multicolumn{3}{|c|}{ Chloromethanes } & \multicolumn{4}{|c|}{ Chloroethanes } & \multicolumn{5}{|c|}{ Chloroethenes } \\
\hline $\begin{array}{c}\text { \% Samples } \\
>\mathrm{DL}\end{array}$ & 25,4 & 73,1 & 35,8 & 52,2 & 19,4 & 22,4 & 19,4 & 58,2 & 23,9 & 11.9 & 14,9 & 16,4 \\
\hline VOCs & CT & CF & $\mathrm{DCM}$ & $\begin{array}{c}1,1,1,2 \\
\text { PCA }\end{array}$ & $\begin{array}{c}1,1,2,2 \\
\text { PCA }\end{array}$ & $\begin{array}{l}1,1,1 \\
\text { TCA }\end{array}$ & $\begin{array}{l}1,1,2 \\
\text { TCA }\end{array}$ & PCE & TCE & $\begin{array}{c}1,1 \\
\text { DCE }\end{array}$ & tDCE & $\mathrm{cDCE}$ \\
\hline Max & 406,37 & 1286,29 & 9053,49 & 848,62 & 1782,11 & 88,91 & 214,93 & 996,73 & 101,06 & 50,61 & 72,12 & 52,35 \\
\hline Min & 1,38 & 2,69 & 2,57 & 2,80 & 2,19 & 2,26 & 2,61 & 1,63 & 2,51 & 2,30 & 2,32 & 2,25 \\
\hline Geom. mean & 7,32 & 122,69 & 23,51 & 19,96 & 6,19 & 6.29 & 6,98 & 20,25 & 6,76 & 5,14 & 5,58 & 5,19 \\
\hline Std. Dev. & 60,93 & 340,31 & 1606,50 & 164,58 & 238,60 & 18,22 & 32,30 & 193,27 & 17,80 & 8,12 & 12,13 & 7,20 \\
\hline $\begin{array}{c}\text { Geom. mean + } \\
2 \text { Std. Dev. }\end{array}$ & 129,18 & 803,30 & $3236,70 \mid$ & 349,11 & 483,40 & 42,73 & 71,58 & 406,78 & 42,35 & 21,39 & 29,84 & 19,60 \\
\hline
\end{tabular}

The maximum concentrations of PCE and TCE in the pore spaces correspond to the values recorded at the S2UB borehole, with 997 and 101 $\mathrm{mg} / \mathrm{L}$ respectively.

The average CT values were similar in both boreholes (S1UB and S2UB) - 11.32 and $7.32 \mathrm{mg} / \mathrm{L}$ respectively - while for $\mathrm{CF}$, they range between 88 and $123 \mathrm{mg} / \mathrm{L}$, corroborating the greater presence of circulating $\mathrm{CF}$ in dissolved forms, as a result of which an important part has penetrated the matrix by molecular diffusion. In the S1UB borehole, the maximum concentrations found for CT are between 8.2 and $8.64 \mathrm{~m}$, between 9.3 and $10.18 \mathrm{~m}$ and at $11.43 \mathrm{~m}$ deep, coinciding with silty levels. In fact, the presence of CT in silty levels is also shown in the aquitard, where maximums are detected at $15.63 \mathrm{~m}$ deep, between 17 and $17.94 \mathrm{~m}$ and at $18.4 \mathrm{~m}$ depth. Based on the sampling in the continuous cores from the boreholes it has been possible to make a semi-quantitative estimate of the diversity and degree of development of the different bacterial communities present in the non saturated zone and in the aquifer environments. Denaturing Gradient Gel Electrophoresis (DGGE) (see Fig. 10) [42], a molecular fingerprinting method that separates polymerase chain reaction (PCR)-generated DNA products was applied on selected samples. The polymerase chain reaction of environmental DNA can generate templates of differing DNA sequence that represent many of the dominant microbial organisms without single 
identifications. As observed for mineral elements, the two boreholes are also characterized by a large degree of spatial and vertical heterogeneities from the microbiological point of view (see Fig. 11).

The piezometric oscillation zone, the capillary zone and, above all, the zones with granulometric contrasts constitute ecotones in which levels with very fine granulometry show the greatest microbial diversity and often the more developed communities.

Chemicals biodegradation takes place not only at the scale of the dissolved fraction but also at the scale of the fraction retained in the finest, low hydraulic conductive, materials. The lower presence of CT in the S2UB borehole than in the S1UB can be attributed to a greater degradation rate due to a higher proportion of fine materials.

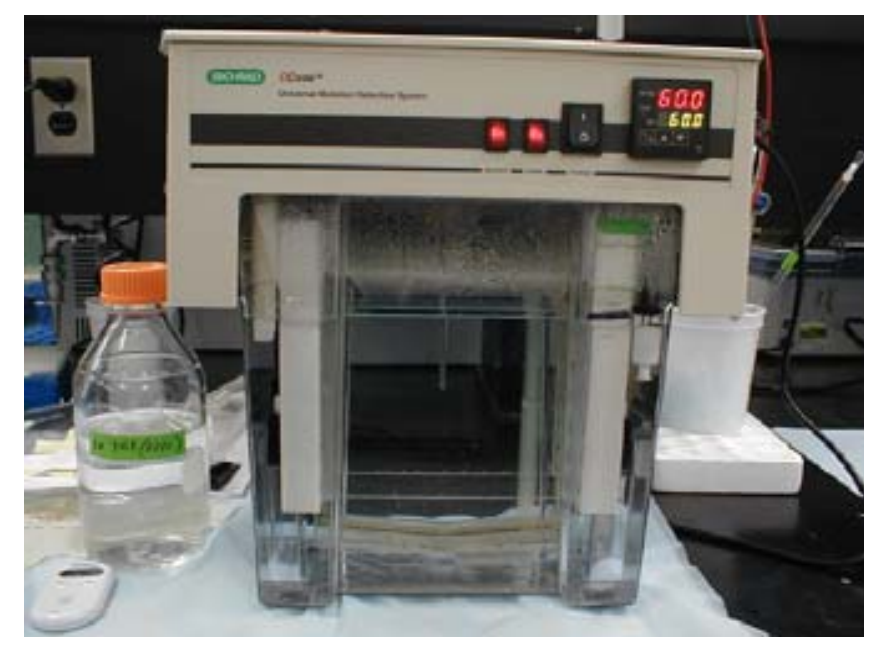

Figure 10. DGGE equipment.

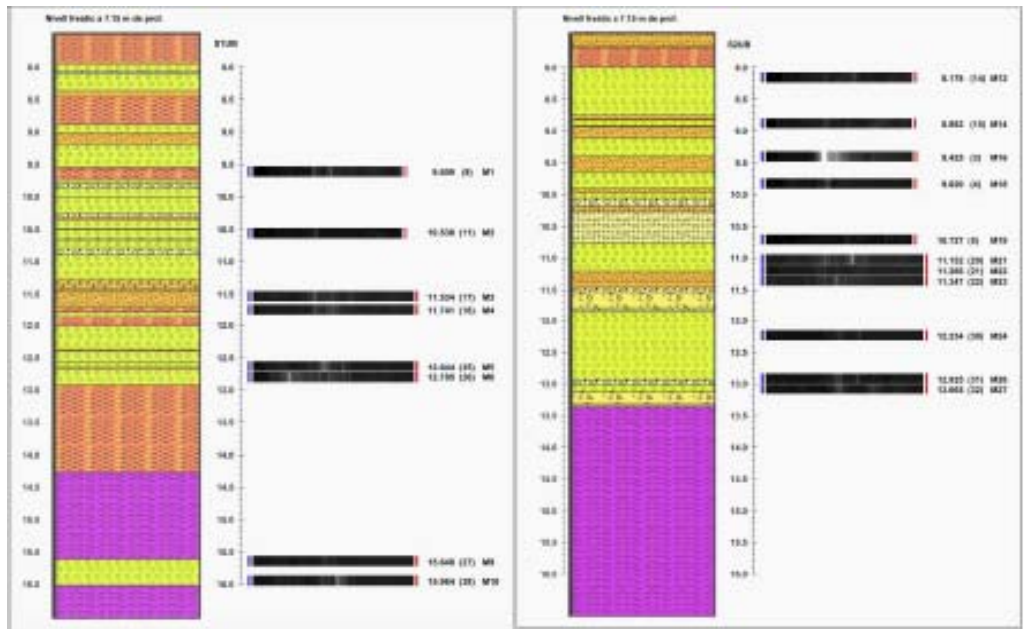

Figure 11. DGGE results in both sampled cores. 


\section{Remediation approach}

Knowledge of chlorinated solvents sources concentration and half-life may be valuable in approximating time to cleanup, long-term monitoring plans, or meeting regulatory criteria for the sites.

Biodegradation processes of the lightest chlorinated solvents affect not only at the dissolved fraction but also at the fractions retained in the very fine, less conductive, materials. The quantification of this pollutant mass is one of the aspects that has been taken into account when designing remedial strategies in order to forecast back-diffusion and additional costs.

The proposed in situ remediation approach for both sites includes a combination of biostimulated microbial bioremediation by addition of hydrogen and nutrients [41], and rhizodegradation in the riparian zone in conjunction with monitored natural attenuation and an apatite barrier placed by infiltration, instead of by injection well, that allows sequestration of other bioavailable contaminants, such as metals, at the non saturated/vadose as well as at the saturated zone. Infiltration of apatite solution, made with an amorphous form of a carbonated hydroxy-apatite that has random nanocrystals of apatite embedded in it, from the soil surface extends the amount of time available for apatite emplacement and immobilizes contaminants in the vadose zone that would not be treated by the proposed techniques. Aluminum, present at site A, is effectively stabilized by apatite treatment.

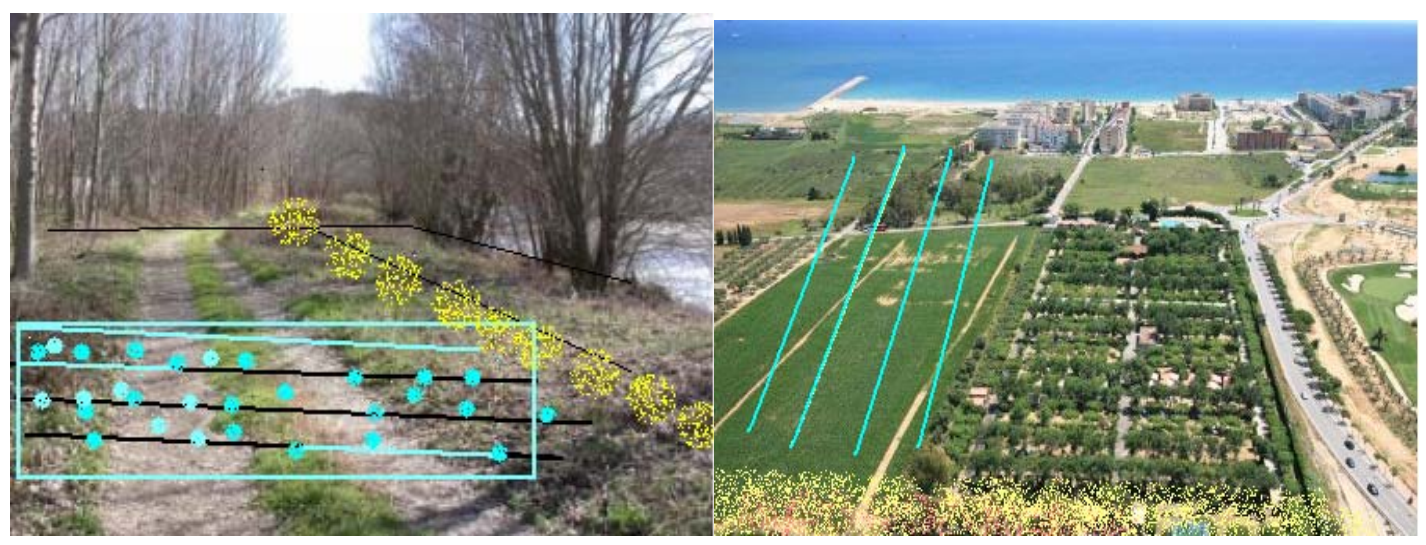

Figure 12. Proposed in situ remediation approach. (in blue groundwater flow; apatite infiltration is in yellow and precede phytoremediation areas).

\section{Phytoremediation: Rhizodegration}

Partial restoration of soil quality with organic matter amendments is a prerequisite for successful phytoremediation. Such amendments are part of the strategies for diminishing the stresses that limit plant growth in the field. 
Important considerations in plant species selection for rhizophytoremediation have included the following items: climatological requirements, tolerance levels with respect to chemicals, tolerance to drought-prone or poorly-drained conditions, depth of the root zone, growth rate, transpiration rate or water use, sensitivity to a wide variety of stressors (salts, calcium carbonate, fire,...), root exudates (specially phenolic compounds), deciduous or evergreen (for trees), native or non-native, maintenance requirements, commercial availability, and ecological relevance.

The more suitable tree species, according to our applied selection criteria, are: hybrid poplar (Trichocarpa X deltoides), eastern cottonwood (Populus deltoides Bartram ex Marsh) and loblolly pine (Pinus taeda). Hybrid poplar is already present in Site A. No pollution has been detected at their leaves but no data is available about pollutants' root concentrations.

Seedling quality improvement for planting in dry sites must be achieved before remediation starts.

Herbaceous plants can be also used for ground cover and erosion control but mowing is necessary; Plantago lanceolata, a very widespread species at Mediterranean polluted sites, can be useful but because of its use in tisanes and other herbal remedies in some villages, attention must be paid to prevent people entering the treated area.

The positive effect of arbuscular mycorrhiza in plant growth and nutrition has been well documented, however the effect of the symbiosis in the up-take of pollutants can vary depending on the fungal isolate, the plant and the contaminant/s concerned.

In a previous published work we have studied the effect of Glomus intraradices (BEG72) on the growth of Plantago lanceolata [43]. We have also tested the good conditions for arbuscular mycorrhiza in the preexisted hybrid poplar field.

Engineering genes, such as haloalkane dehalogenase (DhlA) and haloacid dehalogenase (DhlB), from bacteria that have the ability to dehalogenate a range of halogenated compounds, into high biomass producing plants is a possibility that is reduced nowadays to the laboratory scale.

The number of trees to plant, plant density, or mixtures to be backfilled into the tree holes are important considerations when planning phytoremediation.

Soil nutrients must be supplemented with care; fertilizers applied in slow-release form are recommended. Phosphorous is likely to be the most limiting nutrient in calcareous soils [44]; in these soils, hybrid poplars may respond well to addition of chelated zinc.

Actions can be simplified when using trees that can stand for 20 or 25 years at the sites. 


\section{Conclusion}

Water chlorinated solvents biodegradation rate due to reductive dehalogenation determined in microcosm assays is consistent with the geochemistry of the sites.

A competition for electrons is established during degradation between chlorinated solvents, other organic pollutants, organic matter, and other electron acceptors that can be present at the media such as nitrates and sulphates.

Chlorinated solvents and other organic pollutants may also act as electron donors providing an energy source for certain microorganisms. With the continual exchange of electrons, redox chemistry is an important factor in chlorinated solvents biodegradation.

PCE, TCE, and CT generally require reducing conditions before they will transform to aliphatic compounds. Such conditions require the presence of enough organic substrate to reduce all of the oxygen (below $0,5 \mathrm{mg} / \mathrm{L}$ ), nitrate (below $1 \mathrm{mg} / \mathrm{L}$ ideally), iron, and sulfate ideally (below $20 \mathrm{mg} / \mathrm{L}$ ) before dechlorinating bacteria will successfully compete to reduce chlorinated aliphatic hydrocarbons.

In site A water nitrate concentration oscillates along the year between 5 and $200 \mathrm{mg} / \mathrm{L}$, although concentrations of up to $525 \mathrm{mg} / \mathrm{L}$ may be reached in some dug well. Indicating that in spring or in some autumns biodegradation is not feasible at some points.

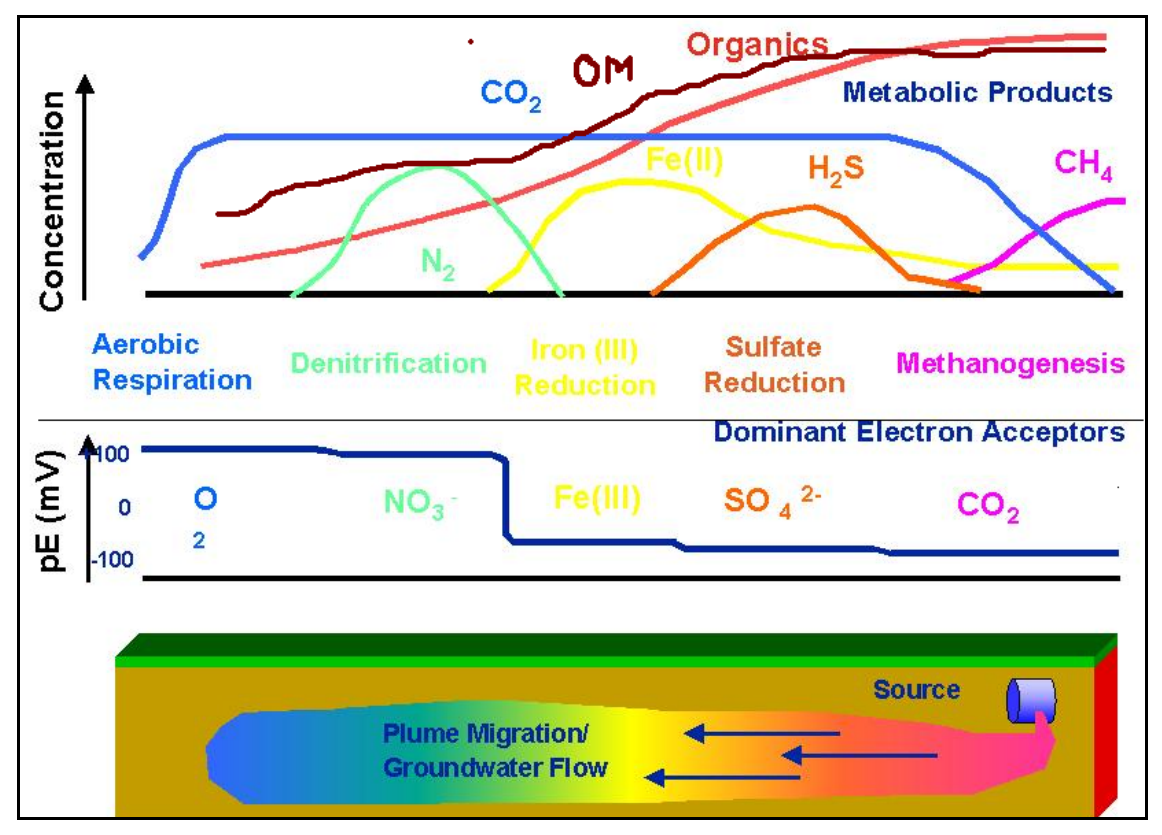

Figure 13. Organic matter and organics competion (a) and electron competition (b) schemes. 

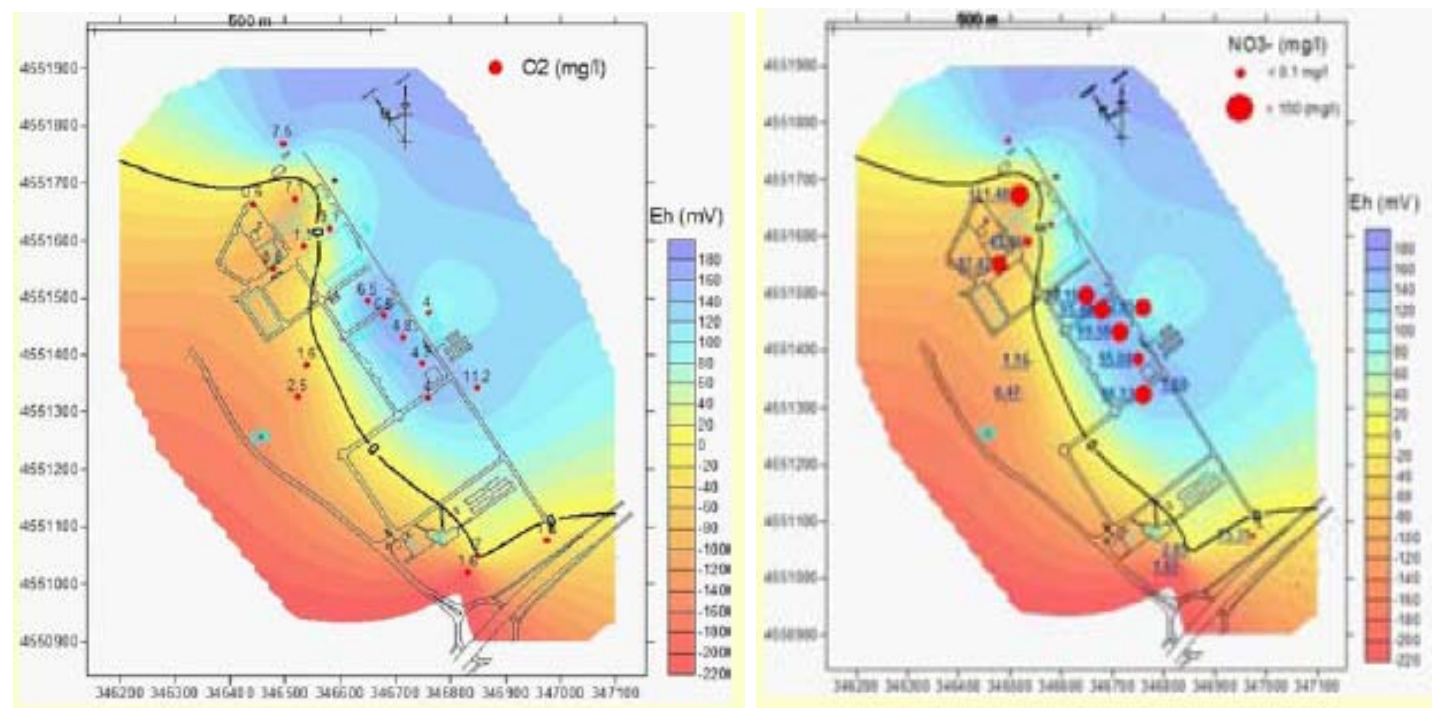

Figure 14. Oxygen and nitrates average concentrations at site B.

At site B (see Fig. 14) nitrate levels are lower, but still reach bad levels for reductive dechlorination. A previous water nitrate reduction treatment is needed in both sites.

The biodegradation of chlorinated solvents in soils, at low concentrations can be enhanced by using adapted mycorrhized trees producing phenolic exudates, but such biodegradation could be also affected by the ionic strength of nitrates or other salts when present.

Plants will also contribute to the remediation via chlorinated solvent uptake and dechlorination in plant systems.

\section{Acknowledgements}

The authors are grateful to Professor Beth Parker of the University of Guelph for her fruitful discussion. We would like to thank the Catalan Water Agency and staff members of some involved companies for the support and cooperation shown while carrying out the field work. We would also like to thank the members of the Department of Geochemistry, Petrology and Geological Prospecting of the University of Barcelona and, most particularly, the members of the Hydrogeology group. We would like to highlight the institutions financing the research carried out within the following research projects: CTM 2005-07824 and CGL 2008- 02164/BTE funded by the Spanish Ministry of Education and Science. 


\section{References}

1. Kueper, B.H., Wealthall, G.P., Smith, J.W.N., Leharne, S.A. \& Lerner, D.N. 2003, Environment Agency. R\&D Publication, 133, 63.

2. Pankow, J.F. and Cherry, J.A. 1996, Waterloo Press, 522.

3. Rivett, M.O., 1995, Ground Water, 33, 84.

4. Arciero, D., Vannelli, T., Logan, M., Hooper, A.B. 1989, Biochem. Biophys. Res. Commun, 159, 640.

5. Wackett, L.P., Brusseau, G.A., Householder, S.R., Hanson, R.S. 1989, Appl. Environ. Microbiol, 55, 2960.

6. Ewers, J., Freierschroder, D., Knackmuss, H.J. 1990, Arch. Microbiol, 154, 410.

7. Folsom, B.R., Chapman, P.J., Pritchard, P.H. 1990, App. Environ. Microbiol, $56,1279$.

8. Fox, B.G., Borneman, J.G., Wackett, L.P., Lipscomb, J.D. 1990, Biochemistry, $29,6419$.

9. Dabrock, B., Riedel, J., Bertram, J., Gottschalk, G. 1992, Arch. Microbiol, 158, 9.

10. Kim, Y., Ayoubi, P., Harker, A.R. 1996, App. Environ. Microbiol, 62, 3227.

11. Smith, K.S., Costello, A.M., Lidstrom, M.E. 1997, App. Environ. Microbiol, 63, 4617.

12. Ensley, B.D. 1991, Annu. Rev. Microbiol, 45, 283.

13. Hull, L.C., Sondrup, A.J. 2003. Idaho Nat. Engineer. and Environ. Lab. http://www.mathesongas.com/msds/MAT04310.pdf [December 2010].

14. Bouwer, E.J., McCarty, P. 1983, App. Environ. Microbiol, 45, 1286.

15. Bouwer, E.J., McCarty, P. 1983, App. Environ. Microbiol, 45, 1295.

16. Mayotte, T. J., Dybas, M. J., Criddle, C. S. 1996, Ground Water, 34, 2, 358.

17. He, J., Sung, Y., Krajmalnik-Brown, R., Ritalahti, K.M., Löffler, F.E. 2005, Environ. Microbiol, 7, 1442.

18. Orchard, B.J., Doucette, W.J., Chard, J.K., Bugbee, B. 2000, Environ. Toxicol. Chem, 19, 895.

19. Shang, T.Q., Newman, L.A., Gordon, M.P. 2003, In Phytoremediation: transformation and control of contaminants. McCutcheon, S.C., and Schnoor, J.L. (eds). pp. 529-560.

20. Anderson, T.A., Guthrie, E.A., Walton, B.T. 1993, Environ. Sci. Technol, 27, 2630.

21. Schnabel, W.E., Dietz, A.C., Burken, J.G., Schnoor, J.L., Alvarez, P.J. 1997, Water Research, 31, 816.

22. Burken, J.G., Schnoor, J.L. 1998, Environ. Sci. Technol, 32, 3379.

23. Wang, X, Dossett, M. P., Gordon, M. P., Strand, S.E. 2004, Sci. Technol, 38,5744 .

24. Newman, L.A., Wang, X., Muiznieks, I.A., Ekuan, G., Ruszaj, M., Cortellucci, R., Domroes, D., Karscig, G., Newman, T., Crampton, R.S:, Hashmonay, R.A., Yost, M.G., Heilman, P. E., Duffy, J., Gordon, M.P. and Strand, S.E. 1999, Environ. Sci. Technol, 33, 2257.

25. Stanhope A, Berry CJ, Brigmon RL. 2008, Int. J. Phytoremediation, 10, 529.

26. Nevius, B.A., Bagwell, C.E., Brigmon, R.L. 2004, J. South Carolina Acad. Sci, 2,25 . 
27. Pilon-Smits, E. 2005, Annu. Rev. Plant Biol, 56, 15.

28. Snyder, K.A., Williams, D.G. 2000, Agricultural and Forest Meteorology, $105,227$.

29. Newman, L.A., Strand, S.E., Choe, N., Duffy, J., Ekuan, G., Ruszaj, M., Shurtleff, B.B., Wilmoth, J., Heilman, P., and Gordon, M.P. 1997, Environ. Sci. Technol, 31, 1062.

30. Ryu, S.B., Davis, L.C., Dana, J., Erickson, L.E. 1996, HSRC/WERC J. Conf. on the Environ.

31. Box, S. 2002, Thesis. Graduate Faculty of the University of Georgia.

32. Gordon, M., Choe, N., Duffy, J., Ekuan, G., Heilman, P., Muiznieks, I., Newman, L., Raszaj, M., Shurtleff, B., Strand, S., and Wilmoth, J. 1997, In: Phytoremediation of Soil and Water Contaminants. Am. Chem. Soc, Washington, DC.

33. Brigmon, R.L., Anderson, T.A., Fliermans, C.B. 1999, Int. J. Phytoremediation, $1,241$.

34. Van Aken, B., Yoon, J.M., Schnoor, J.L. 2004, App. Environ. Microbiol, 70, 508.

35. Van Aken, B., Peres, C.M., Doty, S.L., Yoon, J.M., and Schnoor, J.L. 2004, Int. J. Syst. Evol. Microbiol, 54, 1191.

36. Isebrands, J.G., Karnosky, D.F. 2001, In Poplar Culture in North America Part A. Dickmann, D.I., Isebrands, J.G., Eckenwalder, J.E., and Richardson, J. (eds). Canada: NRC Research Press, pp. 207-218.

37. Brigmon, R.L., Saunders, F.M., Altman, D., Wilde, E., Berry, C.J., Franck, M., McKinsey, P., Burdick, S., Loeffler, F. and Harris, S. 2003, WSRC-TR-200200557.

38. Schnoor, J.L. 1997, In Advances in Innovative Groundwater Rem. Technol.

39. Chappell, J. 1997, Status report. USEPA Technol. Innov. Office.

40. Cunningham, S.D., Anderson, T.A., Schwab, A.P., Hsu, T.C. 1996, Advances in Agronomy, 56, 55.

41. Puigserver, D. 2010, Doctoral Thesis. University of Barcelona.

42. Muyzer, G, Smalla, K. 1998, Antonie van Leeuwenhoek, 73, 127.

43. Estaún, V., Cortés, A., Velianos, K., Camprubí, A., Calvet, C. 2010, SJAR, 8(S1), S109.

44. Cortés, A.; Vergara, P.; Realp, E.; Domenech, J. A. 2005, Consoil Proceedings, 3, 1894. 


\title{
11. Crystallization and resolution of the lipoxygenase of Pseudomonas aeruginosa $42 \mathrm{~A} 2$ and phylogenetic study of the subfamilies of the lipoxygenases
}

\author{
Albert Garreta ${ }^{1,4}$, Xavier Carpena ${ }^{2}$, Montse Busquets ${ }^{3}$ \\ M. Carme Fusté', Ignacio Fita ${ }^{4}$ and Àngels Manresa' \\ 'Departament de Microbiologia i Parasitologia Sanitàries, Universitat de Barcelona, Barcelona, Spain \\ ${ }^{2}$ Institute for Research in Biomedicine (IRB-Barcelona) and Institut Químic de Sarrià, IQS-URL \\ Barcelona, Spain; ${ }^{3}$ Departament de Bioquimica i Biologia Molecular. Universitat de Barcelona \\ Barcelona. Spain; ${ }^{4}$ Institute of Research in Biomedicine (IRB-Barcelona) and Institut de Biologia \\ Molecular (IBMB-CSIC), Parc Científic, Barcelona, Spain
}

\begin{abstract}
Lipoxygenases are non-heme iron enzymes essential in eukaryotes, where they catalyze the formation of the fatty acid hydroperoxides that are required by a large diversity of biological and pathological processes. In prokaryotes, most of them totally lacking in polyunsaturated fatty acids, the possible biological roles of lipoxygenases have remained obscure. The crystal structure, at $1.75 \AA$ resolution, of a lipoxygenase from Pseudomonas aeruginosa (Pa_LOX), the first from a prokaryote, shows significant major differences with respect to available structures from eukaryotic LOX enzymes. An insertion in the catalytic domain of a pair of long antiparallel $\alpha$-helices contributes to forming an enlarged binding
\end{abstract}


pocket containing a bound phospholipid: a phosphatidylethanolamine with well defined chains of 18 and 14 carbons in length. Five carbons of the 18-carbon chain approach the active center iron in a manner suggesting that the catalytic pentadienyl radical intermediate is stabilized by $\pi$-cation (ferrous) interaction and stacking on two His ligands to iron. Overall, the specificity of $\mathrm{Pa} \_\mathrm{LOX}$ for phospholipids also suggests that the role of lipoxygenases in prokaryotes be reformulated.

\section{Introduction}

The oxygenation of polyunsaturated fatty acids mediated by lipoxygenase (LOX) enzymes is the initial step in the formation of a diverse array of lipid mediators [1-4]. LOX products in animals commonly act by signaling through G-protein-coupled receptors. Different eicosanoids appear to be involved in cell homeostasis, proliferation and differentiation, as well as in pathophysiological processes (inflammation and cancer). Generally, plant LOX pathway products are involved in the resistance to environmental stress and defense against microbe and herbivore attack. Some of the volatile products, like jasmonic acid and short-chain aldehydes, have a function in

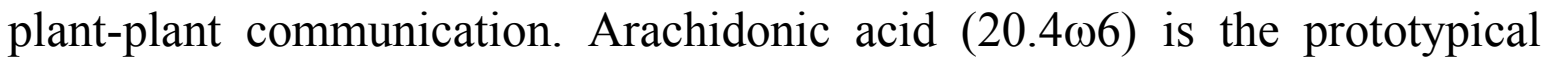
substrate in animals; and linoleic or linolenic acids $(18.2 \omega 6,18.3 \omega 3)$, in plants. The initial products are fatty acid peroxides that give rise to local hormones classified collectively as "eicosanoids" (20-carbon lipids) or by the more general term "oxylipins" [5].

LOX described in plants and animals are non-heme iron enzymes consisting of a single polypeptide chain that is folded into two domains, the mostly $\alpha$-helical catalytic domain and the $\mathrm{N}$-terminal $\beta$ barrel domain that is involved in membrane binding [6-12]. In humans there are five LOX enzymes that act on arachidonic acid and catalyze four distinct oxygenations $(5 \mathrm{~S}, 12 \mathrm{R}, 12 \mathrm{~S}$ or $15 \mathrm{~S})$, the products being individual fatty acid hydroperoxides [1]. There are another five distinct reactions $(5 \mathrm{R}, 8 \mathrm{R}, 8 \mathrm{~S}, 9 \mathrm{~S}$ and 11R) in other animal species [13-15]. A large number of plant LOX enzymes use linoleic or linolenic acid as substrate, many of which are quite specific to these fatty acids $[5,16]$.

The primary structure of LOX genes is highly diverse, with only three of the five amino acids that serve as iron ligands totally conserved within the LOX gene family. There is considerable variation in the polypeptide chain, perhaps not surprisingly in view of the wide spectrum of specific oxygenations that can be catalyzed. Even so, as each specific oxygenation mediated by a LOX involves a 1,4-Z,Z- pentadiene group (a pair of conjugated double bonds) on the fatty acid substrate and its coupling with $\mathrm{O}_{2}$, there may be a common mechanism underlying the different LOX reaction specificities. 


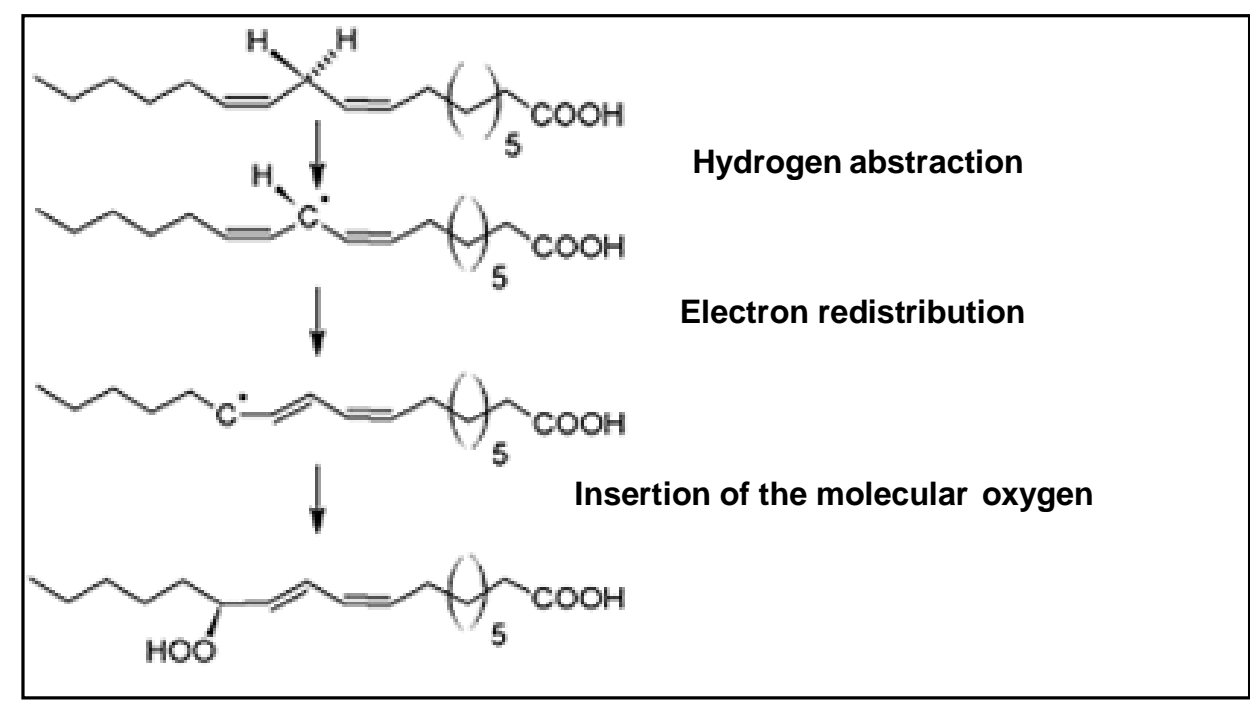

Figure 1. The three consecutive steps of the mechanism of action of lipoxygenases.

The mechanism of LOX (Fig. 1) consists of three consecutive steps. The first is the stereoselective abstraction of one hydrogen of the group 1,4-cis,cis-pentadiene to form a pentadienyl radical. The second step is the electron redistribution modifying the cis-cis pentadiene group to a cis-trans pentadiene group. The third and last step consists of the insertion of the molecular oxygen and the consequent reduction of the hydroperoxide radical.

One of the most interesting aspects is that molecular oxygen is not covalently bound in the enzyme prior to reaction with the substrate. Since polyunsaturated fatty acids, which are the substrate of the reaction, are achiral, a question arises: how do the LOX oxygenate the substrate with such regio- and stereo-selectivity? When trying to describe possible mechanisms for the control of oxygenation in the enzymatic reaction, there are four possible explanations relevant to the LOX [18].

\section{Steric shielding}

The access of the oxygen to the reactive carbon centers of the pentadienyl radical is not free. The structure of the protein restricts the access to one of these reactive carbons.

\section{Oxygen channeling}

The molecular oxygen is directed to the desired site by a channel that starts at the surface of the protein and finishes close to the activated fatty acid substrate, thus controlling position and stereospecificity. 


\section{Selective peroxyl radical trapping}

This hypothesis entails that the oxygenation specificity of the LOX depends on the trapping of the peroxyl radical resulting in the final product. The molecular oxygen reacts with many positions of the pentadiene of the activated fatty acid, but a strategically placed hydrogen donor traps only the desired product by reduction to hydroperoxide.

\section{Radical location}

During oxidation of fatty acids, the pentadienyl radical with the unpaired electron delocalized over all 5 carbons adopts a planar structure. This permits the oxygen to react in multiple positions and in $\mathrm{R}$ or $\mathrm{S}$ configuration. The unpaired electron could be localized if a force is applied and the radical is twisted, with this location of the electron giving way to selective reaction with $\mathrm{O}_{2}$ at this position.

\section{Molecular structure of lipoxygenases}

\subsection{General structure}

Despite more than 60 years of study of lipoxygenases, it was not until 1993 that the first lipoxygenase was crystallized and diffracted [6], soy bean "arachidonic acid 15-lipoxygenase". Later, in 1996, the structure was refined up to $1.4 \AA$ [4]. More recently, other soy bean lipoxygenases have been crystallized, such as 3-lipoxygenase and various complexes. The first crystal structure of an animal lipoxygenase was published in 1997, the rabbit reticulocyte lipoxygenase, a 15-lipoxygenase [8]. The most recent lipoxygenase crystallized was the lipoxygenase of Plexaura homomolla, a coral, in 2005 [12].

Lipoxygenases have a single polypeptide chain (monomeric proteins) with a molecular mass of approximately $75-80 \mathrm{kDa}$ for animal lipoxygenases between 660 and 680 residues [22], and 94-104 $\mathrm{kDa}$ for vegetable lipoxygenases [1] between 839 and 923 residues [23]. Plant and animal LOXs are composed of two domains: a larger $\alpha$-helical catalytic domain and a smaller $N$-terminal $\beta$-barrel domain (Figs. 2 and 3). The fold of the $\mathrm{N}$-terminal $\beta$-barrel domain resembles that of a "C2" module, a kind of calcium-dependent membrane-targeting domain found in many protein kinases and phospholipases. Similarly, the $\beta$-barrel domains of LOXs mediate membrane binding, such as the C2-like membrane-binding domain of gangrene $\alpha$-toxin, a domain with a similar topology to the $\mathrm{N}$-terminal domain of plant and animal LOXs [19]. 


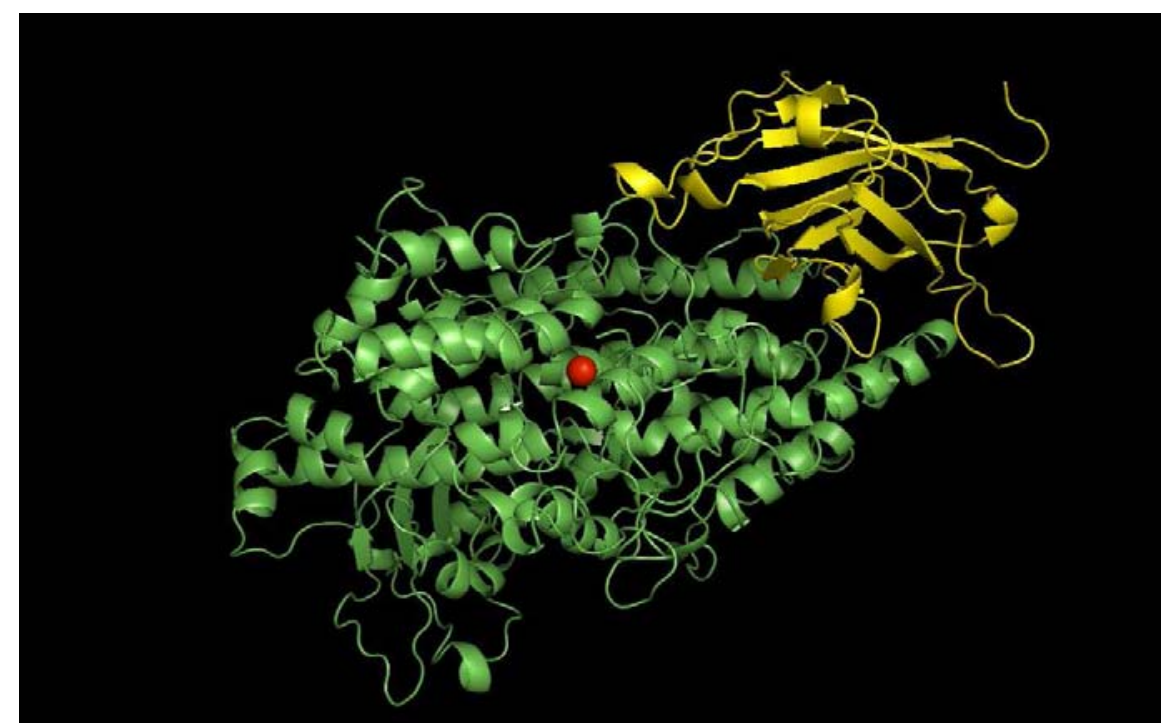

Figure 2. Structure of the soybean lipoxigenaseL-1(Glycine max) from $1.4 \AA$ resolution (PDB code 1YGE). Yellow: N-terminal domain Green: Carboxy-terminal catalytic domain. The red sphere represents an iron atom coordinated in the active site of the protein.

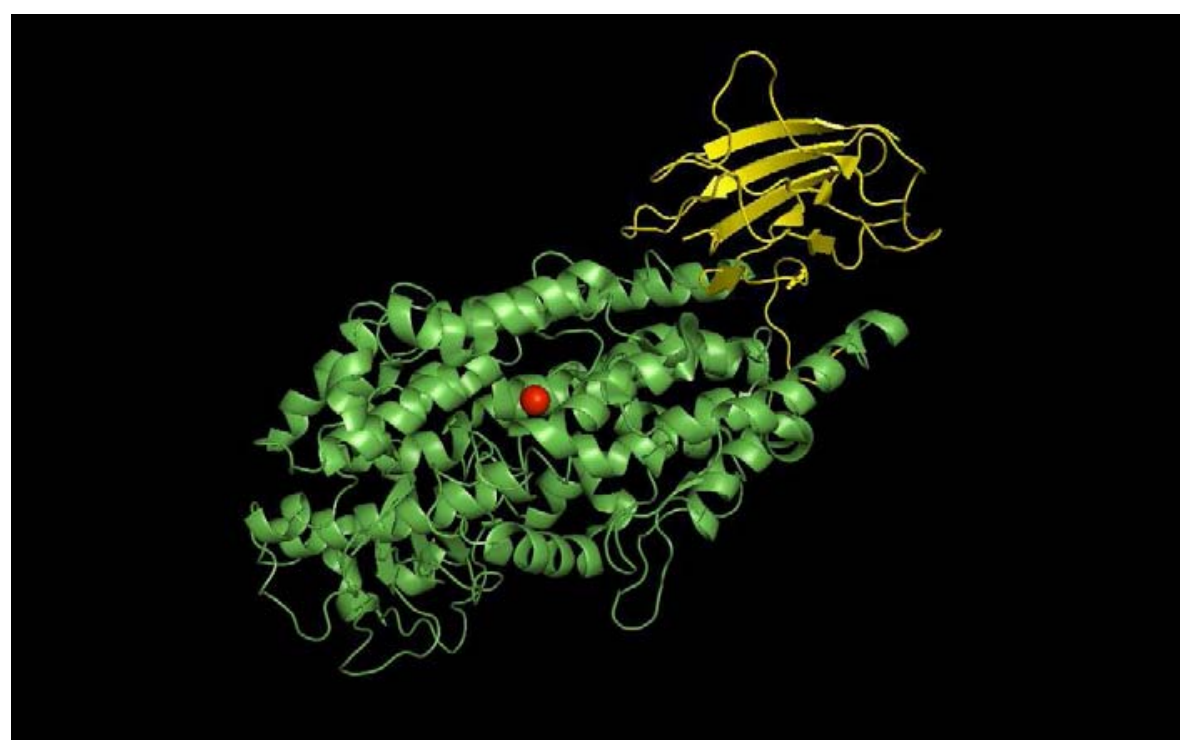

Figure 3. Structure of 8R-lipoxigenase (Plexaura homomolla) from 3.2 Á resolution (PDB code 2FNQ). Yellow: N-terminal domain Green: Carboxy-terminal catalytic domain. The red sphere represents an atom of iron coordinated in the active site of the protein.

The larger $\alpha$-helical catalytic domain contains the non-heme iron positioned in a cavity that accepts the hydrocarbon substrate [8]. This domain consists of $23 \alpha$-helix and 2 anti-parallel $\beta$-sheets for the lipoxygenase of Glycine max [20], and $18 \alpha$-helix and one anti-parallel $\beta$-sheet in the case of animal lipoxygenases [21]. 
Iron is bound to protein by five amino acids and, in some cases, one hydroxyl group. For vegetable lipoxygenases, these five amino acids are three histidines, one asparagine and the carboxyl group of the terminal isoleucine. In the case of soy bean (Glycine max) lipoxygenes, these five amino acids are histidines 499 and 504 of the helix $\alpha 9$, histidine 690 and asparagine 694 of the helix $\alpha 18$ and isoleucine 839 of the extreme carboxy terminal (Fig. 4) [24].

In the case of mammals, lipoxygenase has its iron coordinated by four histidines and, again, the terminal isoleucine [25]. These amino acids, together with a hydroxyl group, form an octahedral arrangement called a slanted bipyramid [1].

To examine the second coordination sphere of iron, soy lipoxygenase was studied. The second coordination sphere consists of two amino acids, glycine 495 and glycine 697, which form a network of hydrogen bonds between the cavity of the substrate and the first coordination sphere (asparagine 694). Some studies reflect how mutants of soy bean lipoxygenase (Fig. 5), like Q495E, Q495A, Q697E or Q697N, affect enzyme activity because the network of hydrogen bonds is modified, affecting the cleavage of the $\mathrm{C}-\mathrm{H}$ bond (Fig. 5 b, c, d, and e). An efficient break of this bond is achieved through the correct position of the substrate with steric interactions with the side chain of Gln495 (Fig. 5a). The network of hydrogen bonds defined by Gln495, Gln697 and Asn694 (Fig. 5a) will be responsible for the reorganization of hydrogen bonds after the substrate binding [26].

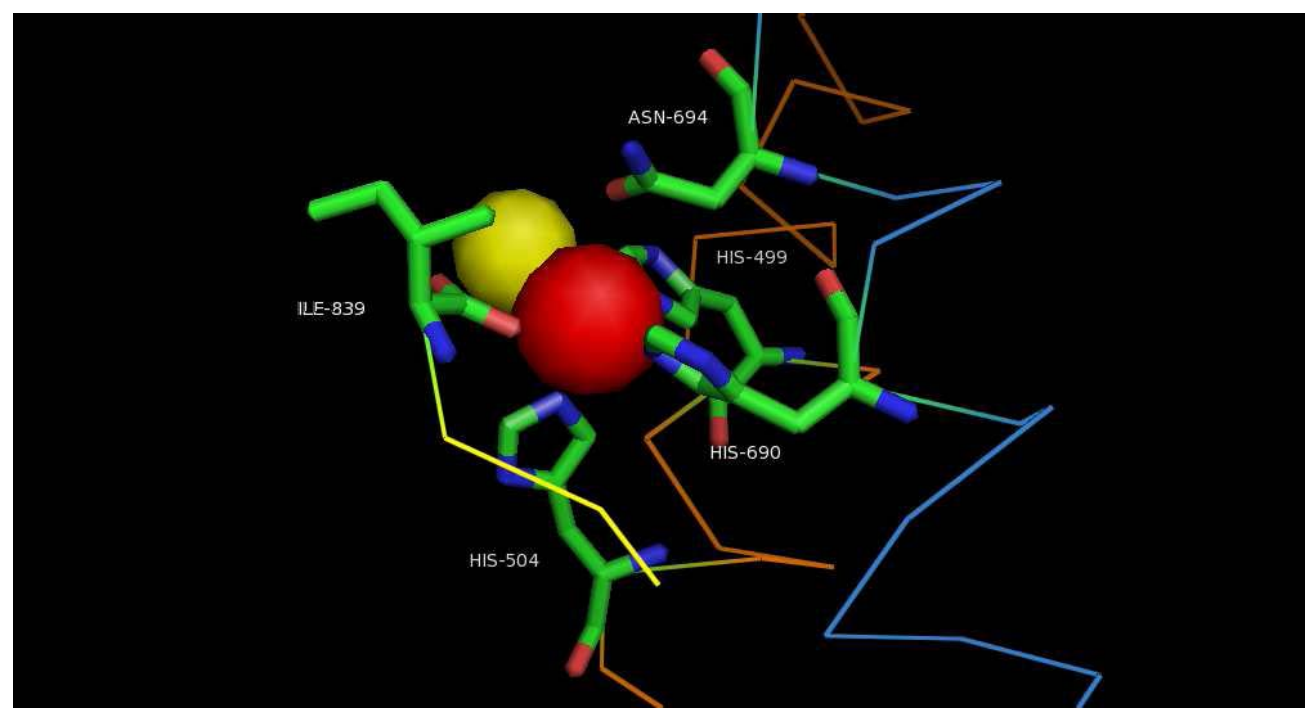

Figure 4. Coordination sphere of the iron atom for lipoxygenase L-1 of soybeans. (Glycine max) from $1.4 \AA$ resolution (PDB code 1YGE). In yellow the hydroxyl group, in red the iron atom. In orange, helix 9, in blue helix 21 and in yellow, part of the chain of residues that located appropriately the terminal isoleucine. 


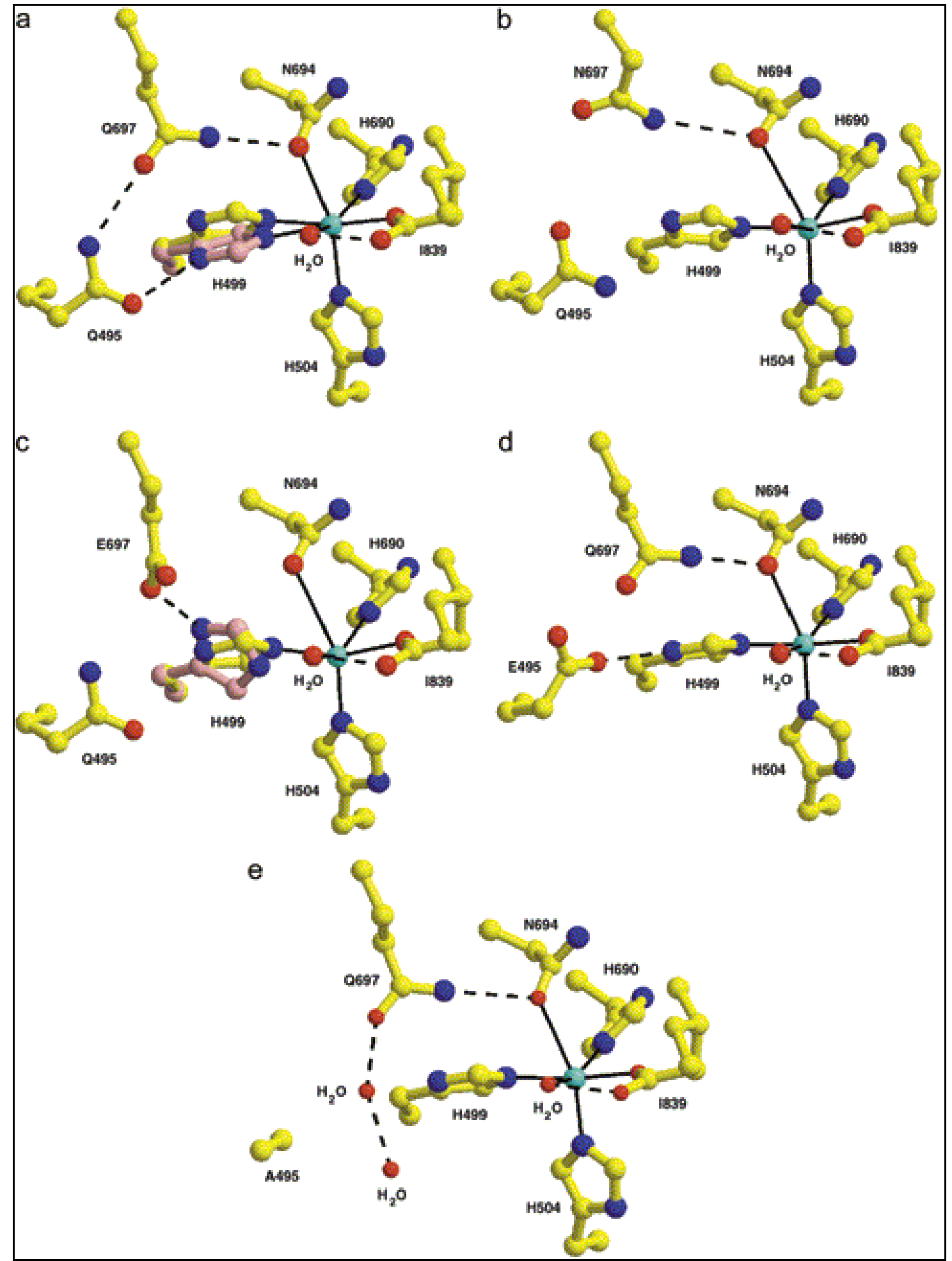

Figure 5. Geometry of the coordination of the $\mathrm{Fe}^{+2}$ of the soybean lipoxygenase-1 (R. Leung and D. Sloane) Native lipoxygenase (a) Q697N (b), Q697E (c), Q495E (d) and Q495A (e). The links between iron and the first coordination sphere are drawn as continuous lines and hydrogen interactions are drawn as dashed lines. The oxygen atoms are drawn in red, nitrogen in blue and carbon in yellow. The iron atom is colored in cyan. The alternative conformations of His 499 are shown in pink [26]. 
The core of the catalytic domain contains two long central helices (Figs. 2 and 3), adopting a conformation of helix $\pi$ for various residues. These sections contain four of the five helical iron lipoxygenase ligands (His 499, His 504, His 690, His 694).

\subsection{Cavities}

As soy bean (Glycine max) lipoxygenase is the most commonly studied of all lipoxygenases, the best option, in order to study this area of the protein, is to take as a reference the cavities of soy bean lipoxygenase. This protein has two cavities (I and II), which were identified in 1993. Both are in the carboxy terminal domain, the catalytic domain of the protein [6].

The residues that define cavity I are: Cys357, Val358, Ile359, Arg360, Tyr409, Ile412, Tyr493, Met497, Ser498, Leu501, Asn502, Thr503, Val570, Asn573, Trp574, Val575, Asp578, Gln579, Leu581, Asp584, Lys587, Arg588, Tyr610, Trp684, Leu689, and Val693.

Cavity I is conical with an opening of about $10 \AA$ at the surface of the protein (Fig. 6). It is defined by the carbonyl group of serine 498 and the side chains of threonine 503, leucine 689 and valine 693: at this point, the presence of a molecule of water is allowed. This area is located at $8 \AA$ of the iron atom. The final part of the cavity was in a line that ends at the iron atom and cut the line between histidines 499 and 690, near the coordination sphere of the iron atom [7].

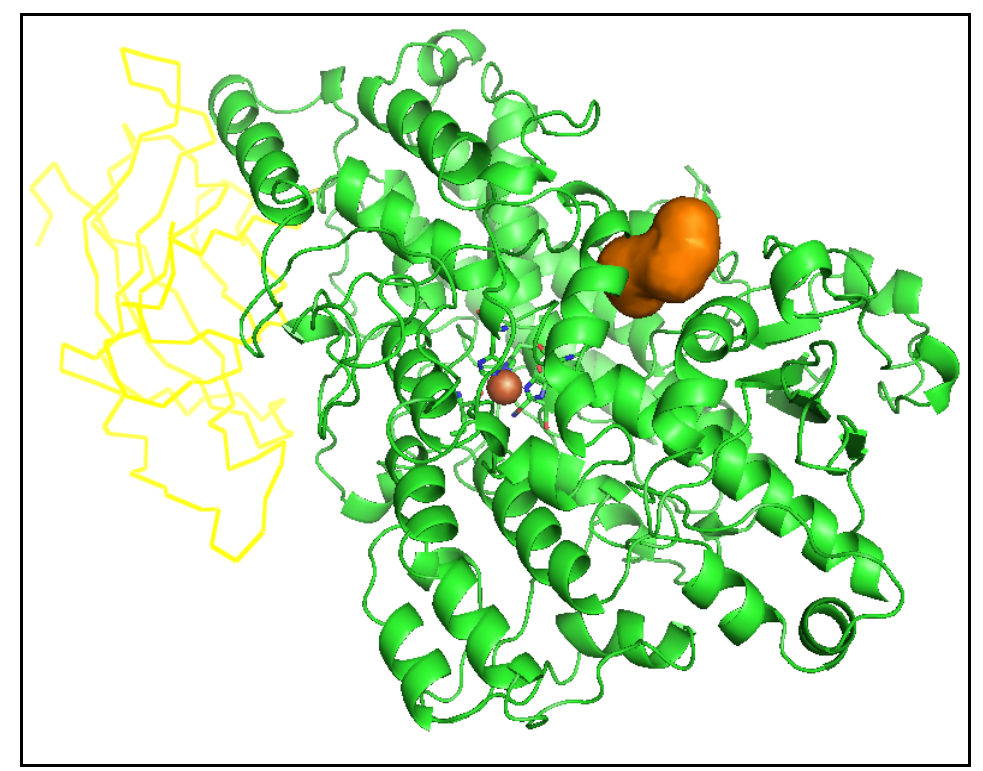

Figure 6. Structure of the soybean lipoxygenase (Glycine max). In orange, the cavity, in yellow, the $\mathrm{N}$-terminal domain, and in green the $\mathrm{C}$-terminal domain. In red, the iron atom with five residues coordinating it. 
Although Boyington [6] held that most of the residues that define the cavity are hydrophobic, it is a mistake to believe that this cavity is hydrophobic, because water molecules form hydrogen bonds with the protein [7]. According to Boyington, the characteristics of this cavity make it ideal to allow the passage of molecular oxygen from outside the protein to one of two vacant sites of coordination of iron [6]. Contrary to the findings of Boyington and according to Minor, the passage of molecular oxygen required a substantial reorganization of the structure of the protein, since a reorientation of the side chain of residue Asn 694 is not enough to facilitate the access of the oxygen to iron [7].

Thus, cavity I may not be not ideal to allow the passage of molecular oxygen into the active site. The most plausible alternative is cavity II, which allows direct access to iron [7].

Cavity II, 40Á long, stretches in two directions (a and b) from the area near iron (Fig. 7). The farthest end of the cavity is defined by the side chain of residue 341 (methionine) and 480 (leucine), which are $30 \AA$ from the iron atom.

Cavity II has two subunits separated by a narrow passage of $3.5 \AA$, determined by arginine 707 and valine 354 . Thus, speaking of cavities IIa and $\mathrm{IIb}$, the first is closest to the iron and is probably the one that links with the fatty acid substrate. Both cavities are interspersed between two layers of helix, helix 9 and 11 on one side and helix 2, 6, 18 and 21 on the other.

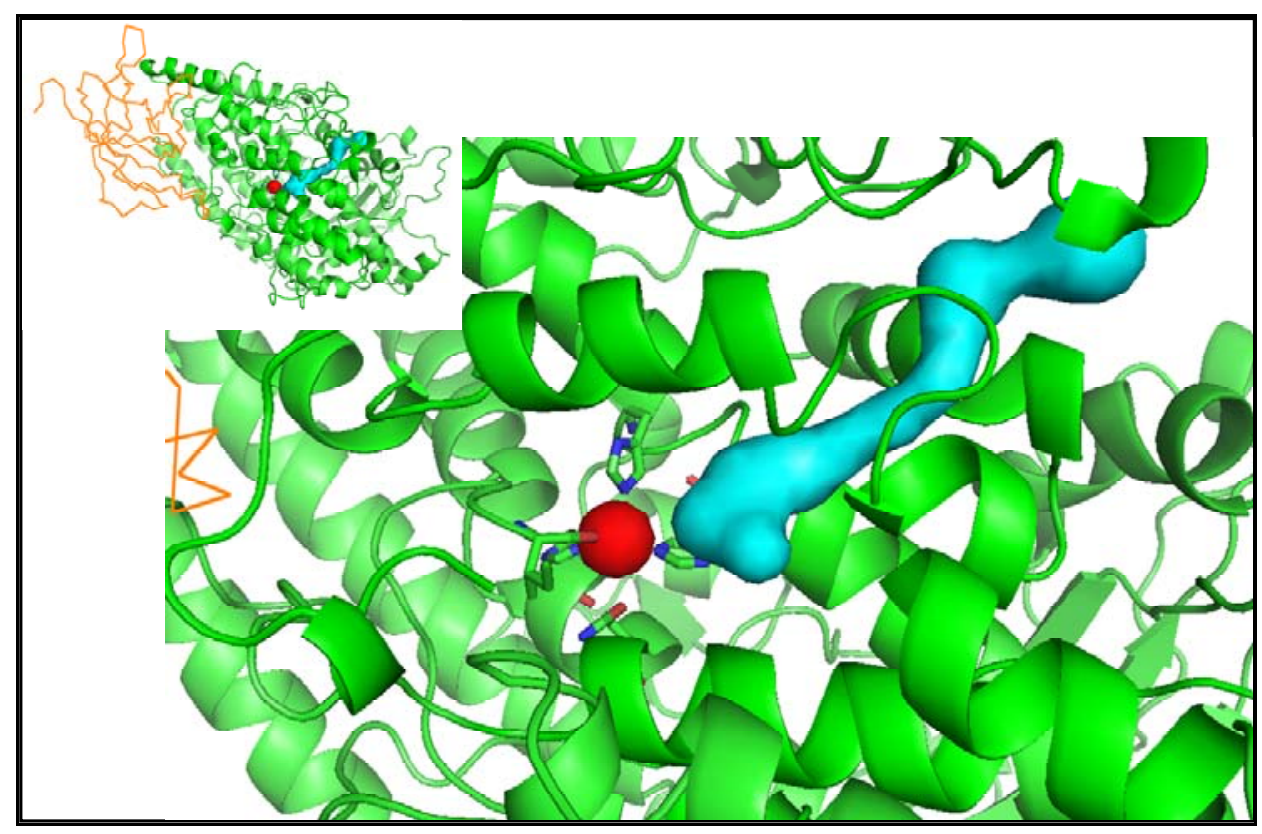

Figure 7. Structure of the soybean lipoxygenase (Glycine max). Top image: orange, $\mathrm{N}$-terminal domain, C-terminal domain in green. In cyan, cavity IIa. In red, the iron atom with five residues that coordinate it. 
The residues that define cavity IIa are Tyr214, Leu255, Glu256, Gly258, Thr259, Leu496, His499, Trp500, His504, Ile538, Leu541, Ala542, Ile547, Ile553, Val564, Ser567, and Ile839.

The residues that define cavity IIb are: Trp340, Met341, Glu345, Phe346, Glu349, Met350, Gly353, Val354, Asn355, Val358, Ile359, Leu407, Leu480, Lys483, Ala484, Val486, Ile487, Asn490, Tyr700, Met705, Asn706, Arg707, Pro708, and Tyr734 [7].

\section{Philogeny of lipoxygenases}

The function of a protein is closely related to its structure. Thus, determining the amino acid sequences of many proteins should enable the evolutionary relationships between them to be described. Ideally, alignments for evolutionary studies should be done with the sequence of the protein and respecting the provision in the same space as its tertiary structure. However, as we do not know the structure of most proteins, this is impossible and, therefore, phylogenetic analysis has to be based on the alignments of the nucleotide or amino acid sequences of the various proteins described.

To perform phylogenetic and evolutionary analysis of a protein family, the mutations produced over time on a sequence are usually analyzed. In phylogenetic studies performed with the proteins of the lipoxygenase family, we observe various subgroups depending on their origin, location and specificity because these are determined by the sequence residues located at the active site. Fig. 8 shows a phylogenetic tree for mammal and plant lipoxygenases, which clearly shows the separation of these two groups and the formation of subgroups. It is remarkable that the formation of a particular product for the catalysis of lipoxygenase is not necessarily associated with similar sequences. In contrast, proteins with functional homology of different species located in different subgroups have sequence identities of between 70 and $95 \%[1]$.

Although the databases of nucleotide sequences contain various sequences described as a lipoxygenase or as potential lipoxygenases for microorganisms (bacteria and fungi), there are very few publications on this field. The most recent shows the presence of lipoxygenases in prokaryotic organisms and suggests that lipoxygenases found in cyanobacteria (Nostoc punctiforme) could be the evolutionary precursors of 13-LOX, the lipoxygenases of the plastids of plants [27] and that the phenomena of horizontal transfer between Cyanobacteria and other bacteria were responsible for the dispersal of this gene between bacteria [27]. 


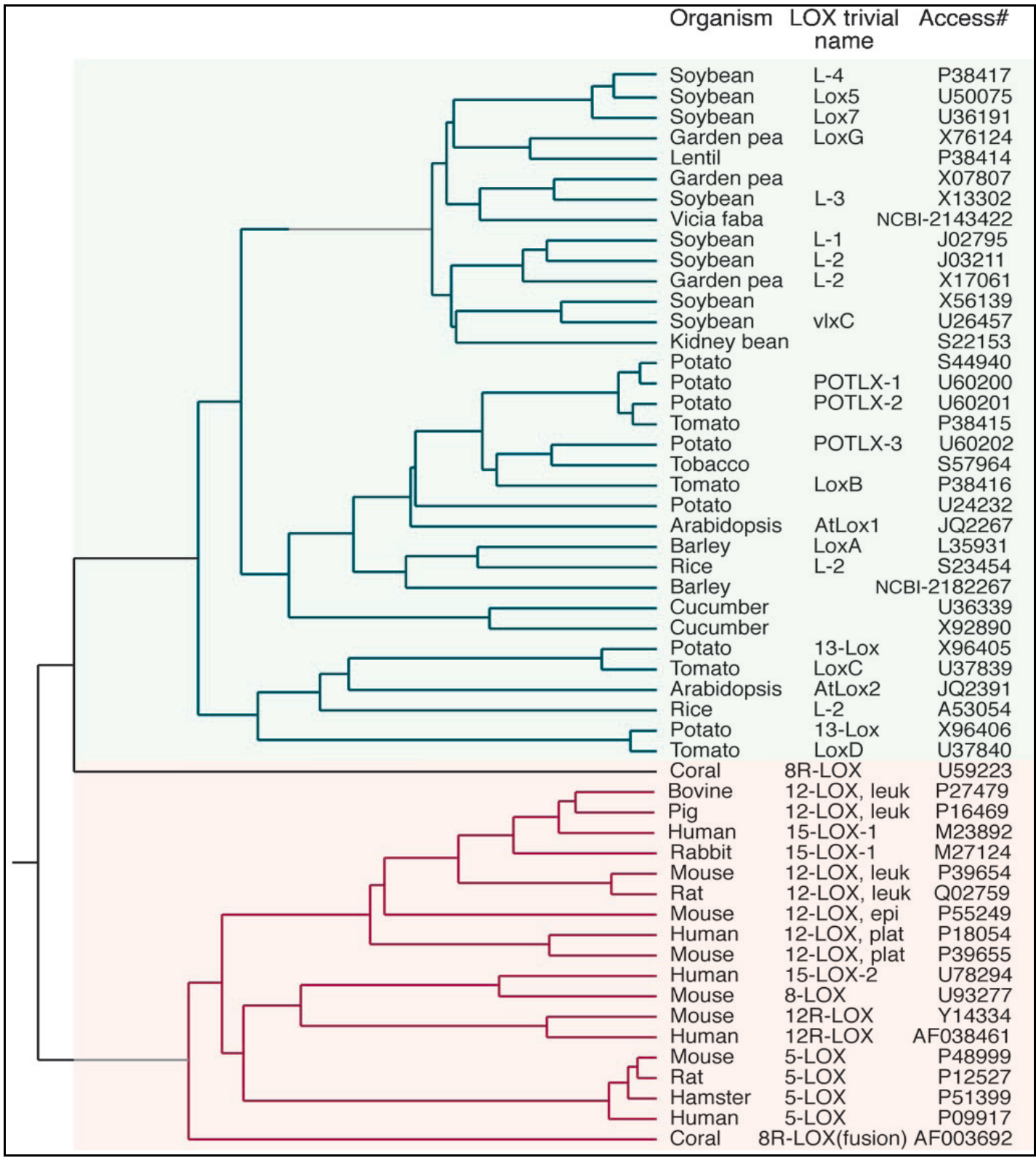

Figure 8. Phylogenetic tree of plant (blue background) and mammals lipoxygenases (pink background) [1].

The phylogenetic tree of Fig. 9 shows the phylogenetic grouping of plant lipoxygenases (13-LOX type 2 groups, 9-LOX 13-LOX type 1 and type 1), animal lipoxygenases, represented only by mammal ones, and the grouping of prokaryotic lipoxygenases. This last group is not very compact and is between the animal and plant lipoxygenases [27]. These results are consistent with those obtained by Porta and Rocha-Sosa in a less detailed phylogenetic study [28].

This phylogenetic tree (Fig. 9) shows the relation between the sequence and the functional properties of each lipoxygenase. 


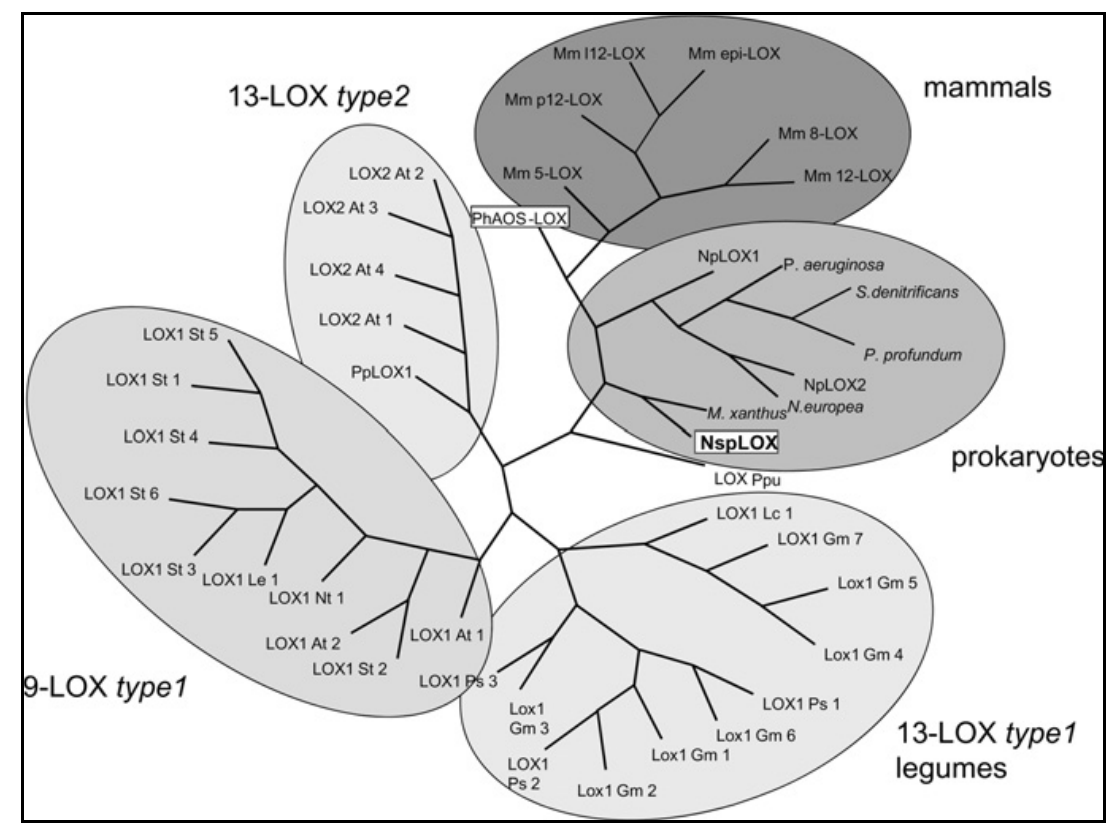

Figure 9. Phylogenetic analysis of sequences of mammals, plants, corals, algae and bacteria lipoxigenases, using the program PHYLIP 3.5 [27]. Access numbers: (i) Mus musculus (Moore E.R.B.): 5-LOX AAC37673; 8-LOX CAA75003; 12R LOX CAA74714; 112-LOX AAA20658; p12LOX AAA20659; e12LOX NP663717; (ii) P. homomalla: PhAOS-LOX O16025; (iii) Arabidopsis thaliana type 2 LOX: LOX2 At1 Q06327; LOX2 At2 CAB56692; LOX2 At3 CAC19364; LOX2 At4 CAG38328; (iv) Physcomitrella patens (Pp):CAE47464; (v) Solanum tuberosum: St1 CAA5572; St2 AAD09202; St3 AAB31252; St4 CAA64766; St5 CAA64765; St6 AAB67860; (vi) A. thaliana type 1 9-LOX: LOX1 At1 NP175900; LOX1 At2 NP188879; (vii) Lycopersicon esculentum: Le1 P38415; (viii) Nicotiana tabacum: Nt1 CAA58859; (ix) Glycine max: Gm1 CAA47717; Gm2 P09439; Gm3 CAA31664; Gm4 P38417; Gm5 AAB67732; Gm6 AAA96817; Gm7 AAC49159; (x) Pisum sativum: Ps1 AAB71759; Ps2 CAA55318; (xi) Lens culinaris: Lc1 CAA50483; (xii) Porphyra purpureum: Ppu AAA61791; (xiii) Pseudomonas aeruginosa: AF479686; (xiv) Nitrosomonas europaea : BX321860; (xv) Shewanella denitrificans OS-217: Q3P217; (xvi) Photobacterium profundum 3TCK: ZP 01218321; (xvii) M. xanthus: DK 1622 hypothetical protein MXAN 1745 YP 629995; (xviii) N. punctiforme (PCC73102): NpLOX1 ZP 00106490; NPLOX2 ZP 00107030; (xvix) Nostoc sp. SAG 25.82: NspLOX NP 478445.

\section{Results}

\subsection{Molecular structure of lipoxygenases of Pseudomonas aeruginosa 42A2}

Obtention of the crystal structure of Pseudomonas aeruginosa 42A2 lipoxygenase would allow the study of its cavities, the active site and the residues that act as iron ligands. 
The crystallographic data indicate that the first 49 amino acids of the $\mathrm{N}$-terminal sequence have no structure (they are highly disordered). It is from amino acid 50, an isoleucine, that one can start to see the structure of the protein. This structure arrangement is maintained until the end of the amino acid sequence, the isoleucine at position 685 (at the carboxyterminal end), with the exception of six amino acids (Thr 201, Gln 202, Gly 203, Gly 204, Gln 205, and Gly 206).

There are several differences between this crystal and the other crystal structures described as lipoxygenases. The most outstanding difference between the crystal structure of the Pseudomonas aeruginosa lipoxygenase and the plant and animal lipoxygenases described so far (Fig. 10) is the absence of PLAT N-terminal domain formed by 8 antiparallel $\beta$ sheets, a non catalytic domain present in all eukaryotic lipoxygenases reported to date of between 110 and 150 residues [29].

The presence of an insertion of 90 amino acids (from alanine 126 to valine 200 ), forming two antiparallel $\alpha$ helices (2A and 2B) of 36 and 38 residues,

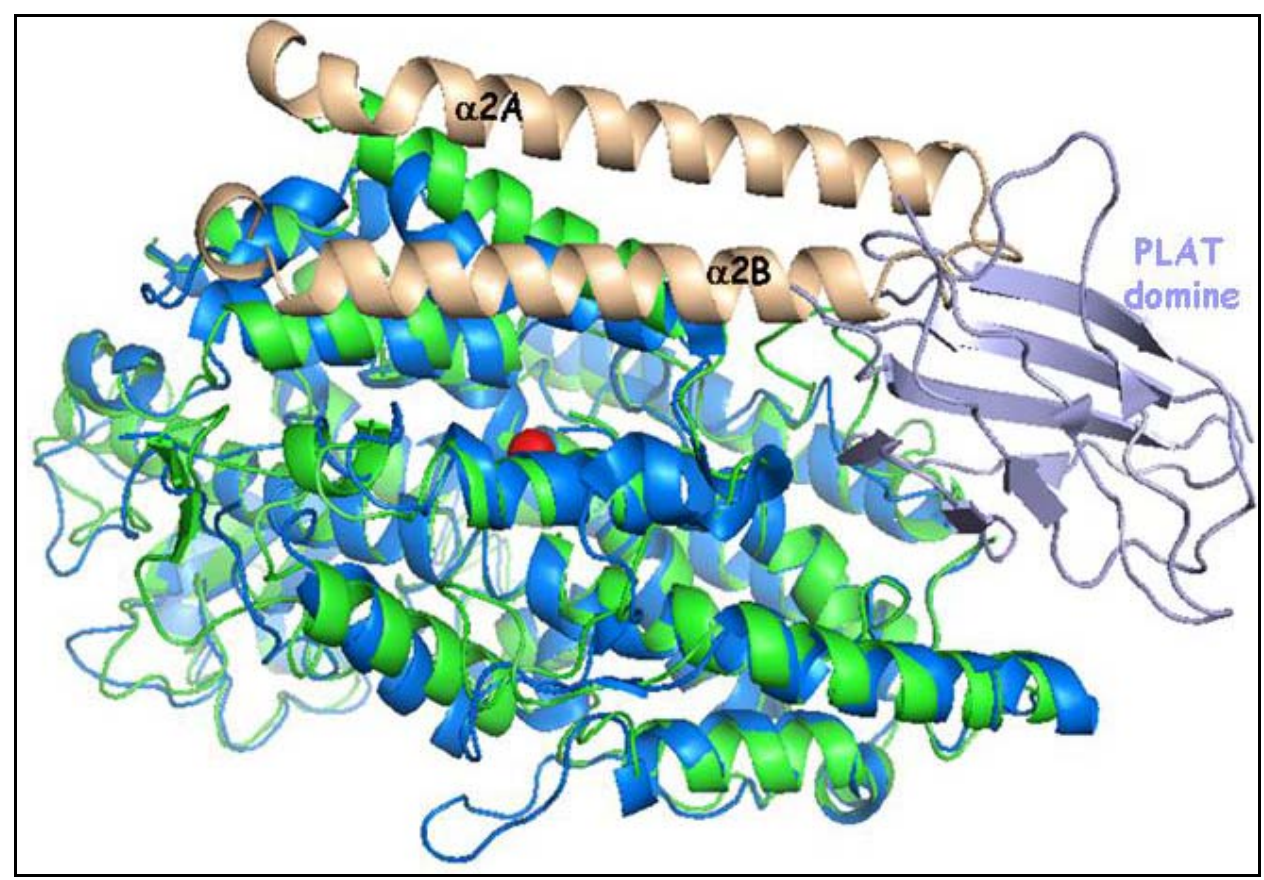

Figure 10. Superposition of the structures of lipoxygenases of Plexaura homomolla (2fnq), in blue, and Pseudomonas aeruginosa 42A2, in green, where it is observed that the part corresponding to the catalytic domain of Plexaura homomolla lipoxygenase is well superimposed on Pseudomonas aeruginosa 42A2 lipoxygenase. In pink, the two helices ( $\alpha 2 \mathrm{~A}$ and $\alpha 2 \mathrm{~B}$ ) formed by the insertion of 90 residues detected in Pseudomonas aeruginosa 42A2 lipoxygenase, and in turquoise blue color, the N-terminal PLAT domain present in lipoxygenase of Plexaura homomolla but absent in Pseudomonas aeruginosa 42A2. 
respectively, is what modifies the general structure of Pseudomonas aeruginosa lipoxygenase, making it a single-protein domain (C-terminal catalytic domain) since the N-terminal PLAT domain is lost (Fig. 10).

This insertion can be seen perfectly in a structural sequence alignment (Fig. 11) between Pseudomonas aeruginosa lipoxygenase, Plexaura homomolla lipoxygenase (2FNQ), rabbit reticulocyte lipoxygenase (1LOX) and Glycine max lipoxygenase (1YGE). In Fig. 11, the sequence of Pseudomonas aeruginosa lipoxygenase (Pa_Lox) corresponding to the insertion of the $\alpha$ helix 2A and the $\alpha$ helix 2B is highlighted in blue.

Another detail of the structure worth mentioning is the absence of the amino acid structure from amino acids 201 to 206 (inclusive). These six amino acids correspond to a loop linking $\alpha$ helix 2A and $\alpha$ helix 3 (Fig. 11). This lack of structure indicates that these residues are highly mobile, which suggests that this structure of two antiparallel helices on the protein can move just like a lid that opens and closes the cavity where the substrate is introduced.

It should be noted that these six amino acids without structure are not comparable with any other structure, as the structure of these two antiparallel $\alpha$ helices has not been described. In Fig. 11, these six amino acids (TQGGQG) are marked in orange, just after the two $\alpha$ helices, 2A and $2 \mathrm{~B}$.

One important aspect of the alignment shown in Fig. 11 is the low sequence homology (22\%) between Pseudomonas aeruginosa and Plexaura homomolla lipoxygenases. This low homology contrasts with the high structural homology (see the overlap of the two structures in Fig. 10) of the catalytic domain of the two proteins, suggesting that this structure is the only one that allows to bind iron, while, at the same time allows the entry and modification of a polyunsaturated fatty acid, either free or ester, by introducing a hydroperoxide group. Moreover, the fact that the coordination of the iron atom is mediated by the carboxyterminal amino acid requires this high conservation of the structure, because, since the terminal isoleucine is not part of any more robust structure, such as a $\beta$ sheet or an $\alpha$ helix, the probability of placing incorrectly this residue in a less preserved structure is very high.

Another pioneering aspect of this structure is the observation of a phospholipid within the protein, a fact unknown until now, because no lipoxygenase complex described to date has contained phospholipids, and only in the case of a complex of soy bean lipoxygenase is there a model with 13 (S) hydroperoxide 9 (Z), 11 (E) octadecenoic acid (Protein Data Bank code 1IK3) in the active center [9]. 


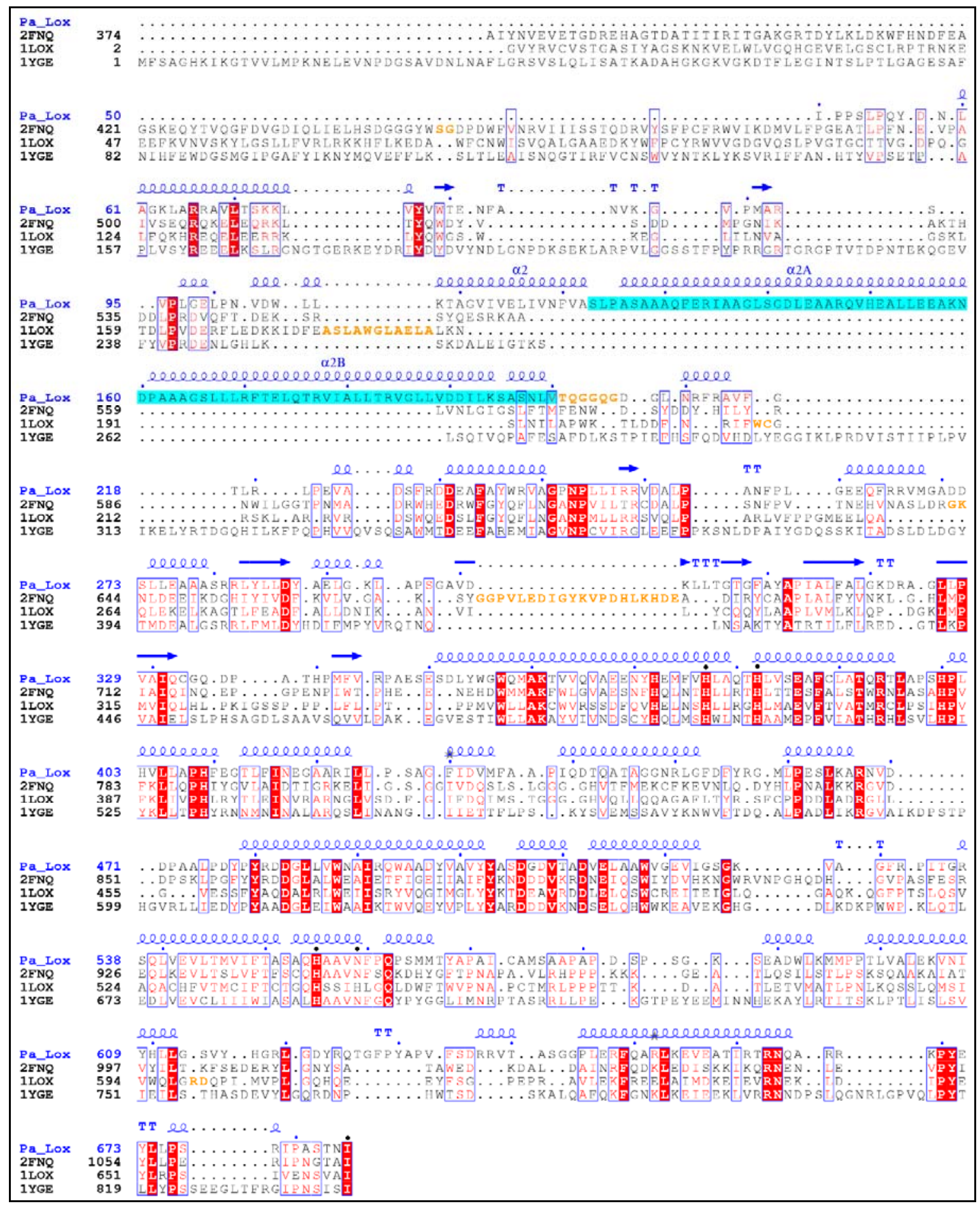

Figure 11. Structural alignment of lipoxygenases of Pseudomonas aeruginosa 42A2 (Pa_Lox), of Plexaura homomolla (2FNQ), of rabbit reticulocyte (1LOX) and from Glycine max (1YGE). It shows the secondary structure determined for Pa_Lox. The residues shown in orange are those that are lost in the alignment with respect to other structures. Lack of PLAT domain and the insertion (blue) after the $\alpha$ helix 2 is more than evident. The black dots indicate the residues that coordinate the iron atom. 


\subsection{Coordination sphere of iron of Pseudomonas aeruginosa 42A2 lipoxygenase}

As the coordination of the iron atom is a highly conserved structure in eukariotic lipoxygenases, it is not surprising that this lipoxygenase of bacterial origin is also conserved.

Thus, the catalytic center in lipoxygenase of Pseudomonas aeruginosa maintains the essential characteristics described for other lipoxygenase enzymes $[1,6,7,29,30]$, an iron atom with slightly distorted octahedral coordination (Fig. 12). The six iron ligands correspond to the three nitrogen atoms of the following histidines: His377, His382, and His555, with coordination distances of $2.24 \AA, 2.18 \AA$, and $2.15 \AA$, respectively, from the oxygen atom of the asparagine Asn559 at $2.37 \AA$, one oxygen atom of the carboxylate group of the terminal isoleucine Ile685 at $2.30 \AA$ and water (W1) at $2.26 \AA$ (Fig. 12). The second carboxylate oxygen atom of isoleucine Ile685 establishes a strong and short hydrogen bond with water W1 (2.46 $\AA)$, completing the geometrical feature of the first coordination sphere of iron in lipoxygenases. The angles of iron-binding ligands of Pseudomonas aeruginosa lipoxygenase are comparable with those published for other lipoxygenases (Table 1).

The atomic temperature factors and electron density map suggest that the iron atom is in ferrous state $\left(\mathrm{Fe}^{2+}\right)$.

Table 1. Comparison among distances from iron to iron ligands and the angles formed between lipoxigenases of Pseudomonas aeruginosa and the Glycine max (1-LOX).

\begin{tabular}{|c|c|c|}
\hline & P. aeruginosa LOX & G. $\max 1$ 1-LOX \\
\hline $\begin{array}{l}\text { Distances ligand-metal } \\
\text { Fe-His }{ }^{499} \mathrm{~N} \text { / Fe-His }{ }^{377} \mathrm{~N} \\
\text { Fe-His }{ }^{504} \mathrm{~N} / \mathrm{Fe}-\mathrm{His} \mathrm{s}^{382} \mathrm{~N} \\
\text { Fe-His }{ }^{690} \mathrm{~N} / \text { Fe-His } \\
\text { Fe-Ile }{ }^{839} \mathrm{O} \text { / Fe-Ile }{ }^{685} \mathrm{O}\end{array}$ & $\begin{array}{l}2.29 \AA \\
2.13 \AA \\
2.19 \AA \\
2.07 \AA\end{array}$ & $\begin{array}{l}2.24 \AA \\
2.18 \AA \\
2.15 \AA \\
2.30 \AA\end{array}$ \\
\hline $\begin{array}{l}\text { Angles ligand-metal-ligand } \\
\mathrm{His}^{499} \mathrm{~N}-\mathrm{Fe}-\mathrm{His}^{504} \mathrm{~N} / \mathrm{His}^{377} \mathrm{~N}-\mathrm{Fe}-\mathrm{His}^{382} \mathrm{~N} \\
\mathrm{His}^{399} \mathrm{~N}-\mathrm{Fe}-\mathrm{His}^{690} \mathrm{~N} / \mathrm{His}^{377} \mathrm{~N}-\mathrm{Fe}-\mathrm{His}^{555} \mathrm{~N} \\
\mathrm{His}^{504} \mathrm{~N}-\mathrm{Fe}-\mathrm{His}^{690} \mathrm{~N} / \mathrm{His}^{382} \mathrm{~N}-\mathrm{Fe}-\mathrm{His}^{555} \mathrm{~N} \\
\mathrm{His}^{690} \mathrm{~N}-\mathrm{Fe}-\mathrm{Ile}^{839} \mathrm{O} / \mathrm{His}^{555} \mathrm{~N}-\mathrm{Fe}-\mathrm{Ile}^{685} \mathrm{O} \\
\mathrm{His}^{504} \mathrm{~N}-\mathrm{Fe}-\mathrm{Ile}^{839} \mathrm{O} / \mathrm{His}^{382} \mathrm{~N}-\mathrm{Fe}-\mathrm{Ile}^{685} \mathrm{O} \\
\mathrm{His}^{499} \mathrm{~N}-\mathrm{Fe}-\mathrm{Ile}^{839} \mathrm{O} / \mathrm{His}^{377} \mathrm{~N} \text {-Fe-Ile }{ }^{685} \mathrm{O}\end{array}$ & $\begin{array}{l}102,6^{\circ} \\
100,9^{\circ} \\
104,0^{\circ} \\
78,1^{\circ} \\
106,0^{\circ} \\
150,8^{\circ}\end{array}$ & $\begin{array}{l}92,0^{\circ} \\
102,2^{\circ} \\
93,7^{\circ} \\
85,1^{\circ} \\
92,6^{\circ} \\
171,7^{\circ}\end{array}$ \\
\hline
\end{tabular}




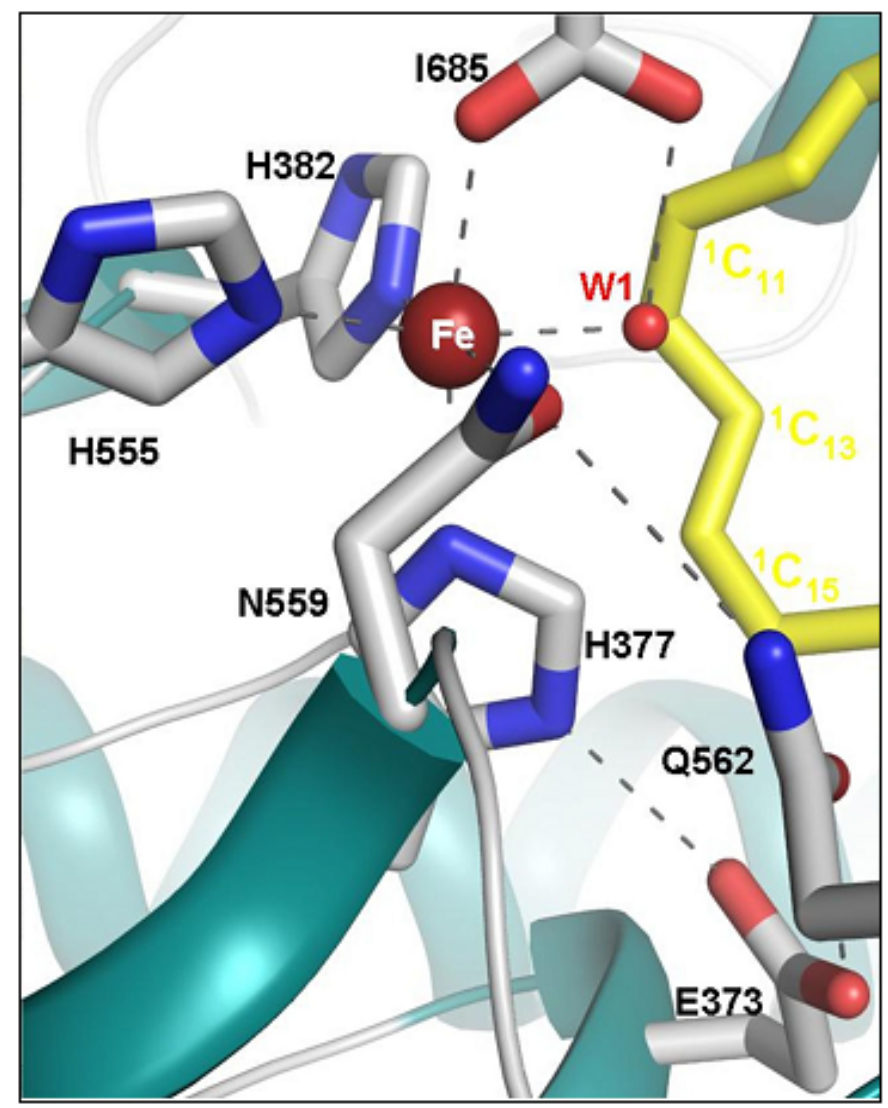

Figure 12. The catalytic center of Pseudomonas aeruginosa lipoxygenase. Displaying the 5 residues and the water that coordinate the iron atom $(\mathrm{H} 377, \mathrm{H} 382, \mathrm{H} 555, \mathrm{~N} 559$, I685, and W1) and the hydrogen bonds (dashed lines) for the first and second coordination sphere (Q562 and E373). It is also shown the approach of the five carbons (yellow) of the chain of the phospholipid to the iron, stacking on two histidines of the coordination sphere. Shown in red, the oxygen atoms, and in blue, the nitrogen atoms.

The preservation of this structure is shown in the alignment of the amino acid sequences in Fig. 13, where it can be seen that the amino acids that coordinate the iron atom are the same, except for asparagine (Asn 559), which, in the animal lipoxygenases is modified by a histidine (His 544 for rabbit reticulocyte lipoxygenase).

This can be seen, as the distance in sequence between the two histidines of the first coordination sphere of iron remains constant in different sequences (separation of four amino acids). The same happens in the second pair of amino acids coordinating iron (His-His for rabbit lipoxygenase or HisAsn for the other lipoxygenases), which are separated by three amino acids. We should mention that the role of the amino terminal in coordinating the iron atom looks very well preserved. 


\begin{tabular}{|c|c|c|}
\hline 1LOX & LP T DP P -------MVWLLAKCWVRSS DFQVHELNSH LLRGH LMAEVFTVATMRCLPS IHP & 384 \\
\hline $2 \mathrm{FNQ}$ & PHEENE------HDWMMAKFWLGVAESNFHQLNT H LLRTHLTTESFA LSTWRNLASAHP & 414 \\
\hline NSPLOX & RPAESESDL-- YWGWQMAKTVVQVAEENYHEMFVH LAQTH LVSEAFCLATQRT LAP SHP & 401 \\
\hline 1YGE & SQVVLPAKEGVEST I WLLAKAYVIVNDSCYHQLMS HWLNTHAAMEPFVIATHRHLSVLHP & 523 \\
\hline $1 \mathrm{LOX}$ & VFKLIVPHLRYT LEINVRARNGLVSDFGI F DQIMSTGGGGHVQLLQQAGAF LT YRSFCPP & 444 \\
\hline $2 \mathrm{FNQ}$ & VFKLLQPHI YGVLA I DT I GRKEL I GSGGIVDQS LS LGGGGHVTFMEKCFKEVNLQDYHLP & 474 \\
\hline NSPLOX & LHVLLAPHFEGT LF INEGAARI LLPSAGF I DVMFAAP IQDTQATAGGNRLGFDFYRGMLP & 461 \\
\hline 1YGE & I YKLLT PHYRNNMN I NALARQSLINANGI I ET TFLP S-KYSVEMS SAVYKNWVFTDQALP & 582 \\
\hline $1 \mathrm{LOX}$ & DDLADRGLLG------------VESSFYAQDALRLWEI I SRYVQGIMGLYYKT DEAVRDD & 492 \\
\hline $2 \mathrm{FNQ}$ & NALKKRGVDDP SK---------LPGFYYRDDG LALWEAI ET FIGEI I AIFYKNDDDVKRD & 525 \\
\hline NSPLOX & ES LKARNVDDP AA-------- LP DYP YRDDG LLVWNAI RQWAADYVAVYYAS DGDVTAD & 512 \\
\hline $1 Y G E$ & ADLIKRGVAIKDPST PHGVRLLI EDYP YAADGLEIWAAI KTWVQEYVP LYYARDDDVKND & 642 \\
\hline 1LOX & LELQSWCREITEIGLQG---AQKQGFP TS LQSVAQACHFVTMCI FTCTGQHSSI H LGQLD & 549 \\
\hline $2 \mathrm{FNQ}$ & NEIQSWI YDVHKNGWRVNPGHQDHGVPASFESREQLKEVLTS LVFTF SCQHAAVNFSQKD & 585 \\
\hline NSPLOX & VELAAWVGEVI GSGKVAG--------FRP I TGRSQLVEVLTMVI FTASAQHAAVNFPQPS & 564 \\
\hline $1 \mathrm{YGE}$ & $\begin{array}{c}\text { SELQHWWKEAVEKGHGDLK---DKPWWPKLQT LEDLVEVCLI I IWIASALHAAVNFGQYP } \\
\star\end{array}$ & 699 \\
\hline $1 \mathrm{LOX}$ & WFTWVPNAPCTMRLP PP TTK--------DATLETVMAT LPNLKQS SLQMS IVWQLGRDQP & 601 \\
\hline $2 \mathrm{FNQ}$ & HYGFT PNAPAVLRHP PP KKKG ------EATLQSI LST LPSKSQAAKA IATVYI LT KFSE & 638 \\
\hline NSPLOX & MMT YAPAICAMSAAPAP DSP S-------GKSEÄDW LKMMPP T LVALEKVNI YHLLGSVYH & 617 \\
\hline $1 \mathrm{YGE}$ & YGGLIMNRPTASRRLLPEKGT PEYEEMINNHEKAYLRT I TSKLPT LI S LSVIEI LSTHAS & 759 \\
\hline $1 \mathrm{LOX}$ & -- --IMVP LGQHQEEYFSGP ---EPRAVLEKFREELAIMDKEIEVRNEK----------L & 644 \\
\hline 2FNQ & ---- DERYLGNYSATAWEDK---DALDAINRFQDKLEDI SKKIKQRNEN- - - - - - - L & 681 \\
\hline NSPLOX & GRLGDYRQT GFP YAPVF SDRRVT ASGGP LERFQARLKEVEAT IRT RNQA----------R & 667 \\
\hline $1 Y G E$ & -- - -DEVYLGQRDNPHWT SD--SKALQAFQKFGNKLKEI EEKLVRRNNDP S LQGNRLGPV & 813 \\
\hline $1 \mathrm{LOX}$ & DIPYEYLRPSI--------VENSVAI 662 & \\
\hline 2FNQ & EVPYIYLLPER--------IPNGTAI 699 & \\
\hline NSPLOX & RKP YEYLLPSR--------IPASTNI 685 & \\
\hline 1YGE & QLPYTLLYPSSEEGLTFRGIPNSISI 839 & \\
\hline
\end{tabular}

Figure 13. Alignment of the amino acid sequence of part of the carboxiterminal domain of lipoxygenases of Pseudomonas aeruginosa 42A2 (NSPLOX), of Plexaura homomolla (2FNQ), of rabbit reticulocyte (1LOX) and from Glycine max (1YGE). The residues shown in red indicate residues that coordinate the iron atom.

\subsection{Cavities and substrate of Pseudomonas aeruginosa $42 \mathrm{~A} 2$ lipoxygenase}

Given the high-resolution crystal which was obtained in lipoxygenase of Pseudomonas aeruginosa, two clearly defined cavities can be seen: one for the substrate (which consists of two subcavities) and one enabling molecular oxygen to reach the catalytic center of the protein. The substrate cavity is perfectly defined by the presence of a phospholipid (Fig. 14).

Given the good quality of the electron density map obtained from the phospholipid, it can be identified as a phosphatidylethanolamine with a chain of 18 carbons in position 1 and another of 14 carbons in position 2 (Fig. 14). The 18-carbon chain might be a vaccenic acid (C18: 1 cis 11), characteristic of the membranes of Escherichia coli [31]. 


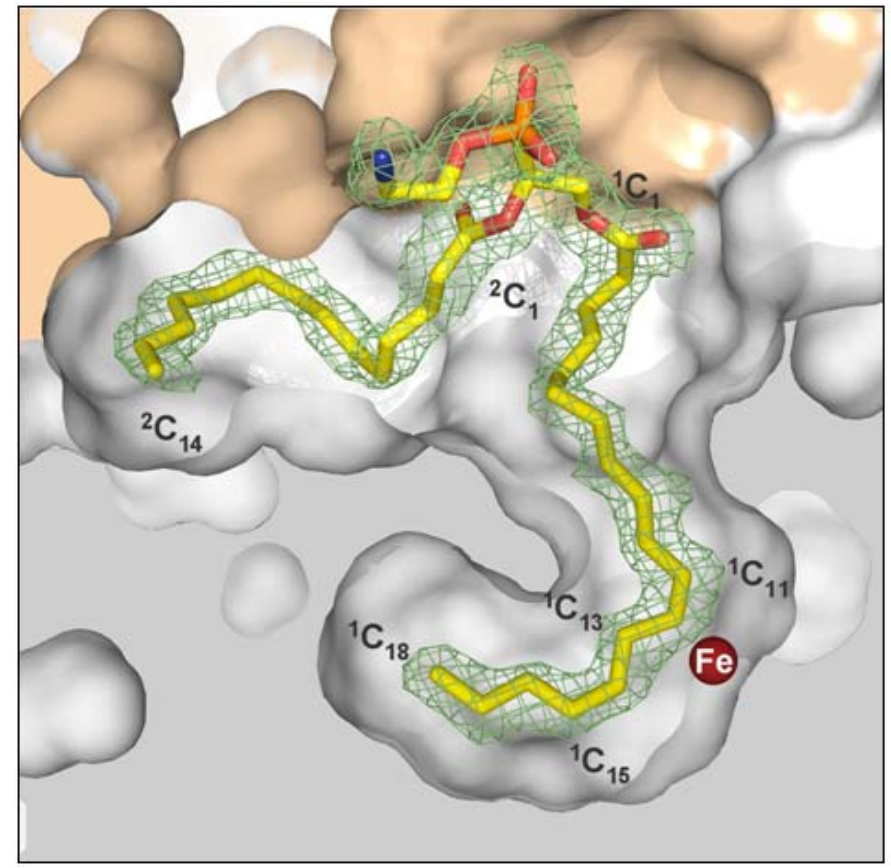

Figure 14. Section of the cavity substrate of Pseudomonas aeruginosa lipoxygenase showing the electron density map of the phosphatidylethanolamine that defines the two subcavitats of substart. It can be seen as the phospholipid polar head is close to the two $\alpha$ helices, 2A and 2B (pink).

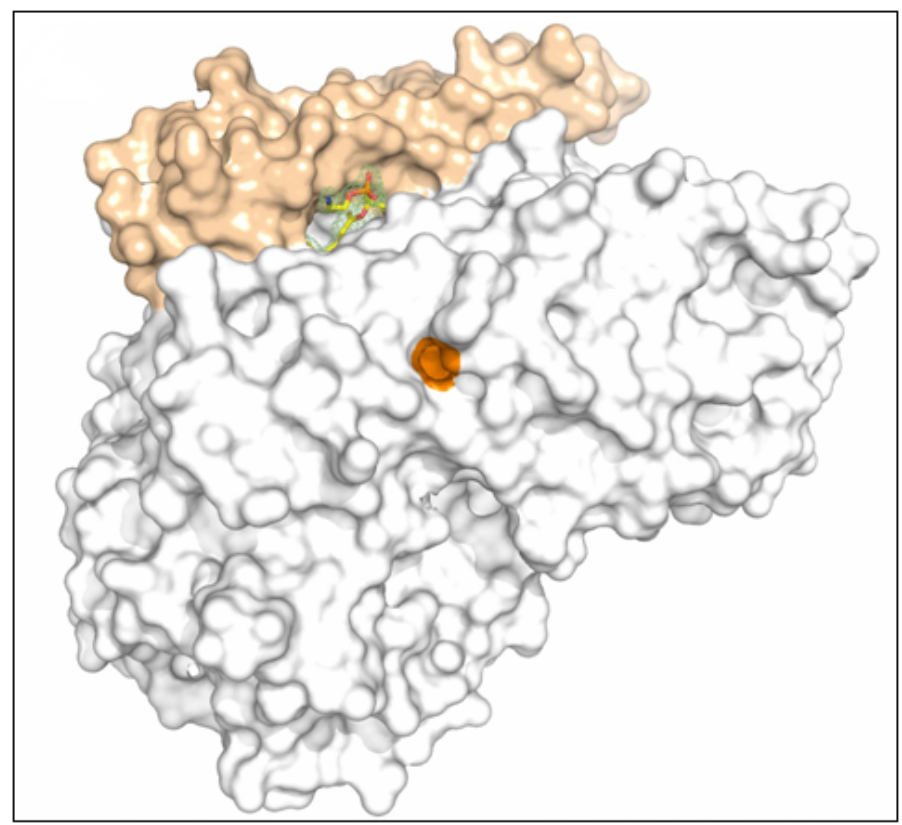

Figure 15. Representation of the surface of Pseudomonas aeruginosa lipoxygenase where can be observed the cavity of the phosphatidylethanolamine and the phospholipid polar head among the area of the two helices $\alpha$ ( $\alpha 2 \mathrm{~A}$ and $\alpha 2 \mathrm{~B}$, pink) and the rest of the protein (white). In orange, the entrance channel that may allow the entry of molecular oxygen to the active site is shown. 
These phospholipids, incorporated into the lipoxygenase during its overexpression in Escherichia coli, must then have maintained full employment during all steps of purification and crystallization. Each of these chains occupies one of the subcavities that form the cavity of the substrate. This cavity is almost completely hydrophobic, except for the presence of water W1, which acts as an iron ligand, and the residues Tyr609 and Asn607 found at the bottom of subcavities 1 and 2, respectively, of the substrate cavity.

The other cavity that is observed is a strongly hydrophobic channel (no visible water on the inside), which starts at the surface of the protein and reaches subcavity 1, near carbon 15 of the chain of 18 carbons of the phosphatidylethanolamine (orange area in Figures 15 and 16). This channel seems very appropriate to allow entry of oxygen to the active site similar to the situation discussed for other lipoxygenases [20]. The residues that define this channel are: Val86, Gly88, Leu383, Glu386, Asn417, Ala421, and Gln443.

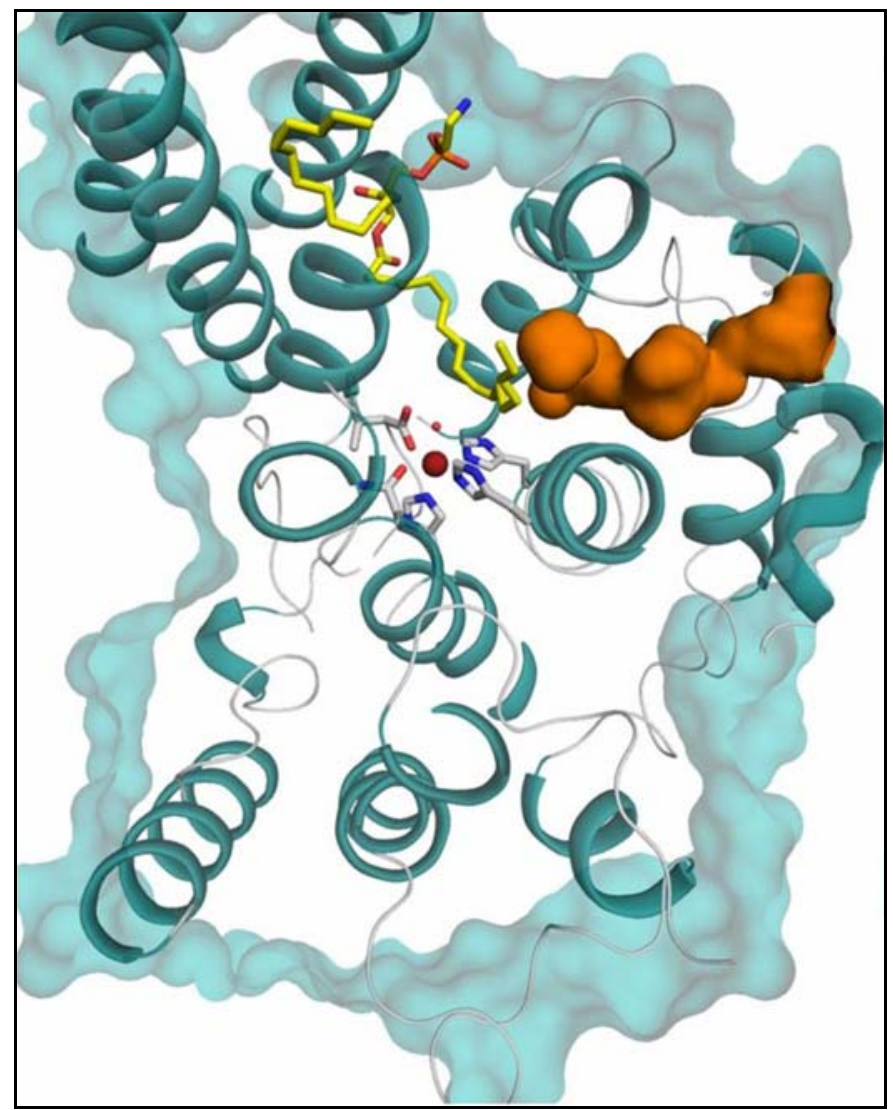

Figure 16. Performance of the approach of the channel (orange) that allows the arrival of molecular oxygen to the active site of lipoxygenase from the surface (blue) to the cavity of the substrate occupied by the chain 1 of the phosphatidylethanolamine (yellow). In red, the iron atom, and also the residues that coordinate the iron are shown. 
The packaging observed at the ends of the aliphatic chains of the phospholipid suggests that both subcavities could accommodate chains of two or four carbons more like arachidonic acid $(C 20: 5,8,11,14)$. The aliphatic chain in position $1(\mathrm{C} 1)$ has a folded conformation with the methyl end curved toward the polar head of the lipid (Fig. 14); the distance between carbon 1 and carbon 18 of this chain is $13.56 \AA$. The five carbons between carbon 10 and carbon 14 are equidistant from the iron by about $5.14 \AA$ (on average); and from the water that coordinates the iron, by about $3.69 \AA$ A. The geometry determined for this chain, taking into account that the position of double bonds was not observed directly because the resolution of the crystal does not allow it to be, suggests that the cavity where it is favors a cis conformation, usually associated with the unsaturated links of fatty acids. Among the carbons in positions 11-12 and 14-15, there is a deviation from the plane of $16^{\circ}$ and $50^{\circ}$, respectively. The alleged double bond between carbons 11 and 12 is the closest to the iron; and the hydrogen associated with these carbons is on the opposite side from the metal. Carbons 14 and 10 are placed on the rings of residues His 382 and His377, respectively, at a distance of $3.9 \AA$ from the nitrogen of these iron ligands. As for the alleged double bond that should be between carbons 14 and 15 and is needed to form the pentadiene group, the $50^{\circ}$ deviation from the plane indicates its absence. This means that the substrate is not right for this enzyme, which would explain why we found this enzyme-substrate construct consolidated without reacting.

The geometry for the second fatty acid chain of the phosphatidylethanolamine that is occupying subcavity 2 of the substrate cavity, the chain of 14 carbons in position 2 , has not been described in any other structure of lipoxygenase. This geometry suggests that the cis conformation between carbon atoms 5 and 6 is the most likely, although in this case the deviation from the plane between them is $31^{\circ}$, which, again, indicates its absence.

On this basis, the most likely phospholipid to bind to Pseudomonas aeruginosa lipoxygenase is O-(sn-1-cis-vaccenil-sn-2- miristoil-sn-glyceric-3phosphoryl) ethanolamine, although a sn-1-estearoil chain is also possible. cisVaccenic acid is a fatty acid of Escherichia coli that could be particularly abundant in cultures incubated at low temperature. A string as short as 14 carbons observed occupying the sn-2 position of the glycerolipid is not common.

The polar head of the phosphatidylethanolamine is partially exposed to solvent and forms two ionic bonds with the protein (Fig. 17). The first one is between the phosphate group of the phosphatidylethanolamine and the guanidine group of residue $\operatorname{Arg} 422$. The second is between the terminal amino group of the phosphatidylethanolamine and the carboxyl group of residue Asp190, which is located in the $\alpha$ helix 2B. 


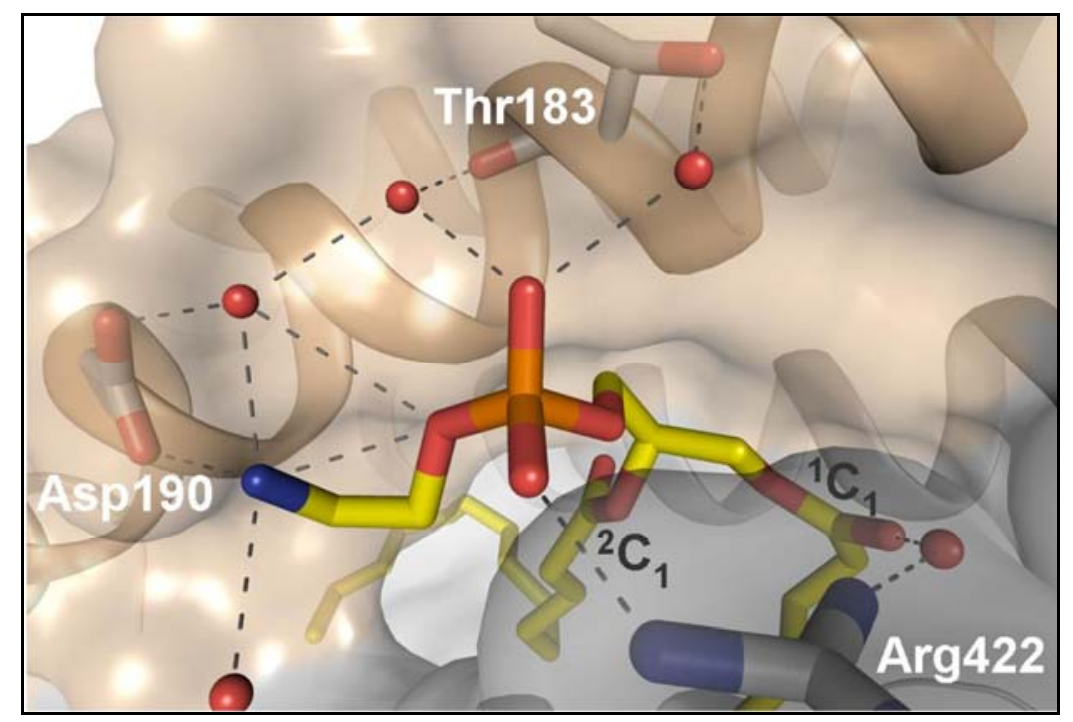

Figure 17. Ionic bonds among the phospholipid polar head and residues Asp190 and Arg422 of Pseudomonas aeruginosa lipoxygenase suggesting a specificity for zwitterionic phospholipids.

The presence of the spatial proximity of residues Asp190 and Arg422 (Fig. 17) seems to indicate that this lipoxygenase is very suitable to capturing zwitterionic phospholipids, particularly with small groups positively charged in terminal position, as it is the case of the phosphatidylethanolamine observed.

The side chain of residue Arg422 has flexibility, which provides versatility when joining the acidic groups of different substrates. In fact, most sequences of lipoxygenases in the equivalent position of the arginine 422 of Pseudomonas aeruginosa lipoxygenase, or the prior residue there, are usually an arginine (Fig. 13, marked in blue). These can interact with the carboxylate group of the corresponding fatty acid substrate [32].

\subsection{Study of the philogeny of lipoxygenases}

For this study we used 14 sequences of bacterial lipoxygenases, 9 animal lipoxygenases, 10 vegetable lipoxygenases and 4 fungi ones. In the case of bacterial and fungal sequences, the nature of the lipoxygenases was confirmed by alignments of the highly conserved amino acids that coordinate the iron atom [33] and an amino acid sequence characteristic of lipoxygenases, WXXAK [24]. The two tables below (Tables 2 and 3) show the positions of the iron ligands in relation to the sequences of bacterial and fungal lipoxygenases and how well they are aligned within the groups concerned. In the case of bacterial sequences, some of the singularities present in the sequences of ATCC 51908 Shewanella woodyi (ZP_01543473.1) should be mentioned. In this case, 
the fourth ligand that normally varies between asparagine $(\mathrm{N})$ or histidine $(\mathrm{H})$ is replaced by an arginine $(\mathrm{R})$.

The fifth ligand, the C-terminal amino acid, is also altered in two of the three sequences of lipoxygenases present in the genome of ATCC 51908 Shewanella woodyi. This position usually has an isoleucine (I) or a valine (V) while, in two of the three lipoxygenase sequences, there is an asparagine $(\mathrm{N})$ (Table 2).

Table 2. Alignment and position of amino acids that bind the iron atom in the sequence typical of lipoxygenases (WXX AK) of all bacterial sequences used in this study.

\begin{tabular}{|c|c|c|c|c|c|}
\hline Species & GenBank acces & pb gen & aa gen & $\mathbf{W}-\mathbf{A ~ K}$ & Ligands of $\mathrm{Fe}$ \\
\hline Burhholderat thatlandensis $\mathrm{E} 264$ & СР000086.1 & 2088 & 695 & 368 WQMAK 372 & $\begin{array}{l}388 \mathrm{H}, 393 \mathrm{H}, 565 \mathrm{H}, \\
569 \mathrm{~N}, 695 \mathrm{I}\end{array}$ \\
\hline Myxococos xanthus DK 1622 & NC_ 008095.1 & 2028 & 675 & 346 WLAAK 350 & $\begin{array}{l}366 \mathrm{H}, 371 \mathrm{H}, 549 \mathrm{H}, \\
553 \mathrm{~N}, 675 \mathrm{~V}\end{array}$ \\
\hline Nitrosomonas europaed ATCC 19718 & NP_841292.1 & 1698 & 565 & 244 WLAAK 248 & $\begin{array}{l}264 \mathrm{H}, 269 \mathrm{H}, 445 \mathrm{H}, \\
449 \mathrm{~N}, 565 \mathrm{~V}\end{array}$ \\
\hline Nitrosospira multiformis ATCC 25196 & NC_007614.1 & 1662 & 553 & 232 WLTAK 236 & $\begin{array}{l}352 \mathrm{H}, 357 \mathrm{H}, 433 \mathrm{H}, \\
437 \mathrm{H}, 553 \mathrm{I}\end{array}$ \\
\hline Photobacterwm profindum $3 \mathrm{TCK}$ & ZP_01218321.1 & 1845 & 614 & 289 WQAAM 293 & $\begin{array}{l}309 \mathrm{H}, 314 \mathrm{H}, 487 \mathrm{H}, \\
491 \mathrm{~N}, 614 \mathrm{I}\end{array}$ \\
\hline Pseudomonas aenginosa $42 \mathrm{~A} 2$ & AAL 85880.2 & 2058 & 685 & 357 WQMAK 361 & $\begin{array}{l}377 \mathrm{H}, 382 \mathrm{H}, 555 \mathrm{H}, \\
559 \mathrm{~N}, 658 \mathrm{I}\end{array}$ \\
\hline Pseudomonas aenginosa $\mathrm{PAO}$ & NC_002516.2 & 2058 & 685 & 357 WQMAK 361 & $\begin{array}{l}377 \mathrm{H}, 382 \mathrm{H}, 555 \mathrm{H}, \\
559 \mathrm{~N}, 658 \mathrm{I}\end{array}$ \\
\hline Pseudomanas aenginasa UCBPP-PA14 & CP000438.1 & 2058 & 685 & 357 WQMAK 361 & $\begin{array}{l}377 \mathrm{H}, 382 \mathrm{H}, 555 \mathrm{H}, \\
559 \mathrm{~N}, 658 \mathrm{I}\end{array}$ \\
\hline Shewanella demitrificans OS 217 & NC_007954.1 & 1857 & 618 & 293 WQAAM 297 & $\begin{array}{l}313 \mathrm{H}, 318 \mathrm{H}, 491 \mathrm{H}, \\
495 \mathrm{~N}, 618 \mathrm{I}\end{array}$ \\
\hline Shew anella woodyi A TCC 51908 & ZP_01543473.1 & 2145 & 714 & 384 WQMAK 388 & $\begin{array}{l}403 \mathrm{H}, 407 \mathrm{H}, 593 \mathrm{H}, \\
597 \mathrm{R}, 714 \mathrm{~N}\end{array}$ \\
\hline Shew anella woody A TCC 51908 & NZ_AAU001000023.1 & 2271 & 756 & 422 WKMAK 426 & $\begin{array}{l}441 \mathrm{H}, 445 \mathrm{H}, 635 \mathrm{H}, \\
639 \mathrm{~N}, 756 \mathrm{~N}\end{array}$ \\
\hline Shew anella woodyi A TCC 51908 & NZ AAUO01000010.1 & 2178 & 725 & 383 WQIAK 387 & $\begin{array}{l}403 \mathrm{H}, 408 \mathrm{H}, 600 \mathrm{H}, \\
604 \mathrm{~N}, 725 \mathrm{I}\end{array}$ \\
\hline
\end{tabular}

Table 3. Alignment and position of amino acids that bind the iron atom in the sequence typical of lipoxygenases (WXXAK) of all sequences of fungi used in this study.

\begin{tabular}{|l|l|c|c|c|c|}
\hline \multicolumn{1}{|c|}{ Species } & GenBank acces & pb gen & aa gen & W - - A K & Ligands of Fe \\
\hline Gibberella zeae & XP_382392.1 & 2238 & 745 & 387 WRYAK 391 & $407 \mathrm{H}, 412 \mathrm{H}, 596 \mathrm{H}, 600 \mathrm{~N}, 745 \mathrm{I}$ \\
Chaetomium globosum & XP_001225066.1 & 2256 & 751 & 409 WRYAK 413 & $429 \mathrm{H}, 434 \mathrm{H}, 611 \mathrm{H}, 615 \mathrm{~N}, 751 \mathrm{I}$ \\
Neurospora crassa & CAD37061.1 & 2268 & 755 & 411 WRYAK 415 & $431 \mathrm{H}, 436 \mathrm{H}, 615 \mathrm{H}, 619 \mathrm{~N}, 755 \mathrm{I}$ \\
Aspergillus fumigatus & XP_746844.1 & 2235 & 744 & 398 WRYAK 402 & $418 \mathrm{H}, 423 \mathrm{H}, 603 \mathrm{H}, 607 \mathrm{~N}$ 744 I \\
\hline
\end{tabular}


Regarding the characteristic sequence of lipoxygenases (WXXAK), the high conservation of tryptophan (W) and alanine (A) is clear, as is, to a lesser extent, the lysine $(\mathrm{K})$, which can be altered by methionine $(\mathrm{M})$ in the case of sequences of bacterial origin (Tables 2 and 3 ).

There was no need to check the animal and plant sequences, as these lipoxygenases have been studied thoroughly and, in some cases, such as the lipoxygenase of Plexaura homomolla, the crystal structure is published [34].

As can be seen in the phylogenetic tree (Fig. 18), the lipoxygenases form a heterogeneous family of enzymes with clearly different subfamilies, corresponding to animal organisms, plant organisms, fungal organisms and bacterial organisms. This kind of grouping (animal, plant and bacterial lipoxygenases) was described in 2007 [35] and is confirmed by the addition of a new lipoxygenase subfamily, the bacterial lipoxygenases consisting of lipoxygenases of Nitrospira, Nitrosomonas, Photobacterium, and Shewanella woodyi.

There are several interesting points about this phylogenetic tree (Fig. 18). First, the case of Myxососcus xanthus, which is outside the group of bacterial lipoxygenases, closer to animal lipoxygenases than the vegetable ones, a phenomenon published in 2008 [27], might suggest, as was proposed in 2001, a case of horizontal transfer [28]. The same occurs for the Plexaura homomolla lipoxygenase that appears outside the group of animal lipoxygenases in a phylogenetic tree published in 2007 [35].

The case of the two Shewanella woodyi sequences ( $a$ and $b$ ), which are clearly outside the group of bacterial lipoxygenases, has never been published. It seems quite reasonable to attribute this to a failure in the annotation of these genes, because although they retain the typical sequence (WXXAK) of lipoxygenases, the typical valine or isoleucine terminal is not present.

The subfamily of bacterial lipoxygenases does not show degrees of similarity to the animal and plant subfamilies, but the subgroup formed by the lipoxygenases of Pseudomonas, Burkholderia, Photobacterium, and Shewanella denitrificans (all these organisms are proteobacteria) do have a degree of similarity with animal and plant lipoxygenase subfamilies.

It is necessary to take into account two key aspects in this kind of analysis. First, the quality of the sequences that are to be worked with must be checked and, second, a high number of sequences is recommended. In the case of this study, this second aspect was improved, due to the few sequences classified as lipoxygenase ones present in databases. As regards the first aspect, it is revealed that, although some sequences have features that are typical of sequences of lipoxygenases, they may not be such sequences. 


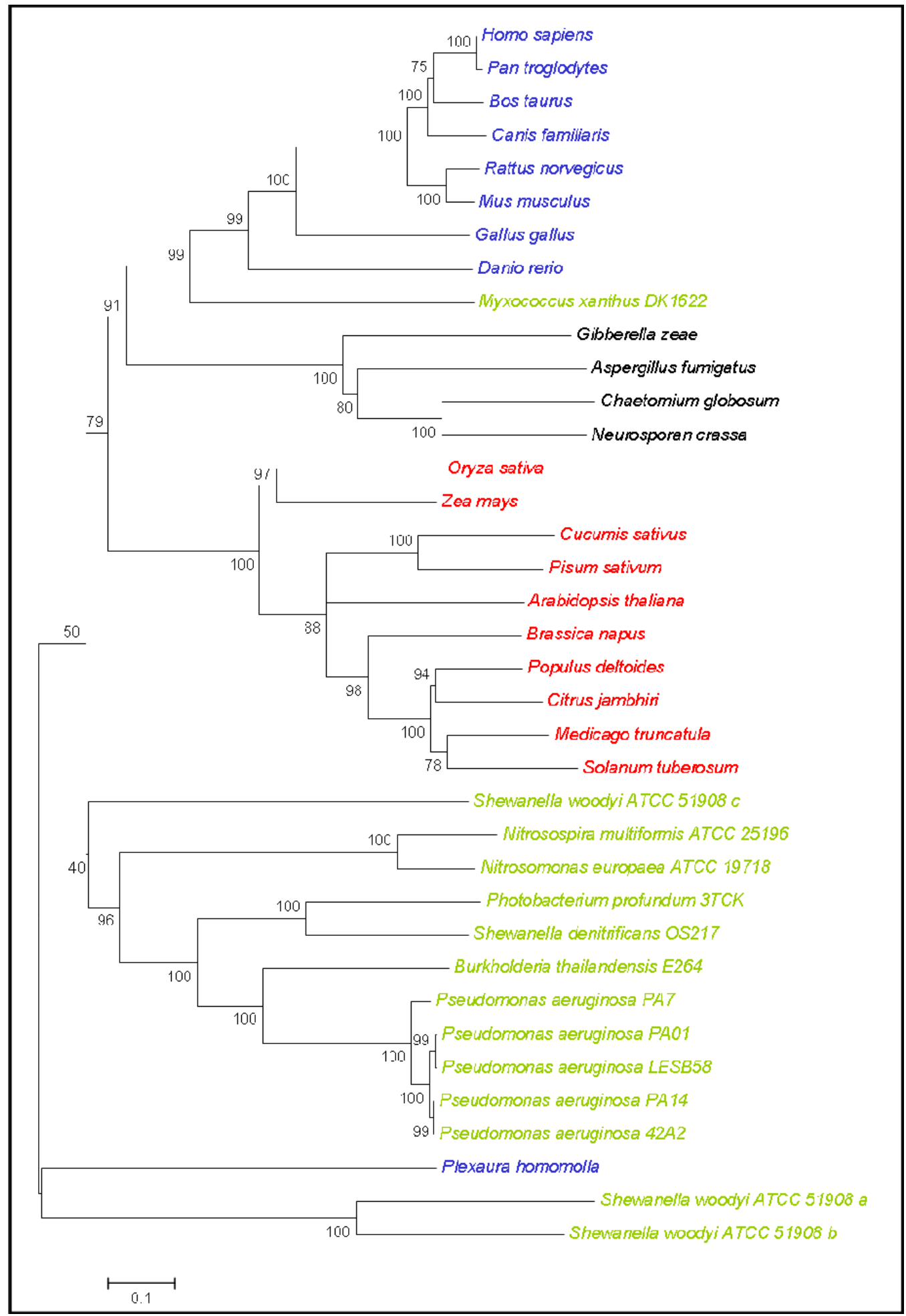

Figure 18. Consensus phylogenetic tree constructed using MEGA4 program under the Neighbor-Joining method with 1000 bootstrap replicates and the Juke-Cantor model (calculation of distance) from an alignment of nucleotide sequences. All positions containing gaps were removed from the dataset (Complete deletion option). In red, vegetal lipoxygenases, in blue, animal lipoxigenases, in black fungal lipoxygenases and in green, bacterial lipoxygenases. 


\section{Conclusions}

1. We have obtained the first crystal of a bacterial lipoxygenase obtaining a new model for crystallographic lipoxygenases. The general features of this crystal with orthorhombic system are:

- Structure consisting of a single-domain unlike lipoxygenases described so far, which show two domains.

- Presence of a structure consisting of two antiparallel $\alpha$ helices adjacent to the opening of the cavity of the substrate which could act as a lid and has not been previously described.

- Presence of a secondary cavity-shaped channel which would allow access of molecular oxygen to the active centre of the enzyme.

2. The determination of the crystal structure has revealed a strong interaction between the catalytic cavity of the enzyme molecule and a phospholipid (O-(sn-1-cis-vaccenil-sn-2-miristoil-sn-glyceric-3-phosphoryl) ethanolamine). The aliphatic chain of 18 carbons of phospholipid reaches the active site of the enzyme while the other aliphatic chain (14 carbons) interacts with the new structure of two antiparallel $\alpha$ helices.

3. The existence of a subfamily of bacterial lipoxygenases has been confirmed. This subfamily has a greater diversity than in the corresponding eukaryotic lipoxygenases.

\section{References}

1. Brash, A. R. 1999, J. Biol. Chem. 274, 23679.

2. Funk, C. D. 2001, Science 294, 1871.

3. Serhan, C. N., Arita, M., Hong, S., Gotlinger, K. 2004, Lipids 39, 1125.

4. Smith, W. L., DeWitt, D. L., Garavito, R. M. 2000, Annu. Rev. Biochem. 69, 145.

5. Liavonchanka, A., and Feussner, I. 2006, Plant Physiol. 163, 348.

6. Boyington, J. C., Gaffney, B. J., Amzel, L. M. 1993, Science 260, 1482.

7. Minor, W., Steczko, J., Stec, B., Otwinowski, Z., Bolin, J. T., Walter, R., Axelrod, B. 1996, Biochemistry 35, 10687.

8. Gillmor, S. A., Villaseñor, A., Fletterick, R., Sigal, E., Browner, M. F. 1997, Nat. Struct. Biol. 4, 1003.

9. Skrzypczak-Jankun, E., Bross, R. A., Carroll, R. T., Dunham, W. R., Funk, M.O., Jr. 2001, J. Am. Chem. Soc. 123, 10814.

10. Dainese, E., Sabatucci, A., van Zadelhoff, G., Angelucci, C. B., Vachette, P., Veldink, G. A., Agro, A. F., Maccarrone, M. 2005, J. Mol. Biol. 349, 143.

11. Hammel, M., Walther, M., Prassl, R., Kuhn, H. 2004, J. Mol. Biol. 343, 917.

12. Oldham, M. L., Brash, A. R., Newcomer, M. E. 2005, J. Biol. Chem. 39, 39545. 
13. Krieg, P., Marks, F., Fürstenberger, G. 2001, Genomics 73, 323.

14. Funk, C. D., Chen, X. S., Johnson, E. N., Zhao, L. 2002, Prostaglandins Other Lipid Mediat. 68-69, 303.

15. Schneider, C., Brash, A. R. 2002, Prostaglandins Other Lipid Mediat. 68-69, 291.

16. Feussner, I., Wasternack, C. 2002, Annu. Rev. Plant Biol. 53, 275.

17. Yin, H., Porter, N. A. 2005, Antioxid. Redox Signal. 7, 170.

18. Schneider, C., Pratt, D. A., Porter, N. A., Brash R. A. 2007, Chemistry \& Biology $14,473$.

19. Rizo, J., Sudhof, T. C. 1998, J. Biol. Chem. 273, 15879.

20. Youn, B., Sellhorn, G. E., Mirchel, R. J., Gaffney, B. J., Grimes, H. D., Kang, C. 2006, Proteins, 65, 1008.

21. Kuhn, H., Saam, J., Eibach, S., Holzhütter, H. G., Ivanov, I., Walther, M. 2005, Biochem. Biophys. Res. Commun., 338, 93

22. Funk, C. D. 1996, Biochim. Biophys. Acta Lipids Lipid Metabol. 1304, 65.

23. Shibata, D., Axelrod, B. 1995, J. Lipid Mediators Cell Signalling 12, 213.

24. Gaffney, B. J. 1996, Annu. Rev. Biophys. Biomol. Struct. 25, 431.

25. Andreou, A., Feussner, I. 2009, Phytochemistry 70, 1504.

26. Tomchick, D. R., Phan, P., Cymborowski, M., Minor, W., Holman, T. R. 2001, Biochemistry 40, 7509.

27. Lang, I., Göbel, C., Porzel, A., Heilmann, I.; Feussner, I., 2008, Biochem. J. 410, 347.

28. Porta, H., Rocha-Sosa, M. 2001, Microbiology 147, 3199.

29. Neau, D. B., Gilbert, N. C., Bartlett, S. G., Boeglin, W., Brash, A. R., Newcomer, M. E., 2009, Biochemistry 48, 7906.

30. Choi, J., Chon, J. K., Kim, S., Shin, W. 2008, Proteins 70, 1023.

31. Ratledge, C., Wilkinson, S. G. 1988, "Microbial lipids Volume 1, Chapter 7: Gram-negative bacteria."

32. Coffa, G., Brash, A. R., 2004, Proc. Natl. Acad. Sci. USA 101, 15579.

33. Solomon, E. I., Zhou, J., Neese, F., Pavel, E. G., 1997, Chem. Biol 4, 795.

34. Brash, A. R., Boeglin, W. E., Chang, M. S., Shieh, B. H. 1996, J. Biol. Chem. 271, 20949.

35. Koeduka, T., Kajiwara, T., Matsui, K. 2007, Curr. Microbiol. 54, 315. 


\title{
12. Cryptosporidium sp. in public swimming pools in Barcelona
}

\author{
Ma Soledad Gómez', Mercedes Gracenea', Laia Angel' \\ and Vicenç Beneyto ${ }^{2}$ \\ 'Laboratory of Parasitology, Faculty of Pharmacy, University of Barcelona. Avda Joan \\ XXIII s/n Barcelona, Spain; ${ }^{2}$ Public Health Agency of Barcelona. Avda. Principe \\ de Asturias 63-65, 08012, Barcelona
}

\begin{abstract}
The presence of Cryptosporidium oocysts in swimming pools in Barcelona was studied. The possible influence of the different parameters related to water conditions, pool structure and users has also been analyzed. Cryptosporidium oocysts were detected in $54 \%$ of the swimming pools analyzed and in $85 \%$ of the sport centers checked. Oocysts concentrations ranged from 0 to 30 oocysts/L. Among parameters related to water conditions, there was no relationship between oocyst presence and water $\mathrm{pH}$, but the influence of temperature was statistically significant $(\mathrm{p}<0.05)$ $\left(\chi^{2}=0.024\right)$. Among parameters related to pool structure, the filter backwash water samples revealed a higher percentage of parasitation $(58.8 \%)$ than the prefilter ones $(42.9 \%)$. With the medium volume pools $\left(100-500 \mathrm{~m}^{3}\right)$, the percentage was higher $(80 \%)$. Covered and uncovered pools presented similar prevalences for Cryptosporidium oocysts. Looking at two of the user related parameters, swimming pools with more users and children's swimming pools showed
\end{abstract}


highest presence of oocysts, $75 \%$ and $66.7 \%$ respectively. Cryptosporidium oocysts were found in all the studied neighborhoods, and this factor exerted a statistically significant influence on the concentration of oocysts $(p<0.05)\left(\chi^{2}=0.021\right)$.

\section{Introduction}

Nowadays, society's concern for our physical and psychological wellbeing has popularized sports and therapies involving water. For this reason, many sport centers include swimming pools and Jacuzzis where the partial recirculation of water and the high concentration of people act as a perfect environment for the development and transmission of some human pathogens, such us Cryptosporidium, which is a significant waterborne protozoa parasite. It is a cosmopolitan human enteropathogen with fecal-oral transmission, capable of surviving in the environment for prolonged periods. Its direct life cycle includes an intracellular intestinal phase inside the host and a stage, oocyst, which is highly resilient in the face of environmental stress and standard water treatment technologies [1,2]. The oocyst is microscopic in size, already infectious at the time of shedding, its infection dose is low and it is excreted in high numbers and for a long period of time [3].

Clinical symptoms of cryptosporidiosis are variable but frequently manifest as an acute or chronic diarrhea that severely affects people with immuno-deficiencies [4]. Cryptosporidium hominis and C. parvum are the most frequent species found in humans [5].

Several waterborne outbreaks of cryptosporidiosis have been reported worldwide [6-8]. Among them, some have been related to swimming pool activities $[5,9,10]$. In swimming pools, several adverse conditions may exist, such as poor hygiene [11], defective filtration systems, animals and rain runoff, all of which favor oocyst occurrence. In addition, many recreational water facilities allow users of any age including, among them, groups that could suffer fecal incontinence.

The objective of this study was to achieve for the first time a qualitative and quantitative determination of presence of Cryptosporidium oocysts in swimming pools in the Barcelona area, as well as to analyze the influence of physical, chemical and social swimming pool parameters over the presence of Cryptosporidium oocysts.

\section{Methods}

Samples were taken in collaboration with Agència de Salut Pública de Barcelona and collected from the filter backwash water or from the prefilter 

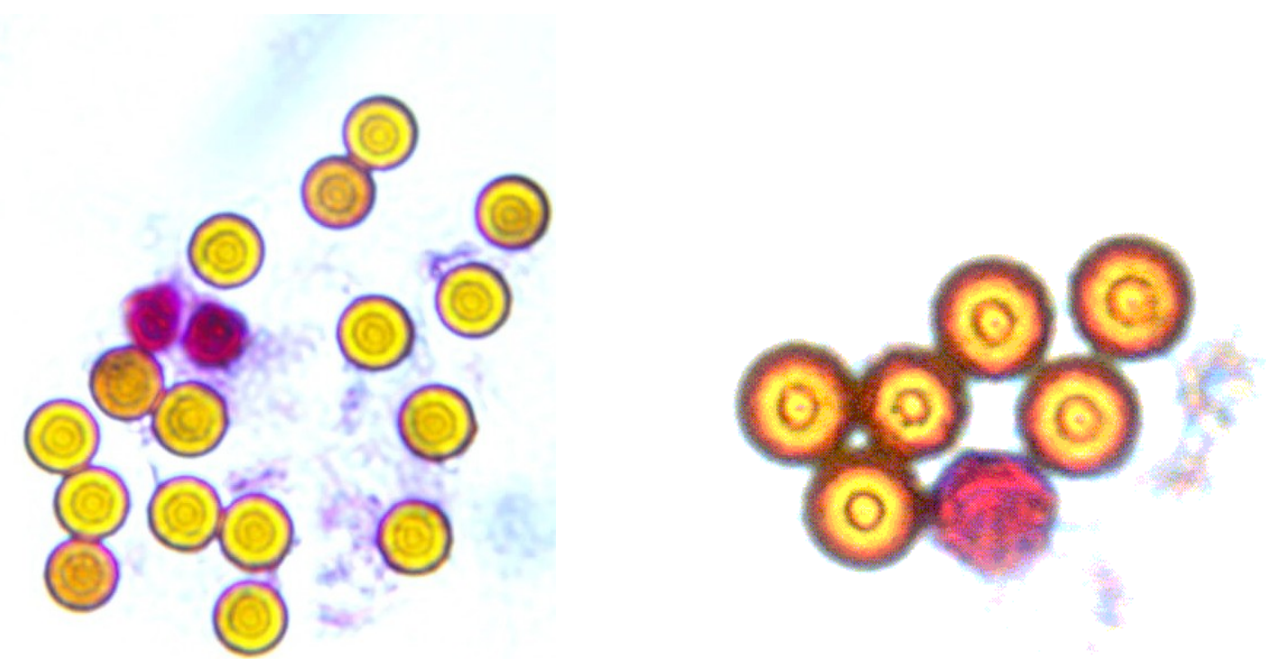

Figure 1. Cryptosporidum oocysts (red) bound to immunomagnetic spheres.

water depending on the facility conditions. Thirteen sport centers and 24 swimming pools were analyzed.

Data about water $\mathrm{pH}$ (6.9-7.9), chemical treatment (chlorine/bromine), temperature $\left(\sim 15{ }^{\circ} \mathrm{C}, 26-28{ }^{\circ} \mathrm{C}\right.$ and $\left.>32{ }^{\circ} \mathrm{C}\right)$, sampling point (filter backwash water/prefilter water), volume $\left(<100,100-750\right.$ and $\left.>750 \mathrm{~m}^{3}\right)$, swimming pool structure (covered/uncovered), average daily user number $(<100,100-500$ and $>500$ ), user age (children/adults), nine neighborhoods and thirteen sport centers were requested. Water was collected in $10 \mathrm{~L}$ drums and transported to the laboratory.

The analytic technique used to detect Cryptosporidium oocysts included a concentration by flocculation with calcium carbonate, isolation by immunomagnetic separation and identification by Ziehl-Neelsen staining (Fig. 1).

Statistical treatment was done with SPSS v12.

\section{Results and discussion}

Cryptosporidium oocysts were detected in $54 \%$ of the checked swimming pools and in $85 \%$ of the sport centers (Table 1). Although one of the swimming pools checked was temporarily out of use when the water sample was taken (number 7 in table 1), it has been considered in this study because it was still in use until one week prior to being checked for the parasite.

A study performed in Netherlands found a presence of $4.6 \%$ oocysts in 7 swimming pools analyzed over one year [12]. In Italy Cryptosporidium oocysts were detected in $40 \%$ of the swimming pools studied in 2004 and $28.6 \%$ in $2006[13,14]$. 
Table 1. Pools vs. temperature, volume, sampling point, daily users, user age, neighborhood and concentration of Cryptosporidium oocysts. (ND) not detected.

\begin{tabular}{|c|c|c|c|c|c|c|c|}
\hline Pools & Temp. & $V\left(m^{3}\right)$ & $\begin{array}{l}\text { Sampling } \\
\text { point }\end{array}$ & $\begin{array}{l}\text { Daily } \\
\text { users }\end{array}$ & User age & Neighbor-hood & Oocysts/L \\
\hline 1 & $26-28^{\circ} \mathrm{C}$ & 1400 & Filter & 400 & Adult & 1 & 30 \\
\hline 2 & $26-28^{\circ} \mathrm{C}$ & 460 & Filter & 400 & Adult, child & 2 & ND \\
\hline 3 & $26-28^{\circ} \mathrm{C}$ & 245 & Filter & 400 & Adult, child & 2 & 5 \\
\hline 4 & $26-28{ }^{\circ} \mathrm{C}$ & 566 & Filter & $150-200$ & Adult, child & 3 & 20 \\
\hline 5 & $26-28^{\circ} \mathrm{C}$ & 625 & Filter & $>100$ & Adult, child & 4 & ND \\
\hline 6 & & 3500 & Filter & 1000 & Adult, child & 5 & 10 \\
\hline 7 & $26-28^{\circ} \mathrm{C}$ & 1500 & Prefilter & No users & No users & 6 & ND \\
\hline 8 & $26-28^{\circ} \mathrm{C}$ & 3500 & Prefilter & 1000 & Adult, child & 6 & ND \\
\hline 9 & $26-28^{\circ} \mathrm{C}$ & 3500 & Prefilter & 1000 & Adult, child & 6 & 2.5 \\
\hline 10 & $26-28^{\circ} \mathrm{C}$ & 250 & Filter & 200 & Adult, child & 6 & 5 \\
\hline 11 & $26-28^{\circ} \mathrm{C}$ & 450 & Filter & 700 & Adult, child & 7 & 5 \\
\hline 12 & $26-28^{\circ} \mathrm{C}$ & 60 & Filter & 100 & Child & 7 & 5 \\
\hline 13 & $26-28^{\circ} \mathrm{C}$ & 200 & Prefilter & 150 & Adult, child & 2 & 20 \\
\hline 14 & $\geq 32{ }^{\circ} \mathrm{C}$ & 48.26 & Filter & 240 & Adult & 8 & ND \\
\hline 15 & $\sim 15^{\circ} \mathrm{C}$ & 14.62 & Filter & 135 & Adult & 8 & ND \\
\hline 16 & $26-28^{\circ} \mathrm{C}$ & 346 & Filter & 80 & Adult, child & 8 & 15 \\
\hline 17 & $26-28^{\circ} \mathrm{C}$ & 28.8 & Filter & 80 & Child & 8 & 5 \\
\hline 18 & $\geq 32{ }^{\circ} \mathrm{C}$ & 75 & Filter & 127 & Adult,child & 9 & ND \\
\hline 19 & $26-28^{\circ} \mathrm{C}$ & 468 & Filter & 169 & Adult, child & 9 & ND \\
\hline 20 & $26-28^{\circ} \mathrm{C}$ & 142 & Filter & 197 & Adult, child & 9 & 5 \\
\hline 21 & $\geq 32{ }^{\circ} \mathrm{C}$ & 1.6 & Prefilter & 40 & Adult, child & 7 & ND \\
\hline 22 & $\geq 32{ }^{\circ} \mathrm{C}$ & 1.6 & Prefilter & 40 & Adult, child & 7 & ND \\
\hline 23 & $26-28^{\circ} \mathrm{C}$ & 430 & Filter & 50 & Adult, child & 7 & 7.5 \\
\hline 24 & $26-28{ }^{\circ} \mathrm{C}$ & 18.9 & Filter & 50 & Child & 7 & ND \\
\hline
\end{tabular}

\section{Water parameters}

Among parameters related to water $(\mathrm{pH}$, chemical treatment and temperature), no statistically significant relation $\left(\mathrm{R}^{2}=0.692\right)$ was observed between the presence of Cryptosporidium oocysts and water $\mathrm{pH}$. The $\mathrm{pH}$ average was 6.9-7.9, close to neutrality, in all pools, so this parameter is not a deciding factor in the presence of oocysts.

Oocysts were present in $60 \%$ of the chlorine treated pools and in $44 \%$ of the bromine treated ones. Consequently, no statistically significant differences have been detected related to chemical water treatment $(p<0.05)$ 
$\left(\chi^{2}=0.459\right)$ but bromine seems to be more efficient than chlorine. In this sense, bromine $(1180 \mathrm{mg} / \mathrm{L}, 60 \mathrm{~min})$ reduces $89 \%$ of oocysts infectivity [15], and the reduction provided by hypochlorite is lower, although it does depend on temperature [16]. Cryptosporidium oocysts are highly resistant to chlorine, which is the typical chemical treatment added to swimming pool waters. A concentration of $30 \mathrm{mg} / \mathrm{L}$ of chlorine during $240 \mathrm{~min}$, a $\mathrm{pH}$ of 7 and a temperature of $25{ }^{\circ} \mathrm{C}$ are necessary in order to inactivate the $99 \%$ of oocysts [17]. To achieve the effective level of disinfection in a standard pool, $1 \mathrm{ppm}$ of chlorine is necessary [18], and it is important to note that oocysts can survive more than 6 days under these conditions [12].

The relationship between the presence of oocysts and temperature was statistically significant $(p<0.05) \quad\left(\chi^{2}=0.024\right)$. Swimming pools with temperatures above $32{ }^{\circ} \mathrm{C}$ and below $15{ }^{\circ} \mathrm{C}$ were negative for Cryptosporidium oocysts that were detected in $68 \%$ of the swimming pools at 26-28 ${ }^{\circ} \mathrm{C}$. The temperature can affect the survival of Cryptosporidium oocysts. Then, temperatures between 5 and $15{ }^{\circ} \mathrm{C}$ are adequate to maintain the infective capacity of oocysts [19]. Temperatures over this range seem to reduce their infective capacity and oocysts are inactivated in 72 hours with temperatures over $30{ }^{\circ} \mathrm{C}$ [20]. In general, the average temperature in swimming pools is $26-28{ }^{\circ} \mathrm{C}$ and Cryptosporidium oocysts can survive 12 to 24 weeks in aquatic environments with temperatures between 20 and $25^{\circ} \mathrm{C}$ [19]. Consequently, it is not expected that swimming pools at $26-28{ }^{\circ} \mathrm{C}$ temperature reduce the presence of the parasite.

\section{Swimming pool structure parameters}

Among parameters related with pool structure (sampling point, volume and covered or uncovered facility), it was observed that samples taken from filter backwash water were more positive $(58.8 \%)$ than those taken from prefilter $(42.9 \%)$ although the oocysts concentration was high in both points with a maximum of 30 and 20 oocysts/L respectively.

There are no statistically significant differences regarding the sampling point and its relation to the presence/absence of oocysts, although it should be pointed out that 3 of the 7 prefilter samples were positive with concentrations ranging from 2.5-20 oocysts/L. These values should be emphasized because they represent the actual concentration of oocysts in the pool. One of the filter backwash water samples had a higher concentration (30 oocysts/L). Although this was not the real swimming pool concentration, the risk of infection could be important in the event of filter washing failure or filter washing while the swimming pool is in use, because oocysts find their way back into the re-circulated water. 
The medium volume pools $\left(100-750 \mathrm{~m}^{3}\right)$ were the most positive $(80 \%)$, followed by big volume pools $(50 \%)$ and small pools $(25 \%)$ but the difference was not statistically significant $(p<0.05)\left(\chi^{2}=0.065\right)$. Shields et al. [21] analyzed swimming pools in Atlanta with different water volumes and no oocysts were detected in swimming pools exceeding $800 \mathrm{~m}^{3}$, while in Barcelona there was an important prevalence found in high volume ( $>1000$ $\mathrm{m}^{3}$ ) swimming pools. The authors found the protozoan in the $5.5 \%$ of the small swimming pools $\left(<20 \mathrm{~m}^{3}\right)$, which is similar to the data found in Barcelona. The medium volume swimming pools in Atlanta showed a $1.8-2.8 \%$ of prevalence while the prevalence was $80 \%$ in Barcelona.

Covered and uncovered pools presented similar prevalence $(54.5 \%$ and $50 \%$ respectively) for Cryptosporidium oocysts. Covered swimming pools prevent exogenous contamination from animal reservoirs such as birds and small mammals (rats, mice, etc.), that frequently carry the parasite and could contaminate the uncovered swimming pools close to their natural environments. Cryptosporidium hominis, a species that infects humans, has been detected even in doves from the Canary Islands, demonstrating capacity of the birds to act as reservoirs [22]. In covered swimming pools, the water contamination has a human origin, mainly due to fecal accidents.

\section{Water user parameters}

Among parameters related to users (average daily user number, ages, neighborhood location and sport centre), the results revealed that swimming pools with more users $(>500)$ were the most positive in oocysts $(75 \%)$ although without statistical significance $(p<0.05)\left(\chi^{2}=0.657\right)$. This seems reasonable bearing in mind that users are the main contamination source. In Atlanta, Shields et al. [21] described the opposite situation, detecting the parasite in swimming pools with less than 10 daily users. They observed prevalence of $4.4 \%$ in low-user swimming pools while in Barcelona it was $50 \%$. But the user number was always higher in Barcelona, with a daily minimum of 40 users.

Prevalence in children-user swimming pools was the most positive (66.7\%), but the same pools also displayed the lowest oocysts concentrations (0-5 oocysts/L), whereas the opposite happened at the adult-user swimming pools $(33.3 \%$ and $0-30$ oocysts/L), although differences were not statistically significant. Data were different than those from Atlanta, where Shields et al. [21] found Cryptosporidium oocysts as follows: when entry was allowed only for children, $3.5 \%$ of pools were positive; in those for children and adults, $2.2 \%$ presented the protozoa; while they did not find oocysts in adult swimming pools. 
Cryptosporidium oocysts were found in all the neighborhoods studied, and the oocyst concentration in them has a statistically significant relation $(p<0.05) \quad\left(\chi^{2}=0.021\right)$. Nevertheless, differences in the parasite presence/absence and in the swimming pool location were not statistically significant. In neighborhood 2, the number of positive swimming pools (2) was higher than the number of negative ones (1). Only one swimming pool was analyzed in each of neighborhoods 1, 3 and 5, and in all three cases they were positive. Neighborhoods 6 and 9 had a minimum positivity (1 of 3 ). As the named neighborhoods have no remarkable differences in terms of demographics, the parasite distribution is not easily explained. Neighborhood 2 stands out for the high prevalence (20 oocysts/L) detected directly in the swimming pool.

The pools are located in 13 sport centers, and among these centers only 2 tested negative for the parasite. Statistically significant differences were not observed in sport centers samples and their Cryptosporidium oocysts presence/absence, but these differences were close to being statistically significant $(\mathrm{p}<0.05)\left(\chi^{2}=0.061\right)$.

\section{Conclusion}

- There was a presence of Cryptosporidium oocysts in more than half of the checked swimming pools in Barcelona.

- There were high parasitic concentrations even in prefilter water which corresponds to the real value in the vessel. The oocyst concentration ranged from 0 to 30 oocysts/L in the filter backwash water.

- Water temperature influenced the presence of Cryptosporidium oocysts.

- Neighborhood location of the pool within Barcelona seems to have had an influence on the Cryptosporidium oocysts concentration.

- The swimming pool volume and the sport centre in which the pool is found may have influenced both the presence and concentration of Cryptosporidium oocysts.

- $\quad \mathrm{pH}$, treatment, structure, sampling point, age and number of users had no relation to either presence or concentration of Cryptosporidium oocysts.

\section{References}

1. Fayer, R. 2008. Biology. In Fayer, R., and Xiao, L. (Eds.), Cryptosporidium and cryptosporidiosis. Second ed. CRC Presss and IWA Publishing, Boca Raton, FL. pp 1-12.

2. Nichols, G., Chalmers, R., Lake, I., Sopwith, W., Regan, M., Hunter, P., Grenfell, P., Harrison, F., and Lane, C. 2006, Cryptosporidiosis, A report on the 
surveillance and epidemiology of Cryptosporidium infection in England and Wales. Drinking water directorate contract number DWI 70/2/201, London.

3. Jokipii, L., and Jokipii, A.M.M. 1986, New Eng. J. Med., 315, 1643.

4. Hunter, P.R., and Nichols, G. 2002, Cl. Mic. Rev., 15, 145.

5. Xiao, L., and Ryan U.M. 2006, Molecular epidemiology. In Fayer, R., Xiao, L. (Eds.), Cryptosporidium and cryptosporidiosis. Second ed. CRC Press and IWA Publishing, Boca Raton, FL. pp 119-171.

6. Craun, G.F., Nwachuku, N., Calderon, R.L., and Craun, M.F. 2002, J. Environ. Health, 65, 16.

7. Karanis, P., Kourenti, V., and Smith, H. 2007, J. Water Health, 51.

8. Slifko, T.R., Smith, H.V., and Rose, J.B. 2000, Int. J. Parasitol., 30, 1370.

9. CDC 1990, Mor. Mort. W. R., 39, 343.

10. Nichols, G., Lake, I., Chalmers, R., Bentham, G., Harrison, F., Hunter, P., Kowats, S., Grundy, C., Anthony, S., Lyons, H., Agnew, M., and Proctor, C. 2009, Intervention in waterborne diseases. In: Giardia and Cryptosporidium: From molecules to disease. CAB International Ed., UK, 227-237.

11. Gerba, C.P. 2000, Q. Microb., 2, 55.

12. Schets, F.M., Engels, G.B., and Evers, E.G. 2004, J. W. Health, 2, 191.

13. Bonadonna, I., Briancesco, R., Magini, V., Orsini, M., and Romano-Spica V. 2004, Ann. di Igiene, 16, 709.

14. Oliveri, R., Di Piazza, F., Marsala, B., Cerame, G., Firenze, A., and Di Benedetto, M.A. 2006, An. di Igiene, 18, 367.

15. Ransome, M.E., Whitmore, T.N., and Carrington, E.G. 1993, W. Supply, 11, 103.

16. Weir, S.C., Pokorny, N.J., Carreno, N.A., Trevors, J.T., and Lee, H. 2002, App. Environ. Microbiol., 68, 2576.

17. World Health Organization recreative waters prevention guide: http://www.who.int/water_sanitation_health/bathing/recreaII-ch3.pdf, (accessed $3^{\text {d }}$ Oct. 2009).

18. National Recreation and Park Association 1999, The Encyclopedia of Aquatic Codes and Standards. NRPA. Ashburn, VA.

19. Fayer, R., Trout, J.M., and Henkins, M.C. 1998, J. Parasit., 84, 1165.

20. King, B.J., Keegan, A.R., Monis, P.T., and Saint, C.P. 2005, Ap. Env. Microbiol., 71,3848 .

21. Shields, J.M., Gleim, E.R., and Beach, M.J. 2008, Em. Infec. Dis., 14, 948.

22. Abreu-Acosta, N., Foronda-Rodríguez, P., López, M., and Valladares, B. 2009, Acta Parasitol., 54, 1. 